\title{
Synthesis, Characterization, and Reactivity of Iron Trisamido-Amine Complexes that Undergo both Metal- and Ligand-Centered Oxidative Transformations
}

Remle Çelenligil-Çetin, ${ }^{\ddagger}$ Patrina Paraskevopoulou, ${ }^{\dagger}$ Rupam Dinda, ${ }^{\dagger}$ Richard J. Staples, ${ }^{\S}$ Ekkehard Sinn, ${ }^{\dagger}$ Nigam P. Rath, ${ }^{\#}$ and Pericles Stavropoulos ${ }^{* \dagger}$

Contribution from the Departments of Chemistry of the University of Missouri-Rolla, Rolla, Missouri 65409, Boston University, Boston, Massachusetts 02215, Harvard University, Cambridge, Massachusetts 02138, and the University of Missouri-St. Louis, St. Louis, Missouri 63121

\section{Experimental Section}

General Considerations. All operations were performed under anaerobic conditions under a pure dinitrogen or argon atmosphere using Schlenk techniques on an inert gas/vacuum manifold or in a dry-box $\left(\mathrm{O}_{2}, \mathrm{H}_{2} \mathrm{O}<1 \mathrm{ppm}\right)$. Anhydrous diethyl ether, dichloromethane, acetonitrile, tetrahydrofuran, hexane, benzene, toluene, dimethylformamide, and dimethylsulfoxide were purchased from Sigma-Aldrich. Ethanol was distilled over the corresponding magnesium alkoxide, and acetone was distilled over drierite. Solvents were degassed by three freeze-pump-thaw cycles. Unless otherwise noted, all other reagents were purchased at the highest purity available. Potassium hydride was provided as dispersion in mineral oil and was thoroughly washed prior to use

with copious amounts of tetrahydrofuran followed by hexane. ${ }^{1} \mathrm{H}-\mathrm{NMR}$ and ${ }^{13} \mathrm{C}-\mathrm{NMR}$ 
spectra were recorded on a Varian XL-400, Varian INOVA/UNITY $400 \mathrm{MHz}$ and Varian 300 Unity Plus FT-HR-NMR spectrometers. FT-IR spectra were obtained on Nicolet Nexus 470, 670, and Magna 750 FT-IR ESP spectrometers. UV-vis spectra were obtained on a Hewlett-Packard 8452A diode array spectrometer and a Varian Cary 50 spectrophotometer. EI and CI mass spectra were obtained on a Finnigan MAT-90 mass spectrometer. X-band EPR spectra were recorded on a Bruker Instruments Model ESP 300 with an EMX upgrade, supported by an APD Cryogenics Co. Cryostat HELI-TRAN model LTR-3, located at the Chemistry Department of Dartmouth College, NH. Microanalyses were done by Quantitative Technologies Inc., Whitehouse, NJ, and on an in-house Perkin-Elmer $2400 \mathrm{CHN}$ analyzer.

Ligand Synthesis. 2,2',2"-Trinitrotriphenylamine. This is a modified literature procedure. A 500-mL three-neck Schlenk flask was charged with 2-nitroaniline (20.0 g, $145 \mathrm{mmol}$ ) and 2-fluoronitrobenzene (62 g, $440 \mathrm{mmol})$ in $250 \mathrm{~mL}$ anhydrous DMSO. To this yellow-orange solution was slowly added oven-dried and pulverized $\mathrm{K}_{2} \mathrm{CO}_{3}(120 \mathrm{~g}$, $870 \mathrm{mmol}$ ) under a dinitrogen atmosphere. The solution turned purple and was continuously heated at $120^{\circ} \mathrm{C}$ in an oil bath for 48 hours under nitrogen. After 48 hours, the solution was allowed to cool down to room temperature and was then poured over ice. The slurry was stirred for about half an hour and filtered. The solid was washed with hot water $(2 \mathrm{~L})$ and filtered to remove the unreacted potassium salt, and was then allowed to dry under vacuum overnight. The resulting solid, which contains the desired product and 2,2'-dinitrodiphenylamine, was dissolved in hot acetone. The residual yellow solid was filtered and recrystallized repeatedly from hot acetone to yield the desired product ( $28 \mathrm{~g}$, 
$51 \%$ yield). ${ }^{1} \mathrm{H}-\mathrm{NMR}\left(\mathrm{CDCl}_{3}, 7.24 \mathrm{ppm}\right): \delta 7.818(\mathrm{~d}, 3 \mathrm{H}, J=1.6 \mathrm{~Hz}), 7.511(\mathrm{t}, 3 \mathrm{H}, J=$ $2.0 \mathrm{~Hz}), 7.276(\mathrm{t}, 3 \mathrm{H}, J=1.2 \mathrm{~Hz}), 7.199(\mathrm{~d}, 3 \mathrm{H}, J=1.2 \mathrm{~Hz}) .{ }^{13} \mathrm{C}-\mathrm{NMR}\left(\mathrm{CDCl}_{3}\right): \delta 144.1$, $138.9,134.1,128.6,126.5,126.2$.

2,2',2"-Triaminotriphenylamine. A Fisher-Porter hydrogenation bottle made of thick glass was charged with $2,2^{\prime}, 2^{\prime \prime}$-trinitrotriphenylamine (5 g, $\left.13 \mathrm{mmol}\right), \mathrm{Pd} / \mathrm{C}$ (50 mg, $10 \% \mathrm{Pd}$ ), and $300 \mathrm{~mL}$ ethanol. A heating mantle was used to warm the flask externally. The flask was pressurized with hydrogen gas to about 30 psig, and kept at $60{ }^{\circ} \mathrm{C}$ (measured by means of a thermometer placed between the heating mantle and the flask) until the color of the solution turned dark gray with formation of an off-white precipitate. The flask was allowed to cool down to room temperature and was then disconnected from the hydrogen feed carefully and connected to a nitrogen atmosphere. The solvent was pumped away under vacuum, and the crude material was purified by dissolving it in degassed hot acetone under strictly anaerobic conditions (otherwise the solid oxidizes to give intractable red-purple solutions), followed by filtering to remove the palladium catalyst. The pale yellow filtrate can then be pumped to dryness under vacuum, suspended in hot hexane and filtered to remove unreacted nitro precursors (1.85 g, $50 \%)$. ${ }^{1} \mathrm{H}-\mathrm{NMR}\left(\mathrm{CDCl}_{3}, 7.24 \mathrm{ppm}\right): \delta 7.967$ (t, 3H, $\left.J=1.2 \mathrm{~Hz}\right), 6.899$ (d, 3H, $\left.J=1.2 \mathrm{~Hz}\right), 6.693$ (m, $6 \mathrm{H}, J=1.2 \mathrm{~Hz}), 3.720\left(\mathrm{~s}, 6 \mathrm{H}, \mathrm{NH}_{2}\right) .{ }^{13} \mathrm{C}-\mathrm{NMR}\left(\mathrm{CDCl}_{3}\right): \delta 141.6,132.7,125.9,125.8$, 119.0, 116.6. Elemental analysis: Calcd. for $\mathrm{C}_{18} \mathrm{H}_{18} \mathrm{~N}_{4} \mathrm{C}, 74.46 ; \mathrm{H}, 6.25 ; \mathrm{N}, 19.29$. Found C, 74.87; H, 6.45; N, 19.53.

[(4-tert-Butyl- $\left.\mathbf{C}_{6} \mathbf{H}_{4}\right)-\mathbf{N H}-\left(\right.$ ortho- $\left.\left.\mathbf{C}_{6} \mathbf{H}_{4}\right)\right]_{3} \mathbf{N}\left(\mathbf{L}^{1} \mathbf{H}_{3}\right)$. A 250-mL Schlenk flask was charged with $\left(2-\mathrm{NH}_{2}-\mathrm{C}_{6} \mathrm{H}_{5}\right)_{3} \mathrm{~N}(1.59 \mathrm{~g}, 5.46 \mathrm{mmol})$, 4-tert-butylbromobenzene $(3.35 \mathrm{~g}$, 
$15.72 \mathrm{mmol})$, and tert-Bu-ONa $(2.36 \mathrm{~g}, 25 \mathrm{mmol})$. The slurry was stirred in anhydrous toluene. In another Schlenk flask, $\operatorname{Pd}_{2}(\mathrm{dba})_{3}(0.125 \mathrm{~g}, 0.137 \mathrm{mmol})$ and BINAP $(0.255 \mathrm{~g}$, $0.41 \mathrm{mmol}$ ) were stirred in anhydrous toluene, and heated until BINAP dissolved. The resulting cherry-red solution was transferred into the former flask via a cannula. The slurry was stirred at $100{ }^{\circ} \mathrm{C}$ for 36 hours to give a brown solution, with precipitation of a solid. The solution was allowed to cool to room temperature followed by addition of ether. The brown solution was filtered, and the filtrate was evaporated under vacuum to give a brown oily residue. The residue was dissolved in minimum amount of hexane$1.5 \%$ ethyl acetate and loaded onto a silica column $(20 \mathrm{~cm}$ length $\times 5 \mathrm{~cm}$ diameter) that had been previously washed with hexane. The column was first eluted with hexane, and then with a hexane- $1.5 \%$ ethyl acetate mixture. The first eluent is the product. All eluents containing the product were combined and evaporated under vacuum to give yellow oil. The oil eventually solidified to give off-white solid (1.87 g, $50 \%) .{ }^{1} \mathrm{H}-\mathrm{NMR}\left(\mathrm{CDCl}_{3}, 7.24\right.$ ppm): $\delta 7.347$ (d, 3H, $J=8 \mathrm{~Hz}), 7.166(\mathrm{~d}, 6 \mathrm{H}, J=8.4 \mathrm{~Hz}), 7.094(\mathrm{~d}, 6 \mathrm{H}, J=7.6 \mathrm{~Hz}), 6.883$ (t, 3H, $J=7.2 \mathrm{~Hz}), 6.686(\mathrm{~d}, 6 \mathrm{H}, J=8.4 \mathrm{~Hz}), 5.594(\mathrm{~s}, 3 \mathrm{H}, \mathrm{NH}), 1.290(\mathrm{~s}, 27 \mathrm{H}$, tertbutyl). ${ }^{13} \mathrm{C}-\mathrm{NMR}\left(\mathrm{CDCl}_{3}\right): \delta 144.26,140.29,138.93,135.93,126.08,125.86,121.28$, 118.96, 118.25, 34.30, 31.66. IR (KBr, $\left.\mathrm{cm}^{-1}\right): 3391,2907,2541,1897,1266,1106,1041$, 920, 844, 752, 613, 589, 548, 480, 457. MS-EI $(\mathrm{m} / \mathrm{z})$ : calcd. 686.99; found 687.3. Elemental analysis: Calcd. for $\mathrm{C}_{48} \mathrm{H}_{54} \mathrm{~N}_{4} \mathrm{C}, 83.92 ; \mathrm{H}, 7.92 ; \mathrm{N}, 8.16$. Found $\mathrm{C}, 84.12 ; \mathrm{H}$, $7.78 ; \mathrm{N}, 8.43$.

Synthesis of Starting Ferrous Complex: [( $\left.\left.\mathrm{L}^{1}\right) \mathrm{Fe}(\mathrm{II})-\mathrm{CH}_{3} \mathrm{CN}\right]\left[\mathrm{Ph}_{4} \mathrm{P}\right] \cdot 3 \mathrm{CH}_{3} \mathrm{CN}$ (1). The ligand $\mathrm{L}^{1} \mathrm{H}_{3}(0.343 \mathrm{~g}, 0.5 \mathrm{mmol})$ was dissolved in degassed THF $(15.0 \mathrm{~mL})$, to which 
$\mathrm{KH}(0.060 \mathrm{~g}, 1.5 \mathrm{mmol})$ was added. The mixture was stirred overnight to afford a yelloworange solution to which $\mathrm{FeCl}_{2}(0.0635 \mathrm{~g}, 0.5 \mathrm{mmol})$ was added as solid. This mixture was stirred for another 8 hours to give a dark green solution. This solution was then pumped to dryness, extracted with hexane $(2 \times 20 \mathrm{~mL})$ and filtered. The hexane filtrate was reduced in volume $(15.0 \mathrm{~mL})$ and was layered with $\mathrm{CH}_{3} \mathrm{CN}(15.0 \mathrm{~mL})$. The green color in the hexane layer was completely transferred to the $\mathrm{CH}_{3} \mathrm{CN}$ layer. The latter was separated and reduced $(10 \mathrm{ml})$. Upon addition of excess $\left[\mathrm{Ph}_{4} \mathrm{P}\right] \mathrm{Cl}$ to this solution a small amount of a light precipitate was formed. This was filtered and the filtrate was allowed to cool at $-40{ }^{\circ} \mathrm{C}$ to afford orange crystals of $1(50 \mathrm{mg}, 10 \%) .{ }^{1} \mathrm{H}-\mathrm{NMR}\left(\mathrm{CD}_{3} \mathrm{CN}, 1.935\right.$ ppm): $\delta 46.206,22.450,18.506,7.868,7.652,1.867,-6.285,-9.118,-32.359$. UV-vis (THF): $\lambda_{\max }\left(\varepsilon\left(\mathrm{M}^{-1} \mathrm{~cm}^{-1}\right)\right) 620$ (1718). Elemental analysis: Calcd. for $\mathrm{C}_{74} \mathrm{H}_{74} \mathrm{FeN}_{5} \mathrm{P}((\mathbf{1})-$ $\left.3 \mathrm{CH}_{3} \mathrm{CN}\right) \mathrm{C}, 79.36 ; \mathrm{H}, 6.61 ; \mathrm{N}, 6.26$. Found $\mathrm{C}, 78.87 ; \mathrm{H}, 6.75 ; \mathrm{N}, 6.53$.

Synthesis of Ferric Complexes: $\left[\left(\mathrm{L}^{1}\right) \mathrm{Fe}(\mathrm{III})-\mathrm{OH}\right]\left[\mathrm{PPh}_{4}\right] \cdot \mathrm{CH}_{3} \mathrm{CN} \cdot 0.5\left(\mathrm{C}_{2} \mathrm{H}_{5}\right)_{2} \mathrm{O}(2)$. The ligand $\mathrm{L}^{1} \mathrm{H}_{3}(0.343 \mathrm{~g}, 0.5 \mathrm{mmol})$ was dissolved in degassed THF $(15.0 \mathrm{~mL})$, to which $\mathrm{KH}(0.060 \mathrm{~g}, 1.5 \mathrm{mmol})$ was added. The mixture was stirred overnight to afford a yelloworange solution to which $\mathrm{FeCl}_{2}(0.0635 \mathrm{~g}, 0.5 \mathrm{mmol})$ was added as solid. This mixture was stirred for another 8 hours to give a dark green solution. This solution was then pumped to dryness, extracted with hexane $(2 \times 20 \mathrm{~mL})$ and filtered. The hexane filtrate was reduced in volume $(15.0 \mathrm{~mL})$ and was layered with $\mathrm{CH}_{3} \mathrm{CN}(15.0 \mathrm{~mL})$. The green color in the hexane layer was completely transferred to the $\mathrm{CH}_{3} \mathrm{CN}$ layer. The latter was separated and reduced $(10 \mathrm{ml})$. Extra dry dioxygen was bubbled through this solution for 5 min to generate a dark blue solution to which an excess of $\mathrm{PPh}_{4} \mathrm{Cl}$ was added and the 
mixture was allowed to stir overnight. The resulting solution was filtered and reduced to $5.0 \mathrm{~mL}$ under vacuum. Diethyl ether $(20.0 \mathrm{~mL})$ was carefully layered over the acetonitrile layer and the system was allowed to mix slowly at $-40{ }^{\circ} \mathrm{C}$ to afford blue, X-ray quality crystals of $2(0.100 \mathrm{~g}, 17 \%)$. IR $\left(\mathrm{KBr}, \mathrm{cm}^{-1}\right)$ : $v_{\mathrm{O}-\mathrm{H}} 3630,3447,3053,3021,2957,2899$, 2864, 1901, 1773, 1687, 1600, 1584, 1504, 1478, 1441, 1392, 1361, 1322, 1300, 1265, 1220, 1184, 1154, 1108, 1041, 997, 928, 880, 839, 739, 724, 689, 617, 558, 528. Elemental Analysis: Calcd. for $\mathrm{C}_{76} \mathrm{H}_{80} \mathrm{~N}_{5} \mathrm{O}_{1.5} \mathrm{FeP}(2)$ : C, 77.73; H, 6.87; N, 5.96. Found: C, 77.82; H, 6.35; N, 5.65.

$\left[\left(\mathbf{L}^{1}\right) \mathbf{F e}(\mathbf{I I I})-\mathbf{O H}\right]\left[\mathbf{K}(\mathbf{D M F})_{3}\right] \cdot \mathbf{3} \mathbf{H}_{2} \mathbf{O}(\mathbf{3})$. The ligand $\mathrm{L}^{1} \mathrm{H}_{3}(0.343 \mathrm{~g}, 0.5 \mathrm{mmol})$ was dissolved in degassed dimethylformamide $(10 \mathrm{~mL})$, to which $\mathrm{KH}(0.060 \mathrm{~g}, 1.5 \mathrm{mmol})$ was added. The mixture was stirred overnight to afford a yellow solution, to which $\mathrm{FeCl}_{2}$ $(0.0635 \mathrm{~g}, 0.5 \mathrm{mmol})$ was added as solid. This mixture was stirred for another 8 hours to give a dark yellow-green solution. This solution was filtered and reduced to $2.0 \mathrm{~mL}$. Extra dry dioxygen (99.9999\%) was passed through the concentrated DMF solution for 5.0 minutes to afford a dark blue solution that was filtered. Acetonitrile was allowed to diffuse slowly into the filtrate at $-25{ }^{\circ} \mathrm{C}$ to afford blue-black crystals of $\mathbf{3}(0.160 \mathrm{~g}, 30$ \%). IR $\left(\mathrm{KBr}, \mathrm{cm}^{-1}\right): v_{\mathrm{O}-\mathrm{H}} 3615,3057,3021,2960,2865,2559,2361,2201,1900,1669$, $1585,1484,1445,1408,1387,1362,1329,1213,1179,1150,1096,1045,1015,927$, $885,840,816,779,739,706,664,624,559,496,471,442,423$. UV-vis (THF): $\lambda_{\max }(\varepsilon$ $\left.\left(\mathrm{M}^{-1} \mathrm{~cm}^{-1}\right)\right)$ : 619 (3385). Elemental Analysis: Calcd. for $\mathrm{C}_{57} \mathrm{H}_{79} \mathrm{FeKN}_{7} \mathrm{O}_{7}$ (3): C, 64.03; H, 7.45; N, 9.17: K, 3.66. Found : C, 64.39; H, 7.84; N, 9.79; K, 3.30. 
$\left[\left(\mathbf{L}^{1}\right) \mathbf{F e}(\mathrm{III})-\mathbf{O H}\right]\left(\mathbf{H}_{3} \mathbf{O}\right) \cdot \mathbf{4} \mathbf{H}_{2} \mathbf{O} \cdot \mathbf{3}\left(\mathrm{Me}_{2} \mathbf{C O}\right)(\mathbf{4})$. The ligand $\mathrm{L}^{1} \mathrm{H}_{3}(0.343 \mathrm{~g}, 0.5 \mathrm{mmol})$ was dissolved in degassed dimethylformamide $(10.0 \mathrm{~mL})$, to which $\mathrm{KH}(0.060 \mathrm{~g}, 1.5$ mmol) was added. The mixture was stirred overnight to afford a yellow solution, to which $\mathrm{FeCl}_{2}(0.0635 \mathrm{~g}, 0.5 \mathrm{mmol})$ was added as solid. This mixture was stirred for another 8 hours to give a dark yellow-green solution. This solution was mixed with ether, containing small amounts of $\mathrm{H}_{2} \mathrm{O} /$ acetone, to afford a white precipitate, which was filtered off. The yellow-green filtrate was purged with wet dioxygen (technical grade) to afford blue crystals of $4(0.060 \mathrm{~g}, 14 \%)$ upon standing at room temperature. IR ( $\mathrm{KBr}$, $\left.\mathrm{cm}^{-1}\right): v_{\mathrm{O}-\mathrm{H}} 3616,3057,3021,2960,2865,2559,2361,2201,1900,1669,1585,1484$, $1445,1408,1387,1362,1329,1213,1179,1150,1096,1045,1015,927,885,840,816$, $779,739,706,664,624,559,496,471,442,423 . \mathrm{UV}$-vis $(\mathrm{THF}): \lambda_{\max }\left(\varepsilon\left(\mathrm{M}^{-1} \mathrm{~cm}^{-1}\right)\right)$ : 620 (3405). Elemental Analysis: Calcd. for $\mathrm{C}_{48} \mathrm{H}_{63} \mathrm{FeN}_{4} \mathrm{O}_{6}$ ((4) - 3Me $\left.{ }_{2} \mathrm{CO}\right)$ : C, 68.00; H, 7.43; N, 6.61. Found: C, 68.31; H, 7.28; N, 6.51.

[(L $\left.\left.\mathbf{L}^{1}\right) \mathbf{F e}(\mathbf{I I I})-\mathbf{D M F}\right]$ (5). The ligand $\mathrm{L}^{1} \mathrm{H}_{3}(0.343 \mathrm{~g}, 0.5 \mathrm{mmol})$ was dissolved in degassed dimethylformamide $(10 \mathrm{~mL})$, to which $\mathrm{KH}(0.060 \mathrm{~g}, 1.5 \mathrm{mmol})$ was added. The mixture was stirred overnight to afford a yellow solution to which $\mathrm{FeCl}_{2}(0.0635 \mathrm{~g}, 0.5$ mmol) was added as solid. This mixture was stirred for an additional 8 hours to give a dark yellow-green solution, which was filtered and evaporated to $2.0 \mathrm{~mL}$. Dichloromethane was allowed to diffuse into the concentrated DMF solution to generate a dark blue solution that deposited X-ray quality crystals of 5 (0.075 g, $16 \%)$. UV-vis (DMF): $\lambda_{\max }\left(\varepsilon\left(\mathrm{M}^{-1} \mathrm{~cm}^{-1}\right)\right): 486$ (873), 680 (1824). Elemental Analysis: Calcd. for $\mathrm{C}_{51} \mathrm{H}_{58} \mathrm{FeN}_{5} \mathrm{O}(\mathbf{5})$ : C, 75.37; H, 7.14; N, 8.62. Found: C, 73.95; H, 7.28; N, 8.21. 
$\left[\left(\mathbf{L}^{1}\right) \mathbf{F e}(\mathbf{I I I})-\mathbf{C H}_{3} \mathbf{C N}\right](\mathbf{6})$. The ligand $\mathrm{L}^{1} \mathrm{H}_{3}(0.343 \mathrm{~g}, 0.5 \mathrm{mmol})$ was dissolved in degassed THF $(15.0 \mathrm{~mL})$, to which $\mathrm{KH}(0.060 \mathrm{~g}, 1.5 \mathrm{mmol})$ was added. The mixture was stirred overnight to afford a yellow-orange solution, to which $\mathrm{FeCl}_{2}(0.0635 \mathrm{~g}, 0.5 \mathrm{mmol})$ was added as solid. This mixture was stirred for another 8 hours to give a yellow-green solution. This solution was pumped to dryness and the residue was dissolved in $\mathrm{CH}_{3} \mathrm{CN}$ $(5.0 \mathrm{~mL})$ to afford a dark green solution. Dichloromethane $(0.1 \mathrm{~mL})$ was then added to acetonitrile to generate a dark blue solution which was layered with hexane to provide blue-black crystals of 6 (0.050 g, $10 \%)$. Elemental Analysis: Calcd. for $\mathrm{C}_{50} \mathrm{H}_{54} \mathrm{FeN}_{5}(\mathbf{6})$ : C, 76.92; H, 6.92; N, 8.97. Found: C, 78.06; H, 6.63; N, 8.48.

$\left[\left(\mathbf{L}^{1}{ }_{\text {re }}\right)(\mathbf{C l}) \mathbf{F e}(\mathbf{I I})\right]\left[\mathbf{E t}_{\mathbf{4}} \mathbf{N}\right]_{2} \mathbf{2}$ (7). The ligand $\mathrm{L}^{1} \mathrm{H}_{3}(0.343 \mathrm{~g}, 0.5 \mathrm{mmol})$ was dissolved in degassed THF $(15.0 \mathrm{~mL})$, to which $\mathrm{KH}(0.060 \mathrm{~g}, 1.5 \mathrm{mmol})$ was added. The mixture was stirred overnight to afford a yellow-orange solution to which $\left[\mathrm{Fe}_{2} \mathrm{OCl}_{6}\right]\left[\mathrm{Et}_{4} \mathrm{~N}\right]_{2}(0.15 \mathrm{~g}$, $0.25 \mathrm{mmol}$ ) was added as solid. This mixture was stirred for an additional 12 hours to generate a dark green solution. The reaction solution was evaporated to dryness and extracted with diethyl ether $(2 \times 20 \mathrm{~mL})$. The ether solution was reduced to $20.0 \mathrm{~mL}$ and hexane $(20.0 \mathrm{~mL})$ was carefully layered over, to afford dark green crystals of $7(0.050 \mathrm{~g}$, $10 \%)$ upon standing at $-40^{\circ} \mathrm{C}$. UV-vis (THF): $\lambda_{\max }\left(\varepsilon\left(\mathrm{M}^{-1} \mathrm{~cm}^{-1}\right)\right) 422(5440), 490$ (3840), 698 (3800). Elemental Analysis: Calcd. for $\mathrm{C}_{64} \mathrm{H}_{91} \mathrm{FeN}{ }_{6} \mathrm{Cl}$ (7): C, 74.24; H, 8.80; N, 8.12. Found: C, 74.88; H, 8.57; N, 8.58.

$\left[\left(\mathbf{L}^{1}{ }_{\text {re }}\right)(\mathbf{C l}) \mathbf{F e}(\mathrm{III})\right]\left[\mathbf{E t}_{\mathbf{4}} \mathbf{N}\right] \cdot\left(\mathbf{C}_{2} \mathbf{H}_{5}\right) \mathbf{O}(\mathbf{8})$. Compound $7(0.050 \mathrm{~g}, 0.047 \mathrm{mmol})$ was dissolved in diethyl ether $(10.0 \mathrm{~mL})$ and a small amount of dichloromethane $(0.1 \mathrm{~mL})$ was added to generate a dark green-blue solution. The solution was filtered and reduced 
to $5.0 \mathrm{~mL}$, which upon prolonged refrigeration at $-40{ }^{\circ} \mathrm{C}$ affords dark blue-green crystals of $8(0.030 \mathrm{~g}, 66 \%)$. UV-vis (THF): $\lambda_{\max }\left(\varepsilon\left(\mathrm{M}^{-1} \mathrm{~cm}^{-1}\right)\right)$ : 500 (4830), 610 (5421). Elemental Analysis: Calcd. for $\mathrm{C}_{60} \mathrm{H}_{81} \mathrm{FeN}_{5} \mathrm{ClO}(\mathbf{8})$ : C, 73.58; H, 8.28; N, 7.15. Found: $\mathrm{C}$, 73.54; H, 7.81; N, 7.18.

$\left[\left(\mathrm{L}^{1}{ }_{\text {re-2 }} \mathrm{H}\right)(\mathrm{Cl}) \mathrm{Fe}(\mathrm{II})-(\mu-\mathrm{Cl})_{2}-\mathrm{Fe}(\mathrm{II})(\mathrm{Cl})\left(\mathrm{L}^{1}{ }_{\text {re-2 }} \mathrm{H}\right)\right] \cdot 0.5 \mathrm{Hexane} \quad$ (9). Compound $\left[\left(\mathrm{L}^{1}\right) \mathrm{Fe}-\mathrm{DMF}\right](5)(0.0207 \mathrm{~g}, 0.026 \mathrm{mmol})$ was dissolved in THF to give a dark blue solution. In another vial, $\mathrm{FeCl}_{3}(0.0041 \mathrm{~g}, 0.025 \mathrm{mmol})$ was dissolved in THF and was added to the THF solution of $\mathbf{5}$ drop-wise. After the addition was complete, the reaction solution was allowed to stir at room temperature overnight. The resulting brown solution was evaporated to dryness. The brown residue was extracted with hexane $(2 \times 20 \mathrm{~mL})$, and filtered. The brown filtrate was reduced to $20.0 \mathrm{~mL}$ and was allowed to stand at room temperature overnight to give X-ray quality crystals of $9(0.030 \mathrm{~g}, 71 \%)$. IR $\left(\mathrm{KBr}, \mathrm{cm}^{-1}\right)$ : $3059,2959,2901,2865,1650,1582,1504,1477,1410,1393,1362,1334,1303,1267$, $1216,1198,1182,1159,1110,1038,1015,939,914,869,829,821,748,706,665,609$, 552, 482, 467. UV-vis (THF): $\lambda_{\max }\left(\varepsilon\left(\mathrm{M}^{-1} \mathrm{~cm}^{-1}\right)\right): 510$ (10183), 870 (14451). Elemental Analysis: Calcd. for $\mathrm{C}_{96} \mathrm{H}_{104} \mathrm{Fe}_{2} \mathrm{~N}_{8} \mathrm{Cl}_{4}((9)-0.5$ Hexane): C, 71.02; H, 6.41; N, 6.90. Found: C, 71.33; H, 6.44; N, 6.96 .

Other Physical Measurements. Cyclic and linear sweep voltammetry was carried out with an Eco Chemie Autolab PGSTAT100 electrochemical workstation fitted in a Dry Box and controlled with a General Purpose Electrochemical Software (GPES). Cyclic voltammetry was performed using a gold disk working electrode $(1.6 \mathrm{~mm}$ diameter) and a $\mathrm{Ag} / \mathrm{Ag}^{+}\left(0.01 \mathrm{M} \mathrm{AgNO}_{3}\right.$ and $0.1 \mathrm{M}\left[(n-\mathrm{Bu})_{4} \mathrm{~N}\right] \mathrm{PF}_{6}$ in acetonitrile or 
DMSO) non-aqueous reference electrode (Bioanalytical Systems, Inc.) with a prolonged bridge $\left(0.1 \mathrm{M}\left[(n-\mathrm{Bu})_{4} \mathrm{~N}\right] \mathrm{PF}_{6}\right.$ in acetonitrile or DMSO). A thin Pt foil or gauge $\left(8 \mathrm{~cm}^{2}\right.$, Sigma-Aldrich) was employed as counter electrode. The working electrode was polished using successively $6,3,1 \mu \mathrm{m}$ diamond paste on a DP-Nap polishing cloth (Struers, Westlake, $\mathrm{OH}$ ), washed with water, acetone and air-dried. The Pt foil and gauge electrodes were cleaned in a $\mathrm{H}_{2} \mathrm{O}_{2} / \mathrm{H}_{2} \mathrm{SO}_{4}$ (conc) solution (1:4 v:v) and oven-dried. The concentration of the samples was $3 \mathrm{mM}$ and that of $\left[(n-\mathrm{Bu})_{4} \mathrm{~N}\right] \mathrm{PF}_{6}$ (supporting electrolyte) was $0.1 \mathrm{M}$. The potential sweep rate varied between $10-1000 \mathrm{mV} / \mathrm{s}$. All voltammograms were compensated for the solution resistance. All potentials are reported versus the ferrocenium/ferrocene $\left(\mathrm{Fc}^{+} / \mathrm{Fc}\right)$ couple.

Mössbauer measurements were recorded on a constant acceleration conventional spectrometer with a source of ${ }^{57} \mathrm{Co}$ (Rh matrix) located at the Institute of Materials Science, NCSR "Demokritos", Greece. Variable-temperature spectra were obtained by using an Oxford cryostat. Isomer shift values are quoted relative to iron foil at $293 \mathrm{~K}$.

Crystallographic data were collected at the Departments of Chemistry at Harvard University, University of Missouri-Rolla, University of Missouri-Columbia and University of Missouri-St. Louis by using a Bruker SMART CCD (charge coupled device) based diffractometer equipped with an Oxford Cryostream low-temperature apparatus operating at variable low temperatures. A suitable crystal was chosen and mounted on a glass fiber using grease. Data were measured using omega scans of $0.3^{\circ}$ per frame for 30 seconds, such that a hemisphere was collected. A total of 1271 frames were collected with a maximum resolution of $0.75 \AA$. The first 50 frames were recollected at 
the end of data collection to monitor for decay. Cell parameters were retrieved using SMART software and refined using SAINT on all observed reflections. Data reduction was performed using the SAINT software, which corrects for Lp and decay. The structures are solved by the direct method using the SHELXS-97 program and refined by least squares method on $\mathrm{F}^{2}$, SHELXL-97, incorporated in SHELXTL-PC V 5.10. All non-hydrogen atoms were refined anisotropically. Hydrogens were calculated by geometrical methods and refined as a riding model. The crystals used for diffraction studies showed no decomposition during data collection. All drawings are done at 50\% ellipsoids unless otherwise stated. 

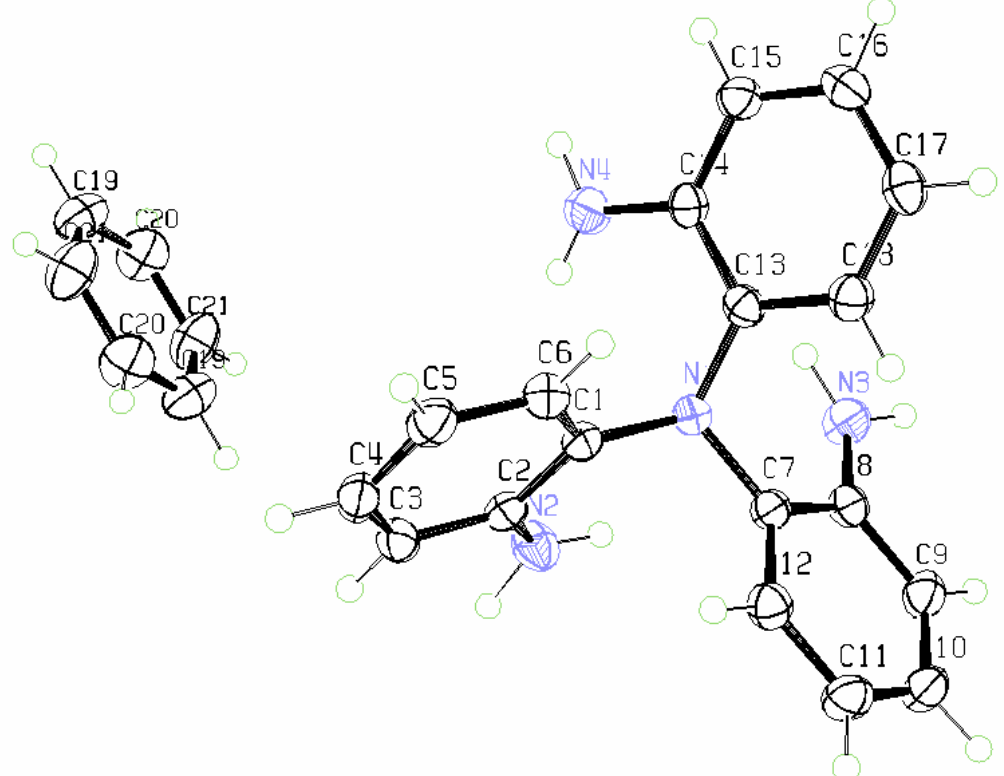

Figure S1. Solid-state structure of $2,2^{\prime}, 2^{\prime \prime}$-triaminotriphenylamine showing $50 \%$ probability ellipsoids and the atom labeling scheme. Selected interatomic distances $(\AA)$ and angles (deg) are: $\mathrm{N}(1)-\mathrm{C}(1)$ 1.4392(17), $\mathrm{N}(1)-\mathrm{C}(13)$ 1.4401(17), $\mathrm{N}(1)-\mathrm{C}(7)$ 1.4422(16), $\quad \mathrm{N}(2)-\mathrm{C}(2) \quad 1.3862(19), \quad \mathrm{C}(1)-\mathrm{N}(1)-\mathrm{C}(13) \quad 116.14(11), \quad \mathrm{C}(1)-\mathrm{N}(1)-\mathrm{C}(7)$ 115.79(11), C(13)-N(1)-C(7) 115.44(10). 


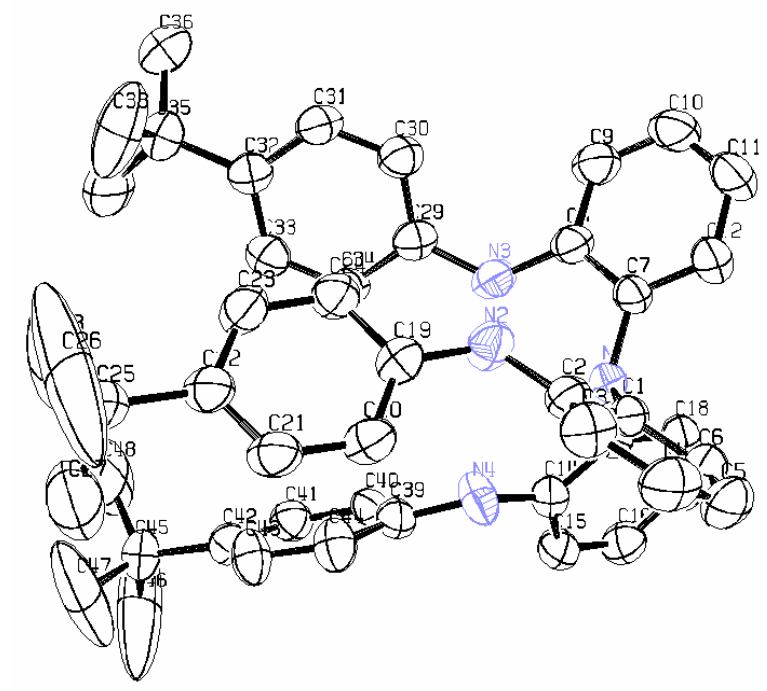

Figure S2. Solid-state structure of $\mathrm{L}^{1} \mathrm{H}_{3}$ showing $50 \%$ probability ellipsoids and the atom labeling scheme. Selected interatomic distances $(\AA)$ and angles (deg) are : N(1)-C(1) 1.432(4), N(1)-C(13) 1.436(4), N(1)-C(7) 1.444(4), N(2)-C(2) 1.403(4), N(2)-C(19) $1.405(4), \mathrm{N}(3)-\mathrm{C}(8) 1.395(4), \mathrm{N}(3)-\mathrm{C}(29) 1.406(4), \mathrm{N}(4)-\mathrm{C}(14) 1.395(4), \mathrm{N}(4)-\mathrm{C}(39)$ 1.408(4), $\quad \mathrm{C}(1)-\mathrm{N}(1)-\mathrm{C}(13) \quad 117.7(3), \quad \mathrm{C}(1)-\mathrm{N}(1)-\mathrm{C}(7) \quad 116.4(3), \quad \mathrm{C}(13)-\mathrm{N}(1)-\mathrm{C}(7)$ 116.0(3), $\mathrm{C}(2)-\mathrm{N}(2)-\mathrm{C}(19) \quad 126.7(3), \quad \mathrm{C}(8)-\mathrm{N}(3)-\mathrm{C}(29) \quad 128.0(3), \quad \mathrm{C}(14)-\mathrm{N}(4)-\mathrm{C}(39)$ 126.7(3). 


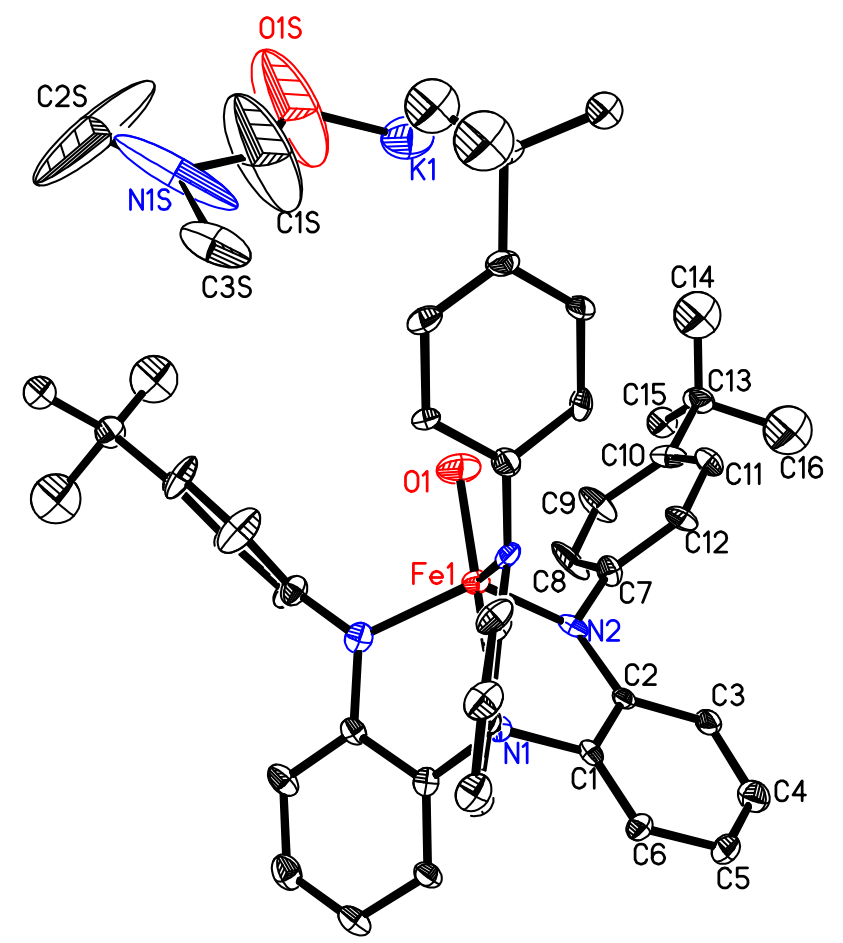

Figure S3. Solid-state structure of $\left[\left(\mathrm{L}^{1}\right) \mathrm{Fe}(\mathrm{III})-\mathrm{OH}\right]\left[\mathrm{K}(\mathrm{DMF})_{3}\right] \cdot 3 \mathrm{H}_{2} \mathrm{O}(\mathbf{3})$ showing $50 \%$ probability ellipsoids and the atom labeling scheme. Selected interatomic distances $(\AA)$ and angles (deg) are : $\mathrm{Fe}(1)-\mathrm{O}(1)$ 1.856(11), $\mathrm{Fe}(1)-\mathrm{N}(2) \# 1$ 1.952(6), $\mathrm{Fe}(1)-\mathrm{N}(2)$ 1.952(6), $\mathrm{Fe}(1)-\mathrm{N}(2) \# 2$ 1.953(6), $\mathrm{Fe}(1)-\mathrm{N}(1)$ 2.321(11), N(1)-C(1)\#1 1.467(8), N(1)$\mathrm{C}(1)$ 1.467(8), N(1)-C(1)\#2 1.467(8), N(2)-C(2) 1.396(10), N(2)-C(7) 1.394(10), O(1)$\mathrm{Fe}(1)-\mathrm{N}(2) \# 1$ 101.98(18), O(1)-Fe(1)-N(2) 101.98(18), N(2)\#1-Fe(1)-N(2) 115.81(12), $\mathrm{O}(1)-\mathrm{Fe}(1)-\mathrm{N}(2) \# 2$ 101.98(18), N(2)\#1-Fe(1)-N(2)\#2 115.81(12), N(2)-Fe(1)-N(2)\#2 115.81(12), N(2)\#1-Fe(1)-N(1) 78.02(18), N(2)-Fe(1)-N(1) 78.02(18), N(2)\#2-Fe(1)$\mathrm{N}(1)$ 78.02(18), $\mathrm{O}(1)-\mathrm{Fe}(1)-\mathrm{N}(1)$ 180.0(5). 
Table S1. Atomic coordinates $\left(\times 10^{4}\right)$ and equivalent isotropic displacement parameters $\left(\AA^{2} \times 10^{3}\right)$ for $2,2^{\prime}, 2^{\prime \prime}$-triaminotriphenylamine. $\mathrm{U}(\mathrm{eq})$ is defined as one third of the trace of the orthogonalized $\mathrm{U}^{\mathrm{ij}}$ tensor

\begin{tabular}{|c|c|c|c|c|}
\hline & $\mathrm{x}$ & $\mathrm{y}$ & $\mathrm{z}$ & $\mathrm{U}(\mathrm{eq})$ \\
\hline $\mathrm{N}(1)$ & $1667(1)$ & $6018(1)$ & $1150(1)$ & $25(1)$ \\
\hline $\mathrm{N}(2)$ & $3174(2)$ & $4613(1)$ & 201(1) & $36(1)$ \\
\hline $\mathrm{N}(3)$ & $-199(2)$ & $6837(1)$ & $-367(1)$ & $35(1)$ \\
\hline $\mathrm{N}(4)$ & $-899(2)$ & $4786(1)$ & 1591(1) & $40(1)$ \\
\hline$C(1)$ & $2808(2)$ & $5337(1)$ & $1607(1)$ & $25(1)$ \\
\hline $\mathrm{C}(2)$ & 3573(2) & $4660(1)$ & $1108(1)$ & $26(1)$ \\
\hline$C(3)$ & $4707(2)$ & 4012(1) & 1551(1) & $32(1)$ \\
\hline $\mathrm{C}(4)$ & $5028(2)$ & $4004(1)$ & $2460(1)$ & $34(1)$ \\
\hline$C(5)$ & $4238(2)$ & $4651(1)$ & $2950(1)$ & $33(1)$ \\
\hline $\mathrm{C}(6)$ & $3146(2)$ & $5318(1)$ & $2518(1)$ & $29(1)$ \\
\hline$C(7)$ & $2427(2)$ & $6715(1)$ & $634(1)$ & $24(1)$ \\
\hline$C(8)$ & $1445(2)$ & $7129(1)$ & $-98(1)$ & $27(1)$ \\
\hline $\mathrm{C}(9)$ & 2192(2) & $7824(1)$ & $-576(1)$ & $33(1)$ \\
\hline$C(10)$ & $3859(2)$ & 8091(1) & $-349(1)$ & $36(1)$ \\
\hline$C(11)$ & $4832(2)$ & $7669(1)$ & $360(1)$ & $35(1)$ \\
\hline$C(12)$ & $4100(2)$ & $6990(1)$ & $847(1)$ & $30(1)$ \\
\hline$C(13)$ & $350(2)$ & $6369(1)$ & $1624(1)$ & $25(1)$ \\
\hline$C(14)$ & $-917(2)$ & $5735(1)$ & $1822(1)$ & $26(1)$ \\
\hline
\end{tabular}




\begin{tabular}{lllll}
$\mathrm{C}(15)$ & $-2198(2)$ & $6086(1)$ & $2269(1)$ & $30(1)$ \\
$\mathrm{C}(16)$ & $-2252(2)$ & $7030(1)$ & $2509(1)$ & $31(1)$ \\
$\mathrm{C}(17)$ & $-1025(2)$ & $7655(1)$ & $2306(1)$ & $31(1)$ \\
$\mathrm{C}(18)$ & $273(2)$ & $7315(1)$ & $1871(1)$ & $29(1)$ \\
$\mathrm{C}(19)$ & $984(2)$ & $594(1)$ & $-435(1)$ & $46(1)$ \\
$\mathrm{C}(20)$ & $1446(2)$ & $389(1)$ & $442(1)$ & $48(1)$ \\
$\mathrm{C}(21)$ & $466(2)$ & $-207(1)$ & $874(1)$ & $48(1)$ \\
\hline
\end{tabular}


Table S2. Bond Distances, $\AA$, for 2,2',2' '-triaminotriphenylamine

\begin{tabular}{|c|c|c|c|}
\hline $\mathrm{N}(1)-\mathrm{C}(1)$ & $1.4392(17)$ & $C(9)-C(10)$ & $1.383(2)$ \\
\hline $\mathrm{N}(1)-\mathrm{C}(13)$ & $1.4401(17)$ & $\mathrm{C}(9)-\mathrm{H}(9)$ & 0.9500 \\
\hline $\mathrm{N}(1)-\mathrm{C}(7)$ & $1.4422(16)$ & $\mathrm{C}(10)-\mathrm{C}(11)$ & $1.384(2)$ \\
\hline $\mathrm{N}(2)-\mathrm{C}(2)$ & $1.3862(19)$ & $\mathrm{C}(10)-\mathrm{H}(10)$ & 0.9500 \\
\hline $\mathrm{N}(2)-\mathrm{H}(2 \mathrm{~N} 2)$ & 0.9788 & $\mathrm{C}(11)-\mathrm{C}(12)$ & $1.387(2)$ \\
\hline $\mathrm{N}(2)-\mathrm{H}(1 \mathrm{~N} 2)$ & $0.851(19)$ & $\mathrm{C}(11)-\mathrm{H}(11)$ & 0.9500 \\
\hline $\mathrm{N}(3)-\mathrm{C}(8)$ & $1.3862(19)$ & $\mathrm{C}(12)-\mathrm{H}(12)$ & 0.9500 \\
\hline N(3)-H(1N3) & 0.9637 & $\mathrm{C}(13)-\mathrm{C}(18)$ & $1.3872(19)$ \\
\hline N(3)-H(2N3) & $0.874(19)$ & $C(13)-C(14)$ & $1.4106(19)$ \\
\hline $\mathrm{N}(4)-\mathrm{C}(14)$ & $1.3822(19)$ & $C(14)-C(15)$ & $1.3929(19)$ \\
\hline $\mathrm{N}(4)-\mathrm{H}(2 \mathrm{~N} 4)$ & 0.9377 & $C(15)-C(16)$ & $1.381(2)$ \\
\hline N(4)-H(1N4) & $0.850(18)$ & $\mathrm{C}(15)-\mathrm{H}(15)$ & 0.9500 \\
\hline$C(1)-C(6)$ & $1.3882(19)$ & $\mathrm{C}(16)-\mathrm{C}(17)$ & $1.381(2)$ \\
\hline$C(1)-C(2)$ & $1.4089(19)$ & $\mathrm{C}(16)-\mathrm{H}(16)$ & 0.9500 \\
\hline$C(2)-C(3)$ & $1.398(2)$ & $\mathrm{C}(17)-\mathrm{C}(18)$ & $1.387(2)$ \\
\hline$C(3)-C(4)$ & $1.383(2)$ & $\mathrm{C}(17)-\mathrm{H}(17)$ & 0.9500 \\
\hline $\mathrm{C}(3)-\mathrm{H}(3)$ & 0.9500 & $\mathrm{C}(18)-\mathrm{H}(18)$ & 0.9500 \\
\hline$C(4)-C(5)$ & $1.383(2)$ & $\mathrm{C}(19)-\mathrm{C}(21) \# 1$ & $1.374(2)$ \\
\hline $\mathrm{C}(4)-\mathrm{H}(4)$ & 0.9500 & C(19)-C(20) & $1.378(2)$ \\
\hline$C(5)-C(6)$ & $1.388(2)$ & $\mathrm{C}(19)-\mathrm{H}(19)$ & 0.9500 \\
\hline $\mathrm{C}(5)-\mathrm{H}(5)$ & 0.9500 & $C(20)-C(21)$ & $1.373(2)$ \\
\hline
\end{tabular}




$\begin{array}{lrrr}\mathrm{C}(6)-\mathrm{H}(6) & 0.9500 & \mathrm{C}(20)-\mathrm{H}(20) & 0.9500 \\ \mathrm{C}(7)-\mathrm{C}(12) & 1.388(2) & \mathrm{C}(21)-\mathrm{C}(19) \# 1 & 1.374(2) \\ \mathrm{C}(7)-\mathrm{C}(8) & 1.4085(19) & \mathrm{C}(21)-\mathrm{H}(21) & 0.9500 \\ \mathrm{C}(8)-\mathrm{C}(9) & 1.4002(19) & & \end{array}$

Symmetry transformations used to generate equivalent atoms:

\#1 -x, -y, -z 
Table S3. Angles, ${ }^{\circ}$, for $2,2^{\prime}, 2^{\prime \prime}$-triaminotriphenylamine

\begin{tabular}{|c|c|c|c|}
\hline $\mathrm{C}(1)-\mathrm{N}(1)-\mathrm{C}(13)$ & $116.14(11)$ & $\mathrm{C}(8)-\mathrm{C}(9)-\mathrm{H}(9)$ & 119.4 \\
\hline $\mathrm{C}(1)-\mathrm{N}(1)-\mathrm{C}(7)$ & $115.79(11)$ & $\mathrm{C}(9)-\mathrm{C}(10)-\mathrm{C}(11)$ & $120.29(14)$ \\
\hline $\mathrm{C}(13)-\mathrm{N}(1)-\mathrm{C}(7)$ & $115.44(10)$ & $\mathrm{C}(9)-\mathrm{C}(10)-\mathrm{H}(10)$ & 119.9 \\
\hline $\mathrm{C}(2)-\mathrm{N}(2)-\mathrm{H}(2 \mathrm{~N} 2)$ & 111.8 & $\mathrm{C}(11)-\mathrm{C}(10)-\mathrm{H}(10)$ & 119.9 \\
\hline $\mathrm{C}(2)-\mathrm{N}(2)-\mathrm{H}(1 \mathrm{~N} 2)$ & $119.4(12)$ & $C(10)-C(11)-C(12)$ & 119.01(14) \\
\hline $\mathrm{H}(2 \mathrm{~N} 2)-\mathrm{N}(2)-\mathrm{H}(1 \mathrm{~N} 2)$ & 123.6 & $\mathrm{C}(10)-\mathrm{C}(11)-\mathrm{H}(11)$ & 120.5 \\
\hline $\mathrm{C}(8)-\mathrm{N}(3)-\mathrm{H}(1 \mathrm{~N} 3)$ & 120.2 & $\mathrm{C}(12)-\mathrm{C}(11)-\mathrm{H}(11)$ & 120.5 \\
\hline $\mathrm{C}(8)-\mathrm{N}(3)-\mathrm{H}(2 \mathrm{~N} 3)$ & $116.5(12)$ & $C(11)-C(12)-C(7)$ & $121.65(14)$ \\
\hline $\mathrm{H}(1 \mathrm{~N} 3)-\mathrm{N}(3)-\mathrm{H}(2 \mathrm{~N} 3)$ & 116.5 & $\mathrm{C}(11)-\mathrm{C}(12)-\mathrm{H}(12)$ & 119.2 \\
\hline $\mathrm{C}(14)-\mathrm{N}(4)-\mathrm{H}(2 \mathrm{~N} 4)$ & 118.6 & $\mathrm{C}(7)-\mathrm{C}(12)-\mathrm{H}(12)$ & 119.2 \\
\hline $\mathrm{C}(14)-\mathrm{N}(4)-\mathrm{H}(1 \mathrm{~N} 4)$ & $122.3(12)$ & $\mathrm{C}(18)-\mathrm{C}(13)-\mathrm{C}(14)$ & $119.40(13)$ \\
\hline $\mathrm{H}(2 \mathrm{~N} 4)-\mathrm{N}(4)-\mathrm{H}(1 \mathrm{~N} 4)$ & 117.8 & $\mathrm{C}(18)-\mathrm{C}(13)-\mathrm{N}(1)$ & $121.82(12)$ \\
\hline$C(6)-C(1)-C(2)$ & $119.39(13)$ & $\mathrm{C}(14)-\mathrm{C}(13)-\mathrm{N}(1)$ & $118.76(12)$ \\
\hline $\mathrm{C}(6)-\mathrm{C}(1)-\mathrm{N}(1)$ & $122.04(12)$ & $\mathrm{N}(4)-\mathrm{C}(14)-\mathrm{C}(15)$ & $120.11(13)$ \\
\hline $\mathrm{C}(2)-\mathrm{C}(1)-\mathrm{N}(1)$ & $118.56(12)$ & $\mathrm{N}(4)-\mathrm{C}(14)-\mathrm{C}(13)$ & $121.66(13)$ \\
\hline $\mathrm{N}(2)-\mathrm{C}(2)-\mathrm{C}(3)$ & $120.42(13)$ & $\mathrm{C}(15)-\mathrm{C}(14)-\mathrm{C}(13)$ & $118.23(13)$ \\
\hline $\mathrm{N}(2)-\mathrm{C}(2)-\mathrm{C}(1)$ & $121.07(13)$ & $C(16)-C(15)-C(14)$ & $121.48(14)$ \\
\hline $\mathrm{C}(3)-\mathrm{C}(2)-\mathrm{C}(1)$ & $118.47(13)$ & $\mathrm{C}(16)-\mathrm{C}(15)-\mathrm{H}(15)$ & 119.3 \\
\hline$C(4)-C(3)-C(2)$ & $121.23(14)$ & $\mathrm{C}(14)-\mathrm{C}(15)-\mathrm{H}(15)$ & 119.3 \\
\hline $\mathrm{C}(4)-\mathrm{C}(3)-\mathrm{H}(3)$ & 119.4 & $\mathrm{C}(15)-\mathrm{C}(16)-\mathrm{C}(17)$ & $120.34(14)$ \\
\hline $\mathrm{C}(2)-\mathrm{C}(3)-\mathrm{H}(3)$ & 119.4 & $\mathrm{C}(15)-\mathrm{C}(16)-\mathrm{H}(16)$ & 119.8 \\
\hline
\end{tabular}




\begin{tabular}{|c|c|c|c|}
\hline$C(3)-C(4)-C(5)$ & $120.19(14)$ & $\mathrm{C}(17)-\mathrm{C}(16)-\mathrm{H}(16)$ & 119.8 \\
\hline $\mathrm{C}(3)-\mathrm{C}(4)-\mathrm{H}(4)$ & 119.9 & $\mathrm{C}(16)-\mathrm{C}(17)-\mathrm{C}(18)$ & $118.99(13)$ \\
\hline $\mathrm{C}(5)-\mathrm{C}(4)-\mathrm{H}(4)$ & 119.9 & $\mathrm{C}(16)-\mathrm{C}(17)-\mathrm{H}(17)$ & 120.5 \\
\hline$C(4)-C(5)-C(6)$ & $119.26(14)$ & $\mathrm{C}(18)-\mathrm{C}(17)-\mathrm{H}(17)$ & 120.5 \\
\hline $\mathrm{C}(4)-\mathrm{C}(5)-\mathrm{H}(5)$ & 120.4 & $\mathrm{C}(17)-\mathrm{C}(18)-\mathrm{C}(13)$ & $121.55(14)$ \\
\hline $\mathrm{C}(6)-\mathrm{C}(5)-\mathrm{H}(5)$ & 120.4 & $\mathrm{C}(17)-\mathrm{C}(18)-\mathrm{H}(18)$ & 119.2 \\
\hline$C(1)-C(6)-C(5)$ & $121.39(14)$ & $\mathrm{C}(13)-\mathrm{C}(18)-\mathrm{H}(18)$ & 119.2 \\
\hline $\mathrm{C}(1)-\mathrm{C}(6)-\mathrm{H}(6)$ & 119.3 & $C(21) \# 1-C(19)-C(20)$ & $119.78(16)$ \\
\hline $\mathrm{C}(5)-\mathrm{C}(6)-\mathrm{H}(6)$ & 119.3 & $\mathrm{C}(21) \# 1-\mathrm{C}(19)-\mathrm{H}(19)$ & 120.1 \\
\hline $\mathrm{C}(12)-\mathrm{C}(7)-\mathrm{C}(8)$ & $119.44(12)$ & $\mathrm{C}(20)-\mathrm{C}(19)-\mathrm{H}(19)$ & 120.1 \\
\hline $\mathrm{C}(12)-\mathrm{C}(7)-\mathrm{N}(1)$ & $121.36(12)$ & $\mathrm{C}(21)-\mathrm{C}(20)-\mathrm{C}(19)$ & $120.03(16)$ \\
\hline $\mathrm{C}(8)-\mathrm{C}(7)-\mathrm{N}(1)$ & $119.20(12)$ & $\mathrm{C}(21)-\mathrm{C}(20)-\mathrm{H}(20)$ & 120.0 \\
\hline $\mathrm{N}(3)-\mathrm{C}(8)-\mathrm{C}(9)$ & $120.16(13)$ & $\mathrm{C}(19)-\mathrm{C}(20)-\mathrm{H}(20)$ & 120.0 \\
\hline $\mathrm{N}(3)-\mathrm{C}(8)-\mathrm{C}(7)$ & $121.48(13)$ & $\mathrm{C}(20)-\mathrm{C}(21)-\mathrm{C}(19) \# 1$ & $120.18(16)$ \\
\hline$C(9)-C(8)-C(7)$ & 118.31(13) & $\mathrm{C}(20)-\mathrm{C}(21)-\mathrm{H}(21)$ & 119.9 \\
\hline $\mathrm{C}(10)-\mathrm{C}(9)-\mathrm{C}(8)$ & $121.29(14)$ & $\mathrm{C}(19) \# 1-\mathrm{C}(21)-\mathrm{H}(21)$ & 119.9 \\
\hline $\mathrm{C}(10)-\mathrm{C}(9)-\mathrm{H}(9)$ & 119.4 & & \\
\hline
\end{tabular}

Symmetry transformations used to generate equivalent atoms:

$\# 1-x,-y,-z$ 
Table S4. Anisotropic displacement parameters $\left(\AA^{2} \times 10^{3}\right)$ for $2,2^{\prime}, 2^{\prime \prime}-$ triaminotriphenylamine. The anisotropic displacement factor exponent takes the form: $-2 \pi^{2}\left[h^{2} a^{* 2} U^{11}+\ldots+2 h k a^{*} b^{*} U^{12}\right]$

\begin{tabular}{|c|c|c|c|c|c|c|}
\hline & $\mathrm{U}^{11}$ & $\mathrm{U}^{22}$ & $\mathrm{U}^{33}$ & $\mathrm{U}^{23}$ & $\mathrm{U}^{13}$ & $\mathrm{U}^{12}$ \\
\hline $\mathrm{N}(1)$ & $24(1)$ & $24(1)$ & $27(1)$ & $3(1)$ & $5(1)$ & $2(1)$ \\
\hline $\mathrm{N}(2)$ & $40(1)$ & $37(1)$ & $32(1)$ & $-4(1)$ & $7(1)$ & $8(1)$ \\
\hline $\mathrm{N}(3)$ & $29(1)$ & $39(1)$ & $34(1)$ & $7(1)$ & $-2(1)$ & $-1(1)$ \\
\hline $\mathrm{N}(4)$ & $35(1)$ & $26(1)$ & 61(1) & $-5(1)$ & $16(1)$ & $-3(1)$ \\
\hline$C(1)$ & $22(1)$ & $22(1)$ & $31(1)$ & $3(1)$ & $4(1)$ & $-1(1)$ \\
\hline$C(2)$ & $24(1)$ & $24(1)$ & $32(1)$ & $0(1)$ & $5(1)$ & $-4(1)$ \\
\hline$C(3)$ & $27(1)$ & $23(1)$ & $46(1)$ & $0(1)$ & $8(1)$ & $0(1)$ \\
\hline$C(4)$ & $26(1)$ & $27(1)$ & $47(1)$ & 11(1) & $0(1)$ & $-1(1)$ \\
\hline$C(5)$ & $29(1)$ & $36(1)$ & $33(1)$ & $8(1)$ & 1(1) & $-4(1)$ \\
\hline$C(6)$ & $28(1)$ & $29(1)$ & $31(1)$ & 1(1) & $5(1)$ & $0(1)$ \\
\hline$C(7)$ & $27(1)$ & $22(1)$ & $25(1)$ & $-1(1)$ & $7(1)$ & $-1(1)$ \\
\hline$C(8)$ & $28(1)$ & $24(1)$ & $28(1)$ & $-2(1)$ & $5(1)$ & $1(1)$ \\
\hline $\mathrm{C}(9)$ & $40(1)$ & $28(1)$ & $31(1)$ & $4(1)$ & $6(1)$ & $2(1)$ \\
\hline$C(10)$ & $42(1)$ & $28(1)$ & $42(1)$ & $4(1)$ & $15(1)$ & $-5(1)$ \\
\hline $\mathrm{C}(11)$ & $30(1)$ & $32(1)$ & $42(1)$ & $-4(1)$ & $6(1)$ & $-8(1)$ \\
\hline$C(12)$ & $30(1)$ & $28(1)$ & $31(1)$ & $-1(1)$ & $2(1)$ & $0(1)$ \\
\hline$C(13)$ & $24(1)$ & $27(1)$ & $23(1)$ & $0(1)$ & 2(1) & $3(1)$ \\
\hline$C(14)$ & $26(1)$ & $25(1)$ & $26(1)$ & $2(1)$ & 1(1) & $3(1)$ \\
\hline
\end{tabular}




\begin{tabular}{llllllr}
$\mathrm{C}(15)$ & $25(1)$ & $33(1)$ & $33(1)$ & $6(1)$ & $5(1)$ & $1(1)$ \\
$\mathrm{C}(16)$ & $27(1)$ & $37(1)$ & $29(1)$ & $-1(1)$ & $5(1)$ & $8(1)$ \\
$\mathrm{C}(17)$ & $37(1)$ & $26(1)$ & $30(1)$ & $-5(1)$ & $2(1)$ & $4(1)$ \\
$\mathrm{C}(18)$ & $30(1)$ & $27(1)$ & $30(1)$ & $-1(1)$ & $3(1)$ & $-1(1)$ \\
$\mathrm{C}(19)$ & $55(1)$ & $42(1)$ & $44(1)$ & $-1(1)$ & $15(1)$ & $-11(1)$ \\
$\mathrm{C}(20)$ & $44(1)$ & $48(1)$ & $48(1)$ & $-1(1)$ & $-4(1)$ & $-10(1)$ \\
$\mathrm{C}(21)$ & $67(1)$ & $43(1)$ & $32(1)$ & $2(1)$ & $-1(1)$ & $-8(1)$ \\
\hline
\end{tabular}


Table S5. Hydrogen coordinates $\left(\times 10^{4}\right)$ and isotropic displacement parameters $\left(\AA^{2} \times 10^{3}\right)$ for $2,2^{\prime}, 2^{\prime \prime}$-triaminotriphenylamine

\begin{tabular}{|c|c|c|c|c|}
\hline & $\mathrm{x}$ & $\mathrm{y}$ & $\mathrm{z}$ & $\mathrm{U}(\mathrm{eq})$ \\
\hline $\mathrm{H}(3)$ & 5268 & 3569 & 1222 & 38 \\
\hline $\mathrm{H}(4)$ & 5793 & 3553 & 2748 & 40 \\
\hline $\mathrm{H}(5)$ & 4440 & 4640 & 3574 & 40 \\
\hline $\mathrm{H}(6)$ & 2618 & 5770 & 2854 & 35 \\
\hline $\mathrm{H}(9)$ & 1541 & 8118 & -1065 & 39 \\
\hline $\mathrm{H}(10)$ & 4339 & 8566 & -681 & 43 \\
\hline $\mathrm{H}(11)$ & 5983 & 7843 & 511 & 41 \\
\hline $\mathrm{H}(12)$ & 4760 & 6706 & 1339 & 36 \\
\hline $\mathrm{H}(15)$ & -3054 & 5666 & 2413 & 36 \\
\hline $\mathrm{H}(16)$ & -3138 & 7252 & 2815 & 37 \\
\hline $\mathrm{H}(17)$ & -1069 & 8307 & 2461 & 38 \\
\hline $\mathrm{H}(18)$ & 1130 & 7740 & 1738 & 35 \\
\hline $\mathrm{H}(19)$ & 1666 & 1002 & -736 & 55 \\
\hline $\mathrm{H}(20)$ & 2443 & 660 & 749 & 57 \\
\hline $\mathrm{H}(21)$ & 792 & -351 & 1478 & 57 \\
\hline $\mathrm{H}(2 \mathrm{~N} 4)$ & -1820 & 4404 & 1687 & $46(5)$ \\
\hline $\mathrm{H}(1 \mathrm{~N} 3)$ & -783 & 6444 & 12 & $70(6)$ \\
\hline $\mathrm{H}(2 \mathrm{~N} 2)$ & 4004 & 4240 & -70 & $55(5)$ \\
\hline $\mathrm{H}(2 \mathrm{~N} 3)$ & $-820(20)$ & $7217(13)$ & $-724(12)$ & $52(6)$ \\
\hline
\end{tabular}




$\begin{array}{lllll}\mathrm{H}(1 \mathrm{~N} 4) & -200(20) & 4562(12) & 1272(11) & 47(5) \\ \mathrm{H}(1 \mathrm{~N} 2) & 2570(20) & 5049(13) & -66(12) & 50(6)\end{array}$


Table S6. Torsion Angles, ${ }^{\circ}$, for 2,2',2' 'triaminotriphenylamine

\begin{tabular}{|c|c|c|c|}
\hline$C(13)-N(1)-C(1)-C(6)$ & $-27.57(18)$ & $\mathrm{C}(7)-\mathrm{C}(8)-\mathrm{C}(9)-\mathrm{C}(10)$ & $1.0(2)$ \\
\hline$C(7)-N(1)-C(1)-C(6)$ & $112.66(14)$ & $\mathrm{C}(8)-\mathrm{C}(9)-\mathrm{C}(10)-\mathrm{C}(11)$ & $0.2(2)$ \\
\hline $\mathrm{C}(13)-\mathrm{N}(1)-\mathrm{C}(1)-\mathrm{C}(2)$ & $151.18(12)$ & $\mathrm{C}(9)-\mathrm{C}(10)-\mathrm{C}(11)-\mathrm{C}(12)$ & $-1.2(2)$ \\
\hline $\mathrm{C}(7)-\mathrm{N}(1)-\mathrm{C}(1)-\mathrm{C}(2)$ & $-68.59(15)$ & $\mathrm{C}(10)-\mathrm{C}(11)-\mathrm{C}(12)-\mathrm{C}(7)$ & $0.9(2)$ \\
\hline $\mathrm{C}(6)-\mathrm{C}(1)-\mathrm{C}(2)-\mathrm{N}(2)$ & $175.32(13)$ & $\mathrm{C}(8)-\mathrm{C}(7)-\mathrm{C}(12)-\mathrm{C}(11)$ & $0.3(2)$ \\
\hline $\mathrm{N}(1)-\mathrm{C}(1)-\mathrm{C}(2)-\mathrm{N}(2)$ & $-3.46(19)$ & $\mathrm{N}(1)-\mathrm{C}(7)-\mathrm{C}(12)-\mathrm{C}(11)$ & $-179.10(13)$ \\
\hline$C(6)-C(1)-C(2)-C(3)$ & $-2.53(19)$ & $\mathrm{C}(1)-\mathrm{N}(1)-\mathrm{C}(13)-\mathrm{C}(18)$ & $114.97(14)$ \\
\hline $\mathrm{N}(1)-\mathrm{C}(1)-\mathrm{C}(2)-\mathrm{C}(3)$ & $178.68(12)$ & $\mathrm{C}(7)-\mathrm{N}(1)-\mathrm{C}(13)-\mathrm{C}(18)$ & $-25.40(18)$ \\
\hline $\mathrm{N}(2)-\mathrm{C}(2)-\mathrm{C}(3)-\mathrm{C}(4)$ & $-175.29(13)$ & $\mathrm{C}(1)-\mathrm{N}(1)-\mathrm{C}(13)-\mathrm{C}(14)$ & $-66.68(16)$ \\
\hline $\mathrm{C}(1)-\mathrm{C}(2)-\mathrm{C}(3)-\mathrm{C}(4)$ & $2.6(2)$ & $\mathrm{C}(7)-\mathrm{N}(1)-\mathrm{C}(13)-\mathrm{C}(14)$ & $152.95(12)$ \\
\hline$C(2)-C(3)-C(4)-C(5)$ & $-0.8(2)$ & $\mathrm{C}(18)-\mathrm{C}(13)-\mathrm{C}(14)-\mathrm{N}(4)$ & $180.00(14)$ \\
\hline$C(3)-C(4)-C(5)-C(6)$ & $-1.1(2)$ & $\mathrm{N}(1)-\mathrm{C}(13)-\mathrm{C}(14)-\mathrm{N}(4)$ & $1.6(2)$ \\
\hline$C(2)-C(1)-C(6)-C(5)$ & $0.7(2)$ & $\mathrm{C}(18)-\mathrm{C}(13)-\mathrm{C}(14)-\mathrm{C}(15)$ & $-0.7(2)$ \\
\hline $\mathrm{N}(1)-\mathrm{C}(1)-\mathrm{C}(6)-\mathrm{C}(5)$ & $179.47(12)$ & $\mathrm{N}(1)-\mathrm{C}(13)-\mathrm{C}(14)-\mathrm{C}(15)$ & $-179.08(12)$ \\
\hline$C(4)-C(5)-C(6)-C(1)$ & $1.1(2)$ & $\mathrm{N}(4)-\mathrm{C}(14)-\mathrm{C}(15)-\mathrm{C}(16)$ & $-179.98(14)$ \\
\hline $\mathrm{C}(1)-\mathrm{N}(1)-\mathrm{C}(7)-\mathrm{C}(12)$ & $-27.26(18)$ & $C(13)-C(14)-C(15)-C(16)$ & $0.7(2)$ \\
\hline $\mathrm{C}(13)-\mathrm{N}(1)-\mathrm{C}(7)-\mathrm{C}(12)$ & $113.24(14)$ & $C(14)-C(15)-C(16)-C(17)$ & $0.2(2)$ \\
\hline $\mathrm{C}(1)-\mathrm{N}(1)-\mathrm{C}(7)-\mathrm{C}(8)$ & $153.32(12)$ & $\mathrm{C}(15)-\mathrm{C}(16)-\mathrm{C}(17)-\mathrm{C}(18)$ & $-1.0(2)$ \\
\hline $\mathrm{C}(13)-\mathrm{N}(1)-\mathrm{C}(7)-\mathrm{C}(8)$ & $-66.17(16)$ & $\mathrm{C}(16)-\mathrm{C}(17)-\mathrm{C}(18)-\mathrm{C}(13)$ & $1.0(2)$ \\
\hline $\mathrm{C}(12)-\mathrm{C}(7)-\mathrm{C}(8)-\mathrm{N}(3)$ & $176.11(13)$ & $\mathrm{C}(14)-\mathrm{C}(13)-\mathrm{C}(18)-\mathrm{C}(17)$ & $-0.2(2)$ \\
\hline $\mathrm{N}(1)-\mathrm{C}(7)-\mathrm{C}(8)-\mathrm{N}(3)$ & $-4.5(2)$ & $\mathrm{N}(1)-\mathrm{C}(13)-\mathrm{C}(18)-\mathrm{C}(17)$ & $178.18(13)$ \\
\hline
\end{tabular}




$\begin{array}{lrlr}\mathrm{C}(12)-\mathrm{C}(7)-\mathrm{C}(8)-\mathrm{C}(9) & -1.2(2) & \mathrm{C}(21) \# 1-\mathrm{C}(19)-\mathrm{C}(20)-\mathrm{C}(21) & 0.5(3) \\ \mathrm{N}(1)-\mathrm{C}(7)-\mathrm{C}(8)-\mathrm{C}(9) & 178.19(12) & \mathrm{C}(19)-\mathrm{C}(20)-\mathrm{C}(21)-\mathrm{C}(19) \# 1 & -0.5(3) \\ \mathrm{N}(3)-\mathrm{C}(8)-\mathrm{C}(9)-\mathrm{C}(10) & -176.40(14) & \end{array}$

Symmetry transformations used to generate equivalent atoms:

$\# 1-\mathrm{x},-\mathrm{y},-\mathrm{z}$ 
Table S7. Atomic coordinates $\left(\times 10^{4}\right)$ and equivalent isotropic displacement parameters $\left(\AA^{2} \times 10^{3}\right)$ for $\mathrm{L}^{1} \mathrm{H}_{3}$. U(eq) is defined as one third of the trace of the orthogonalized $\mathrm{U}^{\mathrm{ij}}$ tensor

\begin{tabular}{|c|c|c|c|c|}
\hline & $\mathrm{x}$ & $\mathrm{y}$ & $\mathrm{z}$ & $\mathrm{U}(\mathrm{eq})$ \\
\hline $\mathrm{N}(1)$ & 10271(2) & 8798(2) & 1983(2) & $41(1)$ \\
\hline $\mathrm{N}(2)$ & $9980(2)$ & 7597(2) & $3061(2)$ & $55(1)$ \\
\hline $\mathrm{N}(3)$ & $8951(2)$ & 7637(2) & $1180(2)$ & $57(1)$ \\
\hline $\mathrm{N}(4)$ & $11175(2)$ & 7097(2) & $1873(2)$ & $58(1)$ \\
\hline $\mathrm{C}(1)$ & $10745(2)$ & 8974(2) & $2737(2)$ & $43(1)$ \\
\hline $\mathrm{C}(2)$ & $10588(2)$ & 8359(3) & $3280(2)$ & $47(1)$ \\
\hline$C(3)$ & $11024(2)$ & 8543(3) & $4018(2)$ & $60(1)$ \\
\hline $\mathrm{C}(4)$ & $11624(3)$ & 9300(3) & $4212(3)$ & $69(1)$ \\
\hline$C(5)$ & 11790(3) & 9880(3) & $3675(3)$ & $69(1)$ \\
\hline $\mathrm{C}(6)$ & $11353(2)$ & 9722(3) & $2947(2)$ & $57(1)$ \\
\hline $\mathrm{C}(7)$ & $9428(2)$ & 9229(2) & $1729(2)$ & $43(1)$ \\
\hline $\mathrm{C}(8)$ & $8764(2)$ & $8624(3)$ & 1301(2) & $48(1)$ \\
\hline $\mathrm{C}(9)$ & $7959(2)$ & 9044(3) & $1006(2)$ & 61(1) \\
\hline$C(10)$ & $7802(3)$ & $10008(3)$ & 1173(3) & $72(1)$ \\
\hline$C(11)$ & $8444(3)$ & 10582(3) & 1631(3) & $74(1)$ \\
\hline$C(12)$ & $9260(3)$ & 10189(3) & $1899(2)$ & 61(1) \\
\hline$C(13)$ & $10735(2)$ & $8767(2)$ & $1455(2)$ & $43(1)$ \\
\hline$C(14)$ & $11198(2)$ & 7900(2) & $1415(2)$ & $47(1)$ \\
\hline
\end{tabular}




\begin{tabular}{|c|c|c|c|c|}
\hline$C(15)$ & $11667(2)$ & $7876(3)$ & $916(2)$ & $55(1)$ \\
\hline$C(16)$ & $11649(2)$ & $8660(3)$ & $451(2)$ & $58(1)$ \\
\hline$C(17)$ & 11171(3) & $9499(3)$ & $472(2)$ & $58(1)$ \\
\hline $\mathrm{C}(18)$ & $10730(2)$ & $9549(3)$ & $977(2)$ & $53(1)$ \\
\hline$C(19)$ & $10047(2)$ & $6624(3)$ & $3345(2)$ & $46(1)$ \\
\hline$C(20)$ & $10798(2)$ & $6216(3)$ & $3795(2)$ & $54(1)$ \\
\hline $\mathrm{C}(21)$ & $10826(2)$ & $5222(3)$ & $4000(2)$ & $58(1)$ \\
\hline $\mathrm{C}(22)$ & $10130(2)$ & $4602(3)$ & $3776(2)$ & $55(1)$ \\
\hline$C(23)$ & $9379(2)$ & $5034(3)$ & $3332(2)$ & $60(1)$ \\
\hline$C(24)$ & $9335(2)$ & $6015(3)$ & $3119(2)$ & $54(1)$ \\
\hline$C(25)$ & 10171(3) & $3503(3)$ & $4002(3)$ & $73(1)$ \\
\hline$C(26)$ & $9991(12)$ & $3389(6)$ & $4650(7)$ & $382(13)$ \\
\hline $\mathrm{C}(27)$ & $10960(5)$ & $3018(4)$ & $4008(5)$ & $152(3)$ \\
\hline $\mathrm{C}(28)$ & $9529(6)$ & $2900(6)$ & $3435(10)$ & $384(13)$ \\
\hline C(29) & $8455(2)$ & $6785(3)$ & $1183(2)$ & $45(1)$ \\
\hline$C(30)$ & $7677(2)$ & $6787(3)$ & $1330(2)$ & $52(1)$ \\
\hline$C(31)$ & $7253(2)$ & $5905(3)$ & $1343(2)$ & $52(1)$ \\
\hline$C(32)$ & $7569(2)$ & 4981(3) & $1218(2)$ & $47(1)$ \\
\hline$C(33)$ & $8353(2)$ & $5000(3)$ & $1090(2)$ & $54(1)$ \\
\hline$C(34)$ & $8780(2)$ & $5872(3)$ & $1073(2)$ & $53(1)$ \\
\hline$C(35)$ & $7077(3)$ & 4019(3) & $1231(2)$ & $59(1)$ \\
\hline$C(36)$ & $6133(3)$ & $4151(4)$ & $850(3)$ & $95(2)$ \\
\hline
\end{tabular}




\begin{tabular}{lrrrr}
$\mathrm{C}(37)$ & $7378(3)$ & $3175(3)$ & $843(4)$ & $105(2)$ \\
$\mathrm{C}(38)$ & $7210(5)$ & $3714(4)$ & $2026(3)$ & $132(3)$ \\
$\mathrm{C}(39)$ & $11225(2)$ & $6079(2)$ & $1711(2)$ & $48(1)$ \\
$\mathrm{C}(40)$ & $10987(2)$ & $5696(3)$ & $1002(2)$ & $49(1)$ \\
$\mathrm{C}(41)$ & $10981(2)$ & $4684(3)$ & $882(2)$ & $51(1)$ \\
$\mathrm{C}(42)$ & $11201(2)$ & $3994(3)$ & $1458(2)$ & $49(1)$ \\
$\mathrm{C}(43)$ & $11453(3)$ & $4391(3)$ & $2157(2)$ & $59(1)$ \\
$\mathrm{C}(44)$ & $11475(3)$ & $5405(3)$ & $2291(2)$ & $56(1)$ \\
$\mathrm{C}(45)$ & $11156(3)$ & $2878(3)$ & $1307(2)$ & $59(1)$ \\
$\mathrm{C}(46)$ & $11621(9)$ & $2607(4)$ & $801(6)$ & $284(9)$ \\
$\mathrm{C}(47)$ & $11545(6)$ & $2282(4)$ & $1962(4)$ & $192(5)$ \\
$\mathrm{C}(48)$ & $10282(5)$ & $2576(4)$ & $1033(8)$ & $288(9)$ \\
\hline
\end{tabular}


Table S8. Bond Distances, $\AA$, for $\mathrm{L}^{1} \mathrm{H}_{3}$

\begin{tabular}{|c|c|c|c|}
\hline $\mathrm{N}(1)-\mathrm{C}(1)$ & $1.432(4)$ & $\mathrm{C}(20)-\mathrm{C}(21)$ & $1.387(5)$ \\
\hline $\mathrm{N}(1)-\mathrm{C}(13)$ & $1.436(4)$ & $C(21)-C(22)$ & $1.375(5)$ \\
\hline $\mathrm{N}(1)-\mathrm{C}(7)$ & $1.444(4)$ & $\mathrm{C}(22)-\mathrm{C}(23)$ & $1.394(5)$ \\
\hline $\mathrm{N}(2)-\mathrm{C}(2)$ & $1.403(4)$ & $\mathrm{C}(22)-\mathrm{C}(25)$ & $1.532(5)$ \\
\hline $\mathrm{N}(2)-\mathrm{C}(19)$ & $1.405(4)$ & $\mathrm{C}(23)-\mathrm{C}(24)$ & $1.373(5)$ \\
\hline $\mathrm{N}(3)-\mathrm{C}(8)$ & $1.395(4)$ & $C(25)-C(26)$ & $1.363(8)$ \\
\hline $\mathrm{N}(3)-\mathrm{C}(29)$ & $1.406(4)$ & $C(25)-C(27)$ & $1.449(7)$ \\
\hline $\mathrm{N}(4)-\mathrm{C}(14)$ & $1.395(4)$ & $\mathrm{C}(25)-\mathrm{C}(28)$ & $1.498(12)$ \\
\hline $\mathrm{N}(4)-\mathrm{C}(39)$ & $1.408(4)$ & $\mathrm{C}(29)-\mathrm{C}(34)$ & $1.378(5)$ \\
\hline$C(1)-C(6)$ & $1.388(5)$ & $C(29)-C(30)$ & $1.392(5)$ \\
\hline$C(1)-C(2)$ & $1.407(5)$ & $\mathrm{C}(30)-\mathrm{C}(31)$ & $1.378(5)$ \\
\hline$C(2)-C(3)$ & $1.394(5)$ & $\mathrm{C}(31)-\mathrm{C}(32)$ & $1.393(5)$ \\
\hline$C(3)-C(4)$ & $1.386(6)$ & $\mathrm{C}(32)-\mathrm{C}(33)$ & $1.384(5)$ \\
\hline$C(4)-C(5)$ & $1.377(6)$ & $\mathrm{C}(32)-\mathrm{C}(35)$ & $1.528(5)$ \\
\hline$C(5)-C(6)$ & $1.372(6)$ & $\mathrm{C}(33)-\mathrm{C}(34)$ & $1.370(5)$ \\
\hline$C(7)-C(12)$ & $1.377(5)$ & $C(35)-C(36)$ & $1.512(6)$ \\
\hline$C(7)-C(8)$ & $1.406(5)$ & $C(35)-C(37)$ & $1.517(6)$ \\
\hline $\mathrm{C}(8)-\mathrm{C}(9)$ & $1.391(5)$ & $\mathrm{C}(35)-\mathrm{C}(38)$ & $1.521(6)$ \\
\hline$C(9)-C(10)$ & $1.375(6)$ & $\mathrm{C}(39)-\mathrm{C}(40)$ & $1.387(5)$ \\
\hline $\mathrm{C}(10)-\mathrm{C}(11)$ & $1.384(6)$ & $\mathrm{C}(39)-\mathrm{C}(44)$ & $1.391(5)$ \\
\hline $\mathrm{C}(11)-\mathrm{C}(12)$ & $1.387(6)$ & $C(40)-C(41)$ & $1.378(5)$ \\
\hline
\end{tabular}




$\begin{array}{lllr}\mathrm{C}(13)-\mathrm{C}(18) & 1.388(5) & \mathrm{C}(41)-\mathrm{C}(42) & 1.398(5) \\ \mathrm{C}(13)-\mathrm{C}(14) & 1.407(5) & \mathrm{C}(42)-\mathrm{C}(43) & 1.377(5) \\ \mathrm{C}(14)-\mathrm{C}(15) & 1.394(5) & \mathrm{C}(42)-\mathrm{C}(45) & 1.524(5) \\ \mathrm{C}(15)-\mathrm{C}(16) & 1.371(5) & \mathrm{C}(43)-\mathrm{C}(44) & 1.383(5) \\ \mathrm{C}(16)-\mathrm{C}(17) & 1.382(5) & \mathrm{C}(45)-\mathrm{C}(48) & 1.433(8) \\ \mathrm{C}(17)-\mathrm{C}(18) & 1.372(5) & \mathrm{C}(45)-\mathrm{C}(46) & 1.448(7) \\ \mathrm{C}(19)-\mathrm{C}(20) & 1.386(5) & \mathrm{C}(45)-\mathrm{C}(47) & 1.457(7) \\ \mathrm{C}(19)-\mathrm{C}(24) & 1.387(5) & & \end{array}$


Table S9. Angles, ${ }^{\circ}$, for $\mathrm{L}^{1} \mathrm{H}_{3}$

\begin{tabular}{|c|c|c|c|}
\hline $\mathrm{C}(1)-\mathrm{N}(1)-\mathrm{C}(13)$ & $117.7(3)$ & $\mathrm{C}(21)-\mathrm{C}(22)-\mathrm{C}(25)$ & $122.3(4)$ \\
\hline $\mathrm{C}(1)-\mathrm{N}(1)-\mathrm{C}(7)$ & $116.4(3)$ & $\mathrm{C}(23)-\mathrm{C}(22)-\mathrm{C}(25)$ & $121.8(3)$ \\
\hline $\mathrm{C}(13)-\mathrm{N}(1)-\mathrm{C}(7)$ & $116.0(3)$ & $\mathrm{C}(24)-\mathrm{C}(23)-\mathrm{C}(22)$ & $122.4(4)$ \\
\hline $\mathrm{C}(2)-\mathrm{N}(2)-\mathrm{C}(19)$ & $126.7(3)$ & $C(23)-C(24)-C(19)$ & $120.6(3)$ \\
\hline $\mathrm{C}(8)-\mathrm{N}(3)-\mathrm{C}(29)$ & $128.0(3)$ & $\mathrm{C}(26)-\mathrm{C}(25)-\mathrm{C}(27)$ & $112.5(7)$ \\
\hline $\mathrm{C}(14)-\mathrm{N}(4)-\mathrm{C}(39)$ & 126.7(3) & $C(26)-C(25)-C(28)$ & 107.1(8) \\
\hline$C(6)-C(1)-C(2)$ & $119.2(3)$ & $\mathrm{C}(27)-\mathrm{C}(25)-\mathrm{C}(28)$ & $101.3(7)$ \\
\hline $\mathrm{C}(6)-\mathrm{C}(1)-\mathrm{N}(1)$ & $122.3(3)$ & $\mathrm{C}(26)-\mathrm{C}(25)-\mathrm{C}(22)$ & $111.1(5)$ \\
\hline $\mathrm{C}(2)-\mathrm{C}(1)-\mathrm{N}(1)$ & $118.5(3)$ & $\mathrm{C}(27)-\mathrm{C}(25)-\mathrm{C}(22)$ & $113.4(4)$ \\
\hline $\mathrm{C}(3)-\mathrm{C}(2)-\mathrm{N}(2)$ & $122.1(3)$ & $\mathrm{C}(28)-\mathrm{C}(25)-\mathrm{C}(22)$ & $110.8(5)$ \\
\hline $\mathrm{C}(3)-\mathrm{C}(2)-\mathrm{C}(1)$ & 119.1(3) & $\mathrm{C}(34)-\mathrm{C}(29)-\mathrm{C}(30)$ & $117.1(3)$ \\
\hline $\mathrm{N}(2)-\mathrm{C}(2)-\mathrm{C}(1)$ & $118.8(3)$ & $\mathrm{C}(34)-\mathrm{C}(29)-\mathrm{N}(3)$ & $118.1(3)$ \\
\hline$C(4)-C(3)-C(2)$ & $120.3(4)$ & $\mathrm{C}(30)-\mathrm{C}(29)-\mathrm{N}(3)$ & $124.7(3)$ \\
\hline $\mathrm{C}(5)-\mathrm{C}(4)-\mathrm{C}(3)$ & $120.2(4)$ & $\mathrm{C}(31)-\mathrm{C}(30)-\mathrm{C}(29)$ & $120.2(3)$ \\
\hline$C(6)-C(5)-C(4)$ & $120.2(4)$ & $C(30)-C(31)-C(32)$ & $123.1(3)$ \\
\hline$C(5)-C(6)-C(1)$ & $120.9(4)$ & $\mathrm{C}(33)-\mathrm{C}(32)-\mathrm{C}(31)$ & $115.4(3)$ \\
\hline$C(12)-C(7)-C(8)$ & 120.1(3) & $C(33)-C(32)-C(35)$ & $123.1(3)$ \\
\hline $\mathrm{C}(12)-\mathrm{C}(7)-\mathrm{N}(1)$ & $122.5(3)$ & $\mathrm{C}(31)-\mathrm{C}(32)-\mathrm{C}(35)$ & $121.5(3)$ \\
\hline $\mathrm{C}(8)-\mathrm{C}(7)-\mathrm{N}(1)$ & $117.5(3)$ & $\mathrm{C}(34)-\mathrm{C}(33)-\mathrm{C}(32)$ & $122.2(3)$ \\
\hline $\mathrm{C}(9)-\mathrm{C}(8)-\mathrm{N}(3)$ & $123.3(3)$ & $\mathrm{C}(33)-\mathrm{C}(34)-\mathrm{C}(29)$ & $122.0(3)$ \\
\hline $\mathrm{C}(9)-\mathrm{C}(8)-\mathrm{C}(7)$ & $118.5(3)$ & $\mathrm{C}(36)-\mathrm{C}(35)-\mathrm{C}(37)$ & $107.0(4)$ \\
\hline
\end{tabular}




\begin{tabular}{|c|c|c|c|}
\hline $\mathrm{N}(3)-\mathrm{C}(8)-\mathrm{C}(7)$ & $118.2(3)$ & $\mathrm{C}(36)-\mathrm{C}(35)-\mathrm{C}(38)$ & $109.1(4)$ \\
\hline $\mathrm{C}(10)-\mathrm{C}(9)-\mathrm{C}(8)$ & $120.6(4)$ & $\mathrm{C}(37)-\mathrm{C}(35)-\mathrm{C}(38)$ & $108.4(4)$ \\
\hline$C(9)-C(10)-C(11)$ & $120.7(4)$ & $C(36)-C(35)-C(32)$ & $111.5(3)$ \\
\hline$C(10)-C(11)-C(12)$ & $119.2(4)$ & $\mathrm{C}(37)-\mathrm{C}(35)-\mathrm{C}(32)$ & $111.7(3)$ \\
\hline$C(7)-C(12)-C(11)$ & $120.6(4)$ & $\mathrm{C}(38)-\mathrm{C}(35)-\mathrm{C}(32)$ & $109.0(3)$ \\
\hline $\mathrm{C}(18)-\mathrm{C}(13)-\mathrm{C}(14)$ & 119.1(3) & $\mathrm{C}(40)-\mathrm{C}(39)-\mathrm{C}(44)$ & $117.5(3)$ \\
\hline $\mathrm{C}(18)-\mathrm{C}(13)-\mathrm{N}(1)$ & $122.6(3)$ & $\mathrm{C}(40)-\mathrm{C}(39)-\mathrm{N}(4)$ & $123.7(3)$ \\
\hline$C(14)-C(13)-N(1)$ & $118.3(3)$ & $\mathrm{C}(44)-\mathrm{C}(39)-\mathrm{N}(4)$ & $118.7(3)$ \\
\hline$C(15)-C(14)-N(4)$ & $122.4(3)$ & $C(41)-C(40)-C(39)$ & $120.9(3)$ \\
\hline $\mathrm{C}(15)-\mathrm{C}(14)-\mathrm{C}(13)$ & $118.5(3)$ & $\mathrm{C}(40)-\mathrm{C}(41)-\mathrm{C}(42)$ & $122.4(4)$ \\
\hline $\mathrm{N}(4)-\mathrm{C}(14)-\mathrm{C}(13)$ & $119.2(3)$ & $\mathrm{C}(43)-\mathrm{C}(42)-\mathrm{C}(41)$ & $115.8(3)$ \\
\hline$C(16)-C(15)-C(14)$ & $121.1(3)$ & $\mathrm{C}(43)-\mathrm{C}(42)-\mathrm{C}(45)$ & $123.1(3)$ \\
\hline$C(15)-C(16)-C(17)$ & $120.5(3)$ & $\mathrm{C}(41)-\mathrm{C}(42)-\mathrm{C}(45)$ & $121.1(3)$ \\
\hline $\mathrm{C}(18)-\mathrm{C}(17)-\mathrm{C}(16)$ & $119.2(3)$ & $\mathrm{C}(42)-\mathrm{C}(43)-\mathrm{C}(44)$ & $122.9(3)$ \\
\hline $\mathrm{C}(17)-\mathrm{C}(18)-\mathrm{C}(13)$ & $121.7(3)$ & $\mathrm{C}(43)-\mathrm{C}(44)-\mathrm{C}(39)$ & $120.6(4)$ \\
\hline $\mathrm{C}(20)-\mathrm{C}(19)-\mathrm{C}(24)$ & $118.0(3)$ & $\mathrm{C}(48)-\mathrm{C}(45)-\mathrm{C}(46)$ & $111.1(8)$ \\
\hline $\mathrm{C}(20)-\mathrm{C}(19)-\mathrm{N}(2)$ & $123.9(3)$ & $\mathrm{C}(48)-\mathrm{C}(45)-\mathrm{C}(47)$ & $106.8(7)$ \\
\hline $\mathrm{C}(24)-\mathrm{C}(19)-\mathrm{N}(2)$ & $117.9(3)$ & $C(46)-C(45)-C(47)$ & $104.5(7)$ \\
\hline $\mathrm{C}(19)-\mathrm{C}(20)-\mathrm{C}(21)$ & $120.1(3)$ & $\mathrm{C}(48)-\mathrm{C}(45)-\mathrm{C}(42)$ & $109.4(4)$ \\
\hline $\mathrm{C}(22)-\mathrm{C}(21)-\mathrm{C}(20)$ & $122.9(4)$ & $C(46)-C(45)-C(42)$ & $111.6(4)$ \\
\hline $\mathrm{C}(21)-\mathrm{C}(22)-\mathrm{C}(23)$ & $115.9(3)$ & $\mathrm{C}(47)-\mathrm{C}(45)-\mathrm{C}(42)$ & $113.2(4)$ \\
\hline
\end{tabular}


Table S10. Anisotropic displacement parameters $\left(\AA^{2} \times 10^{3}\right)$ for $\mathrm{L}^{1} \mathrm{H}_{3}$ The anisotropic displacement factor exponent takes the form: $-2 \pi^{2}\left[h^{2} a^{* 2} U^{11}+\ldots+2 h k a^{*} b^{*} U^{12}\right]$

\begin{tabular}{|c|c|c|c|c|c|c|}
\hline & $\mathrm{U}^{11}$ & $\mathrm{U}^{22}$ & $\mathrm{U}^{33}$ & $\mathrm{U}^{23}$ & $\mathrm{U}^{13}$ & $\mathrm{U}^{12}$ \\
\hline $\mathrm{N}(1)$ & $39(2)$ & $35(2)$ & $49(2)$ & $-2(1)$ & $15(1)$ & $0(1)$ \\
\hline $\mathrm{N}(2)$ & $49(2)$ & $54(2)$ & $52(2)$ & $8(1)$ & $1(2)$ & $-8(1)$ \\
\hline $\mathrm{N}(3)$ & $43(2)$ & $55(2)$ & $75(2)$ & $-14(2)$ & $23(2)$ & $-9(1)$ \\
\hline $\mathrm{N}(4)$ & $82(2)$ & $39(2)$ & $61(2)$ & $5(1)$ & $36(2)$ & $13(2)$ \\
\hline $\mathrm{C}(1)$ & $39(2)$ & $40(2)$ & $50(2)$ & $-10(2)$ & $14(2)$ & $-2(2)$ \\
\hline$C(2)$ & $42(2)$ & $48(2)$ & $52(2)$ & $-7(2)$ & $14(2)$ & $-3(2)$ \\
\hline$C(3)$ & $63(2)$ & $67(3)$ & $49(2)$ & $-7(2)$ & $15(2)$ & $-2(2)$ \\
\hline$C(4)$ & $59(3)$ & $82(3)$ & $60(3)$ & $-23(2)$ & $11(2)$ & $-4(2)$ \\
\hline$C(5)$ & $54(2)$ & $66(3)$ & $80(3)$ & $-26(2)$ & $11(2)$ & $-15(2)$ \\
\hline$C(6)$ & $51(2)$ & $49(2)$ & $70(3)$ & $-8(2)$ & $19(2)$ & $-5(2)$ \\
\hline$C(7)$ & $42(2)$ & $37(2)$ & $53(2)$ & $10(2)$ & $18(2)$ & $6(2)$ \\
\hline$C(8)$ & $40(2)$ & $50(2)$ & $54(2)$ & $8(2)$ & $16(2)$ & $-1(2)$ \\
\hline$C(9)$ & $45(2)$ & $65(3)$ & $71(3)$ & $17(2)$ & $14(2)$ & $1(2)$ \\
\hline$C(10)$ & $49(2)$ & $69(3)$ & $100(4)$ & $34(3)$ & $24(3)$ & $18(2)$ \\
\hline$C(11)$ & $71(3)$ & $49(2)$ & $108(4)$ & $17(2)$ & $37(3)$ & $21(2)$ \\
\hline$C(12)$ & $61(3)$ & $41(2)$ & $86(3)$ & $7(2)$ & $30(2)$ & $6(2)$ \\
\hline$C(13)$ & $39(2)$ & $36(2)$ & $54(2)$ & $-1(2)$ & $14(2)$ & $-2(1)$ \\
\hline$C(14)$ & $48(2)$ & $38(2)$ & $56(2)$ & $0(2)$ & $18(2)$ & $-1(2)$ \\
\hline
\end{tabular}




\begin{tabular}{|c|c|c|c|c|c|c|}
\hline$C(15)$ & $54(2)$ & $47(2)$ & $70(3)$ & $0(2)$ & $29(2)$ & $4(2)$ \\
\hline$C(16)$ & $55(2)$ & $61(3)$ & $66(3)$ & $1(2)$ & $28(2)$ & $-4(2)$ \\
\hline$C(17)$ & $67(3)$ & $49(2)$ & $64(3)$ & $10(2)$ & $28(2)$ & $-3(2)$ \\
\hline$C(18)$ & $56(2)$ & $39(2)$ & $68(3)$ & $5(2)$ & $25(2)$ & $3(2)$ \\
\hline$C(19)$ & $49(2)$ & $54(2)$ & $36(2)$ & $3(2)$ & $16(2)$ & $-5(2)$ \\
\hline$C(20)$ & $45(2)$ & $66(3)$ & $50(2)$ & $6(2)$ & $13(2)$ & $-7(2)$ \\
\hline $\mathrm{C}(21)$ & $43(2)$ & $73(3)$ & $57(2)$ & $18(2)$ & $13(2)$ & $2(2)$ \\
\hline$C(22)$ & $47(2)$ & $66(2)$ & $53(2)$ & $19(2)$ & $19(2)$ & $2(2)$ \\
\hline$C(23)$ & $51(2)$ & $62(3)$ & $63(3)$ & $11(2)$ & $14(2)$ & $-12(2)$ \\
\hline$C(24)$ & $45(2)$ & $57(2)$ & $54(2)$ & $9(2)$ & $8(2)$ & $0(2)$ \\
\hline$C(25)$ & $52(2)$ & $69(3)$ & $99(4)$ & $36(2)$ & $24(2)$ & $4(2)$ \\
\hline$C(26)$ & $860(30)$ & $118(7)$ & $393(17)$ & $160(9)$ & $530(20)$ & $202(13)$ \\
\hline$C(27)$ & $164(7)$ & $77(4)$ & $256(10)$ & $42(5)$ & $125(7)$ & $31(4)$ \\
\hline $\mathrm{C}(28)$ & 177(9) & $99(6)$ & $670(30)$ & $161(11)$ & $-185(14)$ & $-59(6)$ \\
\hline$C(29)$ & $41(2)$ & $54(2)$ & $38(2)$ & $-8(2)$ & $8(2)$ & $-4(2)$ \\
\hline$C(30)$ & $46(2)$ & $55(2)$ & $55(2)$ & $-10(2)$ & $17(2)$ & $-3(2)$ \\
\hline $\mathrm{C}(31)$ & $41(2)$ & $60(2)$ & $56(2)$ & $-7(2)$ & $18(2)$ & $-5(2)$ \\
\hline$C(32)$ & $44(2)$ & $54(2)$ & $41(2)$ & $-2(2)$ & $9(2)$ & $-2(2)$ \\
\hline$C(33)$ & $52(2)$ & $53(2)$ & $60(2)$ & $-3(2)$ & $20(2)$ & $3(2)$ \\
\hline$C(34)$ & $41(2)$ & $60(2)$ & $60(2)$ & $-1(2)$ & $19(2)$ & $0(2)$ \\
\hline$C(35)$ & $63(2)$ & $54(2)$ & $57(3)$ & $-1(2)$ & $17(2)$ & $-9(2)$ \\
\hline$C(36)$ & $60(3)$ & $81(3)$ & $141(5)$ & $-2(3)$ & $28(3)$ & $-17(2)$ \\
\hline
\end{tabular}




\begin{tabular}{lcccccc}
$\mathrm{C}(37)$ & $92(4)$ & $62(3)$ & $168(6)$ & $-34(3)$ & $50(4)$ & $-23(3)$ \\
$\mathrm{C}(38)$ & $195(7)$ & $114(5)$ & $74(4)$ & $20(3)$ & $19(4)$ & $-80(4)$ \\
$\mathrm{C}(39)$ & $47(2)$ & $41(2)$ & $61(2)$ & $4(2)$ & $26(2)$ & $4(2)$ \\
$\mathrm{C}(40)$ & $44(2)$ & $46(2)$ & $55(2)$ & $11(2)$ & $12(2)$ & $10(2)$ \\
$\mathrm{C}(41)$ & $47(2)$ & $52(2)$ & $50(2)$ & $-2(2)$ & $11(2)$ & $0(2)$ \\
$\mathrm{C}(42)$ & $49(2)$ & $45(2)$ & $57(2)$ & $4(2)$ & $21(2)$ & $-1(2)$ \\
$\mathrm{C}(43)$ & $85(3)$ & $43(2)$ & $55(3)$ & $9(2)$ & $30(2)$ & $1(2)$ \\
$\mathrm{C}(44)$ & $77(3)$ & $48(2)$ & $52(2)$ & $2(2)$ & $32(2)$ & $0(2)$ \\
$\mathrm{C}(45)$ & $71(3)$ & $41(2)$ & $65(3)$ & $2(2)$ & $22(2)$ & $-7(2)$ \\
$\mathrm{C}(46)$ & $640(20)$ & $52(4)$ & $334(13)$ & $-45(5)$ & $405(17)$ & $-45(7)$ \\
$\mathrm{C}(47)$ & $299(11)$ & $54(4)$ & $140(6)$ & $-16(4)$ & $-60(7)$ & $54(5)$ \\
$\mathrm{C}(48)$ & $131(7)$ & $49(4)$ & $560(20)$ & $6(7)$ & $-78(10)$ & $-26(4)$ \\
\hline & & & & & & \\
\hline
\end{tabular}


Table S11. Hydrogen coordinates $\left(\times 10^{4}\right)$ and isotropic displacement parameters $\left(\AA^{2} \times 10^{3}\right)$ for $\mathrm{L}^{1} \mathrm{H}_{3}$

\begin{tabular}{|c|c|c|c|c|}
\hline & $\mathrm{x}$ & $y$ & Z & $\overline{\mathrm{U}(\mathrm{eq})}$ \\
\hline $\mathrm{H}(2 \mathrm{~A})$ & 9513 & 7742 & 2714 & 66 \\
\hline $\mathrm{H}(3 \mathrm{~A})$ & 9433 & 7537 & 1093 & 68 \\
\hline $\mathrm{H}(4 \mathrm{~A})$ & 11123 & 7239 & 2303 & 69 \\
\hline $\mathrm{H}(3)$ & 10909 & 8153 & 4387 & 72 \\
\hline $\mathrm{H}(4)$ & 11918 & 9417 & 4711 & 82 \\
\hline $\mathrm{H}(5)$ & 12203 & 10385 & 3809 & 82 \\
\hline $\mathrm{H}(6)$ & 11468 & 10125 & 2586 & 68 \\
\hline $\mathrm{H}(9)$ & 7518 & 8666 & 689 & 73 \\
\hline $\mathrm{H}(10)$ & 7253 & 10279 & 973 & 87 \\
\hline $\mathrm{H}(11)$ & 8329 & 11232 & 1759 & 89 \\
\hline $\mathrm{H}(12)$ & 9703 & 10582 & 2199 & 73 \\
\hline $\mathrm{H}(15)$ & 12001 & 7314 & 899 & 66 \\
\hline $\mathrm{H}(16)$ & 11965 & 8626 & 115 & 70 \\
\hline $\mathrm{H}(17)$ & 11148 & 10030 & 145 & 70 \\
\hline $\mathrm{H}(18)$ & 10415 & 10126 & 999 & 64 \\
\hline $\mathrm{H}(20)$ & 11290 & 6613 & 3961 & 64 \\
\hline $\mathrm{H}(21)$ & 11342 & 4962 & 4305 & 69 \\
\hline $\mathrm{H}(23)$ & 8885 & 4640 & 3173 & 72 \\
\hline $\mathrm{H}(24)$ & 8816 & 6275 & 2816 & 64 \\
\hline
\end{tabular}




\begin{tabular}{|c|c|c|c|c|}
\hline $\mathrm{H}(26 \mathrm{~A})$ & 9892 & 2691 & 4725 & 574 \\
\hline $\mathrm{H}(26 \mathrm{~B})$ & 9484 & 3770 & 4634 & 574 \\
\hline $\mathrm{H}(26 \mathrm{C})$ & 10468 & 3627 & 5052 & 574 \\
\hline $\mathrm{H}(27 \mathrm{~A})$ & 11407 & 3202 & 4452 & 228 \\
\hline $\mathrm{H}(27 \mathrm{~B})$ & 11119 & 3226 & 3580 & 228 \\
\hline $\mathrm{H}(27 \mathrm{C})$ & 10882 & 2301 & 3996 & 228 \\
\hline $\mathrm{H}(28 \mathrm{~A})$ & 9613 & 2199 & 3558 & 576 \\
\hline $\mathrm{H}(28 \mathrm{~B})$ & 9603 & 3015 & 2955 & 576 \\
\hline $\mathrm{H}(28 \mathrm{C})$ & 8958 & 3096 & 3424 & 576 \\
\hline $\mathrm{H}(30)$ & 7439 & 7392 & 1421 & 62 \\
\hline $\mathrm{H}(31)$ & 6727 & 5928 & 1441 & 62 \\
\hline $\mathrm{H}(33)$ & 8600 & 4396 & 1012 & 65 \\
\hline $\mathrm{H}(34)$ & 9311 & 5846 & 984 & 63 \\
\hline $\mathrm{H}(36 \mathrm{~A})$ & 6045 & 4372 & 348 & 142 \\
\hline $\mathrm{H}(36 \mathrm{~B})$ & 5905 & 4644 & 1112 & 142 \\
\hline $\mathrm{H}(36 \mathrm{C})$ & 5842 & 3521 & 845 & 142 \\
\hline $\mathrm{H}(37 \mathrm{~A})$ & 7026 & 2592 & 831 & 157 \\
\hline $\mathrm{H}(37 \mathrm{~B})$ & 7968 & 3019 & 1105 & 157 \\
\hline $\mathrm{H}(37 \mathrm{C})$ & 7332 & 3375 & 343 & 157 \\
\hline $\mathrm{H}(38 \mathrm{~A})$ & 6888 & 3114 & 2039 & 198 \\
\hline $\mathrm{H}(38 \mathrm{~B})$ & 7017 & 4245 & 2282 & 198 \\
\hline $\mathrm{H}(38 \mathrm{C})$ & 7812 & 3590 & 2266 & 198 \\
\hline
\end{tabular}




\begin{tabular}{|c|c|c|c|c|}
\hline $\mathrm{H}(40)$ & 10827 & 6133 & 598 & 59 \\
\hline $\mathrm{H}(41)$ & 10824 & 4448 & 394 & 61 \\
\hline $\mathrm{H}(43)$ & 11617 & 3954 & 2561 & 71 \\
\hline $\mathrm{H}(44)$ & 11661 & 5641 & 2778 & 67 \\
\hline $\mathrm{H}(46 \mathrm{~A})$ & 11281 & 2770 & 302 & 426 \\
\hline $\mathrm{H}(46 \mathrm{~B})$ & 11738 & 1897 & 836 & 426 \\
\hline $\mathrm{H}(46 \mathrm{C})$ & 12155 & 2971 & 924 & 426 \\
\hline $\mathrm{H}(47 \mathrm{~A})$ & 11312 & 2480 & 2352 & 289 \\
\hline $\mathrm{H}(47 \mathrm{~B})$ & 12158 & 2388 & 2122 & 289 \\
\hline $\mathrm{H}(47 \mathrm{C})$ & 11425 & 1583 & 1849 & 289 \\
\hline $\mathrm{H}(48 \mathrm{~A})$ & 10198 & 2182 & 589 & 432 \\
\hline $\mathrm{H}(48 \mathrm{~B})$ & 9919 & 3161 & 920 & 432 \\
\hline $\mathrm{H}(48 \mathrm{C})$ & 10135 & 2179 & 1402 & 432 \\
\hline
\end{tabular}


Table S12. Torsion Angles, ${ }^{\circ}$, for $\mathrm{L}^{1} \mathrm{H}_{3}$

\begin{tabular}{|c|c|c|c|}
\hline $\mathrm{C}(13)-\mathrm{N}(1)-\mathrm{C}(1)-\mathrm{C}(6)$ & $46.5(4)$ & $\mathrm{C}(24)-\mathrm{C}(19)-\mathrm{C}(20)-\mathrm{C}(21)$ & $0.7(5)$ \\
\hline $\mathrm{C}(7)-\mathrm{N}(1)-\mathrm{C}(1)-\mathrm{C}(6)$ & $-98.0(4)$ & $\mathrm{N}(2)-\mathrm{C}(19)-\mathrm{C}(20)-\mathrm{C}(21)$ & $-174.5(3)$ \\
\hline $\mathrm{C}(13)-\mathrm{N}(1)-\mathrm{C}(1)-\mathrm{C}(2)$ & $-133.0(3)$ & $\mathrm{C}(19)-\mathrm{C}(20)-\mathrm{C}(21)-\mathrm{C}(22)$ & $0.0(6)$ \\
\hline $\mathrm{C}(7)-\mathrm{N}(1)-\mathrm{C}(1)-\mathrm{C}(2)$ & $82.6(4)$ & $\mathrm{C}(20)-\mathrm{C}(21)-\mathrm{C}(22)-\mathrm{C}(23)$ & $-0.9(6)$ \\
\hline$C(19)-N(2)-C(2)-C(3)$ & $-43.0(5)$ & $\mathrm{C}(20)-\mathrm{C}(21)-\mathrm{C}(22)-\mathrm{C}(25)$ & $179.5(4)$ \\
\hline $\mathrm{C}(19)-\mathrm{N}(2)-\mathrm{C}(2)-\mathrm{C}(1)$ & $139.0(3)$ & $\mathrm{C}(21)-\mathrm{C}(22)-\mathrm{C}(23)-\mathrm{C}(24)$ & $1.0(6)$ \\
\hline$C(6)-C(1)-C(2)-C(3)$ & $2.7(5)$ & $\mathrm{C}(25)-\mathrm{C}(22)-\mathrm{C}(23)-\mathrm{C}(24)$ & $-179.3(4)$ \\
\hline $\mathrm{N}(1)-\mathrm{C}(1)-\mathrm{C}(2)-\mathrm{C}(3)$ & $-177.8(3)$ & $\mathrm{C}(22)-\mathrm{C}(23)-\mathrm{C}(24)-\mathrm{C}(19)$ & $-0.4(6)$ \\
\hline $\mathrm{C}(6)-\mathrm{C}(1)-\mathrm{C}(2)-\mathrm{N}(2)$ & $-179.2(3)$ & $\mathrm{C}(20)-\mathrm{C}(19)-\mathrm{C}(24)-\mathrm{C}(23)$ & $-0.5(5)$ \\
\hline $\mathrm{N}(1)-\mathrm{C}(1)-\mathrm{C}(2)-\mathrm{N}(2)$ & $0.3(4)$ & $\mathrm{N}(2)-\mathrm{C}(19)-\mathrm{C}(24)-\mathrm{C}(23)$ & $175.0(3)$ \\
\hline $\mathrm{N}(2)-\mathrm{C}(2)-\mathrm{C}(3)-\mathrm{C}(4)$ & $179.6(4)$ & $\mathrm{C}(21)-\mathrm{C}(22)-\mathrm{C}(25)-\mathrm{C}(26)$ & $88.8(10)$ \\
\hline $\mathrm{C}(1)-\mathrm{C}(2)-\mathrm{C}(3)-\mathrm{C}(4)$ & $-2.3(5)$ & $\mathrm{C}(23)-\mathrm{C}(22)-\mathrm{C}(25)-\mathrm{C}(26)$ & $-90.8(10)$ \\
\hline$C(2)-C(3)-C(4)-C(5)$ & $0.5(6)$ & $\mathrm{C}(21)-\mathrm{C}(22)-\mathrm{C}(25)-\mathrm{C}(27)$ & $-39.1(7)$ \\
\hline$C(3)-C(4)-C(5)-C(6)$ & $1.0(6)$ & $\mathrm{C}(23)-\mathrm{C}(22)-\mathrm{C}(25)-\mathrm{C}(27)$ & $141.3(5)$ \\
\hline$C(4)-C(5)-C(6)-C(1)$ & $-0.5(6)$ & $\mathrm{C}(21)-\mathrm{C}(22)-\mathrm{C}(25)-\mathrm{C}(28)$ & $-152.3(9)$ \\
\hline$C(2)-C(1)-C(6)-C(5)$ & $-1.3(5)$ & $\mathrm{C}(23)-\mathrm{C}(22)-\mathrm{C}(25)-\mathrm{C}(28)$ & $28.1(9)$ \\
\hline $\mathrm{N}(1)-\mathrm{C}(1)-\mathrm{C}(6)-\mathrm{C}(5)$ & $179.2(3)$ & $\mathrm{C}(8)-\mathrm{N}(3)-\mathrm{C}(29)-\mathrm{C}(34)$ & $-178.0(3)$ \\
\hline $\mathrm{C}(1)-\mathrm{N}(1)-\mathrm{C}(7)-\mathrm{C}(12)$ & $44.2(4)$ & $\mathrm{C}(8)-\mathrm{N}(3)-\mathrm{C}(29)-\mathrm{C}(30)$ & $-1.7(6)$ \\
\hline $\mathrm{C}(13)-\mathrm{N}(1)-\mathrm{C}(7)-\mathrm{C}(12)$ & $-100.9(4)$ & $\mathrm{C}(34)-\mathrm{C}(29)-\mathrm{C}(30)-\mathrm{C}(31)$ & $-1.5(5)$ \\
\hline $\mathrm{C}(1)-\mathrm{N}(1)-\mathrm{C}(7)-\mathrm{C}(8)$ & $-135.1(3)$ & $\mathrm{N}(3)-\mathrm{C}(29)-\mathrm{C}(30)-\mathrm{C}(31)$ & $-177.9(3)$ \\
\hline $\mathrm{C}(13)-\mathrm{N}(1)-\mathrm{C}(7)-\mathrm{C}(8)$ & $79.8(4)$ & $C(29)-C(30)-C(31)-C(32)$ & $0.3(6)$ \\
\hline
\end{tabular}




\begin{tabular}{|c|c|c|c|}
\hline $\mathrm{C}(29)-\mathrm{N}(3)-\mathrm{C}(8)-\mathrm{C}(9)$ & $-42.2(6)$ & $\mathrm{C}(30)-\mathrm{C}(31)-\mathrm{C}(32)-\mathrm{C}(33)$ & $1.1(5)$ \\
\hline $\mathrm{C}(29)-\mathrm{N}(3)-\mathrm{C}(8)-\mathrm{C}(7)$ & $139.2(4)$ & $\mathrm{C}(30)-\mathrm{C}(31)-\mathrm{C}(32)-\mathrm{C}(35)$ & $-179.5(4)$ \\
\hline $\mathrm{C}(12)-\mathrm{C}(7)-\mathrm{C}(8)-\mathrm{C}(9)$ & $5.3(5)$ & $\mathrm{C}(31)-\mathrm{C}(32)-\mathrm{C}(33)-\mathrm{C}(34)$ & $-1.2(5)$ \\
\hline $\mathrm{N}(1)-\mathrm{C}(7)-\mathrm{C}(8)-\mathrm{C}(9)$ & $-175.4(3)$ & $\mathrm{C}(35)-\mathrm{C}(32)-\mathrm{C}(33)-\mathrm{C}(34)$ & 179.3(4) \\
\hline $\mathrm{C}(12)-\mathrm{C}(7)-\mathrm{C}(8)-\mathrm{N}(3)$ & $-176.0(3)$ & $\mathrm{C}(32)-\mathrm{C}(33)-\mathrm{C}(34)-\mathrm{C}(29)$ & $0.0(6)$ \\
\hline $\mathrm{N}(1)-\mathrm{C}(7)-\mathrm{C}(8)-\mathrm{N}(3)$ & $3.3(5)$ & $\mathrm{C}(30)-\mathrm{C}(29)-\mathrm{C}(34)-\mathrm{C}(33)$ & $1.4(5)$ \\
\hline $\mathrm{N}(3)-\mathrm{C}(8)-\mathrm{C}(9)-\mathrm{C}(10)$ & $176.8(4)$ & $\mathrm{N}(3)-\mathrm{C}(29)-\mathrm{C}(34)-\mathrm{C}(33)$ & $178.0(3)$ \\
\hline$C(7)-C(8)-C(9)-C(10)$ & $-4.6(5)$ & $\mathrm{C}(33)-\mathrm{C}(32)-\mathrm{C}(35)-\mathrm{C}(36)$ & $-139.1(4)$ \\
\hline $\mathrm{C}(8)-\mathrm{C}(9)-\mathrm{C}(10)-\mathrm{C}(11)$ & $0.7(6)$ & $\mathrm{C}(31)-\mathrm{C}(32)-\mathrm{C}(35)-\mathrm{C}(36)$ & $41.4(5)$ \\
\hline $\mathrm{C}(9)-\mathrm{C}(10)-\mathrm{C}(11)-\mathrm{C}(12)$ & $2.5(7)$ & $\mathrm{C}(33)-\mathrm{C}(32)-\mathrm{C}(35)-\mathrm{C}(37)$ & $-19.4(6)$ \\
\hline $\mathrm{C}(8)-\mathrm{C}(7)-\mathrm{C}(12)-\mathrm{C}(11)$ & $-2.1(5)$ & $\mathrm{C}(31)-\mathrm{C}(32)-\mathrm{C}(35)-\mathrm{C}(37)$ & $161.1(4)$ \\
\hline $\mathrm{N}(1)-\mathrm{C}(7)-\mathrm{C}(12)-\mathrm{C}(11)$ & $178.6(3)$ & $\mathrm{C}(33)-\mathrm{C}(32)-\mathrm{C}(35)-\mathrm{C}(38)$ & $100.4(5)$ \\
\hline $\mathrm{C}(10)-\mathrm{C}(11)-\mathrm{C}(12)-\mathrm{C}(7)$ & $-1.8(6)$ & $\mathrm{C}(31)-\mathrm{C}(32)-\mathrm{C}(35)-\mathrm{C}(38)$ & $-79.0(5)$ \\
\hline $\mathrm{C}(1)-\mathrm{N}(1)-\mathrm{C}(13)-\mathrm{C}(18)$ & $-103.8(4)$ & $\mathrm{C}(14)-\mathrm{N}(4)-\mathrm{C}(39)-\mathrm{C}(40)$ & $-25.5(5)$ \\
\hline $\mathrm{C}(7)-\mathrm{N}(1)-\mathrm{C}(13)-\mathrm{C}(18)$ & $40.8(4)$ & $\mathrm{C}(14)-\mathrm{N}(4)-\mathrm{C}(39)-\mathrm{C}(44)$ & $158.7(4)$ \\
\hline $\mathrm{C}(1)-\mathrm{N}(1)-\mathrm{C}(13)-\mathrm{C}(14)$ & $77.6(4)$ & $\mathrm{C}(44)-\mathrm{C}(39)-\mathrm{C}(40)-\mathrm{C}(41)$ & $1.5(5)$ \\
\hline $\mathrm{C}(7)-\mathrm{N}(1)-\mathrm{C}(13)-\mathrm{C}(14)$ & $-137.9(3)$ & $\mathrm{N}(4)-\mathrm{C}(39)-\mathrm{C}(40)-\mathrm{C}(41)$ & $-174.3(3)$ \\
\hline $\mathrm{C}(39)-\mathrm{N}(4)-\mathrm{C}(14)-\mathrm{C}(15)$ & $-32.2(6)$ & $\mathrm{C}(39)-\mathrm{C}(40)-\mathrm{C}(41)-\mathrm{C}(42)$ & $0.8(5)$ \\
\hline $\mathrm{C}(39)-\mathrm{N}(4)-\mathrm{C}(14)-\mathrm{C}(13)$ & $147.8(4)$ & $\mathrm{C}(40)-\mathrm{C}(41)-\mathrm{C}(42)-\mathrm{C}(43)$ & $-2.2(5)$ \\
\hline $\mathrm{C}(18)-\mathrm{C}(13)-\mathrm{C}(14)-\mathrm{C}(15)$ & $2.8(5)$ & $\mathrm{C}(40)-\mathrm{C}(41)-\mathrm{C}(42)-\mathrm{C}(45)$ & $177.7(3)$ \\
\hline $\mathrm{N}(1)-\mathrm{C}(13)-\mathrm{C}(14)-\mathrm{C}(15)$ & $-178.4(3)$ & $\mathrm{C}(41)-\mathrm{C}(42)-\mathrm{C}(43)-\mathrm{C}(44)$ & $1.2(5)$ \\
\hline $\mathrm{C}(18)-\mathrm{C}(13)-\mathrm{C}(14)-\mathrm{N}(4)$ & $-177.2(3)$ & $C(45)-C(42)-C(43)-C(44)$ & $-178.6(4)$ \\
\hline
\end{tabular}




$\begin{array}{lrlr}\mathrm{N}(1)-\mathrm{C}(13)-\mathrm{C}(14)-\mathrm{N}(4) & 1.5(5) & \mathrm{C}(42)-\mathrm{C}(43)-\mathrm{C}(44)-\mathrm{C}(39) & 1.1(6) \\ \mathrm{N}(4)-\mathrm{C}(14)-\mathrm{C}(15)-\mathrm{C}(16) & 177.1(4) & \mathrm{C}(40)-\mathrm{C}(39)-\mathrm{C}(44)-\mathrm{C}(43) & -2.4(5) \\ \mathrm{C}(13)-\mathrm{C}(14)-\mathrm{C}(15)-\mathrm{C}(16) & -2.9(6) & \mathrm{N}(4)-\mathrm{C}(39)-\mathrm{C}(44)-\mathrm{C}(43) & 173.6(3) \\ \mathrm{C}(14)-\mathrm{C}(15)-\mathrm{C}(16)-\mathrm{C}(17) & 0.7(6) & \mathrm{C}(43)-\mathrm{C}(42)-\mathrm{C}(45)-\mathrm{C}(48) & 108.9(8) \\ \mathrm{C}(15)-\mathrm{C}(16)-\mathrm{C}(17)-\mathrm{C}(18) & 1.5(6) & \mathrm{C}(41)-\mathrm{C}(42)-\mathrm{C}(45)-\mathrm{C}(48) & -71.0(8) \\ \mathrm{C}(16)-\mathrm{C}(17)-\mathrm{C}(18)-\mathrm{C}(13) & -1.5(6) & \mathrm{C}(43)-\mathrm{C}(42)-\mathrm{C}(45)-\mathrm{C}(46) & -127.7(7) \\ \mathrm{C}(14)-\mathrm{C}(13)-\mathrm{C}(18)-\mathrm{C}(17) & -0.7(6) & \mathrm{C}(41)-\mathrm{C}(42)-\mathrm{C}(45)-\mathrm{C}(46) & 52.4(8) \\ \mathrm{N}(1)-\mathrm{C}(13)-\mathrm{C}(18)-\mathrm{C}(17) & -179.3(3) & \mathrm{C}(43)-\mathrm{C}(42)-\mathrm{C}(45)-\mathrm{C}(47) & -10.2(7) \\ \mathrm{C}(2)-\mathrm{N}(2)-\mathrm{C}(19)-\mathrm{C}(20) & -12.4(5) & \mathrm{C}(41)-\mathrm{C}(42)-\mathrm{C}(45)-\mathrm{C}(47) & 170.0(6) \\ \mathrm{C}(2)-\mathrm{N}(2)-\mathrm{C}(19)-\mathrm{C}(24) & 172.4(3) & \end{array}$


Table S13. Atomic coordinates $\left(\times 10^{4}\right)$ and equivalent isotropic displacement parameters $\left(\AA^{2} \times 10^{3}\right)$ for $\left[\left(\mathrm{L}^{1}\right) \mathrm{Fe}(\mathrm{II})-\mathrm{NCCH}_{3}\right]\left[\mathrm{Ph}_{4} \mathrm{P}\right](\mathbf{1}) . \mathrm{U}(\mathrm{eq})$ is defined as one third of the trace of the orthogonalized $\mathrm{U}^{\mathrm{ij}}$ tensor

\begin{tabular}{|c|c|c|c|c|}
\hline & $\mathrm{X}$ & $\mathrm{y}$ & $\mathrm{Z}$ & $\mathrm{U}(\mathrm{eq})$ \\
\hline $\mathrm{Fe}(1)$ & $636(1)$ & $3497(1)$ & $3178(1)$ & $22(1)$ \\
\hline $\mathrm{N}(1)$ & $647(3)$ & $3584(2)$ & $2383(2)$ & $21(1)$ \\
\hline $\mathrm{N}(2)$ & $182(4)$ & $2693(2)$ & $2959(2)$ & $27(1)$ \\
\hline $\mathrm{N}(3)$ & $2496(4)$ & $3660(2)$ & $3168(2)$ & $27(1)$ \\
\hline $\mathrm{N}(4)$ & $-663(3)$ & $4108(2)$ & $3009(2)$ & $26(1)$ \\
\hline $\mathrm{N}(5)$ & $608(4)$ & $3366(2)$ & $3924(2)$ & $38(1)$ \\
\hline$C(1)$ & $804(4)$ & $3007(2)$ & $2213(2)$ & $22(1)$ \\
\hline$C(2)$ & $554(4)$ & $2564(2)$ & $2527(2)$ & $26(1)$ \\
\hline$C(3)$ & $664(5)$ & $2014(2)$ & $2341(2)$ & $32(1)$ \\
\hline$C(4)$ & $1054(5)$ & $1916(2)$ & $1907(2)$ & $35(1)$ \\
\hline$C(5)$ & $1356(5)$ & $2348(2)$ & $1619(2)$ & $34(1)$ \\
\hline$C(6)$ & $1217(4)$ & $2898(2)$ & $1783(2)$ & $26(1)$ \\
\hline$C(7)$ & $1770(4)$ & $3935(2)$ & $2353(2)$ & $22(1)$ \\
\hline$C(8)$ & $2747(4)$ & $3910(2)$ & $2760(2)$ & $26(1)$ \\
\hline $\mathrm{C}(9)$ & $3891(5)$ & $4187(2)$ & $2701(2)$ & $34(2)$ \\
\hline$C(10)$ & $4024(5)$ & $4473(2)$ & $2286(3)$ & $39(2)$ \\
\hline $\mathrm{C}(11)$ & $3028(5)$ & $4518(2)$ & $1915(2)$ & $39(2)$ \\
\hline$C(12)$ & $1897(5)$ & $4241(2)$ & $1952(2)$ & $33(1)$ \\
\hline
\end{tabular}




\begin{tabular}{|c|c|c|c|c|}
\hline$C(13)$ & $-565(4)$ & $3847(2)$ & $2194(2)$ & $24(1)$ \\
\hline$C(14)$ & $-1171(4)$ & $4144(2)$ & $2535(2)$ & $20(1)$ \\
\hline$C(15)$ & $-2275(4)$ & $4455(2)$ & $2342(2)$ & $24(1)$ \\
\hline$C(16)$ & $-2753(4)$ & $4441(2)$ & $1864(2)$ & $31(1)$ \\
\hline$C(17)$ & $-2192(5)$ & $4125(2)$ & $1540(2)$ & $32(1)$ \\
\hline$C(18)$ & $-1084(4)$ & $3831(2)$ & $1715(2)$ & $26(1)$ \\
\hline$C(19)$ & $111(5)$ & $2256(2)$ & $3299(2)$ & $31(1)$ \\
\hline$C(20)$ & $-949(5)$ & $2228(2)$ & $3534(2)$ & $34(1)$ \\
\hline$C(21)$ & $-1042(6)$ & $1826(2)$ & $3885(2)$ & $39(2)$ \\
\hline$C(22)$ & $-91(6)$ & $1434(2)$ & $4019(2)$ & $36(1)$ \\
\hline$C(23)$ & $983(6)$ & $1475(2)$ & $3797(2)$ & $42(2)$ \\
\hline$C(24)$ & $1090(5)$ & $1877(2)$ & $3442(2)$ & $38(2)$ \\
\hline$C(25)$ & $-180(7)$ & $977(3)$ & $4401(3)$ & $54(2)$ \\
\hline$C(26)$ & $-114(16)$ & $409(3)$ & $4177(4)$ & $161(7)$ \\
\hline$C(27)$ & $-1396(11)$ & $1018(5)$ & $4601(5)$ & $146(6)$ \\
\hline$C(28)$ & $890(12)$ & $1040(4)$ & $4812(4)$ & $133(5)$ \\
\hline C(29) & $3496(4)$ & $3538(2)$ & $3544(2)$ & $31(1)$ \\
\hline$C(30)$ & $4483(5)$ & $3170(2)$ & $3484(2)$ & $38(2)$ \\
\hline$C(31)$ & $5380(5)$ & $3006(3)$ & $3864(2)$ & $41(2)$ \\
\hline$C(32)$ & $5360(5)$ & $3199(2)$ & $4336(2)$ & $38(2)$ \\
\hline C(33) & $4401(5)$ & $3576(3)$ & $4386(2)$ & $40(2)$ \\
\hline$C(34)$ & $3505(5)$ & $3753(2)$ & $4004(2)$ & $37(2)$ \\
\hline
\end{tabular}




\begin{tabular}{|c|c|c|c|c|}
\hline$C(35)$ & $6344(6)$ & $2989(3)$ & $4752(2)$ & $48(2)$ \\
\hline$C(36)$ & $6122(7)$ & $3240(3)$ & $5234(3)$ & $69(2)$ \\
\hline$C(37)$ & $7685(6)$ & $3155(4)$ & $4663(3)$ & $73(3)$ \\
\hline $\mathrm{C}(38)$ & $6231(8)$ & $2347(3)$ & 4793(3) & $75(3)$ \\
\hline$C(39)$ & $-1235(4)$ & $4377(2)$ & $3367(2)$ & $24(1)$ \\
\hline$C(40)$ & $-2457(4)$ & $4261(2)$ & $3459(2)$ & $30(1)$ \\
\hline$C(41)$ & $-2937(5)$ & $4522(2)$ & $3830(2)$ & $37(2)$ \\
\hline$C(42)$ & $-2229(5)$ & $4910(2)$ & $4136(2)$ & $34(1)$ \\
\hline$C(43)$ & $-998(5)$ & $5002(2)$ & $4052(2)$ & $34(1)$ \\
\hline$C(44)$ & $-509(5)$ & $4743(2)$ & $3679(2)$ & $33(1)$ \\
\hline$C(45)$ & $-2750(6)$ & $5212(3)$ & $4548(3)$ & $45(2)$ \\
\hline$C(46)$ & $-4191(7)$ & $5231(4)$ & $4465(4)$ & $96(3)$ \\
\hline$C(47)$ & $-2326(15)$ & $4917(5)$ & $5008(4)$ & $163(6)$ \\
\hline$C(48)$ & $-2321(10)$ & $5826(4)$ & $4572(5)$ & $135(5)$ \\
\hline C(49) & $857(6)$ & $3128(3)$ & $4271(3)$ & $46(2)$ \\
\hline$C(50)$ & $1209(8)$ & $2804(3)$ & $4719(3)$ & $73(3)$ \\
\hline $\mathrm{P}(1)$ & $5546(1)$ & $1267(1)$ & $2474(1)$ & $26(1)$ \\
\hline$C(51)$ & $7070(5)$ & $981(2)$ & $2416(2)$ & $24(1)$ \\
\hline$C(52)$ & $8005(5)$ & $936(2)$ & $2819(2)$ & $31(1)$ \\
\hline$C(53)$ & $9218(5)$ & $771(2)$ & $2760(2)$ & $36(2)$ \\
\hline$C(54)$ & $9504(5)$ & $652(2)$ & $2310(3)$ & $38(2)$ \\
\hline$C(55)$ & $8571(5)$ & $692(2)$ & $1916(2)$ & $38(2)$ \\
\hline
\end{tabular}




\begin{tabular}{|c|c|c|c|c|}
\hline$C(56)$ & $7350(5)$ & $853(2)$ & $1966(2)$ & $33(1)$ \\
\hline$C(57)$ & $5644(5)$ & $2009(2)$ & $2397(2)$ & $27(1)$ \\
\hline$C(58)$ & $4538(5)$ & $2330(2)$ & $2263(2)$ & $28(1)$ \\
\hline C(59) & $4631(5)$ & $2908(2)$ & $2215(2)$ & $32(1)$ \\
\hline$C(60)$ & $5815(5)$ & $3166(2)$ & $2302(2)$ & $35(1)$ \\
\hline$C(61)$ & $6900(5)$ & $2856(2)$ & $2438(2)$ & $38(2)$ \\
\hline$C(62)$ & $6814(5)$ & $2278(2)$ & $2481(2)$ & $33(1)$ \\
\hline$C(63)$ & $4333(4)$ & $987(2)$ & $2019(2)$ & $29(1)$ \\
\hline$C(64)$ & $4185(5)$ & $1222(2)$ & $1562(2)$ & $33(1)$ \\
\hline$C(65)$ & $3288(5)$ & $1000(2)$ & 1199(2) & $38(2)$ \\
\hline$C(66)$ & $2526(5)$ & $555(2)$ & $1301(3)$ & $41(2)$ \\
\hline$C(67)$ & $2661(5)$ & $336(2)$ & $1754(3)$ & $41(2)$ \\
\hline$C(68)$ & $3561(5)$ & $547(2)$ & $2116(2)$ & $33(1)$ \\
\hline C(69) & $5202(5)$ & $1102(2)$ & $3061(2)$ & $30(1)$ \\
\hline$C(70)$ & $5382(6)$ & $559(2)$ & $3244(2)$ & $40(2)$ \\
\hline $\mathrm{C}(71)$ & $5095(6)$ & $438(3)$ & $3692(3)$ & $51(2)$ \\
\hline$C(72)$ & $4613(6)$ & $847(3)$ & $3958(3)$ & $55(2)$ \\
\hline$C(73)$ & $4451(6)$ & $1386(3)$ & $3785(3)$ & $50(2)$ \\
\hline$C(74)$ & $4734(5)$ & $1521(2)$ & $3338(2)$ & $39(2)$ \\
\hline $\mathrm{N}(6)$ & $8245(11)$ & $2881(5)$ & $564(4)$ & $134(4)$ \\
\hline$C(75)$ & 8093(9) & $2635(4)$ & $901(4)$ & $80(3)$ \\
\hline$C(76)$ & $7909(8)$ & $2358(4)$ & $1328(4)$ & $81(3)$ \\
\hline
\end{tabular}




\begin{tabular}{lrrrr}
$\mathrm{N}(7)$ & $4596(10)$ & $2462(4)$ & $847(3)$ & $109(3)$ \\
$\mathrm{C}(78)$ & $4271(12)$ & $3517(5)$ & $1029(4)$ & $120(4)$ \\
$\mathrm{C}(77)$ & $4442(9)$ & $2927(5)$ & $921(3)$ & $80(3)$ \\
$\mathrm{N}(8)$ & $3415(16)$ & $9676(8)$ & $4594(7)$ & $225(9)$ \\
$\mathrm{C}(79)$ & $4177(18)$ & $9364(7)$ & $4586(5)$ & $133(5)$ \\
$\mathrm{C}(80)$ & $5223(19)$ & $9029(6)$ & $4596(5)$ & $204(9)$ \\
\hline
\end{tabular}


Table S14. Bond Distances, $\AA$, for $\left[\left(\mathrm{L}^{1}\right) \mathrm{Fe}(\mathrm{II})-\mathrm{NCCH}_{3}\right]\left[\mathrm{Ph}_{4} \mathrm{P}\right](\mathbf{1})$

\begin{tabular}{|c|c|c|c|}
\hline $\mathrm{Fe}(1)-\mathrm{N}(4)$ & $2.012(4)$ & $C(33)-C(34)$ & $1.388(8)$ \\
\hline $\mathrm{Fe}(1)-\mathrm{N}(3)$ & $2.024(4)$ & $C(35)-C(36)$ & $1.534(10)$ \\
\hline $\mathrm{Fe}(1)-\mathrm{N}(2)$ & $2.045(4)$ & $\mathrm{C}(35)-\mathrm{C}(38)$ & $1.537(10)$ \\
\hline $\mathrm{Fe}(1)-\mathrm{N}(5)$ & $2.126(6)$ & $C(35)-C(37)$ & $1.539(10)$ \\
\hline $\mathrm{Fe}(1)-\mathrm{N}(1)$ & $2.247(5)$ & $C(39)-C(44)$ & $1.384(7)$ \\
\hline $\mathrm{N}(1)-\mathrm{C}(13)$ & $1.459(5)$ & $C(39)-C(40)$ & $1.394(7)$ \\
\hline $\mathrm{N}(1)-\mathrm{C}(1)$ & $1.470(6)$ & $\mathrm{C}(40)-\mathrm{C}(41)$ & $1.379(8)$ \\
\hline $\mathrm{N}(1)-\mathrm{C}(7)$ & $1.470(6)$ & $\mathrm{C}(41)-\mathrm{C}(42)$ & $1.400(8)$ \\
\hline $\mathrm{N}(2)-\mathrm{C}(2)$ & $1.370(7)$ & $\mathrm{C}(42)-\mathrm{C}(43)$ & $1.386(8)$ \\
\hline $\mathrm{N}(2)-\mathrm{C}(19)$ & $1.422(7)$ & $\mathrm{C}(42)-\mathrm{C}(45)$ & $1.538(8)$ \\
\hline $\mathrm{N}(3)-\mathrm{C}(8)$ & $1.355(7)$ & $C(43)-C(44)$ & $1.386(8)$ \\
\hline N(3)-C(29) & $1.411(6)$ & $C(45)-C(47)$ & $1.480(11)$ \\
\hline $\mathrm{N}(4)-\mathrm{C}(14)$ & $1.363(7)$ & $C(45)-C(46)$ & $1.518(10)$ \\
\hline N(4)-C(39) & $1.406(7)$ & $C(45)-C(48)$ & $1.526(10)$ \\
\hline $\mathrm{N}(5)-\mathrm{C}(49)$ & $1.125(8)$ & $C(49)-C(50)$ & $1.475(10)$ \\
\hline$C(1)-C(6)$ & $1.376(7)$ & $\mathrm{P}(1)-\mathrm{C}(57)$ & $1.781(5)$ \\
\hline$C(1)-C(2)$ & $1.426(7)$ & $\mathrm{P}(1)-\mathrm{C}(69)$ & $1.791(6)$ \\
\hline$C(2)-C(3)$ & $1.419(7)$ & $\mathrm{P}(1)-\mathrm{C}(51)$ & $1.792(5)$ \\
\hline$C(3)-C(4)$ & $1.368(8)$ & $\mathrm{P}(1)-\mathrm{C}(63)$ & $1.799(5)$ \\
\hline$C(4)-C(5)$ & $1.377(8)$ & $C(51)-C(56)$ & $1.376(8)$ \\
\hline$C(5)-C(6)$ & $1.402(7)$ & $\mathrm{C}(51)-\mathrm{C}(52)$ & $1.393(7)$ \\
\hline
\end{tabular}




\begin{tabular}{|c|c|c|c|}
\hline $\mathrm{C}(7)-\mathrm{C}(12)$ & $1.368(8)$ & $\mathrm{C}(52)-\mathrm{C}(53)$ & $1.385(7)$ \\
\hline$C(7)-C(8)$ & $1.424(7)$ & $C(53)-C(54)$ & $1.376(9)$ \\
\hline$C(8)-C(9)$ & $1.419(7)$ & $C(54)-C(55)$ & $1.373(8)$ \\
\hline$C(9)-C(10)$ & $1.375(9)$ & $C(55)-C(56)$ & $1.385(8)$ \\
\hline $\mathrm{C}(10)-\mathrm{C}(11)$ & $1.373(8)$ & $\mathrm{C}(57)-\mathrm{C}(62)$ & $1.389(7)$ \\
\hline $\mathrm{C}(11)-\mathrm{C}(12)$ & $1.391(7)$ & $\mathrm{C}(57)-\mathrm{C}(58)$ & $1.405(6)$ \\
\hline $\mathrm{C}(13)-\mathrm{C}(18)$ & $1.376(8)$ & $\mathrm{C}(58)-\mathrm{C}(59)$ & $1.386(7)$ \\
\hline$C(13)-C(14)$ & $1.424(7)$ & $C(59)-C(60)$ & $1.391(7)$ \\
\hline$C(14)-C(15)$ & $1.423(6)$ & $\mathrm{C}(60)-\mathrm{C}(61)$ & $1.375(7)$ \\
\hline$C(15)-C(16)$ & $1.365(8)$ & $\mathrm{C}(61)-\mathrm{C}(62)$ & $1.381(7)$ \\
\hline$C(16)-C(17)$ & $1.385(8)$ & $\mathrm{C}(63)-\mathrm{C}(68)$ & $1.383(7)$ \\
\hline $\mathrm{C}(17)-\mathrm{C}(18)$ & $1.394(7)$ & $\mathrm{C}(63)-\mathrm{C}(64)$ & $1.389(8)$ \\
\hline $\mathrm{C}(19)-\mathrm{C}(24)$ & $1.389(7)$ & $\mathrm{C}(64)-\mathrm{C}(65)$ & $1.390(7)$ \\
\hline $\mathrm{C}(19)-\mathrm{C}(20)$ & $1.395(8)$ & $C(65)-C(66)$ & $1.389(8)$ \\
\hline $\mathrm{C}(20)-\mathrm{C}(21)$ & $1.388(8)$ & $C(66)-C(67)$ & $1.363(9)$ \\
\hline $\mathrm{C}(21)-\mathrm{C}(22)$ & $1.385(8)$ & $\mathrm{C}(67)-\mathrm{C}(68)$ & $1.382(8)$ \\
\hline $\mathrm{C}(22)-\mathrm{C}(23)$ & $1.389(9)$ & $\mathrm{C}(69)-\mathrm{C}(70)$ & $1.392(7)$ \\
\hline $\mathrm{C}(22)-\mathrm{C}(25)$ & $1.541(8)$ & $\mathrm{C}(69)-\mathrm{C}(74)$ & $1.403(8)$ \\
\hline $\mathrm{C}(23)-\mathrm{C}(24)$ & $1.401(8)$ & $\mathrm{C}(70)-\mathrm{C}(71)$ & $1.370(9)$ \\
\hline$C(25)-C(27)$ & $1.494(13)$ & $\mathrm{C}(71)-\mathrm{C}(72)$ & $1.373(10)$ \\
\hline$C(25)-C(26)$ & $1.494(11)$ & $\mathrm{C}(72)-\mathrm{C}(73)$ & $1.372(9)$ \\
\hline $\mathrm{C}(25)-\mathrm{C}(28)$ & $1.500(12)$ & $\mathrm{C}(73)-\mathrm{C}(74)$ & $1.373(9)$ \\
\hline
\end{tabular}




$\begin{array}{llll}\mathrm{C}(29)-\mathrm{C}(34) & 1.388(8) & \mathrm{N}(6)-\mathrm{C}(75) & 1.146(13) \\ \mathrm{C}(29)-\mathrm{C}(30) & 1.398(7) & \mathrm{C}(75)-\mathrm{C}(76) & 1.409(14) \\ \mathrm{C}(30)-\mathrm{C}(31) & 1.376(8) & \mathrm{N}(7)-\mathrm{C}(77) & 1.141(12) \\ \mathrm{C}(31)-\mathrm{C}(32) & 1.406(8) & \mathrm{C}(78)-\mathrm{C}(77) & 1.451(14) \\ \mathrm{C}(32)-\mathrm{C}(33) & 1.383(8) & \mathrm{N}(8)-\mathrm{C}(79) & 1.102(17) \\ \mathrm{C}(32)-\mathrm{C}(35) & 1.530(8) & \mathrm{C}(79)-\mathrm{C}(80) & 1.366(19)\end{array}$


Table S15. Angles, ${ }^{\circ}$, for $\left[\left(\mathrm{L}^{1}\right) \mathrm{Fe}(\mathrm{II})-\mathrm{NCCH}_{3}\right]\left[\mathrm{Ph}_{4} \mathrm{P}\right](\mathbf{1})$

\begin{tabular}{|c|c|c|c|}
\hline $\mathrm{N}(4)-\mathrm{Fe}(1)-\mathrm{N}(3)$ & $119.92(17)$ & $C(34)-C(29)-N(3)$ & $121.2(5)$ \\
\hline $\mathrm{N}(4)-\mathrm{Fe}(1)-\mathrm{N}(2)$ & 118.79(16) & $\mathrm{C}(30)-\mathrm{C}(29)-\mathrm{N}(3)$ & $122.2(5)$ \\
\hline $\mathrm{N}(3)-\mathrm{Fe}(1)-\mathrm{N}(2)$ & $111.20(17)$ & $C(31)-C(30)-C(29)$ & $121.9(6)$ \\
\hline $\mathrm{N}(4)-\mathrm{Fe}(1)-\mathrm{N}(5)$ & 102.97(19) & $\mathrm{C}(30)-\mathrm{C}(31)-\mathrm{C}(32)$ & $122.1(6)$ \\
\hline $\mathrm{N}(3)-\mathrm{Fe}(1)-\mathrm{N}(5)$ & 101.79(19) & $\mathrm{C}(33)-\mathrm{C}(32)-\mathrm{C}(31)$ & $115.3(5)$ \\
\hline $\mathrm{N}(2)-\mathrm{Fe}(1)-\mathrm{N}(5)$ & $96.99(18)$ & $\mathrm{C}(33)-\mathrm{C}(32)-\mathrm{C}(35)$ & $124.2(6)$ \\
\hline $\mathrm{N}(4)-\mathrm{Fe}(1)-\mathrm{N}(1)$ & $78.94(17)$ & $\mathrm{C}(31)-\mathrm{C}(32)-\mathrm{C}(35)$ & $120.5(6)$ \\
\hline $\mathrm{N}(3)-\mathrm{Fe}(1)-\mathrm{N}(1)$ & $79.25(16)$ & $\mathrm{C}(32)-\mathrm{C}(33)-\mathrm{C}(34)$ & $123.2(6)$ \\
\hline $\mathrm{N}(2)-\mathrm{Fe}(1)-\mathrm{N}(1)$ & $79.82(16)$ & $\mathrm{C}(29)-\mathrm{C}(34)-\mathrm{C}(33)$ & $121.0(5)$ \\
\hline $\mathrm{N}(5)-\mathrm{Fe}(1)-\mathrm{N}(1)$ & $176.79(16)$ & $\mathrm{C}(32)-\mathrm{C}(35)-\mathrm{C}(36)$ & 111.7(6) \\
\hline $\mathrm{C}(13)-\mathrm{N}(1)-\mathrm{C}(1)$ & $114.7(3)$ & $\mathrm{C}(32)-\mathrm{C}(35)-\mathrm{C}(38)$ & $109.2(5)$ \\
\hline $\mathrm{C}(13)-\mathrm{N}(1)-\mathrm{C}(7)$ & $114.8(3)$ & $\mathrm{C}(36)-\mathrm{C}(35)-\mathrm{C}(38)$ & $107.2(6)$ \\
\hline $\mathrm{C}(1)-\mathrm{N}(1)-\mathrm{C}(7)$ & $112.1(4)$ & $\mathrm{C}(32)-\mathrm{C}(35)-\mathrm{C}(37)$ & 109.7(6) \\
\hline $\mathrm{C}(13)-\mathrm{N}(1)-\mathrm{Fe}(1)$ & $105.2(3)$ & $\mathrm{C}(36)-\mathrm{C}(35)-\mathrm{C}(37)$ & $108.5(6)$ \\
\hline $\mathrm{C}(1)-\mathrm{N}(1)-\mathrm{Fe}(1)$ & $104.8(3)$ & $\mathrm{C}(38)-\mathrm{C}(35)-\mathrm{C}(37)$ & $110.6(6)$ \\
\hline $\mathrm{C}(7)-\mathrm{N}(1)-\mathrm{Fe}(1)$ & $103.8(3)$ & $\mathrm{C}(44)-\mathrm{C}(39)-\mathrm{C}(40)$ & $116.9(5)$ \\
\hline $\mathrm{C}(2)-\mathrm{N}(2)-\mathrm{C}(19)$ & $119.1(4)$ & $\mathrm{C}(44)-\mathrm{C}(39)-\mathrm{N}(4)$ & $118.5(5)$ \\
\hline $\mathrm{C}(2)-\mathrm{N}(2)-\mathrm{Fe}(1)$ & $112.9(3)$ & $\mathrm{C}(40)-\mathrm{C}(39)-\mathrm{N}(4)$ & $124.2(5)$ \\
\hline $\mathrm{C}(19)-\mathrm{N}(2)-\mathrm{Fe}(1)$ & $120.9(4)$ & $\mathrm{C}(41)-\mathrm{C}(40)-\mathrm{C}(39)$ & $121.2(5)$ \\
\hline $\mathrm{C}(8)-\mathrm{N}(3)-\mathrm{C}(29)$ & $120.0(4)$ & $\mathrm{C}(40)-\mathrm{C}(41)-\mathrm{C}(42)$ & $122.3(5)$ \\
\hline $\mathrm{C}(8)-\mathrm{N}(3)-\mathrm{Fe}(1)$ & $114.6(3)$ & $\mathrm{C}(43)-\mathrm{C}(42)-\mathrm{C}(41)$ & $115.7(6)$ \\
\hline
\end{tabular}




\begin{tabular}{|c|c|c|c|}
\hline $\mathrm{C}(29)-\mathrm{N}(3)-\mathrm{Fe}(1)$ & $125.4(4)$ & $C(43)-C(42)-C(45)$ & $121.1(5)$ \\
\hline $\mathrm{C}(14)-\mathrm{N}(4)-\mathrm{C}(39)$ & $121.4(4)$ & $\mathrm{C}(41)-\mathrm{C}(42)-\mathrm{C}(45)$ & $123.3(5)$ \\
\hline $\mathrm{C}(14)-\mathrm{N}(4)-\mathrm{Fe}(1)$ & $115.5(3)$ & $C(42)-C(43)-C(44)$ & $122.3(5)$ \\
\hline $\mathrm{C}(39)-\mathrm{N}(4)-\mathrm{Fe}(1)$ & $121.2(3)$ & $\mathrm{C}(39)-\mathrm{C}(44)-\mathrm{C}(43)$ & $121.4(5)$ \\
\hline $\mathrm{C}(49)-\mathrm{N}(5)-\mathrm{Fe}(1)$ & $153.4(6)$ & $\mathrm{C}(47)-\mathrm{C}(45)-\mathrm{C}(46)$ & $108.3(9)$ \\
\hline$C(6)-C(1)-C(2)$ & $121.4(5)$ & $\mathrm{C}(47)-\mathrm{C}(45)-\mathrm{C}(48)$ & $111.5(9)$ \\
\hline $\mathrm{C}(6)-\mathrm{C}(1)-\mathrm{N}(1)$ & $122.0(4)$ & $\mathrm{C}(46)-\mathrm{C}(45)-\mathrm{C}(48)$ & $105.5(7)$ \\
\hline $\mathrm{C}(2)-\mathrm{C}(1)-\mathrm{N}(1)$ & $116.6(5)$ & $\mathrm{C}(47)-\mathrm{C}(45)-\mathrm{C}(42)$ & $109.9(6)$ \\
\hline $\mathrm{N}(2)-\mathrm{C}(2)-\mathrm{C}(3)$ & $125.8(5)$ & $C(46)-C(45)-C(42)$ & $111.8(6)$ \\
\hline $\mathrm{N}(2)-\mathrm{C}(2)-\mathrm{C}(1)$ & $119.3(4)$ & $\mathrm{C}(48)-\mathrm{C}(45)-\mathrm{C}(42)$ & $109.8(6)$ \\
\hline$C(3)-C(2)-C(1)$ & $114.8(5)$ & $\mathrm{N}(5)-\mathrm{C}(49)-\mathrm{C}(50)$ & $178.2(8)$ \\
\hline$C(4)-C(3)-C(2)$ & $122.6(5)$ & $\mathrm{C}(57)-\mathrm{P}(1)-\mathrm{C}(69)$ & $110.7(3)$ \\
\hline$C(3)-C(4)-C(5)$ & $121.9(5)$ & $\mathrm{C}(57)-\mathrm{P}(1)-\mathrm{C}(51)$ & $107.1(2)$ \\
\hline$C(4)-C(5)-C(6)$ & $117.1(6)$ & $\mathrm{C}(69)-\mathrm{P}(1)-\mathrm{C}(51)$ & $108.6(3)$ \\
\hline$C(1)-C(6)-C(5)$ & $122.0(5)$ & $\mathrm{C}(57)-\mathrm{P}(1)-\mathrm{C}(63)$ & $109.2(2)$ \\
\hline$C(12)-C(7)-C(8)$ & $122.1(5)$ & $C(69)-P(1)-C(63)$ & $110.4(3)$ \\
\hline $\mathrm{C}(12)-\mathrm{C}(7)-\mathrm{N}(1)$ & $122.3(5)$ & $\mathrm{C}(51)-\mathrm{P}(1)-\mathrm{C}(63)$ & $110.9(2)$ \\
\hline $\mathrm{C}(8)-\mathrm{C}(7)-\mathrm{N}(1)$ & $115.6(5)$ & $\mathrm{C}(56)-\mathrm{C}(51)-\mathrm{C}(52)$ & $120.1(5)$ \\
\hline $\mathrm{N}(3)-\mathrm{C}(8)-\mathrm{C}(9)$ & $126.1(5)$ & $\mathrm{C}(56)-\mathrm{C}(51)-\mathrm{P}(1)$ & $119.7(4)$ \\
\hline $\mathrm{N}(3)-\mathrm{C}(8)-\mathrm{C}(7)$ & $118.9(4)$ & $\mathrm{C}(52)-\mathrm{C}(51)-\mathrm{P}(1)$ & $119.9(5)$ \\
\hline$C(9)-C(8)-C(7)$ & $114.9(5)$ & $\mathrm{C}(53)-\mathrm{C}(52)-\mathrm{C}(51)$ & $119.2(6)$ \\
\hline $\mathrm{C}(10)-\mathrm{C}(9)-\mathrm{C}(8)$ & $122.1(5)$ & $\mathrm{C}(54)-\mathrm{C}(53)-\mathrm{C}(52)$ & $120.7(5)$ \\
\hline
\end{tabular}




\begin{tabular}{|c|c|c|c|}
\hline $\mathrm{C}(11)-\mathrm{C}(10)-\mathrm{C}(9)$ & $121.0(5)$ & $\mathrm{C}(55)-\mathrm{C}(54)-\mathrm{C}(53)$ & $119.6(5)$ \\
\hline $\mathrm{C}(10)-\mathrm{C}(11)-\mathrm{C}(12)$ & $118.9(6)$ & $\mathrm{C}(54)-\mathrm{C}(55)-\mathrm{C}(56)$ & $120.7(6)$ \\
\hline $\mathrm{C}(7)-\mathrm{C}(12)-\mathrm{C}(11)$ & $120.7(5)$ & $\mathrm{C}(51)-\mathrm{C}(56)-\mathrm{C}(55)$ & $119.7(5)$ \\
\hline $\mathrm{C}(18)-\mathrm{C}(13)-\mathrm{C}(14)$ & $121.2(4)$ & $\mathrm{C}(62)-\mathrm{C}(57)-\mathrm{C}(58)$ & $119.3(4)$ \\
\hline $\mathrm{C}(18)-\mathrm{C}(13)-\mathrm{N}(1)$ & $123.0(5)$ & $\mathrm{C}(62)-\mathrm{C}(57)-\mathrm{P}(1)$ & $120.2(4)$ \\
\hline $\mathrm{C}(14)-\mathrm{C}(13)-\mathrm{N}(1)$ & $115.7(4)$ & $\mathrm{C}(58)-\mathrm{C}(57)-\mathrm{P}(1)$ & $120.4(4)$ \\
\hline $\mathrm{N}(4)-\mathrm{C}(14)-\mathrm{C}(15)$ & $125.8(5)$ & $\mathrm{C}(59)-\mathrm{C}(58)-\mathrm{C}(57)$ & $119.6(4)$ \\
\hline $\mathrm{N}(4)-\mathrm{C}(14)-\mathrm{C}(13)$ & $118.4(4)$ & $\mathrm{C}(58)-\mathrm{C}(59)-\mathrm{C}(60)$ & $119.8(5)$ \\
\hline $\mathrm{C}(15)-\mathrm{C}(14)-\mathrm{C}(13)$ & $115.8(5)$ & $\mathrm{C}(61)-\mathrm{C}(60)-\mathrm{C}(59)$ & $120.8(5)$ \\
\hline$C(16)-C(15)-C(14)$ & $121.6(5)$ & $\mathrm{C}(60)-\mathrm{C}(61)-\mathrm{C}(62)$ & $119.6(5)$ \\
\hline $\mathrm{C}(15)-\mathrm{C}(16)-\mathrm{C}(17)$ & $121.9(4)$ & $\mathrm{C}(61)-\mathrm{C}(62)-\mathrm{C}(57)$ & $120.9(5)$ \\
\hline $\mathrm{C}(16)-\mathrm{C}(17)-\mathrm{C}(18)$ & $117.9(5)$ & $\mathrm{C}(68)-\mathrm{C}(63)-\mathrm{C}(64)$ & $120.0(5)$ \\
\hline $\mathrm{C}(13)-\mathrm{C}(18)-\mathrm{C}(17)$ & $121.5(5)$ & $\mathrm{C}(68)-\mathrm{C}(63)-\mathrm{P}(1)$ & $121.5(5)$ \\
\hline $\mathrm{C}(24)-\mathrm{C}(19)-\mathrm{C}(20)$ & $117.3(5)$ & $\mathrm{C}(64)-\mathrm{C}(63)-\mathrm{P}(1)$ & $118.5(4)$ \\
\hline $\mathrm{C}(24)-\mathrm{C}(19)-\mathrm{N}(2)$ & $123.5(5)$ & $C(63)-C(64)-C(65)$ & $119.6(5)$ \\
\hline $\mathrm{C}(20)-\mathrm{C}(19)-\mathrm{N}(2)$ & $119.0(5)$ & $\mathrm{C}(66)-\mathrm{C}(65)-\mathrm{C}(64)$ & $119.7(6)$ \\
\hline $\mathrm{C}(21)-\mathrm{C}(20)-\mathrm{C}(19)$ & $121.4(5)$ & $\mathrm{C}(67)-\mathrm{C}(66)-\mathrm{C}(65)$ & $120.1(5)$ \\
\hline $\mathrm{C}(22)-\mathrm{C}(21)-\mathrm{C}(20)$ & $121.9(6)$ & $\mathrm{C}(66)-\mathrm{C}(67)-\mathrm{C}(68)$ & $120.8(6)$ \\
\hline $\mathrm{C}(21)-\mathrm{C}(22)-\mathrm{C}(23)$ & $116.6(5)$ & $\mathrm{C}(67)-\mathrm{C}(68)-\mathrm{C}(63)$ & $119.7(6)$ \\
\hline $\mathrm{C}(21)-\mathrm{C}(22)-\mathrm{C}(25)$ & $123.0(6)$ & $\mathrm{C}(70)-\mathrm{C}(69)-\mathrm{C}(74)$ & $119.6(6)$ \\
\hline $\mathrm{C}(23)-\mathrm{C}(22)-\mathrm{C}(25)$ & $120.4(5)$ & $\mathrm{C}(70)-\mathrm{C}(69)-\mathrm{P}(1)$ & $120.6(5)$ \\
\hline $\mathrm{C}(22)-\mathrm{C}(23)-\mathrm{C}(24)$ & $122.2(5)$ & $\mathrm{C}(74)-\mathrm{C}(69)-\mathrm{P}(1)$ & $119.8(4)$ \\
\hline
\end{tabular}




$\begin{array}{lclr}\mathrm{C}(19)-\mathrm{C}(24)-\mathrm{C}(23) & 120.5(6) & \mathrm{C}(71)-\mathrm{C}(70)-\mathrm{C}(69) & 119.7(6) \\ \mathrm{C}(27)-\mathrm{C}(25)-\mathrm{C}(26) & 108.6(9) & \mathrm{C}(70)-\mathrm{C}(71)-\mathrm{C}(72) & 120.5(6) \\ \mathrm{C}(27)-\mathrm{C}(25)-\mathrm{C}(28) & 107.7(10) & \mathrm{C}(73)-\mathrm{C}(72)-\mathrm{C}(71) & 120.3(7) \\ \mathrm{C}(26)-\mathrm{C}(25)-\mathrm{C}(28) & 109.7(8) & \mathrm{C}(72)-\mathrm{C}(73)-\mathrm{C}(74) & 120.7(7) \\ \mathrm{C}(27)-\mathrm{C}(25)-\mathrm{C}(22) & 111.4(6) & \mathrm{C}(73)-\mathrm{C}(74)-\mathrm{C}(69) & 119.2(6) \\ \mathrm{C}(26)-\mathrm{C}(25)-\mathrm{C}(22) & 109.3(7) & \mathrm{N}(6)-\mathrm{C}(75)-\mathrm{C}(76) & 177.1(12) \\ \mathrm{C}(28)-\mathrm{C}(25)-\mathrm{C}(22) & 110.1(6) & \mathrm{N}(7)-\mathrm{C}(77)-\mathrm{C}(78) & 173(2) \\ \mathrm{C}(34)-\mathrm{C}(29)-\mathrm{C}(30) & 116.5(5) & \mathrm{N}(8)-\mathrm{C}(79)-\mathrm{C}(80) & \end{array}$


Table S16. Anisotropic displacement parameters $\left(\AA^{2} \times 10^{3}\right)$ for $\left[\left(\mathrm{L}^{1}\right) \mathrm{Fe}(\mathrm{II})-\mathrm{NCCH}_{3}\right]\left[\mathrm{Ph}_{4} \mathrm{P}\right]$ (1). The anisotropic displacement factor exponent takes the form: $-2 \pi^{2}\left[h^{2} a^{*^{2}} \mathrm{U}^{11}+\ldots+\right.$ $2 \mathrm{~h} \mathrm{k} \mathrm{a}^{*} \mathrm{~b}^{*} \mathrm{U}^{12}$ ]

\begin{tabular}{|c|c|c|c|c|c|c|}
\hline & $\mathrm{U}^{11}$ & $\mathrm{U}^{22}$ & $\mathrm{U}^{33}$ & $\mathrm{U}^{23}$ & $\mathrm{U}^{13}$ & $\mathrm{U}^{12}$ \\
\hline $\mathrm{Fe}(1)$ & $26(1)$ & $24(1)$ & $20(1)$ & $0(1)$ & $10(1)$ & $3(1)$ \\
\hline $\mathrm{N}(1)$ & $25(2)$ & $17(2)$ & $22(3)$ & $-3(2)$ & $5(2)$ & $3(2)$ \\
\hline $\mathrm{N}(2)$ & $38(2)$ & $29(2)$ & $17(3)$ & $5(2)$ & $14(2)$ & $-4(2)$ \\
\hline $\mathrm{N}(3)$ & $28(2)$ & $29(2)$ & $25(3)$ & $-5(2)$ & $8(2)$ & $0(2)$ \\
\hline $\mathrm{N}(4)$ & $24(2)$ & $28(2)$ & $26(3)$ & $-3(2)$ & $9(2)$ & $6(2)$ \\
\hline $\mathrm{N}(5)$ & $43(2)$ & $42(3)$ & $27(4)$ & $-1(2)$ & $5(2)$ & $-2(2)$ \\
\hline $\mathrm{C}(1)$ & $26(2)$ & $22(2)$ & $21(4)$ & $3(2)$ & $11(2)$ & $-2(2)$ \\
\hline $\mathrm{C}(2)$ & $26(2)$ & $24(3)$ & $30(4)$ & $-2(2)$ & $7(2)$ & $-4(2)$ \\
\hline$C(3)$ & $45(3)$ & $23(3)$ & $30(4)$ & $2(2)$ & $16(3)$ & $-5(2)$ \\
\hline$C(4)$ & $47(3)$ & $20(3)$ & $39(5)$ & $-2(2)$ & $15(3)$ & $-1(2)$ \\
\hline$C(5)$ & $39(3)$ & $37(3)$ & $29(4)$ & $-13(2)$ & $13(2)$ & $3(3)$ \\
\hline$C(6)$ & $33(2)$ & $23(3)$ & $23(4)$ & $3(2)$ & $13(2)$ & $-2(2)$ \\
\hline$C(7)$ & $26(2)$ & $20(2)$ & $26(4)$ & $-1(2)$ & $18(2)$ & $1(2)$ \\
\hline$C(8)$ & $28(2)$ & $22(3)$ & $32(4)$ & $-6(2)$ & $16(2)$ & $-2(2)$ \\
\hline$C(9)$ & $29(2)$ & $28(3)$ & $47(5)$ & $-15(3)$ & $12(2)$ & $0(2)$ \\
\hline$C(10)$ & $38(3)$ & $23(3)$ & $62(5)$ & $-6(3)$ & $30(3)$ & $-8(2)$ \\
\hline $\mathrm{C}(11)$ & $45(3)$ & $32(3)$ & $47(5)$ & $4(3)$ & $32(3)$ & $-3(3)$ \\
\hline$C(12)$ & $38(3)$ & $26(3)$ & $36(4)$ & $2(2)$ & $16(3)$ & $2(2)$ \\
\hline
\end{tabular}




\begin{tabular}{|c|c|c|c|c|c|c|}
\hline$C(13)$ & $22(2)$ & $24(3)$ & $29(4)$ & $5(2)$ & $10(2)$ & $-1(2)$ \\
\hline$C(14)$ & $23(2)$ & $16(2)$ & $25(4)$ & $-2(2)$ & $12(2)$ & $-1(2)$ \\
\hline$C(15)$ & $31(2)$ & $20(2)$ & $25(4)$ & $2(2)$ & $14(2)$ & $5(2)$ \\
\hline$C(16)$ & $27(2)$ & $29(3)$ & $39(4)$ & $7(2)$ & $9(2)$ & $7(2)$ \\
\hline$C(17)$ & $35(2)$ & $42(3)$ & $19(4)$ & $4(2)$ & $7(2)$ & $4(3)$ \\
\hline $\mathrm{C}(18)$ & $29(2)$ & $32(3)$ & $21(4)$ & $1(2)$ & $15(2)$ & $-2(2)$ \\
\hline$C(19)$ & $36(3)$ & $22(3)$ & $36(4)$ & $1(2)$ & $10(2)$ & $-7(2)$ \\
\hline$C(20)$ & $38(3)$ & $34(3)$ & $32(4)$ & $-2(2)$ & $12(3)$ & $3(3)$ \\
\hline $\mathrm{C}(21)$ & $51(3)$ & $37(3)$ & $33(5)$ & $2(3)$ & $20(3)$ & $-10(3)$ \\
\hline$C(22)$ & $61(3)$ & $27(3)$ & $22(4)$ & $7(2)$ & $16(3)$ & $-10(3)$ \\
\hline$C(23)$ & $56(3)$ & $38(3)$ & $35(5)$ & $13(3)$ & $11(3)$ & $6(3)$ \\
\hline$C(24)$ & $41(3)$ & $34(3)$ & $41(5)$ & $5(3)$ & $15(3)$ & $0(3)$ \\
\hline$C(25)$ & $79(4)$ & $49(4)$ & $37(5)$ & $19(3)$ & $17(4)$ & $-8(4)$ \\
\hline$C(26)$ & $390(20)$ & $37(5)$ & $70(8)$ & $14(4)$ & $90(11)$ & $-29(8)$ \\
\hline $\mathrm{C}(27)$ & $149(10)$ & $152(11)$ & $157(14)$ & $115(10)$ & $82(9)$ & $17(9)$ \\
\hline $\mathrm{C}(28)$ & $189(11)$ & $135(9)$ & $59(8)$ & $60(6)$ & $-28(8)$ & $-47(8)$ \\
\hline C(29) & $28(2)$ & $42(3)$ & $25(4)$ & $-9(2)$ & $12(2)$ & $-6(2)$ \\
\hline$C(30)$ & $44(3)$ & $48(4)$ & $22(4)$ & $-9(3)$ & $7(3)$ & $10(3)$ \\
\hline$C(31)$ & $44(3)$ & $51(4)$ & $30(5)$ & $-14(3)$ & $9(3)$ & $15(3)$ \\
\hline$C(32)$ & $32(2)$ & $44(3)$ & $38(5)$ & $-3(3)$ & $2(3)$ & $-2(3)$ \\
\hline$C(33)$ & $37(3)$ & $64(4)$ & 21(4) & $-14(3)$ & $11(2)$ & $-6(3)$ \\
\hline$C(34)$ & $30(2)$ & $42(3)$ & $40(5)$ & $-10(3)$ & $11(2)$ & $5(2)$ \\
\hline
\end{tabular}




\begin{tabular}{|c|c|c|c|c|c|c|}
\hline$C(35)$ & $54(3)$ & $66(4)$ & $21(5)$ & $1(3)$ & $0(3)$ & $3(3)$ \\
\hline$C(36)$ & $75(5)$ & $99(6)$ & $27(6)$ & $-1(4)$ & $-5(4)$ & $0(5)$ \\
\hline$C(37)$ & $45(3)$ & $113(7)$ & $58(7)$ & $2(5)$ & $-4(4)$ & $9(4)$ \\
\hline $\mathrm{C}(38)$ & $95(6)$ & $70(5)$ & $52(6)$ & $14(4)$ & $-14(5)$ & $8(5)$ \\
\hline$C(39)$ & $28(2)$ & $21(2)$ & $26(4)$ & $-1(2)$ & $13(2)$ & $10(2)$ \\
\hline$C(40)$ & $30(2)$ & $37(3)$ & $27(4)$ & $-8(2)$ & $11(2)$ & $-3(2)$ \\
\hline$C(41)$ & $31(2)$ & $43(3)$ & $40(5)$ & $1(3)$ & $18(3)$ & $-2(3)$ \\
\hline$C(42)$ & $48(3)$ & $36(3)$ & $21(4)$ & $-2(2)$ & $18(3)$ & $8(3)$ \\
\hline$C(43)$ & $39(3)$ & $37(3)$ & $28(4)$ & $-7(3)$ & $12(3)$ & $-6(3)$ \\
\hline$C(44)$ & $27(2)$ & $35(3)$ & $39(5)$ & $-1(3)$ & $11(2)$ & $-2(2)$ \\
\hline$C(45)$ & $60(4)$ & $48(4)$ & $34(5)$ & $-10(3)$ & $33(3)$ & $10(3)$ \\
\hline$C(46)$ & $70(5)$ & 134(9) & $97(9)$ & $-49(6)$ & $52(5)$ & $8(5)$ \\
\hline$C(47)$ & $272(16)$ & $210(13)$ & $19(7)$ & $17(7)$ & $62(9)$ & $154(12)$ \\
\hline$C(48)$ & $156(9)$ & $97(7)$ & $187(14)$ & $-102(8)$ & $135(9)$ & $-50(7)$ \\
\hline$C(49)$ & $50(3)$ & $46(4)$ & $41(5)$ & $-2(3)$ & $6(3)$ & $-7(3)$ \\
\hline$C(50)$ & $100(6)$ & $67(5)$ & $46(6)$ & $13(4)$ & $-6(5)$ & $-8(5)$ \\
\hline $\mathrm{P}(1)$ & $31(1)$ & $23(1)$ & $25(1)$ & $-1(1)$ & $8(1)$ & $0(1)$ \\
\hline $\mathrm{C}(51)$ & $38(2)$ & $22(2)$ & $14(4)$ & $-4(2)$ & $15(2)$ & $0(2)$ \\
\hline$C(52)$ & $43(3)$ & $26(3)$ & $26(4)$ & $-3(2)$ & $9(2)$ & $1(2)$ \\
\hline$C(53)$ & $32(2)$ & $22(3)$ & $53(5)$ & $3(3)$ & $4(3)$ & $-1(2)$ \\
\hline$C(54)$ & $36(3)$ & $25(3)$ & $59(5)$ & $0(3)$ & $26(3)$ & $2(2)$ \\
\hline$C(55)$ & $53(3)$ & $27(3)$ & $38(5)$ & $-2(2)$ & $25(3)$ & $-6(3)$ \\
\hline
\end{tabular}




\begin{tabular}{|c|c|c|c|c|c|c|}
\hline$C(56)$ & $41(3)$ & $26(3)$ & $35(4)$ & $2(2)$ & $11(3)$ & $-1(2)$ \\
\hline$C(57)$ & $35(2)$ & $24(3)$ & $22(4)$ & $-4(2)$ & $8(2)$ & $3(2)$ \\
\hline $\mathrm{C}(58)$ & $32(2)$ & 21(3) & $32(4)$ & $-3(2)$ & $9(2)$ & $1(2)$ \\
\hline$C(59)$ & $37(3)$ & $27(3)$ & $33(4)$ & $1(2)$ & $14(2)$ & $6(2)$ \\
\hline$C(60)$ & $54(3)$ & $22(3)$ & $34(4)$ & $-2(2)$ & $19(3)$ & $0(3)$ \\
\hline $\mathrm{C}(61)$ & $37(3)$ & $31(3)$ & $47(5)$ & $-5(3)$ & $15(3)$ & $-4(3)$ \\
\hline$C(62)$ & $29(2)$ & 31(3) & $39(4)$ & $-2(2)$ & $8(2)$ & $1(2)$ \\
\hline$C(63)$ & $28(2)$ & $28(3)$ & $31(4)$ & $-5(2)$ & $8(2)$ & $-2(2)$ \\
\hline$C(64)$ & $37(3)$ & $27(3)$ & $36(4)$ & $1(2)$ & $6(3)$ & $-1(2)$ \\
\hline$C(65)$ & $41(3)$ & $44(3)$ & $29(4)$ & $-1(3)$ & $4(3)$ & $9(3)$ \\
\hline$C(66)$ & $30(2)$ & $42(3)$ & $48(5)$ & $-15(3)$ & $2(3)$ & $3(3)$ \\
\hline$C(67)$ & $33(3)$ & $34(3)$ & $58(5)$ & $-9(3)$ & $15(3)$ & $-5(3)$ \\
\hline$C(68)$ & $37(3)$ & $33(3)$ & $32(4)$ & $-3(2)$ & $16(2)$ & $-2(2)$ \\
\hline$C(69)$ & $42(3)$ & $28(3)$ & $24(4)$ & $-2(2)$ & $14(2)$ & $1(2)$ \\
\hline $\mathrm{C}(70)$ & $55(3)$ & $33(3)$ & $35(5)$ & $-1(3)$ & $17(3)$ & $6(3)$ \\
\hline $\mathrm{C}(71)$ & $74(4)$ & $48(4)$ & $37(5)$ & $12(3)$ & $22(4)$ & $2(4)$ \\
\hline$C(72)$ & $66(4)$ & $72(5)$ & $33(5)$ & $6(4)$ & $23(3)$ & $-4(4)$ \\
\hline$C(73)$ & $60(4)$ & $59(4)$ & $35(5)$ & $-12(3)$ & $22(3)$ & $4(3)$ \\
\hline$C(74)$ & $46(3)$ & 39(3) & $33(5)$ & $3(3)$ & $14(3)$ & $1(3)$ \\
\hline $\mathrm{N}(6)$ & 181(10) & 134(9) & $80(10)$ & $-9(6)$ & $-4(8)$ & $-54(8)$ \\
\hline$C(75)$ & $88(6)$ & $80(7)$ & $68(10)$ & $-14(5)$ & $-3(6)$ & $-17(5)$ \\
\hline$C(76)$ & $81(5)$ & $85(6)$ & $75(8)$ & $5(5)$ & $3(5)$ & $-7(5)$ \\
\hline
\end{tabular}




\begin{tabular}{lcccccc}
$\mathrm{N}(7)$ & $130(7)$ & $118(7)$ & $75(7)$ & $-6(6)$ & $4(5)$ & $-15(7)$ \\
$\mathrm{C}(78)$ & $169(11)$ & $119(9)$ & $73(9)$ & $13(6)$ & $25(8)$ & $22(9)$ \\
$\mathrm{C}(77)$ & $78(5)$ & $116(8)$ & $46(7)$ & $7(6)$ & $6(4)$ & $-14(7)$ \\
$\mathrm{N}(8)$ & $201(15)$ & $234(19)$ & $270(20)$ & $116(15)$ & $136(14)$ & $41(13)$ \\
$\mathrm{C}(79)$ & $184(16)$ & $129(13)$ & $102(12)$ & $37(9)$ & $71(11)$ & $20(11)$ \\
$\mathrm{C}(80)$ & $380(30)$ & $172(14)$ & $82(12)$ & $28(9)$ & $115(14)$ & $141(16)$ \\
\hline
\end{tabular}


Table S17. Hydrogen coordinates $\left(\times 10^{4}\right)$ and isotropic displacement parameters $\left(\AA^{2} \times 10^{3}\right)$ for $\left[\left(\mathrm{L}^{1}\right) \mathrm{Fe}(\mathrm{II})-\mathrm{NCCH}_{3}\right]\left[\mathrm{Ph}_{4} \mathrm{P}\right](\mathbf{1})$

\begin{tabular}{|c|c|c|c|c|}
\hline & $\mathrm{x}$ & $\mathrm{y}$ & $\mathrm{z}$ & $\mathrm{U}(\mathrm{eq})$ \\
\hline $\mathrm{H}(3 \mathrm{~A})$ & 464 & 1708 & 2521 & 38 \\
\hline $\mathrm{H}(4 \mathrm{~A})$ & 1118 & 1547 & 1804 & 42 \\
\hline $\mathrm{H}(5 \mathrm{~B})$ & 1639 & 2278 & 1328 & 41 \\
\hline $\mathrm{H}(6 \mathrm{~A})$ & 1411 & 3199 & 1595 & 31 \\
\hline $\mathrm{H}(9 \mathrm{~A})$ & 4574 & 4176 & 2950 & 40 \\
\hline $\mathrm{H}(10 \mathrm{~A})$ & 4800 & 4637 & 2256 & 46 \\
\hline $\mathrm{H}(11 \mathrm{~A})$ & 3108 & 4730 & 1644 & 46 \\
\hline $\mathrm{H}(12 \mathrm{~A})$ & 1220 & 4265 & 1701 & 39 \\
\hline $\mathrm{H}(15 \mathrm{~A})$ & -2679 & 4673 & 2548 & 29 \\
\hline $\mathrm{H}(16 \mathrm{~A})$ & -3476 & 4650 & 1751 & 37 \\
\hline $\mathrm{H}(17 \mathrm{~A})$ & -2543 & 4109 & 1216 & 38 \\
\hline $\mathrm{H}(18 \mathrm{~A})$ & -688 & 3618 & 1503 & 31 \\
\hline $\mathrm{H}(20 \mathrm{~A})$ & -1607 & 2484 & 3453 & 41 \\
\hline $\mathrm{H}(21 \mathrm{~A})$ & -1763 & 1819 & 4034 & 47 \\
\hline $\mathrm{H}(23 \mathrm{~A})$ & 1653 & 1227 & 3888 & 51 \\
\hline $\mathrm{H}(24 \mathrm{~A})$ & 1823 & 1891 & 3300 & 45 \\
\hline $\mathrm{H}(26 \mathrm{~A})$ & -170 & 124 & 4414 & 242 \\
\hline $\mathrm{H}(26 \mathrm{~B})$ & 677 & 372 & 4056 & 242 \\
\hline $\mathrm{H}(26 \mathrm{C})$ & -806 & 368 & 3917 & 242 \\
\hline
\end{tabular}




\begin{tabular}{|c|c|c|c|c|}
\hline $\mathrm{H}(27 \mathrm{~A})$ & -1425 & 727 & 4836 & 219 \\
\hline $\mathrm{H}(27 \mathrm{~B})$ & -2099 & 974 & 4346 & 219 \\
\hline $\mathrm{H}(27 \mathrm{C})$ & -1447 & 1379 & 4750 & 219 \\
\hline $\mathrm{H}(28 \mathrm{~A})$ & 828 & 753 & 5047 & 199 \\
\hline $\mathrm{H}(28 \mathrm{~B})$ & 841 & 1404 & 4957 & 199 \\
\hline $\mathrm{H}(28 \mathrm{C})$ & 1686 & 1005 & 4694 & 199 \\
\hline $\mathrm{H}(30 \mathrm{~A})$ & 4534 & 3031 & 3178 & 46 \\
\hline $\mathrm{H}(31 \mathrm{~A})$ & 6019 & 2760 & 3808 & 49 \\
\hline $\mathrm{H}(33 \mathrm{~A})$ & 4354 & 3718 & 4691 & 48 \\
\hline $\mathrm{H}(34 \mathrm{~A})$ & 2901 & 4018 & 4057 & 44 \\
\hline $\mathrm{H}(36 \mathrm{~A})$ & 5290 & 3138 & 5296 & 103 \\
\hline $\mathrm{H}(36 \mathrm{~B})$ & 6186 & 3643 & 5222 & 103 \\
\hline $\mathrm{H}(36 \mathrm{C})$ & 6749 & 3098 & 5487 & 103 \\
\hline $\mathrm{H}(37 \mathrm{~A})$ & 7739 & 3557 & 4639 & 110 \\
\hline $\mathrm{H}(37 \mathrm{~B})$ & 7853 & 2986 & 4369 & 110 \\
\hline $\mathrm{H}(37 \mathrm{C})$ & 8300 & 3025 & 4926 & 110 \\
\hline $\mathrm{H}(38 \mathrm{~A})$ & 5397 & 2252 & 4856 & 112 \\
\hline $\mathrm{H}(38 \mathrm{~B})$ & 6854 & 2213 & 5052 & 112 \\
\hline $\mathrm{H}(38 \mathrm{C})$ & 6372 & 2175 & 4497 & 112 \\
\hline $\mathrm{H}(40 \mathrm{~A})$ & -2958 & 4003 & 3266 & 36 \\
\hline $\mathrm{H}(41 \mathrm{~A})$ & -3759 & 4437 & 3879 & 45 \\
\hline $\mathrm{H}(43 \mathrm{~A})$ & -480 & 5246 & 4254 & 1 \\
\hline
\end{tabular}




\begin{tabular}{|c|c|c|c|c|}
\hline $\mathrm{H}(44 \mathrm{~A})$ & 324 & 4816 & 3638 & 40 \\
\hline $\mathrm{H}(46 \mathrm{~A})$ & -4519 & 4854 & 4452 & 144 \\
\hline $\mathrm{H}(46 \mathrm{~B})$ & -4484 & 5419 & 4167 & 144 \\
\hline $\mathrm{H}(46 \mathrm{C})$ & -4480 & 5431 & 4724 & 144 \\
\hline $\mathrm{H}(47 \mathrm{~A})$ & -2599 & 4532 & 4980 & 244 \\
\hline $\mathrm{H}(47 \mathrm{~B})$ & -2688 & 5096 & 5261 & 244 \\
\hline $\mathrm{H}(47 \mathrm{C})$ & -1416 & 4930 & 5082 & 244 \\
\hline $\mathrm{H}(48 \mathrm{~A})$ & -1410 & 5842 & 4630 & 203 \\
\hline $\mathrm{H}(48 \mathrm{~B})$ & -2652 & 6013 & 4829 & 203 \\
\hline $\mathrm{H}(48 \mathrm{C})$ & -2632 & 6009 & 4273 & 203 \\
\hline $\mathrm{H}(50 \mathrm{~A})$ & 2049 & 2892 & 4879 & 87 \\
\hline $\mathrm{H}(50 \mathrm{~B})$ & 1117 & 2414 & 4633 & 87 \\
\hline $\mathrm{H}(50 \mathrm{C})$ & 571 & 2884 & 4917 & 87 \\
\hline $\mathrm{H}(52 \mathrm{~A})$ & 7817 & 1017 & 3124 & 37 \\
\hline $\mathrm{H}(53 \mathrm{~A})$ & 9847 & 741 & 3028 & 43 \\
\hline $\mathrm{H}(54 \mathrm{~A})$ & 10323 & 546 & 2273 & 45 \\
\hline $\mathrm{H}(55 \mathrm{~A})$ & 8762 & 610 & 1612 & 45 \\
\hline $\mathrm{H}(56 \mathrm{~A})$ & 6722 & 876 & 1698 & 40 \\
\hline $\mathrm{H}(58 \mathrm{~A})$ & 3748 & 2155 & 2207 & 34 \\
\hline $\mathrm{H}(59 \mathrm{~A})$ & 3903 & 3122 & 2125 & 38 \\
\hline $\mathrm{H}(60 \mathrm{~A})$ & 5874 & 3554 & 2268 & 2 \\
\hline $\mathrm{H}(61 \mathrm{~A})$ & 7686 & 3033 & 2501 & 5 \\
\hline
\end{tabular}




\begin{tabular}{|c|c|c|c|c|}
\hline $\mathrm{H}(62 \mathrm{~A})$ & 7549 & 2067 & 2567 & 39 \\
\hline $\mathrm{H}(64 \mathrm{~A})$ & 4682 & 1526 & 1498 & 40 \\
\hline $\mathrm{H}(65 \mathrm{~A})$ & 3199 & 1148 & 890 & 46 \\
\hline $\mathrm{H}(66 \mathrm{~A})$ & 1922 & 407 & 1060 & 49 \\
\hline $\mathrm{H}(67 \mathrm{~A})$ & 2141 & 41 & 1820 & 49 \\
\hline $\mathrm{H}(68 \mathrm{~A})$ & 3647 & 395 & 2424 & 39 \\
\hline $\mathrm{H}(70 \mathrm{~A})$ & 5695 & 279 & 3064 & 48 \\
\hline $\mathrm{H}(71 \mathrm{~A})$ & 5228 & 77 & 3817 & 62 \\
\hline $\mathrm{H}(72 \mathrm{~A})$ & 4396 & 759 & 4257 & 66 \\
\hline $\mathrm{H}(73 \mathrm{~A})$ & 4147 & 1663 & 3971 & 60 \\
\hline $\mathrm{H}(74 \mathrm{~A})$ & 4616 & 1886 & 3221 & 46 \\
\hline $\mathrm{H}(76 \mathrm{~A})$ & 7016 & 2279 & 1298 & 97 \\
\hline $\mathrm{H}(76 \mathrm{~B})$ & 8120 & 2591 & 1608 & 97 \\
\hline $\mathrm{H}(76 \mathrm{C})$ & 8340 & 2003 & 1374 & 97 \\
\hline $\mathrm{H}(78 \mathrm{~A})$ & 5108 & 3652 & 1005 & 144 \\
\hline H(78B) & 4215 & 3615 & 1357 & 144 \\
\hline $\mathrm{H}(78 \mathrm{C})$ & 3769 & 3746 & 792 & 144 \\
\hline $\mathrm{H}(80 \mathrm{~A})$ & 4978 & 8678 & 4440 & 306 \\
\hline $\mathrm{H}(80 \mathrm{~B})$ & 5593 & 8960 & 4924 & 306 \\
\hline $\mathrm{H}(80 \mathrm{C})$ & 5832 & 9214 & 4432 & 306 \\
\hline
\end{tabular}


Table S18. Torsion Angles, ${ }^{\circ}$, for $\left[\left(\mathrm{L}^{1}\right) \mathrm{Fe}(\mathrm{II})-\mathrm{NCCH}_{3}\right]\left[\mathrm{Ph}_{4} \mathrm{P}\right](\mathbf{1})$

\begin{tabular}{|c|c|c|c|}
\hline N4-Fe1-N1-C13 & $-21.2(3)$ & C20-C21-C22-C23 & $2.1(9)$ \\
\hline N3-Fe1-N1-C13 & $-144.9(3)$ & C20-C21-C22-C25 & $-178.9(6)$ \\
\hline N2-Fe1-N1-C13 & $101.0(3)$ & C21-C22-C23-C24 & $-2.3(9)$ \\
\hline N5-Fe1-N1-C13 & $106(3)$ & $\mathrm{C} 25-\mathrm{C} 22-\mathrm{C} 23-\mathrm{C} 24$ & $178.7(6)$ \\
\hline N4-Fe1-N1-C1 & $-142.6(3)$ & C20-C19-C24-C23 & $1.9(8)$ \\
\hline N3-Fe1-N1-C1 & $93.7(3)$ & N2-C19-C24-C23 & $176.7(6)$ \\
\hline N2-Fe1-N1-C1 & $-20.4(3)$ & C22-C23-C24-C19 & $0.3(10)$ \\
\hline N5-Fe1-N1-C1 & $-16(3)$ & C21-C22-C25-C27 & $-0.4(11)$ \\
\hline N4-Fe1-N1-C7 & $99.7(3)$ & $\mathrm{C} 23-\mathrm{C} 22-\mathrm{C} 25-\mathrm{C} 27$ & $178.6(8)$ \\
\hline N3-Fe1-N1-C7 & $-24.0(3)$ & C21-C22-C25-C26 & 119.6(9) \\
\hline N2-Fe1-N1-C7 & $-138.1(3)$ & $\mathrm{C} 23-\mathrm{C} 22-\mathrm{C} 25-\mathrm{C} 26$ & $-61.5(10)$ \\
\hline N5-Fe1-N1-C7 & $-133(3)$ & C21-C22-C25-C28 & $-119.9(8)$ \\
\hline $\mathrm{N} 4-\mathrm{Fe} 1-\mathrm{N} 2-\mathrm{C} 2$ & $93.4(4)$ & $\mathrm{C} 23-\mathrm{C} 22-\mathrm{C} 25-\mathrm{C} 28$ & $59.1(10)$ \\
\hline N3-Fe1-N2-C2 & $-52.1(4)$ & C8-N3-C29-C34 & $122.2(6)$ \\
\hline N5-Fe1-N2-C2 & $-157.6(3)$ & Fe1-N3-C29-C34 & $-57.3(7)$ \\
\hline N1-Fe1-N2-C2 & $22.1(3)$ & C8-N3-C29-C30 & $-62.0(7)$ \\
\hline N4-Fe1-N2-C19 & $-116.2(4)$ & Fe1-N3-C29-C30 & $118.5(5)$ \\
\hline N3-Fe1-N2-C19 & $98.4(4)$ & C34-C29-C30-C31 & $3.1(9)$ \\
\hline N5-Fe1-N2-C19 & $-7.2(4)$ & N3-C29-C30-C31 & $-172.9(5)$ \\
\hline N1-Fe1-N2-C19 & $172.5(4)$ & C29-C30-C31-C32 & $0.0(10)$ \\
\hline N4-Fe1-N3-C8 & $-50.1(4)$ & C30-C31-C32-C33 & $-1.8(9)$ \\
\hline
\end{tabular}




\begin{tabular}{|c|c|c|c|}
\hline N2-Fe1-N3-C8 & $94.9(4)$ & C30-C31-C32-C35 & $177.6(6)$ \\
\hline N5-Fe1-N3-C8 & $-162.7(3)$ & C31-C32-C33-C34 & $0.6(9)$ \\
\hline N1-Fe1-N3-C8 & $20.4(3)$ & C35-C32-C33-C34 & $-178.8(6)$ \\
\hline N4-Fe1-N3-C29 & $129.4(4)$ & С30-C29-C34-C33 & $-4.2(8)$ \\
\hline N2-Fe1-N3-C29 & $-85.6(4)$ & N3-C29-C34-C33 & $171.8(5)$ \\
\hline N5-Fe1-N3-C29 & $16.8(4)$ & C32-C33-C34-C29 & $2.5(9)$ \\
\hline $\mathrm{N} 1-\mathrm{Fe} 1-\mathrm{N} 3-\mathrm{C} 29$ & $-160.1(4)$ & C33-C32-C35-C36 & $1.0(9)$ \\
\hline N3-Fe1-N4-C14 & $90.1(4)$ & C31-C32-C35-C36 & $-178.3(6)$ \\
\hline $\mathrm{N} 2-\mathrm{Fe} 1-\mathrm{N} 4-\mathrm{C} 14$ & $-52.2(4)$ & C33-C32-C35-C38 & $119.4(7)$ \\
\hline N5-Fe1-N4-C14 & $-157.9(3)$ & C31-C32-C35-C38 & $-60.0(8)$ \\
\hline N1-Fe1-N4-C14 & $19.5(3)$ & C33-C32-C35-C37 & $-119.3(7)$ \\
\hline N3-Fe1-N4-C39 & $-105.5(4)$ & C31-C32-C35-C37 & $61.4(8)$ \\
\hline N2-Fe1-N4-C39 & $112.1(4)$ & C14-N4-C39-C44 & $-125.2(5)$ \\
\hline N5-Fe1-N4-C39 & $6.5(4)$ & Fe1-N4-C39-C44 & $71.4(6)$ \\
\hline N1-Fe1-N4-C39 & $-176.1(4)$ & C14-N4-C39-C40 & $61.1(7)$ \\
\hline N4-Fe1-N5-C49 & $168.7(10)$ & Fe1-N4-C39-C40 & $-102.3(5)$ \\
\hline N3-Fe1-N5-C49 & $-66.5(11)$ & C44-C39-C40-C41 & $3.2(8)$ \\
\hline N2-Fe1-N5-C49 & $46.9(11)$ & N4-C39-C40-C41 & $176.9(5)$ \\
\hline N1-Fe1-N5-C49 & $42(3)$ & C39-C40-C41-C42 & $-0.6(9)$ \\
\hline C13-N1-C1-C6 & $84.3(6)$ & C40-C41-C42-C43 & $-2.1(9)$ \\
\hline C7-N1-C1-C6 & $-48.9(6)$ & C40-C41-C42-C45 & $179.2(6)$ \\
\hline Fe1-N1-C1-C6 & $-160.8(4)$ & C41-C42-C43-C44 & $2.3(9)$ \\
\hline
\end{tabular}




\begin{tabular}{|c|c|c|c|}
\hline C13-N1-C1-C2 & $-98.2(5)$ & C45-C42-C43-C44 & $-179.0(6)$ \\
\hline C7-N1-C1-C2 & $128.6(4)$ & C40-C39-C44-C43 & $-3.0(8)$ \\
\hline Fe1-N1-C1-C2 & $16.7(4)$ & N4-C39-C44-C43 & $-177.1(5)$ \\
\hline C19-N2-C2-C3 & $12.2(7)$ & C42-C43-C44-C39 & $0.2(9)$ \\
\hline Fe1-N2-C2-C3 & $163.3(4)$ & C43-C42-C45-C47 & $-81.3(10)$ \\
\hline C19-N2-C2-C1 & $-171.0(4)$ & C41-C42-C45-C47 & $97.3(10)$ \\
\hline $\mathrm{Fe} 1-\mathrm{N} 2-\mathrm{C} 2-\mathrm{C} 1$ & $-20.0(5)$ & C43-C42-C45-C46 & $158.5(7)$ \\
\hline $\mathrm{C} 6-\mathrm{C} 1-\mathrm{C} 2-\mathrm{N} 2$ & $177.9(4)$ & C41-C42-C45-C46 & $-22.9(9)$ \\
\hline $\mathrm{N} 1-\mathrm{C} 1-\mathrm{C} 2-\mathrm{N} 2$ & $0.4(6)$ & C43-C42-C45-C48 & $41.7(9)$ \\
\hline $\mathrm{C} 6-\mathrm{C} 1-\mathrm{C} 2-\mathrm{C} 3$ & $-5.0(6)$ & C41-C42-C45-C48 & $-139.7(8)$ \\
\hline N1-C1-C2-C3 & $177.5(4)$ & Fe1-N5-C49-C50 & $6(24)$ \\
\hline $\mathrm{N} 2-\mathrm{C} 2-\mathrm{C} 3-\mathrm{C} 4$ & $-179.3(5)$ & C57-P1-C51-C56 & $-85.5(4)$ \\
\hline $\mathrm{C} 1-\mathrm{C} 2-\mathrm{C} 3-\mathrm{C} 4$ & $3.8(7)$ & C69-P1-C51-C56 & $154.9(4)$ \\
\hline $\mathrm{C} 2-\mathrm{C} 3-\mathrm{C} 4-\mathrm{C} 5$ & $-0.6(8)$ & C63-P1-C51-C56 & $33.5(5)$ \\
\hline $\mathrm{C} 3-\mathrm{C} 4-\mathrm{C} 5-\mathrm{C} 6$ & $-1.5(8)$ & C57-P1-C51-C52 & $88.5(5)$ \\
\hline $\mathrm{C} 2-\mathrm{C} 1-\mathrm{C} 6-\mathrm{C} 5$ & $3.2(7)$ & C69-P1-C51-C52 & $-31.1(5)$ \\
\hline N1-C1-C6-C5 & $-179.4(4)$ & C63-P1-C51-C52 & $-152.4(4)$ \\
\hline C4-C5-C6-C1 & $0.2(7)$ & C56-C51-C52-C53 & $0.9(7)$ \\
\hline C13-N1-C7-C12 & $-43.4(7)$ & P1-C51-C52-C53 & $-173.1(4)$ \\
\hline C1-N1-C7-C12 & $89.8(5)$ & C51-C52-C53-C54 & $0.1(8)$ \\
\hline Fe1-N1-C7-C12 & $-157.6(4)$ & C52-C53-C54-C55 & $-0.7(8)$ \\
\hline C13-N1-C7-C8 & $139.8(5)$ & C53-C54-C55-C56 & $0.4(8)$ \\
\hline
\end{tabular}




\begin{tabular}{|c|c|c|c|}
\hline C1-N1-C7-C8 & $-87.0(5)$ & C52-C51-C56-C55 & $-1.2(8)$ \\
\hline $\mathrm{Fe} 1-\mathrm{N} 1-\mathrm{C} 7-\mathrm{C} 8$ & $25.6(5)$ & P1-C51-C56-C55 & $172.8(4)$ \\
\hline C29-N3-C8-C9 & $-15.5(8)$ & C54-C55-C56-C51 & $0.6(8)$ \\
\hline Fe1-N3-C8-C9 & $164.1(4)$ & C69-P1-C57-C62 & $95.2(5)$ \\
\hline C29-N3-C8-C7 & $168.5(5)$ & C51-P1-C57-C62 & $-23.0(5)$ \\
\hline $\mathrm{Fe} 1-\mathrm{N} 3-\mathrm{C} 8-\mathrm{C} 7$ & $-12.0(6)$ & C63-P1-C57-C62 & $-143.1(5)$ \\
\hline $\mathrm{C} 12-\mathrm{C} 7-\mathrm{C} 8-\mathrm{N} 3$ & $171.4(5)$ & C69-P1-C57-C58 & $-83.2(5)$ \\
\hline N1-C7-C8-N3 & $-11.8(7)$ & C51-P1-C57-C58 & $158.6(5)$ \\
\hline C12-C7-C8-C9 & $-5.1(7)$ & C63-P1-C57-C58 & $38.5(5)$ \\
\hline N1-C7-C8-C9 & $171.8(4)$ & C62-C57-C58-C59 & $0.2(8)$ \\
\hline N3-C8-C9-C10 & $-174.2(5)$ & P1-C57-C58-C59 & $178.6(5)$ \\
\hline C7-C8-C9-C10 & $2.0(7)$ & C57-C58-C59-C60 & $-0.3(9)$ \\
\hline C8-C9-C10-C11 & $2.3(9)$ & C58-C59-C60-C61 & $-0.4(9)$ \\
\hline C9-C10-C11-C12 & $-3.8(9)$ & C59-C60-C61-C62 & $1.1(9)$ \\
\hline C8-C7-C12-C11 & $3.8(8)$ & C60-C61-C62-C57 & $-1.2(9)$ \\
\hline N1-C7-C12-C11 & $-172.8(5)$ & C58-C57-C62-C61 & $0.6(9)$ \\
\hline C10-C11-C12-C7 & $0.8(9)$ & P1-C57-C62-C61 & $-177.8(5)$ \\
\hline C1-N1-C13-C18 & $-46.4(6)$ & C57-P1-C63-C68 & $-143.6(4)$ \\
\hline C7-N1-C13-C18 & $85.5(6)$ & C69-P1-C63-C68 & $-21.7(5)$ \\
\hline Fe1-N1-C13-C18 & $-161.1(4)$ & C51-P1-C63-C68 & $98.6(5)$ \\
\hline C1-N1-C13-C14 & $135.7(5)$ & C57-P1-C63-C64 & $36.6(5)$ \\
\hline C7-N1-C13-C14 & $-92.4(5)$ & C69-P1-C63-C64 & $158.5(4)$ \\
\hline
\end{tabular}




\begin{tabular}{|c|c|c|c|}
\hline Fe1-N1-C13-C14 & $21.0(5)$ & C51-P1-C63-C64 & $-81.2(5)$ \\
\hline C39-N4-C14-C15 & $1.8(7)$ & C68-C63-C64-C65 & $-2.3(8)$ \\
\hline Fe1-N4-C14-C15 & $166.1(4)$ & P1-C63-C64-C65 & $177.6(4)$ \\
\hline C39-N4-C14-C13 & $-178.1(4)$ & C63-C64-C65-C66 & $1.8(8)$ \\
\hline Fe1-N4-C14-C13 & $-13.7(5)$ & C64-C65-C66-С67 & $-0.3(8)$ \\
\hline C18-C13-C14-N4 & $174.9(4)$ & C65-C66-С67-C68 & $-0.7(8)$ \\
\hline N1-C13-C14-N4 & $-7.2(6)$ & C66-C67-C68-C63 & $0.2(8)$ \\
\hline C18-C13-C14-C15 & $-5.0(7)$ & C64-C63-C68-C67 & $1.3(8)$ \\
\hline N1-C13-C14-C15 & $172.9(4)$ & P1-C63-C68-C67 & $-178.5(4)$ \\
\hline N4-C14-C15-C16 & $-176.4(5)$ & C57-P1-C69-C70 & $-164.4(4)$ \\
\hline C13-C14-C15-C16 & $3.5(7)$ & C51-P1-C69-C70 & $-47.1(5)$ \\
\hline C14-C15-C16-C17 & $-0.1(8)$ & C63-P1-C69-C70 & $74.6(5)$ \\
\hline C15-C16-C17-C18 & $-2.1(8)$ & C57-P1-C69-C74 & $16.3(5)$ \\
\hline C14-C13-C18-C17 & $3.1(7)$ & C51-P1-C69-C74 & $133.6(4)$ \\
\hline N1-C13-C18-C17 & $-174.7(4)$ & C63-P1-C69-C74 & $-104.7(4)$ \\
\hline C16-C17-C18-C13 & $0.6(8)$ & C74-C69-C70-C71 & $0.2(8)$ \\
\hline C2-N2-C19-C24 & $50.3(7)$ & P1-C69-C70-C71 & $-179.0(5)$ \\
\hline Fe1-N2-C19-C24 & $-98.3(6)$ & C69-C70-C71-C72 & $1.1(10)$ \\
\hline C2-N2-C19-C20 & $-134.9(5)$ & C70-C71-C72-C73 & $-2.1(10)$ \\
\hline Fe1-N2-C19-C20 & $76.5(5)$ & C71-C72-C73-C74 & $1.8(10)$ \\
\hline C24-C19-C20-C21 & $-2.0(8)$ & C72-C73-C74-C69 & $-0.5(9)$ \\
\hline N2-C19-C20-C21 & $-177.1(5)$ & C70-C69-C74-C73 & $-0.5(8)$ \\
\hline
\end{tabular}


Table S19. Atomic coordinates $\left(\times 10^{4}\right)$ and equivalent isotropic displacement parameters $\left(\AA^{2} \times 10^{3}\right)$ for $\left[\left(\mathrm{L}^{1}\right) \mathrm{Fe}(\mathrm{III})-\mathrm{OH}\right]\left[\mathrm{PPh}_{4}\right] \cdot \mathrm{CH}_{3} \mathrm{CN} \cdot 0.5 \mathrm{Et}_{2} \mathrm{O}(\mathbf{2})$. U(eq) is defined as one third of the trace of the orthogonalized $\mathrm{U}^{\mathrm{ij}}$ tensor

\begin{tabular}{|c|c|c|c|c|}
\hline & $\mathrm{x}$ & $\mathrm{y}$ & $\mathrm{Z}$ & $\mathrm{U}(\mathrm{eq})$ \\
\hline $\mathrm{Fe}(1)$ & $722(1)$ & $6500(1)$ & $8281(1)$ & $41(1)$ \\
\hline $\mathrm{O}(1)$ & 499(4) & $6580(2)$ & $8938(2)$ & $56(1)$ \\
\hline $\mathrm{N}(1)$ & $781(4)$ & $6367(2)$ & $7483(2)$ & $35(1)$ \\
\hline $\mathrm{N}(2)$ & $2572(5)$ & $6368(2)$ & $8272(2)$ & $44(1)$ \\
\hline $\mathrm{N}(3)$ & $288(4)$ & $7256(2)$ & $8009(2)$ & $38(1)$ \\
\hline $\mathrm{N}(4)$ & $-488(4)$ & $5873(2)$ & $8096(2)$ & $38(1)$ \\
\hline$C(1)$ & $-1006(6)$ & $5799(2)$ & $7629(2)$ & $34(2)$ \\
\hline$C(2)$ & $-404(6)$ & $6073(2)$ & $7292(2)$ & $36(2)$ \\
\hline$C(3)$ & $-899(6)$ & $6062(3)$ & 6813(2) & $43(2)$ \\
\hline $\mathrm{C}(4)$ & $-2001(6)$ & $5760(3)$ & $6650(3)$ & $48(2)$ \\
\hline$C(5)$ & $-2561(6)$ & $5468(3)$ & $6990(3)$ & $47(2)$ \\
\hline$C(6)$ & $-2100(6)$ & $5479(3)$ & $7459(3)$ & $40(2)$ \\
\hline $\mathrm{C}(7)$ & $919(5)$ & $6929(3)$ & $7287(2)$ & $38(2)$ \\
\hline $\mathrm{C}(8)$ & $1322(6)$ & $7012(3)$ & $6845(2)$ & $42(2)$ \\
\hline$C(9)$ & $1393(6)$ & $7541(3)$ & $6663(3)$ & $49(2)$ \\
\hline$C(10)$ & $1023(6)$ & 7984(3) & $6929(3)$ & $53(2)$ \\
\hline$C(11)$ & $658(6)$ & $7898(3)$ & $7368(3)$ & $47(2)$ \\
\hline$C(12)$ & $627(6)$ & $7371(3)$ & $7569(2)$ & $38(2)$ \\
\hline
\end{tabular}




\begin{tabular}{|c|c|c|c|c|}
\hline$C(13)$ & $182(6)$ & $7709(3)$ & $8327(2)$ & $43(2)$ \\
\hline$C(14)$ & $-926(6)$ & 7781(3) & $8537(2)$ & $49(2)$ \\
\hline$C(15)$ & $-1023(7)$ & $8210(3)$ & $8842(2)$ & $57(2)$ \\
\hline$C(16)$ & $-42(8)$ & $8606(3)$ & $8958(2)$ & $58(2)$ \\
\hline$C(17)$ & $1069(7)$ & $8523(3)$ & $8769(3)$ & $62(2)$ \\
\hline$C(18)$ & $1181(7)$ & $8085(3)$ & $8459(3)$ & $56(2)$ \\
\hline C(19) & $-182(9)$ & $9093(4)$ & $9295(3)$ & $81(3)$ \\
\hline$C(20)$ & $-20(20)$ & $8922(5)$ & $9796(4)$ & $281(12)$ \\
\hline$C(21)$ & $880(20)$ & $9488(7)$ & $9315(9)$ & $366(19)$ \\
\hline$C(22)$ & $-1297(19)$ & $9401(7)$ & $9167(7)$ & $339(16)$ \\
\hline$C(23)$ & 1944(6) & $6023(3)$ & $7477(2)$ & $39(2)$ \\
\hline$C(24)$ & $2129(6)$ & $5693(3)$ & 7101(3) & $48(2)$ \\
\hline$C(25)$ & $3333(8)$ & $5427(3)$ & $7105(3)$ & $60(2)$ \\
\hline$C(26)$ & $4275(7)$ & $5496(3)$ & $7490(3)$ & $59(2)$ \\
\hline$C(27)$ & $4066(6)$ & $5806(3)$ & $7867(3)$ & $49(2)$ \\
\hline$C(28)$ & $2878(6)$ & $6079(3)$ & 7891(3) & $41(2)$ \\
\hline C(29) & $3581(6)$ & $6521(3)$ & $8639(2)$ & $47(2)$ \\
\hline$C(30)$ & $4614(7)$ & $6845(3)$ & $8554(3)$ & $53(2)$ \\
\hline$C(31)$ & $5546(7)$ & $7015(3)$ & $8919(3)$ & $56(2)$ \\
\hline$C(32)$ & $5493(7)$ & $6890(3)$ & $9378(3)$ & $58(2)$ \\
\hline C(33) & $4460(7)$ & $6567(4)$ & $9464(3)$ & $79(3)$ \\
\hline$C(34)$ & $3512(7)$ & $6391(4)$ & $9102(3)$ & $71(2)$ \\
\hline
\end{tabular}




\begin{tabular}{|c|c|c|c|c|}
\hline$C(35)$ & $6524(8)$ & $7105(4)$ & $9774(3)$ & $75(3)$ \\
\hline$C(36)$ & $7819(8)$ & $6890(5)$ & $9707(4)$ & $140(5)$ \\
\hline$C(37)$ & $6603(10)$ & $7730(4)$ & $9749(3)$ & $121(4)$ \\
\hline$C(38)$ & $6238(8)$ & $6964(4)$ & $10273(3)$ & $103(3)$ \\
\hline C(39) & $-1069(6)$ & $5600(3)$ & $8450(2)$ & $39(2)$ \\
\hline$C(40)$ & $-424(6)$ & $5171(3)$ & $8718(2)$ & $48(2)$ \\
\hline$C(41)$ & $-939(8)$ & 4914(3) & $9078(3)$ & $62(2)$ \\
\hline$C(42)$ & $-2112(8)$ & $5067(3)$ & $9206(3)$ & $58(2)$ \\
\hline$C(43)$ & $-2749(7)$ & $5495(4)$ & $8947(3)$ & $67(2)$ \\
\hline$C(44)$ & $-2251(6)$ & $5757(3)$ & $8570(3)$ & $57(2)$ \\
\hline$C(45)$ & $-2622(9)$ & $4784(5)$ & $9629(3)$ & $91(3)$ \\
\hline$C(46)$ & $-3984(10)$ & $4637(6)$ & $9489(4)$ & $171(6)$ \\
\hline$C(47)$ & $-1847(14)$ & $4294(6)$ & $9825(5)$ & $211(8)$ \\
\hline$C(48)$ & $-2569(14)$ & $5180(6)$ & $10020(4)$ & $206(8)$ \\
\hline $\mathrm{P}(1)$ & $510(2)$ & $6348(1)$ & $2465(1)$ & $40(1)$ \\
\hline C(49) & 1999(6) & $6045(2)$ & $2368(3)$ & $41(2)$ \\
\hline$C(50)$ & $2243(6)$ & $5920(3)$ & $1929(3)$ & $48(2)$ \\
\hline$C(51)$ & $3447(8)$ & $5757(3)$ & $1857(3)$ & $58(2)$ \\
\hline$C(52)$ & $4405(7)$ & $5719(3)$ & $2231(3)$ & $59(2)$ \\
\hline$C(53)$ & $4184(7)$ & $5833(3)$ & 2693(3) & $58(2)$ \\
\hline$C(54)$ & $2958(6)$ & 5994(3) & $2764(3)$ & $45(2)$ \\
\hline$C(55)$ & $657(6)$ & 7083(3) & $2398(2)$ & $42(2)$ \\
\hline
\end{tabular}




\begin{tabular}{|c|c|c|c|c|}
\hline$C(56)$ & $-410(6)$ & $7423(3)$ & $2277(2)$ & $49(2)$ \\
\hline$C(57)$ & $-294(6)$ & 7988(3) & $2234(2)$ & $49(2)$ \\
\hline$C(58)$ & $899(7)$ & $8226(3)$ & $2314(2)$ & $53(2)$ \\
\hline C(59) & $1980(7)$ & 7897(3) & $2439(3)$ & $56(2)$ \\
\hline$C(60)$ & $1852(6)$ & $7327(3)$ & $2475(2)$ & $51(2)$ \\
\hline$C(61)$ & $190(6)$ & $6187(3)$ & $3046(2)$ & $43(2)$ \\
\hline$C(62)$ & $-198(6)$ & $6589(3)$ & $3331(3)$ & $52(2)$ \\
\hline$C(63)$ & $-451(7)$ & $6469(4)$ & $3785(3)$ & $69(2)$ \\
\hline$C(64)$ & $-339(8)$ & $5931(4)$ & $3948(3)$ & $77(3)$ \\
\hline$C(65)$ & $57(7)$ & $5514(4)$ & $3658(3)$ & $72(2)$ \\
\hline$C(66)$ & $325(6)$ & $5641(3)$ & $3202(3)$ & $55(2)$ \\
\hline$C(67)$ & $-739(6)$ & $6090(3)$ & $2028(2)$ & $39(2)$ \\
\hline$C(68)$ & $-1496(6)$ & $5653(3)$ & $2131(3)$ & $49(2)$ \\
\hline C(69) & $-2402(7)$ & $5434(3)$ & 1781(3) & $62(2)$ \\
\hline$C(70)$ & $-2552(7)$ & $5652(4)$ & 1331(4) & $70(3)$ \\
\hline$C(71)$ & $-1804(7)$ & $6095(3)$ & $1217(3)$ & $66(2)$ \\
\hline$C(72)$ & $-894(6)$ & $6310(3)$ & $1577(3)$ & $54(2)$ \\
\hline $\mathrm{N}(1 \mathrm{~S})$ & $6000(19)$ & $7873(8)$ & $5957(7)$ & $133(7)$ \\
\hline$C(1 S)$ & $7946(14)$ & $7447(7)$ & $6218(5)$ & $69(5)$ \\
\hline$C(2 S)$ & $6915(19)$ & $7715(8)$ & $6081(6)$ & $81(5)$ \\
\hline $\mathrm{N}(2 \mathrm{~S})$ & $3257(13)$ & $8110(6)$ & $9961(5)$ & $88(4)$ \\
\hline C(3S) & $1521(11)$ & $7434(5)$ & $9725(4)$ & $41(3)$ \\
\hline
\end{tabular}




\begin{tabular}{lrrrr}
$\mathrm{C}(4 \mathrm{~S})$ & $2474(14)$ & $7785(6)$ & $9851(5)$ & $56(4)$ \\
$\mathrm{O}(3 \mathrm{~S})$ & $7606(18)$ & $8920(7)$ & $755(6)$ & $161(6)$ \\
$\mathrm{C}(5 \mathrm{~S})$ & $9580(30)$ & $8174(12)$ & $961(10)$ & $190(12)$ \\
$\mathrm{C}(6 \mathrm{~S})$ & $8800(20)$ & $8568(10)$ & $944(8)$ & $137(8)$ \\
$\mathrm{C}(7 \mathrm{~S})$ & $6390(20)$ & $9107(11)$ & $598(9)$ & $146(9)$ \\
$\mathrm{C}(8 \mathrm{~S})$ & $5450(20)$ & $9352(11)$ & $397(9)$ & $150(9)$ \\
\hline
\end{tabular}


Table S20. Bond Distances, $\AA$, for $\left[\left(\mathrm{L}^{1}\right) \mathrm{Fe}(\mathrm{III})-\mathrm{OH}\right]\left[\mathrm{PPh}_{4}\right] \cdot \mathrm{CH}_{3} \mathrm{CN} \cdot 0.5 \mathrm{Et}_{2} \mathrm{O}$ (2)

\begin{tabular}{|c|c|c|c|}
\hline $\mathrm{Fe}(1)-\mathrm{O}(1)$ & $1.920(4)$ & $\mathrm{C}(40)-\mathrm{C}(41)$ & $1.372(9)$ \\
\hline $\mathrm{Fe}(1)-\mathrm{N}(2)$ & $1.986(5)$ & $\mathrm{C}(40)-\mathrm{H}(40 \mathrm{~A})$ & 0.9300 \\
\hline $\mathrm{Fe}(1)-\mathrm{N}(4)$ & $1.995(5)$ & $C(41)-C(42)$ & $1.395(9)$ \\
\hline $\mathrm{Fe}(1)-\mathrm{N}(3)$ & $2.003(5)$ & $\mathrm{C}(41)-\mathrm{H}(41 \mathrm{~A})$ & 0.9300 \\
\hline $\mathrm{Fe}(1)-\mathrm{N}(1)$ & $2.297(5)$ & $C(42)-C(43)$ & $1.379(10)$ \\
\hline $\mathrm{O}(1)-\mathrm{H}(1 \mathrm{~A})$ & 0.8200 & $C(42)-C(45)$ & $1.544(10)$ \\
\hline $\mathrm{N}(1)-\mathrm{C}(2)$ & $1.471(7)$ & $\mathrm{C}(43)-\mathrm{C}(44)$ & $1.411(9)$ \\
\hline $\mathrm{N}(1)-\mathrm{C}(7)$ & $1.475(7)$ & $\mathrm{C}(43)-\mathrm{H}(43 \mathrm{~A})$ & 0.9300 \\
\hline $\mathrm{N}(1)-\mathrm{C}(23)$ & $1.485(7)$ & $\mathrm{C}(44)-\mathrm{H}(44 \mathrm{~A})$ & 0.9300 \\
\hline $\mathrm{N}(2)-\mathrm{C}(28)$ & $1.366(8)$ & $C(45)-C(48)$ & $1.456(13)$ \\
\hline N(2)-C(29) & $1.422(8)$ & $C(45)-C(46)$ & $1.479(12)$ \\
\hline $\mathrm{N}(3)-\mathrm{C}(12)$ & $1.376(7)$ & $C(45)-C(47)$ & $1.495(14)$ \\
\hline $\mathrm{N}(3)-\mathrm{C}(13)$ & $1.429(7)$ & $\mathrm{C}(46)-\mathrm{H}(46 \mathrm{~A})$ & 0.9600 \\
\hline $\mathrm{N}(4)-\mathrm{C}(1)$ & $1.367(7)$ & $\mathrm{C}(46)-\mathrm{H}(46 \mathrm{~B})$ & 0.9600 \\
\hline $\mathrm{N}(4)-\mathrm{C}(39)$ & $1.415(7)$ & $\mathrm{C}(46)-\mathrm{H}(46 \mathrm{C})$ & 0.9600 \\
\hline $\mathrm{C}(1)-\mathrm{C}(2)$ & $1.391(8)$ & $\mathrm{C}(47)-\mathrm{H}(47 \mathrm{~A})$ & 0.9600 \\
\hline$C(1)-C(6)$ & $1.413(8)$ & $\mathrm{C}(47)-\mathrm{H}(47 \mathrm{~B})$ & 0.9600 \\
\hline$C(2)-C(3)$ & $1.381(8)$ & $\mathrm{C}(47)-\mathrm{H}(47 \mathrm{C})$ & 0.9600 \\
\hline$C(3)-C(4)$ & $1.391(8)$ & $\mathrm{C}(48)-\mathrm{H}(48 \mathrm{~A})$ & 0.9600 \\
\hline $\mathrm{C}(3)-\mathrm{H}(3 \mathrm{~A})$ & 0.9300 & $\mathrm{C}(48)-\mathrm{H}(48 \mathrm{~B})$ & 0.9600 \\
\hline$C(4)-C(5)$ & $1.395(9)$ & $\mathrm{C}(48)-\mathrm{H}(48 \mathrm{C})$ & 0.9600 \\
\hline
\end{tabular}




\begin{tabular}{|c|c|c|c|}
\hline $\mathrm{C}(4)-\mathrm{H}(4 \mathrm{~A})$ & 0.9300 & $\mathrm{P}(1)-\mathrm{C}(61)$ & $1.773(7)$ \\
\hline$C(5)-C(6)$ & $1.348(8)$ & $\mathrm{P}(1)-\mathrm{C}(67)$ & $1.783(7)$ \\
\hline $\mathrm{C}(5)-\mathrm{H}(5 \mathrm{~A})$ & 0.9300 & $\mathrm{P}(1)-\mathrm{C}(55)$ & $1.785(6)$ \\
\hline $\mathrm{C}(6)-\mathrm{H}(6 \mathrm{~A})$ & 0.9300 & $\mathrm{P}(1)-\mathrm{C}(49)$ & $1.794(7)$ \\
\hline$C(7)-C(12)$ & $1.392(8)$ & $C(49)-C(50)$ & $1.342(8)$ \\
\hline$C(7)-C(8)$ & $1.398(8)$ & $\mathrm{C}(49)-\mathrm{C}(54)$ & $1.402(8)$ \\
\hline $\mathrm{C}(8)-\mathrm{C}(9)$ & $1.378(8)$ & $\mathrm{C}(50)-\mathrm{C}(51)$ & $1.377(9)$ \\
\hline $\mathrm{C}(8)-\mathrm{H}(8 \mathrm{~A})$ & 0.9300 & $\mathrm{C}(50)-\mathrm{H}(50 \mathrm{~A})$ & 0.9300 \\
\hline$C(9)-C(10)$ & $1.396(9)$ & $\mathrm{C}(51)-\mathrm{C}(52)$ & $1.355(9)$ \\
\hline $\mathrm{C}(9)-\mathrm{H}(9 \mathrm{~A})$ & 0.9300 & $\mathrm{C}(51)-\mathrm{H}(51 \mathrm{~A})$ & 0.9300 \\
\hline $\mathrm{C}(10)-\mathrm{C}(11)$ & $1.375(9)$ & $C(52)-C(53)$ & $1.392(10)$ \\
\hline $\mathrm{C}(10)-\mathrm{H}(10 \mathrm{~A})$ & 0.9300 & $\mathrm{C}(52)-\mathrm{H}(52 \mathrm{~A})$ & 0.9300 \\
\hline$C(11)-C(12)$ & $1.391(8)$ & $C(53)-C(54)$ & $1.398(8)$ \\
\hline $\mathrm{C}(11)-\mathrm{H}(11 \mathrm{~A})$ & 0.9300 & $\mathrm{C}(53)-\mathrm{H}(53 \mathrm{~A})$ & 0.9300 \\
\hline$C(13)-C(18)$ & $1.398(9)$ & $\mathrm{C}(54)-\mathrm{H}(54 \mathrm{~A})$ & 0.9300 \\
\hline$C(13)-C(14)$ & $1.401(8)$ & $\mathrm{C}(55)-\mathrm{C}(60)$ & $1.381(8)$ \\
\hline$C(14)-C(15)$ & $1.360(9)$ & $\mathrm{C}(55)-\mathrm{C}(56)$ & $1.394(8)$ \\
\hline $\mathrm{C}(14)-\mathrm{H}(14 \mathrm{~A})$ & 0.9300 & $\mathrm{C}(56)-\mathrm{C}(57)$ & $1.372(9)$ \\
\hline$C(15)-C(16)$ & $1.410(9)$ & $\mathrm{C}(56)-\mathrm{H}(56 \mathrm{~A})$ & 0.9300 \\
\hline $\mathrm{C}(15)-\mathrm{H}(15 \mathrm{~A})$ & 0.9300 & $\mathrm{C}(57)-\mathrm{C}(58)$ & $1.373(8)$ \\
\hline$C(16)-C(17)$ & $1.376(9)$ & $\mathrm{C}(57)-\mathrm{H}(57 \mathrm{~A})$ & 0.9300 \\
\hline $\mathrm{C}(16)-\mathrm{C}(19)$ & $1.534(10)$ & $\mathrm{C}(58)-\mathrm{C}(59)$ & $1.392(9)$ \\
\hline
\end{tabular}




\begin{tabular}{|c|c|c|c|}
\hline $\mathrm{C}(17)-\mathrm{C}(18)$ & $1.390(9)$ & $\mathrm{C}(58)-\mathrm{H}(58 \mathrm{~A})$ & 0.9300 \\
\hline $\mathrm{C}(17)-\mathrm{H}(17 \mathrm{~A})$ & 0.9300 & $C(59)-C(60)$ & $1.381(9)$ \\
\hline $\mathrm{C}(18)-\mathrm{H}(18 \mathrm{~A})$ & 0.9300 & $\mathrm{C}(59)-\mathrm{H}(59 \mathrm{~A})$ & 0.9300 \\
\hline$C(19)-C(22)$ & $1.393(14)$ & $\mathrm{C}(60)-\mathrm{H}(60 \mathrm{~A})$ & 0.9300 \\
\hline$C(19)-C(20)$ & $1.465(13)$ & $C(61)-C(62)$ & $1.363(9)$ \\
\hline $\mathrm{C}(19)-\mathrm{C}(21)$ & $1.468(16)$ & $\mathrm{C}(61)-\mathrm{C}(66)$ & $1.387(9)$ \\
\hline $\mathrm{C}(20)-\mathrm{H}(20 \mathrm{~A})$ & 0.9600 & $C(62)-C(63)$ & $1.384(9)$ \\
\hline $\mathrm{C}(20)-\mathrm{H}(20 \mathrm{~B})$ & 0.9600 & $\mathrm{C}(62)-\mathrm{H}(62 \mathrm{~A})$ & 0.9300 \\
\hline $\mathrm{C}(20)-\mathrm{H}(20 \mathrm{C})$ & 0.9600 & $\mathrm{C}(63)-\mathrm{C}(64)$ & $1.373(11)$ \\
\hline $\mathrm{C}(21)-\mathrm{H}(21 \mathrm{~A})$ & 0.9600 & $\mathrm{C}(63)-\mathrm{H}(63 \mathrm{~A})$ & 0.9300 \\
\hline $\mathrm{C}(21)-\mathrm{H}(21 \mathrm{~B})$ & 0.9600 & $\mathrm{C}(64)-\mathrm{C}(65)$ & $1.399(11)$ \\
\hline $\mathrm{C}(21)-\mathrm{H}(21 \mathrm{C})$ & 0.9600 & $\mathrm{C}(64)-\mathrm{H}(64 \mathrm{~A})$ & 0.9300 \\
\hline $\mathrm{C}(22)-\mathrm{H}(22 \mathrm{~A})$ & 0.9600 & $C(65)-C(66)$ & $1.400(9)$ \\
\hline $\mathrm{C}(22)-\mathrm{H}(22 \mathrm{~B})$ & 0.9600 & $\mathrm{C}(65)-\mathrm{H}(65 \mathrm{~A})$ & 0.9300 \\
\hline $\mathrm{C}(22)-\mathrm{H}(22 \mathrm{C})$ & 0.9600 & $\mathrm{C}(66)-\mathrm{H}(66 \mathrm{~A})$ & 0.9300 \\
\hline $\mathrm{C}(23)-\mathrm{C}(24)$ & $1.367(8)$ & $C(67)-C(72)$ & $1.371(9)$ \\
\hline $\mathrm{C}(23)-\mathrm{C}(28)$ & $1.421(9)$ & $\mathrm{C}(67)-\mathrm{C}(68)$ & $1.378(8)$ \\
\hline $\mathrm{C}(24)-\mathrm{C}(25)$ & $1.424(9)$ & $\mathrm{C}(68)-\mathrm{C}(69)$ & $1.378(9)$ \\
\hline $\mathrm{C}(24)-\mathrm{H}(24 \mathrm{~A})$ & 0.9300 & $\mathrm{C}(68)-\mathrm{H}(68 \mathrm{~A})$ & 0.9300 \\
\hline $\mathrm{C}(25)-\mathrm{C}(26)$ & $1.374(10)$ & $\mathrm{C}(69)-\mathrm{C}(70)$ & $1.367(10)$ \\
\hline $\mathrm{C}(25)-\mathrm{H}(25 \mathrm{~A})$ & 0.9300 & $\mathrm{C}(69)-\mathrm{H}(69 \mathrm{~A})$ & 0.9300 \\
\hline $\mathrm{C}(26)-\mathrm{C}(27)$ & $1.349(9)$ & $\mathrm{C}(70)-\mathrm{C}(71)$ & $1.393(10)$ \\
\hline
\end{tabular}




\begin{tabular}{|c|c|c|c|}
\hline $\mathrm{C}(26)-\mathrm{H}(26 \mathrm{~A})$ & 0.9300 & $\mathrm{C}(70)-\mathrm{H}(70 \mathrm{~A})$ & 0.9300 \\
\hline $\mathrm{C}(27)-\mathrm{C}(28)$ & $1.429(8)$ & $\mathrm{C}(71)-\mathrm{C}(72)$ & $1.395(9)$ \\
\hline $\mathrm{C}(27)-\mathrm{H}(27 \mathrm{~A})$ & 0.9300 & $\mathrm{C}(71)-\mathrm{H}(71 \mathrm{~A})$ & 0.9300 \\
\hline $\mathrm{C}(29)-\mathrm{C}(34)$ & $1.363(9)$ & $\mathrm{C}(72)-\mathrm{H}(72 \mathrm{~A})$ & 0.9300 \\
\hline $\mathrm{C}(29)-\mathrm{C}(30)$ & $1.393(8)$ & $\mathrm{N}(1 \mathrm{~S})-\mathrm{C}(2 \mathrm{~S})$ & $1.05(2)$ \\
\hline $\mathrm{C}(30)-\mathrm{C}(31)$ & $1.380(9)$ & $\mathrm{C}(1 \mathrm{~S})-\mathrm{C}(2 \mathrm{~S})$ & $1.28(2)$ \\
\hline $\mathrm{C}(30)-\mathrm{H}(30 \mathrm{~A})$ & 0.9300 & $\mathrm{C}(1 \mathrm{~S})-\mathrm{H}(1 \mathrm{SA})$ & 0.9600 \\
\hline$C(31)-C(32)$ & $1.345(9)$ & $\mathrm{C}(1 \mathrm{~S})-\mathrm{H}(1 \mathrm{SB})$ & 0.9600 \\
\hline $\mathrm{C}(31)-\mathrm{H}(31 \mathrm{~A})$ & 0.9300 & $\mathrm{C}(1 \mathrm{~S})-\mathrm{H}(1 \mathrm{SC})$ & 0.9600 \\
\hline $\mathrm{C}(32)-\mathrm{C}(33)$ & $1.391(9)$ & $\mathrm{N}(2 \mathrm{~S})-\mathrm{C}(4 \mathrm{~S})$ & $1.147(17)$ \\
\hline$C(32)-C(35)$ & $1.533(10)$ & $C(3 S)-C(4 S)$ & $1.322(17)$ \\
\hline $\mathrm{C}(33)-\mathrm{C}(34)$ & $1.389(9)$ & $\mathrm{C}(3 \mathrm{~S})-\mathrm{H}(3 \mathrm{SB})$ & 0.9600 \\
\hline $\mathrm{C}(33)-\mathrm{H}(33 \mathrm{~A})$ & 0.9300 & $\mathrm{C}(3 \mathrm{~S})-\mathrm{H}(3 \mathrm{SC})$ & 0.9600 \\
\hline $\mathrm{C}(34)-\mathrm{H}(34 \mathrm{~A})$ & 0.9300 & $\mathrm{C}(3 \mathrm{~S})-\mathrm{H}(3 \mathrm{SA})$ & 0.9600 \\
\hline $\mathrm{C}(35)-\mathrm{C}(36)$ & $1.503(11)$ & $\mathrm{O}(3 \mathrm{~S})-\mathrm{C}(7 \mathrm{~S})$ & $1.37(2)$ \\
\hline$C(35)-C(37)$ & $1.507(12)$ & $\mathrm{O}(3 \mathrm{~S})-\mathrm{C}(6 \mathrm{~S})$ & $1.55(2)$ \\
\hline $\mathrm{C}(35)-\mathrm{C}(38)$ & $1.529(10)$ & $\mathrm{C}(5 \mathrm{~S})-\mathrm{C}(6 \mathrm{~S})$ & $1.25(2)$ \\
\hline $\mathrm{C}(36)-\mathrm{H}(36 \mathrm{~A})$ & 0.9600 & $\mathrm{C}(5 \mathrm{~S})-\mathrm{H}(5 \mathrm{SA})$ & 0.9600 \\
\hline $\mathrm{C}(36)-\mathrm{H}(36 \mathrm{~B})$ & 0.9600 & $\mathrm{C}(5 \mathrm{~S})-\mathrm{H}(5 \mathrm{SB})$ & 0.9600 \\
\hline $\mathrm{C}(36)-\mathrm{H}(36 \mathrm{C})$ & 0.9600 & $\mathrm{C}(5 \mathrm{~S})-\mathrm{H}(5 \mathrm{SC})$ & 0.9600 \\
\hline $\mathrm{C}(37)-\mathrm{H}(37 \mathrm{~A})$ & 0.9600 & $\mathrm{C}(6 \mathrm{~S})-\mathrm{H}(6 \mathrm{SA})$ & 0.9700 \\
\hline $\mathrm{C}(37)-\mathrm{H}(37 \mathrm{~B})$ & 0.9600 & $\mathrm{C}(6 \mathrm{~S})-\mathrm{H}(6 \mathrm{SB})$ & 0.9700 \\
\hline
\end{tabular}




$\begin{array}{lclr}\mathrm{C}(37)-\mathrm{H}(37 \mathrm{C}) & 0.9600 & \mathrm{C}(7 \mathrm{~S})-\mathrm{C}(8 \mathrm{~S}) & 1.22(2) \\ \mathrm{C}(38)-\mathrm{H}(38 \mathrm{~A}) & 0.9600 & \mathrm{C}(7 \mathrm{~S})-\mathrm{H}(7 \mathrm{SA}) & 0.9701 \\ \mathrm{C}(38)-\mathrm{H}(38 \mathrm{~B}) & 0.9600 & \mathrm{C}(7 \mathrm{~S})-\mathrm{H}(7 \mathrm{SB}) & 0.9700 \\ \mathrm{C}(38)-\mathrm{H}(38 \mathrm{C}) & 0.9600 & \mathrm{C}(8 \mathrm{~S})-\mathrm{H}(8 \mathrm{SA}) & 0.9600 \\ \mathrm{C}(39)-\mathrm{C}(44) & 1.397(8) & \mathrm{C}(8 \mathrm{~S})-\mathrm{H}(8 \mathrm{SB}) & 0.9600 \\ \mathrm{C}(39)-\mathrm{C}(40) & 1.398(8) & \mathrm{C}(8 \mathrm{~S})-\mathrm{H}(8 \mathrm{SC}) & 0.9600\end{array}$


Table S21. Angles, ${ }^{\circ}$, for $\left[\left(\mathrm{L}^{1}\right) \mathrm{Fe}(\mathrm{III})-\mathrm{OH}\right]\left[\mathrm{PPh}_{4}\right] \cdot \mathrm{CH}_{3} \mathrm{CN} \cdot 0.5 \mathrm{Et}_{2} \mathrm{O}(\mathbf{2})$

\begin{tabular}{|c|c|c|c|}
\hline $\mathrm{O}(1)-\mathrm{Fe}(1)-\mathrm{N}(2)$ & $107.0(2)$ & $\mathrm{H}(38 \mathrm{~A})-\mathrm{C}(38)-\mathrm{H}(38 \mathrm{C})$ & 109.5 \\
\hline $\mathrm{O}(1)-\mathrm{Fe}(1)-\mathrm{N}(4)$ & $99.5(2)$ & $\mathrm{H}(38 \mathrm{~B})-\mathrm{C}(38)-\mathrm{H}(38 \mathrm{C})$ & 109.5 \\
\hline $\mathrm{N}(2)-\mathrm{Fe}(1)-\mathrm{N}(4)$ & $118.1(2)$ & $\mathrm{C}(44)-\mathrm{C}(39)-\mathrm{C}(40)$ & $116.7(6)$ \\
\hline $\mathrm{O}(1)-\mathrm{Fe}(1)-\mathrm{N}(3)$ & $103.2(2)$ & $\mathrm{C}(44)-\mathrm{C}(39)-\mathrm{N}(4)$ & $123.2(6)$ \\
\hline $\mathrm{N}(2)-\mathrm{Fe}(1)-\mathrm{N}(3)$ & $108.0(2)$ & $\mathrm{C}(40)-\mathrm{C}(39)-\mathrm{N}(4)$ & $120.0(5)$ \\
\hline $\mathrm{N}(4)-\mathrm{Fe}(1)-\mathrm{N}(3)$ & $118.9(2)$ & $\mathrm{C}(41)-\mathrm{C}(40)-\mathrm{C}(39)$ & $121.4(7)$ \\
\hline $\mathrm{O}(1)-\mathrm{Fe}(1)-\mathrm{N}(1)$ & $174.20(18)$ & $\mathrm{C}(41)-\mathrm{C}(40)-\mathrm{H}(40 \mathrm{~A})$ & 119.3 \\
\hline $\mathrm{N}(2)-\mathrm{Fe}(1)-\mathrm{N}(1)$ & $78.1(2)$ & $\mathrm{C}(39)-\mathrm{C}(40)-\mathrm{H}(40 \mathrm{~A})$ & 119.3 \\
\hline $\mathrm{N}(4)-\mathrm{Fe}(1)-\mathrm{N}(1)$ & $75.31(19)$ & $\mathrm{C}(40)-\mathrm{C}(41)-\mathrm{C}(42)$ & $122.9(7)$ \\
\hline $\mathrm{N}(3)-\mathrm{Fe}(1)-\mathrm{N}(1)$ & $77.51(19)$ & $\mathrm{C}(40)-\mathrm{C}(41)-\mathrm{H}(41 \mathrm{~A})$ & 118.6 \\
\hline $\mathrm{Fe}(1)-\mathrm{O}(1)-\mathrm{H}(1 \mathrm{~A})$ & 109.5 & $\mathrm{C}(42)-\mathrm{C}(41)-\mathrm{H}(41 \mathrm{~A})$ & 118.6 \\
\hline $\mathrm{C}(2)-\mathrm{N}(1)-\mathrm{C}(7)$ & $115.4(5)$ & $\mathrm{C}(43)-\mathrm{C}(42)-\mathrm{C}(41)$ & $116.1(7)$ \\
\hline $\mathrm{C}(2)-\mathrm{N}(1)-\mathrm{C}(23)$ & $113.0(5)$ & $\mathrm{C}(43)-\mathrm{C}(42)-\mathrm{C}(45)$ & $122.9(8)$ \\
\hline $\mathrm{C}(7)-\mathrm{N}(1)-\mathrm{C}(23)$ & $112.2(4)$ & $\mathrm{C}(41)-\mathrm{C}(42)-\mathrm{C}(45)$ & $120.9(8)$ \\
\hline $\mathrm{C}(2)-\mathrm{N}(1)-\mathrm{Fe}(1)$ & $106.4(3)$ & $C(42)-C(43)-C(44)$ & $122.0(7)$ \\
\hline $\mathrm{C}(7)-\mathrm{N}(1)-\mathrm{Fe}(1)$ & $105.2(4)$ & $\mathrm{C}(42)-\mathrm{C}(43)-\mathrm{H}(43 \mathrm{~A})$ & 119.0 \\
\hline $\mathrm{C}(23)-\mathrm{N}(1)-\mathrm{Fe}(1)$ & $103.4(4)$ & $\mathrm{C}(44)-\mathrm{C}(43)-\mathrm{H}(43 \mathrm{~A})$ & 119.0 \\
\hline $\mathrm{C}(28)-\mathrm{N}(2)-\mathrm{C}(29)$ & $118.0(5)$ & $C(39)-C(44)-C(43)$ & $120.8(7)$ \\
\hline $\mathrm{C}(28)-\mathrm{N}(2)-\mathrm{Fe}(1)$ & $116.2(4)$ & $\mathrm{C}(39)-\mathrm{C}(44)-\mathrm{H}(44 \mathrm{~A})$ & 119.6 \\
\hline $\mathrm{C}(29)-\mathrm{N}(2)-\mathrm{Fe}(1)$ & $125.7(4)$ & $\mathrm{C}(43)-\mathrm{C}(44)-\mathrm{H}(44 \mathrm{~A})$ & 119.6 \\
\hline $\mathrm{C}(12)-\mathrm{N}(3)-\mathrm{C}(13)$ & $118.4(5)$ & $\mathrm{C}(48)-\mathrm{C}(45)-\mathrm{C}(46)$ & $106.6(10)$ \\
\hline
\end{tabular}




\begin{tabular}{|c|c|c|c|}
\hline $\mathrm{C}(12)-\mathrm{N}(3)-\mathrm{Fe}(1)$ & $117.1(4)$ & $\mathrm{C}(48)-\mathrm{C}(45)-\mathrm{C}(47)$ & $106.0(11)$ \\
\hline $\mathrm{C}(13)-\mathrm{N}(3)-\mathrm{Fe}(1)$ & $118.9(4)$ & $C(46)-C(45)-C(47)$ & $111.7(10)$ \\
\hline $\mathrm{C}(1)-\mathrm{N}(4)-\mathrm{C}(39)$ & $118.2(5)$ & $\mathrm{C}(48)-\mathrm{C}(45)-\mathrm{C}(42)$ & $108.9(8)$ \\
\hline $\mathrm{C}(1)-\mathrm{N}(4)-\mathrm{Fe}(1)$ & $120.2(4)$ & $\mathrm{C}(46)-\mathrm{C}(45)-\mathrm{C}(42)$ & $109.6(8)$ \\
\hline $\mathrm{C}(39)-\mathrm{N}(4)-\mathrm{Fe}(1)$ & $119.5(4)$ & $\mathrm{C}(47)-\mathrm{C}(45)-\mathrm{C}(42)$ & $113.6(9)$ \\
\hline $\mathrm{N}(4)-\mathrm{C}(1)-\mathrm{C}(2)$ & $116.5(6)$ & $\mathrm{C}(45)-\mathrm{C}(46)-\mathrm{H}(46 \mathrm{~A})$ & 109.5 \\
\hline $\mathrm{N}(4)-\mathrm{C}(1)-\mathrm{C}(6)$ & $126.2(6)$ & $\mathrm{C}(45)-\mathrm{C}(46)-\mathrm{H}(46 \mathrm{~B})$ & 109.5 \\
\hline $\mathrm{C}(2)-\mathrm{C}(1)-\mathrm{C}(6)$ & $117.3(6)$ & $\mathrm{H}(46 \mathrm{~A})-\mathrm{C}(46)-\mathrm{H}(46 \mathrm{~B})$ & 109.5 \\
\hline $\mathrm{C}(3)-\mathrm{C}(2)-\mathrm{C}(1)$ & $121.4(6)$ & $\mathrm{C}(45)-\mathrm{C}(46)-\mathrm{H}(46 \mathrm{C})$ & 109.5 \\
\hline $\mathrm{C}(3)-\mathrm{C}(2)-\mathrm{N}(1)$ & $123.3(6)$ & $\mathrm{H}(46 \mathrm{~A})-\mathrm{C}(46)-\mathrm{H}(46 \mathrm{C})$ & 109.5 \\
\hline $\mathrm{C}(1)-\mathrm{C}(2)-\mathrm{N}(1)$ & $115.3(6)$ & $\mathrm{H}(46 \mathrm{~B})-\mathrm{C}(46)-\mathrm{H}(46 \mathrm{C})$ & 109.5 \\
\hline$C(2)-C(3)-C(4)$ & $120.9(6)$ & $\mathrm{C}(45)-\mathrm{C}(47)-\mathrm{H}(47 \mathrm{~A})$ & 109.5 \\
\hline $\mathrm{C}(2)-\mathrm{C}(3)-\mathrm{H}(3 \mathrm{~A})$ & 119.6 & $\mathrm{C}(45)-\mathrm{C}(47)-\mathrm{H}(47 \mathrm{~B})$ & 109.5 \\
\hline $\mathrm{C}(4)-\mathrm{C}(3)-\mathrm{H}(3 \mathrm{~A})$ & 119.6 & $\mathrm{H}(47 \mathrm{~A})-\mathrm{C}(47)-\mathrm{H}(47 \mathrm{~B})$ & 109.5 \\
\hline$C(3)-C(4)-C(5)$ & $117.0(6)$ & $\mathrm{C}(45)-\mathrm{C}(47)-\mathrm{H}(47 \mathrm{C})$ & 109.5 \\
\hline $\mathrm{C}(3)-\mathrm{C}(4)-\mathrm{H}(4 \mathrm{~A})$ & 121.5 & $\mathrm{H}(47 \mathrm{~A})-\mathrm{C}(47)-\mathrm{H}(47 \mathrm{C})$ & 109.5 \\
\hline $\mathrm{C}(5)-\mathrm{C}(4)-\mathrm{H}(4 \mathrm{~A})$ & 121.5 & $\mathrm{H}(47 \mathrm{~B})-\mathrm{C}(47)-\mathrm{H}(47 \mathrm{C})$ & 109.5 \\
\hline$C(6)-C(5)-C(4)$ & $123.0(6)$ & $\mathrm{C}(45)-\mathrm{C}(48)-\mathrm{H}(48 \mathrm{~A})$ & 109.5 \\
\hline $\mathrm{C}(6)-\mathrm{C}(5)-\mathrm{H}(5 \mathrm{~A})$ & 118.5 & $\mathrm{C}(45)-\mathrm{C}(48)-\mathrm{H}(48 \mathrm{~B})$ & 109.5 \\
\hline $\mathrm{C}(4)-\mathrm{C}(5)-\mathrm{H}(5 \mathrm{~A})$ & 118.5 & $\mathrm{H}(48 \mathrm{~A})-\mathrm{C}(48)-\mathrm{H}(48 \mathrm{~B})$ & 109.5 \\
\hline$C(5)-C(6)-C(1)$ & $120.3(6)$ & $\mathrm{C}(45)-\mathrm{C}(48)-\mathrm{H}(48 \mathrm{C})$ & 109.5 \\
\hline $\mathrm{C}(5)-\mathrm{C}(6)-\mathrm{H}(6 \mathrm{~A})$ & 119.9 & $\mathrm{H}(48 \mathrm{~A})-\mathrm{C}(48)-\mathrm{H}(48 \mathrm{C})$ & 109.5 \\
\hline
\end{tabular}




\begin{tabular}{|c|c|c|c|}
\hline $\mathrm{C}(1)-\mathrm{C}(6)-\mathrm{H}(6 \mathrm{~A})$ & 119.9 & $\mathrm{H}(48 \mathrm{~B})-\mathrm{C}(48)-\mathrm{H}(48 \mathrm{C})$ & 109.5 \\
\hline $\mathrm{C}(12)-\mathrm{C}(7)-\mathrm{C}(8)$ & $122.0(6)$ & $\mathrm{C}(61)-\mathrm{P}(1)-\mathrm{C}(67)$ & $110.1(3)$ \\
\hline $\mathrm{C}(12)-\mathrm{C}(7)-\mathrm{N}(1)$ & $116.1(6)$ & $\mathrm{C}(61)-\mathrm{P}(1)-\mathrm{C}(55)$ & $110.2(3)$ \\
\hline $\mathrm{C}(8)-\mathrm{C}(7)-\mathrm{N}(1)$ & $121.9(6)$ & $C(67)-P(1)-C(55)$ & $109.8(3)$ \\
\hline $\mathrm{C}(9)-\mathrm{C}(8)-\mathrm{C}(7)$ & $120.7(7)$ & $\mathrm{C}(61)-\mathrm{P}(1)-\mathrm{C}(49)$ & $110.3(3)$ \\
\hline $\mathrm{C}(9)-\mathrm{C}(8)-\mathrm{H}(8 \mathrm{~A})$ & 119.7 & $\mathrm{C}(67)-\mathrm{P}(1)-\mathrm{C}(49)$ & $109.2(3)$ \\
\hline $\mathrm{C}(7)-\mathrm{C}(8)-\mathrm{H}(8 \mathrm{~A})$ & 119.7 & $\mathrm{C}(55)-\mathrm{P}(1)-\mathrm{C}(49)$ & $107.1(3)$ \\
\hline$C(8)-C(9)-C(10)$ & $117.8(7)$ & $\mathrm{C}(50)-\mathrm{C}(49)-\mathrm{C}(54)$ & $120.5(6)$ \\
\hline $\mathrm{C}(8)-\mathrm{C}(9)-\mathrm{H}(9 \mathrm{~A})$ & 121.1 & $\mathrm{C}(50)-\mathrm{C}(49)-\mathrm{P}(1)$ & $121.9(6)$ \\
\hline $\mathrm{C}(10)-\mathrm{C}(9)-\mathrm{H}(9 \mathrm{~A})$ & 121.1 & $\mathrm{C}(54)-\mathrm{C}(49)-\mathrm{P}(1)$ & $117.3(5)$ \\
\hline $\mathrm{C}(11)-\mathrm{C}(10)-\mathrm{C}(9)$ & $120.9(6)$ & $\mathrm{C}(49)-\mathrm{C}(50)-\mathrm{C}(51)$ & $120.7(7)$ \\
\hline $\mathrm{C}(11)-\mathrm{C}(10)-\mathrm{H}(10 \mathrm{~A})$ & 119.5 & $\mathrm{C}(49)-\mathrm{C}(50)-\mathrm{H}(50 \mathrm{~A})$ & 119.6 \\
\hline $\mathrm{C}(9)-\mathrm{C}(10)-\mathrm{H}(10 \mathrm{~A})$ & 119.5 & $\mathrm{C}(51)-\mathrm{C}(50)-\mathrm{H}(50 \mathrm{~A})$ & 119.6 \\
\hline $\mathrm{C}(10)-\mathrm{C}(11)-\mathrm{C}(12)$ & $122.4(7)$ & $\mathrm{C}(52)-\mathrm{C}(51)-\mathrm{C}(50)$ & $120.3(8)$ \\
\hline $\mathrm{C}(10)-\mathrm{C}(11)-\mathrm{H}(11 \mathrm{~A})$ & 118.8 & $\mathrm{C}(52)-\mathrm{C}(51)-\mathrm{H}(51 \mathrm{~A})$ & 119.9 \\
\hline $\mathrm{C}(12)-\mathrm{C}(11)-\mathrm{H}(11 \mathrm{~A})$ & 118.8 & $\mathrm{C}(50)-\mathrm{C}(51)-\mathrm{H}(51 \mathrm{~A})$ & 119.9 \\
\hline $\mathrm{N}(3)-\mathrm{C}(12)-\mathrm{C}(11)$ & $125.4(6)$ & $\mathrm{C}(51)-\mathrm{C}(52)-\mathrm{C}(53)$ & $120.8(7)$ \\
\hline $\mathrm{N}(3)-\mathrm{C}(12)-\mathrm{C}(7)$ & $118.6(6)$ & $\mathrm{C}(51)-\mathrm{C}(52)-\mathrm{H}(52 \mathrm{~A})$ & 119.6 \\
\hline $\mathrm{C}(11)-\mathrm{C}(12)-\mathrm{C}(7)$ & $116.0(6)$ & $\mathrm{C}(53)-\mathrm{C}(52)-\mathrm{H}(52 \mathrm{~A})$ & 119.6 \\
\hline $\mathrm{C}(18)-\mathrm{C}(13)-\mathrm{C}(14)$ & $117.0(6)$ & $\mathrm{C}(52)-\mathrm{C}(53)-\mathrm{C}(54)$ & $118.7(7)$ \\
\hline $\mathrm{C}(18)-\mathrm{C}(13)-\mathrm{N}(3)$ & $122.2(6)$ & $\mathrm{C}(52)-\mathrm{C}(53)-\mathrm{H}(53 \mathrm{~A})$ & 120.6 \\
\hline $\mathrm{C}(14)-\mathrm{C}(13)-\mathrm{N}(3)$ & $120.7(6)$ & $\mathrm{C}(54)-\mathrm{C}(53)-\mathrm{H}(53 \mathrm{~A})$ & 120.6 \\
\hline
\end{tabular}




\begin{tabular}{|c|c|c|c|}
\hline$C(15)-C(14)-C(13)$ & $120.7(7)$ & $\mathrm{C}(53)-\mathrm{C}(54)-\mathrm{C}(49)$ & $119.0(7)$ \\
\hline $\mathrm{C}(15)-\mathrm{C}(14)-\mathrm{H}(14 \mathrm{~A})$ & 119.6 & $\mathrm{C}(53)-\mathrm{C}(54)-\mathrm{H}(54 \mathrm{~A})$ & 120.5 \\
\hline $\mathrm{C}(13)-\mathrm{C}(14)-\mathrm{H}(14 \mathrm{~A})$ & 119.6 & $\mathrm{C}(49)-\mathrm{C}(54)-\mathrm{H}(54 \mathrm{~A})$ & 120.5 \\
\hline$C(14)-C(15)-C(16)$ & $122.4(7)$ & $\mathrm{C}(60)-\mathrm{C}(55)-\mathrm{C}(56)$ & $118.6(6)$ \\
\hline $\mathrm{C}(14)-\mathrm{C}(15)-\mathrm{H}(15 \mathrm{~A})$ & 118.8 & $\mathrm{C}(60)-\mathrm{C}(55)-\mathrm{P}(1)$ & $119.8(5)$ \\
\hline $\mathrm{C}(16)-\mathrm{C}(15)-\mathrm{H}(15 \mathrm{~A})$ & 118.8 & $\mathrm{C}(56)-\mathrm{C}(55)-\mathrm{P}(1)$ & $121.7(5)$ \\
\hline$C(17)-C(16)-C(15)$ & $117.0(7)$ & $\mathrm{C}(57)-\mathrm{C}(56)-\mathrm{C}(55)$ & $121.5(6)$ \\
\hline$C(17)-C(16)-C(19)$ & $121.3(8)$ & $\mathrm{C}(57)-\mathrm{C}(56)-\mathrm{H}(56 \mathrm{~A})$ & 119.3 \\
\hline$C(15)-C(16)-C(19)$ & $121.6(7)$ & $\mathrm{C}(55)-\mathrm{C}(56)-\mathrm{H}(56 \mathrm{~A})$ & 119.3 \\
\hline$C(16)-C(17)-C(18)$ & $120.9(7)$ & $\mathrm{C}(56)-\mathrm{C}(57)-\mathrm{C}(58)$ & $119.3(7)$ \\
\hline $\mathrm{C}(16)-\mathrm{C}(17)-\mathrm{H}(17 \mathrm{~A})$ & 119.5 & $\mathrm{C}(56)-\mathrm{C}(57)-\mathrm{H}(57 \mathrm{~A})$ & 120.3 \\
\hline $\mathrm{C}(18)-\mathrm{C}(17)-\mathrm{H}(17 \mathrm{~A})$ & 119.5 & $\mathrm{C}(58)-\mathrm{C}(57)-\mathrm{H}(57 \mathrm{~A})$ & 120.3 \\
\hline$C(17)-C(18)-C(13)$ & $121.8(7)$ & $\mathrm{C}(57)-\mathrm{C}(58)-\mathrm{C}(59)$ & $120.4(6)$ \\
\hline $\mathrm{C}(17)-\mathrm{C}(18)-\mathrm{H}(18 \mathrm{~A})$ & 119.1 & $\mathrm{C}(57)-\mathrm{C}(58)-\mathrm{H}(58 \mathrm{~A})$ & 119.8 \\
\hline $\mathrm{C}(13)-\mathrm{C}(18)-\mathrm{H}(18 \mathrm{~A})$ & 119.1 & $\mathrm{C}(59)-\mathrm{C}(58)-\mathrm{H}(58 \mathrm{~A})$ & 119.8 \\
\hline$C(22)-C(19)-C(20)$ & $111.8(13)$ & $\mathrm{C}(60)-\mathrm{C}(59)-\mathrm{C}(58)$ & $119.7(6)$ \\
\hline $\mathrm{C}(22)-\mathrm{C}(19)-\mathrm{C}(21)$ & $106.4(13)$ & $\mathrm{C}(60)-\mathrm{C}(59)-\mathrm{H}(59 \mathrm{~A})$ & 120.2 \\
\hline$C(20)-C(19)-C(21)$ & $99.7(13)$ & $\mathrm{C}(58)-\mathrm{C}(59)-\mathrm{H}(59 \mathrm{~A})$ & 120.2 \\
\hline$C(22)-C(19)-C(16)$ & $113.6(9)$ & $\mathrm{C}(59)-\mathrm{C}(60)-\mathrm{C}(55)$ & $120.5(6)$ \\
\hline$C(20)-C(19)-C(16)$ & $112.5(8)$ & $\mathrm{C}(59)-\mathrm{C}(60)-\mathrm{H}(60 \mathrm{~A})$ & 119.7 \\
\hline$C(21)-C(19)-C(16)$ & $111.9(8)$ & $\mathrm{C}(55)-\mathrm{C}(60)-\mathrm{H}(60 \mathrm{~A})$ & 119.7 \\
\hline $\mathrm{C}(19)-\mathrm{C}(20)-\mathrm{H}(20 \mathrm{~A})$ & 109.5 & $\mathrm{C}(62)-\mathrm{C}(61)-\mathrm{C}(66)$ & $120.6(7)$ \\
\hline
\end{tabular}




\begin{tabular}{|c|c|c|c|}
\hline$C(19)-C(20)-H(20 B)$ & 109.5 & $\mathrm{C}(62)-\mathrm{C}(61)-\mathrm{P}(1)$ & $120.8(5)$ \\
\hline $\mathrm{H}(20 \mathrm{~A})-\mathrm{C}(20)-\mathrm{H}(20 \mathrm{~B})$ & 109.5 & $\mathrm{C}(66)-\mathrm{C}(61)-\mathrm{P}(1)$ & $118.6(5)$ \\
\hline $\mathrm{C}(19)-\mathrm{C}(20)-\mathrm{H}(20 \mathrm{C})$ & 109.5 & $\mathrm{C}(61)-\mathrm{C}(62)-\mathrm{C}(63)$ & $121.4(7)$ \\
\hline $\mathrm{H}(20 \mathrm{~A})-\mathrm{C}(20)-\mathrm{H}(20 \mathrm{C})$ & 109.5 & $\mathrm{C}(61)-\mathrm{C}(62)-\mathrm{H}(62 \mathrm{~A})$ & 119.3 \\
\hline $\mathrm{H}(20 \mathrm{~B})-\mathrm{C}(20)-\mathrm{H}(20 \mathrm{C})$ & 109.5 & $\mathrm{C}(63)-\mathrm{C}(62)-\mathrm{H}(62 \mathrm{~A})$ & 119.3 \\
\hline $\mathrm{C}(19)-\mathrm{C}(21)-\mathrm{H}(21 \mathrm{~A})$ & 109.5 & $\mathrm{C}(64)-\mathrm{C}(63)-\mathrm{C}(62)$ & $119.4(8)$ \\
\hline $\mathrm{C}(19)-\mathrm{C}(21)-\mathrm{H}(21 \mathrm{~B})$ & 109.5 & $\mathrm{C}(64)-\mathrm{C}(63)-\mathrm{H}(63 \mathrm{~A})$ & 120.3 \\
\hline $\mathrm{H}(21 \mathrm{~A})-\mathrm{C}(21)-\mathrm{H}(21 \mathrm{~B})$ & 109.5 & $\mathrm{C}(62)-\mathrm{C}(63)-\mathrm{H}(63 \mathrm{~A})$ & 120.3 \\
\hline $\mathrm{C}(19)-\mathrm{C}(21)-\mathrm{H}(21 \mathrm{C})$ & 109.5 & $\mathrm{C}(63)-\mathrm{C}(64)-\mathrm{C}(65)$ & $119.8(8)$ \\
\hline $\mathrm{H}(21 \mathrm{~A})-\mathrm{C}(21)-\mathrm{H}(21 \mathrm{C})$ & 109.5 & $\mathrm{C}(63)-\mathrm{C}(64)-\mathrm{H}(64 \mathrm{~A})$ & 120.1 \\
\hline $\mathrm{H}(21 \mathrm{~B})-\mathrm{C}(21)-\mathrm{H}(21 \mathrm{C})$ & 109.5 & $\mathrm{C}(65)-\mathrm{C}(64)-\mathrm{H}(64 \mathrm{~A})$ & 120.1 \\
\hline $\mathrm{C}(19)-\mathrm{C}(22)-\mathrm{H}(22 \mathrm{~A})$ & 109.5 & $\mathrm{C}(64)-\mathrm{C}(65)-\mathrm{C}(66)$ & $120.4(8)$ \\
\hline $\mathrm{C}(19)-\mathrm{C}(22)-\mathrm{H}(22 \mathrm{~B})$ & 109.5 & $\mathrm{C}(64)-\mathrm{C}(65)-\mathrm{H}(65 \mathrm{~A})$ & 119.8 \\
\hline $\mathrm{H}(22 \mathrm{~A})-\mathrm{C}(22)-\mathrm{H}(22 \mathrm{~B})$ & 109.5 & $\mathrm{C}(66)-\mathrm{C}(65)-\mathrm{H}(65 \mathrm{~A})$ & 119.8 \\
\hline $\mathrm{C}(19)-\mathrm{C}(22)-\mathrm{H}(22 \mathrm{C})$ & 109.5 & $\mathrm{C}(61)-\mathrm{C}(66)-\mathrm{C}(65)$ & $118.4(7)$ \\
\hline $\mathrm{H}(22 \mathrm{~A})-\mathrm{C}(22)-\mathrm{H}(22 \mathrm{C})$ & 109.5 & $\mathrm{C}(61)-\mathrm{C}(66)-\mathrm{H}(66 \mathrm{~A})$ & 120.8 \\
\hline $\mathrm{H}(22 \mathrm{~B})-\mathrm{C}(22)-\mathrm{H}(22 \mathrm{C})$ & 109.5 & $\mathrm{C}(65)-\mathrm{C}(66)-\mathrm{H}(66 \mathrm{~A})$ & 120.8 \\
\hline $\mathrm{C}(24)-\mathrm{C}(23)-\mathrm{C}(28)$ & $122.3(6)$ & $\mathrm{C}(72)-\mathrm{C}(67)-\mathrm{C}(68)$ & $120.0(6)$ \\
\hline $\mathrm{C}(24)-\mathrm{C}(23)-\mathrm{N}(1)$ & $123.2(6)$ & $\mathrm{C}(72)-\mathrm{C}(67)-\mathrm{P}(1)$ & $119.1(5)$ \\
\hline $\mathrm{C}(28)-\mathrm{C}(23)-\mathrm{N}(1)$ & $114.5(6)$ & $\mathrm{C}(68)-\mathrm{C}(67)-\mathrm{P}(1)$ & $120.8(6)$ \\
\hline $\mathrm{C}(23)-\mathrm{C}(24)-\mathrm{C}(25)$ & $119.1(7)$ & $\mathrm{C}(67)-\mathrm{C}(68)-\mathrm{C}(69)$ & $120.1(7)$ \\
\hline $\mathrm{C}(23)-\mathrm{C}(24)-\mathrm{H}(24 \mathrm{~A})$ & 120.4 & $\mathrm{C}(67)-\mathrm{C}(68)-\mathrm{H}(68 \mathrm{~A})$ & 119.9 \\
\hline
\end{tabular}




\begin{tabular}{|c|c|c|c|}
\hline $\mathrm{C}(25)-\mathrm{C}(24)-\mathrm{H}(24 \mathrm{~A})$ & 120.4 & $\mathrm{C}(69)-\mathrm{C}(68)-\mathrm{H}(68 \mathrm{~A})$ & 119.9 \\
\hline$C(26)-C(25)-C(24)$ & $119.6(7)$ & $\mathrm{C}(70)-\mathrm{C}(69)-\mathrm{C}(68)$ & $119.8(7)$ \\
\hline $\mathrm{C}(26)-\mathrm{C}(25)-\mathrm{H}(25 \mathrm{~A})$ & 120.2 & $\mathrm{C}(70)-\mathrm{C}(69)-\mathrm{H}(69 \mathrm{~A})$ & 120.1 \\
\hline $\mathrm{C}(24)-\mathrm{C}(25)-\mathrm{H}(25 \mathrm{~A})$ & 120.2 & $\mathrm{C}(68)-\mathrm{C}(69)-\mathrm{H}(69 \mathrm{~A})$ & 120.1 \\
\hline $\mathrm{C}(27)-\mathrm{C}(26)-\mathrm{C}(25)$ & $120.8(7)$ & $\mathrm{C}(69)-\mathrm{C}(70)-\mathrm{C}(71)$ & $121.3(8)$ \\
\hline $\mathrm{C}(27)-\mathrm{C}(26)-\mathrm{H}(26 \mathrm{~A})$ & 119.6 & $\mathrm{C}(69)-\mathrm{C}(70)-\mathrm{H}(70 \mathrm{~A})$ & 119.3 \\
\hline $\mathrm{C}(25)-\mathrm{C}(26)-\mathrm{H}(26 \mathrm{~A})$ & 119.6 & $\mathrm{C}(71)-\mathrm{C}(70)-\mathrm{H}(70 \mathrm{~A})$ & 119.3 \\
\hline $\mathrm{C}(26)-\mathrm{C}(27)-\mathrm{C}(28)$ & $122.7(7)$ & $\mathrm{C}(70)-\mathrm{C}(71)-\mathrm{C}(72)$ & $117.8(8)$ \\
\hline $\mathrm{C}(26)-\mathrm{C}(27)-\mathrm{H}(27 \mathrm{~A})$ & 118.7 & $\mathrm{C}(70)-\mathrm{C}(71)-\mathrm{H}(71 \mathrm{~A})$ & 121.1 \\
\hline $\mathrm{C}(28)-\mathrm{C}(27)-\mathrm{H}(27 \mathrm{~A})$ & 118.7 & $\mathrm{C}(72)-\mathrm{C}(71)-\mathrm{H}(71 \mathrm{~A})$ & 121.1 \\
\hline $\mathrm{N}(2)-\mathrm{C}(28)-\mathrm{C}(23)$ & $118.9(6)$ & $C(67)-C(72)-C(71)$ & $120.9(7)$ \\
\hline $\mathrm{N}(2)-\mathrm{C}(28)-\mathrm{C}(27)$ & $125.8(7)$ & $\mathrm{C}(67)-\mathrm{C}(72)-\mathrm{H}(72 \mathrm{~A})$ & 119.5 \\
\hline $\mathrm{C}(23)-\mathrm{C}(28)-\mathrm{C}(27)$ & $115.3(7)$ & $\mathrm{C}(71)-\mathrm{C}(72)-\mathrm{H}(72 \mathrm{~A})$ & 119.5 \\
\hline $\mathrm{C}(34)-\mathrm{C}(29)-\mathrm{C}(30)$ & $116.8(7)$ & $\mathrm{C}(2 \mathrm{~S})-\mathrm{C}(1 \mathrm{~S})-\mathrm{H}(1 \mathrm{SA})$ & 109.5 \\
\hline $\mathrm{C}(34)-\mathrm{C}(29)-\mathrm{N}(2)$ & $120.2(6)$ & $\mathrm{C}(2 \mathrm{~S})-\mathrm{C}(1 \mathrm{~S})-\mathrm{H}(1 \mathrm{SB})$ & 109.5 \\
\hline $\mathrm{C}(30)-\mathrm{C}(29)-\mathrm{N}(2)$ & $122.8(6)$ & $\mathrm{H}(1 \mathrm{SA})-\mathrm{C}(1 \mathrm{~S})-\mathrm{H}(1 \mathrm{SB})$ & 109.5 \\
\hline $\mathrm{C}(31)-\mathrm{C}(30)-\mathrm{C}(29)$ & $121.8(7)$ & $\mathrm{C}(2 \mathrm{~S})-\mathrm{C}(1 \mathrm{~S})-\mathrm{H}(1 \mathrm{SC})$ & 109.5 \\
\hline $\mathrm{C}(31)-\mathrm{C}(30)-\mathrm{H}(30 \mathrm{~A})$ & 119.1 & $\mathrm{H}(1 \mathrm{SA})-\mathrm{C}(1 \mathrm{~S})-\mathrm{H}(1 \mathrm{SC})$ & 109.5 \\
\hline $\mathrm{C}(29)-\mathrm{C}(30)-\mathrm{H}(30 \mathrm{~A})$ & 119.1 & $\mathrm{H}(1 \mathrm{SB})-\mathrm{C}(1 \mathrm{~S})-\mathrm{H}(1 \mathrm{SC})$ & 109.5 \\
\hline $\mathrm{C}(32)-\mathrm{C}(31)-\mathrm{C}(30)$ & $122.1(7)$ & $\mathrm{N}(1 \mathrm{~S})-\mathrm{C}(2 \mathrm{~S})-\mathrm{C}(1 \mathrm{~S})$ & $171(2)$ \\
\hline $\mathrm{C}(32)-\mathrm{C}(31)-\mathrm{H}(31 \mathrm{~A})$ & 118.9 & $\mathrm{C}(4 \mathrm{~S})-\mathrm{C}(3 \mathrm{~S})-\mathrm{H}(3 \mathrm{SB})$ & 107.2 \\
\hline $\mathrm{C}(30)-\mathrm{C}(31)-\mathrm{H}(31 \mathrm{~A})$ & 118.9 & $\mathrm{C}(4 \mathrm{~S})-\mathrm{C}(3 \mathrm{~S})-\mathrm{H}(3 \mathrm{SC})$ & 107.4 \\
\hline
\end{tabular}




\begin{tabular}{|c|c|c|c|}
\hline $\mathrm{C}(31)-\mathrm{C}(32)-\mathrm{C}(33)$ & $116.2(7)$ & $\mathrm{H}(3 \mathrm{SB})-\mathrm{C}(3 \mathrm{~S})-\mathrm{H}(3 \mathrm{SC})$ & 106.9 \\
\hline $\mathrm{C}(31)-\mathrm{C}(32)-\mathrm{C}(35)$ & $120.4(7)$ & $\mathrm{C}(4 \mathrm{~S})-\mathrm{C}(3 \mathrm{~S})-\mathrm{H}(3 \mathrm{SA})$ & 120.1 \\
\hline$C(33)-C(32)-C(35)$ & $123.4(7)$ & $\mathrm{H}(3 \mathrm{SB})-\mathrm{C}(3 \mathrm{~S})-\mathrm{H}(3 \mathrm{SA})$ & 107.3 \\
\hline $\mathrm{C}(34)-\mathrm{C}(33)-\mathrm{C}(32)$ & $122.7(7)$ & $\mathrm{H}(3 \mathrm{SC})-\mathrm{C}(3 \mathrm{~S})-\mathrm{H}(3 \mathrm{SA})$ & 107.3 \\
\hline $\mathrm{C}(34)-\mathrm{C}(33)-\mathrm{H}(33 \mathrm{~A})$ & 118.7 & $\mathrm{~N}(2 \mathrm{~S})-\mathrm{C}(4 \mathrm{~S})-\mathrm{C}(3 \mathrm{~S})$ & $176.5(16)$ \\
\hline $\mathrm{C}(32)-\mathrm{C}(33)-\mathrm{H}(33 \mathrm{~A})$ & 118.7 & $\mathrm{C}(7 \mathrm{~S})-\mathrm{O}(3 \mathrm{~S})-\mathrm{C}(6 \mathrm{~S})$ & $166(2)$ \\
\hline $\mathrm{C}(29)-\mathrm{C}(34)-\mathrm{C}(33)$ & $120.4(7)$ & $\mathrm{C}(6 \mathrm{~S})-\mathrm{C}(5 \mathrm{~S})-\mathrm{H}(5 \mathrm{SA})$ & 109.5 \\
\hline $\mathrm{C}(29)-\mathrm{C}(34)-\mathrm{H}(34 \mathrm{~A})$ & 119.8 & $\mathrm{C}(6 \mathrm{~S})-\mathrm{C}(5 \mathrm{~S})-\mathrm{H}(5 \mathrm{SB})$ & 109.5 \\
\hline $\mathrm{C}(33)-\mathrm{C}(34)-\mathrm{H}(34 \mathrm{~A})$ & 119.8 & $\mathrm{H}(5 \mathrm{SA})-\mathrm{C}(5 \mathrm{~S})-\mathrm{H}(5 \mathrm{SB})$ & 109.5 \\
\hline $\mathrm{C}(36)-\mathrm{C}(35)-\mathrm{C}(37)$ & $106.2(9)$ & $\mathrm{C}(6 \mathrm{~S})-\mathrm{C}(5 \mathrm{~S})-\mathrm{H}(5 \mathrm{SC})$ & 109.5 \\
\hline$C(36)-C(35)-C(38)$ & $110.5(8)$ & $\mathrm{H}(5 \mathrm{SA})-\mathrm{C}(5 \mathrm{~S})-\mathrm{H}(5 \mathrm{SC})$ & 109.5 \\
\hline $\mathrm{C}(37)-\mathrm{C}(35)-\mathrm{C}(38)$ & $106.5(8)$ & $\mathrm{H}(5 \mathrm{SB})-\mathrm{C}(5 \mathrm{~S})-\mathrm{H}(5 \mathrm{SC})$ & 109.5 \\
\hline$C(36)-C(35)-C(32)$ & $110.7(8)$ & $\mathrm{C}(5 \mathrm{~S})-\mathrm{C}(6 \mathrm{~S})-\mathrm{O}(3 \mathrm{~S})$ & $158(3)$ \\
\hline $\mathrm{C}(37)-\mathrm{C}(35)-\mathrm{C}(32)$ & $109.9(7)$ & $\mathrm{C}(5 \mathrm{~S})-\mathrm{C}(6 \mathrm{~S})-\mathrm{H}(6 \mathrm{SA})$ & 96.9 \\
\hline $\mathrm{C}(38)-\mathrm{C}(35)-\mathrm{C}(32)$ & $112.7(7)$ & $\mathrm{O}(3 \mathrm{~S})-\mathrm{C}(6 \mathrm{~S})-\mathrm{H}(6 \mathrm{SA})$ & 96.9 \\
\hline $\mathrm{C}(35)-\mathrm{C}(36)-\mathrm{H}(36 \mathrm{~A})$ & 109.5 & $\mathrm{C}(5 \mathrm{~S})-\mathrm{C}(6 \mathrm{~S})-\mathrm{H}(6 \mathrm{SB})$ & 96.9 \\
\hline $\mathrm{C}(35)-\mathrm{C}(36)-\mathrm{H}(36 \mathrm{~B})$ & 109.5 & $\mathrm{O}(3 \mathrm{~S})-\mathrm{C}(6 \mathrm{~S})-\mathrm{H}(6 \mathrm{SB})$ & 96.9 \\
\hline $\mathrm{H}(36 \mathrm{~A})-\mathrm{C}(36)-\mathrm{H}(36 \mathrm{~B})$ & 109.5 & $\mathrm{H}(6 \mathrm{SA})-\mathrm{C}(6 \mathrm{~S})-\mathrm{H}(6 \mathrm{SB})$ & 103.5 \\
\hline $\mathrm{C}(35)-\mathrm{C}(36)-\mathrm{H}(36 \mathrm{C})$ & 109.5 & $\mathrm{C}(8 \mathrm{~S})-\mathrm{C}(7 \mathrm{~S})-\mathrm{O}(3 \mathrm{~S})$ & $165(3)$ \\
\hline $\mathrm{H}(36 \mathrm{~A})-\mathrm{C}(36)-\mathrm{H}(36 \mathrm{C})$ & 109.5 & $\mathrm{C}(8 \mathrm{~S})-\mathrm{C}(7 \mathrm{~S})-\mathrm{H}(7 \mathrm{SA})$ & 94.3 \\
\hline $\mathrm{H}(36 \mathrm{~B})-\mathrm{C}(36)-\mathrm{H}(36 \mathrm{C})$ & 109.5 & $\mathrm{O}(3 \mathrm{~S})-\mathrm{C}(7 \mathrm{~S})-\mathrm{H}(7 \mathrm{SA})$ & 94.2 \\
\hline $\mathrm{C}(35)-\mathrm{C}(37)-\mathrm{H}(37 \mathrm{~A})$ & 109.5 & $\mathrm{C}(8 \mathrm{~S})-\mathrm{C}(7 \mathrm{~S})-\mathrm{H}(7 \mathrm{SB})$ & 94. \\
\hline
\end{tabular}




$\begin{array}{lclr}\text { C(35)-C(37)-H(37B) } & 109.5 & \mathrm{O}(3 \mathrm{~S})-\mathrm{C}(7 \mathrm{~S})-\mathrm{H}(7 \mathrm{SB}) & 94.7 \\ \mathrm{H}(37 \mathrm{~A})-\mathrm{C}(37)-\mathrm{H}(37 \mathrm{~B}) & 109.5 & \mathrm{H}(7 \mathrm{SA})-\mathrm{C}(7 \mathrm{~S})-\mathrm{H}(7 \mathrm{SB}) & 103.1 \\ \mathrm{C}(35)-\mathrm{C}(37)-\mathrm{H}(37 \mathrm{C}) & 109.5 & \mathrm{C}(7 \mathrm{~S})-\mathrm{C}(8 \mathrm{~S})-\mathrm{H}(8 \mathrm{SA}) & 109.5 \\ \mathrm{H}(37 \mathrm{~A})-\mathrm{C}(37)-\mathrm{H}(37 \mathrm{C}) & 109.5 & \mathrm{C}(7 \mathrm{~S})-\mathrm{C}(8 \mathrm{~S})-\mathrm{H}(8 \mathrm{SB}) & 109.5 \\ \mathrm{H}(37 \mathrm{~B})-\mathrm{C}(37)-\mathrm{H}(37 \mathrm{C}) & 109.5 & \mathrm{H}(8 \mathrm{SA})-\mathrm{C}(8 \mathrm{~S})-\mathrm{H}(8 \mathrm{SB}) & 109.5 \\ \mathrm{C}(35)-\mathrm{C}(38)-\mathrm{H}(38 \mathrm{~A}) & 109.5 & \mathrm{C}(7 \mathrm{~S})-\mathrm{C}(8 \mathrm{~S})-\mathrm{H}(8 \mathrm{SC}) & 109.5 \\ \mathrm{C}(35)-\mathrm{C}(38)-\mathrm{H}(38 \mathrm{~B}) & 109.5 & \mathrm{H}(8 \mathrm{SA})-\mathrm{C}(8 \mathrm{~S})-\mathrm{H}(8 \mathrm{SC}) & 109.5 \\ \mathrm{H}(38 \mathrm{~A})-\mathrm{C}(38)-\mathrm{H}(38 B) & 109.5 & \mathrm{H}(8 \mathrm{SB})-\mathrm{C}(8 \mathrm{~S})-\mathrm{H}(8 \mathrm{SC}) & \\ \mathrm{C}(35)-\mathrm{C}(38)-\mathrm{H}(38 \mathrm{C}) & 109.5 & \end{array}$


Table S22. Anisotropic displacement parameters $\left(\AA^{2} \times 10^{3}\right)$ for $\left[\left(\mathrm{L}^{1}\right) \mathrm{Fe}(\mathrm{III})-\mathrm{OH}\right]\left[\mathrm{PPh}_{4}\right] \cdot \mathrm{CH}_{3} \mathrm{CN} \cdot 0.5 \mathrm{Et}_{2} \mathrm{O} \quad$ (2). The anisotropic displacement factor exponent takes the form: $-2 \pi^{2}\left[h^{2} a^{* 2} U^{11}+\ldots+2 h k a^{*} b^{*} U^{12}\right]$

\begin{tabular}{|c|c|c|c|c|c|c|}
\hline & $\mathrm{U}^{11}$ & $\mathrm{U}^{22}$ & $\mathrm{U}^{33}$ & $\mathrm{U}^{23}$ & $\mathrm{U}^{13}$ & $\mathrm{U}^{12}$ \\
\hline $\mathrm{Fe}(1)$ & $37(1)$ & $39(1)$ & $49(1)$ & $3(1)$ & $10(1)$ & $-4(1)$ \\
\hline $\mathrm{O}(1)$ & $51(3)$ & $52(3)$ & 61(3) & $-3(3)$ & $2(2)$ & $-15(3)$ \\
\hline $\mathrm{N}(1)$ & $27(3)$ & $30(3)$ & $47(3)$ & $8(3)$ & $7(2)$ & $-1(2)$ \\
\hline $\mathrm{N}(2)$ & $39(3)$ & $47(4)$ & $46(4)$ & $3(3)$ & $4(3)$ & $-4(3)$ \\
\hline $\mathrm{N}(3)$ & $34(3)$ & $37(3)$ & $45(4)$ & $-4(3)$ & $14(3)$ & $-6(3)$ \\
\hline $\mathrm{N}(4)$ & $33(3)$ & $36(3)$ & $46(4)$ & $7(3)$ & $13(3)$ & $-3(3)$ \\
\hline $\mathrm{C}(1)$ & $36(4)$ & $26(4)$ & $43(4)$ & $5(3)$ & $10(3)$ & $4(3)$ \\
\hline $\mathrm{C}(2)$ & $37(4)$ & $25(4)$ & $43(5)$ & $-3(3)$ & $4(3)$ & $7(3)$ \\
\hline$C(3)$ & $47(4)$ & $35(4)$ & $50(5)$ & $-3(3)$ & $14(4)$ & $-3(4)$ \\
\hline$C(4)$ & $41(4)$ & $48(5)$ & $53(5)$ & $-6(4)$ & $-3(4)$ & $-6(4)$ \\
\hline$C(5)$ & $37(4)$ & $44(4)$ & $60(5)$ & $-5(4)$ & $10(4)$ & $-3(4)$ \\
\hline$C(6)$ & $37(4)$ & $30(4)$ & $54(5)$ & $-2(3)$ & 11(4) & $-1(3)$ \\
\hline$C(7)$ & $30(4)$ & $36(4)$ & $49(5)$ & $4(3)$ & $9(3)$ & 1(3) \\
\hline$C(8)$ & $38(4)$ & $39(4)$ & $52(5)$ & $5(4)$ & 11(3) & $-2(3)$ \\
\hline $\mathrm{C}(9)$ & $48(4)$ & $47(5)$ & $54(5)$ & $10(4)$ & $12(4)$ & $-1(4)$ \\
\hline $\mathrm{C}(10)$ & $55(5)$ & $31(4)$ & $71(6)$ & $13(4)$ & $6(4)$ & $-2(4)$ \\
\hline $\mathrm{C}(11)$ & $55(5)$ & $30(4)$ & $59(5)$ & $12(4)$ & $16(4)$ & $9(4)$ \\
\hline
\end{tabular}




\begin{tabular}{|c|c|c|c|c|c|c|}
\hline$C(12)$ & $35(4)$ & $35(4)$ & $45(4)$ & $-4(3)$ & $10(3)$ & $-2(3)$ \\
\hline$C(13)$ & $48(4)$ & $31(4)$ & $50(5)$ & $0(3)$ & $7(4)$ & $2(4)$ \\
\hline$C(14)$ & $45(4)$ & $45(5)$ & $61(5)$ & $-5(4)$ & $20(4)$ & $-2(4)$ \\
\hline$C(15)$ & $62(5)$ & $59(5)$ & $54(5)$ & $-8(4)$ & $26(4)$ & $4(4)$ \\
\hline$C(16)$ & $82(6)$ & $46(5)$ & $46(5)$ & $-1(4)$ & $15(4)$ & $11(5)$ \\
\hline$C(17)$ & $74(5)$ & $47(5)$ & $67(6)$ & $-22(4)$ & $12(4)$ & $-10(5)$ \\
\hline$C(18)$ & $46(4)$ & $52(5)$ & $76(6)$ & $-10(4)$ & $24(4)$ & $-4(4)$ \\
\hline$C(19)$ & $106(7)$ & $49(5)$ & $89(7)$ & $-22(5)$ & $23(6)$ & $7(6)$ \\
\hline$C(20)$ & $670(40)$ & $118(11)$ & $69(9)$ & $-30(8)$ & $120(16)$ & $54(18)$ \\
\hline$C(21)$ & $410(30)$ & $199(18)$ & $570(40)$ & $-280(20)$ & $350(30)$ & $-200(20)$ \\
\hline$C(22)$ & $360(30)$ & $250(20)$ & $350(30)$ & $-232(19)$ & $-160(20)$ & $240(20)$ \\
\hline $\mathrm{C}(23)$ & $38(4)$ & $27(4)$ & $55(5)$ & $5(3)$ & $14(4)$ & $5(3)$ \\
\hline$C(24)$ & $48(4)$ & $39(4)$ & $60(5)$ & $-4(4)$ & $16(4)$ & $-7(4)$ \\
\hline$C(25)$ & $67(6)$ & $33(4)$ & $90(7)$ & $-1(4)$ & $40(5)$ & $14(4)$ \\
\hline$C(26)$ & $47(5)$ & $36(5)$ & $100(7)$ & $9(5)$ & $23(5)$ & $10(4)$ \\
\hline$C(27)$ & $30(4)$ & $37(4)$ & $80(6)$ & $14(4)$ & $8(4)$ & $0(3)$ \\
\hline$C(28)$ & $41(4)$ & $35(4)$ & $47(5)$ & $15(4)$ & $7(4)$ & $-7(4)$ \\
\hline$C(29)$ & $38(4)$ & $52(4)$ & $51(5)$ & $13(4)$ & $3(4)$ & $-5(4)$ \\
\hline$C(30)$ & $54(5)$ & $59(5)$ & $46(5)$ & $14(4)$ & $11(4)$ & $-8(4)$ \\
\hline $\mathrm{C}(31)$ & $49(5)$ & $70(6)$ & $48(5)$ & $13(4)$ & $1(4)$ & $-23(4)$ \\
\hline$C(32)$ & $49(5)$ & $78(6)$ & $46(5)$ & $14(4)$ & $3(4)$ & $-15(4)$ \\
\hline$C(33)$ & $65(5)$ & $117(8)$ & $53(6)$ & $22(5)$ & $2(4)$ & $-24(6)$ \\
\hline
\end{tabular}




\begin{tabular}{|c|c|c|c|c|c|c|}
\hline$C(34)$ & $56(5)$ & $102(7)$ & $53(5)$ & $27(5)$ & $-2(4)$ & $-24(5)$ \\
\hline$C(35)$ & $59(6)$ & 101(8) & $63(6)$ & $15(5)$ & $-2(4)$ & $-21(5)$ \\
\hline$C(36)$ & $52(6)$ & $241(15)$ & 119(9) & $-44(9)$ & $-13(6)$ & $7(8)$ \\
\hline$C(37)$ & 133(9) & $123(10)$ & $99(8)$ & $-10(7)$ & $-14(7)$ & $-44(8)$ \\
\hline $\mathrm{C}(38)$ & $93(7)$ & $142(9)$ & $66(7)$ & $-6(6)$ & $-12(5)$ & $-8(7)$ \\
\hline C(39) & $36(4)$ & $39(4)$ & $45(4)$ & $1(3)$ & $12(3)$ & $0(3)$ \\
\hline$C(40)$ & $49(4)$ & $45(4)$ & $54(5)$ & $7(4)$ & $22(4)$ & $-1(4)$ \\
\hline$C(41)$ & $76(6)$ & $56(5)$ & $55(5)$ & $11(4)$ & $14(4)$ & $4(5)$ \\
\hline$C(42)$ & $65(5)$ & $69(6)$ & $45(5)$ & $-2(4)$ & $22(4)$ & $-23(5)$ \\
\hline$C(43)$ & $48(5)$ & $91(7)$ & $70(6)$ & $-3(5)$ & $27(4)$ & $-16(5)$ \\
\hline$C(44)$ & $38(4)$ & $66(5)$ & $68(6)$ & $5(4)$ & $15(4)$ & $6(4)$ \\
\hline$C(45)$ & $102(8)$ & 115(9) & $63(7)$ & $-5(6)$ & $35(6)$ & $-58(7)$ \\
\hline$C(46)$ & $135(10)$ & $268(17)$ & $116(10)$ & 41(10) & $39(8)$ & $-119(11)$ \\
\hline$C(47)$ & $244(16)$ & $194(15)$ & $232(17)$ & $160(14)$ & $154(14)$ & $62(13)$ \\
\hline$C(48)$ & $288(18)$ & $282(19)$ & $67(8)$ & $-44(10)$ & $89(10)$ & $-173(16)$ \\
\hline $\mathrm{P}(1)$ & $39(1)$ & $32(1)$ & $50(1)$ & $1(1)$ & $8(1)$ & $1(1)$ \\
\hline C(49) & $39(4)$ & $24(4)$ & $63(5)$ & $1(4)$ & $13(4)$ & $-2(3)$ \\
\hline$C(50)$ & $44(4)$ & $47(5)$ & $55(5)$ & $-4(4)$ & $18(4)$ & $0(4)$ \\
\hline $\mathrm{C}(51)$ & $67(6)$ & $45(5)$ & $65(6)$ & $0(4)$ & $22(5)$ & $-2(4)$ \\
\hline$C(52)$ & $51(5)$ & $38(5)$ & $93(7)$ & $4(5)$ & $30(5)$ & $1(4)$ \\
\hline$C(53)$ & $45(5)$ & $39(5)$ & $90(7)$ & $15(4)$ & $7(4)$ & $1(4)$ \\
\hline$C(54)$ & $43(4)$ & $34(4)$ & $61(5)$ & $-1(4)$ & $14(4)$ & $2(4)$ \\
\hline
\end{tabular}




\begin{tabular}{|c|c|c|c|c|c|c|}
\hline$C(55)$ & $33(4)$ & $28(4)$ & $66(5)$ & $-4(3)$ & $11(3)$ & $0(3)$ \\
\hline$C(56)$ & $38(4)$ & $42(5)$ & $66(5)$ & $-4(4)$ & $8(4)$ & $1(4)$ \\
\hline$C(57)$ & $39(4)$ & $36(4)$ & $75(6)$ & $-3(4)$ & $14(4)$ & $3(4)$ \\
\hline$C(58)$ & $62(5)$ & $27(4)$ & $74(6)$ & $-1(4)$ & $27(4)$ & $4(4)$ \\
\hline$C(59)$ & $36(4)$ & $41(4)$ & $95(6)$ & $1(4)$ & $17(4)$ & $-9(4)$ \\
\hline$C(60)$ & $33(4)$ & $37(4)$ & $82(6)$ & $1(4)$ & $5(4)$ & $-1(4)$ \\
\hline $\mathrm{C}(61)$ & $44(4)$ & $36(4)$ & $51(5)$ & $4(4)$ & $17(3)$ & $10(4)$ \\
\hline$C(62)$ & $46(4)$ & $54(5)$ & $56(5)$ & $3(4)$ & $9(4)$ & $9(4)$ \\
\hline$C(63)$ & $73(5)$ & $77(6)$ & $60(6)$ & $-7(5)$ & $19(4)$ & $19(5)$ \\
\hline$C(64)$ & $72(6)$ & $112(8)$ & $50(6)$ & $10(6)$ & $21(4)$ & $9(6)$ \\
\hline$C(65)$ & $64(5)$ & $62(6)$ & $91(7)$ & $29(5)$ & $21(5)$ & $8(5)$ \\
\hline$C(66)$ & $54(5)$ & $58(5)$ & $58(5)$ & $3(4)$ & $22(4)$ & $1(4)$ \\
\hline$C(67)$ & $38(4)$ & $28(4)$ & $50(5)$ & $0(3)$ & $4(3)$ & $0(3)$ \\
\hline $\mathrm{C}(68)$ & $45(4)$ & $44(4)$ & $58(5)$ & $-9(4)$ & $9(4)$ & $2(4)$ \\
\hline$C(69)$ & $51(5)$ & $51(5)$ & $90(7)$ & $-12(5)$ & $28(5)$ & $-9(4)$ \\
\hline$C(70)$ & $34(4)$ & $63(6)$ & $109(8)$ & $-35(6)$ & $4(5)$ & $-1(4)$ \\
\hline$C(71)$ & $67(6)$ & $59(5)$ & $66(6)$ & $-2(5)$ & $-5(5)$ & $10(5)$ \\
\hline $\mathrm{C}(72)$ & $44(4)$ & $41(4)$ & $73(6)$ & $-11(4)$ & $-3(4)$ & $-6(4)$ \\
\hline
\end{tabular}


Table S23. Hydrogen coordinates $\left(\times 10^{4}\right)$ and isotropic displacement parameters $\left(\AA^{2} \times 10^{3}\right)$ for $\left[\left(\mathrm{L}^{1}\right) \mathrm{Fe}(\mathrm{III})-\mathrm{OH}\right]\left[\mathrm{PPh}_{4}\right] \cdot \mathrm{CH}_{3} \mathrm{CN} \cdot 0.5 \mathrm{Et}_{2} \mathrm{O}(2)$

\begin{tabular}{|c|c|c|c|c|}
\hline & $\mathrm{x}$ & $\mathrm{y}$ & $\mathrm{z}$ & $\overline{\mathrm{U}(\mathrm{eq})}$ \\
\hline $\mathrm{H}(1 \mathrm{~A})$ & 1150 & 6713 & 9089 & 83 \\
\hline $\mathrm{H}(3 \mathrm{~A})$ & -491 & 6258 & 6596 & 52 \\
\hline $\mathrm{H}(4 \mathrm{~A})$ & -2348 & 5754 & 6328 & 58 \\
\hline $\mathrm{H}(5 \mathrm{~A})$ & -3286 & 5256 & 6888 & 56 \\
\hline $\mathrm{H}(6 \mathrm{~A})$ & -2504 & 5276 & 7672 & 48 \\
\hline $\mathrm{H}(8 \mathrm{~A})$ & 1544 & 6708 & 6672 & 51 \\
\hline $\mathrm{H}(9 \mathrm{~A})$ & 1678 & 7600 & 6372 & 59 \\
\hline $\mathrm{H}(10 \mathrm{~A})$ & 1024 & 8344 & 6807 & 63 \\
\hline $\mathrm{H}(11 \mathrm{~A})$ & 423 & 8203 & 7537 & 57 \\
\hline $\mathrm{H}(14 \mathrm{~A})$ & -1601 & 7532 & 8466 & 59 \\
\hline $\mathrm{H}(15 \mathrm{~A})$ & -1764 & 8243 & 8980 & 68 \\
\hline $\mathrm{H}(17 \mathrm{~A})$ & 1754 & 8765 & 8851 & 75 \\
\hline $\mathrm{H}(18 \mathrm{~A})$ & 1941 & 8040 & 8335 & 68 \\
\hline $\mathrm{H}(20 \mathrm{~A})$ & -136 & 9238 & 9992 & 421 \\
\hline $\mathrm{H}(20 \mathrm{~B})$ & 818 & 8771 & 9887 & 421 \\
\hline $\mathrm{H}(20 \mathrm{C})$ & -649 & 8644 & 9837 & 421 \\
\hline $\mathrm{H}(21 \mathrm{~A})$ & 755 & 9789 & 9525 & 548 \\
\hline $\mathrm{H}(21 \mathrm{~B})$ & 920 & 9631 & 9002 & 548 \\
\hline $\mathrm{H}(21 \mathrm{C})$ & 1670 & 9302 & 9432 & 548 \\
\hline
\end{tabular}




\begin{tabular}{|c|c|c|c|c|}
\hline $\mathrm{H}(22 \mathrm{~A})$ & -1319 & 9702 & 9389 & 508 \\
\hline $\mathrm{H}(22 \mathrm{~B})$ & -2026 & 9165 & 9174 & 508 \\
\hline $\mathrm{H}(22 \mathrm{C})$ & -1312 & 9548 & 8852 & 508 \\
\hline $\mathrm{H}(24 \mathrm{~A})$ & 1476 & 5642 & 6847 & 58 \\
\hline $\mathrm{H}(25 \mathrm{~A})$ & 3481 & 5208 & 6848 & 73 \\
\hline $\mathrm{H}(26 \mathrm{~A})$ & 5066 & 5327 & 7490 & 71 \\
\hline $\mathrm{H}(27 \mathrm{~A})$ & 4723 & 5843 & 8123 & 59 \\
\hline $\mathrm{H}(30 \mathrm{~A})$ & 4679 & 6950 & 8243 & 64 \\
\hline $\mathrm{H}(31 \mathrm{~A})$ & 6232 & 7223 & 8845 & 68 \\
\hline $\mathrm{H}(33 \mathrm{~A})$ & 4402 & 6465 & 9776 & 95 \\
\hline $\mathrm{H}(34 \mathrm{~A})$ & 2826 & 6184 & 9177 & 86 \\
\hline $\mathrm{H}(36 \mathrm{~A})$ & 7996 & 6992 & 9396 & 209 \\
\hline $\mathrm{H}(36 \mathrm{~B})$ & 7833 & 6492 & 9737 & 209 \\
\hline $\mathrm{H}(36 \mathrm{C})$ & 8457 & 7048 & 9946 & 209 \\
\hline $\mathrm{H}(37 \mathrm{~A})$ & 6810 & 7838 & 9444 & 182 \\
\hline $\mathrm{H}(37 \mathrm{~B})$ & 7254 & 7861 & 9997 & 182 \\
\hline $\mathrm{H}(37 \mathrm{C})$ & 5794 & 7888 & 9792 & 182 \\
\hline $\mathrm{H}(38 \mathrm{~A})$ & 6899 & 7113 & 10507 & 154 \\
\hline $\mathrm{H}(38 \mathrm{~B})$ & 6204 & 6567 & 10308 & 154 \\
\hline $\mathrm{H}(38 \mathrm{C})$ & 5430 & 7122 & 10316 & 154 \\
\hline $\mathrm{H}(40 \mathrm{~A})$ & 371 & 5057 & 8652 & 57 \\
\hline $\mathrm{H}(41 \mathrm{~A})$ & -486 & 4625 & 9243 & 4 \\
\hline
\end{tabular}




\begin{tabular}{|c|c|c|c|c|}
\hline $\mathrm{H}(43 \mathrm{~A})$ & -3530 & 5613 & 9024 & 81 \\
\hline $\mathrm{H}(44 \mathrm{~A})$ & -2716 & 6039 & 8399 & 68 \\
\hline $\mathrm{H}(46 \mathrm{~A})$ & -4303 & 4467 & 9754 & 256 \\
\hline $\mathrm{H}(46 \mathrm{~B})$ & -4066 & 4381 & 9226 & 256 \\
\hline $\mathrm{H}(46 \mathrm{C})$ & -4467 & 4967 & 9396 & 256 \\
\hline $\mathrm{H}(47 \mathrm{~A})$ & -2204 & 4141 & 10089 & 317 \\
\hline $\mathrm{H}(47 \mathrm{~B})$ & -982 & 4409 & 9931 & 317 \\
\hline $\mathrm{H}(47 \mathrm{C})$ & -1856 & 4016 & 9581 & 317 \\
\hline $\mathrm{H}(48 \mathrm{~A})$ & -2884 & 5007 & 10284 & 309 \\
\hline $\mathrm{H}(48 \mathrm{~B})$ & -3086 & 5498 & 9917 & 309 \\
\hline $\mathrm{H}(48 \mathrm{C})$ & -1700 & 5296 & 10116 & 309 \\
\hline $\mathrm{H}(50 \mathrm{~A})$ & 1592 & 5945 & 1670 & 57 \\
\hline $\mathrm{H}(51 \mathrm{~A})$ & 3604 & 5672 & 1551 & 69 \\
\hline $\mathrm{H}(52 \mathrm{~A})$ & 5220 & 5616 & 2178 & 70 \\
\hline $\mathrm{H}(53 \mathrm{~A})$ & 4840 & 5802 & 2949 & 70 \\
\hline $\mathrm{H}(54 \mathrm{~A})$ & 2780 & 6067 & 3069 & 54 \\
\hline $\mathrm{H}(56 \mathrm{~A})$ & -1219 & 7262 & 2224 & 58 \\
\hline $\mathrm{H}(57 \mathrm{~A})$ & -1016 & 8208 & 2152 & 59 \\
\hline $\mathrm{H}(58 \mathrm{~A})$ & 985 & 8608 & 2285 & 63 \\
\hline $\mathrm{H}(59 \mathrm{~A})$ & 2786 & 8060 & 2498 & 68 \\
\hline $\mathrm{H}(60 \mathrm{~A})$ & 2575 & 7107 & 2551 & 61 \\
\hline $\mathrm{H}(62 \mathrm{~A})$ & -295 & 6953 & 3218 & 62 \\
\hline
\end{tabular}




\begin{tabular}{|c|c|c|c|c|}
\hline $\mathrm{H}(63 \mathrm{~A})$ & -694 & 6751 & 3978 & 83 \\
\hline $\mathrm{H}(64 \mathrm{~A})$ & -526 & 5844 & 4249 & 92 \\
\hline $\mathrm{H}(65 \mathrm{~A})$ & 143 & 5150 & 3770 & 86 \\
\hline $\mathrm{H}(66 \mathrm{~A})$ & 588 & 5365 & 3008 & 66 \\
\hline $\mathrm{H}(68 \mathrm{~A})$ & -1395 & 5506 & 2438 & 58 \\
\hline $\mathrm{H}(69 \mathrm{~A})$ & -2911 & 5138 & 1851 & 75 \\
\hline $\mathrm{H}(70 \mathrm{~A})$ & -3164 & 5502 & 1096 & 83 \\
\hline $\mathrm{H}(71 \mathrm{~A})$ & -1909 & 6243 & 910 & 79 \\
\hline $\mathrm{H}(72 \mathrm{~A})$ & -385 & 6607 & 1511 & 65 \\
\hline $\mathrm{H}(1 \mathrm{SA})$ & 8631 & 7705 & 6309 & 104 \\
\hline $\mathrm{H}(1 \mathrm{SB})$ & 8145 & 7218 & 5962 & 104 \\
\hline $\mathrm{H}(1 \mathrm{SC})$ & 7838 & 7217 & 6486 & 104 \\
\hline $\mathrm{H}(3 \mathrm{SB})$ & 1289 & 7282 & 10013 & 49 \\
\hline $\mathrm{H}(3 \mathrm{SC})$ & 1848 & 7133 & 9557 & 49 \\
\hline $\mathrm{H}(3 \mathrm{SA})$ & 748 & 7561 & 9534 & 49 \\
\hline $\mathrm{H}(5 \mathrm{SA})$ & 10363 & 8276 & 1156 & 285 \\
\hline $\mathrm{H}(5 \mathrm{SB})$ & 9740 & 8090 & 644 & 285 \\
\hline $\mathrm{H}(5 \mathrm{SC})$ & 9224 & 7853 & 1094 & 285 \\
\hline $\mathrm{H}(6 \mathrm{SA})$ & 9425 & 8865 & 952 & 164 \\
\hline $\mathrm{H}(6 \mathrm{SB})$ & 8652 & 8543 & 1273 & 164 \\
\hline $\mathrm{H}(7 \mathrm{SA})$ & 6059 & 8740 & 506 & 175 \\
\hline $\mathrm{H}(7 \mathrm{SB})$ & 6168 & 9153 & 916 & 5 \\
\hline
\end{tabular}




\begin{tabular}{lcccc}
$\mathrm{H}(8 \mathrm{SA})$ & 4691 & 9172 & 469 & 225 \\
$\mathrm{H}(8 \mathrm{SB})$ & 5454 & 9345 & 59 & 225 \\
$\mathrm{H}(8 \mathrm{SC})$ & 5466 & 9731 & 506 & 225 \\
\hline
\end{tabular}


Table S24. Torsion Angles, ${ }^{\circ}$, for $\left[\left(\mathrm{L}^{1}\right) \mathrm{Fe}(\mathrm{III})-\mathrm{OH}\right]\left[\mathrm{PPh}_{4}\right] \cdot \mathrm{CH}_{3} \mathrm{CN} \cdot 0.5 \mathrm{Et}_{2} \mathrm{O}(\mathbf{2})$

\begin{tabular}{|c|c|c|c|}
\hline $\mathrm{O}(1)-\mathrm{Fe}(1)-\mathrm{N}(1)-\mathrm{C}(2)$ & $-7(2)$ & $\mathrm{C}(29)-\mathrm{N}(2)-\mathrm{C}(28)-\mathrm{C}(23)$ & $-166.9(6)$ \\
\hline $\mathrm{N}(2)-\mathrm{Fe}(1)-\mathrm{N}(1)-\mathrm{C}(2)$ & $144.5(4)$ & $\mathrm{Fe}(1)-\mathrm{N}(2)-\mathrm{C}(28)-\mathrm{C}(23)$ & $16.5(7)$ \\
\hline $\mathrm{N}(4)-\mathrm{Fe}(1)-\mathrm{N}(1)-\mathrm{C}(2)$ & $21.0(3)$ & $\mathrm{C}(29)-\mathrm{N}(2)-\mathrm{C}(28)-\mathrm{C}(27)$ & $14.9(9)$ \\
\hline $\mathrm{N}(3)-\mathrm{Fe}(1)-\mathrm{N}(1)-\mathrm{C}(2)$ & $-103.7(4)$ & $\mathrm{Fe}(1)-\mathrm{N}(2)-\mathrm{C}(28)-\mathrm{C}(27)$ & $-161.7(5)$ \\
\hline $\mathrm{O}(1)-\mathrm{Fe}(1)-\mathrm{N}(1)-\mathrm{C}(7)$ & $116.4(18)$ & $\mathrm{C}(24)-\mathrm{C}(23)-\mathrm{C}(28)-\mathrm{N}(2)$ & $-173.0(6)$ \\
\hline $\mathrm{N}(2)-\mathrm{Fe}(1)-\mathrm{N}(1)-\mathrm{C}(7)$ & $-92.6(4)$ & $\mathrm{N}(1)-\mathrm{C}(23)-\mathrm{C}(28)-\mathrm{N}(2)$ & $9.0(8)$ \\
\hline $\mathrm{N}(4)-\mathrm{Fe}(1)-\mathrm{N}(1)-\mathrm{C}(7)$ & $143.9(4)$ & $C(24)-C(23)-C(28)-C(27)$ & $5.4(9)$ \\
\hline $\mathrm{N}(3)-\mathrm{Fe}(1)-\mathrm{N}(1)-\mathrm{C}(7)$ & $19.2(3)$ & $\mathrm{N}(1)-\mathrm{C}(23)-\mathrm{C}(28)-\mathrm{C}(27)$ & $-172.6(5)$ \\
\hline $\mathrm{O}(1)-\mathrm{Fe}(1)-\mathrm{N}(1)-\mathrm{C}(23)$ & $-125.7(18)$ & $\mathrm{C}(26)-\mathrm{C}(27)-\mathrm{C}(28)-\mathrm{N}(2)$ & $175.5(6)$ \\
\hline $\mathrm{N}(2)-\mathrm{Fe}(1)-\mathrm{N}(1)-\mathrm{C}(23)$ & $25.3(3)$ & $C(26)-C(27)-C(28)-C(23)$ & $-2.8(9)$ \\
\hline $\mathrm{N}(4)-\mathrm{Fe}(1)-\mathrm{N}(1)-\mathrm{C}(23)$ & $-98.3(4)$ & $\mathrm{C}(28)-\mathrm{N}(2)-\mathrm{C}(29)-\mathrm{C}(34)$ & $-127.6(7)$ \\
\hline $\mathrm{N}(3)-\mathrm{Fe}(1)-\mathrm{N}(1)-\mathrm{C}(23)$ & 137.0(4) & $\mathrm{Fe}(1)-\mathrm{N}(2)-\mathrm{C}(29)-\mathrm{C}(34)$ & $48.7(9)$ \\
\hline $\mathrm{O}(1)-\mathrm{Fe}(1)-\mathrm{N}(2)-\mathrm{C}(28)$ & 153.6(4) & $\mathrm{C}(28)-\mathrm{N}(2)-\mathrm{C}(29)-\mathrm{C}(30)$ & $58.2(9)$ \\
\hline $\mathrm{N}(4)-\mathrm{Fe}(1)-\mathrm{N}(2)-\mathrm{C}(28)$ & $42.6(5)$ & $\mathrm{Fe}(1)-\mathrm{N}(2)-\mathrm{C}(29)-\mathrm{C}(30)$ & $-125.6(6)$ \\
\hline $\mathrm{N}(3)-\mathrm{Fe}(1)-\mathrm{N}(2)-\mathrm{C}(28)$ & $-95.9(5)$ & $C(34)-C(29)-C(30)-C(31)$ & $1.8(11)$ \\
\hline $\mathrm{N}(1)-\mathrm{Fe}(1)-\mathrm{N}(2)-\mathrm{C}(28)$ & $-23.4(4)$ & $N(2)-C(29)-C(30)-C(31)$ & $176.3(7)$ \\
\hline $\mathrm{O}(1)-\mathrm{Fe}(1)-\mathrm{N}(2)-\mathrm{C}(29)$ & $-22.7(6)$ & $\mathrm{C}(29)-\mathrm{C}(30)-\mathrm{C}(31)-\mathrm{C}(32)$ & $-1.8(12)$ \\
\hline $\mathrm{N}(4)-\mathrm{Fe}(1)-\mathrm{N}(2)-\mathrm{C}(29)$ & $-133.7(5)$ & $\mathrm{C}(30)-\mathrm{C}(31)-\mathrm{C}(32)-\mathrm{C}(33)$ & $1.5(12)$ \\
\hline $\mathrm{N}(3)-\mathrm{Fe}(1)-\mathrm{N}(2)-\mathrm{C}(29)$ & $87.8(5)$ & $\mathrm{C}(30)-\mathrm{C}(31)-\mathrm{C}(32)-\mathrm{C}(35)$ & $-177.8(7)$ \\
\hline $\mathrm{N}(1)-\mathrm{Fe}(1)-\mathrm{N}(2)-\mathrm{C}(29)$ & $160.3(5)$ & $\mathrm{C}(31)-\mathrm{C}(32)-\mathrm{C}(33)-\mathrm{C}(34)$ & $-1.5(13)$ \\
\hline $\mathrm{O}(1)-\mathrm{Fe}(1)-\mathrm{N}(3)-\mathrm{C}(12)$ & $165.9(4)$ & $\mathrm{C}(35)-\mathrm{C}(32)-\mathrm{C}(33)-\mathrm{C}(34)$ & $177.8(8)$ \\
\hline
\end{tabular}




\begin{tabular}{|c|c|c|c|}
\hline $\mathrm{N}(2)-\mathrm{Fe}(1)-\mathrm{N}(3)-\mathrm{C}(12)$ & $52.8(5)$ & $C(30)-C(29)-C(34)-C(33)$ & $-1.7(12)$ \\
\hline $\mathrm{N}(4)-\mathrm{Fe}(1)-\mathrm{N}(3)-\mathrm{C}(12)$ & $-85.3(5)$ & $\mathrm{N}(2)-\mathrm{C}(29)-\mathrm{C}(34)-\mathrm{C}(33)$ & $-176.4(7)$ \\
\hline $\mathrm{N}(1)-\mathrm{Fe}(1)-\mathrm{N}(3)-\mathrm{C}(12)$ & $-20.0(4)$ & $\mathrm{C}(32)-\mathrm{C}(33)-\mathrm{C}(34)-\mathrm{C}(29)$ & $1.7(13)$ \\
\hline $\mathrm{O}(1)-\mathrm{Fe}(1)-\mathrm{N}(3)-\mathrm{C}(13)$ & $12.6(5)$ & $\mathrm{C}(31)-\mathrm{C}(32)-\mathrm{C}(35)-\mathrm{C}(36)$ & $-60.8(11)$ \\
\hline $\mathrm{N}(2)-\mathrm{Fe}(1)-\mathrm{N}(3)-\mathrm{C}(13)$ & $-100.4(5)$ & $\mathrm{C}(33)-\mathrm{C}(32)-\mathrm{C}(35)-\mathrm{C}(36)$ & $120.0(10)$ \\
\hline $\mathrm{N}(4)-\mathrm{Fe}(1)-\mathrm{N}(3)-\mathrm{C}(13)$ & $121.5(4)$ & $\mathrm{C}(31)-\mathrm{C}(32)-\mathrm{C}(35)-\mathrm{C}(37)$ & $56.3(11)$ \\
\hline $\mathrm{N}(1)-\mathrm{Fe}(1)-\mathrm{N}(3)-\mathrm{C}(13)$ & $-173.3(5)$ & $\mathrm{C}(33)-\mathrm{C}(32)-\mathrm{C}(35)-\mathrm{C}(37)$ & $-123.0(9)$ \\
\hline $\mathrm{O}(1)-\mathrm{Fe}(1)-\mathrm{N}(4)-\mathrm{C}(1)$ & $156.1(4)$ & $\mathrm{C}(31)-\mathrm{C}(32)-\mathrm{C}(35)-\mathrm{C}(38)$ & $174.9(8)$ \\
\hline $\mathrm{N}(2)-\mathrm{Fe}(1)-\mathrm{N}(4)-\mathrm{C}(1)$ & $-88.8(5)$ & $\mathrm{C}(33)-\mathrm{C}(32)-\mathrm{C}(35)-\mathrm{C}(38)$ & $-4.3(12)$ \\
\hline $\mathrm{N}(3)-\mathrm{Fe}(1)-\mathrm{N}(4)-\mathrm{C}(1)$ & $45.2(5)$ & $\mathrm{C}(1)-\mathrm{N}(4)-\mathrm{C}(39)-\mathrm{C}(44)$ & $-71.8(8)$ \\
\hline $\mathrm{N}(1)-\mathrm{Fe}(1)-\mathrm{N}(4)-\mathrm{C}(1)$ & $-21.2(4)$ & $\mathrm{Fe}(1)-\mathrm{N}(4)-\mathrm{C}(39)-\mathrm{C}(44)$ & $91.8(7)$ \\
\hline $\mathrm{O}(1)-\mathrm{Fe}(1)-\mathrm{N}(4)-\mathrm{C}(39)$ & $-7.2(5)$ & $\mathrm{C}(1)-\mathrm{N}(4)-\mathrm{C}(39)-\mathrm{C}(40)$ & $112.2(7)$ \\
\hline $\mathrm{N}(2)-\mathrm{Fe}(1)-\mathrm{N}(4)-\mathrm{C}(39)$ & $108.0(4)$ & $\mathrm{Fe}(1)-\mathrm{N}(4)-\mathrm{C}(39)-\mathrm{C}(40)$ & $-84.2(7)$ \\
\hline $\mathrm{N}(3)-\mathrm{Fe}(1)-\mathrm{N}(4)-\mathrm{C}(39)$ & $-118.1(4)$ & $\mathrm{C}(44)-\mathrm{C}(39)-\mathrm{C}(40)-\mathrm{C}(41)$ & $0.9(10)$ \\
\hline $\mathrm{N}(1)-\mathrm{Fe}(1)-\mathrm{N}(4)-\mathrm{C}(39)$ & $175.5(5)$ & $\mathrm{N}(4)-\mathrm{C}(39)-\mathrm{C}(40)-\mathrm{C}(41)$ & $177.2(6)$ \\
\hline$C(39)-N(4)-C(1)-C(2)$ & $-179.8(5)$ & $C(39)-C(40)-C(41)-C(42)$ & $-1.4(12)$ \\
\hline $\mathrm{Fe}(1)-\mathrm{N}(4)-\mathrm{C}(1)-\mathrm{C}(2)$ & $16.7(7)$ & $C(40)-C(41)-C(42)-C(43)$ & $0.6(12)$ \\
\hline$C(39)-N(4)-C(1)-C(6)$ & $1.3(9)$ & $\mathrm{C}(40)-\mathrm{C}(41)-\mathrm{C}(42)-\mathrm{C}(45)$ & $-177.1(7)$ \\
\hline $\mathrm{Fe}(1)-\mathrm{N}(4)-\mathrm{C}(1)-\mathrm{C}(6)$ & $-162.2(5)$ & $\mathrm{C}(41)-\mathrm{C}(42)-\mathrm{C}(43)-\mathrm{C}(44)$ & $0.8(12)$ \\
\hline $\mathrm{N}(4)-\mathrm{C}(1)-\mathrm{C}(2)-\mathrm{C}(3)$ & $-175.1(5)$ & $\mathrm{C}(45)-\mathrm{C}(42)-\mathrm{C}(43)-\mathrm{C}(44)$ & $178.4(7)$ \\
\hline$C(6)-C(1)-C(2)-C(3)$ & $3.9(8)$ & $\mathrm{C}(40)-\mathrm{C}(39)-\mathrm{C}(44)-\mathrm{C}(43)$ & $0.4(10)$ \\
\hline $\mathrm{N}(4)-\mathrm{C}(1)-\mathrm{C}(2)-\mathrm{N}(1)$ & $5.2(7)$ & $\mathrm{N}(4)-\mathrm{C}(39)-\mathrm{C}(44)-\mathrm{C}(43)$ & $-175.7(6)$ \\
\hline
\end{tabular}




\begin{tabular}{|c|c|c|c|}
\hline$C(6)-C(1)-C(2)-N(1)$ & $-175.8(5)$ & $C(42)-C(43)-C(44)-C(39)$ & $-1.3(12)$ \\
\hline$C(7)-N(1)-C(2)-C(3)$ & $44.1(8)$ & $\mathrm{C}(43)-\mathrm{C}(42)-\mathrm{C}(45)-\mathrm{C}(48)$ & $-68.0(12)$ \\
\hline $\mathrm{C}(23)-\mathrm{N}(1)-\mathrm{C}(2)-\mathrm{C}(3)$ & $-86.9(7)$ & $\mathrm{C}(41)-\mathrm{C}(42)-\mathrm{C}(45)-\mathrm{C}(48)$ & $109.4(11)$ \\
\hline $\mathrm{Fe}(1)-\mathrm{N}(1)-\mathrm{C}(2)-\mathrm{C}(3)$ & $160.4(5)$ & $\mathrm{C}(43)-\mathrm{C}(42)-\mathrm{C}(45)-\mathrm{C}(46)$ & $48.3(12)$ \\
\hline $\mathrm{C}(7)-\mathrm{N}(1)-\mathrm{C}(2)-\mathrm{C}(1)$ & $-136.2(5)$ & $\mathrm{C}(41)-\mathrm{C}(42)-\mathrm{C}(45)-\mathrm{C}(46)$ & $-134.2(10)$ \\
\hline $\mathrm{C}(23)-\mathrm{N}(1)-\mathrm{C}(2)-\mathrm{C}(1)$ & $92.8(6)$ & $\mathrm{C}(43)-\mathrm{C}(42)-\mathrm{C}(45)-\mathrm{C}(47)$ & $174.1(10)$ \\
\hline $\mathrm{Fe}(1)-\mathrm{N}(1)-\mathrm{C}(2)-\mathrm{C}(1)$ & $-20.0(6)$ & $\mathrm{C}(41)-\mathrm{C}(42)-\mathrm{C}(45)-\mathrm{C}(47)$ & $-8.4(13)$ \\
\hline$C(1)-C(2)-C(3)-C(4)$ & $-1.9(9)$ & $\mathrm{C}(61)-\mathrm{P}(1)-\mathrm{C}(49)-\mathrm{C}(50)$ & $154.5(5)$ \\
\hline $\mathrm{N}(1)-\mathrm{C}(2)-\mathrm{C}(3)-\mathrm{C}(4)$ & $177.7(6)$ & $C(67)-P(1)-C(49)-C(50)$ & $33.3(6)$ \\
\hline$C(2)-C(3)-C(4)-C(5)$ & $-0.9(9)$ & $\mathrm{C}(55)-\mathrm{P}(1)-\mathrm{C}(49)-\mathrm{C}(50)$ & $-85.5(6)$ \\
\hline$C(3)-C(4)-C(5)-C(6)$ & $1.6(10)$ & $\mathrm{C}(61)-\mathrm{P}(1)-\mathrm{C}(49)-\mathrm{C}(54)$ & $-31.9(6)$ \\
\hline$C(4)-C(5)-C(6)-C(1)$ & $0.4(10)$ & $\mathrm{C}(67)-\mathrm{P}(1)-\mathrm{C}(49)-\mathrm{C}(54)$ & $-153.0(5)$ \\
\hline $\mathrm{N}(4)-\mathrm{C}(1)-\mathrm{C}(6)-\mathrm{C}(5)$ & $175.8(6)$ & $\mathrm{C}(55)-\mathrm{P}(1)-\mathrm{C}(49)-\mathrm{C}(54)$ & $88.1(5)$ \\
\hline $\mathrm{C}(2)-\mathrm{C}(1)-\mathrm{C}(6)-\mathrm{C}(5)$ & $-3.1(9)$ & $C(54)-C(49)-C(50)-C(51)$ & $-2.0(10)$ \\
\hline $\mathrm{C}(2)-\mathrm{N}(1)-\mathrm{C}(7)-\mathrm{C}(12)$ & $99.9(6)$ & $\mathrm{P}(1)-\mathrm{C}(49)-\mathrm{C}(50)-\mathrm{C}(51)$ & $171.4(5)$ \\
\hline $\mathrm{C}(23)-\mathrm{N}(1)-\mathrm{C}(7)-\mathrm{C}(12)$ & $-128.7(6)$ & $\mathrm{C}(49)-\mathrm{C}(50)-\mathrm{C}(51)-\mathrm{C}(52)$ & $0.0(11)$ \\
\hline $\mathrm{Fe}(1)-\mathrm{N}(1)-\mathrm{C}(7)-\mathrm{C}(12)$ & $-17.0(6)$ & $\mathrm{C}(50)-\mathrm{C}(51)-\mathrm{C}(52)-\mathrm{C}(53)$ & $1.4(11)$ \\
\hline $\mathrm{C}(2)-\mathrm{N}(1)-\mathrm{C}(7)-\mathrm{C}(8)$ & $-80.2(7)$ & $\mathrm{C}(51)-\mathrm{C}(52)-\mathrm{C}(53)-\mathrm{C}(54)$ & $-0.8(10)$ \\
\hline $\mathrm{C}(23)-\mathrm{N}(1)-\mathrm{C}(7)-\mathrm{C}(8)$ & $51.1(8)$ & $\mathrm{C}(52)-\mathrm{C}(53)-\mathrm{C}(54)-\mathrm{C}(49)$ & $-1.1(9)$ \\
\hline $\mathrm{Fe}(1)-\mathrm{N}(1)-\mathrm{C}(7)-\mathrm{C}(8)$ & $162.9(5)$ & $\mathrm{C}(50)-\mathrm{C}(49)-\mathrm{C}(54)-\mathrm{C}(53)$ & $2.5(9)$ \\
\hline $\mathrm{C}(12)-\mathrm{C}(7)-\mathrm{C}(8)-\mathrm{C}(9)$ & $-2.7(10)$ & $\mathrm{P}(1)-\mathrm{C}(49)-\mathrm{C}(54)-\mathrm{C}(53)$ & $-171.2(5)$ \\
\hline $\mathrm{N}(1)-\mathrm{C}(7)-\mathrm{C}(8)-\mathrm{C}(9)$ & $177.4(5)$ & $C(61)-P(1)-C(55)-C(60)$ & $95.1(6)$ \\
\hline
\end{tabular}




\begin{tabular}{|c|c|c|c|}
\hline $\mathrm{C}(7)-\mathrm{C}(8)-\mathrm{C}(9)-\mathrm{C}(10)$ & $-1.4(9)$ & $\mathrm{C}(67)-\mathrm{P}(1)-\mathrm{C}(55)-\mathrm{C}(60)$ & $-143.3(6)$ \\
\hline$C(8)-C(9)-C(10)-C(11)$ & $3.0(10)$ & $\mathrm{C}(49)-\mathrm{P}(1)-\mathrm{C}(55)-\mathrm{C}(60)$ & $-24.9(7)$ \\
\hline $\mathrm{C}(9)-\mathrm{C}(10)-\mathrm{C}(11)-\mathrm{C}(12)$ & $-0.4(11)$ & $\mathrm{C}(61)-\mathrm{P}(1)-\mathrm{C}(55)-\mathrm{C}(56)$ & $-84.0(6)$ \\
\hline $\mathrm{C}(13)-\mathrm{N}(3)-\mathrm{C}(12)-\mathrm{C}(11)$ & $-12.2(9)$ & $\mathrm{C}(67)-\mathrm{P}(1)-\mathrm{C}(55)-\mathrm{C}(56)$ & $37.5(6)$ \\
\hline $\mathrm{Fe}(1)-\mathrm{N}(3)-\mathrm{C}(12)-\mathrm{C}(11)$ & $-165.6(5)$ & $\mathrm{C}(49)-\mathrm{P}(1)-\mathrm{C}(55)-\mathrm{C}(56)$ & $156.0(6)$ \\
\hline $\mathrm{C}(13)-\mathrm{N}(3)-\mathrm{C}(12)-\mathrm{C}(7)$ & $170.5(5)$ & $\mathrm{C}(60)-\mathrm{C}(55)-\mathrm{C}(56)-\mathrm{C}(57)$ & $-0.2(10)$ \\
\hline $\mathrm{Fe}(1)-\mathrm{N}(3)-\mathrm{C}(12)-\mathrm{C}(7)$ & 17.1(7) & $P(1)-C(55)-C(56)-C(57)$ & $178.9(5)$ \\
\hline $\mathrm{C}(10)-\mathrm{C}(11)-\mathrm{C}(12)-\mathrm{N}(3)$ & $179.1(6)$ & $\mathrm{C}(55)-\mathrm{C}(56)-\mathrm{C}(57)-\mathrm{C}(58)$ & $-0.2(11)$ \\
\hline$C(10)-C(11)-C(12)-C(7)$ & $-3.5(10)$ & $\mathrm{C}(56)-\mathrm{C}(57)-\mathrm{C}(58)-\mathrm{C}(59)$ & $-0.2(10)$ \\
\hline $\mathrm{C}(8)-\mathrm{C}(7)-\mathrm{C}(12)-\mathrm{N}(3)$ & $-177.3(5)$ & $\mathrm{C}(57)-\mathrm{C}(58)-\mathrm{C}(59)-\mathrm{C}(60)$ & $1.0(11)$ \\
\hline $\mathrm{N}(1)-\mathrm{C}(7)-\mathrm{C}(12)-\mathrm{N}(3)$ & $2.5(8)$ & $\mathrm{C}(58)-\mathrm{C}(59)-\mathrm{C}(60)-\mathrm{C}(55)$ & $-1.4(11)$ \\
\hline$C(8)-C(7)-C(12)-C(11)$ & $5.1(9)$ & $C(56)-C(55)-C(60)-C(59)$ & $1.1(10)$ \\
\hline $\mathrm{N}(1)-\mathrm{C}(7)-\mathrm{C}(12)-\mathrm{C}(11)$ & $-175.0(5)$ & $\mathrm{P}(1)-\mathrm{C}(55)-\mathrm{C}(60)-\mathrm{C}(59)$ & $-178.1(6)$ \\
\hline $\mathrm{C}(12)-\mathrm{N}(3)-\mathrm{C}(13)-\mathrm{C}(18)$ & $-54.7(8)$ & $\mathrm{C}(67)-\mathrm{P}(1)-\mathrm{C}(61)-\mathrm{C}(62)$ & $-106.2(6)$ \\
\hline $\mathrm{Fe}(1)-\mathrm{N}(3)-\mathrm{C}(13)-\mathrm{C}(18)$ & $98.2(7)$ & $\mathrm{C}(55)-\mathrm{P}(1)-\mathrm{C}(61)-\mathrm{C}(62)$ & $15.1(7)$ \\
\hline $\mathrm{C}(12)-\mathrm{N}(3)-\mathrm{C}(13)-\mathrm{C}(14)$ & $127.9(6)$ & $\mathrm{C}(49)-\mathrm{P}(1)-\mathrm{C}(61)-\mathrm{C}(62)$ & $133.2(6)$ \\
\hline $\mathrm{Fe}(1)-\mathrm{N}(3)-\mathrm{C}(13)-\mathrm{C}(14)$ & $-79.2(7)$ & $\mathrm{C}(67)-\mathrm{P}(1)-\mathrm{C}(61)-\mathrm{C}(66)$ & $72.9(6)$ \\
\hline $\mathrm{C}(18)-\mathrm{C}(13)-\mathrm{C}(14)-\mathrm{C}(15)$ & $2.0(10)$ & $\mathrm{C}(55)-\mathrm{P}(1)-\mathrm{C}(61)-\mathrm{C}(66)$ & $-165.8(5)$ \\
\hline $\mathrm{N}(3)-\mathrm{C}(13)-\mathrm{C}(14)-\mathrm{C}(15)$ & $179.5(6)$ & $\mathrm{C}(49)-\mathrm{P}(1)-\mathrm{C}(61)-\mathrm{C}(66)$ & $-47.7(6)$ \\
\hline$C(13)-C(14)-C(15)-C(16)$ & $1.0(11)$ & $C(66)-C(61)-C(62)-C(63)$ & $0.9(11)$ \\
\hline$C(14)-C(15)-C(16)-C(17)$ & $-3.8(11)$ & $P(1)-C(61)-C(62)-C(63)$ & $-180.0(5)$ \\
\hline$C(14)-C(15)-C(16)-C(19)$ & $178.7(7)$ & $\mathrm{C}(61)-\mathrm{C}(62)-\mathrm{C}(63)-\mathrm{C}(64)$ & $-1.6(11)$ \\
\hline
\end{tabular}




\begin{tabular}{|c|c|c|c|}
\hline $\mathrm{C}(15)-\mathrm{C}(16)-\mathrm{C}(17)-\mathrm{C}(18)$ & $3.4(11)$ & $\mathrm{C}(62)-\mathrm{C}(63)-\mathrm{C}(64)-\mathrm{C}(65)$ & $1.5(12)$ \\
\hline $\mathrm{C}(19)-\mathrm{C}(16)-\mathrm{C}(17)-\mathrm{C}(18)$ & $-179.1(7)$ & $\mathrm{C}(63)-\mathrm{C}(64)-\mathrm{C}(65)-\mathrm{C}(66)$ & $-0.8(12)$ \\
\hline $\mathrm{C}(16)-\mathrm{C}(17)-\mathrm{C}(18)-\mathrm{C}(13)$ & $-0.4(12)$ & $C(62)-C(61)-C(66)-C(65)$ & $-0.2(10)$ \\
\hline $\mathrm{C}(14)-\mathrm{C}(13)-\mathrm{C}(18)-\mathrm{C}(17)$ & $-2.4(10)$ & $\mathrm{P}(1)-\mathrm{C}(61)-\mathrm{C}(66)-\mathrm{C}(65)$ & $-179.3(5)$ \\
\hline $\mathrm{N}(3)-\mathrm{C}(13)-\mathrm{C}(18)-\mathrm{C}(17)$ & $-179.8(7)$ & $C(64)-C(65)-C(66)-C(61)$ & $0.1(11)$ \\
\hline $\mathrm{C}(17)-\mathrm{C}(16)-\mathrm{C}(19)-\mathrm{C}(22)$ & $130.3(14)$ & $\mathrm{C}(61)-\mathrm{P}(1)-\mathrm{C}(67)-\mathrm{C}(72)$ & $159.9(5)$ \\
\hline$C(15)-C(16)-C(19)-C(22)$ & $-52.3(16)$ & $\mathrm{C}(55)-\mathrm{P}(1)-\mathrm{C}(67)-\mathrm{C}(72)$ & $38.3(6)$ \\
\hline$C(17)-C(16)-C(19)-C(20)$ & $-101.5(13)$ & $\mathrm{C}(49)-\mathrm{P}(1)-\mathrm{C}(67)-\mathrm{C}(72)$ & $-78.8(6)$ \\
\hline$C(15)-C(16)-C(19)-C(20)$ & $75.9(14)$ & $\mathrm{C}(61)-\mathrm{P}(1)-\mathrm{C}(67)-\mathrm{C}(68)$ & $-23.8(6)$ \\
\hline$C(17)-C(16)-C(19)-C(21)$ & $9.8(17)$ & $\mathrm{C}(55)-\mathrm{P}(1)-\mathrm{C}(67)-\mathrm{C}(68)$ & $-145.4(5)$ \\
\hline $\mathrm{C}(15)-\mathrm{C}(16)-\mathrm{C}(19)-\mathrm{C}(21)$ & $-172.8(14)$ & $\mathrm{C}(49)-\mathrm{P}(1)-\mathrm{C}(67)-\mathrm{C}(68)$ & $97.5(6)$ \\
\hline $\mathrm{C}(2)-\mathrm{N}(1)-\mathrm{C}(23)-\mathrm{C}(24)$ & $42.6(8)$ & $\mathrm{C}(72)-\mathrm{C}(67)-\mathrm{C}(68)-\mathrm{C}(69)$ & $0.5(10)$ \\
\hline $\mathrm{C}(7)-\mathrm{N}(1)-\mathrm{C}(23)-\mathrm{C}(24)$ & $-89.9(7)$ & $\mathrm{P}(1)-\mathrm{C}(67)-\mathrm{C}(68)-\mathrm{C}(69)$ & $-175.7(5)$ \\
\hline $\mathrm{Fe}(1)-\mathrm{N}(1)-\mathrm{C}(23)-\mathrm{C}(24)$ & $157.2(5)$ & $\mathrm{C}(67)-\mathrm{C}(68)-\mathrm{C}(69)-\mathrm{C}(70)$ & $-0.2(10)$ \\
\hline $\mathrm{C}(2)-\mathrm{N}(1)-\mathrm{C}(23)-\mathrm{C}(28)$ & $-139.4(5)$ & $\mathrm{C}(68)-\mathrm{C}(69)-\mathrm{C}(70)-\mathrm{C}(71)$ & $-0.1(11)$ \\
\hline $\mathrm{C}(7)-\mathrm{N}(1)-\mathrm{C}(23)-\mathrm{C}(28)$ & $88.1(6)$ & $\mathrm{C}(69)-\mathrm{C}(70)-\mathrm{C}(71)-\mathrm{C}(72)$ & $0.1(11)$ \\
\hline $\mathrm{Fe}(1)-\mathrm{N}(1)-\mathrm{C}(23)-\mathrm{C}(28)$ & $-24.8(6)$ & $\mathrm{C}(68)-\mathrm{C}(67)-\mathrm{C}(72)-\mathrm{C}(71)$ & $-0.6(10)$ \\
\hline $\mathrm{C}(28)-\mathrm{C}(23)-\mathrm{C}(24)-\mathrm{C}(25)$ & $-4.9(9)$ & $\mathrm{P}(1)-\mathrm{C}(67)-\mathrm{C}(72)-\mathrm{C}(71)$ & $175.8(5)$ \\
\hline $\mathrm{N}(1)-\mathrm{C}(23)-\mathrm{C}(24)-\mathrm{C}(25)$ & $172.9(5)$ & $\mathrm{C}(70)-\mathrm{C}(71)-\mathrm{C}(72)-\mathrm{C}(67)$ & $0.3(10)$ \\
\hline$C(23)-C(24)-C(25)-C(26)$ & $1.6(10)$ & $\mathrm{C}(7 \mathrm{~S})-\mathrm{O}(3 \mathrm{~S})-\mathrm{C}(6 \mathrm{~S})-\mathrm{C}(5 \mathrm{~S})$ & $54(12)$ \\
\hline$C(24)-C(25)-C(26)-C(27)$ & $1.0(11)$ & $\mathrm{C}(6 \mathrm{~S})-\mathrm{O}(3 \mathrm{~S})-\mathrm{C}(7 \mathrm{~S})-\mathrm{C}(8 \mathrm{~S})$ & $-148(9)$ \\
\hline$C(25)-C(26)-C(27)-C(28)$ & $-0.2(11)$ & & \\
\hline
\end{tabular}


Table S25. Atomic coordinates $\left(\times 10^{4}\right)$ and equivalent isotropic displacement parameters $\left(\AA^{2} \times 10^{3}\right)$ for $\left[\left(\mathrm{L}^{1}\right) \mathrm{Fe}(\mathrm{III})-\mathrm{OH}\right]\left[\mathrm{K}(\mathrm{DMF})_{3}\right] \cdot 3 \mathrm{H}_{2} \mathrm{O}(3) . \mathrm{U}(\mathrm{eq})$ is defined as one third of the trace of the orthogonalized $\mathrm{U}^{\mathrm{ij}}$ tensor

\begin{tabular}{|c|c|c|c|c|}
\hline & $\mathrm{x}$ & $\mathrm{y}$ & $\mathrm{z}$ & $\mathrm{U}(\mathrm{eq})$ \\
\hline $\mathrm{Fe}(1)$ & $7869(1)$ & $2869(1)$ & 2131(1) & $31(1)$ \\
\hline $\mathrm{O}(1)$ & $8349(3)$ & $3349(3)$ & $1651(3)$ & $69(4)$ \\
\hline $\mathrm{N}(1)$ & $7268(3)$ & $2268(3)$ & $2732(3)$ & $28(3)$ \\
\hline $\mathrm{N}(2)$ & $8086(3)$ & 2066(3) & $1861(3)$ & $33(2)$ \\
\hline $\mathrm{C}(1)$ & $7553(3)$ & $1676(3)$ & $2712(3)$ & $27(2)$ \\
\hline $\mathrm{C}(2)$ & 7959(3) & $1582(3)$ & $2237(3)$ & $27(2)$ \\
\hline$C(3)$ & $8223(4)$ & $1002(4)$ & $2189(4)$ & $40(2)$ \\
\hline $\mathrm{C}(4)$ & $8062(4)$ & $567(4)$ & $2596(5)$ & $49(3)$ \\
\hline$C(5)$ & $7684(4)$ & $658(4)$ & $3049(5)$ & $43(2)$ \\
\hline $\mathrm{C}(6)$ & $7417(4)$ & $1234(4)$ & $3112(4)$ & $34(2)$ \\
\hline$C(7)$ & $8536(4)$ & 1991(4) & $1434(4)$ & $35(2)$ \\
\hline $\mathrm{C}(8)$ & $8413(4)$ & $2053(6)$ & $839(4)$ & $62(3)$ \\
\hline $\mathrm{C}(9)$ & $8849(4)$ & $2040(5)$ & $399(4)$ & $54(3)$ \\
\hline$C(10)$ & $9444(4)$ & $1939(4)$ & $539(4)$ & $41(2)$ \\
\hline $\mathrm{C}(11)$ & $9576(4)$ & 1891(4) & $1132(4)$ & $42(2)$ \\
\hline$C(12)$ & $9134(3)$ & 1914(4) & $1576(4)$ & $35(2)$ \\
\hline$C(13)$ & $9924(4)$ & 1894(4) & $41(5)$ & $46(2)$ \\
\hline $\mathrm{K}(1)$ & $9837(1)$ & $4837(1)$ & 163(1) & $118(2)$ \\
\hline
\end{tabular}




\begin{tabular}{lrrrr}
$\mathrm{N}(1 \mathrm{~S})$ & $8534(6)$ & $6026(15)$ & $1129(6)$ & $320(20)$ \\
$\mathrm{O}(1 \mathrm{~S})$ & $9401(11)$ & $5650(20)$ & $500(12)$ & $440(20)$ \\
$\mathrm{C}(1 \mathrm{~S})$ & $9033(16)$ & $5640(20)$ & $892(15)$ & $440(20)$ \\
$\mathrm{C}(2 \mathrm{~S})$ & $8360(30)$ & $6574(13)$ & $910(30)$ & $670(90)$ \\
$\mathrm{C}(3 \mathrm{~S})$ & $8352(7)$ & $5605(9)$ & $1703(7)$ & $122(7)$ \\
$\mathrm{O}(2 \mathrm{~S})$ & $8951(4)$ & $4251(4)$ & $1661(5)$ & $92(3)$ \\
$\mathrm{C}(14)$ & $10359(17)$ & $2363(17)$ & $100(19)$ & $117(11)$ \\
$\mathrm{C}(15)$ & $9662(9)$ & $1789(9)$ & $-563(9)$ & $51(5)$ \\
$\mathrm{C}(16)$ & $10208(19)$ & $1265(15)$ & $133(18)$ & $129(12)$ \\
$\mathrm{C}\left(14^{\prime}\right)$ & $10039(11)$ & $2571(9)$ & $-152(11)$ & $64(6)$ \\
$\mathrm{C}\left(15^{\prime}\right)$ & $9875(14)$ & $1397(13)$ & $-313(13)$ & $87(8)$ \\
$\mathrm{C}\left(16^{\prime}\right)$ & $10543(10)$ & $1771(10)$ & $357(11)$ & $65(6)$ \\
\hline
\end{tabular}


Table S26. Bond Distances, $\AA$, for $\left[\left(\mathrm{L}^{1}\right) \mathrm{Fe}(\mathrm{III})-\mathrm{OH}\right]\left[\mathrm{K}(\mathrm{DMF})_{3}\right] \cdot 3 \mathrm{H}_{2} \mathrm{O}$ (3)

\begin{tabular}{|c|c|c|c|}
\hline $\mathrm{Fe}(1)-\mathrm{O}(1)$ & $1.856(11)$ & $\mathrm{C}(11)-\mathrm{H}(11 \mathrm{~A})$ & 0.9500 \\
\hline $\mathrm{Fe}(1)-\mathrm{N}(2) \# 1$ & $1.952(6)$ & $\mathrm{C}(12)-\mathrm{H}(12 \mathrm{~A})$ & 0.9500 \\
\hline $\mathrm{Fe}(1)-\mathrm{N}(2)$ & $1.952(6)$ & $C(13)-C\left(15^{\prime}\right)$ & $1.37(3)$ \\
\hline $\mathrm{Fe}(1)-\mathrm{N}(2) \# 2$ & $1.953(6)$ & $C(13)-C(14)$ & $1.43(4)$ \\
\hline $\mathrm{Fe}(1)-\mathrm{N}(1)$ & $2.321(11)$ & $\mathrm{C}(13)-\mathrm{C}(15)$ & $1.49(2)$ \\
\hline $\mathrm{N}(1)-\mathrm{C}(1) \# 1$ & $1.467(8)$ & $C(13)-C(16)$ & $1.55(4)$ \\
\hline $\mathrm{N}(1)-\mathrm{C}(1)$ & $1.467(8)$ & $\mathrm{C}(13)-\mathrm{C}\left(16^{\prime}\right)$ & $1.57(3)$ \\
\hline $\mathrm{N}(1)-\mathrm{C}(1) \# 2$ & $1.467(8)$ & $C(13)-C\left(14^{\prime}\right)$ & $1.59(2)$ \\
\hline $\mathrm{N}(2)-\mathrm{C}(7)$ & $1.394(10)$ & $\mathrm{K}(1)-\mathrm{K}(1) \# 3$ & $1.259(11)$ \\
\hline $\mathrm{N}(2)-\mathrm{C}(2)$ & $1.396(10)$ & $\mathrm{K}(1)-\mathrm{O}(1 \mathrm{~S}) \# 2$ & $2.19(4)$ \\
\hline$C(1)-C(6)$ & $1.365(11)$ & $\mathrm{K}(1)-\mathrm{O}(1 \mathrm{~S})$ & $2.19(4)$ \\
\hline $\mathrm{C}(1)-\mathrm{C}(2)$ & $1.411(11)$ & $\mathrm{K}(1)-\mathrm{O}(1 \mathrm{~S}) \# 1$ & $2.19(4)$ \\
\hline$C(2)-C(3)$ & $1.426(11)$ & $\mathrm{K}(1)-\mathrm{O}(1 \mathrm{~S}) \# 4$ & $2.50(2)$ \\
\hline$C(3)-C(4)$ & $1.379(14)$ & $\mathrm{K}(1)-\mathrm{O}(1 \mathrm{~S}) \# 5$ & $2.50(2)$ \\
\hline $\mathrm{C}(3)-\mathrm{H}(3 \mathrm{~A})$ & 0.9500 & $\mathrm{~K}(1)-\mathrm{O}(1 \mathrm{~S}) \# 3$ & $2.50(2)$ \\
\hline$C(4)-C(5)$ & $1.333(13)$ & $\mathrm{K}(1)-\mathrm{C}(1 \mathrm{~S}) \# 2$ & $3.02(3)$ \\
\hline $\mathrm{C}(4)-\mathrm{H}(4 \mathrm{~A})$ & 0.9500 & $K(1)-C(1 S)$ & $3.02(3)$ \\
\hline$C(5)-C(6)$ & $1.424(12)$ & $\mathrm{K}(1)-\mathrm{C}(1 \mathrm{~S}) \# 1$ & $3.02(3)$ \\
\hline $\mathrm{C}(5)-\mathrm{H}(5 \mathrm{~A})$ & 0.9500 & $\mathrm{~N}(1 \mathrm{~S})-\mathrm{C}(2 \mathrm{~S})$ & $1.37(4)$ \\
\hline $\mathrm{C}(6)-\mathrm{H}(6 \mathrm{~A})$ & 0.9500 & $\mathrm{~N}(1 \mathrm{~S})-\mathrm{C}(1 \mathrm{~S})$ & $1.498(5)$ \\
\hline $\mathrm{C}(7)-\mathrm{C}(8)$ & $1.362(13)$ & $\mathrm{N}(1 \mathrm{~S})-\mathrm{C}(3 \mathrm{~S})$ & $1.64(3)$ \\
\hline
\end{tabular}




$\begin{array}{lrll}\mathrm{C}(7)-\mathrm{C}(12) & 1.383(12) & \mathrm{O}(1 \mathrm{~S})-\mathrm{C}(1 \mathrm{~S}) & 1.200(5) \\ \mathrm{C}(8)-\mathrm{C}(9) & 1.381(13) & \mathrm{O}(1 \mathrm{~S})-\mathrm{K}(1) \mathrm{H} 3 & 2.50(2) \\ \mathrm{C}(8)-\mathrm{H}(8 \mathrm{~A}) & 0.9500 & \mathrm{C}(1 \mathrm{~S})-\mathrm{H}(1 \mathrm{SA}) & 0.9500 \\ \mathrm{C}(9)-\mathrm{C}(10) & 1.383(14) & \mathrm{C}(2 \mathrm{~S})-\mathrm{H}(2 \mathrm{SA}) & 0.9800 \\ \mathrm{C}(9)-\mathrm{H}(9 \mathrm{~A}) & 0.9500 & \mathrm{C}(2 \mathrm{~S})-\mathrm{H}(2 \mathrm{SB}) & 0.9800 \\ \mathrm{C}(10)-\mathrm{C}(11) & 1.358(14) & \mathrm{C}(2 \mathrm{~S})-\mathrm{H}(2 \mathrm{SC}) & 0.9800 \\ \mathrm{C}(10)-\mathrm{C}(13) & 1.547(12) & \mathrm{C}(3 \mathrm{~S})-\mathrm{H}(3 \mathrm{SA}) & 0.9800 \\ \mathrm{C}(11)-\mathrm{C}(12) & 1.399(12) & \mathrm{C}(3 \mathrm{~S})-\mathrm{H}(3 \mathrm{SB}) & 0.9800 \\ \mathrm{C}(3 \mathrm{~S})-\mathrm{H}(3 \mathrm{SC}) & 0.9800 & \mathrm{C}\left(14^{\prime}\right)-\mathrm{H}(14 \mathrm{D}) & 0.9800 \\ \mathrm{C}(14)-\mathrm{H}(14 \mathrm{~A}) & 0.9800 & \mathrm{C}\left(14^{\prime}\right)-\mathrm{H}(14 \mathrm{E}) & 0.9800 \\ \mathrm{C}(14)-\mathrm{H}(14 \mathrm{~B}) & 0.9800 & \mathrm{C}\left(14^{\prime}\right)-\mathrm{H}(14 \mathrm{~F}) & 0.9800 \\ \mathrm{C}(14)-\mathrm{H}(14 \mathrm{C}) & 0.9800 & \mathrm{C}\left(15^{\prime}\right)-\mathrm{H}(15 \mathrm{D}) & 0.9800 \\ \mathrm{C}(15)-\mathrm{H}(15 \mathrm{~A}) & 0.9800 & \mathrm{C}\left(15^{\prime}\right)-\mathrm{H}(15 \mathrm{E}) & 0.9800 \\ \mathrm{C}(15)-\mathrm{H}(15 \mathrm{~B}) & 0.9800 & \mathrm{C}\left(15^{\prime}\right)-\mathrm{H}(15 \mathrm{~F}) & 0.9800 \\ \mathrm{C}(15)-\mathrm{H}(15 \mathrm{C}) & 0.9800 & \mathrm{C}\left(16^{\prime}\right)-\mathrm{H}(16 \mathrm{D}) & 0.9800 \\ \mathrm{C}(16)-\mathrm{H}(16 \mathrm{~A}) & 0.9800 & \mathrm{C}\left(16^{\prime}\right)-\mathrm{H}(16 \mathrm{E}) & 0.9800 \\ \mathrm{C}(16)-\mathrm{H}(16 \mathrm{~B}) & 0.9800 & \mathrm{C}\left(16^{\prime}\right)-\mathrm{H}(16 \mathrm{~F}) & 0.9800 \\ \mathrm{C}(16)-\mathrm{H}(16 \mathrm{C}) & 0.9800 & & \\ & & & \\ & & & \\ & & & \end{array}$

Symmetry transformations used to generate equivalent atoms:

$\# 1-z+1, x-1 / 2,-y+1 / 2 \quad \# 2 y+1 / 2,-z+1 / 2,-x+1 \quad \# 3-x+2,-y+1,-z$

$\# 4 \mathrm{z}+1,-\mathrm{x}+3 / 2, \mathrm{y}-1 / 2 \quad \# 5-\mathrm{y}+3 / 2, \mathrm{z}+1 / 2, x-1$ 
Table S27. Angles, ${ }^{\circ}$, for $\left[\left(\mathrm{L}^{1}\right) \mathrm{Fe}(\mathrm{III})-\mathrm{OH}\right]\left[\mathrm{K}(\mathrm{DMF})_{3}\right] \cdot 3 \mathrm{H}_{2} \mathrm{O}(\mathbf{3})$

\begin{tabular}{|c|c|c|c|}
\hline $\mathrm{O}(1)-\mathrm{Fe}(1)-\mathrm{N}(2) \# 1$ & $101.98(18)$ & $\mathrm{K}(1) \# 3-\mathrm{K}(1)-\mathrm{O}(1 \mathrm{~S}) \# 3$ & $61.1(9)$ \\
\hline $\mathrm{O}(1)-\mathrm{Fe}(1)-\mathrm{N}(2)$ & $101.98(18)$ & $\mathrm{O}(1 \mathrm{~S}) \# 2-\mathrm{K}(1)-\mathrm{O}(1 \mathrm{~S}) \# 3$ & $63.4(6)$ \\
\hline $\mathrm{N}(2) \# 1-\mathrm{Fe}(1)-\mathrm{N}(2)$ & $115.81(12)$ & $\mathrm{O}(1 \mathrm{~S})-\mathrm{K}(1)-\mathrm{O}(1 \mathrm{~S}) \# 3$ & $149.8(4)$ \\
\hline $\mathrm{O}(1)-\mathrm{Fe}(1)-\mathrm{N}(2) \# 2$ & $101.98(18)$ & $\mathrm{O}(1 \mathrm{~S}) \# 1-\mathrm{K}(1)-\mathrm{O}(1 \mathrm{~S}) \# 3$ & $63.4(6)$ \\
\hline $\mathrm{N}(2) \# 1-\mathrm{Fe}(1)-\mathrm{N}(2) \# 2$ & $115.81(12)$ & $\mathrm{O}(1 \mathrm{~S}) \# 4-\mathrm{K}(1)-\mathrm{O}(1 \mathrm{~S}) \# 3$ & $98.6(11)$ \\
\hline $\mathrm{N}(2)-\mathrm{Fe}(1)-\mathrm{N}(2) \# 2$ & $115.81(12)$ & $\mathrm{O}(1 \mathrm{~S}) \# 5-\mathrm{K}(1)-\mathrm{O}(1 \mathrm{~S}) \# 3$ & $98.6(11)$ \\
\hline $\mathrm{O}(1)-\mathrm{Fe}(1)-\mathrm{N}(1)$ & $180.0(5)$ & $\mathrm{K}(1) \# 3-\mathrm{K}(1)-\mathrm{C}(1 \mathrm{~S}) \# 2$ & $108.1(8)$ \\
\hline $\mathrm{N}(2) \# 1-\mathrm{Fe}(1)-\mathrm{N}(1)$ & $78.02(18)$ & $\mathrm{O}(1 \mathrm{~S}) \# 2-\mathrm{K}(1)-\mathrm{C}(1 \mathrm{~S}) \# 2$ & $19.4(8)$ \\
\hline $\mathrm{N}(2)-\mathrm{Fe}(1)-\mathrm{N}(1)$ & $78.02(18)$ & $\mathrm{O}(1 \mathrm{~S})-\mathrm{K}(1)-\mathrm{C}(1 \mathrm{~S}) \# 2$ & $120.5(12)$ \\
\hline $\mathrm{N}(2) \# 2-\mathrm{Fe}(1)-\mathrm{N}(1)$ & $78.02(18)$ & $\mathrm{O}(1 \mathrm{~S}) \# 1-\mathrm{K}(1)-\mathrm{C}(1 \mathrm{~S}) \# 2$ & $117.2(13)$ \\
\hline $\mathrm{C}(1) \# 1-\mathrm{N}(1)-\mathrm{C}(1)$ & $113.8(4)$ & $\mathrm{O}(1 \mathrm{~S}) \# 4-\mathrm{K}(1)-\mathrm{C}(1 \mathrm{~S}) \# 2$ & $75.9(11)$ \\
\hline $\mathrm{C}(1) \# 1-\mathrm{N}(1)-\mathrm{C}(1) \# 2$ & $113.8(4)$ & $\mathrm{O}(1 \mathrm{~S}) \# 5-\mathrm{K}(1)-\mathrm{C}(1 \mathrm{~S}) \# 2$ & $169.0(12)$ \\
\hline $\mathrm{C}(1)-\mathrm{N}(1)-\mathrm{C}(1) \# 2$ & $113.8(4)$ & $\mathrm{O}(1 \mathrm{~S}) \# 3-\mathrm{K}(1)-\mathrm{C}(1 \mathrm{~S}) \# 2$ & $73.3(12)$ \\
\hline $\mathrm{C}(1) \# 1-\mathrm{N}(1)-\mathrm{Fe}(1)$ & $104.6(5)$ & $\mathrm{K}(1) \# 3-\mathrm{K}(1)-\mathrm{C}(1 \mathrm{~S})$ & $108.1(8)$ \\
\hline $\mathrm{C}(1)-\mathrm{N}(1)-\mathrm{Fe}(1)$ & $104.6(5)$ & $\mathrm{O}(1 \mathrm{~S}) \# 2-\mathrm{K}(1)-\mathrm{C}(1 \mathrm{~S})$ & $117.2(13)$ \\
\hline $\mathrm{C}(1) \# 2-\mathrm{N}(1)-\mathrm{Fe}(1)$ & $104.6(5)$ & $\mathrm{O}(1 \mathrm{~S})-\mathrm{K}(1)-\mathrm{C}(1 \mathrm{~S})$ & $19.4(8)$ \\
\hline $\mathrm{C}(7)-\mathrm{N}(2)-\mathrm{C}(2)$ & $117.7(7)$ & $\mathrm{O}(1 \mathrm{~S}) \# 1-\mathrm{K}(1)-\mathrm{C}(1 \mathrm{~S})$ & $120.5(12)$ \\
\hline $\mathrm{C}(7)-\mathrm{N}(2)-\mathrm{Fe}(1)$ & $120.0(5)$ & $\mathrm{O}(1 \mathrm{~S}) \# 4-\mathrm{K}(1)-\mathrm{C}(1 \mathrm{~S})$ & $73.2(12)$ \\
\hline $\mathrm{C}(2)-\mathrm{N}(2)-\mathrm{Fe}(1)$ & $118.2(5)$ & $\mathrm{O}(1 \mathrm{~S}) \# 5-\mathrm{K}(1)-\mathrm{C}(1 \mathrm{~S})$ & $75.9(11)$ \\
\hline$C(6)-C(1)-C(2)$ & $121.8(7)$ & $\mathrm{O}(1 \mathrm{~S}) \# 3-\mathrm{K}(1)-\mathrm{C}(1 \mathrm{~S})$ & $169.0(12)$ \\
\hline $\mathrm{C}(6)-\mathrm{C}(1)-\mathrm{N}(1)$ & $122.4(7)$ & $\mathrm{C}(1 \mathrm{~S}) \# 2-\mathrm{K}(1)-\mathrm{C}(1 \mathrm{~S})$ & $110.9(7)$ \\
\hline
\end{tabular}




\begin{tabular}{|c|c|c|c|}
\hline $\mathrm{C}(2)-\mathrm{C}(1)-\mathrm{N}(1)$ & $115.7(7)$ & $\mathrm{K}(1) \# 3-\mathrm{K}(1)-\mathrm{C}(1 \mathrm{~S}) \# 1$ & $108.0(8)$ \\
\hline $\mathrm{N}(2)-\mathrm{C}(2)-\mathrm{C}(1)$ & $117.9(7)$ & $\mathrm{O}(1 \mathrm{~S}) \# 2-\mathrm{K}(1)-\mathrm{C}(1 \mathrm{~S}) \# 1$ & $120.5(12)$ \\
\hline $\mathrm{N}(2)-\mathrm{C}(2)-\mathrm{C}(3)$ & $124.9(7)$ & $\mathrm{O}(1 \mathrm{~S})-\mathrm{K}(1)-\mathrm{C}(1 \mathrm{~S}) \# 1$ & $117.2(13)$ \\
\hline $\mathrm{C}(1)-\mathrm{C}(2)-\mathrm{C}(3)$ & $117.2(7)$ & $\mathrm{O}(1 \mathrm{~S}) \# 1-\mathrm{K}(1)-\mathrm{C}(1 \mathrm{~S}) \# 1$ & $19.4(8)$ \\
\hline$C(4)-C(3)-C(2)$ & $118.8(8)$ & $\mathrm{O}(1 \mathrm{~S}) \# 4-\mathrm{K}(1)-\mathrm{C}(1 \mathrm{~S}) \# 1$ & $169.0(12)$ \\
\hline $\mathrm{C}(4)-\mathrm{C}(3)-\mathrm{H}(3 \mathrm{~A})$ & 120.6 & $\mathrm{O}(1 \mathrm{~S}) \# 5-\mathrm{K}(1)-\mathrm{C}(1 \mathrm{~S}) \# 1$ & $73.2(12)$ \\
\hline $\mathrm{C}(2)-\mathrm{C}(3)-\mathrm{H}(3 \mathrm{~A})$ & 120.6 & $\mathrm{O}(1 \mathrm{~S}) \# 3-\mathrm{K}(1)-\mathrm{C}(1 \mathrm{~S}) \# 1$ & $75.9(11)$ \\
\hline$C(5)-C(4)-C(3)$ & $123.9(10)$ & $\mathrm{C}(1 \mathrm{~S}) \# 2-\mathrm{K}(1)-\mathrm{C}(1 \mathrm{~S}) \# 1$ & $110.9(7)$ \\
\hline $\mathrm{C}(5)-\mathrm{C}(4)-\mathrm{H}(4 \mathrm{~A})$ & 118.0 & $\mathrm{C}(1 \mathrm{~S})-\mathrm{K}(1)-\mathrm{C}(1 \mathrm{~S}) \# 1$ & $110.9(7)$ \\
\hline $\mathrm{C}(3)-\mathrm{C}(4)-\mathrm{H}(4 \mathrm{~A})$ & 118.0 & $\mathrm{C}(2 \mathrm{~S})-\mathrm{N}(1 \mathrm{~S})-\mathrm{C}(1 \mathrm{~S})$ & $127(4)$ \\
\hline$C(4)-C(5)-C(6)$ & $118.5(9)$ & $\mathrm{C}(2 \mathrm{~S})-\mathrm{N}(1 \mathrm{~S})-\mathrm{C}(3 \mathrm{~S})$ & $136(4)$ \\
\hline $\mathrm{C}(4)-\mathrm{C}(5)-\mathrm{H}(5 \mathrm{~A})$ & 120.7 & $\mathrm{C}(1 \mathrm{~S})-\mathrm{N}(1 \mathrm{~S})-\mathrm{C}(3 \mathrm{~S})$ & $97.7(16)$ \\
\hline $\mathrm{C}(6)-\mathrm{C}(5)-\mathrm{H}(5 \mathrm{~A})$ & 120.7 & $\mathrm{C}(1 \mathrm{~S})-\mathrm{O}(1 \mathrm{~S})-\mathrm{K}(1)$ & $123(3)$ \\
\hline$C(1)-C(6)-C(5)$ & 119.7(8) & $\mathrm{C}(1 \mathrm{~S})-\mathrm{O}(1 \mathrm{~S})-\mathrm{K}(1) \# 3$ & $153(3)$ \\
\hline $\mathrm{C}(1)-\mathrm{C}(6)-\mathrm{H}(6 \mathrm{~A})$ & 120.2 & $\mathrm{~K}(1)-\mathrm{O}(1 \mathrm{~S})-\mathrm{K}(1) \# 3$ & $30.2(4)$ \\
\hline $\mathrm{C}(5)-\mathrm{C}(6)-\mathrm{H}(6 \mathrm{~A})$ & 120.2 & $\mathrm{O}(1 \mathrm{~S})-\mathrm{C}(1 \mathrm{~S})-\mathrm{N}(1 \mathrm{~S})$ & $140(3)$ \\
\hline $\mathrm{C}(8)-\mathrm{C}(7)-\mathrm{C}(12)$ & $115.5(7)$ & $\mathrm{O}(1 \mathrm{~S})-\mathrm{C}(1 \mathrm{~S})-\mathrm{K}(1)$ & $37(2)$ \\
\hline $\mathrm{C}(8)-\mathrm{C}(7)-\mathrm{N}(2)$ & $120.6(8)$ & $\mathrm{N}(1 \mathrm{~S})-\mathrm{C}(1 \mathrm{~S})-\mathrm{K}(1)$ & $166(3)$ \\
\hline $\mathrm{C}(12)-\mathrm{C}(7)-\mathrm{N}(2)$ & $123.6(8)$ & $\mathrm{O}(1 \mathrm{~S})-\mathrm{C}(1 \mathrm{~S})-\mathrm{H}(1 \mathrm{SA})$ & 110.2 \\
\hline $\mathrm{C}(7)-\mathrm{C}(8)-\mathrm{C}(9)$ & $123.2(8)$ & $\mathrm{N}(1 \mathrm{~S})-\mathrm{C}(1 \mathrm{~S})-\mathrm{H}(1 \mathrm{SA})$ & 110.2 \\
\hline $\mathrm{C}(7)-\mathrm{C}(8)-\mathrm{H}(8 \mathrm{~A})$ & 118.4 & $\mathrm{~K}(1)-\mathrm{C}(1 \mathrm{~S})-\mathrm{H}(1 \mathrm{SA})$ & 75.3 \\
\hline $\mathrm{C}(9)-\mathrm{C}(8)-\mathrm{H}(8 \mathrm{~A})$ & 118.4 & $\mathrm{~N}(1 \mathrm{~S})-\mathrm{C}(2 \mathrm{~S})-\mathrm{H}(2 \mathrm{SA})$ & 109.5 \\
\hline
\end{tabular}




\begin{tabular}{|c|c|c|c|}
\hline$C(8)-C(9)-C(10)$ & 121.3(9) & $\mathrm{N}(1 \mathrm{~S})-\mathrm{C}(2 \mathrm{~S})-\mathrm{H}(2 \mathrm{SB})$ & 109.5 \\
\hline $\mathrm{C}(8)-\mathrm{C}(9)-\mathrm{H}(9 \mathrm{~A})$ & 119.4 & $\mathrm{H}(2 \mathrm{SA})-\mathrm{C}(2 \mathrm{~S})-\mathrm{H}(2 \mathrm{SB})$ & 109.5 \\
\hline $\mathrm{C}(10)-\mathrm{C}(9)-\mathrm{H}(9 \mathrm{~A})$ & 119.4 & $\mathrm{~N}(1 \mathrm{~S})-\mathrm{C}(2 \mathrm{~S})-\mathrm{H}(2 \mathrm{SC})$ & 109.5 \\
\hline$C(11)-C(10)-C(9)$ & $116.1(7)$ & $\mathrm{H}(2 \mathrm{SA})-\mathrm{C}(2 \mathrm{~S})-\mathrm{H}(2 \mathrm{SC})$ & 109.5 \\
\hline$C(11)-C(10)-C(13)$ & $123.0(9)$ & $\mathrm{H}(2 \mathrm{SB})-\mathrm{C}(2 \mathrm{~S})-\mathrm{H}(2 \mathrm{SC})$ & 109.5 \\
\hline$C(9)-C(10)-C(13)$ & 120.9(9) & $\mathrm{N}(1 \mathrm{~S})-\mathrm{C}(3 \mathrm{~S})-\mathrm{H}(3 \mathrm{SA})$ & 109.5 \\
\hline $\mathrm{C}(10)-\mathrm{C}(11)-\mathrm{C}(12)$ & $122.3(8)$ & $\mathrm{N}(1 \mathrm{~S})-\mathrm{C}(3 \mathrm{~S})-\mathrm{H}(3 \mathrm{SB})$ & 109.5 \\
\hline $\mathrm{C}(10)-\mathrm{C}(11)-\mathrm{H}(11 \mathrm{~A})$ & 118.8 & $\mathrm{H}(3 \mathrm{SA})-\mathrm{C}(3 \mathrm{~S})-\mathrm{H}(3 \mathrm{SB})$ & 109.5 \\
\hline $\mathrm{C}(12)-\mathrm{C}(11)-\mathrm{H}(11 \mathrm{~A})$ & 118.8 & $\mathrm{~N}(1 \mathrm{~S})-\mathrm{C}(3 \mathrm{~S})-\mathrm{H}(3 \mathrm{SC})$ & 109.5 \\
\hline$C(7)-C(12)-C(11)$ & $121.4(8)$ & $\mathrm{H}(3 \mathrm{SA})-\mathrm{C}(3 \mathrm{~S})-\mathrm{H}(3 \mathrm{SC})$ & 109.5 \\
\hline $\mathrm{C}(7)-\mathrm{C}(12)-\mathrm{H}(12 \mathrm{~A})$ & 119.3 & $\mathrm{H}(3 \mathrm{SB})-\mathrm{C}(3 \mathrm{~S})-\mathrm{H}(3 \mathrm{SC})$ & 109.5 \\
\hline $\mathrm{C}(11)-\mathrm{C}(12)-\mathrm{H}(12 \mathrm{~A})$ & 119.3 & $\mathrm{C}(13)-\mathrm{C}(14)-\mathrm{H}(14 \mathrm{~A})$ & 109.5 \\
\hline $\mathrm{C}\left(15^{\prime}\right)-\mathrm{C}(13)-\mathrm{C}(14)$ & $134(2)$ & $\mathrm{C}(13)-\mathrm{C}(14)-\mathrm{H}(14 \mathrm{~B})$ & 109.5 \\
\hline$C\left(15^{\prime}\right)-C(13)-C(15)$ & $46.9(14)$ & $\mathrm{H}(14 \mathrm{~A})-\mathrm{C}(14)-\mathrm{H}(14 \mathrm{~B})$ & 109.5 \\
\hline$C(14)-C(13)-C(15)$ & 117.6(19) & $\mathrm{C}(13)-\mathrm{C}(14)-\mathrm{H}(14 \mathrm{C})$ & 109.5 \\
\hline$C\left(15^{\prime}\right)-C(13)-C(10)$ & $114.5(14)$ & $\mathrm{H}(14 \mathrm{~A})-\mathrm{C}(14)-\mathrm{H}(14 \mathrm{C})$ & 109.5 \\
\hline$C(14)-C(13)-C(10)$ & $110.8(18)$ & $\mathrm{H}(14 \mathrm{~B})-\mathrm{C}(14)-\mathrm{H}(14 \mathrm{C})$ & 109.5 \\
\hline$C(15)-C(13)-C(10)$ & $113.0(11)$ & $\mathrm{C}(13)-\mathrm{C}(15)-\mathrm{H}(15 \mathrm{~A})$ & 109.5 \\
\hline$C\left(15^{\prime}\right)-C(13)-C(16)$ & $51.3(16)$ & $\mathrm{C}(13)-\mathrm{C}(15)-\mathrm{H}(15 \mathrm{~B})$ & 109.5 \\
\hline$C(14)-C(13)-C(16)$ & $112(2)$ & $\mathrm{H}(15 \mathrm{~A})-\mathrm{C}(15)-\mathrm{H}(15 \mathrm{~B})$ & 109.5 \\
\hline$C(15)-C(13)-C(16)$ & $97.9(17)$ & $\mathrm{C}(13)-\mathrm{C}(15)-\mathrm{H}(15 \mathrm{C})$ & 109.5 \\
\hline $\mathrm{C}(10)-\mathrm{C}(13)-\mathrm{C}(16)$ & $104.3(17)$ & $\mathrm{H}(15 \mathrm{~A})-\mathrm{C}(15)-\mathrm{H}(15 \mathrm{C})$ & 109.5 \\
\hline
\end{tabular}




\begin{tabular}{|c|c|c|c|}
\hline $\mathrm{C}\left(15^{\prime}\right)-\mathrm{C}(13)-\mathrm{C}\left(16^{\prime}\right)$ & $100.7(17)$ & $\mathrm{H}(15 \mathrm{~B})-\mathrm{C}(15)-\mathrm{H}(15 \mathrm{C})$ & 109.5 \\
\hline$C(14)-C(13)-C\left(16^{\prime}\right)$ & $59.5(17)$ & $C(13)-C(16)-H(16 A)$ & 109.5 \\
\hline$C(15)-C(13)-C\left(16^{\prime}\right)$ & $136.2(13)$ & $\mathrm{C}(13)-\mathrm{C}(16)-\mathrm{H}(16 \mathrm{~B})$ & 109.5 \\
\hline $\mathrm{C}(10)-\mathrm{C}(13)-\mathrm{C}\left(16^{\prime}\right)$ & $107.3(12)$ & $\mathrm{H}(16 \mathrm{~A})-\mathrm{C}(16)-\mathrm{H}(16 \mathrm{~B})$ & 109.5 \\
\hline $\mathrm{C}(16)-\mathrm{C}(13)-\mathrm{C}\left(16^{\prime}\right)$ & $54.9(15)$ & $\mathrm{C}(13)-\mathrm{C}(16)-\mathrm{H}(16 \mathrm{C})$ & 109.5 \\
\hline $\mathrm{C}\left(15^{\prime}\right)-\mathrm{C}(13)-\mathrm{C}\left(14^{\prime}\right)$ & $128.8(17)$ & $\mathrm{H}(16 \mathrm{~A})-\mathrm{C}(16)-\mathrm{H}(16 \mathrm{C})$ & 109.5 \\
\hline $\mathrm{C}(14)-\mathrm{C}(13)-\mathrm{C}\left(14^{\prime}\right)$ & $39.0(16)$ & H(16B)-C(16)-H(16C) & 109.5 \\
\hline$C(15)-C(13)-C\left(14^{\prime}\right)$ & $88.1(13)$ & $\mathrm{C}(13)-\mathrm{C}\left(14^{\prime}\right)-\mathrm{H}(14 \mathrm{D})$ & 109.5 \\
\hline $\mathrm{C}(10)-\mathrm{C}(13)-\mathrm{C}\left(14^{\prime}\right)$ & $104.2(10)$ & $\mathrm{C}(13)-\mathrm{C}\left(14^{\prime}\right)-\mathrm{H}(14 \mathrm{E})$ & 109.5 \\
\hline $\mathrm{C}(16)-\mathrm{C}(13)-\mathrm{C}\left(14^{\prime}\right)$ & $145.8(19)$ & $\mathrm{H}(14 \mathrm{D})-\mathrm{C}\left(14^{\prime}\right)-\mathrm{H}(14 \mathrm{E})$ & 109.5 \\
\hline$C\left(16^{\prime}\right)-C(13)-C\left(14^{\prime}\right)$ & $98.4(13)$ & $\mathrm{C}(13)-\mathrm{C}\left(14^{\prime}\right)-\mathrm{H}(14 \mathrm{~F})$ & 109.5 \\
\hline $\mathrm{K}(1) \# 3-\mathrm{K}(1)-\mathrm{O}(1 \mathrm{~S}) \# 2$ & $88.7(7)$ & H(14D)-C(14')-H(14F) & 109.5 \\
\hline $\mathrm{K}(1) \# 3-\mathrm{K}(1)-\mathrm{O}(1 \mathrm{~S})$ & $88.7(7)$ & $\mathrm{H}(14 \mathrm{E})-\mathrm{C}\left(14{ }^{\prime}\right)-\mathrm{H}(14 \mathrm{~F})$ & 109.5 \\
\hline $\mathrm{O}(1 \mathrm{~S}) \# 2-\mathrm{K}(1)-\mathrm{O}(1 \mathrm{~S})$ & $119.95(6)$ & $\mathrm{C}(13)-\mathrm{C}\left(15^{\prime}\right)-\mathrm{H}(15 \mathrm{D})$ & 109.5 \\
\hline $\mathrm{K}(1) \# 3-\mathrm{K}(1)-\mathrm{O}(1 \mathrm{~S}) \# 1$ & $88.7(7)$ & $\mathrm{C}(13)-\mathrm{C}\left(15^{\prime}\right)-\mathrm{H}(15 \mathrm{E})$ & 109.5 \\
\hline $\mathrm{O}(1 \mathrm{~S}) \# 2-\mathrm{K}(1)-\mathrm{O}(1 \mathrm{~S}) \# 1$ & $119.95(6)$ & $\mathrm{H}(15 \mathrm{D})-\mathrm{C}\left(15^{\prime}\right)-\mathrm{H}(15 \mathrm{E})$ & 109.5 \\
\hline $\mathrm{O}(1 \mathrm{~S})-\mathrm{K}(1)-\mathrm{O}(1 \mathrm{~S}) \# 1$ & $119.95(6)$ & $\mathrm{C}(13)-\mathrm{C}\left(15^{\prime}\right)-\mathrm{H}(15 \mathrm{~F})$ & 109.5 \\
\hline $\mathrm{K}(1) \# 3-\mathrm{K}(1)-\mathrm{O}(1 \mathrm{~S}) \# 4$ & $61.1(9)$ & $\mathrm{H}(15 \mathrm{D})-\mathrm{C}\left(15^{\prime}\right)-\mathrm{H}(15 \mathrm{~F})$ & 109.5 \\
\hline $\mathrm{O}(1 \mathrm{~S}) \# 2-\mathrm{K}(1)-\mathrm{O}(1 \mathrm{~S}) \# 4$ & $63.4(6)$ & $\mathrm{H}(15 \mathrm{E})-\mathrm{C}\left(15^{\prime}\right)-\mathrm{H}(15 \mathrm{~F})$ & 109.5 \\
\hline $\mathrm{O}(1 \mathrm{~S})-\mathrm{K}(1)-\mathrm{O}(1 \mathrm{~S}) \# 4$ & $63.4(6)$ & $\mathrm{C}(13)-\mathrm{C}\left(16^{\prime}\right)-\mathrm{H}(16 \mathrm{D})$ & 109.5 \\
\hline $\mathrm{O}(1 \mathrm{~S}) \# 1-\mathrm{K}(1)-\mathrm{O}(1 \mathrm{~S}) \# 4$ & $149.8(4)$ & $\mathrm{C}(13)-\mathrm{C}\left(16^{\prime}\right)-\mathrm{H}(16 \mathrm{E})$ & 109.5 \\
\hline $\mathrm{K}(1) \# 3-\mathrm{K}(1)-\mathrm{O}(1 \mathrm{~S}) \# 5$ & $61.1(9)$ & $\mathrm{H}(16 \mathrm{D})-\mathrm{C}\left(16^{\prime}\right)-\mathrm{H}(16 \mathrm{E})$ & 109.5 \\
\hline
\end{tabular}




$\begin{array}{lccr}\mathrm{O}(1 \mathrm{~S}) \# 2-\mathrm{K}(1)-\mathrm{O}(1 \mathrm{~S}) \# 5 & 149.8(4) & \mathrm{C}(13)-\mathrm{C}\left(16^{\prime}\right)-\mathrm{H}(16 \mathrm{~F}) & 109.5 \\ \mathrm{O}(1 \mathrm{~S})-\mathrm{K}(1)-\mathrm{O}(1 \mathrm{~S}) \# 5 & 63.4(6) & \mathrm{H}(16 \mathrm{D})-\mathrm{C}\left(16^{\prime}\right)-\mathrm{H}(16 \mathrm{~F}) & 109.5 \\ \mathrm{O}(1 \mathrm{~S}) \# 1-\mathrm{K}(1)-\mathrm{O}(1 \mathrm{~S}) \# 5 & 63.4(6) & \mathrm{H}(16 \mathrm{E})-\mathrm{C}\left(16^{\prime}\right)-\mathrm{H}(16 \mathrm{~F}) & 109.5 \\ \mathrm{O}(1 \mathrm{~S}) \# 4-\mathrm{K}(1)-\mathrm{O}(1 \mathrm{~S}) \# 5 & 98.6(11) & & \end{array}$

Symmetry transformations used to generate equivalent atoms:

$\# 1-\mathrm{z}+1, \mathrm{x}-1 / 2,-\mathrm{y}+1 / 2 \quad \# 2 \mathrm{y}+1 / 2,-\mathrm{z}+1 / 2,-\mathrm{x}+1 \quad \# 3-\mathrm{x}+2,-\mathrm{y}+1,-\mathrm{z}$

$\# 4 \mathrm{z}+1,-\mathrm{x}+3 / 2, \mathrm{y}-1 / 2 \quad \# 5-\mathrm{y}+3 / 2, \mathrm{z}+1 / 2, \mathrm{x}-1$ 
Table S28. Anisotropic displacement parameters $\left(\AA^{2} \times 10^{3}\right)$ for $\left[\left(\mathrm{L}^{1}\right) \mathrm{Fe}(\mathrm{III})-\right.$ $\mathrm{OH}]\left[\mathrm{K}(\mathrm{DMF})_{3}\right] \cdot 3 \mathrm{H}_{2} \mathrm{O}(\mathbf{3})$. The anisotropic displacement factor exponent takes the form: $-2 \pi^{2}\left[h^{2} a^{* 2} U^{11}+\ldots+2 h k a^{*} b^{*} U^{12}\right]$

\begin{tabular}{|c|c|c|c|c|c|c|}
\hline & $\mathrm{U}^{11}$ & $\mathrm{U}^{22}$ & $\mathrm{U}^{33}$ & $\mathrm{U}^{23}$ & $\mathrm{U}^{13}$ & $\mathrm{U}^{12}$ \\
\hline $\mathrm{Fe}(1)$ & $31(1)$ & $31(1)$ & $31(1)$ & $4(1)$ & $4(1)$ & $-4(1)$ \\
\hline $\mathrm{O}(1)$ & $69(4)$ & $69(4)$ & $69(4)$ & $20(3)$ & $20(3)$ & $-20(3)$ \\
\hline $\mathrm{N}(1)$ & $28(3)$ & $28(3)$ & $28(3)$ & $0(2)$ & $0(2)$ & $0(2)$ \\
\hline $\mathrm{N}(2)$ & $17(3)$ & $44(4)$ & $36(4)$ & $-6(3)$ & 2(3) & $-7(3)$ \\
\hline$C(1)$ & $17(4)$ & $33(4)$ & $29(4)$ & $-4(3)$ & $-3(3)$ & 1(3) \\
\hline $\mathrm{C}(2)$ & $16(4)$ & $24(4)$ & $40(5)$ & $-4(3)$ & $0(3)$ & $-3(3)$ \\
\hline$C(3)$ & $25(4)$ & $27(4)$ & $69(6)$ & $-4(4)$ & $6(4)$ & 2(3) \\
\hline$C(4)$ & $40(5)$ & $40(5)$ & $67(7)$ & $-11(5)$ & $5(5)$ & $2(4)$ \\
\hline$C(5)$ & $44(5)$ & $25(5)$ & $60(6)$ & 2(4) & $-3(5)$ & $-1(4)$ \\
\hline$C(6)$ & $35(5)$ & $23(4)$ & $43(5)$ & 2(4) & $3(4)$ & $0(3)$ \\
\hline$C(7)$ & $32(5)$ & $43(5)$ & $30(5)$ & $-2(4)$ & $0(3)$ & $8(4)$ \\
\hline$C(8)$ & $19(4)$ & $133(10)$ & $35(5)$ & $1(6)$ & $-14(4)$ & $1(5)$ \\
\hline$C(9)$ & $27(5)$ & $110(9)$ & $24(5)$ & $-14(5)$ & $7(4)$ & $1(5)$ \\
\hline$C(10)$ & $40(5)$ & $44(5)$ & $41(6)$ & $-15(4)$ & $15(4)$ & $-18(4)$ \\
\hline$C(11)$ & $24(4)$ & $51(5)$ & $51(6)$ & $-1(4)$ & $9(4)$ & 4(4) \\
\hline$C(12)$ & $22(4)$ & $56(6)$ & $28(4)$ & $-3(4)$ & $8(3)$ & $-7(4)$ \\
\hline$C(13)$ & $33(6)$ & $48(5)$ & $56(7)$ & $-4(5)$ & $18(4)$ & $-2(4)$ \\
\hline $\mathrm{K}(1)$ & $118(2)$ & $118(2)$ & $118(2)$ & $22(2)$ & $22(2)$ & $-22(2)$ \\
\hline
\end{tabular}




\begin{tabular}{lcccccc}
$\mathrm{N}(1 \mathrm{~S})$ & $83(10)$ & $820(60)$ & $62(8)$ & $-180(20)$ & $46(8)$ & $-180(20)$ \\
$\mathrm{O}(1 \mathrm{~S})$ & $200(20)$ & $830(70)$ & $300(30)$ & $270(40)$ & $165(19)$ & $230(30)$ \\
$\mathrm{C}(1 \mathrm{~S})$ & $200(20)$ & $830(70)$ & $300(30)$ & $270(40)$ & $165(19)$ & $230(30)$ \\
$\mathrm{C}(2 \mathrm{~S})$ & $1110(170)$ & $120(20)$ & $770(120)$ & $190(40)$ & $-840(140)$ & $-250(50)$ \\
$\mathrm{C}(3 \mathrm{~S})$ & $78(10)$ & $200(19)$ & $87(11)$ & $-86(12)$ & $24(8)$ & $-51(11)$ \\
$\mathrm{O}(2 \mathrm{~S})$ & $107(7)$ & $61(5)$ & $109(7)$ & $-2(5)$ & $-17(6)$ & $-27(5)$ \\
\hline
\end{tabular}


Table S29. Hydrogen coordinates $\left(\times 10^{4}\right)$ and isotropic displacement parameters $\left(\AA^{2} \times 10^{3}\right)$ for $\left[\left(\mathrm{L}^{1}\right) \mathrm{Fe}(\mathrm{III})-\mathrm{OH}\right]\left[\mathrm{K}(\mathrm{DMF})_{3}\right] \cdot 3 \mathrm{H}_{2} \mathrm{O}(\mathbf{3})$

\begin{tabular}{|c|c|c|c|c|}
\hline & $\mathrm{x}$ & $\mathrm{y}$ & $\mathrm{Z}$ & $\mathrm{U}(\mathrm{eq})$ \\
\hline $\mathrm{H}(3 \mathrm{~A})$ & 8505 & 918 & 1881 & 48 \\
\hline $\mathrm{H}(4 \mathrm{~A})$ & 8230 & 178 & 2551 & 59 \\
\hline $\mathrm{H}(5 \mathrm{~A})$ & 7594 & 345 & 3324 & 51 \\
\hline $\mathrm{H}(6 \mathrm{~A})$ & 7146 & 1310 & 3431 & 40 \\
\hline $\mathrm{H}(8 \mathrm{~A})$ & 8008 & 2108 & 721 & 75 \\
\hline $\mathrm{H}(9 \mathrm{~A})$ & 8737 & 2102 & -7 & 64 \\
\hline $\mathrm{H}(11 \mathrm{~A})$ & 9982 & 1841 & 1248 & 50 \\
\hline $\mathrm{H}(12 \mathrm{~A})$ & 9248 & 1874 & 1984 & 43 \\
\hline H(1SA) & 9071 & 5290 & 1125 & 531 \\
\hline $\mathrm{H}(2 \mathrm{SA})$ & 8565 & 6654 & 534 & 998 \\
\hline $\mathrm{H}(2 \mathrm{SB})$ & 8464 & 6885 & 1205 & 998 \\
\hline $\mathrm{H}(2 \mathrm{SC})$ & 7926 & 6574 & 848 & 998 \\
\hline $\mathrm{H}(3 \mathrm{SA})$ & 8573 & 5226 & 1683 & 183 \\
\hline $\mathrm{H}(3 \mathrm{SB})$ & 7921 & 5523 & 1694 & 183 \\
\hline $\mathrm{H}(3 \mathrm{SC})$ & 8453 & 5814 & 2076 & 183 \\
\hline $\mathrm{H}(14 \mathrm{~A})$ & 10203 & 2732 & -81 & 175 \\
\hline $\mathrm{H}(14 \mathrm{~B})$ & 10730 & 2246 & -104 & 175 \\
\hline $\mathrm{H}(14 \mathrm{C})$ & 10443 & 2433 & 526 & 175 \\
\hline $\mathrm{H}(15 \mathrm{~A})$ & 9240 & 1910 & -563 & 77 \\
\hline
\end{tabular}




\begin{tabular}{lrrrr}
$\mathrm{H}(15 B)$ & 9692 & 1363 & -664 & 77 \\
$\mathrm{H}(15 \mathrm{C})$ & 9882 & 2025 & -861 & 77 \\
$\mathrm{H}(16 \mathrm{~A})$ & 9979 & 966 & -93 & 193 \\
$\mathrm{H}(16 \mathrm{~B})$ & 10201 & 1162 & 560 & 193 \\
$\mathrm{H}(16 \mathrm{C})$ & 10623 & 1269 & -10 & 193 \\
$\mathrm{H}(14 \mathrm{D})$ & 9697 & 2717 & -386 & 96 \\
$\mathrm{H}(14 \mathrm{E})$ & 10404 & 2594 & -396 & 96 \\
$\mathrm{H}(14 \mathrm{~F})$ & 10087 & 2820 & 207 & 96 \\
$\mathrm{H}(15 \mathrm{D})$ & 9493 & 1408 & -528 & 130 \\
$\mathrm{H}(15 \mathrm{E})$ & 9892 & 1037 & -62 & 98 \\
$\mathrm{H}(15 \mathrm{~F})$ & 10206 & 1390 & -601 & 130 \\
$\mathrm{H}(16 \mathrm{D})$ & 10545 & 1363 & 520 & 98 \\
$\mathrm{H}(16 \mathrm{E})$ & 10601 & 2060 & 683 & 98 \\
$\mathrm{H}(16 \mathrm{~F})$ & 10867 & 1813 & 64 & \\
\hline
\end{tabular}


Table S30. Torsion Angles, ${ }^{\circ}$, for $\left[\left(\mathrm{L}^{1}\right) \mathrm{Fe}(\mathrm{III})-\mathrm{OH}\right]\left[\mathrm{K}(\mathrm{DMF})_{3}\right] \cdot 3 \mathrm{H}_{2} \mathrm{O}(\mathbf{3})$

\begin{tabular}{|c|c|c|c|}
\hline O1-Fe1-N1-C1 & $107(100)$ & $\mathrm{C} 10-\mathrm{C} 11-\mathrm{C} 12-\mathrm{C} 7$ & $-0.6(14)$ \\
\hline N2-Fe1-N1-C1 & 19.7(3) & $\mathrm{C} 11-\mathrm{C} 10-\mathrm{C} 13-\mathrm{C} 15^{\prime}$ & $112.4(18)$ \\
\hline N2-Fe1-N1-C1 & $139.7(3)$ & C9-C10-C13-C15' & $-68.5(19)$ \\
\hline N2-Fe1-N1-C1 & $-100.3(3)$ & C11-C10-C13-C14 & $-62(2)$ \\
\hline O1-Fe1-N1-C1 & $-13(100)$ & C9-C10-C13-C14 & $117(2)$ \\
\hline N2-Fe1-N1-C1 & $-100.3(3)$ & C11-C10-C13-C15 & $163.9(11)$ \\
\hline N2-Fe1-N1-C1 & $19.7(3)$ & C9-C10-C13-C15 & $-16.9(14)$ \\
\hline N2-Fe1-N1-C1 & 139.7(3) & $\mathrm{C} 11-\mathrm{C} 10-\mathrm{C} 13-\mathrm{C} 16$ & $58.7(17)$ \\
\hline O1-Fe1-N1-C1 & $-133(100)$ & C9-C10-C13-C16 & $-122.1(17)$ \\
\hline N2-Fe1-N1-C1 & $139.7(3)$ & $\mathrm{C} 11-\mathrm{C} 10-\mathrm{C} 13-\mathrm{C} 16^{\prime}$ & $1.5(13)$ \\
\hline N2-Fe1-N1-C1 & $-100.3(3)$ & C9-C10-C13-C16' & $-179.3(12)$ \\
\hline N2-Fe1-N1-C1 & 19.7(3) & C11-C10-C13-C14' & $-102.1(13)$ \\
\hline O1-Fe1-N2-C7 & $3.6(6)$ & C9-C10-C13-C14' & $77.0(14)$ \\
\hline N2-Fe1-N2-C7 & $-106.1(7)$ & K1-K1-O1S-C1S & $-175(4)$ \\
\hline N2-Fe1-N2-C7 & $113.4(7)$ & O1S-K1-O1S-C1S & $-87(4)$ \\
\hline N1-Fe1-N2-C7 & $-176.4(6)$ & O1S-K1-O1S-C1S & $97(4)$ \\
\hline $\mathrm{O} 1-\mathrm{Fe} 1-\mathrm{N} 2-\mathrm{C} 2$ & $160.5(5)$ & O1S-K1-O1S-C1S & $-117(4)$ \\
\hline N2-Fe1-N2-C2 & $50.7(6)$ & O1S-K1-O1S-C1S & $127(4)$ \\
\hline N2-Fe1-N2-C2 & $-89.8(5)$ & O1S-K1-O1S-C1S & $-175(4)$ \\
\hline N1-Fe1-N2-C2 & $-19.5(5)$ & C1S-K1-O1S-C1S & $-65(4)$ \\
\hline C1-N1-C1-C6 & $51.1(10)$ & C1S-K1-O1S-C1S & $75(4)$ \\
\hline
\end{tabular}




\begin{tabular}{|c|c|c|c|}
\hline C1-N1-C1-C6 & $-81.7(8)$ & O1S-K1-O1S-K1 & $87.7(13)$ \\
\hline Fe1-N1-C1-C6 & $164.7(6)$ & O1S-K1-O1S-K1 & $-87.7(13)$ \\
\hline C1-N1-C1-C2 & $-131.6(8)$ & O1S-K1-O1S-K1 & $58.0(13)$ \\
\hline C1-N1-C1-C2 & $95.7(10)$ & O1S-K1-O1S-K1 & $-58.0(13)$ \\
\hline Fe1-N1-C1-C2 & $-18.0(6)$ & O1S-K1-O1S-K1 & $-0.001(1)$ \\
\hline C7-N2-C2-C1 & $173.0(7)$ & C1S-K1-O1S-K1 & $110.3(10)$ \\
\hline Fe1-N2-C2-C1 & $15.7(9)$ & C1S-K1-O1S-K1 & $175(4)$ \\
\hline $\mathrm{C} 7-\mathrm{N} 2-\mathrm{C} 2-\mathrm{C} 3$ & $-5.0(11)$ & C1S-K1-O1S-K1 & $-109.7(11)$ \\
\hline $\mathrm{Fe} 1-\mathrm{N} 2-\mathrm{C} 2-\mathrm{C} 3$ & $-162.4(6)$ & K1-O1S-C1S-N1S & $-158(5)$ \\
\hline $\mathrm{C} 6-\mathrm{C} 1-\mathrm{C} 2-\mathrm{N} 2$ & $-178.0(7)$ & K1-O1S-C1S-N1S & $-164(4)$ \\
\hline $\mathrm{N} 1-\mathrm{C} 1-\mathrm{C} 2-\mathrm{N} 2$ & $4.6(9)$ & K1-O1S-C1S-K1 & $-6(5)$ \\
\hline $\mathrm{C} 6-\mathrm{C} 1-\mathrm{C} 2-\mathrm{C} 3$ & $0.2(11)$ & C2S-N1S-C1S-O1S & $-1(9)$ \\
\hline $\mathrm{N} 1-\mathrm{C} 1-\mathrm{C} 2-\mathrm{C} 3$ & $-177.1(6)$ & C3S-N1S-C1S-O1S & $-177(7)$ \\
\hline N2-C2-C3-C4 & 179.2(8) & C2S-N1S-C1S-K1 & $-70(10)$ \\
\hline C1-C2-C3-C4 & $1.2(12)$ & C3S-N1S-C1S-K1 & $114(8)$ \\
\hline $\mathrm{C} 2-\mathrm{C} 3-\mathrm{C} 4-\mathrm{C} 5$ & $-2.1(15)$ & K1-K1-C1S-O1S & $5(4)$ \\
\hline C3-C4-C5-C6 & $1.5(16)$ & O1S-K1-C1S-O1S & $103(4)$ \\
\hline $\mathrm{C} 2-\mathrm{C} 1-\mathrm{C} 6-\mathrm{C} 5$ & $-0.8(12)$ & O1S-K1-C1S-O1S & $-94(4)$ \\
\hline N1-C1-C6-C5 & $176.4(7)$ & O1S-K1-C1S-O1S & $56(3)$ \\
\hline $\mathrm{C} 4-\mathrm{C} 5-\mathrm{C} 6-\mathrm{C} 1$ & $-0.1(14)$ & O1S-K1-C1S-O1S & $-47(4)$ \\
\hline C2-N2-C7-C8 & $120.5(10)$ & O1S-K1-C1S-O1S & $13(9)$ \\
\hline $\mathrm{Fe} 1-\mathrm{N} 2-\mathrm{C} 7-\mathrm{C} 8$ & $-82.6(10)$ & C1S-K1-C1S-O1S & $124(4)$ \\
\hline
\end{tabular}




$\begin{array}{lrlr}\text { C2-N2-C7-C12 } & -66.3(11) & \text { C1S-K1-C1S-O1S } & -113(4) \\ \text { Fe1-N2-C7-C12 } & 90.6(9) & \text { K1-K1-C1S-N1S } & 91(8) \\ \text { C12-C7-C8-C9 } & 0.1(16) & \text { O1S-K1-C1S-N1S } & -171(8) \\ \text { N2-C7-C8-C9 } & 173.7(10) & \text { O1S-K1-C1S-N1S } & 86(7) \\ \text { C7-C8-C9-C10 } & 2.5(19) & \text { O1S-K1-C1S-N1S } & -8(9) \\ \text { C8-C9-C10-C11 } & -3.9(15) & \text { O1S-K1-C1S-N1S } & 142(8) \\ \text { C8-C9-C10-C13 } & 176.9(10) & \text { O1S-K1-C1S-N1S } & 38(8) \\ \text { C9-C10-C11-C12 } & 3.0(13) & \text { O1S-K1-C1S-N1S } & 99(12) \\ \text { C13-C10-C11-C12 } & -177.8(8) & \text { C1S-K1-C1S-N1S } & -151(8) \\ \text { C8-C7-C12-C11 } & -1.0(13) & \text { C1S-K1-C1S-N1S } & -27(9) \\ \text { N2-C7-C12-C11 } & -174.5(8) & & \end{array}$


Table S31. Atomic coordinates $\left(\times 10^{4}\right)$ and equivalent isotropic displacement parameters $\left(\AA^{2} \times 10^{3}\right)$ for $\left[\left(\mathrm{L}^{1}\right) \mathrm{Fe}(\mathrm{III})-\mathrm{OH}\right]\left(\mathrm{H}_{3} \mathrm{O}\right) \cdot 4 \mathrm{H}_{2} \mathrm{O}(4) . \mathrm{U}(\mathrm{eq})$ is defined as one third of the trace of the orthogonalized $\mathrm{U}^{\mathrm{ij}}$ tensor

\begin{tabular}{|c|c|c|c|c|}
\hline & $\mathrm{x}$ & $\mathrm{y}$ & $\mathrm{z}$ & $\mathrm{U}(\mathrm{eq})$ \\
\hline $\mathrm{Fe}(1)$ & 2121(1) & $2880(1)$ & $2120(1)$ & $53(1)$ \\
\hline $\mathrm{O}(1)$ & 1641(2) & $3356(2)$ & $1643(3)$ & $89(2)$ \\
\hline $\mathrm{N}(1)$ & $2722(2)$ & $2280(2)$ & $2724(2)$ & $41(1)$ \\
\hline $\mathrm{N}(2)$ & 2923(2) & $3144(2)$ & $1901(2)$ & $52(1)$ \\
\hline $\mathrm{N}(3)$ & $1906(2)$ & $2078(3)$ & $1852(2)$ & $51(1)$ \\
\hline $\mathrm{N}(4)$ & $1856(2)$ & $3094(2)$ & 2924(3) & $52(2)$ \\
\hline$C(1)$ & $3301(3)$ & $2316(3)$ & $2451(3)$ & $48(2)$ \\
\hline$C(2)$ & $3399(3)$ & $2786(3)$ & $2040(3)$ & $54(2)$ \\
\hline$C(3)$ & $3975(3)$ & $2844(4)$ & $1797(4)$ & $68(2)$ \\
\hline $\mathrm{C}(4)$ & $4426(4)$ & $2452(4)$ & $1942(4)$ & $77(2)$ \\
\hline$C(5)$ & $4328(3)$ & $1980(4)$ & $2331(4)$ & $68(2)$ \\
\hline$C(6)$ & $3767(3)$ & $1915(3)$ & $2578(3)$ & $59(2)$ \\
\hline$C(7)$ & $2449(3)$ & $1699(3)$ & $2688(3)$ & $48(2)$ \\
\hline$C(8)$ & 2038(3) & $1601(3)$ & $2209(3)$ & $52(2)$ \\
\hline$C(9)$ & $1797(3)$ & $1029(3)$ & $2159(4)$ & $68(2)$ \\
\hline$C(10)$ & $1939(4)$ & $573(4)$ & $2543(4)$ & $76(2)$ \\
\hline$C(11)$ & $2333(4)$ & $674(3)$ & $3012(4)$ & $71(2)$ \\
\hline$C(12)$ & $2583(3)$ & $1233(3)$ & $3084(3)$ & $59(2)$ \\
\hline
\end{tabular}




\begin{tabular}{|c|c|c|c|c|}
\hline$C(13)$ & 2684(3) & 2549(3) & $3302(3)$ & $48(2)$ \\
\hline$C(14)$ & $2215(3)$ & 2960(3) & $3404(3)$ & $51(2)$ \\
\hline$C(15)$ & $2162(4)$ & $3202(3)$ & $3971(3)$ & $67(2)$ \\
\hline$C(16)$ & 2543(4) & $3060(4)$ & $4419(4)$ & $78(2)$ \\
\hline$C(17)$ & 3019(4) & 2668(4) & $4320(3)$ & $70(2)$ \\
\hline$C(18)$ & 3081(3) & 2424(3) & $3770(3)$ & $58(2)$ \\
\hline$C(19)$ & 3001(3) & $3572(3)$ & 1444(3) & $53(2)$ \\
\hline$C(20)$ & 2958(4) & 4169(4) & $1569(3)$ & 83(3) \\
\hline$C(21)$ & 2992(4) & 4592(4) & 1134(4) & 91(3) \\
\hline$C(22)$ & $3064(3)$ & $4445(3)$ & $536(4)$ & $62(2)$ \\
\hline$C(23)$ & $3101(4)$ & 3851(4) & $414(4)$ & $77(2)$ \\
\hline$C(25)$ & $3100(4)$ & 4910(4) & $56(4)$ & $75(2)$ \\
\hline$C(24)$ & 3069(3) & $3421(3)$ & $862(3)$ & $65(2)$ \\
\hline$C(28)$ & 1454(3) & 2003(3) & $1426(3)$ & $56(2)$ \\
\hline$C(29)$ & $859(3)$ & 1921(4) & 1581(3) & 71(2) \\
\hline $\mathrm{C}(30)$ & $421(3)$ & 1893(4) & $1146(4)$ & $75(2)$ \\
\hline $\mathrm{C}(31)$ & $545(3)$ & 1935(3) & $551(3)$ & $62(2)$ \\
\hline$C(32)$ & $1138(4)$ & 2022(4) & $405(4)$ & $90(3)$ \\
\hline$C(33)$ & $1566(3)$ & 2050(4) & $828(4)$ & $87(3)$ \\
\hline$C(34)$ & $59(4)$ & 1904(4) & $86(4)$ & $79(2)$ \\
\hline$C(37)$ & $1425(3)$ & $3552(3)$ & $3000(3)$ & $55(2)$ \\
\hline $\mathrm{C}(38)$ & $826(3)$ & $3436(3)$ & 2961(4) & $86(3)$ \\
\hline
\end{tabular}




\begin{tabular}{|c|c|c|c|c|}
\hline C(39) & $408(3)$ & $3856(4)$ & $2988(5)$ & $91(3)$ \\
\hline$C(40)$ & $554(3)$ & $4453(3)$ & $3062(3)$ & $63(2)$ \\
\hline $\mathrm{C}(41)$ & $1150(4)$ & $4587(3)$ & $3105(4)$ & $76(3)$ \\
\hline$C(42)$ & $1578(3)$ & $4142(3)$ & $3070(4)$ & $71(2)$ \\
\hline$C(43)$ & $86(4)$ & $4941(4)$ & $3107(4)$ & $77(2)$ \\
\hline$C(34 A)$ & $18(9)$ & $2495(9)$ & $-221(9)$ & $105(6)$ \\
\hline$C(35 \mathrm{~A})$ & $68(10)$ & $1309(9)$ & $-207(10)$ & $111(7)$ \\
\hline$C(36 A)$ & $-597(7)$ & $1865(9)$ & $390(8)$ & $79(5)$ \\
\hline$C(34 B)$ & $325(8)$ & $1777(8)$ & $-563(9)$ & $96(6)$ \\
\hline$C(35 B)$ & $-375(16)$ & $1326(16)$ & $219(16)$ & $218(15)$ \\
\hline$C(36 B)$ & $-393(13)$ & $2311(14)$ & $98(14)$ & $169(11)$ \\
\hline$C(43 C)$ & $-202(9)$ & $5018(9)$ & $2532(9)$ & $101(6)$ \\
\hline$C(44 C)$ & $-232(10)$ & $4899(10)$ & $3688(10)$ & $110(7)$ \\
\hline$C(45 C)$ & $391(8)$ & $5576(8)$ & $3194(9)$ & $90(6)$ \\
\hline$C(43 D)$ & 161(15) & $5446(14)$ & $2751(15)$ & $184(12)$ \\
\hline$C(44 D)$ & $-568(8)$ & $4680(8)$ & $3237(9)$ & $95(6)$ \\
\hline$C(45 D)$ & $169(13)$ & $5267(13)$ & $3768(13)$ & $167(11)$ \\
\hline$C(25 E)$ & $2508(9)$ & $5269(9)$ & $38(10)$ & $109(7)$ \\
\hline$C(26 E)$ & $3671(10)$ & $5237(10)$ & $100(10)$ & $114(7)$ \\
\hline$C(27 E)$ & $3140(9)$ & $4621(8)$ & $-568(8)$ & $88(5)$ \\
\hline $\mathrm{C}(25 \mathrm{~F})$ & $3221(9)$ & $5578(8)$ & $312(9)$ & $98(6)$ \\
\hline$C(26 F)$ & $3709(14)$ & $4838(14)$ & $-292(15)$ & $187(12)$ \\
\hline
\end{tabular}




\begin{tabular}{|c|c|c|c|c|}
\hline $\mathrm{C}(27 \mathrm{~F})$ & $2605(14)$ & $4938(14)$ & $-345(14)$ & $179(12)$ \\
\hline $\mathrm{O}(1 \mathrm{~S})$ & $1029(5)$ & $4249(5)$ & $1553(6)$ & $206(4)$ \\
\hline $\mathrm{O}(2 \mathrm{~S})$ & $753(5)$ & $3445(5)$ & $1026(5)$ & 205(4) \\
\hline $\mathrm{O}(3 \mathrm{~S})$ & $1557(5)$ & $3969(5)$ & $749(5)$ & 201(4) \\
\hline $\mathrm{O}(4 \mathrm{~S})$ & $164(3)$ & $4839(3)$ & $164(3)$ & $97(2)$ \\
\hline $\mathrm{O}(5 \mathrm{~S})$ & $1561(8)$ & $5497(8)$ & $1688(8)$ & $317(9)$ \\
\hline $\mathrm{O}(6 \mathrm{~S})$ & $9342(18)$ & $4252(17)$ & $965(14)$ & $630(30)$ \\
\hline $\mathrm{O}(7 \mathrm{~S})$ & $739(18)$ & $4079(15)$ & $9248(18)$ & $640(30)$ \\
\hline$C(1 S)$ & $3323(8)$ & $1528(8)$ & $968(8)$ & $216(8)$ \\
\hline $\mathrm{C}(2 \mathrm{~S})$ & $3577(6)$ & $948(8)$ & $1120(7)$ & $147(6)$ \\
\hline$C(3 S)$ & $4058(15)$ & $726(17)$ & $816(16)$ & $480(30)$ \\
\hline$C(4 S)$ & $8535(10)$ & $4009(6)$ & $1648(10)$ & $236(11)$ \\
\hline$C(5 S)$ & $9098(10)$ & $3867(10)$ & $1425(8)$ & 176(9) \\
\hline$C(6 S)$ & $9522(9)$ & $3331(8)$ & $1553(9)$ & $250(12)$ \\
\hline$C(7 S)$ & $985(7)$ & $3354(10)$ & $8519(12)$ & $251(12)$ \\
\hline $\mathrm{C}(8 \mathrm{~S})$ & $1154(10)$ & $3579(8)$ & $9115(10)$ & $169(8)$ \\
\hline $\mathrm{C}(9 \mathrm{~S})$ & $1668(8)$ & $3432(8)$ & $9501(9)$ & $259(13)$ \\
\hline
\end{tabular}


Table S32. Bond Distances, $\AA$, for $\left[\left(\mathrm{L}^{1}\right) \mathrm{Fe}(\mathrm{III})-\mathrm{OH}\right]\left(\mathrm{H}_{3} \mathrm{O}\right) \cdot 4 \mathrm{H}_{2} \mathrm{O}(4)$

\begin{tabular}{|c|c|c|c|}
\hline $\mathrm{Fe}(1)-\mathrm{O}(1)$ & $1.863(5)$ & $\mathrm{C}(34 \mathrm{~B})-\mathrm{H}(35 \mathrm{~A})$ & 1.0451 \\
\hline $\mathrm{Fe}(1)-\mathrm{N}(3)$ & $1.963(6)$ & $\mathrm{C}(34 \mathrm{~B})-\mathrm{H}(34 \mathrm{D})$ & 0.9601 \\
\hline $\mathrm{Fe}(1)-\mathrm{N}(2)$ & $1.964(6)$ & $\mathrm{C}(34 \mathrm{~B})-\mathrm{H}(34 \mathrm{E})$ & 0.9601 \\
\hline $\mathrm{Fe}(1)-\mathrm{N}(4)$ & $1.966(6)$ & $\mathrm{C}(34 \mathrm{~B})-\mathrm{H}(34 \mathrm{~F})$ & 0.9600 \\
\hline $\mathrm{Fe}(1)-\mathrm{N}(1)$ & $2.346(5)$ & $\mathrm{C}(35 \mathrm{~B})-\mathrm{H}(35 \mathrm{~B})$ & 1.4290 \\
\hline $\mathrm{N}(1)-\mathrm{C}(13)$ & $1.440(8)$ & $\mathrm{C}(35 \mathrm{~B})-\mathrm{H}(35 \mathrm{C})$ & 1.4189 \\
\hline $\mathrm{N}(1)-\mathrm{C}(1)$ & $1.445(8)$ & $\mathrm{C}(35 \mathrm{~B})-\mathrm{H}(36 \mathrm{~B})$ & 1.4468 \\
\hline $\mathrm{N}(1)-\mathrm{C}(7)$ & $1.447(8)$ & $\mathrm{C}(35 \mathrm{~B})-\mathrm{H}(36 \mathrm{C})$ & 1.3795 \\
\hline $\mathrm{N}(2)-\mathrm{C}(2)$ & $1.379(8)$ & $\mathrm{C}(35 \mathrm{~B})-\mathrm{H}(35 \mathrm{D})$ & 0.9597 \\
\hline $\mathrm{N}(2)-\mathrm{C}(19)$ & $1.419(8)$ & $\mathrm{C}(35 \mathrm{~B})-\mathrm{H}(35 \mathrm{E})$ & 0.9600 \\
\hline $\mathrm{N}(3)-\mathrm{C}(8)$ & $1.375(8)$ & $\mathrm{C}(35 \mathrm{~B})-\mathrm{H}(35 \mathrm{~F})$ & 0.9601 \\
\hline $\mathrm{N}(3)-\mathrm{C}(28)$ & $1.409(8)$ & $\mathrm{C}(36 \mathrm{~B})-\mathrm{H}(34 \mathrm{C})$ & 0.9502 \\
\hline $\mathrm{N}(4)-\mathrm{C}(14)$ & $1.386(8)$ & $\mathrm{C}(36 \mathrm{~B})-\mathrm{H}(36 \mathrm{~A})$ & 1.2073 \\
\hline $\mathrm{N}(4)-\mathrm{C}(37)$ & $1.427(8)$ & $\mathrm{C}(36 \mathrm{~B})-\mathrm{H}(36 \mathrm{D})$ & 0.9602 \\
\hline $\mathrm{C}(1)-\mathrm{C}(6)$ & $1.414(9)$ & $\mathrm{C}(36 \mathrm{~B})-\mathrm{H}(36 \mathrm{E})$ & 0.9599 \\
\hline $\mathrm{C}(1)-\mathrm{C}(2)$ & $1.423(9)$ & $\mathrm{C}(36 \mathrm{~B})-\mathrm{H}(36 \mathrm{~F})$ & 0.9601 \\
\hline$C(2)-C(3)$ & $1.412(10)$ & $\mathrm{C}(43 \mathrm{C})-\mathrm{H}(43 \mathrm{~A})$ & 0.9598 \\
\hline$C(3)-C(4)$ & $1.385(10)$ & $\mathrm{C}(43 \mathrm{C})-\mathrm{H}(43 \mathrm{~B})$ & 0.9599 \\
\hline $\mathrm{C}(3)-\mathrm{H}(3)$ & 0.9300 & $\mathrm{C}(43 \mathrm{C})-\mathrm{H}(43 \mathrm{C})$ & 0.9599 \\
\hline$C(4)-C(5)$ & $1.394(10)$ & $\mathrm{C}(43 \mathrm{C})-\mathrm{H}(43 \mathrm{D})$ & 1.0562 \\
\hline $\mathrm{C}(4)-\mathrm{H}(4)$ & 0.9300 & $\mathrm{C}(44 \mathrm{C})-\mathrm{H}(44 \mathrm{~A})$ & 0.9600 \\
\hline
\end{tabular}




\begin{tabular}{|c|c|c|c|}
\hline$C(5)-C(6)$ & $1.389(9)$ & $\mathrm{C}(44 \mathrm{C})-\mathrm{H}(44 \mathrm{~B})$ & 0.9599 \\
\hline $\mathrm{C}(5)-\mathrm{H}(5)$ & 0.9300 & $\mathrm{C}(44 \mathrm{C})-\mathrm{H}(44 \mathrm{C})$ & 0.9601 \\
\hline $\mathrm{C}(6)-\mathrm{H}(6)$ & 0.9300 & $\mathrm{C}(44 \mathrm{C})-\mathrm{H}(44 \mathrm{E})$ & 1.3361 \\
\hline$C(7)-C(12)$ & $1.410(9)$ & $\mathrm{C}(44 \mathrm{C})-\mathrm{H}(45 \mathrm{E})$ & 1.5141 \\
\hline$C(7)-C(8)$ & $1.439(9)$ & $\mathrm{C}(44 \mathrm{C})-\mathrm{H}(45 \mathrm{~F})$ & 1.2185 \\
\hline $\mathrm{C}(8)-\mathrm{C}(9)$ & $1.401(10)$ & $\mathrm{C}(45 \mathrm{C})-\mathrm{H}(45 \mathrm{~A})$ & 0.9600 \\
\hline $\mathrm{C}(9)-\mathrm{C}(10)$ & $1.380(11)$ & $\mathrm{C}(45 \mathrm{C})-\mathrm{H}(45 \mathrm{~B})$ & 0.9600 \\
\hline $\mathrm{C}(9)-\mathrm{H}(9)$ & 0.9300 & $\mathrm{C}(45 \mathrm{C})-\mathrm{H}(45 \mathrm{C})$ & 0.9599 \\
\hline $\mathrm{C}(10)-\mathrm{C}(11)$ & $1.397(11)$ & $\mathrm{C}(45 \mathrm{C})-\mathrm{H}(43 \mathrm{E})$ & 1.5035 \\
\hline $\mathrm{C}(10)-\mathrm{H}(10)$ & 0.9300 & $\mathrm{C}(45 \mathrm{C})-\mathrm{H}(43 \mathrm{~F})$ & 0.9517 \\
\hline $\mathrm{C}(11)-\mathrm{C}(12)$ & $1.390(9)$ & $\mathrm{C}(45 \mathrm{C})-\mathrm{H}(45 \mathrm{D})$ & 1.4169 \\
\hline $\mathrm{C}(11)-\mathrm{H}(11)$ & 0.9300 & $\mathrm{C}(43 \mathrm{D})-\mathrm{H}(43 \mathrm{C})$ & 1.0455 \\
\hline $\mathrm{C}(12)-\mathrm{H}(12)$ & 0.9300 & $\mathrm{C}(43 \mathrm{D})-\mathrm{H}(45 \mathrm{~A})$ & 0.9957 \\
\hline $\mathrm{C}(13)-\mathrm{C}(18)$ & $1.410(9)$ & $\mathrm{C}(43 \mathrm{D})-\mathrm{H}(43 \mathrm{D})$ & 0.9599 \\
\hline $\mathrm{C}(13)-\mathrm{C}(14)$ & $1.421(9)$ & $\mathrm{C}(43 \mathrm{D})-\mathrm{H}(43 \mathrm{E})$ & 0.9601 \\
\hline$C(14)-C(15)$ & $1.394(10)$ & $\mathrm{C}(43 \mathrm{D})-\mathrm{H}(43 \mathrm{~F})$ & 0.9600 \\
\hline$C(15)-C(16)$ & $1.363(10)$ & $\mathrm{C}(44 \mathrm{D})-\mathrm{H}(44 \mathrm{~A})$ & 0.9531 \\
\hline $\mathrm{C}(15)-\mathrm{H}(15)$ & 0.9300 & $\mathrm{C}(44 \mathrm{D})-\mathrm{H}(44 \mathrm{D})$ & 0.9601 \\
\hline$C(16)-C(17)$ & $1.404(10)$ & $\mathrm{C}(44 \mathrm{D})-\mathrm{H}(44 \mathrm{E})$ & 0.9600 \\
\hline $\mathrm{C}(16)-\mathrm{H}(16)$ & 0.9300 & $\mathrm{C}(44 \mathrm{D})-\mathrm{H}(44 \mathrm{~F})$ & 0.9601 \\
\hline $\mathrm{C}(17)-\mathrm{C}(18)$ & $1.362(9)$ & $\mathrm{C}(45 \mathrm{D})-\mathrm{H}(44 \mathrm{~B})$ & 1.2441 \\
\hline C(17)-H(17) & 0.9300 & $\mathrm{C}(45 \mathrm{D})-\mathrm{H}(44 \mathrm{C})$ & 1.2828 \\
\hline
\end{tabular}




\begin{tabular}{|c|c|c|c|}
\hline $\mathrm{C}(18)-\mathrm{H}(18)$ & 0.9300 & $\mathrm{C}(45 \mathrm{D})-\mathrm{H}(45 \mathrm{D})$ & 0.9603 \\
\hline$C(19)-C(24)$ & $1.365(9)$ & $\mathrm{C}(45 \mathrm{D})-\mathrm{H}(45 \mathrm{E})$ & 0.9601 \\
\hline$C(19)-C(20)$ & $1.376(10)$ & $\mathrm{C}(45 \mathrm{D})-\mathrm{H}(45 \mathrm{~F})$ & 0.9601 \\
\hline$C(20)-C(21)$ & $1.369(11)$ & $\mathrm{C}(25 \mathrm{E})-\mathrm{H}(25 \mathrm{~A})$ & 0.9599 \\
\hline $\mathrm{C}(20)-\mathrm{H}(20)$ & 0.9300 & $\mathrm{C}(25 \mathrm{E})-\mathrm{H}(25 \mathrm{~B})$ & 0.9599 \\
\hline $\mathrm{C}(21)-\mathrm{C}(22)$ & $1.395(11)$ & $\mathrm{C}(25 \mathrm{E})-\mathrm{H}(25 \mathrm{C})$ & 0.9602 \\
\hline $\mathrm{C}(21)-\mathrm{H}(21)$ & 0.9300 & $\mathrm{C}(25 \mathrm{E})-\mathrm{H}(27 \mathrm{D})$ & 1.0982 \\
\hline$C(22)-C(23)$ & $1.369(10)$ & $\mathrm{C}(25 \mathrm{E})-\mathrm{H}(27 \mathrm{E})$ & 1.5053 \\
\hline $\mathrm{C}(22)-\mathrm{C}(25)$ & $1.507(10)$ & $\mathrm{C}(26 \mathrm{E})-\mathrm{H}(26 \mathrm{~A})$ & 0.9600 \\
\hline $\mathrm{C}(23)-\mathrm{C}(24)$ & $1.400(10)$ & $\mathrm{C}(26 \mathrm{E})-\mathrm{H}(26 \mathrm{~B})$ & 0.9600 \\
\hline $\mathrm{C}(23)-\mathrm{H}(23)$ & 0.9300 & $\mathrm{C}(26 \mathrm{E})-\mathrm{H}(26 \mathrm{C})$ & 0.9599 \\
\hline $\mathrm{C}(25)-\mathrm{C}(27 \mathrm{~F})$ & $1.44(3)$ & $\mathrm{C}(26 \mathrm{E})-\mathrm{H}(25 \mathrm{E})$ & 1.3455 \\
\hline$C(25)-C(26 E)$ & $1.49(2)$ & $\mathrm{C}(26 \mathrm{E})-\mathrm{H}(26 \mathrm{E})$ & 1.5464 \\
\hline$C(25)-C(27 E)$ & $1.554(19)$ & $\mathrm{C}(26 \mathrm{E})-\mathrm{H}(26 \mathrm{~F})$ & 1.1217 \\
\hline$C(25)-C(25 E)$ & $1.56(2)$ & $\mathrm{C}(27 \mathrm{E})-\mathrm{H}(27 \mathrm{~A})$ & 0.9599 \\
\hline$C(25)-C(26 F)$ & $1.59(3)$ & $\mathrm{C}(27 \mathrm{E})-\mathrm{H}(27 \mathrm{~B})$ & 0.9602 \\
\hline $\mathrm{C}(25)-\mathrm{C}(25 \mathrm{~F})$ & $1.63(2)$ & $\mathrm{C}(27 \mathrm{E})-\mathrm{H}(27 \mathrm{C})$ & 0.9601 \\
\hline $\mathrm{C}(24)-\mathrm{H}(24)$ & 0.9300 & $\mathrm{C}(27 \mathrm{E})-\mathrm{H}(26 \mathrm{D})$ & 1.4389 \\
\hline $\mathrm{C}(28)-\mathrm{C}(33)$ & $1.375(10)$ & $\mathrm{C}(27 \mathrm{E})-\mathrm{H}(27 \mathrm{~F})$ & 1.1025 \\
\hline $\mathrm{C}(28)-\mathrm{C}(29)$ & $1.397(9)$ & $\mathrm{C}(25 \mathrm{~F})-\mathrm{H}(25 \mathrm{~B})$ & 1.5504 \\
\hline $\mathrm{C}(29)-\mathrm{C}(30)$ & $1.390(10)$ & $\mathrm{C}(25 \mathrm{~F})-\mathrm{H}(26 \mathrm{~A})$ & 0.9308 \\
\hline C(29)-H(29) & 0.9300 & $\mathrm{C}(25 \mathrm{~F})-\mathrm{H}(25 \mathrm{D})$ & 0.9601 \\
\hline
\end{tabular}




\begin{tabular}{|c|c|c|c|}
\hline$C(30)-C(31)$ & $1.373(10)$ & $\mathrm{C}(25 \mathrm{~F})-\mathrm{H}(25 \mathrm{E})$ & 0.9599 \\
\hline $\mathrm{C}(30)-\mathrm{H}(30)$ & 0.9300 & $\mathrm{C}(25 \mathrm{~F})-\mathrm{H}(25 \mathrm{~F})$ & 0.9600 \\
\hline$C(31)-C(32)$ & $1.390(10)$ & $\mathrm{C}(26 \mathrm{~F})-\mathrm{H}(26 \mathrm{~B})$ & 1.3530 \\
\hline $\mathrm{C}(31)-\mathrm{C}(34)$ & $1.517(10)$ & $\mathrm{C}(26 \mathrm{~F})-\mathrm{H}(26 \mathrm{C})$ & 1.2810 \\
\hline$C(32)-C(33)$ & $1.357(11)$ & $\mathrm{C}(26 \mathrm{~F})-\mathrm{H}(27 \mathrm{~B})$ & 1.5508 \\
\hline $\mathrm{C}(32)-\mathrm{H}(32)$ & 0.9300 & $\mathrm{C}(26 \mathrm{~F})-\mathrm{H}(27 \mathrm{C})$ & 1.5486 \\
\hline $\mathrm{C}(33)-\mathrm{H}(33)$ & 0.9300 & $\mathrm{C}(26 \mathrm{~F})-\mathrm{H}(26 \mathrm{D})$ & 0.9601 \\
\hline$C(34)-C(36 B)$ & $1.37(3)$ & $\mathrm{C}(26 \mathrm{~F})-\mathrm{H}(26 \mathrm{E})$ & 0.9599 \\
\hline$C(34)-C(35 A)$ & $1.49(2)$ & $\mathrm{C}(26 \mathrm{~F})-\mathrm{H}(26 \mathrm{~F})$ & 0.9599 \\
\hline$C(34)-C(34 A)$ & $1.50(2)$ & $\mathrm{C}(27 \mathrm{~F})-\mathrm{H}(25 \mathrm{C})$ & 0.8286 \\
\hline$C(34)-C(34 B)$ & $1.61(2)$ & $\mathrm{C}(27 \mathrm{~F})-\mathrm{H}(27 \mathrm{~A})$ & 1.3174 \\
\hline$C(34)-C(36 A)$ & $1.630(18)$ & $\mathrm{C}(27 \mathrm{~F})-\mathrm{H}(27 \mathrm{D})$ & 0.9601 \\
\hline$C(34)-C(35 B)$ & $1.65(3)$ & $\mathrm{C}(27 \mathrm{~F})-\mathrm{H}(27 \mathrm{E})$ & 0.9599 \\
\hline $\mathrm{C}(37)-\mathrm{C}(38)$ & $1.376(10)$ & $\mathrm{C}(27 \mathrm{~F})-\mathrm{H}(27 \mathrm{~F})$ & 0.9600 \\
\hline$C(37)-C(42)$ & $1.382(9)$ & $\mathrm{O}(4 \mathrm{~S})-\mathrm{O}(4 \mathrm{~S}) \# 1$ & $1.271(10)$ \\
\hline $\mathrm{C}(38)-\mathrm{C}(39)$ & $1.337(10)$ & $\mathrm{O}(5 \mathrm{~S})-\mathrm{C}(2 \mathrm{~S}) \# 2$ & $1.66(2)$ \\
\hline $\mathrm{C}(38)-\mathrm{H}(38)$ & 0.9300 & $\mathrm{O}(6 \mathrm{~S})-\mathrm{C}(5 \mathrm{~S})$ & $1.46(4)$ \\
\hline$C(39)-C(40)$ & $1.393(10)$ & $\mathrm{O}(7 \mathrm{~S})-\mathrm{C}(8 \mathrm{~S})$ & $1.49(4)$ \\
\hline C(39)-H(39) & 0.9300 & $\mathrm{C}(1 \mathrm{~S})-\mathrm{C}(2 \mathrm{~S})$ & $1.47(2)$ \\
\hline$C(40)-C(41)$ & $1.379(10)$ & $\mathrm{C}(1 \mathrm{~S})-\mathrm{H}(1 \mathrm{~S} 1)$ & 0.9601 \\
\hline$C(40)-C(43)$ & $1.526(10)$ & $\mathrm{C}(1 \mathrm{~S})-\mathrm{H}(1 \mathrm{~S} 2)$ & 0.9600 \\
\hline$C(41)-C(42)$ & $1.394(10)$ & $\mathrm{C}(1 \mathrm{~S})-\mathrm{H}(1 \mathrm{~S} 3)$ & 0.9600 \\
\hline
\end{tabular}




\begin{tabular}{|c|c|c|c|}
\hline $\mathrm{C}(41)-\mathrm{H}(41)$ & 0.9300 & $C(2 S)-C(3 S)$ & $1.38(3)$ \\
\hline $\mathrm{C}(42)-\mathrm{H}(42)$ & 0.9300 & $\mathrm{C}(2 \mathrm{~S})-\mathrm{O}(5 \mathrm{~S}) \# 3$ & $1.66(2)$ \\
\hline$C(43)-C(43 D)$ & $1.40(3)$ & $\mathrm{C}(3 \mathrm{~S})-\mathrm{H}(3 \mathrm{SA})$ & 0.9601 \\
\hline$C(43)-C(43 C)$ & $1.46(2)$ & $\mathrm{C}(3 \mathrm{~S})-\mathrm{H}(3 \mathrm{SB})$ & 0.9601 \\
\hline$C(43)-C(44 C)$ & $1.49(2)$ & $\mathrm{C}(3 \mathrm{~S})-\mathrm{H}(3 \mathrm{SC})$ & 0.9598 \\
\hline$C(43)-C(45 C)$ & $1.60(2)$ & $C(4 S)-C(5 S)$ & $1.40(2)$ \\
\hline$C(43)-C(44 D)$ & $1.61(2)$ & $\mathrm{C}(4 \mathrm{~S})-\mathrm{H}(4 \mathrm{~S} 1)$ & 0.9598 \\
\hline$C(43)-C(45 D)$ & $1.67(3)$ & $\mathrm{C}(4 \mathrm{~S})-\mathrm{H}(4 \mathrm{~S} 2)$ & 0.9601 \\
\hline $\mathrm{C}(34 \mathrm{~A})-\mathrm{H}(34 \mathrm{~A})$ & 0.9600 & $\mathrm{C}(4 \mathrm{~S})-\mathrm{H}(4 \mathrm{~S} 3)$ & 0.9601 \\
\hline $\mathrm{C}(34 \mathrm{~A})-\mathrm{H}(34 \mathrm{~B})$ & 0.9598 & $\mathrm{C}(5 \mathrm{~S})-\mathrm{C}(6 \mathrm{~S})$ & $1.57(3)$ \\
\hline $\mathrm{C}(34 \mathrm{~A})-\mathrm{H}(34 \mathrm{C})$ & 0.9601 & $\mathrm{C}(6 \mathrm{~S})-\mathrm{H}(6 \mathrm{~S} 1)$ & 0.9599 \\
\hline $\mathrm{C}(34 \mathrm{~A})-\mathrm{H}(36 \mathrm{D})$ & 0.9644 & $\mathrm{C}(6 \mathrm{~S})-\mathrm{H}(6 \mathrm{~S} 2)$ & 0.9600 \\
\hline $\mathrm{C}(35 \mathrm{~A})-\mathrm{H}(35 \mathrm{~A})$ & 0.9601 & $\mathrm{C}(6 \mathrm{~S})-\mathrm{H}(6 \mathrm{~S} 3)$ & 0.9599 \\
\hline $\mathrm{C}(35 \mathrm{~A})-\mathrm{H}(35 \mathrm{~B})$ & 0.9601 & $\mathrm{C}(7 \mathrm{~S})-\mathrm{C}(8 \mathrm{~S})$ & $1.49(3)$ \\
\hline $\mathrm{C}(35 \mathrm{~A})-\mathrm{H}(35 \mathrm{C})$ & 0.9600 & $\mathrm{C}(7 \mathrm{~S})-\mathrm{H}(7 \mathrm{~S} 1)$ & 0.9599 \\
\hline $\mathrm{C}(35 \mathrm{~A})-\mathrm{H}(34 \mathrm{E})$ & 1.3893 & $\mathrm{C}(7 \mathrm{~S})-\mathrm{H}(7 \mathrm{~S} 2)$ & 0.9600 \\
\hline $\mathrm{C}(35 \mathrm{~A})-\mathrm{H}(35 \mathrm{~F})$ & 1.1923 & $\mathrm{C}(7 \mathrm{~S})-\mathrm{H}(7 \mathrm{~S} 3)$ & 0.9602 \\
\hline $\mathrm{C}(36 \mathrm{~A})-\mathrm{H}(36 \mathrm{~A})$ & 0.9600 & $\mathrm{C}(8 \mathrm{~S})-\mathrm{C}(9 \mathrm{~S})$ & $1.48(3)$ \\
\hline $\mathrm{C}(36 \mathrm{~A})-\mathrm{H}(36 \mathrm{~B})$ & 0.9600 & $\mathrm{C}(9 \mathrm{~S})-\mathrm{H}(9 \mathrm{~S} 1)$ & 0.9599 \\
\hline $\mathrm{C}(36 \mathrm{~A})-\mathrm{H}(36 \mathrm{C})$ & 0.9600 & $\mathrm{C}(9 \mathrm{~S})-\mathrm{H}(9 \mathrm{~S} 2)$ & 0.9600 \\
\hline $\mathrm{C}(36 \mathrm{~A})-\mathrm{H}(35 \mathrm{D})$ & 1.3305 & $\mathrm{C}(9 \mathrm{~S})-\mathrm{H}(9 \mathrm{~S} 3)$ & 0.9603 \\
\hline $\mathrm{C}(36 \mathrm{~A})-\mathrm{H}(36 \mathrm{~F})$ & 0.9392 & & \\
\hline
\end{tabular}


Symmetry transformations used to generate equivalent atoms:

$\# 1-x,-y+1,-z \quad \# 2-x+1 / 2, y+1 / 2, z \quad \# 3-x+1 / 2, y-1 / 2, z$ 
Table S33. Angles, ${ }^{\circ}$, for $\left[\left(\mathrm{L}^{1}\right) \mathrm{Fe}(\mathrm{III})-\mathrm{OH}\right]\left(\mathrm{H}_{3} \mathrm{O}\right) \cdot 4 \mathrm{H}_{2} \mathrm{O}$ (4)

\begin{tabular}{|c|c|c|c|}
\hline $\mathrm{O}(1)-\mathrm{Fe}(1)-\mathrm{N}(3)$ & $102.1(2)$ & $\mathrm{H}(35 \mathrm{~B})-\mathrm{C}(35 \mathrm{~B})-\mathrm{H}(35 \mathrm{~F})$ & 20.1 \\
\hline $\mathrm{O}(1)-\mathrm{Fe}(1)-\mathrm{N}(2)$ & $102.3(2)$ & $\mathrm{H}(35 \mathrm{C})-\mathrm{C}(35 \mathrm{~B})-\mathrm{H}(35 \mathrm{~F})$ & 74.9 \\
\hline $\mathrm{N}(3)-\mathrm{Fe}(1)-\mathrm{N}(2)$ & $115.3(2)$ & $\mathrm{H}(36 \mathrm{~B})-\mathrm{C}(35 \mathrm{~B})-\mathrm{H}(35 \mathrm{~F})$ & 162.8 \\
\hline $\mathrm{O}(1)-\mathrm{Fe}(1)-\mathrm{N}(4)$ & $102.3(2)$ & $\mathrm{H}(36 \mathrm{C})-\mathrm{C}(35 \mathrm{~B})-\mathrm{H}(35 \mathrm{~F})$ & 127.0 \\
\hline $\mathrm{N}(3)-\mathrm{Fe}(1)-\mathrm{N}(4)$ & $115.7(2)$ & $\mathrm{H}(35 \mathrm{D})-\mathrm{C}(35 \mathrm{~B})-\mathrm{H}(35 \mathrm{~F})$ & 109.5 \\
\hline $\mathrm{N}(2)-\mathrm{Fe}(1)-\mathrm{N}(4)$ & $115.8(2)$ & $\mathrm{H}(35 \mathrm{E})-\mathrm{C}(35 \mathrm{~B})-\mathrm{H}(35 \mathrm{~F})$ & 109.5 \\
\hline $\mathrm{O}(1)-\mathrm{Fe}(1)-\mathrm{N}(1)$ & $179.7(2)$ & $\mathrm{C}(34)-\mathrm{C}(36 \mathrm{~B})-\mathrm{H}(34 \mathrm{C})$ & 119.6 \\
\hline $\mathrm{N}(3)-\mathrm{Fe}(1)-\mathrm{N}(1)$ & $77.9(2)$ & $\mathrm{C}(34)-\mathrm{C}(36 \mathrm{~B})-\mathrm{H}(36 \mathrm{~A})$ & 113.4 \\
\hline $\mathrm{N}(2)-\mathrm{Fe}(1)-\mathrm{N}(1)$ & $77.89(19)$ & $\mathrm{H}(34 \mathrm{C})-\mathrm{C}(36 \mathrm{~B})-\mathrm{H}(36 \mathrm{~A})$ & 121.4 \\
\hline $\mathrm{N}(4)-\mathrm{Fe}(1)-\mathrm{N}(1)$ & $77.5(2)$ & $\mathrm{C}(34)-\mathrm{C}(36 \mathrm{~B})-\mathrm{H}(36 \mathrm{D})$ & 108.7 \\
\hline $\mathrm{C}(13)-\mathrm{N}(1)-\mathrm{C}(1)$ & $114.5(5)$ & $\mathrm{H}(34 \mathrm{C})-\mathrm{C}(36 \mathrm{~B})-\mathrm{H}(36 \mathrm{D})$ & 26.6 \\
\hline C(13)-N(1)-C(7) & $113.9(5)$ & $\mathrm{H}(36 \mathrm{~A})-\mathrm{C}(36 \mathrm{~B})-\mathrm{H}(36 \mathrm{D})$ & 113.6 \\
\hline $\mathrm{C}(1)-\mathrm{N}(1)-\mathrm{C}(7)$ & $114.3(5)$ & $\mathrm{C}(34)-\mathrm{C}(36 \mathrm{~B})-\mathrm{H}(36 \mathrm{E})$ & 111.5 \\
\hline $\mathrm{C}(13)-\mathrm{N}(1)-\mathrm{Fe}(1)$ & $104.3(4)$ & $\mathrm{H}(34 \mathrm{C})-\mathrm{C}(36 \mathrm{~B})-\mathrm{H}(36 \mathrm{E})$ & 82.9 \\
\hline $\mathrm{C}(1)-\mathrm{N}(1)-\mathrm{Fe}(1)$ & $104.0(4)$ & $H(36 A)-C(36 B)-H(36 E)$ & 99.9 \\
\hline $\mathrm{C}(7)-\mathrm{N}(1)-\mathrm{Fe}(1)$ & $104.1(4)$ & $\mathrm{H}(36 \mathrm{D})-\mathrm{C}(36 \mathrm{~B})-\mathrm{H}(36 \mathrm{E})$ & 109.5 \\
\hline C(2)-N(2)-C(19) & $117.7(6)$ & $\mathrm{C}(34)-\mathrm{C}(36 \mathrm{~B})-\mathrm{H}(36 \mathrm{~F})$ & 108.2 \\
\hline $\mathrm{C}(2)-\mathrm{N}(2)-\mathrm{Fe}(1)$ & $118.7(4)$ & $\mathrm{H}(34 \mathrm{C})-\mathrm{C}(36 \mathrm{~B})-\mathrm{H}(36 \mathrm{~F})$ & 121.7 \\
\hline $\mathrm{C}(19)-\mathrm{N}(2)-\mathrm{Fe}(1)$ & $120.1(4)$ & $\mathrm{H}(36 \mathrm{~A})-\mathrm{C}(36 \mathrm{~B})-\mathrm{H}(36 \mathrm{~F})$ & 9.6 \\
\hline $\mathrm{C}(8)-\mathrm{N}(3)-\mathrm{C}(28)$ & $117.3(6)$ & $H(36 D)-C(36 B)-H(36 F)$ & 109.4 \\
\hline $\mathrm{C}(8)-\mathrm{N}(3)-\mathrm{Fe}(1)$ & $119.1(4)$ & $\mathrm{H}(36 \mathrm{E})-\mathrm{C}(36 \mathrm{~B})-\mathrm{H}(36 \mathrm{~F})$ & 109.5 \\
\hline
\end{tabular}




\begin{tabular}{|c|c|c|c|}
\hline $\mathrm{C}(28)-\mathrm{N}(3)-\mathrm{Fe}(1)$ & $119.8(4)$ & $\mathrm{C}(43)-\mathrm{C}(43 \mathrm{C})-\mathrm{H}(43 \mathrm{~A})$ & 109.1 \\
\hline $\mathrm{C}(14)-\mathrm{N}(4)-\mathrm{C}(37)$ & $117.5(6)$ & $\mathrm{C}(43)-\mathrm{C}(43 \mathrm{C})-\mathrm{H}(43 \mathrm{~B})$ & 109.6 \\
\hline $\mathrm{C}(14)-\mathrm{N}(4)-\mathrm{Fe}(1)$ & $119.2(4)$ & $\mathrm{H}(43 \mathrm{~A})-\mathrm{C}(43 \mathrm{C})-\mathrm{H}(43 \mathrm{~B})$ & 109.5 \\
\hline $\mathrm{C}(37)-\mathrm{N}(4)-\mathrm{Fe}(1)$ & 119.7(4) & $\mathrm{C}(43)-\mathrm{C}(43 \mathrm{C})-\mathrm{H}(43 \mathrm{C})$ & 109.7 \\
\hline$C(6)-C(1)-C(2)$ & $119.5(6)$ & $\mathrm{H}(43 \mathrm{~A})-\mathrm{C}(43 \mathrm{C})-\mathrm{H}(43 \mathrm{C})$ & 109.5 \\
\hline $\mathrm{C}(6)-\mathrm{C}(1)-\mathrm{N}(1)$ & $123.3(6)$ & $\mathrm{H}(43 \mathrm{~B})-\mathrm{C}(43 \mathrm{C})-\mathrm{H}(43 \mathrm{C})$ & 109.5 \\
\hline $\mathrm{C}(2)-\mathrm{C}(1)-\mathrm{N}(1)$ & $117.3(6)$ & $\mathrm{C}(43)-\mathrm{C}(43 \mathrm{C})-\mathrm{H}(43 \mathrm{D})$ & 95.9 \\
\hline $\mathrm{N}(2)-\mathrm{C}(2)-\mathrm{C}(3)$ & $124.9(7)$ & $\mathrm{H}(43 \mathrm{~A})-\mathrm{C}(43 \mathrm{C})-\mathrm{H}(43 \mathrm{D})$ & 93.0 \\
\hline $\mathrm{N}(2)-\mathrm{C}(2)-\mathrm{C}(1)$ & $117.6(6)$ & $\mathrm{H}(43 \mathrm{~B})-\mathrm{C}(43 \mathrm{C})-\mathrm{H}(43 \mathrm{D})$ & 137.0 \\
\hline$C(3)-C(2)-C(1)$ & $117.5(7)$ & $\mathrm{H}(43 \mathrm{C})-\mathrm{C}(43 \mathrm{C})-\mathrm{H}(43 \mathrm{D})$ & 27.6 \\
\hline$C(4)-C(3)-C(2)$ & $121.6(7)$ & $\mathrm{C}(43)-\mathrm{C}(44 \mathrm{C})-\mathrm{H}(44 \mathrm{~A})$ & 108.8 \\
\hline $\mathrm{C}(4)-\mathrm{C}(3)-\mathrm{H}(3)$ & 119.2 & $\mathrm{C}(43)-\mathrm{C}(44 \mathrm{C})-\mathrm{H}(44 \mathrm{~B})$ & 108.4 \\
\hline $\mathrm{C}(2)-\mathrm{C}(3)-\mathrm{H}(3)$ & 119.2 & $\mathrm{H}(44 \mathrm{~A})-\mathrm{C}(44 \mathrm{C})-\mathrm{H}(44 \mathrm{~B})$ & 109.5 \\
\hline$C(3)-C(4)-C(5)$ & $121.1(7)$ & $\mathrm{C}(43)-\mathrm{C}(44 \mathrm{C})-\mathrm{H}(44 \mathrm{C})$ & 111.1 \\
\hline $\mathrm{C}(3)-\mathrm{C}(4)-\mathrm{H}(4)$ & 119.4 & $\mathrm{H}(44 \mathrm{~A})-\mathrm{C}(44 \mathrm{C})-\mathrm{H}(44 \mathrm{C})$ & 109.5 \\
\hline $\mathrm{C}(5)-\mathrm{C}(4)-\mathrm{H}(4)$ & 119.4 & $\mathrm{H}(44 \mathrm{~B})-\mathrm{C}(44 \mathrm{C})-\mathrm{H}(44 \mathrm{C})$ & 109.5 \\
\hline$C(6)-C(5)-C(4)$ & $118.4(7)$ & $\mathrm{C}(43)-\mathrm{C}(44 \mathrm{C})-\mathrm{H}(44 \mathrm{E})$ & 97.6 \\
\hline $\mathrm{C}(6)-\mathrm{C}(5)-\mathrm{H}(5)$ & 120.8 & $\mathrm{H}(44 \mathrm{~A})-\mathrm{C}(44 \mathrm{C})-\mathrm{H}(44 \mathrm{E})$ & 15.6 \\
\hline $\mathrm{C}(4)-\mathrm{C}(5)-\mathrm{H}(5)$ & 120.8 & $\mathrm{H}(44 \mathrm{~B})-\mathrm{C}(44 \mathrm{C})-\mathrm{H}(44 \mathrm{E})$ & 104.4 \\
\hline$C(5)-C(6)-C(1)$ & $121.8(7)$ & $\mathrm{H}(44 \mathrm{C})-\mathrm{C}(44 \mathrm{C})-\mathrm{H}(44 \mathrm{E})$ & 124.6 \\
\hline $\mathrm{C}(5)-\mathrm{C}(6)-\mathrm{H}(6)$ & 119.1 & $\mathrm{C}(43)-\mathrm{C}(44 \mathrm{C})-\mathrm{H}(45 \mathrm{E})$ & 93.2 \\
\hline $\mathrm{C}(1)-\mathrm{C}(6)-\mathrm{H}(6)$ & 119.1 & $\mathrm{H}(44 \mathrm{~A})-\mathrm{C}(44 \mathrm{C})-\mathrm{H}(45 \mathrm{E})$ & 140.5 \\
\hline
\end{tabular}




\begin{tabular}{|c|c|c|c|}
\hline$C(12)-C(7)-C(8)$ & $119.8(6)$ & $\mathrm{H}(44 \mathrm{~B})-\mathrm{C}(44 \mathrm{C})-\mathrm{H}(45 \mathrm{E})$ & 93.1 \\
\hline $\mathrm{C}(12)-\mathrm{C}(7)-\mathrm{N}(1)$ & $123.2(6)$ & $\mathrm{H}(44 \mathrm{C})-\mathrm{C}(44 \mathrm{C})-\mathrm{H}(45 \mathrm{E})$ & 31.0 \\
\hline $\mathrm{C}(8)-\mathrm{C}(7)-\mathrm{N}(1)$ & $117.0(6)$ & $\mathrm{H}(44 \mathrm{E})-\mathrm{C}(44 \mathrm{C})-\mathrm{H}(45 \mathrm{E})$ & 155.3 \\
\hline $\mathrm{N}(3)-\mathrm{C}(8)-\mathrm{C}(9)$ & $126.0(6)$ & $\mathrm{C}(43)-\mathrm{C}(44 \mathrm{C})-\mathrm{H}(45 \mathrm{~F})$ & 105.9 \\
\hline $\mathrm{N}(3)-\mathrm{C}(8)-\mathrm{C}(7)$ & $117.1(6)$ & $\mathrm{H}(44 \mathrm{~A})-\mathrm{C}(44 \mathrm{C})-\mathrm{H}(45 \mathrm{~F})$ & 130.6 \\
\hline $\mathrm{C}(9)-\mathrm{C}(8)-\mathrm{C}(7)$ & $116.8(7)$ & $\mathrm{H}(44 \mathrm{~B})-\mathrm{C}(44 \mathrm{C})-\mathrm{H}(45 \mathrm{~F})$ & 24.2 \\
\hline $\mathrm{C}(10)-\mathrm{C}(9)-\mathrm{C}(8)$ & $122.9(7)$ & $\mathrm{H}(44 \mathrm{C})-\mathrm{C}(44 \mathrm{C})-\mathrm{H}(45 \mathrm{~F})$ & 89.0 \\
\hline $\mathrm{C}(10)-\mathrm{C}(9)-\mathrm{H}(9)$ & 118.5 & $\mathrm{H}(44 \mathrm{E})-\mathrm{C}(44 \mathrm{C})-\mathrm{H}(45 \mathrm{~F})$ & 128.1 \\
\hline $\mathrm{C}(8)-\mathrm{C}(9)-\mathrm{H}(9)$ & 118.5 & $\mathrm{H}(45 \mathrm{E})-\mathrm{C}(44 \mathrm{C})-\mathrm{H}(45 \mathrm{~F})$ & 69.1 \\
\hline$C(9)-C(10)-C(11)$ & $120.0(7)$ & $\mathrm{C}(43)-\mathrm{C}(45 \mathrm{C})-\mathrm{H}(45 \mathrm{~A})$ & 107.2 \\
\hline $\mathrm{C}(9)-\mathrm{C}(10)-\mathrm{H}(10)$ & 120.0 & $\mathrm{C}(43)-\mathrm{C}(45 \mathrm{C})-\mathrm{H}(45 \mathrm{~B})$ & 110.7 \\
\hline $\mathrm{C}(11)-\mathrm{C}(10)-\mathrm{H}(10)$ & 120.0 & $\mathrm{H}(45 \mathrm{~A})-\mathrm{C}(45 \mathrm{C})-\mathrm{H}(45 \mathrm{~B})$ & 109.5 \\
\hline $\mathrm{C}(12)-\mathrm{C}(11)-\mathrm{C}(10)$ & $119.5(7)$ & $\mathrm{C}(43)-\mathrm{C}(45 \mathrm{C})-\mathrm{H}(45 \mathrm{C})$ & 110.6 \\
\hline $\mathrm{C}(12)-\mathrm{C}(11)-\mathrm{H}(11)$ & 120.2 & $\mathrm{H}(45 \mathrm{~A})-\mathrm{C}(45 \mathrm{C})-\mathrm{H}(45 \mathrm{C})$ & 109.5 \\
\hline $\mathrm{C}(10)-\mathrm{C}(11)-\mathrm{H}(11)$ & 120.2 & $\mathrm{H}(45 \mathrm{~B})-\mathrm{C}(45 \mathrm{C})-\mathrm{H}(45 \mathrm{C})$ & 109.5 \\
\hline $\mathrm{C}(11)-\mathrm{C}(12)-\mathrm{C}(7)$ & $120.9(7)$ & $\mathrm{C}(43)-\mathrm{C}(45 \mathrm{C})-\mathrm{H}(43 \mathrm{E})$ & 78.7 \\
\hline $\mathrm{C}(11)-\mathrm{C}(12)-\mathrm{H}(12)$ & 119.6 & $\mathrm{H}(45 \mathrm{~A})-\mathrm{C}(45 \mathrm{C})-\mathrm{H}(43 \mathrm{E})$ & 68.9 \\
\hline $\mathrm{C}(7)-\mathrm{C}(12)-\mathrm{H}(12)$ & 119.6 & $\mathrm{H}(45 \mathrm{~B})-\mathrm{C}(45 \mathrm{C})-\mathrm{H}(43 \mathrm{E})$ & 63.5 \\
\hline $\mathrm{C}(18)-\mathrm{C}(13)-\mathrm{C}(14)$ & $118.6(6)$ & $\mathrm{H}(45 \mathrm{C})-\mathrm{C}(45 \mathrm{C})-\mathrm{H}(43 \mathrm{E})$ & 170.4 \\
\hline $\mathrm{C}(18)-\mathrm{C}(13)-\mathrm{N}(1)$ & $123.7(6)$ & $\mathrm{C}(43)-\mathrm{C}(45 \mathrm{C})-\mathrm{H}(43 \mathrm{~F})$ & 98.2 \\
\hline $\mathrm{C}(14)-\mathrm{C}(13)-\mathrm{N}(1)$ & $117.7(6)$ & $\mathrm{H}(45 \mathrm{~A})-\mathrm{C}(45 \mathrm{C})-\mathrm{H}(43 \mathrm{~F})$ & 13.2 \\
\hline $\mathrm{N}(4)-\mathrm{C}(14)-\mathrm{C}(15)$ & $125.4(6)$ & $\mathrm{H}(45 \mathrm{~B})-\mathrm{C}(45 \mathrm{C})-\mathrm{H}(43 \mathrm{~F})$ & 122.4 \\
\hline
\end{tabular}




\begin{tabular}{|c|c|c|c|}
\hline $\mathrm{N}(4)-\mathrm{C}(14)-\mathrm{C}(13)$ & $116.7(6)$ & $\mathrm{H}(45 \mathrm{C})-\mathrm{C}(45 \mathrm{C})-\mathrm{H}(43 \mathrm{~F})$ & 104.8 \\
\hline$C(15)-C(14)-C(13)$ & $117.9(7)$ & $H(43 E)-C(45 C)-H(43 F)$ & 75.7 \\
\hline$C(16)-C(15)-C(14)$ & $122.0(7)$ & $\mathrm{C}(43)-\mathrm{C}(45 \mathrm{C})-\mathrm{H}(45 \mathrm{D})$ & 93.6 \\
\hline $\mathrm{C}(16)-\mathrm{C}(15)-\mathrm{H}(15)$ & 119.0 & $\mathrm{H}(45 \mathrm{~A})-\mathrm{C}(45 \mathrm{C})-\mathrm{H}(45 \mathrm{D})$ & 148.5 \\
\hline $\mathrm{C}(14)-\mathrm{C}(15)-\mathrm{H}(15)$ & 119.0 & $\mathrm{H}(45 \mathrm{~B})-\mathrm{C}(45 \mathrm{C})-\mathrm{H}(45 \mathrm{D})$ & 83.8 \\
\hline $\mathrm{C}(15)-\mathrm{C}(16)-\mathrm{C}(17)$ & $120.8(8)$ & $\mathrm{H}(45 \mathrm{C})-\mathrm{C}(45 \mathrm{C})-\mathrm{H}(45 \mathrm{D})$ & 39.6 \\
\hline $\mathrm{C}(15)-\mathrm{C}(16)-\mathrm{H}(16)$ & 119.6 & $\mathrm{H}(43 \mathrm{E})-\mathrm{C}(45 \mathrm{C})-\mathrm{H}(45 \mathrm{D})$ & 140.1 \\
\hline $\mathrm{C}(17)-\mathrm{C}(16)-\mathrm{H}(16)$ & 119.6 & $\mathrm{H}(43 \mathrm{~F})-\mathrm{C}(45 \mathrm{C})-\mathrm{H}(45 \mathrm{D})$ & 144.1 \\
\hline $\mathrm{C}(18)-\mathrm{C}(17)-\mathrm{C}(16)$ & $118.4(7)$ & $\mathrm{C}(43)-\mathrm{C}(43 \mathrm{D})-\mathrm{H}(43 \mathrm{C})$ & 108.6 \\
\hline $\mathrm{C}(18)-\mathrm{C}(17)-\mathrm{H}(17)$ & 120.8 & $\mathrm{C}(43)-\mathrm{C}(43 \mathrm{D})-\mathrm{H}(45 \mathrm{~A})$ & 120.9 \\
\hline $\mathrm{C}(16)-\mathrm{C}(17)-\mathrm{H}(17)$ & 120.8 & $\mathrm{H}(43 \mathrm{C})-\mathrm{C}(43 \mathrm{D})-\mathrm{H}(45 \mathrm{~A})$ & 126.1 \\
\hline $\mathrm{C}(17)-\mathrm{C}(18)-\mathrm{C}(13)$ & $122.2(7)$ & $\mathrm{C}(43)-\mathrm{C}(43 \mathrm{D})-\mathrm{H}(43 \mathrm{D})$ & 104.5 \\
\hline $\mathrm{C}(17)-\mathrm{C}(18)-\mathrm{H}(18)$ & 118.9 & $\mathrm{H}(43 \mathrm{C})-\mathrm{C}(43 \mathrm{D})-\mathrm{H}(43 \mathrm{D})$ & 27.9 \\
\hline $\mathrm{C}(13)-\mathrm{C}(18)-\mathrm{H}(18)$ & 118.9 & $\mathrm{H}(45 \mathrm{~A})-\mathrm{C}(43 \mathrm{D})-\mathrm{H}(43 \mathrm{D})$ & 113.2 \\
\hline $\mathrm{C}(24)-\mathrm{C}(19)-\mathrm{C}(20)$ & $116.6(7)$ & $\mathrm{C}(43)-\mathrm{C}(43 \mathrm{D})-\mathrm{H}(43 \mathrm{E})$ & 111.6 \\
\hline $\mathrm{C}(24)-\mathrm{C}(19)-\mathrm{N}(2)$ & $122.8(6)$ & $\mathrm{H}(43 \mathrm{C})-\mathrm{C}(43 \mathrm{D})-\mathrm{H}(43 \mathrm{E})$ & 82.8 \\
\hline $\mathrm{C}(20)-\mathrm{C}(19)-\mathrm{N}(2)$ & $120.4(7)$ & $\mathrm{H}(45 \mathrm{~A})-\mathrm{C}(43 \mathrm{D})-\mathrm{H}(43 \mathrm{E})$ & 96.9 \\
\hline $\mathrm{C}(21)-\mathrm{C}(20)-\mathrm{C}(19)$ & $122.0(8)$ & $\mathrm{H}(43 \mathrm{D})-\mathrm{C}(43 \mathrm{D})-\mathrm{H}(43 \mathrm{E})$ & 109.5 \\
\hline $\mathrm{C}(21)-\mathrm{C}(20)-\mathrm{H}(20)$ & 119.0 & $\mathrm{C}(43)-\mathrm{C}(43 \mathrm{D})-\mathrm{H}(43 \mathrm{~F})$ & 112.2 \\
\hline $\mathrm{C}(19)-\mathrm{C}(20)-\mathrm{H}(20)$ & 119.0 & $\mathrm{H}(43 \mathrm{C})-\mathrm{C}(43 \mathrm{D})-\mathrm{H}(43 \mathrm{~F})$ & 128.2 \\
\hline $\mathrm{C}(20)-\mathrm{C}(21)-\mathrm{C}(22)$ & $122.2(8)$ & $\mathrm{H}(45 \mathrm{~A})-\mathrm{C}(43 \mathrm{D})-\mathrm{H}(43 \mathrm{~F})$ & 12.8 \\
\hline $\mathrm{C}(20)-\mathrm{C}(21)-\mathrm{H}(21)$ & 118.9 & $\mathrm{H}(43 \mathrm{D})-\mathrm{C}(43 \mathrm{D})-\mathrm{H}(43 \mathrm{~F})$ & 109.5 \\
\hline
\end{tabular}




\begin{tabular}{|c|c|c|c|}
\hline $\mathrm{C}(22)-\mathrm{C}(21)-\mathrm{H}(21)$ & 118.9 & $\mathrm{H}(43 \mathrm{E})-\mathrm{C}(43 \mathrm{D})-\mathrm{H}(43 \mathrm{~F})$ & 109.5 \\
\hline$C(23)-C(22)-C(21)$ & $115.6(7)$ & $\mathrm{C}(43)-\mathrm{C}(44 \mathrm{D})-\mathrm{H}(44 \mathrm{~A})$ & 100.7 \\
\hline$C(23)-C(22)-C(25)$ & $122.1(8)$ & $\mathrm{C}(43)-\mathrm{C}(44 \mathrm{D})-\mathrm{H}(44 \mathrm{D})$ & 108.9 \\
\hline $\mathrm{C}(21)-\mathrm{C}(22)-\mathrm{C}(25)$ & $122.3(7)$ & H(44A)-C(44D)-H(44D) & 137.2 \\
\hline $\mathrm{C}(22)-\mathrm{C}(23)-\mathrm{C}(24)$ & $121.9(7)$ & $\mathrm{C}(43)-\mathrm{C}(44 \mathrm{D})-\mathrm{H}(44 \mathrm{E})$ & 109.3 \\
\hline $\mathrm{C}(22)-\mathrm{C}(23)-\mathrm{H}(23)$ & 119.0 & $\mathrm{H}(44 \mathrm{~A})-\mathrm{C}(44 \mathrm{D})-\mathrm{H}(44 \mathrm{E})$ & 29.4 \\
\hline $\mathrm{C}(24)-\mathrm{C}(23)-\mathrm{H}(23)$ & 119.0 & H(44D)-C(44D)-H(44E) & 109.5 \\
\hline$C(27 F)-C(25)-C(26 E)$ & $133.8(16)$ & $\mathrm{C}(43)-\mathrm{C}(44 \mathrm{D})-\mathrm{H}(44 \mathrm{~F})$ & 110.2 \\
\hline $\mathrm{C}(27 \mathrm{~F})-\mathrm{C}(25)-\mathrm{C}(22)$ & $116.0(14)$ & $\mathrm{H}(44 \mathrm{~A})-\mathrm{C}(44 \mathrm{D})-\mathrm{H}(44 \mathrm{~F})$ & 87.4 \\
\hline$C(26 E)-C(25)-C(22)$ & $110.2(10)$ & $\mathrm{H}(44 \mathrm{D})-\mathrm{C}(44 \mathrm{D})-\mathrm{H}(44 \mathrm{~F})$ & 109.5 \\
\hline$C(27 F)-C(25)-C(27 E)$ & $59.5(14)$ & $\mathrm{H}(44 \mathrm{E})-\mathrm{C}(44 \mathrm{D})-\mathrm{H}(44 \mathrm{~F})$ & 109.5 \\
\hline$C(26 E)-C(25)-C(27 E)$ & $102.7(12)$ & $\mathrm{C}(43)-\mathrm{C}(45 \mathrm{D})-\mathrm{H}(44 \mathrm{~B})$ & 86.2 \\
\hline$C(22)-C(25)-C(27 E)$ & 111.1(9) & $\mathrm{C}(43)-\mathrm{C}(45 \mathrm{D})-\mathrm{H}(44 \mathrm{C})$ & 86.7 \\
\hline$C(27 F)-C(25)-C(25 E)$ & $45.4(13)$ & $\mathrm{H}(44 \mathrm{~B})-\mathrm{C}(45 \mathrm{D})-\mathrm{H}(44 \mathrm{C})$ & 76.7 \\
\hline$C(26 E)-C(25)-C(25 E)$ & $119.0(13)$ & $\mathrm{C}(43)-\mathrm{C}(45 \mathrm{D})-\mathrm{H}(45 \mathrm{D})$ & 110.8 \\
\hline$C(22)-C(25)-C(25 E)$ & $109.4(10)$ & $\mathrm{H}(44 \mathrm{~B})-\mathrm{C}(45 \mathrm{D})-\mathrm{H}(45 \mathrm{D})$ & 125.9 \\
\hline$C(27 E)-C(25)-C(25 E)$ & $104.0(12)$ & $\mathrm{H}(44 \mathrm{C})-\mathrm{C}(45 \mathrm{D})-\mathrm{H}(45 \mathrm{D})$ & 150.9 \\
\hline$C(27 F)-C(25)-C(26 F)$ & $111.4(19)$ & $\mathrm{C}(43)-\mathrm{C}(45 \mathrm{D})-\mathrm{H}(45 \mathrm{E})$ & 109.4 \\
\hline$C(26 E)-C(25)-C(26 F)$ & $48.4(12)$ & $\mathrm{H}(44 \mathrm{~B})-\mathrm{C}(45 \mathrm{D})-\mathrm{H}(45 \mathrm{E})$ & 112.2 \\
\hline $\mathrm{C}(22)-\mathrm{C}(25)-\mathrm{C}(26 \mathrm{~F})$ & $109.3(12)$ & $\mathrm{H}(44 \mathrm{C})-\mathrm{C}(45 \mathrm{D})-\mathrm{H}(45 \mathrm{E})$ & 41.5 \\
\hline$C(27 E)-C(25)-C(26 F)$ & $57.4(13)$ & $\mathrm{H}(45 \mathrm{D})-\mathrm{C}(45 \mathrm{D})-\mathrm{H}(45 \mathrm{E})$ & 109.5 \\
\hline$C(25 E)-C(25)-C(26 F)$ & $141.1(14)$ & $\mathrm{C}(43)-\mathrm{C}(45 \mathrm{D})-\mathrm{H}(45 \mathrm{~F})$ & 108.2 \\
\hline
\end{tabular}




\begin{tabular}{|c|c|c|c|}
\hline$C(27 F)-C(25)-C(25 F)$ & $108.2(15)$ & $\mathrm{H}(44 \mathrm{~B})-\mathrm{C}(45 \mathrm{D})-\mathrm{H}(45 \mathrm{~F})$ & 23.1 \\
\hline$C(26 E)-C(25)-C(25 F)$ & $51.4(10)$ & $\mathrm{H}(44 \mathrm{C})-\mathrm{C}(45 \mathrm{D})-\mathrm{H}(45 \mathrm{~F})$ & 85.3 \\
\hline$C(22)-C(25)-C(25 F)$ & $113.4(9)$ & $\mathrm{H}(45 \mathrm{D})-\mathrm{C}(45 \mathrm{D})-\mathrm{H}(45 \mathrm{~F})$ & 109.5 \\
\hline$C(27 E)-C(25)-C(25 F)$ & $134.1(11)$ & $\mathrm{H}(45 \mathrm{E})-\mathrm{C}(45 \mathrm{D})-\mathrm{H}(45 \mathrm{~F})$ & 109.5 \\
\hline$C(25 E)-C(25)-C(25 F)$ & $71.0(11)$ & $\mathrm{C}(25)-\mathrm{C}(25 \mathrm{E})-\mathrm{H}(25 \mathrm{~A})$ & 111.8 \\
\hline$C(26 F)-C(25)-C(25 F)$ & $97.1(14)$ & $\mathrm{C}(25)-\mathrm{C}(25 \mathrm{E})-\mathrm{H}(25 \mathrm{~B})$ & 111.3 \\
\hline$C(19)-C(24)-C(23)$ & $121.7(7)$ & $\mathrm{H}(25 \mathrm{~A})-\mathrm{C}(25 \mathrm{E})-\mathrm{H}(25 \mathrm{~B})$ & 109.5 \\
\hline $\mathrm{C}(19)-\mathrm{C}(24)-\mathrm{H}(24)$ & 119.1 & $\mathrm{C}(25)-\mathrm{C}(25 \mathrm{E})-\mathrm{H}(25 \mathrm{C})$ & 105.2 \\
\hline $\mathrm{C}(23)-\mathrm{C}(24)-\mathrm{H}(24)$ & 119.1 & $\mathrm{H}(25 \mathrm{~A})-\mathrm{C}(25 \mathrm{E})-\mathrm{H}(25 \mathrm{C})$ & 109.5 \\
\hline$C(33)-C(28)-C(29)$ & $115.5(7)$ & $\mathrm{H}(25 \mathrm{~B})-\mathrm{C}(25 \mathrm{E})-\mathrm{H}(25 \mathrm{C})$ & 109.5 \\
\hline $\mathrm{C}(33)-\mathrm{C}(28)-\mathrm{N}(3)$ & $121.7(7)$ & $\mathrm{C}(25)-\mathrm{C}(25 \mathrm{E})-\mathrm{H}(27 \mathrm{D})$ & 102.5 \\
\hline $\mathrm{C}(29)-\mathrm{C}(28)-\mathrm{N}(3)$ & $122.7(7)$ & $\mathrm{H}(25 \mathrm{~A})-\mathrm{C}(25 \mathrm{E})-\mathrm{H}(27 \mathrm{D})$ & 73.9 \\
\hline$C(30)-C(29)-C(28)$ & $120.8(7)$ & $H(25 B)-C(25 E)-H(27 D)$ & 140.7 \\
\hline $\mathrm{C}(30)-\mathrm{C}(29)-\mathrm{H}(29)$ & 119.6 & $\mathrm{H}(25 \mathrm{C})-\mathrm{C}(25 \mathrm{E})-\mathrm{H}(27 \mathrm{D})$ & 40.1 \\
\hline $\mathrm{C}(28)-\mathrm{C}(29)-\mathrm{H}(29)$ & 119.6 & $\mathrm{C}(25)-\mathrm{C}(25 \mathrm{E})-\mathrm{H}(27 \mathrm{E})$ & 78.6 \\
\hline $\mathrm{C}(31)-\mathrm{C}(30)-\mathrm{C}(29)$ & $122.7(7)$ & $\mathrm{H}(25 \mathrm{~A})-\mathrm{C}(25 \mathrm{E})-\mathrm{H}(27 \mathrm{E})$ & 145.9 \\
\hline $\mathrm{C}(31)-\mathrm{C}(30)-\mathrm{H}(30)$ & 118.7 & $\mathrm{H}(25 \mathrm{~B})-\mathrm{C}(25 \mathrm{E})-\mathrm{H}(27 \mathrm{E})$ & 95.2 \\
\hline $\mathrm{C}(29)-\mathrm{C}(30)-\mathrm{H}(30)$ & 118.7 & $\mathrm{H}(25 \mathrm{C})-\mathrm{C}(25 \mathrm{E})-\mathrm{H}(27 \mathrm{E})$ & 37.9 \\
\hline$C(30)-C(31)-C(32)$ & $115.8(7)$ & $\mathrm{H}(27 \mathrm{D})-\mathrm{C}(25 \mathrm{E})-\mathrm{H}(27 \mathrm{E})$ & 72.1 \\
\hline $\mathrm{C}(30)-\mathrm{C}(31)-\mathrm{C}(34)$ & $121.7(7)$ & $\mathrm{C}(25)-\mathrm{C}(26 \mathrm{E})-\mathrm{H}(26 \mathrm{~A})$ & 108.7 \\
\hline$C(32)-C(31)-C(34)$ & $122.5(8)$ & $C(25)-C(26 E)-H(26 B)$ & 108.7 \\
\hline$C(33)-C(32)-C(31)$ & $121.7(8)$ & $H(26 A)-C(26 E)-H(26 B)$ & 109. \\
\hline
\end{tabular}




\begin{tabular}{|c|c|c|c|}
\hline $\mathrm{C}(33)-\mathrm{C}(32)-\mathrm{H}(32)$ & 119.2 & $C(25)-C(26 E)-H(26 C)$ & 111.0 \\
\hline $\mathrm{C}(31)-\mathrm{C}(32)-\mathrm{H}(32)$ & 119.2 & $\mathrm{H}(26 \mathrm{~A})-\mathrm{C}(26 \mathrm{E})-\mathrm{H}(26 \mathrm{C})$ & 109.5 \\
\hline $\mathrm{C}(32)-\mathrm{C}(33)-\mathrm{C}(28)$ & $123.6(7)$ & $\mathrm{H}(26 \mathrm{~B})-\mathrm{C}(26 \mathrm{E})-\mathrm{H}(26 \mathrm{C})$ & 109.5 \\
\hline $\mathrm{C}(32)-\mathrm{C}(33)-\mathrm{H}(33)$ & 118.2 & $\mathrm{C}(25)-\mathrm{C}(26 \mathrm{E})-\mathrm{H}(25 \mathrm{E})$ & 98.6 \\
\hline $\mathrm{C}(28)-\mathrm{C}(33)-\mathrm{H}(33)$ & 118.2 & $\mathrm{H}(26 \mathrm{~A})-\mathrm{C}(26 \mathrm{E})-\mathrm{H}(25 \mathrm{E})$ & 14.6 \\
\hline $\mathrm{C}(36 \mathrm{~B})-\mathrm{C}(34)-\mathrm{C}(35 \mathrm{~A})$ & $128.4(16)$ & $\mathrm{H}(26 \mathrm{~B})-\mathrm{C}(26 \mathrm{E})-\mathrm{H}(25 \mathrm{E})$ & 104.0 \\
\hline $\mathrm{C}(36 \mathrm{~B})-\mathrm{C}(34)-\mathrm{C}(34 \mathrm{~A})$ & $51.0(14)$ & $\mathrm{H}(26 \mathrm{C})-\mathrm{C}(26 \mathrm{E})-\mathrm{H}(25 \mathrm{E})$ & 123.8 \\
\hline $\mathrm{C}(35 \mathrm{~A})-\mathrm{C}(34)-\mathrm{C}(34 \mathrm{~A})$ & $126.4(13)$ & $\mathrm{C}(25)-\mathrm{C}(26 \mathrm{E})-\mathrm{H}(26 \mathrm{E})$ & 89.4 \\
\hline$C(36 B)-C(34)-C(31)$ & $119.4(14)$ & $H(26 A)-C(26 E)-H(26 E)$ & 139.7 \\
\hline$C(35 A)-C(34)-C(31)$ & $109.6(10)$ & $H(26 B)-C(26 E)-H(26 E)$ & 97.5 \\
\hline$C(34 A)-C(34)-C(31)$ & $108.7(10)$ & $H(26 C)-C(26 E)-H(26 E)$ & 31.1 \\
\hline $\mathrm{C}(36 \mathrm{~B})-\mathrm{C}(34)-\mathrm{C}(34 \mathrm{~B})$ & $114.6(16)$ & $\mathrm{H}(25 \mathrm{E})-\mathrm{C}(26 \mathrm{E})-\mathrm{H}(26 \mathrm{E})$ & 153.1 \\
\hline $\mathrm{C}(35 \mathrm{~A})-\mathrm{C}(34)-\mathrm{C}(34 \mathrm{~B})$ & $55.5(10)$ & $\mathrm{C}(25)-\mathrm{C}(26 \mathrm{E})-\mathrm{H}(26 \mathrm{~F})$ & 101.5 \\
\hline $\mathrm{C}(34 \mathrm{~A})-\mathrm{C}(34)-\mathrm{C}(34 \mathrm{~B})$ & $76.3(11)$ & $H(26 A)-C(26 E)-H(26 F)$ & 135.9 \\
\hline $\mathrm{C}(31)-\mathrm{C}(34)-\mathrm{C}(34 \mathrm{~B})$ & $111.5(9)$ & $\mathrm{H}(26 \mathrm{~B})-\mathrm{C}(26 \mathrm{E})-\mathrm{H}(26 \mathrm{~F})$ & 28.3 \\
\hline$C(36 B)-C(34)-C(36 A)$ & $49.8(14)$ & $\mathrm{H}(26 \mathrm{C})-\mathrm{C}(26 \mathrm{E})-\mathrm{H}(26 \mathrm{~F})$ & 87.8 \\
\hline$C(35 A)-C(34)-C(36 A)$ & $98.6(11)$ & $\mathrm{H}(25 \mathrm{E})-\mathrm{C}(26 \mathrm{E})-\mathrm{H}(26 \mathrm{~F})$ & 132.3 \\
\hline$C(34 A)-C(34)-C(36 A)$ & $100.7(11)$ & $\mathrm{H}(26 \mathrm{E})-\mathrm{C}(26 \mathrm{E})-\mathrm{H}(26 \mathrm{~F})$ & 69.9 \\
\hline$C(31)-C(34)-C(36 A)$ & $111.4(9)$ & $\mathrm{C}(25)-\mathrm{C}(27 \mathrm{E})-\mathrm{H}(27 \mathrm{~A})$ & 110.7 \\
\hline $\mathrm{C}(34 \mathrm{~B})-\mathrm{C}(34)-\mathrm{C}(36 \mathrm{~A})$ & $135.4(10)$ & $\mathrm{C}(25)-\mathrm{C}(27 \mathrm{E})-\mathrm{H}(27 \mathrm{~B})$ & 109.7 \\
\hline $\mathrm{C}(36 \mathrm{~B})-\mathrm{C}(34)-\mathrm{C}(35 \mathrm{~B})$ & $94.9(19)$ & $\mathrm{H}(27 \mathrm{~A})-\mathrm{C}(27 \mathrm{E})-\mathrm{H}(27 \mathrm{~B})$ & 109.5 \\
\hline $\mathrm{C}(35 \mathrm{~A})-\mathrm{C}(34)-\mathrm{C}(35 \mathrm{~B})$ & $51.9(14)$ & $\mathrm{C}(25)-\mathrm{C}(27 \mathrm{E})-\mathrm{H}(27 \mathrm{C})$ & 108.0 \\
\hline
\end{tabular}




\begin{tabular}{|c|c|c|c|}
\hline $\mathrm{C}(34 \mathrm{~A})-\mathrm{C}(34)-\mathrm{C}(35 \mathrm{~B})$ & $138.1(16)$ & $\mathrm{H}(27 \mathrm{~A})-\mathrm{C}(27 \mathrm{E})-\mathrm{H}(27 \mathrm{C})$ & 109.5 \\
\hline $\mathrm{C}(31)-\mathrm{C}(34)-\mathrm{C}(35 \mathrm{~B})$ & $109.7(14)$ & $\mathrm{H}(27 \mathrm{~B})-\mathrm{C}(27 \mathrm{E})-\mathrm{H}(27 \mathrm{C})$ & 109.5 \\
\hline $\mathrm{C}(34 \mathrm{~B})-\mathrm{C}(34)-\mathrm{C}(35 \mathrm{~B})$ & $104.3(16)$ & $\mathrm{C}(25)-\mathrm{C}(27 \mathrm{E})-\mathrm{H}(26 \mathrm{D})$ & 92.4 \\
\hline $\mathrm{C}(36 \mathrm{~A})-\mathrm{C}(34)-\mathrm{C}(35 \mathrm{~B})$ & $49.2(13)$ & $\mathrm{H}(27 \mathrm{~A})-\mathrm{C}(27 \mathrm{E})-\mathrm{H}(26 \mathrm{D})$ & 147.0 \\
\hline $\mathrm{C}(38)-\mathrm{C}(37)-\mathrm{C}(42)$ & $115.8(6)$ & $\mathrm{H}(27 \mathrm{~B})-\mathrm{C}(27 \mathrm{E})-\mathrm{H}(26 \mathrm{D})$ & 82.8 \\
\hline $\mathrm{C}(38)-\mathrm{C}(37)-\mathrm{N}(4)$ & $121.4(6)$ & $\mathrm{H}(27 \mathrm{C})-\mathrm{C}(27 \mathrm{E})-\mathrm{H}(26 \mathrm{D})$ & 38.7 \\
\hline $\mathrm{C}(42)-\mathrm{C}(37)-\mathrm{N}(4)$ & $122.5(6)$ & $\mathrm{C}(25)-\mathrm{C}(27 \mathrm{E})-\mathrm{H}(27 \mathrm{~F})$ & 88.0 \\
\hline $\mathrm{C}(39)-\mathrm{C}(38)-\mathrm{C}(37)$ & $123.5(7)$ & $\mathrm{H}(27 \mathrm{~A})-\mathrm{C}(27 \mathrm{E})-\mathrm{H}(27 \mathrm{~F})$ & 22.7 \\
\hline $\mathrm{C}(39)-\mathrm{C}(38)-\mathrm{H}(38)$ & 118.2 & $\mathrm{H}(27 \mathrm{~B})-\mathrm{C}(27 \mathrm{E})-\mathrm{H}(27 \mathrm{~F})$ & 117.8 \\
\hline $\mathrm{C}(37)-\mathrm{C}(38)-\mathrm{H}(38)$ & 118.2 & $\mathrm{H}(27 \mathrm{C})-\mathrm{C}(27 \mathrm{E})-\mathrm{H}(27 \mathrm{~F})$ & 120.8 \\
\hline $\mathrm{C}(38)-\mathrm{C}(39)-\mathrm{C}(40)$ & $121.5(7)$ & $\mathrm{H}(26 \mathrm{D})-\mathrm{C}(27 \mathrm{E})-\mathrm{H}(27 \mathrm{~F})$ & 157.9 \\
\hline $\mathrm{C}(38)-\mathrm{C}(39)-\mathrm{H}(39)$ & 119.2 & $\mathrm{C}(25)-\mathrm{C}(25 \mathrm{~F})-\mathrm{H}(25 \mathrm{~B})$ & 82.9 \\
\hline C(40)-C(39)-H(39) & 119.2 & $\mathrm{C}(25)-\mathrm{C}(25 \mathrm{~F})-\mathrm{H}(26 \mathrm{~A})$ & 99.6 \\
\hline $\mathrm{C}(41)-\mathrm{C}(40)-\mathrm{C}(39)$ & 116.7(7) & $\mathrm{H}(25 \mathrm{~B})-\mathrm{C}(25 \mathrm{~F})-\mathrm{H}(26 \mathrm{~A})$ & 170.0 \\
\hline $\mathrm{C}(41)-\mathrm{C}(40)-\mathrm{C}(43)$ & $120.6(7)$ & $\mathrm{C}(25)-\mathrm{C}(25 \mathrm{~F})-\mathrm{H}(25 \mathrm{D})$ & 109.0 \\
\hline$C(39)-C(40)-C(43)$ & $122.7(7)$ & $\mathrm{H}(25 \mathrm{~B})-\mathrm{C}(25 \mathrm{~F})-\mathrm{H}(25 \mathrm{D})$ & 48.4 \\
\hline $\mathrm{C}(40)-\mathrm{C}(41)-\mathrm{C}(42)$ & $120.8(7)$ & $\mathrm{H}(26 \mathrm{~A})-\mathrm{C}(25 \mathrm{~F})-\mathrm{H}(25 \mathrm{D})$ & 137.7 \\
\hline $\mathrm{C}(40)-\mathrm{C}(41)-\mathrm{H}(41)$ & 119.6 & $\mathrm{C}(25)-\mathrm{C}(25 \mathrm{~F})-\mathrm{H}(25 \mathrm{E})$ & 109.1 \\
\hline $\mathrm{C}(42)-\mathrm{C}(41)-\mathrm{H}(41)$ & 119.6 & $\mathrm{H}(25 \mathrm{~B})-\mathrm{C}(25 \mathrm{~F})-\mathrm{H}(25 \mathrm{E})$ & 157.9 \\
\hline $\mathrm{C}(37)-\mathrm{C}(42)-\mathrm{C}(41)$ & $121.6(7)$ & $\mathrm{H}(26 \mathrm{~A})-\mathrm{C}(25 \mathrm{~F})-\mathrm{H}(25 \mathrm{E})$ & 29.5 \\
\hline $\mathrm{C}(37)-\mathrm{C}(42)-\mathrm{H}(42)$ & 119.2 & $\mathrm{H}(25 \mathrm{D})-\mathrm{C}(25 \mathrm{~F})-\mathrm{H}(25 \mathrm{E})$ & 109.5 \\
\hline $\mathrm{C}(41)-\mathrm{C}(42)-\mathrm{H}(42)$ & 119.2 & $\mathrm{C}(25)-\mathrm{C}(25 \mathrm{~F})-\mathrm{H}(25 \mathrm{~F})$ & 110.3 \\
\hline
\end{tabular}




\begin{tabular}{|c|c|c|c|}
\hline$C(43 D)-C(43)-C(43 C)$ & $56.6(15)$ & $\mathrm{H}(25 \mathrm{~B})-\mathrm{C}(25 \mathrm{~F})-\mathrm{H}(25 \mathrm{~F})$ & 82.1 \\
\hline$C(43 D)-C(43)-C(44 C)$ & $127.6(17)$ & $\mathrm{H}(26 \mathrm{~A})-\mathrm{C}(25 \mathrm{~F})-\mathrm{H}(25 \mathrm{~F})$ & 88.0 \\
\hline$C(43 C)-C(43)-C(44 C)$ & $124.8(13)$ & $\mathrm{H}(25 \mathrm{D})-\mathrm{C}(25 \mathrm{~F})-\mathrm{H}(25 \mathrm{~F})$ & 109.5 \\
\hline$C(43 D)-C(43)-C(40)$ & $117.6(15)$ & $\mathrm{H}(25 \mathrm{E})-\mathrm{C}(25 \mathrm{~F})-\mathrm{H}(25 \mathrm{~F})$ & 109.5 \\
\hline$C(43 C)-C(43)-C(40)$ & $109.5(10)$ & $\mathrm{C}(25)-\mathrm{C}(26 \mathrm{~F})-\mathrm{H}(26 \mathrm{~B})$ & 85.9 \\
\hline$C(44 C)-C(43)-C(40)$ & $110.1(10)$ & $\mathrm{C}(25)-\mathrm{C}(26 \mathrm{~F})-\mathrm{H}(26 \mathrm{C})$ & 89.9 \\
\hline$C(43 D)-C(43)-C(45 C)$ & $45.0(14)$ & $\mathrm{H}(26 \mathrm{~B})-\mathrm{C}(26 \mathrm{~F})-\mathrm{H}(26 \mathrm{C})$ & 73.0 \\
\hline$C(43 C)-C(43)-C(45 C)$ & $101.2(12)$ & $\mathrm{C}(25)-\mathrm{C}(26 \mathrm{~F})-\mathrm{H}(27 \mathrm{~B})$ & 83.2 \\
\hline$C(44 C)-C(43)-C(45 C)$ & $98.9(12)$ & $H(26 B)-C(26 F)-H(27 B)$ & 160.2 \\
\hline$C(40)-C(43)-C(45 C)$ & $110.9(9)$ & $H(26 C)-C(26 F)-H(27 B)$ & 90.5 \\
\hline$C(43 D)-C(43)-C(44 D)$ & $120.7(16)$ & $\mathrm{C}(25)-\mathrm{C}(26 \mathrm{~F})-\mathrm{H}(27 \mathrm{C})$ & 82.3 \\
\hline$C(43 C)-C(43)-C(44 D)$ & $78.4(11)$ & $\mathrm{H}(26 \mathrm{~B})-\mathrm{C}(26 \mathrm{~F})-\mathrm{H}(27 \mathrm{C})$ & 133.7 \\
\hline$C(44 C)-C(43)-C(44 D)$ & $51.7(10)$ & $\mathrm{H}(26 \mathrm{C})-\mathrm{C}(26 \mathrm{~F})-\mathrm{H}(27 \mathrm{C})$ & 150.9 \\
\hline$C(40)-C(43)-C(44 D)$ & $112.3(9)$ & $\mathrm{H}(27 \mathrm{~B})-\mathrm{C}(26 \mathrm{~F})-\mathrm{H}(27 \mathrm{C})$ & 60.8 \\
\hline$C(45 C)-C(43)-C(44 D)$ & $134.1(11)$ & $\mathrm{C}(25)-\mathrm{C}(26 \mathrm{~F})-\mathrm{H}(26 \mathrm{D})$ & 113.9 \\
\hline$C(43 D)-C(43)-C(45 D)$ & $98.1(18)$ & $\mathrm{H}(26 \mathrm{~B})-\mathrm{C}(26 \mathrm{~F})-\mathrm{H}(26 \mathrm{D})$ & 122.9 \\
\hline$C(43 C)-C(43)-C(45 D)$ & $142.1(14)$ & $\mathrm{H}(26 \mathrm{C})-\mathrm{C}(26 \mathrm{~F})-\mathrm{H}(26 \mathrm{D})$ & 151.0 \\
\hline$C(44 C)-C(43)-C(45 D)$ & $45.7(11)$ & $\mathrm{H}(27 \mathrm{~B})-\mathrm{C}(26 \mathrm{~F})-\mathrm{H}(26 \mathrm{D})$ & 76.7 \\
\hline$C(40)-C(43)-C(45 D)$ & $107.3(11)$ & $\mathrm{H}(27 \mathrm{C})-\mathrm{C}(26 \mathrm{~F})-\mathrm{H}(26 \mathrm{D})$ & 33.3 \\
\hline$C(45 C)-C(43)-C(45 D)$ & $56.6(11)$ & $\mathrm{C}(25)-\mathrm{C}(26 \mathrm{~F})-\mathrm{H}(26 \mathrm{E})$ & 111.3 \\
\hline$C(44 D)-C(43)-C(45 D)$ & $95.8(13)$ & $\mathrm{H}(26 \mathrm{~B})-\mathrm{C}(26 \mathrm{~F})-\mathrm{H}(26 \mathrm{E})$ & 111.4 \\
\hline $\mathrm{C}(34)-\mathrm{C}(34 \mathrm{~A})-\mathrm{H}(34 \mathrm{~A})$ & 110.5 & $H(26 C)-C(26 F)-H(26 E)$ & 43. \\
\hline
\end{tabular}




\begin{tabular}{|c|c|c|c|}
\hline $\mathrm{C}(34)-\mathrm{C}(34 \mathrm{~A})-\mathrm{H}(34 \mathrm{~B})$ & 110.1 & $\mathrm{H}(27 \mathrm{~B})-\mathrm{C}(26 \mathrm{~F})-\mathrm{H}(26 \mathrm{E})$ & 58.3 \\
\hline $\mathrm{H}(34 \mathrm{~A})-\mathrm{C}(34 \mathrm{~A})-\mathrm{H}(34 \mathrm{~B})$ & 109.5 & $\mathrm{H}(27 \mathrm{C})-\mathrm{C}(26 \mathrm{~F})-\mathrm{H}(26 \mathrm{E})$ & 114.5 \\
\hline $\mathrm{C}(34)-\mathrm{C}(34 \mathrm{~A})-\mathrm{H}(34 \mathrm{C})$ & 107.8 & $\mathrm{H}(26 \mathrm{D})-\mathrm{C}(26 \mathrm{~F})-\mathrm{H}(26 \mathrm{E})$ & 109.5 \\
\hline $\mathrm{H}(34 \mathrm{~A})-\mathrm{C}(34 \mathrm{~A})-\mathrm{H}(34 \mathrm{C})$ & 109.5 & $\mathrm{C}(25)-\mathrm{C}(26 \mathrm{~F})-\mathrm{H}(26 \mathrm{~F})$ & 103.1 \\
\hline $\mathrm{H}(34 \mathrm{~B})-\mathrm{C}(34 \mathrm{~A})-\mathrm{H}(34 \mathrm{C})$ & 109.5 & $\mathrm{H}(26 \mathrm{~B})-\mathrm{C}(26 \mathrm{~F})-\mathrm{H}(26 \mathrm{~F})$ & 18.1 \\
\hline $\mathrm{C}(34)-\mathrm{C}(34 \mathrm{~A})-\mathrm{H}(36 \mathrm{D})$ & 98.9 & $\mathrm{H}(26 \mathrm{C})-\mathrm{C}(26 \mathrm{~F})-\mathrm{H}(26 \mathrm{~F})$ & 79.1 \\
\hline $\mathrm{H}(34 \mathrm{~A})-\mathrm{C}(34 \mathrm{~A})-\mathrm{H}(36 \mathrm{D})$ & 90.6 & $\mathrm{H}(27 \mathrm{~B})-\mathrm{C}(26 \mathrm{~F})-\mathrm{H}(26 \mathrm{~F})$ & 167.8 \\
\hline $\mathrm{H}(34 \mathrm{~B})-\mathrm{C}(34 \mathrm{~A})-\mathrm{H}(36 \mathrm{D})$ & 134.9 & $\mathrm{H}(27 \mathrm{C})-\mathrm{C}(26 \mathrm{~F})-\mathrm{H}(26 \mathrm{~F})$ & 130.0 \\
\hline $\mathrm{H}(34 \mathrm{C})-\mathrm{C}(34 \mathrm{~A})-\mathrm{H}(36 \mathrm{D})$ & 26.4 & $\mathrm{H}(26 \mathrm{D})-\mathrm{C}(26 \mathrm{~F})-\mathrm{H}(26 \mathrm{~F})$ & 109.5 \\
\hline $\mathrm{C}(34)-\mathrm{C}(35 \mathrm{~A})-\mathrm{H}(35 \mathrm{~A})$ & 106.7 & $\mathrm{H}(26 \mathrm{E})-\mathrm{C}(26 \mathrm{~F})-\mathrm{H}(26 \mathrm{~F})$ & 109.5 \\
\hline $\mathrm{C}(34)-\mathrm{C}(35 \mathrm{~A})-\mathrm{H}(35 \mathrm{~B})$ & 109.5 & $\mathrm{C}(25)-\mathrm{C}(27 \mathrm{~F})-\mathrm{H}(25 \mathrm{C})$ & 125.6 \\
\hline $\mathrm{H}(35 \mathrm{~A})-\mathrm{C}(35 \mathrm{~A})-\mathrm{H}(35 \mathrm{~B})$ & 109.4 & $\mathrm{C}(25)-\mathrm{C}(27 \mathrm{~F})-\mathrm{H}(27 \mathrm{~A})$ & 98.9 \\
\hline $\mathrm{C}(34)-\mathrm{C}(35 \mathrm{~A})-\mathrm{H}(35 \mathrm{C})$ & 112.2 & $\mathrm{H}(25 \mathrm{C})-\mathrm{C}(27 \mathrm{~F})-\mathrm{H}(27 \mathrm{~A})$ & 134.8 \\
\hline $\mathrm{H}(35 \mathrm{~A})-\mathrm{C}(35 \mathrm{~A})-\mathrm{H}(35 \mathrm{C})$ & 109.5 & $\mathrm{C}(25)-\mathrm{C}(27 \mathrm{~F})-\mathrm{H}(27 \mathrm{D})$ & 120.4 \\
\hline $\mathrm{H}(35 \mathrm{~B})-\mathrm{C}(35 \mathrm{~A})-\mathrm{H}(35 \mathrm{C})$ & 109.5 & $\mathrm{H}(25 \mathrm{C})-\mathrm{C}(27 \mathrm{~F})-\mathrm{H}(27 \mathrm{D})$ & 46.5 \\
\hline $\mathrm{C}(34)-\mathrm{C}(35 \mathrm{~A})-\mathrm{H}(34 \mathrm{E})$ & 94.5 & $\mathrm{H}(27 \mathrm{~A})-\mathrm{C}(27 \mathrm{~F})-\mathrm{H}(27 \mathrm{D})$ & 119.9 \\
\hline $\mathrm{H}(35 \mathrm{~A})-\mathrm{C}(35 \mathrm{~A})-\mathrm{H}(34 \mathrm{E})$ & 13.4 & $\mathrm{C}(25)-\mathrm{C}(27 \mathrm{~F})-\mathrm{H}(27 \mathrm{E})$ & 106.5 \\
\hline $\mathrm{H}(35 \mathrm{~B})-\mathrm{C}(35 \mathrm{~A})-\mathrm{H}(34 \mathrm{E})$ & 109.8 & $\mathrm{H}(25 \mathrm{C})-\mathrm{C}(27 \mathrm{~F})-\mathrm{H}(27 \mathrm{E})$ & 63.9 \\
\hline $\mathrm{H}(35 \mathrm{C})-\mathrm{C}(35 \mathrm{~A})-\mathrm{H}(34 \mathrm{E})$ & 120.4 & $\mathrm{H}(27 \mathrm{~A})-\mathrm{C}(27 \mathrm{~F})-\mathrm{H}(27 \mathrm{E})$ & 98.9 \\
\hline $\mathrm{C}(34)-\mathrm{C}(35 \mathrm{~A})-\mathrm{H}(35 \mathrm{~F})$ & 99.9 & $\mathrm{H}(27 \mathrm{D})-\mathrm{C}(27 \mathrm{~F})-\mathrm{H}(27 \mathrm{E})$ & 109.5 \\
\hline $\mathrm{H}(35 \mathrm{~A})-\mathrm{C}(35 \mathrm{~A})-\mathrm{H}(35 \mathrm{~F})$ & 139.5 & $\mathrm{C}(25)-\mathrm{C}(27 \mathrm{~F})-\mathrm{H}(27 \mathrm{~F})$ & 100.9 \\
\hline $\mathrm{H}(35 \mathrm{~B})-\mathrm{C}(35 \mathrm{~A})-\mathrm{H}(35 \mathrm{~F})$ & 31.3 & $\mathrm{H}(25 \mathrm{C})-\mathrm{C}(27 \mathrm{~F})-\mathrm{H}(27 \mathrm{~F})$ & 133. \\
\hline
\end{tabular}




\begin{tabular}{|c|c|c|c|}
\hline $\mathrm{H}(35 \mathrm{C})-\mathrm{C}(35 \mathrm{~A})-\mathrm{H}(35 \mathrm{~F})$ & 87.1 & $\mathrm{H}(27 \mathrm{~A})-\mathrm{C}(27 \mathrm{~F})-\mathrm{H}(27 \mathrm{~F})$ & 12.2 \\
\hline $\mathrm{H}(34 \mathrm{E})-\mathrm{C}(35 \mathrm{~A})-\mathrm{H}(35 \mathrm{~F})$ & 141.0 & $\mathrm{H}(27 \mathrm{D})-\mathrm{C}(27 \mathrm{~F})-\mathrm{H}(27 \mathrm{~F})$ & 109.5 \\
\hline $\mathrm{C}(34)-\mathrm{C}(36 \mathrm{~A})-\mathrm{H}(36 \mathrm{~A})$ & 110.0 & $\mathrm{H}(27 \mathrm{E})-\mathrm{C}(27 \mathrm{~F})-\mathrm{H}(27 \mathrm{~F})$ & 109.5 \\
\hline $\mathrm{C}(34)-\mathrm{C}(36 \mathrm{~A})-\mathrm{H}(36 \mathrm{~B})$ & 109.7 & $\mathrm{C}(2 \mathrm{~S})-\mathrm{C}(1 \mathrm{~S})-\mathrm{H}(1 \mathrm{~S} 1)$ & 110.4 \\
\hline $\mathrm{H}(36 \mathrm{~A})-\mathrm{C}(36 \mathrm{~A})-\mathrm{H}(36 \mathrm{~B})$ & 109.5 & $\mathrm{C}(2 \mathrm{~S})-\mathrm{C}(1 \mathrm{~S})-\mathrm{H}(1 \mathrm{~S} 2)$ & 109.4 \\
\hline $\mathrm{C}(34)-\mathrm{C}(36 \mathrm{~A})-\mathrm{H}(36 \mathrm{C})$ & 108.7 & $\mathrm{H}(1 \mathrm{~S} 1)-\mathrm{C}(1 \mathrm{~S})-\mathrm{H}(1 \mathrm{~S} 2)$ & 109.5 \\
\hline $\mathrm{H}(36 \mathrm{~A})-\mathrm{C}(36 \mathrm{~A})-\mathrm{H}(36 \mathrm{C})$ & 109.5 & $\mathrm{C}(2 \mathrm{~S})-\mathrm{C}(1 \mathrm{~S})-\mathrm{H}(1 \mathrm{~S} 3)$ & 108.6 \\
\hline $\mathrm{H}(36 \mathrm{~B})-\mathrm{C}(36 \mathrm{~A})-\mathrm{H}(36 \mathrm{C})$ & 109.5 & $\mathrm{H}(1 \mathrm{~S} 1)-\mathrm{C}(1 \mathrm{~S})-\mathrm{H}(1 \mathrm{~S} 3)$ & 109.5 \\
\hline $\mathrm{C}(34)-\mathrm{C}(36 \mathrm{~A})-\mathrm{H}(35 \mathrm{D})$ & 99.5 & $\mathrm{H}(1 \mathrm{~S} 2)-\mathrm{C}(1 \mathrm{~S})-\mathrm{H}(1 \mathrm{~S} 3)$ & 109.5 \\
\hline $\mathrm{H}(36 \mathrm{~A})-\mathrm{C}(36 \mathrm{~A})-\mathrm{H}(35 \mathrm{D})$ & 139.3 & $C(3 S)-C(2 S)-C(1 S)$ & $121(2)$ \\
\hline $\mathrm{H}(36 \mathrm{~B})-\mathrm{C}(36 \mathrm{~A})-\mathrm{H}(35 \mathrm{D})$ & 84.9 & $\mathrm{C}(3 \mathrm{~S})-\mathrm{C}(2 \mathrm{~S})-\mathrm{O}(5 \mathrm{~S}) \# 3$ & $108(2)$ \\
\hline $\mathrm{H}(36 \mathrm{C})-\mathrm{C}(36 \mathrm{~A})-\mathrm{H}(35 \mathrm{D})$ & 32.0 & $\mathrm{C}(1 \mathrm{~S})-\mathrm{C}(2 \mathrm{~S})-\mathrm{O}(5 \mathrm{~S}) \# 3$ & $130.6(15)$ \\
\hline $\mathrm{C}(34)-\mathrm{C}(36 \mathrm{~A})-\mathrm{H}(36 \mathrm{~F})$ & 91.5 & $\mathrm{C}(2 \mathrm{~S})-\mathrm{C}(3 \mathrm{~S})-\mathrm{H}(3 \mathrm{SA})$ & 112.0 \\
\hline $\mathrm{H}(36 \mathrm{~A})-\mathrm{C}(36 \mathrm{~A})-\mathrm{H}(36 \mathrm{~F})$ & 18.5 & $\mathrm{C}(2 \mathrm{~S})-\mathrm{C}(3 \mathrm{~S})-\mathrm{H}(3 \mathrm{SB})$ & 109.0 \\
\hline $\mathrm{H}(36 \mathrm{~B})-\mathrm{C}(36 \mathrm{~A})-\mathrm{H}(36 \mathrm{~F})$ & 119.0 & $\mathrm{H}(3 \mathrm{SA})-\mathrm{C}(3 \mathrm{~S})-\mathrm{H}(3 \mathrm{SB})$ & 109.5 \\
\hline $\mathrm{H}(36 \mathrm{C})-\mathrm{C}(36 \mathrm{~A})-\mathrm{H}(36 \mathrm{~F})$ & 116.5 & $\mathrm{C}(2 \mathrm{~S})-\mathrm{C}(3 \mathrm{~S})-\mathrm{H}(3 \mathrm{SC})$ & 107.3 \\
\hline $\mathrm{H}(35 \mathrm{D})-\mathrm{C}(36 \mathrm{~A})-\mathrm{H}(36 \mathrm{~F})$ & 148.5 & $\mathrm{H}(3 \mathrm{SA})-\mathrm{C}(3 \mathrm{~S})-\mathrm{H}(3 \mathrm{SC})$ & 109.5 \\
\hline $\mathrm{C}(34)-\mathrm{C}(34 \mathrm{~B})-\mathrm{H}(35 \mathrm{~A})$ & 95.3 & H(3SB)-C(3S)-H(3SC) & 109.5 \\
\hline $\mathrm{C}(34)-\mathrm{C}(34 \mathrm{~B})-\mathrm{H}(34 \mathrm{D})$ & 110.3 & $\mathrm{C}(5 \mathrm{~S})-\mathrm{C}(4 \mathrm{~S})-\mathrm{H}(4 \mathrm{~S} 1)$ & 112.5 \\
\hline $\mathrm{H}(35 \mathrm{~A})-\mathrm{C}(34 \mathrm{~B})-\mathrm{H}(34 \mathrm{D})$ & 138.1 & $\mathrm{C}(5 \mathrm{~S})-\mathrm{C}(4 \mathrm{~S})-\mathrm{H}(4 \mathrm{~S} 2)$ & 108.3 \\
\hline $\mathrm{C}(34)-\mathrm{C}(34 \mathrm{~B})-\mathrm{H}(34 \mathrm{E})$ & 108.7 & $\mathrm{H}(4 \mathrm{~S} 1)-\mathrm{C}(4 \mathrm{~S})-\mathrm{H}(4 \mathrm{~S} 2)$ & 109.5 \\
\hline $\mathrm{H}(35 \mathrm{~A})-\mathrm{C}(34 \mathrm{~B})-\mathrm{H}(34 \mathrm{E})$ & 28.9 & $\mathrm{C}(5 \mathrm{~S})-\mathrm{C}(4 \mathrm{~S})-\mathrm{H}(4 \mathrm{~S} 3)$ & 107.6 \\
\hline
\end{tabular}




\begin{tabular}{|c|c|c|c|}
\hline $\mathrm{H}(34 \mathrm{D})-\mathrm{C}(34 \mathrm{~B})-\mathrm{H}(34 \mathrm{E})$ & 109.5 & $\mathrm{H}(4 \mathrm{~S} 1)-\mathrm{C}(4 \mathrm{~S})-\mathrm{H}(4 \mathrm{~S} 3)$ & 109.5 \\
\hline $\mathrm{C}(34)-\mathrm{C}(34 \mathrm{~B})-\mathrm{H}(34 \mathrm{~F})$ & 109.4 & $\mathrm{H}(4 \mathrm{~S} 2)-\mathrm{C}(4 \mathrm{~S})-\mathrm{H}(4 \mathrm{~S} 3)$ & 109.5 \\
\hline $\mathrm{H}(35 \mathrm{~A})-\mathrm{C}(34 \mathrm{~B})-\mathrm{H}(34 \mathrm{~F})$ & 91.4 & $\mathrm{C}(4 \mathrm{~S})-\mathrm{C}(5 \mathrm{~S})-\mathrm{O}(6 \mathrm{~S})$ & $117(3)$ \\
\hline $\mathrm{H}(34 \mathrm{D})-\mathrm{C}(34 \mathrm{~B})-\mathrm{H}(34 \mathrm{~F})$ & 109.5 & $\mathrm{C}(4 \mathrm{~S})-\mathrm{C}(5 \mathrm{~S})-\mathrm{C}(6 \mathrm{~S})$ & $132(2)$ \\
\hline $\mathrm{H}(34 \mathrm{E})-\mathrm{C}(34 \mathrm{~B})-\mathrm{H}(34 \mathrm{~F})$ & 109.5 & $\mathrm{O}(6 \mathrm{~S})-\mathrm{C}(5 \mathrm{~S})-\mathrm{C}(6 \mathrm{~S})$ & $111(2)$ \\
\hline $\mathrm{C}(34)-\mathrm{C}(35 \mathrm{~B})-\mathrm{H}(35 \mathrm{~B})$ & 81.9 & $\mathrm{C}(5 \mathrm{~S})-\mathrm{C}(6 \mathrm{~S})-\mathrm{H}(6 \mathrm{~S} 1)$ & 114.9 \\
\hline $\mathrm{C}(34)-\mathrm{C}(35 \mathrm{~B})-\mathrm{H}(35 \mathrm{C})$ & 83.7 & $\mathrm{C}(5 \mathrm{~S})-\mathrm{C}(6 \mathrm{~S})-\mathrm{H}(6 \mathrm{~S} 2)$ & 109.2 \\
\hline $\mathrm{H}(35 \mathrm{~B})-\mathrm{C}(35 \mathrm{~B})-\mathrm{H}(35 \mathrm{C})$ & 66.8 & $\mathrm{H}(6 \mathrm{~S} 1)-\mathrm{C}(6 \mathrm{~S})-\mathrm{H}(6 \mathrm{~S} 2)$ & 109.5 \\
\hline $\mathrm{C}(34)-\mathrm{C}(35 \mathrm{~B})-\mathrm{H}(36 \mathrm{~B})$ & 87.6 & $\mathrm{C}(5 \mathrm{~S})-\mathrm{C}(6 \mathrm{~S})-\mathrm{H}(6 \mathrm{~S} 3)$ & 104.0 \\
\hline $\mathrm{H}(35 \mathrm{~B})-\mathrm{C}(35 \mathrm{~B})-\mathrm{H}(36 \mathrm{~B})$ & 156.7 & $\mathrm{H}(6 \mathrm{~S} 1)-\mathrm{C}(6 \mathrm{~S})-\mathrm{H}(6 \mathrm{~S} 3)$ & 109.5 \\
\hline $\mathrm{H}(35 \mathrm{C})-\mathrm{C}(35 \mathrm{~B})-\mathrm{H}(36 \mathrm{~B})$ & 91.5 & $\mathrm{H}(6 \mathrm{~S} 2)-\mathrm{C}(6 \mathrm{~S})-\mathrm{H}(6 \mathrm{~S} 3)$ & 109.5 \\
\hline $\mathrm{C}(34)-\mathrm{C}(35 \mathrm{~B})-\mathrm{H}(36 \mathrm{C})$ & 89.3 & $\mathrm{C}(8 \mathrm{~S})-\mathrm{C}(7 \mathrm{~S})-\mathrm{H}(7 \mathrm{~S} 1)$ & 108.4 \\
\hline $\mathrm{H}(35 \mathrm{~B})-\mathrm{C}(35 \mathrm{~B})-\mathrm{H}(36 \mathrm{C})$ & 132.9 & $\mathrm{C}(8 \mathrm{~S})-\mathrm{C}(7 \mathrm{~S})-\mathrm{H}(7 \mathrm{~S} 2)$ & 110.0 \\
\hline $\mathrm{H}(35 \mathrm{C})-\mathrm{C}(35 \mathrm{~B})-\mathrm{H}(36 \mathrm{C})$ & 158.0 & $\mathrm{H}(7 \mathrm{~S} 1)-\mathrm{C}(7 \mathrm{~S})-\mathrm{H}(7 \mathrm{~S} 2)$ & 109.5 \\
\hline $\mathrm{H}(36 \mathrm{~B})-\mathrm{C}(35 \mathrm{~B})-\mathrm{H}(36 \mathrm{C})$ & 67.3 & $\mathrm{C}(8 \mathrm{~S})-\mathrm{C}(7 \mathrm{~S})-\mathrm{H}(7 \mathrm{~S} 3)$ & 110.1 \\
\hline $\mathrm{C}(34)-\mathrm{C}(35 \mathrm{~B})-\mathrm{H}(35 \mathrm{D})$ & 117.9 & H(7S1)-C(7S)-H(7S3) & 109.5 \\
\hline $\mathrm{H}(35 \mathrm{~B})-\mathrm{C}(35 \mathrm{~B})-\mathrm{H}(35 \mathrm{D})$ & 124.7 & $\mathrm{H}(7 \mathrm{~S} 2)-\mathrm{C}(7 \mathrm{~S})-\mathrm{H}(7 \mathrm{~S} 3)$ & 109.5 \\
\hline $\mathrm{H}(35 \mathrm{C})-\mathrm{C}(35 \mathrm{~B})-\mathrm{H}(35 \mathrm{D})$ & 155.4 & $\mathrm{C}(9 \mathrm{~S})-\mathrm{C}(8 \mathrm{~S})-\mathrm{C}(7 \mathrm{~S})$ & $131(2)$ \\
\hline $\mathrm{H}(36 \mathrm{~B})-\mathrm{C}(35 \mathrm{~B})-\mathrm{H}(35 \mathrm{D})$ & 78.6 & $\mathrm{C}(9 \mathrm{~S})-\mathrm{C}(8 \mathrm{~S})-\mathrm{O}(7 \mathrm{~S})$ & $123(2)$ \\
\hline $\mathrm{H}(36 \mathrm{C})-\mathrm{C}(35 \mathrm{~B})-\mathrm{H}(35 \mathrm{D})$ & 29.8 & $\mathrm{C}(7 \mathrm{~S})-\mathrm{C}(8 \mathrm{~S})-\mathrm{O}(7 \mathrm{~S})$ & $106(3)$ \\
\hline $\mathrm{C}(34)-\mathrm{C}(35 \mathrm{~B})-\mathrm{H}(35 \mathrm{E})$ & 109.0 & $\mathrm{C}(8 \mathrm{~S})-\mathrm{C}(9 \mathrm{~S})-\mathrm{H}(9 \mathrm{~S} 1)$ & 110.1 \\
\hline $\mathrm{H}(35 \mathrm{~B})-\mathrm{C}(35 \mathrm{~B})-\mathrm{H}(35 \mathrm{E})$ & 110.9 & $\mathrm{C}(8 \mathrm{~S})-\mathrm{C}(9 \mathrm{~S})-\mathrm{H}(9 \mathrm{~S} 2)$ & 106.7 \\
\hline
\end{tabular}




$\begin{array}{lccr}\text { H(35C)-C(35B)-H(35E) } & 48.5 & \text { H(9S1)-C(9S)-H(9S2) } & 109.5 \\ \text { H(36B)-C(35B)-H(35E) } & 53.3 & \mathrm{C}(8 \mathrm{~S})-\mathrm{C}(9 \mathrm{~S})-\mathrm{H}(9 \mathrm{~S} 3) & 111.5 \\ \mathrm{H}(36 \mathrm{C})-\mathrm{C}(35 \mathrm{~B})-\mathrm{H}(35 \mathrm{E}) & 115.8 & \mathrm{H}(9 \mathrm{~S} 1)-\mathrm{C}(9 \mathrm{~S})-\mathrm{H}(9 \mathrm{~S} 3) & 109.5 \\ \mathrm{H}(35 \mathrm{D})-\mathrm{C}(35 \mathrm{~B})-\mathrm{H}(35 \mathrm{E}) & 109.5 & \mathrm{H}(9 \mathrm{~S} 2)-\mathrm{C}(9 \mathrm{~S})-\mathrm{H}(9 \mathrm{~S} 3) & 109.5 \\ \mathrm{C}(34)-\mathrm{C}(35 \mathrm{~B})-\mathrm{H}(35 \mathrm{~F}) & 101.1 & & \end{array}$

Symmetry transformations used to generate equivalent atoms:

$\# 1-x,-y+1,-z \quad \# 2-x+1 / 2, y+1 / 2, z \quad \# 3-x+1 / 2, y-1 / 2, z$ 
Table S34. Anisotropic displacement parameters $\left(\AA^{2} \times 10^{3}\right)$ for $\left[\left(\mathrm{L}^{1}\right) \mathrm{Fe}(\mathrm{III})-\right.$ $\mathrm{OH}]\left(\mathrm{H}_{3} \mathrm{O}\right) \cdot 4 \mathrm{H}_{2} \mathrm{O}$ (4). The anisotropic displacement factor exponent takes the form: $-2 \pi^{2}\left[h^{2} a^{* 2} U^{11}+\ldots+2 h k a^{*} b^{*} U^{12}\right]$

\begin{tabular}{|c|c|c|c|c|c|c|}
\hline & $\mathrm{U}^{11}$ & $\mathrm{U}^{22}$ & $\mathrm{U}^{33}$ & $\mathrm{U}^{23}$ & $\mathrm{U}^{13}$ & $\mathrm{U}^{12}$ \\
\hline $\mathrm{Fe}(1)$ & $51(1)$ & $54(1)$ & $54(1)$ & $4(1)$ & $-4(1)$ & $4(1)$ \\
\hline $\mathrm{O}(1)$ & $86(4)$ & $87(4)$ & 93(4) & $18(4)$ & $-19(3)$ & $19(3)$ \\
\hline $\mathrm{N}(1)$ & $38(3)$ & $42(3)$ & $43(3)$ & $-2(3)$ & $-1(2)$ & $-1(2)$ \\
\hline $\mathrm{N}(2)$ & $55(4)$ & $51(3)$ & $48(3)$ & $12(3)$ & $0(3)$ & $7(3)$ \\
\hline $\mathrm{N}(3)$ & $44(3)$ & $59(4)$ & $50(3)$ & $-1(3)$ & $-13(3)$ & 2(3) \\
\hline $\mathrm{N}(4)$ & $49(3)$ & $46(3)$ & $62(4)$ & $0(3)$ & $0(3)$ & $11(3)$ \\
\hline$C(1)$ & $45(4)$ & $53(4)$ & 44(4) & 2(4) & $-5(3)$ & 1(3) \\
\hline$C(2)$ & $57(4)$ & $59(4)$ & $46(4)$ & $-3(4)$ & $0(4)$ & $-6(4)$ \\
\hline$C(3)$ & $58(5)$ & $78(5)$ & $69(5)$ & $21(5)$ & $-2(4)$ & $-7(4)$ \\
\hline$C(4)$ & $53(5)$ & $104(7)$ & $76(6)$ & $8(5)$ & $8(4)$ & $-11(5)$ \\
\hline$C(5)$ & $48(5)$ & $82(6)$ & $75(6)$ & $5(5)$ & $-1(4)$ & $8(4)$ \\
\hline$C(6)$ & $56(5)$ & $66(5)$ & $53(5)$ & $1(4)$ & $5(4)$ & $-6(4)$ \\
\hline $\mathrm{C}(7)$ & $40(4)$ & $49(4)$ & $54(4)$ & $0(4)$ & $-1(3)$ & $7(3)$ \\
\hline $\mathrm{C}(8)$ & $41(4)$ & $54(4)$ & $60(5)$ & $-5(4)$ & $-3(4)$ & $3(3)$ \\
\hline $\mathrm{C}(9)$ & $68(5)$ & $58(5)$ & $78(6)$ & $-9(5)$ & $-19(5)$ & 1(4) \\
\hline$C(10)$ & $74(6)$ & $58(5)$ & $96(7)$ & $-14(5)$ & $-10(5)$ & $-12(4)$ \\
\hline$C(11)$ & $77(5)$ & $51(5)$ & $84(6)$ & 11(4) & $-2(5)$ & $5(4)$ \\
\hline $\mathrm{C}(12)$ & $53(4)$ & $55(5)$ & $68(5)$ & $0(4)$ & $-1(4)$ & $-3(4)$ \\
\hline
\end{tabular}




\begin{tabular}{|c|c|c|c|c|c|c|}
\hline$C(13)$ & $52(4)$ & $46(4)$ & $45(4)$ & $5(3)$ & $2(3)$ & $3(3)$ \\
\hline$C(14)$ & $57(4)$ & $43(4)$ & $54(4)$ & $3(4)$ & $3(4)$ & $2(3)$ \\
\hline$C(15)$ & $76(5)$ & $69(5)$ & $56(5)$ & $-1(4)$ & $5(5)$ & $21(4)$ \\
\hline$C(16)$ & $104(7)$ & $78(6)$ & $51(5)$ & $-8(5)$ & $10(5)$ & $3(5)$ \\
\hline$C(17)$ & $82(6)$ & $75(5)$ & $52(5)$ & $0(5)$ & $-7(4)$ & $11(5)$ \\
\hline $\mathrm{C}(18)$ & $61(5)$ & $53(4)$ & $61(5)$ & $-7(4)$ & $7(4)$ & $7(4)$ \\
\hline$C(19)$ & $63(5)$ & $47(4)$ & $48(4)$ & $3(4)$ & $0(4)$ & $5(3)$ \\
\hline $\mathrm{C}(20)$ & 151(9) & $63(5)$ & $37(4)$ & $-3(4)$ & $-7(5)$ & $-12(5)$ \\
\hline $\mathrm{C}(21)$ & 153(9) & $50(5)$ & $70(6)$ & $4(5)$ & $-19(6)$ & $-8(5)$ \\
\hline$C(22)$ & $79(5)$ & $52(5)$ & $54(5)$ & $16(4)$ & $-13(4)$ & $-5(4)$ \\
\hline $\mathrm{C}(23)$ & $106(7)$ & $76(6)$ & $48(5)$ & $5(5)$ & 11(4) & $8(5)$ \\
\hline$C(25)$ & $86(6)$ & $75(5)$ & $66(6)$ & $20(5)$ & $5(5)$ & $-6(5)$ \\
\hline$C(24)$ & $104(6)$ & $43(4)$ & $50(5)$ & $-2(4)$ & $13(4)$ & $7(4)$ \\
\hline $\mathrm{C}(28)$ & $48(4)$ & $64(5)$ & $55(5)$ & $-1(4)$ & $-5(4)$ & $2(3)$ \\
\hline $\mathrm{C}(29)$ & $51(5)$ & 113(7) & $49(5)$ & $5(5)$ & $4(4)$ & $-17(4)$ \\
\hline$C(30)$ & $42(4)$ & $110(7)$ & $74(6)$ & $-2(5)$ & $-5(4)$ & $-12(4)$ \\
\hline$C(31)$ & $54(5)$ & $78(5)$ & $53(5)$ & $-8(4)$ & $-18(4)$ & $8(4)$ \\
\hline$C(32)$ & $61(6)$ & 153(9) & $57(5)$ & $-4(6)$ & $-5(4)$ & $23(6)$ \\
\hline$C(33)$ & $44(4)$ & 157(9) & $58(5)$ & $-1(6)$ & $6(4)$ & $9(5)$ \\
\hline$C(34)$ & $66(5)$ & $86(6)$ & $84(6)$ & $-11(5)$ & $-20(5)$ & $0(5)$ \\
\hline$C(37)$ & $53(4)$ & $48(4)$ & $64(5)$ & $5(4)$ & $-3(4)$ & $5(3)$ \\
\hline$C(38)$ & $54(5)$ & $42(4)$ & $163(10)$ & $11(5)$ & $8(6)$ & $-6(4)$ \\
\hline
\end{tabular}




\begin{tabular}{|c|c|c|c|c|c|c|}
\hline C(39) & $51(5)$ & $61(5)$ & $160(10)$ & $22(6)$ & $6(5)$ & $3(4)$ \\
\hline$C(40)$ & $57(5)$ & $56(5)$ & $76(6)$ & $4(4)$ & $5(4)$ & $20(4)$ \\
\hline$C(41)$ & $69(6)$ & $45(4)$ & $114(8)$ & $-11(5)$ & $-3(5)$ & $8(4)$ \\
\hline$C(42)$ & $48(4)$ & $55(5)$ & $112(7)$ & $-13(5)$ & $-7(4)$ & $-1(4)$ \\
\hline$C(43)$ & $79(6)$ & $70(6)$ & $81(6)$ & $-2(5)$ & $10(5)$ & $22(5)$ \\
\hline $\mathrm{O}(1 \mathrm{~S})$ & $183(10)$ & 177(9) & $256(12)$ & $29(9)$ & $16(9)$ & $36(7)$ \\
\hline $\mathrm{O}(2 \mathrm{~S})$ & $193(10)$ & $246(11)$ & $174(10)$ & $-12(8)$ & $-43(8)$ & $33(9)$ \\
\hline $\mathrm{O}(3 \mathrm{~S})$ & $232(11)$ & 181(9) & 191(10) & $36(8)$ & $-19(8)$ & $-10(8)$ \\
\hline $\mathrm{O}(4 \mathrm{~S})$ & $95(5)$ & $93(5)$ & $105(6)$ & $7(4)$ & $-13(4)$ & $13(3)$ \\
\hline $\mathrm{O}(5 \mathrm{~S})$ & $320(20)$ & $400(20)$ & $233(15)$ & $-26(15)$ & $48(13)$ & $167(18)$ \\
\hline $\mathrm{O}(6 \mathrm{~S})$ & $830(70)$ & $580(50)$ & $480(40)$ & $-170(40)$ & $350(40)$ & $-20(50)$ \\
\hline $\mathrm{O}(7 \mathrm{~S})$ & $620(60)$ & $470(40)$ & $820(70)$ & $-280(40)$ & $130(50)$ & $-180(40)$ \\
\hline $\mathrm{C}(2 \mathrm{~S})$ & 104(9) & $218(17)$ & $120(11)$ & $-71(11)$ & $-11(8)$ & $5(10)$ \\
\hline$C(3 S)$ & $410(50)$ & $610(70)$ & $440(50)$ & $-110(50)$ & $-150(40)$ & $230(50)$ \\
\hline$C(4 S)$ & $290(30)$ & $105(11)$ & $310(30)$ & $12(15)$ & $-110(20)$ & $-74(16)$ \\
\hline$C(5 S)$ & 197(18) & $220(20)$ & $110(12)$ & $-48(13)$ & $16(13)$ & $-93(18)$ \\
\hline $\mathrm{C}(6 \mathrm{~S})$ & $330(30)$ & $154(14)$ & $270(20)$ & $-47(15)$ & $-190(20)$ & $1(16)$ \\
\hline$C(7 S)$ & $121(14)$ & $240(20)$ & $390(40)$ & $110(20)$ & $90(20)$ & $-3(14)$ \\
\hline $\mathrm{C}(8 \mathrm{~S})$ & $180(18)$ & $109(11)$ & $220(20)$ & $-3(14)$ & $86(17)$ & $-17(11)$ \\
\hline $\mathrm{C}(9 \mathrm{~S})$ & $133(13)$ & $280(20)$ & $370(30)$ & $220(20)$ & $18(16)$ & $-13(14)$ \\
\hline
\end{tabular}


Table S35. Hydrogen coordinates $\left(\times 10^{4}\right)$ and isotropic displacement parameters $\left(\AA^{2} \times 10^{3}\right)$ for $\left[\left(\mathrm{L}^{1}\right) \mathrm{Fe}(\mathrm{III})-\mathrm{OH}\right]\left(\mathrm{H}_{3} \mathrm{O}\right) \cdot 4 \mathrm{H}_{2} \mathrm{O}(4)$

\begin{tabular}{|c|c|c|c|c|}
\hline & $\mathrm{x}$ & $y$ & $\mathrm{Z}$ & $\mathrm{U}(\mathrm{eq})$ \\
\hline $\mathrm{H}(3)$ & 4052 & 3152 & 1534 & 82 \\
\hline $\mathrm{H}(4)$ & 4801 & 2505 & 1778 & 93 \\
\hline $\mathrm{H}(5)$ & 4632 & 1715 & 2422 & 82 \\
\hline $\mathrm{H}(6)$ & 3696 & 1599 & 2834 & 70 \\
\hline $\mathrm{H}(9)$ & 1529 & 954 & 1854 & 82 \\
\hline $\mathrm{H}(10)$ & 1773 & 199 & 2489 & 91 \\
\hline $\mathrm{H}(11)$ & 2426 & 368 & 3274 & 85 \\
\hline $\mathrm{H}(12)$ & 2843 & 1301 & 3398 & 70 \\
\hline $\mathrm{H}(15)$ & 1856 & 3469 & 4046 & 80 \\
\hline $\mathrm{H}(16)$ & 2489 & 3224 & 4794 & 93 \\
\hline $\mathrm{H}(17)$ & 3284 & 2578 & 4623 & 84 \\
\hline $\mathrm{H}(18)$ & 3396 & 2166 & 3701 & 70 \\
\hline $\mathrm{H}(20)$ & 2904 & 4288 & 1961 & 100 \\
\hline $\mathrm{H}(21)$ & 2967 & 4990 & 1240 & 110 \\
\hline $\mathrm{H}(23)$ & 3148 & 3729 & 22 & 92 \\
\hline $\mathrm{H}(24)$ & 3096 & 3022 & 759 & 79 \\
\hline $\mathrm{H}(29)$ & 754 & 1885 & 1978 & 85 \\
\hline $\mathrm{H}(30)$ & 28 & 1843 & 1264 & 90 \\
\hline $\mathrm{H}(32)$ & 1244 & 2061 & 8 & 109 \\
\hline
\end{tabular}




\begin{tabular}{|c|c|c|c|c|}
\hline $\mathrm{H}(33)$ & 1957 & 2104 & 706 & 104 \\
\hline $\mathrm{H}(38)$ & 706 & 3043 & 2913 & 104 \\
\hline $\mathrm{H}(39)$ & 11 & 3748 & 2957 & 109 \\
\hline $\mathrm{H}(41)$ & 1267 & 4979 & 3157 & 91 \\
\hline $\mathrm{H}(42)$ & 1978 & 4245 & 3094 & 86 \\
\hline $\mathrm{H}(34 \mathrm{~A})$ & 352 & 2740 & -120 & 157 \\
\hline $\mathrm{H}(34 \mathrm{~B})$ & 4 & 2441 & -643 & 157 \\
\hline $\mathrm{H}(34 \mathrm{C})$ & -341 & 2685 & -90 & 157 \\
\hline $\mathrm{H}(35 \mathrm{~A})$ & 378 & 1320 & -499 & 166 \\
\hline $\mathrm{H}(35 \mathrm{~B})$ & 157 & 1009 & 83 & 166 \\
\hline $\mathrm{H}(35 \mathrm{C})$ & -303 & 1217 & -397 & 166 \\
\hline $\mathrm{H}(36 \mathrm{~A})$ & -721 & 2252 & 518 & 119 \\
\hline $\mathrm{H}(36 \mathrm{~B})$ & -877 & 1711 & 108 & 119 \\
\hline $\mathrm{H}(36 \mathrm{C})$ & -576 & 1604 & 727 & 119 \\
\hline $\mathrm{H}(34 \mathrm{D})$ & 559 & 2108 & -694 & 144 \\
\hline $\mathrm{H}(34 \mathrm{E})$ & 570 & 1428 & -545 & 144 \\
\hline $\mathrm{H}(34 \mathrm{~F})$ & 6 & 1712 & -837 & 144 \\
\hline $\mathrm{H}(35 \mathrm{D})$ & -577 & 1311 & 594 & 327 \\
\hline $\mathrm{H}(35 \mathrm{E})$ & -659 & 1289 & -97 & 327 \\
\hline $\mathrm{H}(35 \mathrm{~F})$ & -94 & 1006 & 196 & 327 \\
\hline $\mathrm{H}(36 \mathrm{D})$ & -225 & 2702 & 66 & 253 \\
\hline$H(36 E)$ & -669 & 2248 & -220 & 253 \\
\hline
\end{tabular}




\begin{tabular}{|c|c|c|c|c|}
\hline $\mathrm{H}(36 \mathrm{~F})$ & -595 & 2274 & 471 & 253 \\
\hline $\mathrm{H}(43 \mathrm{~A})$ & -95 & 4694 & 2277 & 151 \\
\hline $\mathrm{H}(43 \mathrm{~B})$ & -625 & 5024 & 2584 & 151 \\
\hline $\mathrm{H}(43 \mathrm{C})$ & -75 & 5385 & 2356 & 151 \\
\hline $\mathrm{H}(44 \mathrm{~A})$ & -543 & 4610 & 3654 & 165 \\
\hline $\mathrm{H}(44 \mathrm{~B})$ & 46 & 4774 & 3985 & 165 \\
\hline $\mathrm{H}(44 \mathrm{C})$ & -399 & 5275 & 3799 & 165 \\
\hline $\mathrm{H}(45 \mathrm{~A})$ & 508 & 5714 & 2808 & 135 \\
\hline $\mathrm{H}(45 \mathrm{~B})$ & 116 & 5853 & 3366 & 135 \\
\hline $\mathrm{H}(45 \mathrm{C})$ & 735 & 5544 & 3444 & 135 \\
\hline $\mathrm{H}(43 \mathrm{D})$ & 125 & 5301 & 2352 & 276 \\
\hline $\mathrm{H}(43 \mathrm{E})$ & -141 & 5737 & 2825 & 276 \\
\hline $\mathrm{H}(43 \mathrm{~F})$ & 545 & 5624 & 2803 & 276 \\
\hline $\mathrm{H}(44 \mathrm{D})$ & -704 & 4470 & 2892 & 142 \\
\hline $\mathrm{H}(44 \mathrm{E})$ & -549 & 4412 & 3568 & 142 \\
\hline $\mathrm{H}(44 \mathrm{~F})$ & -838 & 4997 & 3326 & 142 \\
\hline $\mathrm{H}(45 \mathrm{D})$ & 547 & 5463 & 3793 & 250 \\
\hline $\mathrm{H}(45 \mathrm{E})$ & -143 & 5551 & 3827 & 250 \\
\hline $\mathrm{H}(45 \mathrm{~F})$ & 146 & 4966 & 4069 & 250 \\
\hline $\mathrm{H}(25 \mathrm{~A})$ & 2230 & 5131 & 331 & 163 \\
\hline $\mathrm{H}(25 \mathrm{~B})$ & 2577 & 5686 & 94 & 163 \\
\hline $\mathrm{H}(25 \mathrm{C})$ & 2349 & 5202 & -351 & 163 \\
\hline
\end{tabular}




\begin{tabular}{|c|c|c|c|c|}
\hline $\mathrm{H}(26 \mathrm{~A})$ & 3627 & 5552 & 385 & 170 \\
\hline $\mathrm{H}(26 \mathrm{~B})$ & 3974 & 4967 & 232 & 170 \\
\hline $\mathrm{H}(26 \mathrm{C})$ & 3783 & 5402 & -277 & 170 \\
\hline $\mathrm{H}(27 \mathrm{~A})$ & 2754 & 4499 & -702 & 132 \\
\hline $\mathrm{H}(27 \mathrm{~B})$ & 3305 & 4899 & -846 & 132 \\
\hline $\mathrm{H}(27 \mathrm{C})$ & 3394 & 4280 & -540 & 132 \\
\hline $\mathrm{H}(25 \mathrm{D})$ & 2873 & 5714 & 516 & 147 \\
\hline$H(25 E)$ & 3549 & 5566 & 584 & 147 \\
\hline $\mathrm{H}(25 \mathrm{~F})$ & 3311 & 5845 & -8 & 147 \\
\hline $\mathrm{H}(26 \mathrm{D})$ & 3753 & 4462 & -486 & 281 \\
\hline $\mathrm{H}(26 \mathrm{E})$ & 3763 & 5152 & -575 & 281 \\
\hline $\mathrm{H}(26 \mathrm{~F})$ & 4001 & 4874 & 17 & 281 \\
\hline $\mathrm{H}(27 \mathrm{D})$ & 2207 & 4964 & -194 & 269 \\
\hline $\mathrm{H}(27 \mathrm{E})$ & 2680 & 5265 & -607 & 269 \\
\hline $\mathrm{H}(27 \mathrm{~F})$ & 2653 & 4571 & -557 & 269 \\
\hline $\mathrm{H}(1 \mathrm{~S} 1)$ & 3529 & 1697 & 635 & 323 \\
\hline $\mathrm{H}(1 \mathrm{~S} 2)$ & 3357 & 1789 & 1304 & 323 \\
\hline $\mathrm{H}(1 \mathrm{~S} 3)$ & 2912 & 1475 & 869 & 323 \\
\hline $\mathrm{H}(3 \mathrm{SA})$ & 4009 & 314 & 719 & 725 \\
\hline H(3SB) & 4408 & 775 & 1055 & 725 \\
\hline $\mathrm{H}(3 \mathrm{SC})$ & 4097 & 953 & 458 & 725 \\
\hline $\mathrm{H}(4 \mathrm{~S} 1)$ & 8481 & 3880 & 2051 & 354 \\
\hline
\end{tabular}




\begin{tabular}{lcccc}
$\mathrm{H}(4 \mathrm{~S} 2)$ & 8242 & 3825 & 1399 & 354 \\
$\mathrm{H}(4 \mathrm{~S} 3)$ & 8492 & 4433 & 1628 & 354 \\
$\mathrm{H}(6 \mathrm{~S} 1)$ & 9938 & 3418 & 1519 & 376 \\
$\mathrm{H}(6 \mathrm{~S} 2)$ & 9423 & 3008 & 1294 & 376 \\
$\mathrm{H}(6 \mathrm{~S} 3)$ & 9433 & 3222 & 1956 & 376 \\
$\mathrm{H}(7 \mathrm{~S} 1)$ & 577 & 3230 & 8533 & 377 \\
$\mathrm{H}(7 \mathrm{~S} 2)$ & 1231 & 3022 & 8413 & 377 \\
$\mathrm{H}(7 \mathrm{~S} 3)$ & 1029 & 3663 & 8228 & 377 \\
$\mathrm{H}(9 \mathrm{~S} 1)$ & 2009 & 3660 & 9384 & 388 \\
$\mathrm{H}(9 \mathrm{~S} 2)$ & 1749 & 3017 & 9446 & 388 \\
$\mathrm{H}(9 \mathrm{~S} 3)$ & 1582 & 3507 & 9912 & 388 \\
\hline
\end{tabular}


Table S36. Torsion Angles, ${ }^{\circ}$, for $\left[\left(\mathrm{L}^{1}\right) \mathrm{Fe}(\mathrm{III})-\mathrm{OH}\right]\left(\mathrm{H}_{3} \mathrm{O}\right) \cdot 4 \mathrm{H}_{2} \mathrm{O}$ (4)

\begin{tabular}{|c|c|c|c|}
\hline $\mathrm{O}(1)-\mathrm{Fe}(1)-\mathrm{N}(1)-\mathrm{C}(13)$ & $-48(62)$ & $\mathrm{N}(1)-\mathrm{C}(13)-\mathrm{C}(14)-\mathrm{N}(4)$ & $-4.1(8)$ \\
\hline $\mathrm{N}(3)-\mathrm{Fe}(1)-\mathrm{N}(1)-\mathrm{C}(13)$ & $-137.9(4)$ & $\mathrm{C}(18)-\mathrm{C}(13)-\mathrm{C}(14)-\mathrm{C}(15)$ & $-2.1(10)$ \\
\hline $\mathrm{N}(2)-\mathrm{Fe}(1)-\mathrm{N}(1)-\mathrm{C}(13)$ & $102.5(4)$ & $\mathrm{N}(1)-\mathrm{C}(13)-\mathrm{C}(14)-\mathrm{C}(15)$ & $177.3(6)$ \\
\hline $\mathrm{N}(4)-\mathrm{Fe}(1)-\mathrm{N}(1)-\mathrm{C}(13)$ & $-17.7(4)$ & $\mathrm{N}(4)-\mathrm{C}(14)-\mathrm{C}(15)-\mathrm{C}(16)$ & $-178.1(7)$ \\
\hline $\mathrm{O}(1)-\mathrm{Fe}(1)-\mathrm{N}(1)-\mathrm{C}(1)$ & $-168(100)$ & $\mathrm{C}(13)-\mathrm{C}(14)-\mathrm{C}(15)-\mathrm{C}(16)$ & $0.4(11)$ \\
\hline $\mathrm{N}(3)-\mathrm{Fe}(1)-\mathrm{N}(1)-\mathrm{C}(1)$ & $101.8(4)$ & $C(14)-C(15)-C(16)-C(17)$ & $1.4(13)$ \\
\hline $\mathrm{N}(2)-\mathrm{Fe}(1)-\mathrm{N}(1)-\mathrm{C}(1)$ & $-17.8(4)$ & $\mathrm{C}(15)-\mathrm{C}(16)-\mathrm{C}(17)-\mathrm{C}(18)$ & $-1.4(12)$ \\
\hline $\mathrm{N}(4)-\mathrm{Fe}(1)-\mathrm{N}(1)-\mathrm{C}(1)$ & $-138.0(4)$ & $\mathrm{C}(16)-\mathrm{C}(17)-\mathrm{C}(18)-\mathrm{C}(13)$ & $-0.3(12)$ \\
\hline $\mathrm{O}(1)-\mathrm{Fe}(1)-\mathrm{N}(1)-\mathrm{C}(7)$ & $72(62)$ & $\mathrm{C}(14)-\mathrm{C}(13)-\mathrm{C}(18)-\mathrm{C}(17)$ & $2.1(10)$ \\
\hline $\mathrm{N}(3)-\mathrm{Fe}(1)-\mathrm{N}(1)-\mathrm{C}(7)$ & $-18.1(4)$ & $\mathrm{N}(1)-\mathrm{C}(13)-\mathrm{C}(18)-\mathrm{C}(17)$ & $-177.3(6)$ \\
\hline $\mathrm{N}(2)-\mathrm{Fe}(1)-\mathrm{N}(1)-\mathrm{C}(7)$ & $-137.7(4)$ & $\mathrm{C}(2)-\mathrm{N}(2)-\mathrm{C}(19)-\mathrm{C}(24)$ & $69.0(9)$ \\
\hline $\mathrm{N}(4)-\mathrm{Fe}(1)-\mathrm{N}(1)-\mathrm{C}(7)$ & $102.0(4)$ & $\mathrm{Fe}(1)-\mathrm{N}(2)-\mathrm{C}(19)-\mathrm{C}(24)$ & $-89.8(7)$ \\
\hline $\mathrm{O}(1)-\mathrm{Fe}(1)-\mathrm{N}(2)-\mathrm{C}(2)$ & $-162.4(5)$ & $\mathrm{C}(2)-\mathrm{N}(2)-\mathrm{C}(19)-\mathrm{C}(20)$ & $-116.9(8)$ \\
\hline $\mathrm{N}(3)-\mathrm{Fe}(1)-\mathrm{N}(2)-\mathrm{C}(2)$ & $-52.4(5)$ & $\mathrm{Fe}(1)-\mathrm{N}(2)-\mathrm{C}(19)-\mathrm{C}(20)$ & $84.3(8)$ \\
\hline $\mathrm{N}(4)-\mathrm{Fe}(1)-\mathrm{N}(2)-\mathrm{C}(2)$ & $87.3(5)$ & $C(24)-C(19)-C(20)-C(21)$ & $-1.2(13)$ \\
\hline $\mathrm{N}(1)-\mathrm{Fe}(1)-\mathrm{N}(2)-\mathrm{C}(2)$ & $17.7(5)$ & $\mathrm{N}(2)-\mathrm{C}(19)-\mathrm{C}(20)-\mathrm{C}(21)$ & $-175.6(8)$ \\
\hline $\mathrm{O}(1)-\mathrm{Fe}(1)-\mathrm{N}(2)-\mathrm{C}(19)$ & $-3.8(5)$ & $C(19)-C(20)-C(21)-C(22)$ & $1.0(15)$ \\
\hline $\mathrm{N}(3)-\mathrm{Fe}(1)-\mathrm{N}(2)-\mathrm{C}(19)$ & $106.1(5)$ & $\mathrm{C}(20)-\mathrm{C}(21)-\mathrm{C}(22)-\mathrm{C}(23)$ & $-0.3(13)$ \\
\hline $\mathrm{N}(4)-\mathrm{Fe}(1)-\mathrm{N}(2)-\mathrm{C}(19)$ & $-114.2(5)$ & $\mathrm{C}(20)-\mathrm{C}(21)-\mathrm{C}(22)-\mathrm{C}(25)$ & $179.9(8)$ \\
\hline $\mathrm{N}(1)-\mathrm{Fe}(1)-\mathrm{N}(2)-\mathrm{C}(19)$ & $176.3(5)$ & $\mathrm{C}(21)-\mathrm{C}(22)-\mathrm{C}(23)-\mathrm{C}(24)$ & $-0.1(12)$ \\
\hline $\mathrm{O}(1)-\mathrm{Fe}(1)-\mathrm{N}(3)-\mathrm{C}(8)$ & $-161.5(5)$ & $\mathrm{C}(25)-\mathrm{C}(22)-\mathrm{C}(23)-\mathrm{C}(24)$ & $179.7(8)$ \\
\hline
\end{tabular}




\begin{tabular}{|c|c|c|c|}
\hline $\mathrm{N}(2)-\mathrm{Fe}(1)-\mathrm{N}(3)-\mathrm{C}(8)$ & $88.4(5)$ & $\mathrm{C}(23)-\mathrm{C}(22)-\mathrm{C}(25)-\mathrm{C}(27 \mathrm{~F})$ & $69.2(18)$ \\
\hline $\mathrm{N}(4)-\mathrm{Fe}(1)-\mathrm{N}(3)-\mathrm{C}(8)$ & $-51.3(5)$ & $\mathrm{C}(21)-\mathrm{C}(22)-\mathrm{C}(25)-\mathrm{C}(27 \mathrm{~F})$ & $-111.0(18)$ \\
\hline $\mathrm{N}(1)-\mathrm{Fe}(1)-\mathrm{N}(3)-\mathrm{C}(8)$ & $18.2(4)$ & $C(23)-C(22)-C(25)-C(26 E)$ & $-109.3(13)$ \\
\hline $\mathrm{O}(1)-\mathrm{Fe}(1)-\mathrm{N}(3)-\mathrm{C}(28)$ & $-3.8(6)$ & $\mathrm{C}(21)-\mathrm{C}(22)-\mathrm{C}(25)-\mathrm{C}(26 \mathrm{E})$ & $70.5(14)$ \\
\hline $\mathrm{N}(2)-\mathrm{Fe}(1)-\mathrm{N}(3)-\mathrm{C}(28)$ & $-113.8(5)$ & $\mathrm{C}(23)-\mathrm{C}(22)-\mathrm{C}(25)-\mathrm{C}(27 \mathrm{E})$ & $3.8(13)$ \\
\hline $\mathrm{N}(4)-\mathrm{Fe}(1)-\mathrm{N}(3)-\mathrm{C}(28)$ & $106.4(5)$ & $\mathrm{C}(21)-\mathrm{C}(22)-\mathrm{C}(25)-\mathrm{C}(27 \mathrm{E})$ & $-176.4(10)$ \\
\hline $\mathrm{N}(1)-\mathrm{Fe}(1)-\mathrm{N}(3)-\mathrm{C}(28)$ & $176.0(5)$ & $\mathrm{C}(23)-\mathrm{C}(22)-\mathrm{C}(25)-\mathrm{C}(25 \mathrm{E})$ & $118.1(12)$ \\
\hline $\mathrm{O}(1)-\mathrm{Fe}(1)-\mathrm{N}(4)-\mathrm{C}(14)$ & $-162.1(5)$ & $\mathrm{C}(21)-\mathrm{C}(22)-\mathrm{C}(25)-\mathrm{C}(25 \mathrm{E})$ & $-62.1(13)$ \\
\hline $\mathrm{N}(3)-\mathrm{Fe}(1)-\mathrm{N}(4)-\mathrm{C}(14)$ & $87.8(5)$ & $\mathrm{C}(23)-\mathrm{C}(22)-\mathrm{C}(25)-\mathrm{C}(26 \mathrm{~F})$ & $-57.7(16)$ \\
\hline $\mathrm{N}(2)-\mathrm{Fe}(1)-\mathrm{N}(4)-\mathrm{C}(14)$ & $-51.7(5)$ & $\mathrm{C}(21)-\mathrm{C}(22)-\mathrm{C}(25)-\mathrm{C}(26 \mathrm{~F})$ & $122.1(16)$ \\
\hline $\mathrm{N}(1)-\mathrm{Fe}(1)-\mathrm{N}(4)-\mathrm{C}(14)$ & $18.0(4)$ & $\mathrm{C}(23)-\mathrm{C}(22)-\mathrm{C}(25)-\mathrm{C}(25 \mathrm{~F})$ & $-164.8(10)$ \\
\hline $\mathrm{O}(1)-\mathrm{Fe}(1)-\mathrm{N}(4)-\mathrm{C}(37)$ & $-3.9(5)$ & $\mathrm{C}(21)-\mathrm{C}(22)-\mathrm{C}(25)-\mathrm{C}(25 \mathrm{~F})$ & $15.0(13)$ \\
\hline $\mathrm{N}(3)-\mathrm{Fe}(1)-\mathrm{N}(4)-\mathrm{C}(37)$ & $-114.0(5)$ & $\mathrm{C}(20)-\mathrm{C}(19)-\mathrm{C}(24)-\mathrm{C}(23)$ & $0.7(12)$ \\
\hline $\mathrm{N}(2)-\mathrm{Fe}(1)-\mathrm{N}(4)-\mathrm{C}(37)$ & $106.4(5)$ & $\mathrm{N}(2)-\mathrm{C}(19)-\mathrm{C}(24)-\mathrm{C}(23)$ & $175.1(7)$ \\
\hline $\mathrm{N}(1)-\mathrm{Fe}(1)-\mathrm{N}(4)-\mathrm{C}(37)$ & $176.2(5)$ & $\mathrm{C}(22)-\mathrm{C}(23)-\mathrm{C}(24)-\mathrm{C}(19)$ & $-0.1(13)$ \\
\hline $\mathrm{C}(13)-\mathrm{N}(1)-\mathrm{C}(1)-\mathrm{C}(6)$ & $82.6(8)$ & $\mathrm{C}(8)-\mathrm{N}(3)-\mathrm{C}(28)-\mathrm{C}(33)$ & $-117.8(8)$ \\
\hline $\mathrm{C}(7)-\mathrm{N}(1)-\mathrm{C}(1)-\mathrm{C}(6)$ & $-51.3(8)$ & $\mathrm{Fe}(1)-\mathrm{N}(3)-\mathrm{C}(28)-\mathrm{C}(33)$ & $84.1(8)$ \\
\hline $\mathrm{Fe}(1)-\mathrm{N}(1)-\mathrm{C}(1)-\mathrm{C}(6)$ & $-164.2(5)$ & $\mathrm{C}(8)-\mathrm{N}(3)-\mathrm{C}(28)-\mathrm{C}(29)$ & $67.2(9)$ \\
\hline $\mathrm{C}(13)-\mathrm{N}(1)-\mathrm{C}(1)-\mathrm{C}(2)$ & $-96.7(7)$ & $\mathrm{Fe}(1)-\mathrm{N}(3)-\mathrm{C}(28)-\mathrm{C}(29)$ & $-91.0(8)$ \\
\hline $\mathrm{C}(7)-\mathrm{N}(1)-\mathrm{C}(1)-\mathrm{C}(2)$ & $129.3(6)$ & $\mathrm{C}(33)-\mathrm{C}(28)-\mathrm{C}(29)-\mathrm{C}(30)$ & $-0.2(12)$ \\
\hline $\mathrm{Fe}(1)-\mathrm{N}(1)-\mathrm{C}(1)-\mathrm{C}(2)$ & $16.5(6)$ & $\mathrm{N}(3)-\mathrm{C}(28)-\mathrm{C}(29)-\mathrm{C}(30)$ & $175.2(7)$ \\
\hline $\mathrm{C}(19)-\mathrm{N}(2)-\mathrm{C}(2)-\mathrm{C}(3)$ & $5.6(10)$ & $\mathrm{C}(28)-\mathrm{C}(29)-\mathrm{C}(30)-\mathrm{C}(31)$ & $0.9(13)$ \\
\hline
\end{tabular}




\begin{tabular}{|c|c|c|c|}
\hline $\mathrm{Fe}(1)-\mathrm{N}(2)-\mathrm{C}(2)-\mathrm{C}(3)$ & $164.7(6)$ & $C(29)-C(30)-C(31)-C(32)$ & $-1.4(12)$ \\
\hline $\mathrm{C}(19)-\mathrm{N}(2)-\mathrm{C}(2)-\mathrm{C}(1)$ & $-173.3(6)$ & $\mathrm{C}(29)-\mathrm{C}(30)-\mathrm{C}(31)-\mathrm{C}(34)$ & $-179.9(8)$ \\
\hline $\mathrm{Fe}(1)-\mathrm{N}(2)-\mathrm{C}(2)-\mathrm{C}(1)$ & $-14.3(8)$ & $\mathrm{C}(30)-\mathrm{C}(31)-\mathrm{C}(32)-\mathrm{C}(33)$ & $1.3(13)$ \\
\hline $\mathrm{C}(6)-\mathrm{C}(1)-\mathrm{C}(2)-\mathrm{N}(2)$ & $176.1(6)$ & $C(34)-C(31)-C(32)-C(33)$ & $179.8(9)$ \\
\hline $\mathrm{N}(1)-\mathrm{C}(1)-\mathrm{C}(2)-\mathrm{N}(2)$ & $-4.5(9)$ & $\mathrm{C}(31)-\mathrm{C}(32)-\mathrm{C}(33)-\mathrm{C}(28)$ & $-0.8(15)$ \\
\hline $\mathrm{C}(6)-\mathrm{C}(1)-\mathrm{C}(2)-\mathrm{C}(3)$ & $-2.9(10)$ & $\mathrm{C}(29)-\mathrm{C}(28)-\mathrm{C}(33)-\mathrm{C}(32)$ & $0.2(13)$ \\
\hline $\mathrm{N}(1)-\mathrm{C}(1)-\mathrm{C}(2)-\mathrm{C}(3)$ & $176.5(6)$ & $\mathrm{N}(3)-\mathrm{C}(28)-\mathrm{C}(33)-\mathrm{C}(32)$ & $-175.2(8)$ \\
\hline $\mathrm{N}(2)-\mathrm{C}(2)-\mathrm{C}(3)-\mathrm{C}(4)$ & $-177.6(7)$ & $\mathrm{C}(30)-\mathrm{C}(31)-\mathrm{C}(34)-\mathrm{C}(36 \mathrm{~B})$ & $59(2)$ \\
\hline $\mathrm{C}(1)-\mathrm{C}(2)-\mathrm{C}(3)-\mathrm{C}(4)$ & $1.3(11)$ & $\mathrm{C}(32)-\mathrm{C}(31)-\mathrm{C}(34)-\mathrm{C}(36 \mathrm{~B})$ & $-119.4(19)$ \\
\hline$C(2)-C(3)-C(4)-C(5)$ & $0.6(13)$ & $\mathrm{C}(30)-\mathrm{C}(31)-\mathrm{C}(34)-\mathrm{C}(35 \mathrm{~A})$ & $-104.1(13)$ \\
\hline$C(3)-C(4)-C(5)-C(6)$ & $-1.0(12)$ & $\mathrm{C}(32)-\mathrm{C}(31)-\mathrm{C}(34)-\mathrm{C}(35 \mathrm{~A})$ & $77.5(14)$ \\
\hline $\mathrm{C}(4)-\mathrm{C}(5)-\mathrm{C}(6)-\mathrm{C}(1)$ & $-0.7(11)$ & $\mathrm{C}(30)-\mathrm{C}(31)-\mathrm{C}(34)-\mathrm{C}(34 \mathrm{~A})$ & $114.1(12)$ \\
\hline$C(2)-C(1)-C(6)-C(5)$ & $2.6(10)$ & $\mathrm{C}(32)-\mathrm{C}(31)-\mathrm{C}(34)-\mathrm{C}(34 \mathrm{~A})$ & $-64.3(13)$ \\
\hline $\mathrm{N}(1)-\mathrm{C}(1)-\mathrm{C}(6)-\mathrm{C}(5)$ & $-176.7(6)$ & $\mathrm{C}(30)-\mathrm{C}(31)-\mathrm{C}(34)-\mathrm{C}(34 \mathrm{~B})$ & $-163.7(10)$ \\
\hline $\mathrm{C}(13)-\mathrm{N}(1)-\mathrm{C}(7)-\mathrm{C}(12)$ & $-52.3(8)$ & $\mathrm{C}(32)-\mathrm{C}(31)-\mathrm{C}(34)-\mathrm{C}(34 \mathrm{~B})$ & $17.9(13)$ \\
\hline $\mathrm{C}(1)-\mathrm{N}(1)-\mathrm{C}(7)-\mathrm{C}(12)$ & $81.9(8)$ & $\mathrm{C}(30)-\mathrm{C}(31)-\mathrm{C}(34)-\mathrm{C}(36 \mathrm{~A})$ & $4.0(13)$ \\
\hline $\mathrm{Fe}(1)-\mathrm{N}(1)-\mathrm{C}(7)-\mathrm{C}(12)$ & $-165.3(5)$ & $\mathrm{C}(32)-\mathrm{C}(31)-\mathrm{C}(34)-\mathrm{C}(36 \mathrm{~A})$ & $-174.4(10)$ \\
\hline $\mathrm{C}(13)-\mathrm{N}(1)-\mathrm{C}(7)-\mathrm{C}(8)$ & $129.8(6)$ & $\mathrm{C}(30)-\mathrm{C}(31)-\mathrm{C}(34)-\mathrm{C}(35 \mathrm{~B})$ & $-48.7(18)$ \\
\hline $\mathrm{C}(1)-\mathrm{N}(1)-\mathrm{C}(7)-\mathrm{C}(8)$ & $-96.0(6)$ & $\mathrm{C}(32)-\mathrm{C}(31)-\mathrm{C}(34)-\mathrm{C}(35 \mathrm{~B})$ & $132.8(17)$ \\
\hline $\mathrm{Fe}(1)-\mathrm{N}(1)-\mathrm{C}(7)-\mathrm{C}(8)$ & $16.8(6)$ & $\mathrm{C}(14)-\mathrm{N}(4)-\mathrm{C}(37)-\mathrm{C}(38)$ & $-116.7(8)$ \\
\hline $\mathrm{C}(28)-\mathrm{N}(3)-\mathrm{C}(8)-\mathrm{C}(9)$ & $5.4(10)$ & $\mathrm{Fe}(1)-\mathrm{N}(4)-\mathrm{C}(37)-\mathrm{C}(38)$ & $84.8(8)$ \\
\hline $\mathrm{Fe}(1)-\mathrm{N}(3)-\mathrm{C}(8)-\mathrm{C}(9)$ & $163.7(6)$ & $\mathrm{C}(14)-\mathrm{N}(4)-\mathrm{C}(37)-\mathrm{C}(42)$ & $68.6(9)$ \\
\hline
\end{tabular}




\begin{tabular}{|c|c|c|c|}
\hline $\mathrm{C}(28)-\mathrm{N}(3)-\mathrm{C}(8)-\mathrm{C}(7)$ & $-172.9(6)$ & $\mathrm{Fe}(1)-\mathrm{N}(4)-\mathrm{C}(37)-\mathrm{C}(42)$ & $-89.9(8)$ \\
\hline $\mathrm{Fe}(1)-\mathrm{N}(3)-\mathrm{C}(8)-\mathrm{C}(7)$ & $-14.6(8)$ & $\mathrm{C}(42)-\mathrm{C}(37)-\mathrm{C}(38)-\mathrm{C}(39)$ & $-0.1(14)$ \\
\hline $\mathrm{C}(12)-\mathrm{C}(7)-\mathrm{C}(8)-\mathrm{N}(3)$ & $177.4(6)$ & $\mathrm{N}(4)-\mathrm{C}(37)-\mathrm{C}(38)-\mathrm{C}(39)$ & $-175.2(9)$ \\
\hline $\mathrm{N}(1)-\mathrm{C}(7)-\mathrm{C}(8)-\mathrm{N}(3)$ & $-4.5(8)$ & $\mathrm{C}(37)-\mathrm{C}(38)-\mathrm{C}(39)-\mathrm{C}(40)$ & $-0.3(16)$ \\
\hline $\mathrm{C}(12)-\mathrm{C}(7)-\mathrm{C}(8)-\mathrm{C}(9)$ & $-1.1(9)$ & $\mathrm{C}(38)-\mathrm{C}(39)-\mathrm{C}(40)-\mathrm{C}(41)$ & $0.1(14)$ \\
\hline $\mathrm{N}(1)-\mathrm{C}(7)-\mathrm{C}(8)-\mathrm{C}(9)$ & $177.0(6)$ & $\mathrm{C}(38)-\mathrm{C}(39)-\mathrm{C}(40)-\mathrm{C}(43)$ & $-178.8(9)$ \\
\hline $\mathrm{N}(3)-\mathrm{C}(8)-\mathrm{C}(9)-\mathrm{C}(10)$ & $-178.5(7)$ & $\mathrm{C}(39)-\mathrm{C}(40)-\mathrm{C}(41)-\mathrm{C}(42)$ & $0.5(12)$ \\
\hline$C(7)-C(8)-C(9)-C(10)$ & $-0.1(11)$ & $\mathrm{C}(43)-\mathrm{C}(40)-\mathrm{C}(41)-\mathrm{C}(42)$ & $179.4(8)$ \\
\hline $\mathrm{C}(8)-\mathrm{C}(9)-\mathrm{C}(10)-\mathrm{C}(11)$ & $1.0(13)$ & $\mathrm{C}(38)-\mathrm{C}(37)-\mathrm{C}(42)-\mathrm{C}(41)$ & $0.7(12)$ \\
\hline $\mathrm{C}(9)-\mathrm{C}(10)-\mathrm{C}(11)-\mathrm{C}(12)$ & $-0.7(12)$ & $\mathrm{N}(4)-\mathrm{C}(37)-\mathrm{C}(42)-\mathrm{C}(41)$ & $175.7(7)$ \\
\hline $\mathrm{C}(10)-\mathrm{C}(11)-\mathrm{C}(12)-\mathrm{C}(7)$ & $-0.5(11)$ & $\mathrm{C}(40)-\mathrm{C}(41)-\mathrm{C}(42)-\mathrm{C}(37)$ & $-0.9(13)$ \\
\hline $\mathrm{C}(8)-\mathrm{C}(7)-\mathrm{C}(12)-\mathrm{C}(11)$ & $1.4(10)$ & $\mathrm{C}(41)-\mathrm{C}(40)-\mathrm{C}(43)-\mathrm{C}(43 \mathrm{D})$ & $50(2)$ \\
\hline $\mathrm{N}(1)-\mathrm{C}(7)-\mathrm{C}(12)-\mathrm{C}(11)$ & $-176.5(6)$ & $\mathrm{C}(39)-\mathrm{C}(40)-\mathrm{C}(43)-\mathrm{C}(43 \mathrm{D})$ & $-131.3(19)$ \\
\hline $\mathrm{C}(1)-\mathrm{N}(1)-\mathrm{C}(13)-\mathrm{C}(18)$ & $-51.3(8)$ & $\mathrm{C}(41)-\mathrm{C}(40)-\mathrm{C}(43)-\mathrm{C}(43 \mathrm{C})$ & $111.6(12)$ \\
\hline $\mathrm{C}(7)-\mathrm{N}(1)-\mathrm{C}(13)-\mathrm{C}(18)$ & $82.8(8)$ & $C(39)-C(40)-C(43)-C(43 C)$ & $-69.6(13)$ \\
\hline $\mathrm{Fe}(1)-\mathrm{N}(1)-\mathrm{C}(13)-\mathrm{C}(18)$ & $-164.3(5)$ & $\mathrm{C}(41)-\mathrm{C}(40)-\mathrm{C}(43)-\mathrm{C}(44 \mathrm{C})$ & $-107.6(13)$ \\
\hline $\mathrm{C}(1)-\mathrm{N}(1)-\mathrm{C}(13)-\mathrm{C}(14)$ & $129.3(6)$ & $\mathrm{C}(39)-\mathrm{C}(40)-\mathrm{C}(43)-\mathrm{C}(44 \mathrm{C})$ & $71.2(14)$ \\
\hline $\mathrm{C}(7)-\mathrm{N}(1)-\mathrm{C}(13)-\mathrm{C}(14)$ & $-96.6(6)$ & $\mathrm{C}(41)-\mathrm{C}(40)-\mathrm{C}(43)-\mathrm{C}(45 \mathrm{C})$ & $0.8(13)$ \\
\hline $\mathrm{Fe}(1)-\mathrm{N}(1)-\mathrm{C}(13)-\mathrm{C}(14)$ & $16.3(6)$ & $C(39)-C(40)-C(43)-C(45 C)$ & $179.6(11)$ \\
\hline $\mathrm{C}(37)-\mathrm{N}(4)-\mathrm{C}(14)-\mathrm{C}(15)$ & $5.1(10)$ & $\mathrm{C}(41)-\mathrm{C}(40)-\mathrm{C}(43)-\mathrm{C}(44 \mathrm{D})$ & $-163.3(10)$ \\
\hline $\mathrm{Fe}(1)-\mathrm{N}(4)-\mathrm{C}(14)-\mathrm{C}(15)$ & $163.8(6)$ & $\mathrm{C}(39)-\mathrm{C}(40)-\mathrm{C}(43)-\mathrm{C}(44 \mathrm{D})$ & $15.5(13)$ \\
\hline $\mathrm{C}(37)-\mathrm{N}(4)-\mathrm{C}(14)-\mathrm{C}(13)$ & $-173.4(6)$ & $\mathrm{C}(41)-\mathrm{C}(40)-\mathrm{C}(43)-\mathrm{C}(45 \mathrm{D})$ & $-59.3(15)$ \\
\hline
\end{tabular}




$\begin{array}{llll}\mathrm{Fe}(1)-\mathrm{N}(4)-\mathrm{C}(14)-\mathrm{C}(13) & -14.8(8) & \mathrm{C}(39)-\mathrm{C}(40)-\mathrm{C}(43)-\mathrm{C}(45 \mathrm{D}) & 119.5(14) \\ \mathrm{C}(18)-\mathrm{C}(13)-\mathrm{C}(14)-\mathrm{N}(4) & 176.5(6)\end{array}$

Symmetry transformations used to generate equivalent atoms:

$\# 1-x,-y+1,-z \quad \# 2-x+1 / 2, y+1 / 2, z \quad \# 3-x+1 / 2, y-1 / 2, z$ 
Table S37. Atomic coordinates $\left(\times 10^{4}\right)$ and equivalent isotropic displacement parameters $\left(\AA^{2} \times 10^{3}\right)$ for $\left[\left(\mathrm{L}^{1}\right) \mathrm{Fe}(\mathrm{III})-\mathrm{DMF}\right](\mathbf{5})$. $\mathrm{U}(\mathrm{eq})$ is defined as one third of the trace of the orthogonalized $\mathrm{U}^{\mathrm{ij}}$ tensor

\begin{tabular}{|c|c|c|c|c|}
\hline & $\mathrm{x}$ & $\mathrm{y}$ & $\mathrm{z}$ & $\overline{U(e q)}$ \\
\hline $\mathrm{Fe}(1)$ & 3333 & 6667 & $10138(1)$ & $\overline{50(1)}$ \\
\hline $\mathrm{N}(1)$ & 3333 & 6667 & $10757(2)$ & $31(1)$ \\
\hline $\mathrm{N}(2)$ & $4759(3)$ & 7802(3) & $10242(1)$ & $43(1)$ \\
\hline $\mathrm{C}(1)$ & $4147(3)$ & $7730(3)$ & $10849(1)$ & $34(1)$ \\
\hline$C(2)$ & 4193(4) & $8183(4)$ & $11182(1)$ & $42(1)$ \\
\hline$C(3)$ & $5019(4)$ & $9176(4)$ & $11263(1)$ & $54(1)$ \\
\hline$C(4)$ & $5771(4)$ & $9698(4)$ & 11011(1) & $55(1)$ \\
\hline $\mathrm{C}(5)$ & $5725(4)$ & $9287(4)$ & 10664(1) & $44(1)$ \\
\hline$C(6)$ & 4892(3) & $8278(3)$ & $10578(1)$ & $35(1)$ \\
\hline$C(7)$ & $5475(3)$ & $8324(3)$ & 9952(1) & $39(1)$ \\
\hline $\mathrm{C}(8)$ & $5434(4)$ & $9093(4)$ & $9749(1)$ & $50(1)$ \\
\hline $\mathrm{C}(9)$ & $6079(4)$ & $9547(4)$ & $9454(1)$ & $51(1)$ \\
\hline $\mathrm{C}(10)$ & $6771(4)$ & $9236(4)$ & $9338(1)$ & $48(1)$ \\
\hline $\mathrm{C}(11)$ & $6798(5)$ & $8458(4)$ & $9539(2)$ & $67(2)$ \\
\hline$C(12)$ & $6181(4)$ & $8011(4)$ & $9841(2)$ & $59(1)$ \\
\hline$C(13)$ & $7440(5)$ & $9731(4)$ & $8997(2)$ & $66(2)$ \\
\hline$C(14)$ & $8047(5)$ & $10940(4)$ & $9034(2)$ & $82(2)$ \\
\hline$C(15)$ & $8239(6)$ & $9352(6)$ & $8933(2)$ & $100(3)$ \\
\hline
\end{tabular}




\begin{tabular}{rrrrr}
$\mathrm{C}(16)$ & $6724(6)$ & $9446(5)$ & $8664(2)$ & $91(2)$ \\
$\mathrm{O}(1 \mathrm{D})$ & 3333 & 6667 & $9589(2)$ & $69(2)$ \\
$\mathrm{N}(1 \mathrm{D})$ & 3333 & 6667 & $8987(2)$ & $65(2)$ \\
$\mathrm{C}(1 \mathrm{D})$ & $3390(50)$ & $7075(16)$ & $9333(4)$ & $78(7)$ \\
$\mathrm{C}(2 \mathrm{D})$ & $2467(15)$ & $5960(20)$ & $8700(6)$ & $227(11)$ \\
\hline
\end{tabular}


Table S38. Bond Distances, $\AA$, for $\left[\left(\mathrm{L}^{1}\right) \mathrm{Fe}(\mathrm{III})-\mathrm{DMF}\right]$ (5)

\begin{tabular}{|c|c|c|c|}
\hline $\mathrm{Fe}(1)-\mathrm{N}(2) \# 1$ & $1.961(4)$ & $\mathrm{C}(10)-\mathrm{C}(11)$ & $1.380(7)$ \\
\hline $\mathrm{Fe}(1)-\mathrm{N}(2)$ & $1.961(4)$ & $C(10)-C(13)$ & $1.529(7)$ \\
\hline $\mathrm{Fe}(1)-\mathrm{N}(2) \# 2$ & $1.961(4)$ & $\mathrm{C}(11)-\mathrm{C}(12)$ & $1.373(7)$ \\
\hline $\mathrm{Fe}(1)-\mathrm{O}(1 \mathrm{D})$ & $2.007(7)$ & $C(13)-C(16)$ & $1.530(10)$ \\
\hline $\mathrm{Fe}(1)-\mathrm{N}(1)$ & $2.267(6)$ & $C(13)-C(14)$ & $1.550(8)$ \\
\hline $\mathrm{N}(1)-\mathrm{C}(1) \# 1$ & $1.459(5)$ & $C(13)-C(15)$ & $1.553(9)$ \\
\hline $\mathrm{N}(1)-\mathrm{C}(1)$ & $1.459(5)$ & $\mathrm{O}(1 \mathrm{D})-\mathrm{C}(1 \mathrm{D})$ & $1.095(16)$ \\
\hline $\mathrm{N}(1)-\mathrm{C}(1) \# 2$ & $1.459(5)$ & $\mathrm{O}(1 \mathrm{D})-\mathrm{C}(1 \mathrm{D}) \# 1$ & $1.095(16)$ \\
\hline $\mathrm{N}(2)-\mathrm{C}(6)$ & $1.380(6)$ & $\mathrm{O}(1 \mathrm{D})-\mathrm{C}(1 \mathrm{D}) \# 2$ & $1.095(16)$ \\
\hline $\mathrm{N}(2)-\mathrm{C}(7)$ & $1.423(5)$ & $\mathrm{N}(1 \mathrm{D})-\mathrm{C}(1 \mathrm{D}) \# 1$ & $1.386(17)$ \\
\hline $\mathrm{C}(1)-\mathrm{C}(2)$ & $1.376(6)$ & $\mathrm{N}(1 \mathrm{D})-\mathrm{C}(1 \mathrm{D})$ & $1.386(17)$ \\
\hline$C(1)-C(6)$ & $1.398(6)$ & $\mathrm{N}(1 \mathrm{D})-\mathrm{C}(1 \mathrm{D}) \# 2$ & $1.386(16)$ \\
\hline$C(2)-C(3)$ & $1.390(7)$ & $\mathrm{N}(1 \mathrm{D})-\mathrm{C}(2 \mathrm{D}) \# 2$ & $1.58(2)$ \\
\hline$C(3)-C(4)$ & $1.350(8)$ & $\mathrm{N}(1 \mathrm{D})-\mathrm{C}(2 \mathrm{D})$ & $1.58(2)$ \\
\hline$C(4)-C(5)$ & $1.394(7)$ & $\mathrm{N}(1 \mathrm{D})-\mathrm{C}(2 \mathrm{D}) \# 1$ & $1.58(2)$ \\
\hline$C(5)-C(6)$ & $1.411(6)$ & $\mathrm{C}(1 \mathrm{D})-\mathrm{C}(1 \mathrm{D}) \# 1$ & $0.98(3)$ \\
\hline$C(7)-C(8)$ & $1.381(7)$ & $\mathrm{C}(1 \mathrm{D})-\mathrm{C}(1 \mathrm{D}) \# 2$ & $0.98(3)$ \\
\hline$C(7)-C(12)$ & $1.392(7)$ & $\mathrm{C}(2 \mathrm{D})-\mathrm{C}(2 \mathrm{D}) \# 2$ & $2.04(3)$ \\
\hline$C(8)-C(9)$ & $1.373(7)$ & $\mathrm{C}(2 \mathrm{D})-\mathrm{C}(2 \mathrm{D}) \# 1$ & $2.04(3)$ \\
\hline$C(9)-C(10)$ & $1.379(7)$ & & \\
\hline
\end{tabular}


Symmetry transformations used to generate equivalent atoms:

$\# 1-y+1, x-y+1, z \# 2-x+y,-x+1, z$ 
Table S39. Angles, ${ }^{o}$, for $\left[\left(\mathrm{L}^{1}\right) \mathrm{Fe}(\mathrm{III})-\mathrm{DMF}\right](\mathbf{5})$

\begin{tabular}{|c|c|c|c|}
\hline $\mathrm{N}(2) \# 1-\mathrm{Fe}(1)-\mathrm{N}(2)$ & $116.28(7)$ & $C(11)-C(12)-C(7)$ & $120.0(5)$ \\
\hline $\mathrm{N}(2) \# 1-\mathrm{Fe}(1)-\mathrm{N}(2) \# 2$ & $116.28(7)$ & $C(10)-C(13)-C(16)$ & $109.2(5)$ \\
\hline $\mathrm{N}(2)-\mathrm{Fe}(1)-\mathrm{N}(2) \# 2$ & $116.28(7)$ & $\mathrm{C}(10)-\mathrm{C}(13)-\mathrm{C}(14)$ & $110.0(4)$ \\
\hline $\mathrm{N}(2) \# 1-\mathrm{Fe}(1)-\mathrm{O}(1 \mathrm{D})$ & $101.27(11)$ & $\mathrm{C}(16)-\mathrm{C}(13)-\mathrm{C}(14)$ & $107.9(5)$ \\
\hline $\mathrm{N}(2)-\mathrm{Fe}(1)-\mathrm{O}(1 \mathrm{D})$ & $101.27(11)$ & $\mathrm{C}(10)-\mathrm{C}(13)-\mathrm{C}(15)$ & $112.1(5)$ \\
\hline $\mathrm{N}(2) \# 2-\mathrm{Fe}(1)-\mathrm{O}(1 \mathrm{D})$ & $101.27(11)$ & $\mathrm{C}(16)-\mathrm{C}(13)-\mathrm{C}(15)$ & $108.8(6)$ \\
\hline $\mathrm{N}(2) \# 1-\mathrm{Fe}(1)-\mathrm{N}(1)$ & $78.73(11)$ & $\mathrm{C}(14)-\mathrm{C}(13)-\mathrm{C}(15)$ & $108.8(6)$ \\
\hline $\mathrm{N}(2)-\mathrm{Fe}(1)-\mathrm{N}(1)$ & $78.73(11)$ & $\mathrm{C}(1 \mathrm{D})-\mathrm{O}(1 \mathrm{D})-\mathrm{C}(1 \mathrm{D}) \# 1$ & $52.9(14)$ \\
\hline $\mathrm{N}(2) \# 2-\mathrm{Fe}(1)-\mathrm{N}(1)$ & $78.73(11)$ & $\mathrm{C}(1 \mathrm{D})-\mathrm{O}(1 \mathrm{D})-\mathrm{C}(1 \mathrm{D}) \# 2$ & $52.9(15)$ \\
\hline $\mathrm{O}(1 \mathrm{D})-\mathrm{Fe}(1)-\mathrm{N}(1)$ & $180.000(3)$ & $\mathrm{C}(1 \mathrm{D}) \# 1-\mathrm{O}(1 \mathrm{D})-\mathrm{C}(1 \mathrm{D}) \# 2$ & $52.9(14)$ \\
\hline $\mathrm{C}(1) \# 1-\mathrm{N}(1)-\mathrm{C}(1)$ & $114.83(19)$ & $\mathrm{C}(1 \mathrm{D})-\mathrm{O}(1 \mathrm{D})-\mathrm{Fe}(1)$ & $149.0(9)$ \\
\hline $\mathrm{C}(1) \# 1-\mathrm{N}(1)-\mathrm{C}(1) \# 2$ & $114.83(19)$ & $\mathrm{C}(1 \mathrm{D}) \# 1-\mathrm{O}(1 \mathrm{D})-\mathrm{Fe}(1)$ & $149.0(9)$ \\
\hline $\mathrm{C}(1)-\mathrm{N}(1)-\mathrm{C}(1) \# 2$ & $114.83(19)$ & $\mathrm{C}(1 \mathrm{D}) \# 2-\mathrm{O}(1 \mathrm{D})-\mathrm{Fe}(1)$ & $149.0(9)$ \\
\hline $\mathrm{C}(1) \# 1-\mathrm{N}(1)-\mathrm{Fe}(1)$ & $103.4(3)$ & $\mathrm{C}(1 \mathrm{D}) \# 1-\mathrm{N}(1 \mathrm{D})-\mathrm{C}(1 \mathrm{D})$ & $41.2(12)$ \\
\hline $\mathrm{C}(1)-\mathrm{N}(1)-\mathrm{Fe}(1)$ & $103.4(3)$ & $\mathrm{C}(1 \mathrm{D}) \# 1-\mathrm{N}(1 \mathrm{D})-\mathrm{C}(1 \mathrm{D}) \# 2$ & $41.2(12)$ \\
\hline $\mathrm{C}(1) \# 2-\mathrm{N}(1)-\mathrm{Fe}(1)$ & $103.4(3)$ & $\mathrm{C}(1 \mathrm{D})-\mathrm{N}(1 \mathrm{D})-\mathrm{C}(1 \mathrm{D}) \# 2$ & $41.2(12)$ \\
\hline$C(6)-N(2)-C(7)$ & $120.9(4)$ & $\mathrm{C}(1 \mathrm{D}) \# 1-\mathrm{N}(1 \mathrm{D})-\mathrm{C}(2 \mathrm{D}) \# 2$ & $140(3)$ \\
\hline $\mathrm{C}(6)-\mathrm{N}(2)-\mathrm{Fe}(1)$ & $115.3(3)$ & $\mathrm{C}(1 \mathrm{D})-\mathrm{N}(1 \mathrm{D})-\mathrm{C}(2 \mathrm{D}) \# 2$ & $107.8(10)$ \\
\hline $\mathrm{C}(7)-\mathrm{N}(2)-\mathrm{Fe}(1)$ & $120.2(3)$ & $\mathrm{C}(1 \mathrm{D}) \# 2-\mathrm{N}(1 \mathrm{D})-\mathrm{C}(2 \mathrm{D}) \# 2$ & $139(3)$ \\
\hline$C(2)-C(1)-C(6)$ & $121.0(4)$ & $\mathrm{C}(1 \mathrm{D}) \# 1-\mathrm{N}(1 \mathrm{D})-\mathrm{C}(2 \mathrm{D})$ & $107.8(11)$ \\
\hline $\mathrm{C}(2)-\mathrm{C}(1)-\mathrm{N}(1)$ & $122.7(4)$ & $\mathrm{C}(1 \mathrm{D})-\mathrm{N}(1 \mathrm{D})-\mathrm{C}(2 \mathrm{D})$ & $139(3)$ \\
\hline
\end{tabular}




$\begin{array}{lllr}\mathrm{C}(6)-\mathrm{C}(1)-\mathrm{N}(1) & 116.3(4) & \mathrm{C}(1 \mathrm{D}) \# 2-\mathrm{N}(1 \mathrm{D})-\mathrm{C}(2 \mathrm{D}) & 140(3) \\ \mathrm{C}(1)-\mathrm{C}(2)-\mathrm{C}(3) & 120.3(4) & \mathrm{C}(2 \mathrm{D}) \# 2-\mathrm{N}(1 \mathrm{D})-\mathrm{C}(2 \mathrm{D}) & 80.4(12) \\ \mathrm{C}(4)-\mathrm{C}(3)-\mathrm{C}(2) & 119.4(4) & \mathrm{C}(1 \mathrm{D}) \# 1-\mathrm{N}(1 \mathrm{D})-\mathrm{C}(2 \mathrm{D}) \# 1 & 139(3) \\ \mathrm{C}(3)-\mathrm{C}(4)-\mathrm{C}(5) & 121.9(5) & \mathrm{C}(1 \mathrm{D})-\mathrm{N}(1 \mathrm{D})-\mathrm{C}(2 \mathrm{D}) \# 1 & 140(3) \\ \mathrm{C}(4)-\mathrm{C}(5)-\mathrm{C}(6) & 119.2(4) & \mathrm{C}(1 \mathrm{D}) \# 2-\mathrm{N}(1 \mathrm{D})-\mathrm{C}(2 \mathrm{D}) \# 1 & 107.8(10) \\ \mathrm{N}(2)-\mathrm{C}(6)-\mathrm{C}(1) & 117.9(4) & \mathrm{C}(2 \mathrm{D}) \# 2-\mathrm{N}(1 \mathrm{D})-\mathrm{C}(2 \mathrm{D}) \# 1 & 80.4(12) \\ \mathrm{N}(2)-\mathrm{C}(6)-\mathrm{C}(5) & 124.1(4) & \mathrm{C}(2 \mathrm{D})-\mathrm{N}(1 \mathrm{D})-\mathrm{C}(2 \mathrm{D}) \# 1 & 80.4(12) \\ \mathrm{C}(1)-\mathrm{C}(6)-\mathrm{C}(5) & 118.0(4) & \mathrm{C}(1 \mathrm{D}) \# 1-\mathrm{C}(1 \mathrm{D})-\mathrm{C}(1 \mathrm{D}) \# 2 & 60.00(4) \\ \mathrm{C}(8)-\mathrm{C}(7)-\mathrm{C}(12) & 117.6(4) & \mathrm{C}(1 \mathrm{D}) \# 1-\mathrm{C}(1 \mathrm{D})-\mathrm{O}(1 \mathrm{D}) & 63.5(7) \\ \mathrm{C}(8)-\mathrm{C}(7)-\mathrm{N}(2) & 121.3(4) & \mathrm{C}(1 \mathrm{D}) \# 2-\mathrm{C}(1 \mathrm{D})-\mathrm{O}(1 \mathrm{D}) & 63.5(7) \\ \mathrm{C}(12)-\mathrm{C}(7)-\mathrm{N}(2) & 120.9(4) & \mathrm{C}(1 \mathrm{D}) \# 1-\mathrm{C}(1 \mathrm{D})-\mathrm{N}(1 \mathrm{D}) & 69.4(6) \\ \mathrm{C}(9)-\mathrm{C}(8)-\mathrm{C}(7) & 121.3(4) & \mathrm{C}(1 \mathrm{D}) \# 2-\mathrm{C}(1 \mathrm{D})-\mathrm{N}(1 \mathrm{D}) & 69.4(6) \\ \mathrm{C}(8)-\mathrm{C}(9)-\mathrm{C}(10) & 121.8(4) & \mathrm{O}(1 \mathrm{D})-\mathrm{C}(1 \mathrm{D})-\mathrm{N}(1 \mathrm{D}) & 125.1(15) \\ \mathrm{C}(9)-\mathrm{C}(10)-\mathrm{C}(11) & 116.4(4) & \mathrm{N}(1 \mathrm{D})-\mathrm{C}(2 \mathrm{D})-\mathrm{C}(2 \mathrm{D}) \# 2 & 49.8(6) \\ \mathrm{C}(9)-\mathrm{C}(10)-\mathrm{C}(13) & 120.0(4) & \mathrm{N}(1 \mathrm{D})-\mathrm{C}(2 \mathrm{D})-\mathrm{C}(2 \mathrm{D}) \# 1 & 49.8(6) \\ \mathrm{C}(11)-\mathrm{C}(10)-\mathrm{C}(13) & 123.5(4) & \mathrm{C}(2 \mathrm{D}) \# 2-\mathrm{C}(2 \mathrm{D})-\mathrm{C}(2 \mathrm{D}) \# 1 & 60.000(13) \\ \mathrm{C}(12)-\mathrm{C}(11)-\mathrm{C}(10) & 122.8(5) & & \end{array}$

Symmetry transformations used to generate equivalent atoms:

$\# 1-y+1, x-y+1, z \# 2-x+y,-x+1, z$ 
Table S40. Anisotropic displacement parameters $\left(\AA^{2} \times 10^{3}\right)$ for $\left[\left(\mathrm{L}^{1}\right) \mathrm{Fe}(\mathrm{III})-\mathrm{DMF}\right](\mathbf{5})$. The anisotropic displacement factor exponent takes the form: $-2 \pi^{2}\left[h^{2} a^{* 2} U^{11}+\ldots+2 h k\right.$ $a^{*} b^{*} U^{12}$ ]

\begin{tabular}{|c|c|c|c|c|c|c|}
\hline & $\mathrm{U}^{11}$ & $\mathrm{U}^{22}$ & $\mathrm{U}^{33}$ & $\mathrm{U}^{23}$ & $\mathrm{U}^{13}$ & $\mathrm{U}^{12}$ \\
\hline $\mathrm{Fe}(1)$ & $59(1)$ & $59(1)$ & $31(1)$ & 0 & 0 & $30(1)$ \\
\hline $\mathrm{N}(1)$ & $36(2)$ & $36(2)$ & $21(3)$ & 0 & 0 & $18(1)$ \\
\hline $\mathrm{N}(2)$ & $47(2)$ & $47(2)$ & $30(2)$ & $3(2)$ & 10(2) & $20(2)$ \\
\hline $\mathrm{C}(1)$ & $37(2)$ & $40(2)$ & $26(2)$ & $-1(2)$ & $-5(2)$ & $20(2)$ \\
\hline$C(2)$ & $49(3)$ & 49(3) & $24(2)$ & $-3(2)$ & $-2(2)$ & $22(2)$ \\
\hline$C(3)$ & 61(3) & $56(3)$ & $38(3)$ & $-14(2)$ & $-9(2)$ & $24(3)$ \\
\hline $\mathrm{C}(4)$ & $59(3)$ & $44(3)$ & $48(3)$ & $-10(2)$ & $-13(2)$ & $16(2)$ \\
\hline$C(5)$ & $44(2)$ & $43(2)$ & $42(3)$ & $3(2)$ & $0(2)$ & $19(2)$ \\
\hline$C(6)$ & $35(2)$ & $39(2)$ & $33(2)$ & $0(2)$ & $-3(2)$ & $19(2)$ \\
\hline $\mathrm{C}(7)$ & $40(2)$ & $42(2)$ & $32(2)$ & $3(2)$ & $6(2)$ & $18(2)$ \\
\hline $\mathrm{C}(8)$ & $58(3)$ & $52(3)$ & $51(3)$ & $9(2)$ & $16(2)$ & $37(2)$ \\
\hline $\mathrm{C}(9)$ & $65(3)$ & $47(3)$ & $49(3)$ & $9(2)$ & $13(2)$ & $34(3)$ \\
\hline$C(10)$ & $57(3)$ & $40(2)$ & $48(3)$ & $8(2)$ & $19(2)$ & $25(2)$ \\
\hline $\mathrm{C}(11)$ & $81(4)$ & $63(3)$ & $78(4)$ & $24(3)$ & $43(3)$ & $53(3)$ \\
\hline$C(12)$ & $67(3)$ & $59(3)$ & $66(4)$ & $29(3)$ & $30(3)$ & $42(3)$ \\
\hline$C(13)$ & $91(4)$ & $46(3)$ & $62(4)$ & $16(2)$ & $40(3)$ & $34(3)$ \\
\hline$C(14)$ & $96(5)$ & $50(3)$ & $85(5)$ & $16(3)$ & $38(4)$ & $25(3)$ \\
\hline$C(15)$ & $121(6)$ & $85(4)$ & $109(6)$ & $38(4)$ & $86(5)$ & $62(4)$ \\
\hline
\end{tabular}




\begin{tabular}{lrrrccr}
$\mathrm{C}(16)$ & $130(6)$ & $77(4)$ & $46(4)$ & $10(3)$ & $29(4)$ & $36(4)$ \\
$\mathrm{O}(1 \mathrm{D})$ & $89(3)$ & $89(3)$ & $31(3)$ & 0 & 0 & $44(2)$ \\
$\mathrm{N}(1 \mathrm{D})$ & $84(4)$ & $84(4)$ & $28(4)$ & 0 & 0 & $42(2)$ \\
$\mathrm{C}(1 \mathrm{D})$ & $137(15)$ & $49(13)$ & $34(8)$ & $3(7)$ & $19(13)$ & $40(20)$ \\
$\mathrm{C}(2 \mathrm{D})$ & $170(20)$ & $240(30)$ & $180(20)$ & $47(18)$ & $-17(16)$ & $33(17)$ \\
\hline
\end{tabular}


Table S41. Hydrogen coordinates $\left(\times 10^{4}\right)$ and isotropic displacement parameters $\left(\AA^{2} \times 10^{3}\right)$ for $\left[\left(\mathrm{L}^{1}\right) \mathrm{Fe}(\mathrm{III})-\mathrm{DMF}\right](\mathbf{5})$

\begin{tabular}{|c|c|c|c|c|}
\hline & $\mathrm{x}$ & $\mathrm{y}$ & $\mathrm{Z}$ & $\mathrm{U}(\mathrm{eq})$ \\
\hline $\mathrm{H}(2 \mathrm{~A})$ & 3669 & 7823 & 11354 & 50 \\
\hline $\mathrm{H}(3 \mathrm{~A})$ & 5054 & 9479 & 11490 & 65 \\
\hline $\mathrm{H}(4 \mathrm{~A})$ & 6337 & 10350 & 11070 & 66 \\
\hline $\mathrm{H}(5 \mathrm{~A})$ & 6237 & 9673 & 10491 & 53 \\
\hline $\mathrm{H}(8 \mathrm{~A})$ & 4960 & 9307 & 9814 & 60 \\
\hline $\mathrm{H}(9 \mathrm{~A})$ & 6048 & 10080 & 9328 & 61 \\
\hline $\mathrm{H}(11 \mathrm{~A})$ & 7254 & 8228 & 9468 & 80 \\
\hline $\mathrm{H}(12 \mathrm{~A})$ & 6234 & 7498 & 9972 & 71 \\
\hline $\mathrm{H}(14 \mathrm{~A})$ & 7560 & 11183 & 9073 & 123 \\
\hline $\mathrm{H}(14 \mathrm{~B})$ & 8437 & 11246 & 8815 & 123 \\
\hline $\mathrm{H}(14 \mathrm{C})$ & 8519 & 11140 & 9238 & 123 \\
\hline $\mathrm{H}(15 \mathrm{~A})$ & 7872 & 8603 & 8912 & 150 \\
\hline $\mathrm{H}(15 \mathrm{~B})$ & 8718 & 9563 & 9135 & 150 \\
\hline $\mathrm{H}(15 \mathrm{C})$ & 8621 & 9658 & 8712 & 150 \\
\hline $\mathrm{H}(16 \mathrm{~A})$ & 6227 & 9677 & 8701 & 137 \\
\hline $\mathrm{H}(16 \mathrm{~B})$ & 6359 & 8701 & 8629 & 137 \\
\hline $\mathrm{H}(16 \mathrm{C})$ & 7138 & 9783 & 8451 & 137 \\
\hline $\mathrm{H}(1 \mathrm{DA})$ & 3585 & 7799 & 9349 & 94 \\
\hline $\mathrm{H}(2 \mathrm{DA})$ & 2834 & 5878 & 8498 & 341 \\
\hline
\end{tabular}




$\begin{array}{llrrr}\mathrm{H}(2 \mathrm{DB}) & 1963 & 5286 & 8793 & 341 \\ \mathrm{H}(2 \mathrm{DC}) & 2115 & 6326 & 8620 & 341\end{array}$


Table S42. Torsion Angles, ${ }^{\circ}$, for $\left[\left(\mathrm{L}^{1}\right) \mathrm{Fe}(\mathrm{III})-\mathrm{DMF}\right](\mathbf{5})$

\begin{tabular}{|c|c|c|c|}
\hline N2-Fe1-N1-C1 & 24.1(2) & C13-C10-C11-C12 & $-179.0(6)$ \\
\hline N2-Fe1-N1-C1 & $144.1(2)$ & $\mathrm{C} 10-\mathrm{C} 11-\mathrm{C} 12-\mathrm{C} 7$ & $1.2(10)$ \\
\hline N2-Fe1-N1-C1 & $-95.9(2)$ & C8-C7-C12-C11 & $-0.5(8)$ \\
\hline O1D-Fe1-N1-C1 & $-77(100)$ & N2-C7-C12-C11 & $174.0(5)$ \\
\hline N2-Fe1-N1-C1 & $-95.9(2)$ & C9-C10-C13-C16 & $-64.6(7)$ \\
\hline N2-Fe1-N1-C1 & $24.1(2)$ & C11-C10-C13-C16 & $114.0(7)$ \\
\hline N2-Fe1-N1-C1 & $144.1(2)$ & C9-C10-C13-C14 & $53.6(8)$ \\
\hline O1D-Fe1-N1-C1 & $163(100)$ & C11-C10-C13-C14 & $-127.8(7)$ \\
\hline N2-Fe1-N1-C1 & 144.1(2) & C9-C10-C13-C15 & $174.7(6)$ \\
\hline N2-Fe1-N1-C1 & $-95.9(2)$ & C11-C10-C13-C15 & $-6.7(9)$ \\
\hline N2-Fe1-N1-C1 & 24.1(2) & N2-Fe1-O1D-C1D & $57(6)$ \\
\hline O1D-Fe1-N1-C1 & $43(100)$ & N2-Fe1-O1D-C1D & $-63(6)$ \\
\hline N2-Fe1-N2-C6 & $46.9(4)$ & N2-Fe1-O1D-C1D & $177(6)$ \\
\hline N2-Fe1-N2-C6 & $-95.7(3)$ & N1-Fe1-O1D-C1D & $158(100)$ \\
\hline O1D-Fe1-N2-C6 & $155.6(3)$ & N2-Fe1-O1D-C1D & $-63(6)$ \\
\hline N1-Fe1-N2-C6 & $-24.4(3)$ & N2-Fe1-O1D-C1D & $177(6)$ \\
\hline $\mathrm{N} 2-\mathrm{Fe} 1-\mathrm{N} 2-\mathrm{C} 7$ & $-111.8(4)$ & N2-Fe1-O1D-C1D & $57(6)$ \\
\hline N2-Fe1-N2-C7 & $105.6(4)$ & N1-Fe1-O1D-C1D & $38(100)$ \\
\hline O1D-Fe1-N2-C7 & $-3.1(3)$ & N2-Fe1-O1D-C1D & $177(6)$ \\
\hline N1-Fe1-N2-C7 & 176.9(3) & N2-Fe1-O1D-C1D & $57(6)$ \\
\hline $\mathrm{C} 1-\mathrm{N} 1-\mathrm{C} 1-\mathrm{C} 2$ & $46.7(6)$ & N2-Fe1-O1D-C1D & $-63(6)$ \\
\hline
\end{tabular}




\begin{tabular}{|c|c|c|c|}
\hline C1-N1-C1-C2 & $-89.7(4)$ & N1-Fe1-O1D-C1D & $-82(100)$ \\
\hline Fe1-N1-C1-C2 & $158.5(4)$ & C1D-O1D-C1D-C1D & $67.9(5)$ \\
\hline C1-N1-C1-C6 & $-133.3(4)$ & Fe1-O1D-C1D-C1D & $-146.0(3)$ \\
\hline C1-N1-C1-C6 & $90.3(6)$ & C1D-O1D-C1D-C1D & $-67.9(5)$ \\
\hline Fe1-N1-C1-C6 & $-21.5(3)$ & Fe1-O1D-C1D-C1D & $146.0(2)$ \\
\hline $\mathrm{C} 6-\mathrm{C} 1-\mathrm{C} 2-\mathrm{C} 3$ & $-3.7(7)$ & C1D-O1D-C1D-N1D & $-34.0(3)$ \\
\hline $\mathrm{N} 1-\mathrm{C} 1-\mathrm{C} 2-\mathrm{C} 3$ & $176.2(4)$ & C1D-O1D-C1D-N1D & $34.0(2)$ \\
\hline $\mathrm{C} 1-\mathrm{C} 2-\mathrm{C} 3-\mathrm{C} 4$ & $0.7(8)$ & Fe1-O1D-C1D-N1D & $180.00(7)$ \\
\hline $\mathrm{C} 2-\mathrm{C} 3-\mathrm{C} 4-\mathrm{C} 5$ & $2.5(8)$ & C1D-N1D-C1D-C1D & $-64.6(3)$ \\
\hline $\mathrm{C} 3-\mathrm{C} 4-\mathrm{C} 5-\mathrm{C} 6$ & $-2.7(8)$ & C2D-N1D-C1D-C1D & $149(5)$ \\
\hline C7-N2-C6-C1 & $178.6(4)$ & C2D-N1D-C1D-C1D & $54(6)$ \\
\hline Fe1-N2-C6-C1 & $20.1(5)$ & C2D-N1D-C1D-C1D & $-114(6)$ \\
\hline C7-N2-C6-C5 & $-0.6(6)$ & C1D-N1D-C1D-C1D & $64.6(4)$ \\
\hline Fe1-N2-C6-C5 & $-159.1(3)$ & C2D-N1D-C1D-C1D & $-146(5)$ \\
\hline $\mathrm{C} 2-\mathrm{C} 1-\mathrm{C} 6-\mathrm{N} 2$ & $-175.8(4)$ & C2D-N1D-C1D-C1D & $118(7)$ \\
\hline N1-C1-C6-N2 & $4.2(5)$ & C2D-N1D-C1D-C1D & $-49(6)$ \\
\hline $\mathrm{C} 2-\mathrm{C} 1-\mathrm{C} 6-\mathrm{C} 5$ & $3.4(6)$ & C1D-N1D-C1D-O1D & $32.29(15)$ \\
\hline N1-C1-C6-C5 & $-176.5(3)$ & C1D-N1D-C1D-O1D & $-32.29(14)$ \\
\hline $\mathrm{C} 4-\mathrm{C} 5-\mathrm{C} 6-\mathrm{N} 2$ & $178.9(4)$ & C2D-N1D-C1D-O1D & $-178(5)$ \\
\hline C4-C5-C6-C1 & $-0.3(6)$ & C2D-N1D-C1D-O1D & $86(6)$ \\
\hline C6-N2-C7-C8 & $-78.0(6)$ & C2D-N1D-C1D-O1D & $-82(6)$ \\
\hline Fe1-N2-C7-C8 & $79.5(5)$ & C1D-N1D-C2D-C2D & $140(3)$ \\
\hline
\end{tabular}




$\begin{array}{lrlr}\text { C6-N2-C7-C12 } & 107.7(5) & \text { C1D-N1D-C2D-C2D } & 106.2(12) \\ \text { Fe1-N2-C7-C12 } & -94.8(5) & \text { C1D-N1D-C2D-C2D } & 171.7(14) \\ \text { C12-C7-C8-C9 } & -1.2(8) & \text { C2D-N1D-C2D-C2D } & -81.8(9) \\ \text { N2-C7-C8-C9 } & -175.7(5) & \text { C1D-N1D-C2D-C2D } & -138(3) \\ \text { C7-C8-C9-C10 } & 2.2(9) & \text { C1D-N1D-C2D-C2D } & -172.0(16) \\ \text { C8-C9-C10-C11 } & -1.4(8) & \text { C1D-N1D-C2D-C2D } & -106.5(10) \\ \text { C8-C9-C10-C13 } & 177.3(5) & \text { C2D-N1D-C2D-C2D } & 81.8(9) \\ \text { C9-C10-C11-C12 } & -0.3(9) & & \end{array}$


Table S43. Atomic coordinates $\left(\times 10^{4}\right)$ and equivalent isotropic displacement parameters $\left(\AA^{2} \times 10^{3}\right)$ for $\left[\left(\mathrm{L}^{1}\right) \mathrm{Fe}(\mathrm{III})-\mathrm{MeCN}\right](\mathbf{6}) . \mathrm{U}(\mathrm{eq})$ is defined as one third of the trace of the orthogonalized $\mathrm{U}^{\mathrm{ij}}$ tensor

\begin{tabular}{|c|c|c|c|c|}
\hline & $\mathrm{x}$ & $\mathrm{y}$ & $\mathrm{z}$ & $\mathrm{U}(\mathrm{eq})$ \\
\hline $\mathrm{Fe}(1)$ & 0 & 0 & $1523(1)$ & $108(1)$ \\
\hline $\mathrm{N}(1)$ & 0 & 0 & $902(2)$ & $66(3)$ \\
\hline $\mathrm{N}(2)$ & $1116(5)$ & $1433(5)$ & $1408(2)$ & $93(2)$ \\
\hline $\mathrm{C}(1)$ & $1065(6)$ & $790(6)$ & $812(2)$ & $66(2)$ \\
\hline$C(2)$ & 1609(7) & $1555(6)$ & $1074(2)$ & 71(2) \\
\hline$C(3)$ & 2614(7) & $2366(7)$ & $995(2)$ & $83(2)$ \\
\hline $\mathrm{C}(4)$ & $3052(6)$ & 2392(7) & $650(3)$ & $97(3)$ \\
\hline$C(5)$ & 2502(9) & $1630(8)$ & $403(2)$ & 99(3) \\
\hline$C(6)$ & $1514(7)$ & $823(7)$ & $485(2)$ & $80(2)$ \\
\hline$C(7)$ & 1649(7) & $2168(7)$ & $1698(2)$ & $83(2)$ \\
\hline $\mathrm{C}(8)$ & 2370(7) & 2094(7) & 1916(3) & 98(3) \\
\hline $\mathrm{C}(9)$ & 2816(7) & $2726(7)$ & $2213(2)$ & 94(3) \\
\hline$C(10)$ & $2545(6)$ & $3469(7)$ & $2308(2)$ & $82(2)$ \\
\hline $\mathrm{C}(11)$ & 1883(8) & $3568(7)$ & $2087(3)$ & $109(3)$ \\
\hline$C(12)$ & 1432(7) & $2950(8)$ & $1786(2)$ & $103(3)$ \\
\hline$C(13)$ & $3043(8)$ & $4122(8)$ & 2661(3) & $112(3)$ \\
\hline$C(14)$ & $2627(10)$ & $4841(10)$ & 2734(3) & $190(6)$ \\
\hline$C(15)$ & 4219(7) & 4649(9) & 2643(3) & $162(5)$ \\
\hline
\end{tabular}




\begin{tabular}{lrccc}
$\mathrm{C}(16)$ & $2674(9)$ & $3320(10)$ & $2997(3)$ & $181(5)$ \\
$\mathrm{N}(3)$ & 0 & 0 & $2083(4)$ & $154(6)$ \\
$\mathrm{C}(17)$ & 0 & 0 & $2425(8)$ & $286(18)$ \\
$\mathrm{C}(18)$ & 0 & 0 & $2809(5)$ & $370(30)$ \\
\hline
\end{tabular}


Table S44. Bond Distances, $\AA$, for $\left[\left(\mathrm{L}^{1}\right) \mathrm{Fe}(\mathrm{III})-\mathrm{MeCN}\right](\mathbf{6})$

\begin{tabular}{|c|c|c|c|}
\hline $\mathrm{Fe}(1)-\mathrm{N}(2)$ & $1.964(6)$ & $\mathrm{C}(9)-\mathrm{C}(10)$ & $1.383(10)$ \\
\hline $\mathrm{Fe}(1)-\mathrm{N}(2) \# 1$ & $1.964(6)$ & $\mathrm{C}(9)-\mathrm{H}(9 \mathrm{~A})$ & 0.9300 \\
\hline $\mathrm{Fe}(1)-\mathrm{N}(2) \# 2$ & $1.964(6)$ & $C(10)-C(11)$ & $1.328(10)$ \\
\hline $\mathrm{Fe}(1)-\mathrm{N}(3)$ & $2.053(17)$ & $C(10)-C(13)$ & $1.560(11)$ \\
\hline $\mathrm{Fe}(1)-\mathrm{N}(1)$ & $2.272(9)$ & $C(11)-C(12)$ & $1.371(10)$ \\
\hline $\mathrm{N}(1)-\mathrm{C}(1)$ & $1.447(7)$ & $\mathrm{C}(11)-\mathrm{H}(11 \mathrm{~A})$ & 0.9300 \\
\hline $\mathrm{N}(1)-\mathrm{C}(1) \# 2$ & $1.447(7)$ & $\mathrm{C}(12)-\mathrm{H}(12 \mathrm{~A})$ & 0.9300 \\
\hline $\mathrm{N}(1)-\mathrm{C}(1) \# 1$ & $1.447(7)$ & $C(13)-C(14)$ & $1.486(12)$ \\
\hline $\mathrm{N}(2)-\mathrm{C}(2)$ & $1.388(8)$ & $C(13)-C(15)$ & $1.502(11)$ \\
\hline $\mathrm{N}(2)-\mathrm{C}(7)$ & $1.437(9)$ & $C(13)-C(16)$ & $1.599(14)$ \\
\hline$C(1)-C(6)$ & $1.356(8)$ & $\mathrm{C}(14)-\mathrm{H}(14 \mathrm{~A})$ & 0.9600 \\
\hline $\mathrm{C}(1)-\mathrm{C}(2)$ & $1.388(9)$ & $\mathrm{C}(14)-\mathrm{H}(14 \mathrm{~B})$ & 0.9600 \\
\hline $\mathrm{C}(2)-\mathrm{C}(3)$ & $1.388(10)$ & $\mathrm{C}(14)-\mathrm{H}(14 \mathrm{C})$ & 0.9600 \\
\hline $\mathrm{C}(3)-\mathrm{C}(4)$ & $1.411(10)$ & $\mathrm{C}(15)-\mathrm{H}(15 \mathrm{~A})$ & 0.9600 \\
\hline $\mathrm{C}(3)-\mathrm{H}(3 \mathrm{~A})$ & 0.9300 & $\mathrm{C}(15)-\mathrm{H}(15 \mathrm{~B})$ & 0.9600 \\
\hline$C(4)-C(5)$ & $1.351(11)$ & $\mathrm{C}(15)-\mathrm{H}(15 \mathrm{C})$ & 0.9600 \\
\hline $\mathrm{C}(4)-\mathrm{H}(4 \mathrm{~A})$ & 0.9300 & $\mathrm{C}(16)-\mathrm{H}(16 \mathrm{~A})$ & 0.9600 \\
\hline$C(5)-C(6)$ & $1.374(10)$ & $\mathrm{C}(16)-\mathrm{H}(16 \mathrm{~B})$ & 0.9600 \\
\hline $\mathrm{C}(5)-\mathrm{H}(5 \mathrm{~A})$ & 0.9300 & $\mathrm{C}(16)-\mathrm{H}(16 \mathrm{C})$ & 0.9600 \\
\hline $\mathrm{C}(6)-\mathrm{H}(6 \mathrm{~A})$ & 0.9300 & $\mathrm{~N}(3)-\mathrm{C}(17)$ & $1.25(3)$ \\
\hline $\mathrm{C}(7)-\mathrm{C}(8)$ & $1.373(10)$ & $\mathrm{C}(17)-\mathrm{C}(18)$ & $1.41(3)$ \\
\hline
\end{tabular}




$\begin{array}{lrrr}\mathrm{C}(7)-\mathrm{C}(12) & 1.378(10) & \mathrm{C}(18)-\mathrm{H}(18 \mathrm{~A}) & 0.9600 \\ \mathrm{C}(8)-\mathrm{C}(9) & 1.369(10) & \mathrm{C}(18)-\mathrm{H}(18 \mathrm{~B}) & 0.9600 \\ \mathrm{C}(8)-\mathrm{H}(8 \mathrm{~A}) & 0.9300 & \mathrm{C}(18)-\mathrm{H}(18 \mathrm{C}) & 0.9600\end{array}$

Symmetry transformations used to generate equivalent atoms:

\#1 -y, x-y, z \#2-x+y, -x, z 
Table S45. Angles, ${ }^{\circ}$, for $\left[\left(\mathrm{L}^{1}\right) \mathrm{Fe}(\mathrm{III})-\mathrm{MeCN}\right](6)$

\begin{tabular}{|c|c|c|c|}
\hline $\mathrm{N}(2)-\mathrm{Fe}(1)-\mathrm{N}(2) \# 1$ & $115.55(13)$ & $\mathrm{C}(8)-\mathrm{C}(9)-\mathrm{H}(9 \mathrm{~A})$ & 119.8 \\
\hline $\mathrm{N}(2)-\mathrm{Fe}(1)-\mathrm{N}(2) \# 2$ & $115.55(13)$ & $\mathrm{C}(10)-\mathrm{C}(9)-\mathrm{H}(9 \mathrm{~A})$ & 119.8 \\
\hline $\mathrm{N}(2) \# 1-\mathrm{Fe}(1)-\mathrm{N}(2) \# 2$ & $115.55(13)$ & $\mathrm{C}(11)-\mathrm{C}(10)-\mathrm{C}(9)$ & $116.9(7)$ \\
\hline $\mathrm{N}(2)-\mathrm{Fe}(1)-\mathrm{N}(3)$ & 102.36(19) & $\mathrm{C}(11)-\mathrm{C}(10)-\mathrm{C}(13)$ & $125.6(9)$ \\
\hline $\mathrm{N}(2) \# 1-\mathrm{Fe}(1)-\mathrm{N}(3)$ & $102.36(19)$ & $C(9)-C(10)-C(13)$ & $117.5(8)$ \\
\hline $\mathrm{N}(2) \# 2-\mathrm{Fe}(1)-\mathrm{N}(3)$ & $102.36(19)$ & $\mathrm{C}(10)-\mathrm{C}(11)-\mathrm{C}(12)$ & $123.7(8)$ \\
\hline $\mathrm{N}(2)-\mathrm{Fe}(1)-\mathrm{N}(1)$ & 77.64(19) & $\mathrm{C}(10)-\mathrm{C}(11)-\mathrm{H}(11 \mathrm{~A})$ & 118.2 \\
\hline $\mathrm{N}(2) \# 1-\mathrm{Fe}(1)-\mathrm{N}(1)$ & 77.64(19) & $\mathrm{C}(12)-\mathrm{C}(11)-\mathrm{H}(11 \mathrm{~A})$ & 118.2 \\
\hline $\mathrm{N}(2) \# 2-\mathrm{Fe}(1)-\mathrm{N}(1)$ & 77.64(19) & $\mathrm{C}(11)-\mathrm{C}(12)-\mathrm{C}(7)$ & $120.4(8)$ \\
\hline $\mathrm{N}(3)-\mathrm{Fe}(1)-\mathrm{N}(1)$ & $180.000(1)$ & $\mathrm{C}(11)-\mathrm{C}(12)-\mathrm{H}(12 \mathrm{~A})$ & 119.8 \\
\hline $\mathrm{C}(1)-\mathrm{N}(1)-\mathrm{C}(1) \# 2$ & $114.9(3)$ & $\mathrm{C}(7)-\mathrm{C}(12)-\mathrm{H}(12 \mathrm{~A})$ & 119.8 \\
\hline $\mathrm{C}(1)-\mathrm{N}(1)-\mathrm{C}(1) \# 1$ & $114.9(3)$ & $\mathrm{C}(14)-\mathrm{C}(13)-\mathrm{C}(15)$ & $114.7(9)$ \\
\hline $\mathrm{C}(1) \# 2-\mathrm{N}(1)-\mathrm{C}(1) \# 1$ & $114.9(3)$ & $\mathrm{C}(14)-\mathrm{C}(13)-\mathrm{C}(10)$ & $110.7(8)$ \\
\hline $\mathrm{C}(1)-\mathrm{N}(1)-\mathrm{Fe}(1)$ & $103.3(4)$ & $C(15)-C(13)-C(10)$ & $110.3(8)$ \\
\hline $\mathrm{C}(1) \# 2-\mathrm{N}(1)-\mathrm{Fe}(1)$ & $103.3(4)$ & $\mathrm{C}(14)-\mathrm{C}(13)-\mathrm{C}(16)$ & $106.1(9)$ \\
\hline $\mathrm{C}(1) \# 1-\mathrm{N}(1)-\mathrm{Fe}(1)$ & $103.3(4)$ & $C(15)-C(13)-C(16)$ & $107.1(9)$ \\
\hline $\mathrm{C}(2)-\mathrm{N}(2)-\mathrm{C}(7)$ & $120.1(6)$ & $\mathrm{C}(10)-\mathrm{C}(13)-\mathrm{C}(16)$ & $107.6(8)$ \\
\hline $\mathrm{C}(2)-\mathrm{N}(2)-\mathrm{Fe}(1)$ & $115.4(5)$ & $\mathrm{C}(13)-\mathrm{C}(14)-\mathrm{H}(14 \mathrm{~A})$ & 109.5 \\
\hline $\mathrm{C}(7)-\mathrm{N}(2)-\mathrm{Fe}(1)$ & $119.9(5)$ & $\mathrm{C}(13)-\mathrm{C}(14)-\mathrm{H}(14 \mathrm{~B})$ & 109.5 \\
\hline$C(6)-C(1)-C(2)$ & $120.5(7)$ & $\mathrm{H}(14 \mathrm{~A})-\mathrm{C}(14)-\mathrm{H}(14 \mathrm{~B})$ & 109.5 \\
\hline $\mathrm{C}(6)-\mathrm{C}(1)-\mathrm{N}(1)$ & $122.9(8)$ & $\mathrm{C}(13)-\mathrm{C}(14)-\mathrm{H}(14 \mathrm{C})$ & 109.5 \\
\hline
\end{tabular}




\begin{tabular}{|c|c|c|c|}
\hline $\mathrm{C}(2)-\mathrm{C}(1)-\mathrm{N}(1)$ & $116.5(7)$ & $\mathrm{H}(14 \mathrm{~A})-\mathrm{C}(14)-\mathrm{H}(14 \mathrm{C})$ & 109.5 \\
\hline $\mathrm{N}(2)-\mathrm{C}(2)-\mathrm{C}(1)$ & $116.9(7)$ & $\mathrm{H}(14 \mathrm{~B})-\mathrm{C}(14)-\mathrm{H}(14 \mathrm{C})$ & 109.5 \\
\hline $\mathrm{N}(2)-\mathrm{C}(2)-\mathrm{C}(3)$ & $123.8(7)$ & $\mathrm{C}(13)-\mathrm{C}(15)-\mathrm{H}(15 \mathrm{~A})$ & 109.5 \\
\hline $\mathrm{C}(1)-\mathrm{C}(2)-\mathrm{C}(3)$ & $119.2(7)$ & $\mathrm{C}(13)-\mathrm{C}(15)-\mathrm{H}(15 \mathrm{~B})$ & 109.5 \\
\hline$C(2)-C(3)-C(4)$ & $119.0(8)$ & $\mathrm{H}(15 \mathrm{~A})-\mathrm{C}(15)-\mathrm{H}(15 \mathrm{~B})$ & 109.5 \\
\hline $\mathrm{C}(2)-\mathrm{C}(3)-\mathrm{H}(3 \mathrm{~A})$ & 120.5 & $\mathrm{C}(13)-\mathrm{C}(15)-\mathrm{H}(15 \mathrm{C})$ & 109.5 \\
\hline $\mathrm{C}(4)-\mathrm{C}(3)-\mathrm{H}(3 \mathrm{~A})$ & 120.5 & $\mathrm{H}(15 \mathrm{~A})-\mathrm{C}(15)-\mathrm{H}(15 \mathrm{C})$ & 109.5 \\
\hline $\mathrm{C}(5)-\mathrm{C}(4)-\mathrm{C}(3)$ & $120.2(8)$ & $\mathrm{H}(15 \mathrm{~B})-\mathrm{C}(15)-\mathrm{H}(15 \mathrm{C})$ & 109.5 \\
\hline $\mathrm{C}(5)-\mathrm{C}(4)-\mathrm{H}(4 \mathrm{~A})$ & 119.9 & $\mathrm{C}(13)-\mathrm{C}(16)-\mathrm{H}(16 \mathrm{~A})$ & 109.5 \\
\hline $\mathrm{C}(3)-\mathrm{C}(4)-\mathrm{H}(4 \mathrm{~A})$ & 119.9 & $\mathrm{C}(13)-\mathrm{C}(16)-\mathrm{H}(16 \mathrm{~B})$ & 109.5 \\
\hline$C(4)-C(5)-C(6)$ & $120.3(8)$ & $\mathrm{H}(16 \mathrm{~A})-\mathrm{C}(16)-\mathrm{H}(16 \mathrm{~B})$ & 109.5 \\
\hline $\mathrm{C}(4)-\mathrm{C}(5)-\mathrm{H}(5 \mathrm{~A})$ & 119.8 & $\mathrm{C}(13)-\mathrm{C}(16)-\mathrm{H}(16 \mathrm{C})$ & 109.5 \\
\hline $\mathrm{C}(6)-\mathrm{C}(5)-\mathrm{H}(5 \mathrm{~A})$ & 119.8 & $\mathrm{H}(16 \mathrm{~A})-\mathrm{C}(16)-\mathrm{H}(16 \mathrm{C})$ & 109.5 \\
\hline$C(1)-C(6)-C(5)$ & $120.7(7)$ & $\mathrm{H}(16 \mathrm{~B})-\mathrm{C}(16)-\mathrm{H}(16 \mathrm{C})$ & 109.5 \\
\hline$C(1)-C(6)-H(6 A)$ & 119.6 & $\mathrm{C}(17)-\mathrm{N}(3)-\mathrm{Fe}(1)$ & $180.000(2)$ \\
\hline $\mathrm{C}(5)-\mathrm{C}(6)-\mathrm{H}(6 \mathrm{~A})$ & 119.6 & $\mathrm{~N}(3)-\mathrm{C}(17)-\mathrm{C}(18)$ & $180.000(3)$ \\
\hline C(8)-C(7)-C(12) & $116.1(8)$ & $\mathrm{C}(17)-\mathrm{C}(18)-\mathrm{H}(18 \mathrm{~A})$ & 109.5 \\
\hline $\mathrm{C}(8)-\mathrm{C}(7)-\mathrm{N}(2)$ & $121.5(9)$ & $\mathrm{C}(17)-\mathrm{C}(18)-\mathrm{H}(18 \mathrm{~B})$ & 109.5 \\
\hline $\mathrm{C}(12)-\mathrm{C}(7)-\mathrm{N}(2)$ & $122.3(9)$ & $\mathrm{H}(18 \mathrm{~A})-\mathrm{C}(18)-\mathrm{H}(18 \mathrm{~B})$ & 109.5 \\
\hline $\mathrm{C}(9)-\mathrm{C}(8)-\mathrm{C}(7)$ & $122.4(8)$ & $\mathrm{C}(17)-\mathrm{C}(18)-\mathrm{H}(18 \mathrm{C})$ & 109.5 \\
\hline $\mathrm{C}(9)-\mathrm{C}(8)-\mathrm{H}(8 \mathrm{~A})$ & 118.8 & $\mathrm{H}(18 \mathrm{~A})-\mathrm{C}(18)-\mathrm{H}(18 \mathrm{C})$ & 109.5 \\
\hline $\mathrm{C}(7)-\mathrm{C}(8)-\mathrm{H}(8 \mathrm{~A})$ & 118.8 & $\mathrm{H}(18 \mathrm{~B})-\mathrm{C}(18)-\mathrm{H}(18 \mathrm{C})$ & 109.5 \\
\hline
\end{tabular}


Symmetry transformations used to generate equivalent atoms:

\#1 -y, x-y, z \#2-x+y, -x, z 
Table S46. Anisotropic displacement parameters $\left(\AA^{2} \times 10^{3}\right)$ for $\left[\left(\mathrm{L}^{1}\right) \mathrm{Fe}(\mathrm{III})-\mathrm{MeCN}\right](6)$. The anisotropic displacement factor exponent takes the form $-2 \pi^{2}\left[\mathrm{~h}^{2} \mathrm{a}^{*^{2}} \mathrm{U}^{11}+\ldots+2 \mathrm{hk}\right.$ $\left.a^{*} b^{*} U^{12}\right]$

\begin{tabular}{|c|c|c|c|c|c|c|}
\hline & $\mathrm{U}^{11}$ & $\mathrm{U}^{22}$ & $\mathrm{U}^{33}$ & $\mathrm{U}^{23}$ & $\mathrm{U}^{13}$ & $\mathrm{U}^{12}$ \\
\hline $\mathrm{Fe}(1)$ & $124(1)$ & $124(1)$ & $76(2)$ & 0 & 0 & $62(1)$ \\
\hline $\mathrm{N}(1)$ & $71(4)$ & $71(4)$ & $56(6)$ & 0 & 0 & $35(2)$ \\
\hline $\mathrm{N}(2)$ & $88(5)$ & $95(5)$ & $60(4)$ & $-16(4)$ & $5(4)$ & $20(4)$ \\
\hline$C(1)$ & $71(5)$ & $79(5)$ & $43(4)$ & $1(4)$ & $-1(4)$ & $35(5)$ \\
\hline$C(2)$ & $71(6)$ & $82(6)$ & $63(5)$ & $5(5)$ & $3(5)$ & $41(5)$ \\
\hline$C(3)$ & $87(7)$ & $100(7)$ & $74(6)$ & $2(5)$ & $1(5)$ & $56(6)$ \\
\hline$C(4)$ & $76(6)$ & $102(7)$ & $105(7)$ & $27(6)$ & $10(6)$ & $40(6)$ \\
\hline$C(5)$ & 127(9) & $118(8)$ & $54(5)$ & $1(6)$ & $8(6)$ & $61(7)$ \\
\hline$C(6)$ & $76(6)$ & $88(6)$ & $61(5)$ & $3(4)$ & $-2(5)$ & $31(5)$ \\
\hline$C(7)$ & $86(7)$ & $93(7)$ & $63(5)$ & $-2(5)$ & $0(5)$ & $38(6)$ \\
\hline$C(8)$ & 118(8) & $95(7)$ & $96(6)$ & $-25(6)$ & $-6(6)$ & $65(6)$ \\
\hline$C(9)$ & $101(7)$ & $106(7)$ & $81(6)$ & $-18(5)$ & $-21(5)$ & $56(6)$ \\
\hline$C(10)$ & $78(6)$ & $98(7)$ & $75(6)$ & $-26(5)$ & $-7(5)$ & $49(5)$ \\
\hline$C(11)$ & $135(9)$ & $116(8)$ & $117(7)$ & $-50(6)$ & $-49(7)$ & $94(7)$ \\
\hline$C(12)$ & $111(8)$ & $119(8)$ & $104(7)$ & $-21(6)$ & $-38(6)$ & $77(7)$ \\
\hline$C(13)$ & $99(8)$ & $126(8)$ & $109(8)$ & $-59(7)$ & $-26(6)$ & $56(7)$ \\
\hline$C(14)$ & $203(13)$ & $243(14)$ & $190(11)$ & $-150(10)$ & $-81(10)$ & 161(12) \\
\hline$C(15)$ & $90(8)$ & $196(12)$ & $161(10)$ & $-83(8)$ & $-33(7)$ & $42(8)$ \\
\hline
\end{tabular}




\begin{tabular}{lcccccc}
$\mathrm{C}(16)$ & $176(11)$ & $243(15)$ & $84(7)$ & $-26(8)$ & $4(7)$ & $73(11)$ \\
$\mathrm{N}(3)$ & $167(9)$ & $167(9)$ & $127(12)$ & 0 & 0 & $83(5)$ \\
$\mathrm{C}(17)$ & $350(30)$ & $350(30)$ & $150(20)$ & 0 & 0 & $176(15)$ \\
$\mathrm{C}(18)$ & $520(40)$ & $520(40)$ & $64(14)$ & 0 & 0 & $260(20)$ \\
\hline
\end{tabular}


Table S47. Hydrogen coordinates $\left(\times 10^{4}\right)$ and isotropic displacement parameters $\left(\AA^{2} \times 10^{3}\right)$ for $\left[\left(\mathrm{L}^{1}\right) \mathrm{Fe}(\mathrm{III})-\mathrm{MeCN}\right](\mathbf{6})$

\begin{tabular}{|c|c|c|c|c|}
\hline & $\mathrm{x}$ & $\mathrm{y}$ & $\mathrm{Z}$ & $\mathrm{U}(\mathrm{eq})$ \\
\hline $\mathrm{H}(3 \mathrm{~A})$ & 2992 & 2884 & 1167 & 100 \\
\hline $\mathrm{H}(4 \mathrm{~A})$ & 3721 & 2935 & 593 & 116 \\
\hline $\mathrm{H}(5 \mathrm{~A})$ & 2795 & 1651 & 175 & 119 \\
\hline $\mathrm{H}(6 \mathrm{~A})$ & 1149 & 294 & 315 & 95 \\
\hline $\mathrm{H}(8 \mathrm{~A})$ & 2561 & 1596 & 1859 & 118 \\
\hline $\mathrm{H}(9 \mathrm{~A})$ & 3306 & 2656 & 2353 & 113 \\
\hline $\mathrm{H}(11 \mathrm{~A})$ & 1716 & 4086 & 2140 & 130 \\
\hline $\mathrm{H}(12 \mathrm{~A})$ & 976 & 3059 & 1641 & 123 \\
\hline $\mathrm{H}(14 \mathrm{~A})$ & 2941 & 5233 & 2953 & 285 \\
\hline $\mathrm{H}(14 \mathrm{~B})$ & 1879 & 4438 & 2764 & 285 \\
\hline $\mathrm{H}(14 \mathrm{C})$ & 2793 & 5315 & 2533 & 285 \\
\hline $\mathrm{H}(15 \mathrm{~A})$ & 4472 & 5154 & 2448 & 243 \\
\hline $\mathrm{H}(15 \mathrm{~B})$ & 4422 & 4132 & 2599 & 243 \\
\hline $\mathrm{H}(15 \mathrm{C})$ & 4514 & 4999 & 2870 & 243 \\
\hline $\mathrm{H}(16 \mathrm{~A})$ & 2964 & 3702 & 3219 & 272 \\
\hline $\mathrm{H}(16 \mathrm{~B})$ & 2913 & 2828 & 2961 & 272 \\
\hline $\mathrm{H}(16 \mathrm{C})$ & 1921 & 2950 & 3012 & 272 \\
\hline $\mathrm{H}(18 \mathrm{~A})$ & 706 & 285 & 2896 & 554 \\
\hline $\mathrm{H}(18 \mathrm{~B})$ & -421 & -706 & 2896 & 554 \\
\hline
\end{tabular}


$\mathrm{H}(18 \mathrm{C})$

$-285$

421

2896

554 
Table S48. Torsion Angles, ${ }^{\circ}$, for $\left[\left(\mathrm{L}^{1}\right) \mathrm{Fe}(\mathrm{III})-\mathrm{MeCN}\right](\mathbf{6})$

\begin{tabular}{|c|c|c|c|}
\hline $\mathrm{N}(2)-\mathrm{Fe}(1)-\mathrm{N}(1)-\mathrm{C}(1)$ & $26.5(3)$ & $\mathrm{N}(1)-\mathrm{C}(1)-\mathrm{C}(2)-\mathrm{C}(3)$ & $-176.6(6)$ \\
\hline $\mathrm{N}(2) \# 1-\mathrm{Fe}(1)-\mathrm{N}(1)-\mathrm{C}(1)$ & $146.5(3)$ & $\mathrm{N}(2)-\mathrm{C}(2)-\mathrm{C}(3)-\mathrm{C}(4)$ & $177.8(7)$ \\
\hline $\mathrm{N}(2) \# 2-\mathrm{Fe}(1)-\mathrm{N}(1)-\mathrm{C}(1)$ & $-93.5(3)$ & $C(1)-C(2)-C(3)-C(4)$ & $0.2(11)$ \\
\hline $\mathrm{N}(3)-\mathrm{Fe}(1)-\mathrm{N}(1)-\mathrm{C}(1)$ & $0(39)$ & $C(2)-C(3)-C(4)-C(5)$ & $-0.7(12)$ \\
\hline $\mathrm{N}(2)-\mathrm{Fe}(1)-\mathrm{N}(1)-\mathrm{C}(1) \# 2$ & $146.5(3)$ & $C(3)-C(4)-C(5)-C(6)$ & $-0.1(13)$ \\
\hline $\mathrm{N}(2) \# 1-\mathrm{Fe}(1)-\mathrm{N}(1)-\mathrm{C}(1) \# 2$ & $-93.5(3)$ & $C(2)-C(1)-C(6)-C(5)$ & $-2.0(12)$ \\
\hline $\mathrm{N}(2) \# 2-\mathrm{Fe}(1)-\mathrm{N}(1)-\mathrm{C}(1) \# 2$ & $26.5(3)$ & $\mathrm{N}(1)-\mathrm{C}(1)-\mathrm{C}(6)-\mathrm{C}(5)$ & $175.6(7)$ \\
\hline $\mathrm{N}(3)-\mathrm{Fe}(1)-\mathrm{N}(1)-\mathrm{C}(1) \# 2$ & $0(100)$ & $C(4)-C(5)-C(6)-C(1)$ & $1.5(13)$ \\
\hline $\mathrm{N}(2)-\mathrm{Fe}(1)-\mathrm{N}(1)-\mathrm{C}(1) \# 1$ & $-93.5(3)$ & $\mathrm{C}(2)-\mathrm{N}(2)-\mathrm{C}(7)-\mathrm{C}(8)$ & $-81.1(10)$ \\
\hline $\mathrm{N}(2) \# 1-\mathrm{Fe}(1)-\mathrm{N}(1)-\mathrm{C}(1) \# 1$ & $26.5(3)$ & $\mathrm{Fe}(1)-\mathrm{N}(2)-\mathrm{C}(7)-\mathrm{C}(8)$ & $73.9(9)$ \\
\hline $\mathrm{N}(2) \# 2-\mathrm{Fe}(1)-\mathrm{N}(1)-\mathrm{C}(1) \# 1$ & $146.5(3)$ & $\mathrm{C}(2)-\mathrm{N}(2)-\mathrm{C}(7)-\mathrm{C}(12)$ & $102.3(10)$ \\
\hline $\mathrm{N}(3)-\mathrm{Fe}(1)-\mathrm{N}(1)-\mathrm{C}(1) \# 1$ & $0(100)$ & $\mathrm{Fe}(1)-\mathrm{N}(2)-\mathrm{C}(7)-\mathrm{C}(12)$ & $-102.7(8)$ \\
\hline $\mathrm{N}(2) \# 1-\mathrm{Fe}(1)-\mathrm{N}(2)-\mathrm{C}(2)$ & $-96.1(5)$ & $\mathrm{C}(12)-\mathrm{C}(7)-\mathrm{C}(8)-\mathrm{C}(9)$ & $2.7(12)$ \\
\hline $\mathrm{N}(2) \# 2-\mathrm{Fe}(1)-\mathrm{N}(2)-\mathrm{C}(2)$ & $43.2(6)$ & $\mathrm{N}(2)-\mathrm{C}(7)-\mathrm{C}(8)-\mathrm{C}(9)$ & $-174.0(7)$ \\
\hline $\mathrm{N}(3)-\mathrm{Fe}(1)-\mathrm{N}(2)-\mathrm{C}(2)$ & $153.6(5)$ & $C(7)-C(8)-C(9)-C(10)$ & $0.5(13)$ \\
\hline $\mathrm{N}(1)-\mathrm{Fe}(1)-\mathrm{N}(2)-\mathrm{C}(2)$ & $-26.4(5)$ & $C(8)-C(9)-C(10)-C(11)$ & $-3.4(13)$ \\
\hline $\mathrm{N}(2) \# 1-\mathrm{Fe}(1)-\mathrm{N}(2)-\mathrm{C}(7)$ & $107.8(7)$ & $C(8)-C(9)-C(10)-C(13)$ & $177.0(8)$ \\
\hline $\mathrm{N}(2) \# 2-\mathrm{Fe}(1)-\mathrm{N}(2)-\mathrm{C}(7)$ & $-112.9(7)$ & $\mathrm{C}(9)-\mathrm{C}(10)-\mathrm{C}(11)-\mathrm{C}(12)$ & $2.9(14)$ \\
\hline $\mathrm{N}(3)-\mathrm{Fe}(1)-\mathrm{N}(2)-\mathrm{C}(7)$ & $-2.5(7)$ & $\mathrm{C}(13)-\mathrm{C}(10)-\mathrm{C}(11)-\mathrm{C}(12)$ & $-177.4(9)$ \\
\hline $\mathrm{N}(1)-\mathrm{Fe}(1)-\mathrm{N}(2)-\mathrm{C}(7)$ & $177.5(7)$ & $\mathrm{C}(10)-\mathrm{C}(11)-\mathrm{C}(12)-\mathrm{C}(7)$ & $0.4(15)$ \\
\hline $\mathrm{C}(1) \# 2-\mathrm{N}(1)-\mathrm{C}(1)-\mathrm{C}(6)$ & $46.4(10)$ & $\mathrm{C}(8)-\mathrm{C}(7)-\mathrm{C}(12)-\mathrm{C}(11)$ & $-3.2(13)$ \\
\hline
\end{tabular}




$\begin{array}{lclr}\mathrm{C}(1) \# 1-\mathrm{N}(1)-\mathrm{C}(1)-\mathrm{C}(6) & -90.3(8) & \mathrm{N}(2)-\mathrm{C}(7)-\mathrm{C}(12)-\mathrm{C}(11) & 173.6(7) \\ \mathrm{Fe}(1)-\mathrm{N}(1)-\mathrm{C}(1)-\mathrm{C}(6) & 158.0(6) & \mathrm{C}(11)-\mathrm{C}(10)-\mathrm{C}(13)-\mathrm{C}(14) & 3.4(14) \\ \mathrm{C}(1) \# 2-\mathrm{N}(1)-\mathrm{C}(1)-\mathrm{C}(2) & -136.0(8) & \mathrm{C}(9)-\mathrm{C}(10)-\mathrm{C}(13)-\mathrm{C}(14) & -176.9(9) \\ \mathrm{C}(1) \# 1-\mathrm{N}(1)-\mathrm{C}(1)-\mathrm{C}(2) & 87.4(10) & \mathrm{C}(11)-\mathrm{C}(10)-\mathrm{C}(13)-\mathrm{C}(15) & -124.6(10) \\ \mathrm{Fe}(1)-\mathrm{N}(1)-\mathrm{C}(1)-\mathrm{C}(2) & -24.3(6) & \mathrm{C}(9)-\mathrm{C}(10)-\mathrm{C}(13)-\mathrm{C}(15) & 55.1(12) \\ \mathrm{C}(7)-\mathrm{N}(2)-\mathrm{C}(2)-\mathrm{C}(1) & 177.3(7) & \mathrm{C}(11)-\mathrm{C}(10)-\mathrm{C}(13)-\mathrm{C}(16) & 118.9(10) \\ \mathrm{Fe}(1)-\mathrm{N}(2)-\mathrm{C}(2)-\mathrm{C}(1) & 21.2(8) & \mathrm{C}(9)-\mathrm{C}(10)-\mathrm{C}(13)-\mathrm{C}(16) & -61.4(11) \\ \mathrm{C}(7)-\mathrm{N}(2)-\mathrm{C}(2)-\mathrm{C}(3) & -0.3(12) & \mathrm{N}(2)-\mathrm{Fe}(1)-\mathrm{N}(3)-\mathrm{C}(17) & 0(100) \\ \mathrm{Fe}(1)-\mathrm{N}(2)-\mathrm{C}(2)-\mathrm{C}(3) & -156.4(6) & \mathrm{N}(2) \# 1-\mathrm{Fe}(1)-\mathrm{N}(3)-\mathrm{C}(17) & 0(100) \\ \mathrm{C}(6)-\mathrm{C}(1)-\mathrm{C}(2)-\mathrm{N}(2) & -176.6(6) & \mathrm{N}(2) \# 2-\mathrm{Fe}(1)-\mathrm{N}(3)-\mathrm{C}(17) & 0(100) \\ \mathrm{N}(1)-\mathrm{C}(1)-\mathrm{C}(2)-\mathrm{N}(2) & 5.7(9) & \mathrm{N}(1)-\mathrm{Fe}(1)-\mathrm{N}(3)-\mathrm{C}(17) & 0(100) \\ \mathrm{C}(6)-\mathrm{C}(1)-\mathrm{C}(2)-\mathrm{C}(3) & 1.1(11) & \mathrm{Fe}(1)-\mathrm{N}(3)-\mathrm{C}(17)-\mathrm{C}(18) & 0(100)\end{array}$

Symmetry transformations used to generate equivalent atoms:

$\# 1-y, x-y, z \quad \# 2-x+y,-x, z$ 
Table S49. Atomic coordinates $\left(\times 10^{4}\right)$ and equivalent isotropic displacement parameters $\left(\AA^{2} \times 10^{3}\right)$ for $\left[\left(\mathrm{L}^{1}{ }_{\mathrm{re}}\right)(\mathrm{Cl}) \mathrm{Fe}(\mathrm{II})\right]\left[\mathrm{Et}_{4} \mathrm{~N}\right]_{2}(7) . \mathrm{U}(\mathrm{eq})$ is defined as one third of the trace of the orthogonalized $\mathrm{U}^{\mathrm{ij}}$ tensor

\begin{tabular}{|c|c|c|c|c|}
\hline & $\mathrm{x}$ & $\mathrm{y}$ & $\mathrm{Z}$ & $\mathrm{U}(\mathrm{eq})$ \\
\hline $\mathrm{Fe}(1)$ & $581(2)$ & 2592(1) & $1309(1)$ & $43(1)$ \\
\hline $\mathrm{Cl}(1)$ & $2366(3)$ & $2410(1)$ & $772(2)$ & $59(1)$ \\
\hline $\mathrm{N}(1)$ & $-238(9)$ & $3109(3)$ & $772(5)$ & $42(3)$ \\
\hline $\mathrm{N}(2)$ & $-992(9)$ & 2282(3) & $877(5)$ & $46(3)$ \\
\hline $\mathrm{N}(3)$ & $304(9)$ & $1825(3)$ & $1861(5)$ & $42(3)$ \\
\hline $\mathrm{N}(4)$ & $689(9)$ & $2670(3)$ & $2304(5)$ & $44(3)$ \\
\hline $\mathrm{C}(1)$ & $-1336(12)$ & $3020(4)$ & $476(6)$ & $52(2)$ \\
\hline$C(2)$ & $-1771(11)$ & $2565(5)$ & $487(6)$ & $54(2)$ \\
\hline$C(3)$ & $-2992(11)$ & $2437(5)$ & $165(6)$ & $59(2)$ \\
\hline $\mathrm{C}(4)$ & $-3714(14)$ & $2727(5)$ & $-199(7)$ & $63(3)$ \\
\hline$C(5)$ & $-3324(13)$ & $3156(5)$ & $-227(7)$ & $63(3)$ \\
\hline$C(6)$ & $-2153(12)$ & $3307(4)$ & $85(6)$ & $59(2)$ \\
\hline $\mathrm{C}(7)$ & $-1010(10)$ & $1846(5)$ & $835(6)$ & $47(2)$ \\
\hline$C(8)$ & $-380(11)$ & $1602(4)$ & $1333(7)$ & $47(2)$ \\
\hline$C(9)$ & $-406(11)$ & $1175(4)$ & $1323(7)$ & $55(2)$ \\
\hline$C(10)$ & $-998(12)$ & $962(5)$ & $802(7)$ & $59(2)$ \\
\hline $\mathrm{C}(11)$ & $-1583(12)$ & 1173(4) & $287(7)$ & $58(2)$ \\
\hline$C(12)$ & $-1599(11)$ & $1620(4)$ & $269(7)$ & $56(2)$ \\
\hline
\end{tabular}




\begin{tabular}{|c|c|c|c|c|}
\hline$C(13)$ & $-442(11)$ & $1982(4)$ & $2371(6)$ & $42(2)$ \\
\hline$C(14)$ & $-135(11)$ & $2412(4)$ & $2651(6)$ & $42(2)$ \\
\hline$C(15)$ & $-771(10)$ & $2533(4)$ & $3223(6)$ & $45(2)$ \\
\hline$C(16)$ & $-1641(11)$ & 2291(4) & $3482(6)$ & $46(2)$ \\
\hline$C(17)$ & $-1879(10)$ & $1885(4)$ & $3228(6)$ & $48(2)$ \\
\hline $\mathrm{C}(18)$ & $-1317(10)$ & $1724(4)$ & $2673(6)$ & $45(2)$ \\
\hline C(19) & $209(15)$ & $3523(5)$ & $800(7)$ & $68(3)$ \\
\hline $\mathrm{C}(20)$ & $1414(14)$ & $3590(5)$ & $720(7)$ & $70(3)$ \\
\hline $\mathrm{C}(21)$ & $1985(15)$ & $3987(5)$ & $778(7)$ & $78(3)$ \\
\hline$C(22)$ & $1308(16)$ & $4345(5)$ & $895(8)$ & $88(2)$ \\
\hline $\mathrm{C}(23)$ & $-6(15)$ & $4275(5)$ & $967(8)$ & $85(3)$ \\
\hline $\mathrm{C}(24)$ & $-516(14)$ & $3872(5)$ & $956(7)$ & $75(3)$ \\
\hline$C(25)$ & $1799(17)$ & $4812(6)$ & $973(10)$ & $101(3)$ \\
\hline$C(26)$ & $2568(17)$ & $4830(5)$ & $1623(9)$ & $131(5)$ \\
\hline $\mathrm{C}(27)$ & $980(16)$ & $5154(5)$ & 994(9) & $117(5)$ \\
\hline $\mathrm{C}(28)$ & $2483(16)$ & $4926(5)$ & $416(9)$ & $117(5)$ \\
\hline C(29) & $1426(11)$ & $1623(4)$ & $2115(7)$ & $48(2)$ \\
\hline$C(30)$ & $2222(11)$ & $1456(4)$ & $1699(7)$ & $51(2)$ \\
\hline$C(31)$ & $3279(12)$ & $1240(4)$ & $1940(7)$ & $55(2)$ \\
\hline$C(32)$ & $3660(13)$ & $1204(4)$ & $2611(8)$ & $58(2)$ \\
\hline$C(33)$ & $2793(12)$ & $1402(4)$ & $3049(7)$ & $58(2)$ \\
\hline$C(34)$ & $1744(11)$ & $1618(4)$ & $2805(7)$ & $54(2)$ \\
\hline
\end{tabular}




\begin{tabular}{|c|c|c|c|c|}
\hline$C(35)$ & $4756(14)$ & $941(5)$ & 2904(8) & $69(3$ \\
\hline$C(36)$ & $5853(12)$ & 1011(5) & $2502(8)$ & $80(4$ \\
\hline$C(37)$ & $4319(13)$ & $458(4)$ & $2859(8)$ & $89(4$ \\
\hline$C(38)$ & $5146(13)$ & 1013(5) & $3628(8)$ & $87(4$ \\
\hline$C(39)$ & $1327(12)$ & 2993(4) & $2684(6)$ & $48(2$ \\
\hline$C(40)$ & $1482(11)$ & $3384(4)$ & $2396(7)$ & $54(2$ \\
\hline$C(41)$ & 2141(11) & $3686(4)$ & 2791(7) & $59(2$ \\
\hline$C(42)$ & 2741(12) & $3600(4)$ & $3427(7)$ & $59(2$ \\
\hline$C(43)$ & $2621(11)$ & $3206(4)$ & $3648(7)$ & $55(2$ \\
\hline$C(44)$ & $1896(11)$ & 2906(4) & $3318(6)$ & $49(2$ \\
\hline$C(45)$ & $3476(14)$ & $3948(5)$ & $3815(8)$ & $73(3$ \\
\hline$C(46)$ & 4791(13) & $3776(5)$ & 4013(8) & $95(4$ \\
\hline$C(47)$ & 2813(14) & $4069(5)$ & $4411(8)$ & $101(4$ \\
\hline$C(48)$ & $3670(14)$ & $4337(4)$ & $3424(8)$ & $95(4$ \\
\hline $\mathrm{Fe}(1 \mathrm{~A})$ & $6941(2)$ & 6841(1) & $3142(1)$ & $42(1$ \\
\hline $\mathrm{Cl}(1 \mathrm{~A})$ & $8721(3)$ & 7011(1) & $2589(2)$ & $60(1$ \\
\hline $\mathrm{N}(1 \mathrm{~A})$ & $6133(10)$ & $6343(3)$ & $2612(5)$ & $55(3$ \\
\hline $\mathrm{N}(2 \mathrm{~A})$ & $5382(9)$ & $7128(4)$ & $2700(5)$ & $48(3$ \\
\hline $\mathrm{N}(3 \mathrm{~A})$ & $6654(8)$ & 7592(4) & $3714(5)$ & $42(3$ \\
\hline $\mathrm{N}(4 \mathrm{~A})$ & $7010(9)$ & $6772(3)$ & $4150(5)$ & $41(3$ \\
\hline$C(1 \mathrm{~A})$ & $4942(12)$ & $6444(4)$ & $2286(7)$ & $57(2$ \\
\hline $\mathrm{C}(2 \mathrm{~A})$ & $4591(11)$ & $6863(5)$ & $2295(6)$ & $54(2$ \\
\hline
\end{tabular}




\begin{tabular}{|c|c|c|c|c|}
\hline$C(3 A)$ & $3477(11)$ & $6960(4)$ & $1972(6)$ & $61(2)$ \\
\hline $\mathrm{C}(4 \mathrm{~A})$ & 2704(13) & $6656(5)$ & $1630(7)$ & $64(3)$ \\
\hline$C(5 A)$ & $3017(13)$ & $6281(5)$ & $1595(7)$ & $65(3)$ \\
\hline$C(6 A)$ & $4158(12)$ & $6157(4)$ & $1911(7)$ & $62(3)$ \\
\hline$C(7 A)$ & $5338(10)$ & $7568(5)$ & $2659(6)$ & $43(2)$ \\
\hline $\mathrm{C}(8 \mathrm{~A})$ & $5949(11)$ & $7809(4)$ & $3173(7)$ & $45(2)$ \\
\hline$C(9 A)$ & $5915(11)$ & $8255(4)$ & $3163(7)$ & $54(2)$ \\
\hline$C(10 A)$ & $5353(12)$ & $8484(4)$ & $2636(7)$ & $56(2)$ \\
\hline$C(11 A)$ & $4784(12)$ & $8230(4)$ & $2096(7)$ & $55(2)$ \\
\hline$C(12 A)$ & $4815(11)$ & $7802(4)$ & $2126(7)$ & $50(2)$ \\
\hline$C(13 A)$ & $5907(11)$ & $7433(4)$ & $4240(6)$ & $44(2)$ \\
\hline $\mathrm{C}(14 \mathrm{~A})$ & $6205(11)$ & $7036(4)$ & $4468(6)$ & $46(2)$ \\
\hline$C(15 A)$ & $5600(10)$ & $6887(5)$ & $5031(6)$ & $50(2)$ \\
\hline$C(16 A)$ & $4791(11)$ & $7178(4)$ & $5309(7)$ & $53(2)$ \\
\hline$C(17 A)$ & $4499(10)$ & $7573(5)$ & $5042(6)$ & $52(2)$ \\
\hline $\mathrm{C}(18 \mathrm{~A})$ & $5086(10)$ & $7679(4)$ & $4534(6)$ & $48(2)$ \\
\hline$C(19 A)$ & $6569(12)$ & $5922(4)$ & $2664(7)$ & $54(2)$ \\
\hline$C(20 A)$ & $7822(12)$ & $5824(4)$ & $2568(6)$ & $54(2)$ \\
\hline$C(21 A)$ & $8293(13)$ & $5426(4)$ & $2634(7)$ & $60(2)$ \\
\hline $\mathrm{C}(22 \mathrm{~A})$ & $7647(14)$ & $5081(5)$ & $2816(8)$ & $72(2)$ \\
\hline$C(23 A)$ & $6392(13)$ & $5160(4)$ & $2939(7)$ & $65(2)$ \\
\hline$C(24 A)$ & $5912(13)$ & $5557(4)$ & $2831(7)$ & $59(2)$ \\
\hline
\end{tabular}




\begin{tabular}{|c|c|c|c|c|}
\hline$C(25 \mathrm{~A})$ & $8244(17)$ & $4632(5)$ & 2987(9) & $94(3)$ \\
\hline$C(26 A)$ & $7674(17)$ & $4341(6)$ & $3384(10)$ & $135(5)$ \\
\hline$C(27 A)$ & $9403(16)$ & $4600(5)$ & $2877(10)$ & $131(5)$ \\
\hline $\mathrm{C}(28 \mathrm{~A})$ & $7760(40)$ & $4320(9)$ & $2251(13)$ & $330(20)$ \\
\hline$C(29 A)$ & $7818(11)$ & $7790(4)$ & $3988(7)$ & $43(2)$ \\
\hline$C(30 A)$ & 8593(11) & 7964(4) & $3556(7)$ & $47(2)$ \\
\hline$C(31 \mathrm{~A})$ & $9637(12)$ & $8196(4)$ & $3811(7)$ & $49(2)$ \\
\hline$C(32 A)$ & $9943(12)$ & $8240(4)$ & $4487(7)$ & $51(2)$ \\
\hline$C(33 A)$ & $9249(11)$ & $8021(4)$ & $4888(7)$ & $47(2)$ \\
\hline$C(34 A)$ & $8168(10)$ & $7817(4)$ & $4635(6)$ & $45(2)$ \\
\hline$C(35 \mathrm{~A})$ & $11115(12)$ & $8504(4)$ & $4710(7)$ & $59(2)$ \\
\hline$C(36 A)$ & $12232(12)$ & $8414(5)$ & $4310(7)$ & $74(4)$ \\
\hline$C(37 A)$ & 10747(13) & $8978(4)$ & $4628(8)$ & $82(4)$ \\
\hline $\mathrm{C}(38 \mathrm{~A})$ & $11503(13)$ & $8372(5)$ & $5456(7)$ & $76(4)$ \\
\hline$C(39 A)$ & $7717(12)$ & $6472(4)$ & $4541(7)$ & $55(2)$ \\
\hline $\mathrm{C}(40 \mathrm{~A})$ & $8010(12)$ & $6070(4)$ & $4297(7)$ & $61(2)$ \\
\hline$C(41 \mathrm{~A})$ & $8773(12)$ & $5764(4)$ & $4611(8)$ & $66(2)$ \\
\hline $\mathrm{C}(42 \mathrm{~A})$ & $9295(14)$ & $5833(5)$ & $5238(8)$ & $74(2)$ \\
\hline$C(43 A)$ & $9027(12)$ & $6255(4)$ & $5519(8)$ & $66(2)$ \\
\hline $\mathrm{C}(44 \mathrm{~A})$ & $8309(12)$ & $6550(4)$ & $5166(7)$ & $58(2)$ \\
\hline$C(45 A)$ & $10077(17)$ & $5535(6)$ & $5660(10)$ & $97(3)$ \\
\hline$C(46 A)$ & $10946(17)$ & $5299(6)$ & $5267(10)$ & $137(5)$ \\
\hline
\end{tabular}




\begin{tabular}{|c|c|c|c|c|}
\hline $\mathrm{C}(47 \mathrm{~A})$ & $9463(16)$ & $5232(6)$ & $5930(10)$ & $137(5)$ \\
\hline$C(48 \mathrm{~A})$ & $11113(17)$ & $5688(6)$ & 6099(9) & $134(5)$ \\
\hline $\mathrm{N}(7)$ & $2028(10)$ & $6639(4)$ & $3798(6)$ & $58(3)$ \\
\hline$C(65)$ & $2809(14)$ & $6352(5)$ & $4272(8)$ & $82(4)$ \\
\hline$C(66)$ & $3906(14)$ & 6171(5) & $3978(8)$ & $88(5)$ \\
\hline$C(67)$ & $1670(13)$ & $6429(5)$ & $3136(8)$ & $81(4)$ \\
\hline $\mathrm{C}(68)$ & $1052(14)$ & $5997(5)$ & $3180(8)$ & $90(5)$ \\
\hline $\mathrm{C}(69)$ & $816(12)$ & $6753(5)$ & $4116(7)$ & $68(4)$ \\
\hline $\mathrm{C}(70)$ & 1086(13) & $6960(5)$ & $4792(7)$ & $81(4)$ \\
\hline $\mathrm{C}(71)$ & $2807(12)$ & 7019(4) & $3687(7)$ & $58(4)$ \\
\hline $\mathrm{C}(72)$ & $2117(13)$ & $7350(5)$ & $3317(8)$ & $74(4)$ \\
\hline $\mathrm{N}(5)$ & $2958(11)$ & 1241(4) & $9498(6)$ & $72(4)$ \\
\hline $\mathrm{C}(49)$ & $3706(14)$ & $1263(6)$ & 10159(8) & $98(5)$ \\
\hline$C(50)$ & $5108(14)$ & $1138(6)$ & 10126(8) & $105(5)$ \\
\hline $\mathrm{C}(51)$ & $3483(16)$ & $1504(5)$ & $8935(9)$ & $91(5)$ \\
\hline $\mathrm{C}(52)$ & $3675(16)$ & $1947(5)$ & $9142(8)$ & $110(5)$ \\
\hline$C(53)$ & $1587(14)$ & $1376(5)$ & $9636(8)$ & $82(4)$ \\
\hline$C(54)$ & $645(14)$ & $1346(5)$ & $8999(8)$ & $92(5)$ \\
\hline$C(55)$ & $3110(20)$ & $766(6)$ & 9246(9) & $145(7)$ \\
\hline$C(56)$ & $2660(20)$ & $402(5)$ & 9701(9) & $155(7)$ \\
\hline $\mathrm{N}(6)$ & $5680(9)$ & $2808(3)$ & $1998(5)$ & $45(3)$ \\
\hline$C(57)$ & $6543(12)$ & $3082(4)$ & $2419(7)$ & $61(4)$ \\
\hline
\end{tabular}




\begin{tabular}{|c|c|c|c|c|}
\hline$C(58)$ & $7650(12)$ & $3239(4)$ & $2116(7)$ & $66(4)$ \\
\hline$C(59)$ & $6349(12)$ & $2403(4)$ & $1856(6)$ & $57(4)$ \\
\hline$C(60)$ & $5560(12)$ & $2007(5)$ & $1499(7)$ & $77(4)$ \\
\hline $\mathrm{C}(61)$ & $4544(12)$ & $2702(5)$ & $2389(8)$ & $72(4)$ \\
\hline$C(62)$ & $4743(13)$ & $2433(5)$ & $3007(8)$ & $79(4)$ \\
\hline$C(63)$ & $5293(13)$ & $3010(4)$ & $1352(7)$ & $61(4)$ \\
\hline$C(64)$ & $4696(13)$ & $3459(4)$ & $1419(8)$ & $79(4)$ \\
\hline $\mathrm{N}(8)$ & $9186(11)$ & $8182(4)$ & $1340(6)$ & $61(3)$ \\
\hline$C(73)$ & $10032(13)$ & $8106(5)$ & $1973(7)$ & $76(4)$ \\
\hline$C(74)$ & $11386(13)$ & $8172(5)$ & 1917(8) & $93(4)$ \\
\hline$C(75)$ & $9168(15)$ & $8655(5)$ & $1119(10)$ & $102(5)$ \\
\hline$C(76)$ & $8615(15)$ & $8930(5)$ & $1602(10)$ & $116(5)$ \\
\hline$C(77)$ & $7927(12)$ & $8014(5)$ & $1474(8)$ & $79(4)$ \\
\hline $\mathrm{C}(78)$ & $6989(12)$ & $8068(5)$ & $903(8)$ & $88(5)$ \\
\hline$C(79)$ & $9750(14)$ & $7915(5)$ & $799(8)$ & $82(4)$ \\
\hline $\mathrm{C}(80)$ & $9914(14)$ & $7468(5)$ & $906(8)$ & $85(4)$ \\
\hline $\mathrm{O}(1 \mathrm{~S})$ & $5536(12)$ & $9756(4)$ & $4286(7)$ & $138(5)$ \\
\hline$C(1 S)$ & $7005(19)$ & $9455(7)$ & $3493(11)$ & $174(8)$ \\
\hline $\mathrm{C}(2 \mathrm{~S})$ & $5770(20)$ & $9420(7)$ & $3809(11)$ & $179(8)$ \\
\hline$C(3 S)$ & $4334(17)$ & $9796(6)$ & $4504(11)$ & $149(7)$ \\
\hline$C(4 S)$ & $4342(19)$ & $10139(6)$ & $4948(11)$ & $170(8)$ \\
\hline
\end{tabular}


Table S50. Bond Distances, $\AA$, for $\left[\left(\mathrm{L}^{1}{ }_{\mathrm{re}}\right)(\mathrm{Cl}) \mathrm{Fe}(\mathrm{II})\right]\left[\mathrm{Et}_{4} \mathrm{~N}\right]_{2}(7)$

\begin{tabular}{|c|c|c|c|}
\hline $\mathrm{Fe}(1)-\mathrm{N}(4)$ & $2.019(10)$ & $C(4 A)-C(5 A)$ & $1.225(17)$ \\
\hline $\mathrm{Fe}(1)-\mathrm{N}(2)$ & $2.090(10)$ & $C(5 A)-C(6 A)$ & $1.397(17)$ \\
\hline $\mathrm{Fe}(1)-\mathrm{N}(1)$ & $2.106(9)$ & $\mathrm{C}(7 \mathrm{~A})-\mathrm{C}(12 \mathrm{~A})$ & $1.385(16)$ \\
\hline $\mathrm{Fe}(1)-\mathrm{Cl}(1)$ & $2.357(4)$ & $\mathrm{C}(7 \mathrm{~A})-\mathrm{C}(8 \mathrm{~A})$ & $1.405(17)$ \\
\hline $\mathrm{Fe}(1)-\mathrm{N}(3)$ & $2.679(10)$ & $\mathrm{C}(8 \mathrm{~A})-\mathrm{C}(9 \mathrm{~A})$ & $1.401(16)$ \\
\hline $\mathrm{N}(1)-\mathrm{C}(1)$ & $1.316(14)$ & $\mathrm{C}(9 \mathrm{~A})-\mathrm{C}(10 \mathrm{~A})$ & $1.383(16)$ \\
\hline $\mathrm{N}(1)-\mathrm{C}(19)$ & $1.384(16)$ & $C(10 A)-C(11 A)$ & $1.448(17)$ \\
\hline $\mathrm{N}(2)-\mathrm{C}(7)$ & $1.368(15)$ & $C(11 \mathrm{~A})-\mathrm{C}(12 \mathrm{~A})$ & $1.343(16)$ \\
\hline $\mathrm{N}(2)-\mathrm{C}(2)$ & $1.417(14)$ & $\mathrm{C}(13 \mathrm{~A})-\mathrm{C}(18 \mathrm{~A})$ & $1.349(16)$ \\
\hline $\mathrm{N}(3)-\mathrm{C}(29)$ & $1.427(14)$ & $C(13 A)-C(14 A)$ & $1.356(17)$ \\
\hline $\mathrm{N}(3)-\mathrm{C}(8)$ & $1.431(15)$ & $C(14 A)-C(15 A)$ & $1.433(16)$ \\
\hline $\mathrm{N}(3)-\mathrm{C}(13)$ & $1.444(14)$ & $C(15 A)-C(16 A)$ & $1.410(16)$ \\
\hline $\mathrm{N}(4)-\mathrm{C}(39)$ & $1.416(14)$ & $C(16 A)-C(17 A)$ & $1.378(17)$ \\
\hline $\mathrm{N}(4)-\mathrm{C}(14)$ & $1.427(14)$ & $\mathrm{C}(17 \mathrm{~A})-\mathrm{C}(18 \mathrm{~A})$ & $1.290(15)$ \\
\hline$C(1)-C(6)$ & $1.450(16)$ & $C(19 A)-C(24 A)$ & $1.404(17)$ \\
\hline $\mathrm{C}(1)-\mathrm{C}(2)$ & $1.504(18)$ & $C(19 A)-C(20 A)$ & $1.417(16)$ \\
\hline $\mathrm{C}(2)-\mathrm{C}(3)$ & $1.480(16)$ & $C(20 A)-C(21 A)$ & $1.351(16)$ \\
\hline$C(3)-C(4)$ & $1.374(17)$ & $C(21 A)-C(22 A)$ & $1.352(17)$ \\
\hline $\mathrm{C}(4)-\mathrm{C}(5)$ & $1.412(18)$ & $C(22 A)-C(23 A)$ & $1.420(17)$ \\
\hline$C(5)-C(6)$ & $1.448(18)$ & $C(22 \mathrm{~A})-\mathrm{C}(25 \mathrm{~A})$ & $1.58(2)$ \\
\hline$C(7)-C(8)$ & $1.398(17)$ & $\mathrm{C}(23 \mathrm{~A})-\mathrm{C}(24 \mathrm{~A})$ & $1.359(17)$ \\
\hline
\end{tabular}




\begin{tabular}{|c|c|c|c|}
\hline$C(7)-C(12)$ & $1.449(16)$ & $\mathrm{C}(25 \mathrm{~A})-\mathrm{C}(27 \mathrm{~A})$ & $1.292(19)$ \\
\hline $\mathrm{C}(8)-\mathrm{C}(9)$ & $1.340(16)$ & $C(25 A)-C(26 A)$ & $1.39(2)$ \\
\hline $\mathrm{C}(9)-\mathrm{C}(10)$ & $1.362(16)$ & $\mathrm{C}(25 \mathrm{~A})-\mathrm{C}(28 \mathrm{~A})$ & $1.82(3)$ \\
\hline $\mathrm{C}(10)-\mathrm{C}(11)$ & $1.348(17)$ & $\mathrm{C}(29 \mathrm{~A})-\mathrm{C}(34 \mathrm{~A})$ & $1.335(16)$ \\
\hline $\mathrm{C}(11)-\mathrm{C}(12)$ & $1.401(17)$ & $C(29 A)-C(30 A)$ & $1.368(16)$ \\
\hline $\mathrm{C}(13)-\mathrm{C}(18)$ & $1.419(15)$ & $C(30 A)-C(31 A)$ & $1.406(15)$ \\
\hline$C(13)-C(14)$ & $1.490(16)$ & $C(31 \mathrm{~A})-\mathrm{C}(32 \mathrm{~A})$ & $1.385(17)$ \\
\hline$C(14)-C(15)$ & $1.441(16)$ & $C(32 A)-C(33 A)$ & $1.336(17)$ \\
\hline $\mathrm{C}(15)-\mathrm{C}(16)$ & $1.345(15)$ & $\mathrm{C}(32 \mathrm{~A})-\mathrm{C}(35 \mathrm{~A})$ & $1.549(17)$ \\
\hline$C(16)-C(17)$ & $1.388(16)$ & $\mathrm{C}(33 \mathrm{~A})-\mathrm{C}(34 \mathrm{~A})$ & $1.392(15)$ \\
\hline $\mathrm{C}(17)-\mathrm{C}(18)$ & $1.410(15)$ & $C(35 A)-C(36 A)$ & $1.532(18)$ \\
\hline$C(19)-C(20)$ & $1.342(18)$ & $C(35 A)-C(37 A)$ & $1.546(18)$ \\
\hline $\mathrm{C}(19)-\mathrm{C}(24)$ & $1.396(19)$ & $\mathrm{C}(35 \mathrm{~A})-\mathrm{C}(38 \mathrm{~A})$ & $1.585(18)$ \\
\hline $\mathrm{C}(20)-\mathrm{C}(21)$ & $1.390(18)$ & $C(39 A)-C(44 A)$ & $1.391(17)$ \\
\hline $\mathrm{C}(21)-\mathrm{C}(22)$ & $1.371(19)$ & $C(39 A)-C(40 A)$ & $1.399(17)$ \\
\hline $\mathrm{C}(22)-\mathrm{C}(23)$ & $1.45(2)$ & $C(40 A)-C(41 A)$ & $1.385(16)$ \\
\hline$C(22)-C(25)$ & $1.56(2)$ & $C(41 A)-C(42 A)$ & $1.362(18)$ \\
\hline $\mathrm{C}(23)-\mathrm{C}(24)$ & $1.379(18)$ & $\mathrm{C}(42 \mathrm{~A})-\mathrm{C}(43 \mathrm{~A})$ & $1.477(19)$ \\
\hline $\mathrm{C}(25)-\mathrm{C}(27)$ & $1.39(2)$ & $\mathrm{C}(42 \mathrm{~A})-\mathrm{C}(45 \mathrm{~A})$ & $1.48(2)$ \\
\hline$C(25)-C(28)$ & $1.44(2)$ & $C(43 A)-C(44 A)$ & $1.369(17)$ \\
\hline$C(25)-C(26)$ & $1.50(2)$ & $\mathrm{C}(45 \mathrm{~A})-\mathrm{C}(47 \mathrm{~A})$ & $1.30(2)$ \\
\hline C(29)-C(30) & $1.355(16)$ & $\mathrm{C}(45 \mathrm{~A})-\mathrm{C}(48 \mathrm{~A})$ & $1.45(2)$ \\
\hline
\end{tabular}




\begin{tabular}{|c|c|c|c|}
\hline$C(29)-C(34)$ & $1.409(17)$ & $C(45 A)-C(46 A)$ & $1.48(2)$ \\
\hline$C(30)-C(31)$ & $1.384(16)$ & $\mathrm{N}(7)-\mathrm{C}(71)$ & $1.489(15)$ \\
\hline$C(31)-C(32)$ & $1.389(18)$ & $\mathrm{N}(7)-\mathrm{C}(67)$ & $1.512(17)$ \\
\hline$C(32)-C(33)$ & $1.474(18)$ & $\mathrm{N}(7)-\mathrm{C}(65)$ & $1.519(17)$ \\
\hline$C(32)-C(35)$ & $1.524(18)$ & $\mathrm{N}(7)-\mathrm{C}(69)$ & $1.545(15)$ \\
\hline$C(33)-C(34)$ & $1.378(15)$ & $C(65)-C(66)$ & $1.481(18)$ \\
\hline$C(35)-C(36)$ & $1.505(19)$ & $\mathrm{C}(67)-\mathrm{C}(68)$ & $1.515(18)$ \\
\hline$C(35)-C(38)$ & $1.506(19)$ & $\mathrm{C}(69)-\mathrm{C}(70)$ & $1.521(17)$ \\
\hline$C(35)-C(37)$ & $1.587(19)$ & $\mathrm{C}(71)-\mathrm{C}(72)$ & $1.449(17)$ \\
\hline$C(39)-C(40)$ & $1.373(16)$ & $\mathrm{N}(5)-\mathrm{C}(49)$ & $1.505(17)$ \\
\hline$C(39)-C(44)$ & $1.402(17)$ & $\mathrm{N}(5)-\mathrm{C}(51)$ & $1.549(18)$ \\
\hline$C(40)-C(41)$ & $1.396(16)$ & $\mathrm{N}(5)-\mathrm{C}(53)$ & $1.586(18)$ \\
\hline $\mathrm{C}(41)-\mathrm{C}(42)$ & $1.417(18)$ & $\mathrm{N}(5)-\mathrm{C}(55)$ & $1.587(19)$ \\
\hline$C(42)-C(43)$ & $1.325(17)$ & $\mathrm{C}(49)-\mathrm{C}(50)$ & $1.572(19)$ \\
\hline$C(42)-C(45)$ & $1.529(18)$ & $\mathrm{C}(51)-\mathrm{C}(52)$ & $1.46(2)$ \\
\hline$C(43)-C(44)$ & $1.363(15)$ & $C(53)-C(54)$ & $1.578(18)$ \\
\hline$C(45)-C(48)$ & $1.477(18)$ & $C(55)-C(56)$ & $1.57(2)$ \\
\hline$C(45)-C(47)$ & $1.498(19)$ & $\mathrm{N}(6)-\mathrm{C}(63)$ & $1.479(15)$ \\
\hline$C(45)-C(46)$ & $1.542(19)$ & $\mathrm{N}(6)-\mathrm{C}(57)$ & $1.485(15)$ \\
\hline $\mathrm{Fe}(1 \mathrm{~A})-\mathrm{N}(4 \mathrm{~A})$ & $2.043(10)$ & $\mathrm{N}(6)-\mathrm{C}(59)$ & $1.501(15)$ \\
\hline $\mathrm{Fe}(1 \mathrm{~A})-\mathrm{N}(1 \mathrm{~A})$ & $2.048(10)$ & $\mathrm{N}(6)-\mathrm{C}(61)$ & $1.548(15)$ \\
\hline $\mathrm{Fe}(1 \mathrm{~A})-\mathrm{N}(2 \mathrm{~A})$ & $2.050(9)$ & $\mathrm{C}(57)-\mathrm{C}(58)$ & $1.470(16)$ \\
\hline
\end{tabular}




\begin{tabular}{|c|c|c|c|}
\hline $\mathrm{Fe}(1 \mathrm{~A})-\mathrm{Cl}(1 \mathrm{~A})$ & $2.359(4)$ & $\mathrm{C}(59)-\mathrm{C}(60)$ & $1.641(17)$ \\
\hline $\mathrm{Fe}(1 \mathrm{~A})-\mathrm{N}(3 \mathrm{~A})$ & $2.653(11)$ & $\mathrm{C}(61)-\mathrm{C}(62)$ & $1.506(17)$ \\
\hline $\mathrm{N}(1 \mathrm{~A})-\mathrm{C}(19 \mathrm{~A})$ & $1.404(15)$ & $\mathrm{C}(63)-\mathrm{C}(64)$ & $1.559(17)$ \\
\hline $\mathrm{N}(1 \mathrm{~A})-\mathrm{C}(1 \mathrm{~A})$ & $1.433(15)$ & $\mathrm{N}(8)-\mathrm{C}(77)$ & $1.503(16)$ \\
\hline $\mathrm{N}(2 \mathrm{~A})-\mathrm{C}(7 \mathrm{~A})$ & $1.385(15)$ & $\mathrm{N}(8)-\mathrm{C}(73)$ & $1.531(16)$ \\
\hline $\mathrm{N}(2 \mathrm{~A})-\mathrm{C}(2 \mathrm{~A})$ & $1.406(15)$ & $\mathrm{N}(8)-\mathrm{C}(79)$ & $1.540(17)$ \\
\hline $\mathrm{N}(3 \mathrm{~A})-\mathrm{C}(8 \mathrm{~A})$ & $1.449(14)$ & $\mathrm{N}(8)-\mathrm{C}(75)$ & $1.547(17)$ \\
\hline $\mathrm{N}(3 \mathrm{~A})-\mathrm{C}(29 \mathrm{~A})$ & $1.471(14)$ & $\mathrm{C}(73)-\mathrm{C}(74)$ & $1.490(18)$ \\
\hline $\mathrm{N}(3 \mathrm{~A})-\mathrm{C}(13 \mathrm{~A})$ & $1.471(15)$ & $\mathrm{C}(75)-\mathrm{C}(76)$ & $1.46(2)$ \\
\hline $\mathrm{N}(4 \mathrm{~A})-\mathrm{C}(14 \mathrm{~A})$ & $1.394(15)$ & $\mathrm{C}(77)-\mathrm{C}(78)$ & $1.483(19)$ \\
\hline $\mathrm{N}(4 \mathrm{~A})-\mathrm{C}(39 \mathrm{~A})$ & $1.412(15)$ & $\mathrm{C}(79)-\mathrm{C}(80)$ & $1.428(19)$ \\
\hline $\mathrm{C}(1 \mathrm{~A})-\mathrm{C}(2 \mathrm{~A})$ & $1.369(17)$ & $\mathrm{O}(1 \mathrm{~S})-\mathrm{C}(3 \mathrm{~S})$ & $1.409(15)$ \\
\hline$C(1 \mathrm{~A})-\mathrm{C}(6 \mathrm{~A})$ & $1.414(16)$ & $\mathrm{O}(1 \mathrm{~S})-\mathrm{C}(2 \mathrm{~S})$ & $1.461(15)$ \\
\hline$C(2 A)-C(3 A)$ & $1.358(15)$ & $\mathrm{C}(1 \mathrm{~S})-\mathrm{C}(2 \mathrm{~S})$ & $1.529(16)$ \\
\hline$C(3 A)-C(4 A)$ & $1.412(16)$ & $C(3 S)-C(4 S)$ & $1.397(16)$ \\
\hline
\end{tabular}


Table S51. Angles, ${ }^{\circ}$, for $\left[\left(\mathrm{L}^{1}{ }_{\mathrm{re}}\right)(\mathrm{Cl}) \mathrm{Fe}(\mathrm{II})\right]\left[\mathrm{Et}_{4} \mathrm{~N}\right]_{2}(7)$

\begin{tabular}{|c|c|c|c|}
\hline $\mathrm{N}(4)-\mathrm{Fe}(1)-\mathrm{N}(2)$ & $116.9(4)$ & $C(3 \mathrm{~A})-\mathrm{C}(2 \mathrm{~A})-\mathrm{C}(1 \mathrm{~A})$ & $116.4(13)$ \\
\hline $\mathrm{N}(4)-\mathrm{Fe}(1)-\mathrm{N}(1)$ & $114.1(4)$ & $\mathrm{C}(3 \mathrm{~A})-\mathrm{C}(2 \mathrm{~A})-\mathrm{N}(2 \mathrm{~A})$ & $128.5(14)$ \\
\hline $\mathrm{N}(2)-\mathrm{Fe}(1)-\mathrm{N}(1)$ & $81.3(4)$ & $\mathrm{C}(1 \mathrm{~A})-\mathrm{C}(2 \mathrm{~A})-\mathrm{N}(2 \mathrm{~A})$ & $114.7(12)$ \\
\hline $\mathrm{N}(4)-\mathrm{Fe}(1)-\mathrm{Cl}(1)$ & $120.1(3)$ & $\mathrm{C}(14 \mathrm{~A})-\mathrm{N}(4 \mathrm{~A})-\mathrm{C}(39 \mathrm{~A})$ & $118.0(11)$ \\
\hline $\mathrm{N}(2)-\mathrm{Fe}(1)-\mathrm{Cl}(1)$ & 111.3(3) & $\mathrm{C}(14 \mathrm{~A})-\mathrm{N}(4 \mathrm{~A})-\mathrm{Fe}(1 \mathrm{~A})$ & $114.9(8)$ \\
\hline $\mathrm{N}(1)-\mathrm{Fe}(1)-\mathrm{Cl}(1)$ & $106.1(3)$ & $\mathrm{C}(39 \mathrm{~A})-\mathrm{N}(4 \mathrm{~A})-\mathrm{Fe}(1 \mathrm{~A})$ & $127.1(9)$ \\
\hline $\mathrm{N}(4)-\mathrm{Fe}(1)-\mathrm{N}(3)$ & $72.2(3)$ & $\mathrm{C}(2 \mathrm{~A})-\mathrm{C}(1 \mathrm{~A})-\mathrm{C}(6 \mathrm{~A})$ & $117.5(13)$ \\
\hline $\mathrm{N}(2)-\mathrm{Fe}(1)-\mathrm{N}(3)$ & $69.0(4)$ & $\mathrm{C}(2 \mathrm{~A})-\mathrm{C}(1 \mathrm{~A})-\mathrm{N}(1 \mathrm{~A})$ & $116.4(12)$ \\
\hline $\mathrm{N}(1)-\mathrm{Fe}(1)-\mathrm{N}(3)$ & $148.2(3)$ & $C(6 A)-C(1 A)-N(1 A)$ & $125.8(13)$ \\
\hline $\mathrm{Cl}(1)-\mathrm{Fe}(1)-\mathrm{N}(3)$ & $95.5(2)$ & $\mathrm{C}(2 \mathrm{~A})-\mathrm{C}(3 \mathrm{~A})-\mathrm{C}(4 \mathrm{~A})$ & $123.4(13)$ \\
\hline $\mathrm{C}(1)-\mathrm{N}(1)-\mathrm{C}(19)$ & $121.2(11)$ & $C(5 A)-C(4 A)-C(3 A)$ & $121.4(14)$ \\
\hline $\mathrm{C}(1)-\mathrm{N}(1)-\mathrm{Fe}(1)$ & $113.4(8)$ & $C(4 A)-C(5 A)-C(6 A)$ & $118.5(15)$ \\
\hline $\mathrm{C}(19)-\mathrm{N}(1)-\mathrm{Fe}(1)$ & $124.7(9)$ & $C(5 A)-C(6 A)-C(1 A)$ & $122.5(14)$ \\
\hline $\mathrm{C}(7)-\mathrm{N}(2)-\mathrm{C}(2)$ & $125.9(11)$ & $\mathrm{C}(12 \mathrm{~A})-\mathrm{C}(7 \mathrm{~A})-\mathrm{N}(2 \mathrm{~A})$ & $125.8(12)$ \\
\hline $\mathrm{C}(7)-\mathrm{N}(2)-\mathrm{Fe}(1)$ & $119.9(8)$ & $\mathrm{C}(12 \mathrm{~A})-\mathrm{C}(7 \mathrm{~A})-\mathrm{C}(8 \mathrm{~A})$ & $115.4(13)$ \\
\hline $\mathrm{C}(2)-\mathrm{N}(2)-\mathrm{Fe}(1)$ & $111.5(8)$ & $\mathrm{N}(2 \mathrm{~A})-\mathrm{C}(7 \mathrm{~A})-\mathrm{C}(8 \mathrm{~A})$ & $118.7(12)$ \\
\hline $\mathrm{C}(29)-\mathrm{N}(3)-\mathrm{C}(8)$ & $115.2(11)$ & $\mathrm{C}(9 \mathrm{~A})-\mathrm{C}(8 \mathrm{~A})-\mathrm{C}(7 \mathrm{~A})$ & $121.0(13)$ \\
\hline $\mathrm{C}(29)-\mathrm{N}(3)-\mathrm{C}(13)$ & $113.7(9)$ & $\mathrm{C}(9 \mathrm{~A})-\mathrm{C}(8 \mathrm{~A})-\mathrm{N}(3 \mathrm{~A})$ & $119.3(12)$ \\
\hline $\mathrm{C}(8)-\mathrm{N}(3)-\mathrm{C}(13)$ & $114.4(9)$ & $\mathrm{C}(7 \mathrm{~A})-\mathrm{C}(8 \mathrm{~A})-\mathrm{N}(3 \mathrm{~A})$ & $119.6(12)$ \\
\hline $\mathrm{C}(29)-\mathrm{N}(3)-\mathrm{Fe}(1)$ & $115.4(8)$ & $C(10 A)-C(9 A)-C(8 A)$ & $122.6(14)$ \\
\hline $\mathrm{C}(8)-\mathrm{N}(3)-\mathrm{Fe}(1)$ & $101.4(7)$ & $\mathrm{C}(9 \mathrm{~A})-\mathrm{C}(10 \mathrm{~A})-\mathrm{C}(11 \mathrm{~A})$ & $115.4(13)$ \\
\hline
\end{tabular}




\begin{tabular}{|c|c|c|c|}
\hline $\mathrm{C}(13)-\mathrm{N}(3)-\mathrm{Fe}(1)$ & $94.4(7)$ & $C(12 A)-C(11 A)-C(10 A)$ & $120.5(13)$ \\
\hline $\mathrm{C}(39)-\mathrm{N}(4)-\mathrm{C}(14)$ & $115.9(10)$ & $C(11 A)-C(12 A)-C(7 A)$ & $124.9(14)$ \\
\hline $\mathrm{C}(39)-\mathrm{N}(4)-\mathrm{Fe}(1)$ & $127.8(8)$ & $\mathrm{C}(18 \mathrm{~A})-\mathrm{C}(13 \mathrm{~A})-\mathrm{C}(14 \mathrm{~A})$ & $121.5(13)$ \\
\hline $\mathrm{C}(14)-\mathrm{N}(4)-\mathrm{Fe}(1)$ & $115.5(8)$ & $\mathrm{C}(18 \mathrm{~A})-\mathrm{C}(13 \mathrm{~A})-\mathrm{N}(3 \mathrm{~A})$ & $122.7(12)$ \\
\hline $\mathrm{N}(1)-\mathrm{C}(1)-\mathrm{C}(6)$ & 127.1(13) & $\mathrm{C}(14 \mathrm{~A})-\mathrm{C}(13 \mathrm{~A})-\mathrm{N}(3 \mathrm{~A})$ & $115.4(12)$ \\
\hline $\mathrm{N}(1)-\mathrm{C}(1)-\mathrm{C}(2)$ & $117.8(11)$ & $\mathrm{C}(13 \mathrm{~A})-\mathrm{C}(14 \mathrm{~A})-\mathrm{N}(4 \mathrm{~A})$ & $122.0(12)$ \\
\hline $\mathrm{C}(6)-\mathrm{C}(1)-\mathrm{C}(2)$ & $114.9(12)$ & $\mathrm{C}(13 \mathrm{~A})-\mathrm{C}(14 \mathrm{~A})-\mathrm{C}(15 \mathrm{~A})$ & $117.3(13)$ \\
\hline $\mathrm{N}(2)-\mathrm{C}(2)-\mathrm{C}(3)$ & $122.9(13)$ & $\mathrm{N}(4 \mathrm{~A})-\mathrm{C}(14 \mathrm{~A})-\mathrm{C}(15 \mathrm{~A})$ & $120.7(13)$ \\
\hline $\mathrm{N}(2)-\mathrm{C}(2)-\mathrm{C}(1)$ & $115.5(11)$ & $C(16 A)-C(15 A)-C(14 A)$ & $116.1(14)$ \\
\hline$C(3)-C(2)-C(1)$ & $121.3(12)$ & $\mathrm{C}(17 \mathrm{~A})-\mathrm{C}(16 \mathrm{~A})-\mathrm{C}(15 \mathrm{~A})$ & $123.8(14)$ \\
\hline$C(4)-C(3)-C(2)$ & $120.3(14)$ & $C(18 A)-C(17 A)-C(16 A)$ & $115.6(14)$ \\
\hline$C(3)-C(4)-C(5)$ & $119.6(14)$ & $\mathrm{C}(17 \mathrm{~A})-\mathrm{C}(18 \mathrm{~A})-\mathrm{C}(13 \mathrm{~A})$ & $125.7(15)$ \\
\hline$C(4)-C(5)-C(6)$ & $123.3(13)$ & $\mathrm{N}(1 \mathrm{~A})-\mathrm{C}(19 \mathrm{~A})-\mathrm{C}(24 \mathrm{~A})$ & $127.9(12)$ \\
\hline$C(5)-C(6)-C(1)$ & $120.4(13)$ & $\mathrm{N}(1 \mathrm{~A})-\mathrm{C}(19 \mathrm{~A})-\mathrm{C}(20 \mathrm{~A})$ & $120.9(12)$ \\
\hline $\mathrm{N}(2)-\mathrm{C}(7)-\mathrm{C}(8)$ & $119.9(12)$ & $\mathrm{C}(24 \mathrm{~A})-\mathrm{C}(19 \mathrm{~A})-\mathrm{C}(20 \mathrm{~A})$ & $111.2(13)$ \\
\hline $\mathrm{N}(2)-\mathrm{C}(7)-\mathrm{C}(12)$ & $122.7(13)$ & $C(21 A)-C(20 A)-C(19 A)$ & $123.0(13)$ \\
\hline $\mathrm{C}(8)-\mathrm{C}(7)-\mathrm{C}(12)$ & $117.2(13)$ & $C(20 A)-C(21 A)-C(22 A)$ & $124.5(14)$ \\
\hline$C(9)-C(8)-C(7)$ & $121.9(13)$ & $C(21 A)-C(22 A)-C(23 A)$ & $115.4(14)$ \\
\hline $\mathrm{C}(9)-\mathrm{C}(8)-\mathrm{N}(3)$ & $120.6(13)$ & $\mathrm{C}(21 \mathrm{~A})-\mathrm{C}(22 \mathrm{~A})-\mathrm{C}(25 \mathrm{~A})$ & $124.2(14)$ \\
\hline $\mathrm{C}(7)-\mathrm{C}(8)-\mathrm{N}(3)$ & $117.5(12)$ & $C(23 A)-C(22 A)-C(25 A)$ & $119.8(13)$ \\
\hline $\mathrm{C}(8)-\mathrm{C}(9)-\mathrm{C}(10)$ & $120.7(14)$ & $\mathrm{C}(24 \mathrm{~A})-\mathrm{C}(23 \mathrm{~A})-\mathrm{C}(22 \mathrm{~A})$ & $119.3(13)$ \\
\hline$C(11)-C(10)-C(9)$ & $121.2(14)$ & $\mathrm{C}(23 \mathrm{~A})-\mathrm{C}(24 \mathrm{~A})-\mathrm{C}(19 \mathrm{~A})$ & $126.3(13)$ \\
\hline
\end{tabular}




\begin{tabular}{|c|c|c|c|}
\hline$C(10)-C(11)-C(12)$ & $121.1(14)$ & $C(27 A)-C(25 A)-C(26 A)$ & $121.8(18)$ \\
\hline$C(11)-C(12)-C(7)$ & $117.7(13)$ & $\mathrm{C}(27 \mathrm{~A})-\mathrm{C}(25 \mathrm{~A})-\mathrm{C}(22 \mathrm{~A})$ & $114.6(16)$ \\
\hline $\mathrm{C}(18)-\mathrm{C}(13)-\mathrm{N}(3)$ & $122.7(11)$ & $C(26 A)-C(25 A)-C(22 A)$ & $121.4(15)$ \\
\hline$C(18)-C(13)-C(14)$ & $119.3(12)$ & $\mathrm{C}(27 \mathrm{~A})-\mathrm{C}(25 \mathrm{~A})-\mathrm{C}(28 \mathrm{~A})$ & $92.1(18)$ \\
\hline $\mathrm{N}(3)-\mathrm{C}(13)-\mathrm{C}(14)$ & $117.4(11)$ & $\mathrm{C}(26 \mathrm{~A})-\mathrm{C}(25 \mathrm{~A})-\mathrm{C}(28 \mathrm{~A})$ & $90.3(17)$ \\
\hline $\mathrm{N}(4)-\mathrm{C}(14)-\mathrm{C}(15)$ & $126.8(11)$ & $\mathrm{C}(22 \mathrm{~A})-\mathrm{C}(25 \mathrm{~A})-\mathrm{C}(28 \mathrm{~A})$ & $102.5(15)$ \\
\hline $\mathrm{N}(4)-\mathrm{C}(14)-\mathrm{C}(13)$ & $117.2(11)$ & $C(34 A)-C(29 A)-C(30 A)$ & $117.2(12)$ \\
\hline$C(15)-C(14)-C(13)$ & $115.9(12)$ & $\mathrm{C}(34 \mathrm{~A})-\mathrm{C}(29 \mathrm{~A})-\mathrm{N}(3 \mathrm{~A})$ & $124.3(12)$ \\
\hline$C(16)-C(15)-C(14)$ & $123.7(13)$ & $C(30 A)-C(29 A)-N(3 A)$ & $118.5(11)$ \\
\hline$C(15)-C(16)-C(17)$ & $119.3(13)$ & $C(29 A)-C(30 A)-C(31 A)$ & $119.1(12)$ \\
\hline$C(16)-C(17)-C(18)$ & $122.9(12)$ & $\mathrm{C}(32 \mathrm{~A})-\mathrm{C}(31 \mathrm{~A})-\mathrm{C}(30 \mathrm{~A})$ & $122.3(13)$ \\
\hline $\mathrm{C}(17)-\mathrm{C}(18)-\mathrm{C}(13)$ & $118.7(12)$ & $\mathrm{C}(33 \mathrm{~A})-\mathrm{C}(32 \mathrm{~A})-\mathrm{C}(31 \mathrm{~A})$ & $116.3(13)$ \\
\hline $\mathrm{C}(20)-\mathrm{C}(19)-\mathrm{N}(1)$ & $118.7(15)$ & $\mathrm{C}(33 \mathrm{~A})-\mathrm{C}(32 \mathrm{~A})-\mathrm{C}(35 \mathrm{~A})$ & $125.7(13)$ \\
\hline$C(20)-C(19)-C(24)$ & $118.1(15)$ & $\mathrm{C}(31 \mathrm{~A})-\mathrm{C}(32 \mathrm{~A})-\mathrm{C}(35 \mathrm{~A})$ & $117.8(13)$ \\
\hline $\mathrm{N}(1)-\mathrm{C}(19)-\mathrm{C}(24)$ & $123.0(14)$ & $\mathrm{C}(32 \mathrm{~A})-\mathrm{C}(33 \mathrm{~A})-\mathrm{C}(34 \mathrm{~A})$ & $120.6(13)$ \\
\hline$C(19)-C(20)-C(21)$ & $124.0(16)$ & $\mathrm{C}(29 \mathrm{~A})-\mathrm{C}(34 \mathrm{~A})-\mathrm{C}(33 \mathrm{~A})$ & $123.6(13)$ \\
\hline$C(22)-C(21)-C(20)$ & $120.5(16)$ & $C(36 \mathrm{~A})-\mathrm{C}(35 \mathrm{~A})-\mathrm{C}(37 \mathrm{~A})$ & $109.1(12)$ \\
\hline$C(21)-C(22)-C(23)$ & $115.7(15)$ & $C(36 A)-C(35 A)-C(32 A)$ & $114.1(12)$ \\
\hline$C(21)-C(22)-C(25)$ & 127.1(16) & $\mathrm{C}(37 \mathrm{~A})-\mathrm{C}(35 \mathrm{~A})-\mathrm{C}(32 \mathrm{~A})$ & $106.6(11)$ \\
\hline $\mathrm{C}(23)-\mathrm{C}(22)-\mathrm{C}(25)$ & $117.2(15)$ & $\mathrm{C}(36 \mathrm{~A})-\mathrm{C}(35 \mathrm{~A})-\mathrm{C}(38 \mathrm{~A})$ & $107.0(11)$ \\
\hline $\mathrm{C}(24)-\mathrm{C}(23)-\mathrm{C}(22)$ & $121.8(16)$ & $\mathrm{C}(37 \mathrm{~A})-\mathrm{C}(35 \mathrm{~A})-\mathrm{C}(38 \mathrm{~A})$ & $113.6(12)$ \\
\hline$C(23)-C(24)-C(19)$ & $119.5(15)$ & $\mathrm{C}(32 \mathrm{~A})-\mathrm{C}(35 \mathrm{~A})-\mathrm{C}(38 \mathrm{~A})$ & $106.6(11)$ \\
\hline
\end{tabular}




\begin{tabular}{|c|c|c|c|}
\hline$C(27)-C(25)-C(28)$ & $101.4(15)$ & $\mathrm{C}(44 \mathrm{~A})-\mathrm{C}(39 \mathrm{~A})-\mathrm{C}(40 \mathrm{~A})$ & $112.2(13)$ \\
\hline$C(27)-C(25)-C(26)$ & $104.7(17)$ & $\mathrm{C}(44 \mathrm{~A})-\mathrm{C}(39 \mathrm{~A})-\mathrm{N}(4 \mathrm{~A})$ & $125.4(13)$ \\
\hline$C(28)-C(25)-C(26)$ & $113.1(16)$ & $\mathrm{C}(40 \mathrm{~A})-\mathrm{C}(39 \mathrm{~A})-\mathrm{N}(4 \mathrm{~A})$ & $122.1(13)$ \\
\hline$C(27)-C(25)-C(22)$ & $120.9(16)$ & $\mathrm{C}(41 \mathrm{~A})-\mathrm{C}(40 \mathrm{~A})-\mathrm{C}(39 \mathrm{~A})$ & $127.3(14)$ \\
\hline $\mathrm{C}(28)-\mathrm{C}(25)-\mathrm{C}(22)$ & $110.3(16)$ & $\mathrm{C}(42 \mathrm{~A})-\mathrm{C}(41 \mathrm{~A})-\mathrm{C}(40 \mathrm{~A})$ & $120.2(15)$ \\
\hline$C(26)-C(25)-C(22)$ & $106.5(14)$ & $\mathrm{C}(41 \mathrm{~A})-\mathrm{C}(42 \mathrm{~A})-\mathrm{C}(43 \mathrm{~A})$ & $114.8(14)$ \\
\hline$C(30)-C(29)-C(34)$ & $119.5(12)$ & $\mathrm{C}(41 \mathrm{~A})-\mathrm{C}(42 \mathrm{~A})-\mathrm{C}(45 \mathrm{~A})$ & $127.5(16)$ \\
\hline$C(30)-C(29)-N(3)$ & $120.8(12)$ & $\mathrm{C}(43 \mathrm{~A})-\mathrm{C}(42 \mathrm{~A})-\mathrm{C}(45 \mathrm{~A})$ & $117.7(15)$ \\
\hline $\mathrm{C}(34)-\mathrm{C}(29)-\mathrm{N}(3)$ & $119.6(12)$ & $\mathrm{C}(44 \mathrm{~A})-\mathrm{C}(43 \mathrm{~A})-\mathrm{C}(42 \mathrm{~A})$ & $121.7(14)$ \\
\hline$C(29)-C(30)-C(31)$ & $121.2(13)$ & $\mathrm{C}(43 \mathrm{~A})-\mathrm{C}(44 \mathrm{~A})-\mathrm{C}(39 \mathrm{~A})$ & $123.7(14)$ \\
\hline$C(30)-C(31)-C(32)$ & $123.8(13)$ & $\mathrm{C}(47 \mathrm{~A})-\mathrm{C}(45 \mathrm{~A})-\mathrm{C}(48 \mathrm{~A})$ & $112.3(18)$ \\
\hline $\mathrm{C}(31)-\mathrm{C}(32)-\mathrm{C}(33)$ & $113.4(13)$ & $\mathrm{C}(47 \mathrm{~A})-\mathrm{C}(45 \mathrm{~A})-\mathrm{C}(46 \mathrm{~A})$ & $103.0(17)$ \\
\hline $\mathrm{C}(31)-\mathrm{C}(32)-\mathrm{C}(35)$ & $125.7(14)$ & $\mathrm{C}(48 \mathrm{~A})-\mathrm{C}(45 \mathrm{~A})-\mathrm{C}(46 \mathrm{~A})$ & $90.0(15)$ \\
\hline$C(33)-C(32)-C(35)$ & $120.5(13)$ & $C(47 A)-C(45 A)-C(42 A)$ & $114.5(16)$ \\
\hline$C(34)-C(33)-C(32)$ & $122.5(13)$ & $C(48 A)-C(45 A)-C(42 A)$ & $121.2(16)$ \\
\hline$C(33)-C(34)-C(29)$ & 119.2(13) & $C(46 \mathrm{~A})-\mathrm{C}(45 \mathrm{~A})-\mathrm{C}(42 \mathrm{~A})$ & $111.4(16)$ \\
\hline $\mathrm{C}(36)-\mathrm{C}(35)-\mathrm{C}(38)$ & $109.2(13)$ & $\mathrm{C}(71)-\mathrm{N}(7)-\mathrm{C}(67)$ & $108.9(11)$ \\
\hline$C(36)-C(35)-C(32)$ & $109.4(13)$ & $\mathrm{C}(71)-\mathrm{N}(7)-\mathrm{C}(65)$ & $106.0(10)$ \\
\hline $\mathrm{C}(38)-\mathrm{C}(35)-\mathrm{C}(32)$ & $116.4(13)$ & $\mathrm{C}(67)-\mathrm{N}(7)-\mathrm{C}(65)$ & $113.0(12)$ \\
\hline$C(36)-C(35)-C(37)$ & $110.6(13)$ & $\mathrm{C}(71)-\mathrm{N}(7)-\mathrm{C}(69)$ & $112.6(10)$ \\
\hline $\mathrm{C}(38)-\mathrm{C}(35)-\mathrm{C}(37)$ & $105.0(13)$ & $\mathrm{C}(67)-\mathrm{N}(7)-\mathrm{C}(69)$ & $107.6(10)$ \\
\hline$C(32)-C(35)-C(37)$ & $106.1(12)$ & $\mathrm{C}(65)-\mathrm{N}(7)-\mathrm{C}(69)$ & $108.8(11)$ \\
\hline
\end{tabular}




\begin{tabular}{|c|c|c|c|}
\hline C(40)-C(39)-C(44) & $120.1(12)$ & $\mathrm{C}(66)-\mathrm{C}(65)-\mathrm{N}(7)$ & $113.4(14)$ \\
\hline $\mathrm{C}(40)-\mathrm{C}(39)-\mathrm{N}(4)$ & $118.7(12)$ & N(7)-C(67)-C(68) & $114.9(13)$ \\
\hline $\mathrm{C}(44)-\mathrm{C}(39)-\mathrm{N}(4)$ & $121.0(12)$ & $\mathrm{C}(70)-\mathrm{C}(69)-\mathrm{N}(7)$ & $111.3(11)$ \\
\hline $\mathrm{C}(39)-\mathrm{C}(40)-\mathrm{C}(41)$ & $115.9(13)$ & $\mathrm{C}(72)-\mathrm{C}(71)-\mathrm{N}(7)$ & $112.2(11)$ \\
\hline $\mathrm{C}(40)-\mathrm{C}(41)-\mathrm{C}(42)$ & $124.5(13)$ & $\mathrm{C}(49)-\mathrm{N}(5)-\mathrm{C}(51)$ & $115.0(13)$ \\
\hline $\mathrm{C}(43)-\mathrm{C}(42)-\mathrm{C}(41)$ & $115.6(13)$ & $\mathrm{C}(49)-\mathrm{N}(5)-\mathrm{C}(53)$ & $106.0(12)$ \\
\hline$C(43)-C(42)-C(45)$ & $123.7(14)$ & $\mathrm{C}(51)-\mathrm{N}(5)-\mathrm{C}(53)$ & $112.8(12)$ \\
\hline $\mathrm{C}(41)-\mathrm{C}(42)-\mathrm{C}(45)$ & $120.7(13)$ & $\mathrm{C}(49)-\mathrm{N}(5)-\mathrm{C}(55)$ & $105.5(13)$ \\
\hline$C(42)-C(43)-C(44)$ & $123.1(14)$ & $\mathrm{C}(51)-\mathrm{N}(5)-\mathrm{C}(55)$ & $102.2(12)$ \\
\hline $\mathrm{C}(43)-\mathrm{C}(44)-\mathrm{C}(39)$ & $120.4(13)$ & $\mathrm{C}(53)-\mathrm{N}(5)-\mathrm{C}(55)$ & $115.3(13)$ \\
\hline$C(48)-C(45)-C(47)$ & $108.5(14)$ & N(5)-C(49)-C(50) & $113.8(13)$ \\
\hline $\mathrm{C}(48)-\mathrm{C}(45)-\mathrm{C}(42)$ & $114.0(13)$ & $\mathrm{C}(52)-\mathrm{C}(51)-\mathrm{N}(5)$ & $110.5(14)$ \\
\hline $\mathrm{C}(47)-\mathrm{C}(45)-\mathrm{C}(42)$ & $109.4(12)$ & $\mathrm{C}(54)-\mathrm{C}(53)-\mathrm{N}(5)$ & $113.1(13)$ \\
\hline $\mathrm{C}(48)-\mathrm{C}(45)-\mathrm{C}(46)$ & $105.0(13)$ & $\mathrm{C}(56)-\mathrm{C}(55)-\mathrm{N}(5)$ & $116.8(17)$ \\
\hline $\mathrm{C}(47)-\mathrm{C}(45)-\mathrm{C}(46)$ & $111.7(13)$ & $\mathrm{C}(63)-\mathrm{N}(6)-\mathrm{C}(57)$ & $112.2(11)$ \\
\hline$C(42)-C(45)-C(46)$ & $108.3(12)$ & $\mathrm{C}(63)-\mathrm{N}(6)-\mathrm{C}(59)$ & $107.5(10)$ \\
\hline $\mathrm{N}(4 \mathrm{~A})-\mathrm{Fe}(1 \mathrm{~A})-\mathrm{N}(1 \mathrm{~A})$ & $114.8(4)$ & $\mathrm{C}(57)-\mathrm{N}(6)-\mathrm{C}(59)$ & $108.0(10)$ \\
\hline $\mathrm{N}(4 \mathrm{~A})-\mathrm{Fe}(1 \mathrm{~A})-\mathrm{N}(2 \mathrm{~A})$ & $116.4(4)$ & $\mathrm{C}(63)-\mathrm{N}(6)-\mathrm{C}(61)$ & $111.2(10)$ \\
\hline $\mathrm{N}(1 \mathrm{~A})-\mathrm{Fe}(1 \mathrm{~A})-\mathrm{N}(2 \mathrm{~A})$ & $78.8(4)$ & $\mathrm{C}(57)-\mathrm{N}(6)-\mathrm{C}(61)$ & $108.6(10)$ \\
\hline $\mathrm{N}(4 \mathrm{~A})-\mathrm{Fe}(1 \mathrm{~A})-\mathrm{Cl}(1 \mathrm{~A})$ & $121.9(3)$ & $\mathrm{C}(59)-\mathrm{N}(6)-\mathrm{C}(61)$ & $109.1(10)$ \\
\hline $\mathrm{N}(1 \mathrm{~A})-\mathrm{Fe}(1 \mathrm{~A})-\mathrm{Cl}(1 \mathrm{~A})$ & $104.8(3)$ & $\mathrm{C}(58)-\mathrm{C}(57)-\mathrm{N}(6)$ & $116.7(12)$ \\
\hline $\mathrm{N}(2 \mathrm{~A})-\mathrm{Fe}(1 \mathrm{~A})-\mathrm{Cl}(1 \mathrm{~A})$ & $111.6(3)$ & $\mathrm{N}(6)-\mathrm{C}(59)-\mathrm{C}(60)$ & $118.9(10)$ \\
\hline
\end{tabular}




\begin{tabular}{|c|c|c|c|}
\hline $\mathrm{N}(4 \mathrm{~A})-\mathrm{Fe}(1 \mathrm{~A})-\mathrm{N}(3 \mathrm{~A})$ & $69.9(3)$ & $\mathrm{C}(62)-\mathrm{C}(61)-\mathrm{N}(6)$ & $118.4(11)$ \\
\hline $\mathrm{N}(1 \mathrm{~A})-\mathrm{Fe}(1 \mathrm{~A})-\mathrm{N}(3 \mathrm{~A})$ & $147.6(4)$ & $\mathrm{N}(6)-\mathrm{C}(63)-\mathrm{C}(64)$ & $113.5(12)$ \\
\hline $\mathrm{N}(2 \mathrm{~A})-\mathrm{Fe}(1 \mathrm{~A})-\mathrm{N}(3 \mathrm{~A})$ & $71.3(4)$ & $\mathrm{C}(77)-\mathrm{N}(8)-\mathrm{C}(73)$ & $106.5(11)$ \\
\hline $\mathrm{Cl}(1 \mathrm{~A})-\mathrm{Fe}(1 \mathrm{~A})-\mathrm{N}(3 \mathrm{~A})$ & $97.7(2)$ & $\mathrm{C}(77)-\mathrm{N}(8)-\mathrm{C}(79)$ & $110.6(11)$ \\
\hline $\mathrm{C}(19 \mathrm{~A})-\mathrm{N}(1 \mathrm{~A})-\mathrm{C}(1 \mathrm{~A})$ & $121.6(11)$ & $\mathrm{C}(73)-\mathrm{N}(8)-\mathrm{C}(79)$ & $105.2(11)$ \\
\hline $\mathrm{C}(19 \mathrm{~A})-\mathrm{N}(1 \mathrm{~A})-\mathrm{Fe}(1 \mathrm{~A})$ & $123.5(9)$ & $\mathrm{C}(77)-\mathrm{N}(8)-\mathrm{C}(75)$ & $113.4(11)$ \\
\hline $\mathrm{C}(1 \mathrm{~A})-\mathrm{N}(1 \mathrm{~A})-\mathrm{Fe}(1 \mathrm{~A})$ & $113.7(8)$ & $\mathrm{C}(73)-\mathrm{N}(8)-\mathrm{C}(75)$ & $112.6(12)$ \\
\hline $\mathrm{C}(7 \mathrm{~A})-\mathrm{N}(2 \mathrm{~A})-\mathrm{C}(2 \mathrm{~A})$ & $122.5(11)$ & $\mathrm{C}(79)-\mathrm{N}(8)-\mathrm{C}(75)$ & $108.3(12)$ \\
\hline $\mathrm{C}(7 \mathrm{~A})-\mathrm{N}(2 \mathrm{~A})-\mathrm{Fe}(1 \mathrm{~A})$ & 119.1(8) & $\mathrm{C}(74)-\mathrm{C}(73)-\mathrm{N}(8)$ & $116.1(13)$ \\
\hline $\mathrm{C}(2 \mathrm{~A})-\mathrm{N}(2 \mathrm{~A})-\mathrm{Fe}(1 \mathrm{~A})$ & $115.8(9)$ & $\mathrm{C}(76)-\mathrm{C}(75)-\mathrm{N}(8)$ & $111.8(15)$ \\
\hline $\mathrm{C}(8 \mathrm{~A})-\mathrm{N}(3 \mathrm{~A})-\mathrm{C}(29 \mathrm{~A})$ & $118.0(10)$ & $\mathrm{C}(78)-\mathrm{C}(77)-\mathrm{N}(8)$ & $113.1(13)$ \\
\hline $\mathrm{C}(8 \mathrm{~A})-\mathrm{N}(3 \mathrm{~A})-\mathrm{C}(13 \mathrm{~A})$ & $114.7(9)$ & $\mathrm{C}(80)-\mathrm{C}(79)-\mathrm{N}(8)$ & $118.5(14)$ \\
\hline $\mathrm{C}(29 \mathrm{~A})-\mathrm{N}(3 \mathrm{~A})-\mathrm{C}(13 \mathrm{~A})$ & $111.9(9)$ & $\mathrm{C}(3 \mathrm{~S})-\mathrm{O}(1 \mathrm{~S})-\mathrm{C}(2 \mathrm{~S})$ & $118.8(15)$ \\
\hline $\mathrm{C}(8 \mathrm{~A})-\mathrm{N}(3 \mathrm{~A})-\mathrm{Fe}(1 \mathrm{~A})$ & $99.2(7)$ & $\mathrm{O}(1 \mathrm{~S})-\mathrm{C}(2 \mathrm{~S})-\mathrm{C}(1 \mathrm{~S})$ & $115.2(18)$ \\
\hline $\mathrm{C}(29 \mathrm{~A})-\mathrm{N}(3 \mathrm{~A})-\mathrm{Fe}(1 \mathrm{~A})$ & $114.3(7)$ & $\mathrm{C}(4 \mathrm{~S})-\mathrm{C}(3 \mathrm{~S})-\mathrm{O}(1 \mathrm{~S})$ & $108.1(18)$ \\
\hline $\mathrm{C}(13 \mathrm{~A})-\mathrm{N}(3 \mathrm{~A})-\mathrm{Fe}(1 \mathrm{~A})$ & $95.8(7)$ & & \\
\hline
\end{tabular}


Table S52. Anisotropic displacement parameters $\left(\AA^{2} \times 10^{3}\right)$ for $\left[\left(\mathrm{L}_{\mathrm{re}}^{1}\right)(\mathrm{Cl}) \mathrm{Fe}(\mathrm{II})\right]\left[\mathrm{Et}_{4} \mathrm{~N}\right]_{2}$ (7). The anisotropic displacement factor exponent takes the form: $-2 \pi^{2}\left[h^{2} a^{* 2} U^{11}+\ldots+2\right.$ $\mathrm{hka} \mathrm{k}^{*} \mathrm{U}^{12}$ ]

\begin{tabular}{|c|c|c|c|c|c|c|}
\hline & $\mathrm{U}^{11}$ & $\mathrm{U}^{22}$ & $\mathrm{U}^{33}$ & $\mathrm{U}^{23}$ & $\mathrm{U}^{13}$ & $\mathrm{U}^{12}$ \\
\hline $\mathrm{Fe}(1)$ & $37(1)$ & $57(1)$ & $35(1)$ & $-1(1)$ & $0(1)$ & $2(1)$ \\
\hline $\mathrm{Cl}(1)$ & $43(2)$ & $71(2)$ & $63(3)$ & $4(2)$ & $14(2)$ & $6(2)$ \\
\hline $\mathrm{N}(1)$ & $34(6)$ & $41(7)$ & $50(8)$ & $10(5)$ & $-3(5)$ & $1(5)$ \\
\hline $\mathrm{N}(2)$ & $41(6)$ & $63(8)$ & $33(7)$ & $10(6)$ & $2(5)$ & $-7(6)$ \\
\hline $\mathrm{N}(3)$ & $43(6)$ & $61(7)$ & $23(7)$ & $7(6)$ & $12(5)$ & $15(6)$ \\
\hline $\mathrm{N}(4)$ & $47(7)$ & $45(7)$ & $36(7)$ & $-1(5)$ & $-8(5)$ & $3(5)$ \\
\hline$C(1)$ & $57(5)$ & $69(5)$ & $29(5)$ & $7(5)$ & $-2(4)$ & $20(5)$ \\
\hline$C(2)$ & $52(5)$ & $76(5)$ & $32(5)$ & $6(5)$ & $-4(4)$ & $15(5)$ \\
\hline $\mathrm{C}(3)$ & $55(5)$ & $81(6)$ & $38(5)$ & $1(5)$ & $-10(4)$ & $12(5)$ \\
\hline$C(4)$ & $60(5)$ & $83(6)$ & $43(5)$ & $0(5)$ & $-8(4)$ & $16(5)$ \\
\hline$C(5)$ & $63(5)$ & $83(6)$ & $40(5)$ & $5(5)$ & $-6(4)$ & $23(5)$ \\
\hline$C(6)$ & $65(5)$ & $76(6)$ & $37(5)$ & $5(5)$ & $-1(4)$ & $18(5)$ \\
\hline$C(7)$ & $37(5)$ & $55(5)$ & $48(5)$ & $-9(4)$ & $2(4)$ & $-6(4)$ \\
\hline $\mathrm{C}(8)$ & $41(5)$ & $53(5)$ & $46(5)$ & $-10(4)$ & $-1(4)$ & $-8(4)$ \\
\hline$C(9)$ & $52(5)$ & $57(5)$ & $53(5)$ & $-8(5)$ & $-5(4)$ & $-8(5)$ \\
\hline$C(10)$ & $57(5)$ & $61(5)$ & $58(6)$ & $-9(5)$ & $-3(4)$ & $-9(4)$ \\
\hline $\mathrm{C}(11)$ & $51(5)$ & $64(5)$ & $59(6)$ & $-15(5)$ & $-5(4)$ & $-8(5)$ \\
\hline$C(12)$ & $47(5)$ & $65(5)$ & $54(5)$ & $-8(5)$ & $-1(4)$ & $-3(4)$ \\
\hline
\end{tabular}




\begin{tabular}{|c|c|c|c|c|c|c|}
\hline$C(13)$ & $37(4)$ & $50(5)$ & $37(5)$ & $2(4)$ & $-5(4)$ & $-2(4)$ \\
\hline$C(14)$ & $38(4)$ & $51(5)$ & $37(5)$ & $0(4)$ & $-5(4)$ & $-2(4)$ \\
\hline$C(15)$ & $42(4)$ & $53(5)$ & $41(5)$ & $-1(4)$ & $4(4)$ & $-3(4)$ \\
\hline$C(16)$ & $39(4)$ & $61(5)$ & $39(5)$ & $3(4)$ & $7(4)$ & $-3(4)$ \\
\hline$C(17)$ & $41(4)$ & $61(5)$ & $42(5)$ & $8(4)$ & $4(4)$ & $-8(4)$ \\
\hline$C(18)$ & $41(4)$ & $54(5)$ & $39(5)$ & $4(4)$ & $-2(4)$ & $-4(4)$ \\
\hline C(19) & $77(6)$ & $55(5)$ & $70(5)$ & $16(5)$ & $-5(5)$ & $-3(5)$ \\
\hline$C(20)$ & $80(6)$ & $56(5)$ & $70(5)$ & $16(5)$ & $-12(5)$ & $-2(5)$ \\
\hline $\mathrm{C}(21)$ & $87(6)$ & $63(5)$ & $82(5)$ & $19(5)$ & $-10(5)$ & $-1(5)$ \\
\hline$C(22)$ & $100(6)$ & $67(5)$ & $96(5)$ & $17(5)$ & $-4(5)$ & $-5(5)$ \\
\hline $\mathrm{C}(23)$ & $95(6)$ & $65(5)$ & $91(6)$ & $11(5)$ & $-2(5)$ & $-2(5)$ \\
\hline$C(24)$ & $86(6)$ & $57(5)$ & $82(6)$ & $14(5)$ & $0(5)$ & $-5(5)$ \\
\hline$C(25)$ & $115(6)$ & $75(5)$ & $112(6)$ & $23(6)$ & $1(5)$ & $-12(5)$ \\
\hline$C(26)$ & 151(10) & $105(9)$ & $134(10)$ & $14(9)$ & $-4(9)$ & $-38(9)$ \\
\hline$C(27)$ & $137(10)$ & $78(9)$ & $137(10)$ & $26(9)$ & $8(9)$ & $1(8)$ \\
\hline$C(28)$ & $136(10)$ & $86(9)$ & $128(10)$ & $41(8)$ & $0(9)$ & $-12(8)$ \\
\hline C(29) & $40(5)$ & $63(5)$ & $41(5)$ & $3(4)$ & $2(4)$ & $8(4)$ \\
\hline$C(30)$ & $45(5)$ & $67(5)$ & $43(5)$ & $-1(4)$ & $5(4)$ & $13(4)$ \\
\hline $\mathrm{C}(31)$ & $51(5)$ & $66(5)$ & $49(5)$ & $0(4)$ & $7(4)$ & $11(4)$ \\
\hline$C(32)$ & $50(4)$ & $65(4)$ & $58(5)$ & $7(4)$ & $3(4)$ & $13(4)$ \\
\hline$C(33)$ & $50(5)$ & $71(5)$ & $54(5)$ & $12(4)$ & $1(4)$ & $9(4)$ \\
\hline $\mathrm{C}(34)$ & $42(5)$ & $68(5)$ & $50(5)$ & $7(5)$ & $1(4)$ & $10(4)$ \\
\hline
\end{tabular}




\begin{tabular}{|c|c|c|c|c|c|c|}
\hline$C(35)$ & $54(5)$ & $77(5)$ & $75(6)$ & $7(5)$ & $1(5)$ & $17(4)$ \\
\hline$C(36)$ & $48(7)$ & $97(8)$ & $95(9)$ & $11(8)$ & $16(7)$ & $13(7)$ \\
\hline$C(37)$ & $70(8)$ & $76(8)$ & $117(9)$ & $22(8)$ & $-11(8)$ & $11(7)$ \\
\hline$C(38)$ & $63(8)$ & 103(8) & $89(9)$ & $7(8)$ & $-21(7)$ & $35(7)$ \\
\hline C(39) & $48(5)$ & $56(5)$ & $40(5)$ & $-4(4)$ & $2(4)$ & $-11(4)$ \\
\hline$C(40)$ & $55(5)$ & $58(5)$ & $47(5)$ & $-3(4)$ & $-1(4)$ & $-17(4)$ \\
\hline$C(41)$ & $60(5)$ & $63(5)$ & $54(5)$ & $-3(4)$ & $5(4)$ & $-16(4)$ \\
\hline$C(42)$ & $57(4)$ & $67(5)$ & $53(5)$ & $-8(4)$ & $5(4)$ & $-20(4)$ \\
\hline$C(43)$ & $54(5)$ & $67(5)$ & $45(5)$ & $-6(4)$ & $1(4)$ & $-17(4)$ \\
\hline$C(44)$ & $50(5)$ & $58(5)$ & $40(5)$ & $-5(4)$ & $6(4)$ & $-16(4)$ \\
\hline$C(45)$ & $71(5)$ & $79(5)$ & $69(6)$ & $-14(5)$ & $2(5)$ & $-25(5)$ \\
\hline$C(46)$ & $81(8)$ & $106(9)$ & $95(9)$ & $-22(8)$ & $-18(8)$ & $-31(8)$ \\
\hline$C(47)$ & $98(9)$ & $114(9)$ & $93(9)$ & $-31(8)$ & $9(8)$ & $-38(8)$ \\
\hline$C(48)$ & $96(8)$ & $83(8)$ & 103(9) & $-10(8)$ & $-7(8)$ & $-38(7)$ \\
\hline $\mathrm{Fe}(1 \mathrm{~A})$ & $35(1)$ & $56(1)$ & $34(1)$ & $0(1)$ & $0(1)$ & $-3(1)$ \\
\hline $\mathrm{Cl}(1 \mathrm{~A})$ & $47(2)$ & $69(3)$ & $66(3)$ & $-4(2)$ & $12(2)$ & $-8(2)$ \\
\hline $\mathrm{N}(1 \mathrm{~A})$ & $53(7)$ & $46(7)$ & $65(9)$ & $-14(6)$ & $0(6)$ & $2(6)$ \\
\hline $\mathrm{N}(2 \mathrm{~A})$ & $31(6)$ & $74(8)$ & $37(8)$ & $17(6)$ & $-8(5)$ & $-7(6)$ \\
\hline $\mathrm{N}(3 \mathrm{~A})$ & $27(6)$ & $58(7)$ & $38(7)$ & $16(6)$ & $-10(5)$ & $-11(6)$ \\
\hline $\mathrm{N}(4 \mathrm{~A})$ & $51(7)$ & $52(7)$ & $20(7)$ & $3(6)$ & $9(5)$ & $12(6)$ \\
\hline$C(1 \mathrm{~A})$ & $46(5)$ & $64(5)$ & $59(6)$ & $1(5)$ & $-4(4)$ & $-16(5)$ \\
\hline$C(2 A)$ & $43(5)$ & $60(5)$ & $58(5)$ & $5(5)$ & $-9(4)$ & $-13(5)$ \\
\hline
\end{tabular}




\begin{tabular}{|c|c|c|c|c|c|c|}
\hline$C(3 \mathrm{~A})$ & $52(5)$ & $66(5)$ & $62(6)$ & $7(5)$ & $-15(4)$ & $-10(5)$ \\
\hline$C(4 A)$ & $55(5)$ & $70(6)$ & $64(6)$ & $4(5)$ & $-17(4)$ & $-14(5)$ \\
\hline$C(5 \mathrm{~A})$ & $56(5)$ & $74(6)$ & $63(6)$ & $1(5)$ & $-14(4)$ & $-18(5)$ \\
\hline$C(6 A)$ & $55(5)$ & $70(5)$ & $60(6)$ & $1(5)$ & $-4(4)$ & $-15(5)$ \\
\hline$C(7 A)$ & $41(5)$ & $51(5)$ & $37(5)$ & $2(4)$ & $1(4)$ & $0(4)$ \\
\hline$C(8 A)$ & $45(5)$ & $55(5)$ & $36(5)$ & $8(4)$ & $1(4)$ & $4(4)$ \\
\hline$C(9 A)$ & $54(5)$ & $62(5)$ & $46(5)$ & $8(4)$ & $-3(4)$ & $5(5)$ \\
\hline$C(10 A)$ & $60(5)$ & $59(5)$ & $49(5)$ & $14(4)$ & $0(4)$ & $5(5)$ \\
\hline$C(11 A)$ & $56(5)$ & $61(5)$ & $46(5)$ & $14(5)$ & $-2(4)$ & $5(5)$ \\
\hline$C(12 A)$ & $52(5)$ & $57(5)$ & $41(5)$ & $4(4)$ & $-1(4)$ & $0(4)$ \\
\hline$C(13 A)$ & $30(4)$ & $68(6)$ & $35(5)$ & $2(4)$ & $0(4)$ & $-9(4)$ \\
\hline $\mathrm{C}(14 \mathrm{~A})$ & $31(4)$ & $74(5)$ & $32(5)$ & $2(5)$ & $-4(4)$ & $-8(4)$ \\
\hline$C(15 A)$ & $35(4)$ & $80(6)$ & $34(5)$ & $4(5)$ & $-1(4)$ & $-6(4)$ \\
\hline$C(16 A)$ & $39(5)$ & $86(6)$ & $34(5)$ & $1(5)$ & $4(4)$ & $-7(5)$ \\
\hline $\mathrm{C}(17 \mathrm{~A})$ & $38(4)$ & $78(5)$ & $40(5)$ & $0(5)$ & $6(4)$ & $-4(5)$ \\
\hline $\mathrm{C}(18 \mathrm{~A})$ & $35(5)$ & $72(6)$ & $37(5)$ & $4(5)$ & $5(4)$ & $-5(4)$ \\
\hline$C(19 A)$ & $54(5)$ & $61(5)$ & $48(5)$ & $-2(5)$ & $-8(5)$ & $-8(5)$ \\
\hline$C(20 A)$ & $55(5)$ & $60(5)$ & $48(5)$ & $1(5)$ & $21(4)$ & $-8(5)$ \\
\hline$C(21 \mathrm{~A})$ & $60(5)$ & $63(5)$ & $61(5)$ & $-1(5)$ & $-5(4)$ & $-5(4)$ \\
\hline $\mathrm{C}(22 \mathrm{~A})$ & $74(5)$ & $64(5)$ & $83(5)$ & $4(4)$ & $32(4)$ & $-2(4)$ \\
\hline$C(23 A)$ & $68(5)$ & $58(5)$ & $72(5)$ & $3(5)$ & $33(5)$ & $-8(5)$ \\
\hline $\mathrm{C}(24 \mathrm{~A})$ & $60(5)$ & $62(5)$ & $60(5)$ & $1(5)$ & $32(4)$ & $-4(5)$ \\
\hline
\end{tabular}




\begin{tabular}{|c|c|c|c|c|c|c|}
\hline$C(25 \mathrm{~A})$ & $91(6)$ & $81(6)$ & $115(6)$ & $13(5)$ & $42(6)$ & $6(5)$ \\
\hline$C(26 A)$ & $144(10)$ & $120(9)$ & $148(10)$ & $39(9)$ & $52(9)$ & $25(9)$ \\
\hline$C(27 \mathrm{~A})$ & $115(10)$ & $112(9)$ & $172(11)$ & $28(9)$ & $45(10)$ & $21(9)$ \\
\hline $\mathrm{C}(28 \mathrm{~A})$ & $620(70)$ & $190(30)$ & $160(30)$ & $-60(20)$ & $-130(40)$ & $-10(40)$ \\
\hline$C(29 A)$ & $36(4)$ & $54(5)$ & $38(5)$ & $0(4)$ & $1(4)$ & $-5(4)$ \\
\hline$C(30 A)$ & $39(4)$ & $61(5)$ & $42(5)$ & $0(4)$ & $1(4)$ & $-6(4)$ \\
\hline $\mathrm{C}(31 \mathrm{~A})$ & $40(4)$ & $62(5)$ & $45(5)$ & $-1(4)$ & $2(4)$ & $-7(4)$ \\
\hline$C(32 A)$ & $40(4)$ & $61(4)$ & $50(5)$ & $-8(4)$ & $-3(4)$ & $-3(4)$ \\
\hline$C(33 A)$ & $38(4)$ & $61(5)$ & $43(5)$ & $-5(4)$ & $-4(4)$ & $-4(4)$ \\
\hline$C(34 \mathrm{~A})$ & $36(4)$ & $57(5)$ & $40(5)$ & $1(4)$ & $0(4)$ & $-4(4)$ \\
\hline $\mathrm{C}(35 \mathrm{~A})$ & $48(5)$ & $64(5)$ & $63(5)$ & $-10(5)$ & $-5(4)$ & $-5(4)$ \\
\hline$C(36 A)$ & $47(7)$ & $87(8)$ & $88(9)$ & $-11(7)$ & $1(7)$ & $-19(7)$ \\
\hline$C(37 A)$ & $67(8)$ & $78(8)$ & $99(9)$ & $-12(8)$ & $-6(7)$ & $-16(7)$ \\
\hline $\mathrm{C}(38 \mathrm{~A})$ & $68(8)$ & $85(8)$ & $70(8)$ & $-13(7)$ & $-19(7)$ & $-8(7)$ \\
\hline$C(39 A)$ & $54(5)$ & $52(5)$ & $56(6)$ & $3(5)$ & $-7(4)$ & $-8(4)$ \\
\hline$C(40 A)$ & $62(5)$ & $58(5)$ & $60(6)$ & $4(5)$ & $-10(4)$ & $-7(4)$ \\
\hline$C(41 A)$ & $65(5)$ & $60(5)$ & $71(6)$ & $9(5)$ & $-11(4)$ & $0(4)$ \\
\hline$C(42 A)$ & $70(5)$ & $70(5)$ & $79(5)$ & $14(4)$ & $-13(4)$ & $3(4)$ \\
\hline$C(43 A)$ & $60(5)$ & $68(5)$ & $66(6)$ & $4(5)$ & $-9(4)$ & $-1(4)$ \\
\hline $\mathrm{C}(44 \mathrm{~A})$ & $56(5)$ & $58(5)$ & $57(6)$ & $0(5)$ & $-7(4)$ & $-2(4)$ \\
\hline$C(45 A)$ & $92(6)$ & $89(6)$ & $106(6)$ & $26(5)$ & $-22(5)$ & $10(5)$ \\
\hline$C(46 A)$ & $139(10)$ & $137(10)$ & $132(10)$ & $36(9)$ & $-9(9)$ & $43(9)$ \\
\hline
\end{tabular}




\begin{tabular}{|c|c|c|c|c|c|c|}
\hline $\mathrm{C}(47 \mathrm{~A})$ & $122(10)$ & $123(10)$ & 159(11) & $67(9)$ & $-35(9)$ & $15(9)$ \\
\hline $\mathrm{C}(48 \mathrm{~A})$ & $135(10)$ & $118(10)$ & $137(10)$ & $2(9)$ & $-59(9)$ & $22(9)$ \\
\hline $\mathrm{N}(7)$ & $54(8)$ & $83(9)$ & $39(8)$ & $-17(7)$ & $9(6)$ & $-13(7)$ \\
\hline$C(65)$ & $69(10)$ & $103(11)$ & $78(11)$ & $-23(9)$ & $21(8)$ & $-18(9)$ \\
\hline$C(66)$ & $68(10)$ & 111(11) & $86(11)$ & $-34(9)$ & $16(8)$ & $-6(9)$ \\
\hline$C(67)$ & $53(8)$ & $104(11)$ & $88(11)$ & $-27(9)$ & $19(8)$ & $-31(8)$ \\
\hline$C(68)$ & $69(9)$ & 103(11) & $100(11)$ & $-33(9)$ & $12(8)$ & $-28(8)$ \\
\hline C(69) & $60(8)$ & $81(10)$ & $70(10)$ & $-21(7)$ & $42(8)$ & $-26(7)$ \\
\hline$C(70)$ & $62(8)$ & $97(10)$ & $88(10)$ & $-26(8)$ & $33(8)$ & $-32(8)$ \\
\hline$C(71)$ & $46(8)$ & $55(9)$ & $72(10)$ & $-7(7)$ & $5(7)$ & $-11(7)$ \\
\hline$C(72)$ & $71(9)$ & $62(9)$ & $89(10)$ & $-21(8)$ & $11(8)$ & $-6(7)$ \\
\hline $\mathrm{N}(5)$ & $52(8)$ & $90(10)$ & $72(10)$ & $-24(8)$ & $-13(7)$ & $11(7)$ \\
\hline C(49) & $79(9)$ & $148(12)$ & $65(10)$ & $-26(9)$ & $-14(9)$ & $6(10)$ \\
\hline$C(50)$ & $74(9)$ & $159(13)$ & $78(10)$ & $-20(9)$ & $-13(9)$ & $22(10)$ \\
\hline $\mathrm{C}(51)$ & 121(11) & $77(11)$ & $83(12)$ & $16(10)$ & $51(9)$ & $4(10)$ \\
\hline$C(52)$ & $137(11)$ & $95(11)$ & $105(12)$ & $14(10)$ & $46(10)$ & $-10(10)$ \\
\hline$C(53)$ & $84(10)$ & $103(10)$ & $57(10)$ & $5(8)$ & $1(8)$ & $-16(9)$ \\
\hline$C(54)$ & $84(10)$ & $114(11)$ & $72(10)$ & $13(9)$ & $-23(8)$ & $-18(9)$ \\
\hline$C(55)$ & $246(19)$ & $91(13)$ & $90(14)$ & $2(9)$ & $-48(14)$ & $-17(13)$ \\
\hline$C(56)$ & $250(19)$ & $94(13)$ & $110(14)$ & $14(9)$ & $-51(14)$ & $-15(13)$ \\
\hline $\mathrm{N}(6)$ & $35(6)$ & $56(7)$ & $47(8)$ & $-3(6)$ & $11(6)$ & $15(6)$ \\
\hline$C(57)$ & $56(8)$ & $64(8)$ & $66(9)$ & $-15(7)$ & $18(7)$ & $-5(7)$ \\
\hline
\end{tabular}




\begin{tabular}{|c|c|c|c|c|c|c|}
\hline$C(58)$ & $53(8)$ & $67(8)$ & $82(10)$ & $-19(7)$ & $21(7)$ & $-17(7)$ \\
\hline$C(59)$ & $43(7)$ & $76(9)$ & $51(9)$ & $-4(7)$ & $-2(6)$ & $14(7)$ \\
\hline$C(60)$ & $75(9)$ & $87(10)$ & $68(9)$ & $-12(8)$ & $0(7)$ & $9(7)$ \\
\hline$C(61)$ & $45(7)$ & $89(11)$ & $82(11)$ & $14(8)$ & $3(8)$ & $4(7)$ \\
\hline$C(62)$ & $56(8)$ & $94(10)$ & $88(11)$ & $30(8)$ & $11(8)$ & $6(7)$ \\
\hline$C(63)$ & $72(9)$ & $56(8)$ & $55(9)$ & $8(7)$ & $1(7)$ & $5(7)$ \\
\hline$C(64)$ & $80(9)$ & $76(9)$ & $78(10)$ & $10(8)$ & $-7(8)$ & $8(8)$ \\
\hline $\mathrm{N}(8)$ & $69(9)$ & $77(9)$ & $38(8)$ & $-2(7)$ & $1(6)$ & $17(7)$ \\
\hline$C(73)$ & $58(8)$ & $108(10)$ & $61(9)$ & $9(8)$ & $-8(8)$ & $-21(8)$ \\
\hline$C(74)$ & $68(8)$ & $123(10)$ & $85(10)$ & $20(8)$ & $-14(8)$ & $-31(8)$ \\
\hline$C(75)$ & 87(11) & $68(11)$ & $-153(17)$ & $4(10)$ & $24(10)$ & $-16(9)$ \\
\hline$C(76)$ & $103(11)$ & $76(11)$ & $168(17)$ & $-17(10)$ & $9(10)$ & $-3(9)$ \\
\hline$C(77)$ & $32(8)$ & $113(11)$ & $95(12)$ & $-33(9)$ & $17(7)$ & $-7(8)$ \\
\hline $\mathrm{C}(78)$ & $36(8)$ & $116(11)$ & $114(13)$ & $-34(10)$ & $6(7)$ & $-5(8)$ \\
\hline$C(79)$ & $71(8)$ & $94(11)$ & $80(10)$ & $-10(10)$ & $6(8)$ & $5(9)$ \\
\hline$C(80)$ & $79(8)$ & $90(11)$ & $89(10)$ & $-8(9)$ & $25(8)$ & $1(9)$ \\
\hline
\end{tabular}


Table S53. Hydrogen coordinates $\left(\times 10^{4}\right)$ and isotropic displacement parameters $\left(\AA^{2} \times 10^{3}\right)$ for $\left[\left(\mathrm{L}^{1}{ }_{\mathrm{re}}\right)(\mathrm{Cl}) \mathrm{Fe}(\mathrm{II})\right]\left[\mathrm{Et}_{4} \mathrm{~N}\right]_{2}(7)$

\begin{tabular}{|c|c|c|c|c|}
\hline & $\mathrm{X}$ & $\mathrm{y}$ & Z & $\mathrm{U}(\mathrm{eq})$ \\
\hline $\mathrm{H}(3 \mathrm{~A})$ & -3273 & 2158 & 210 & 70 \\
\hline $\mathrm{H}(4 \mathrm{~A})$ & -4454 & 2641 & -427 & 75 \\
\hline $\mathrm{H}(5 \mathrm{~A})$ & -3845 & 3351 & -456 & 75 \\
\hline $\mathrm{H}(6 \mathrm{~A})$ & -1924 & 3591 & 35 & 71 \\
\hline $\mathrm{H}(9 \mathrm{~A})$ & -16 & 1022 & 1675 & 66 \\
\hline $\mathrm{H}(10 \mathrm{~A})$ & -999 & 665 & 801 & 71 \\
\hline $\mathrm{H}(11 \mathrm{~A})$ & -1982 & 1020 & -63 & 70 \\
\hline $\mathrm{H}(12 \mathrm{~A})$ & -1975 & 1766 & -94 & 67 \\
\hline $\mathrm{H}(15 \mathrm{~A})$ & -569 & 2793 & 3425 & 55 \\
\hline $\mathrm{H}(16 \mathrm{~A})$ & -2079 & 2394 & 3827 & 55 \\
\hline $\mathrm{H}(17 \mathrm{~A})$ & -2434 & 1712 & 3434 & 58 \\
\hline $\mathrm{H}(18 \mathrm{~A})$ & -1516 & 1453 & 2508 & 54 \\
\hline $\mathrm{H}(20 \mathrm{~A})$ & 1897 & 3357 & 620 & 84 \\
\hline $\mathrm{H}(21 \mathrm{~A})$ & 2834 & 4010 & 737 & 94 \\
\hline $\mathrm{H}(23 \mathrm{~A})$ & -517 & 4510 & 1021 & 101 \\
\hline $\mathrm{H}(24 \mathrm{~A})$ & -1336 & 3833 & 1052 & 90 \\
\hline $\mathrm{H}(26 \mathrm{~A})$ & 2887 & 5113 & 1694 & 196 \\
\hline $\mathrm{H}(26 \mathrm{~B})$ & 3245 & 4632 & 1616 & 196 \\
\hline $\mathrm{H}(26 \mathrm{C})$ & 2063 & 4755 & 1976 & 196 \\
\hline
\end{tabular}




\begin{tabular}{|c|c|c|c|}
\hline $\mathrm{H}(27 \mathrm{~A})$ & 483 & 5172 & 579 \\
\hline $\mathrm{H}(27 \mathrm{~B})$ & 1441 & 5413 & 1068 \\
\hline $\mathrm{H}(27 \mathrm{C})$ & 450 & 5111 & 1348 \\
\hline $\mathrm{H}(28 \mathrm{~A})$ & 1946 & 4909 & 14 \\
\hline $\mathrm{H}(28 \mathrm{~B})$ & 3168 & 4734 & 389 \\
\hline $\mathrm{H}(28 \mathrm{C})$ & 2789 & 5212 & 473 \\
\hline $\mathrm{H}(30 \mathrm{~A})$ & 2055 & 1487 & 1242 \\
\hline $\mathrm{H}(31 \mathrm{~A})$ & 3763 & 1111 & 1636 \\
\hline $\mathrm{H}(33 \mathrm{~A})$ & 2960 & 1380 & 3507 \\
\hline $\mathrm{H}(34 \mathrm{~A})$ & 1252 & 1759 & 3093 \\
\hline $\mathrm{H}(36 \mathrm{~A})$ & 5611 & 961 & 2041 \\
\hline $\mathrm{H}(36 \mathrm{~B})$ & 6507 & 818 & 2648 \\
\hline $\mathrm{H}(36 \mathrm{C})$ & 6141 & 1299 & 2560 \\
\hline $\mathrm{H}(37 \mathrm{~A})$ & 4099 & 385 & 2403 \\
\hline $\mathrm{H}(37 \mathrm{~B})$ & 3611 & 420 & 3112 \\
\hline $\mathrm{H}(37 \mathrm{C})$ & 4982 & 277 & 3034 \\
\hline $\mathrm{H}(38 \mathrm{~A})$ & 5835 & 831 & 3761 \\
\hline $\mathrm{H}(38 \mathrm{~B})$ & 4465 & 949 & 3890 \\
\hline $\mathrm{H}(38 \mathrm{C})$ & 5388 & 1305 & 3695 \\
\hline $\mathrm{H}(40 \mathrm{~A})$ & 1167 & 3445 & 1964 \\
\hline $\mathrm{H}(41 \mathrm{~A})$ & 2190 & 3961 & 2626 \\
\hline $\mathrm{H}(43 \mathrm{~A})$ & 3050 & 3130 & 4048 \\
\hline
\end{tabular}




\begin{tabular}{|c|c|c|c|c|}
\hline $\mathrm{H}(44 \mathrm{~A})$ & 1780 & 2642 & 3513 & 59 \\
\hline $\mathrm{H}(46 \mathrm{~A})$ & 5195 & 3709 & 3619 & 143 \\
\hline $\mathrm{H}(46 \mathrm{~B})$ & 4734 & 3524 & 4278 & 143 \\
\hline $\mathrm{H}(46 \mathrm{C})$ & 5263 & 3989 & 4264 & 143 \\
\hline $\mathrm{H}(47 \mathrm{~A})$ & 2014 & 4186 & 4270 & 152 \\
\hline $\mathrm{H}(47 \mathrm{~B})$ & 3292 & 4278 & 4667 & 152 \\
\hline $\mathrm{H}(47 \mathrm{C})$ & 2707 & 3821 & 4680 & 152 \\
\hline $\mathrm{H}(48 \mathrm{~A})$ & 2884 & 4469 & 3301 & 142 \\
\hline $\mathrm{H}(48 \mathrm{~B})$ & 4067 & 4263 & 3031 & 142 \\
\hline $\mathrm{H}(48 \mathrm{C})$ & 4187 & 4532 & 3687 & 142 \\
\hline $\mathrm{H}(3 \mathrm{AA})$ & 3208 & 7242 & 1976 & 73 \\
\hline $\mathrm{H}(4 \mathrm{AA})$ & 1942 & 6741 & 1426 & 77 \\
\hline $\mathrm{H}(5 \mathrm{AA})$ & 2505 & 6084 & 1365 & 78 \\
\hline $\mathrm{H}(6 \mathrm{AA})$ & 4411 & 5876 & 1872 & 74 \\
\hline $\mathrm{H}(9 \mathrm{AA})$ & 6285 & 8404 & 3525 & 65 \\
\hline $\mathrm{H}(10 \mathrm{~B})$ & 5341 & 8780 & 2629 & 67 \\
\hline $\mathrm{H}(11 \mathrm{~B})$ & 4394 & 8364 & 1725 & 66 \\
\hline $\mathrm{H}(12 \mathrm{C})$ & 4458 & 7652 & 1762 & 60 \\
\hline $\mathrm{H}(15 \mathrm{~B})$ & 5732 & 6614 & 5202 & 60 \\
\hline $\mathrm{H}(16 \mathrm{~B})$ & 4435 & 7099 & 5695 & 63 \\
\hline $\mathrm{H}(17 \mathrm{~B})$ & 3916 & 7751 & 5215 & 62 \\
\hline $\mathrm{H}(18 \mathrm{~B})$ & 4930 & 7949 & 4356 & 58 \\
\hline
\end{tabular}




\begin{tabular}{|c|c|c|c|c|}
\hline $\mathrm{H}(20 \mathrm{~B})$ & 8344 & 6043 & 2455 & 64 \\
\hline $\mathrm{H}(21 \mathrm{~B})$ & 9118 & 5386 & 2548 & 72 \\
\hline $\mathrm{H}(23 \mathrm{~B})$ & 5903 & 4942 & 3091 & 78 \\
\hline $\mathrm{H}(24 \mathrm{C})$ & 5066 & 5589 & 2873 & 71 \\
\hline $\mathrm{H}(26 \mathrm{D})$ & 7815 & 4423 & 3842 & 203 \\
\hline $\mathrm{H}(26 \mathrm{E})$ & 8015 & 4062 & 3324 & 203 \\
\hline $\mathrm{H}(26 \mathrm{~F})$ & 6797 & 4336 & 3260 & 203 \\
\hline $\mathrm{H}(27 \mathrm{D})$ & 9903 & 4733 & 3233 & 197 \\
\hline $\mathrm{H}(27 \mathrm{E})$ & 9541 & 4739 & 2466 & 197 \\
\hline $\mathrm{H}(27 \mathrm{~F})$ & 9624 & 4305 & 2851 & 197 \\
\hline $\mathrm{H}(28 \mathrm{D})$ & 8083 & 4035 & 2303 & 497 \\
\hline $\mathrm{H}(28 \mathrm{E})$ & 8075 & 4449 & 1867 & 497 \\
\hline $\mathrm{H}(28 \mathrm{~F})$ & 6871 & 4310 & 2195 & 497 \\
\hline $\mathrm{H}(30 \mathrm{~B})$ & 8431 & 7929 & 3100 & 57 \\
\hline $\mathrm{H}(31 \mathrm{~B})$ & 10140 & 8325 & 3516 & 59 \\
\hline $\mathrm{H}(33 \mathrm{~B})$ & 9491 & 8004 & 5340 & 57 \\
\hline $\mathrm{H}(34 \mathrm{~B})$ & 7662 & 7694 & 4934 & 53 \\
\hline $\mathrm{H}(36 \mathrm{D})$ & 12499 & 8124 & 4382 & 111 \\
\hline $\mathrm{H}(36 \mathrm{E})$ & 12899 & 8604 & 4450 & 111 \\
\hline $\mathrm{H}(36 \mathrm{~F})$ & 12001 & 8457 & 3846 & 111 \\
\hline $\mathrm{H}(37 \mathrm{D})$ & 11449 & 9155 & 4762 & 123 \\
\hline $\mathrm{H}(37 \mathrm{E})$ & 10080 & 9041 & 4900 & 123 \\
\hline
\end{tabular}




\begin{tabular}{|c|c|c|c|}
\hline $\mathrm{H}(37 \mathrm{~F})$ & 10484 & 9034 & 4171 \\
\hline $\mathrm{H}(38 \mathrm{D})$ & 11731 & 8076 & 5472 \\
\hline $\mathrm{H}(38 \mathrm{E})$ & 10817 & 8418 & 5722 \\
\hline $\mathrm{H}(38 \mathrm{~F})$ & 12196 & 8542 & 5625 \\
\hline $\mathrm{H}(40 \mathrm{C})$ & 7654 & 6000 & 3877 \\
\hline $\mathrm{H}(41 \mathrm{~B})$ & 8928 & 5511 & 4393 \\
\hline $\mathrm{H}(43 \mathrm{~B})$ & 9354 & 6323 & 5946 \\
\hline $\mathrm{H}(44 \mathrm{~B})$ & 8212 & 6817 & 5356 \\
\hline $\mathrm{H}(46 \mathrm{D})$ & 11421 & 5103 & 5551 \\
\hline $\mathrm{H}(46 \mathrm{E})$ & 11497 & 5497 & 5077 \\
\hline $\mathrm{H}(46 \mathrm{~F})$ & 10487 & 5144 & 4919 \\
\hline $\mathrm{H}(47 \mathrm{D})$ & 8932 & 5353 & 6240 \\
\hline $\mathrm{H}(47 \mathrm{E})$ & 10036 & 5038 & 6158 \\
\hline $\mathrm{H}(47 \mathrm{~F})$ & 8969 & 5081 & 5590 \\
\hline $\mathrm{H}(48 \mathrm{D})$ & 10815 & 5887 & 6411 \\
\hline $\mathrm{H}(48 \mathrm{E})$ & 11705 & 5827 & 5841 \\
\hline $\mathrm{H}(48 \mathrm{~F})$ & 11502 & 5452 & 6335 \\
\hline $\mathrm{H}(65 \mathrm{~A})$ & 3087 & 6515 & 4664 \\
\hline $\mathrm{H}(65 \mathrm{~B})$ & 2295 & 6121 & 4412 \\
\hline $\mathrm{H}(66 \mathrm{~A})$ & 4368 & 5998 & 4303 \\
\hline $\mathrm{H}(66 \mathrm{~B})$ & 4423 & 6398 & 3841 \\
\hline $\mathrm{H}(66 \mathrm{C})$ & 3637 & 5999 & 3600 \\
\hline
\end{tabular}




\begin{tabular}{|c|c|c|c|c|}
\hline $\mathrm{H}(67 \mathrm{~A})$ & 2411 & 6396 & 2900 & 97 \\
\hline $\mathrm{H}(67 \mathrm{~B})$ & 1111 & 6617 & 2876 & 97 \\
\hline $\mathrm{H}(68 \mathrm{~A})$ & 827 & 5892 & 2740 & 136 \\
\hline $\mathrm{H}(68 \mathrm{~B})$ & 320 & 6024 & 3417 & 136 \\
\hline $\mathrm{H}(68 \mathrm{C})$ & 1618 & 5801 & 3410 & 136 \\
\hline $\mathrm{H}(69 \mathrm{~A})$ & 328 & 6946 & 3825 & 82 \\
\hline H(69B) & 331 & 6496 & 4164 & 82 \\
\hline $\mathrm{H}(70 \mathrm{~A})$ & 319 & 7036 & 4972 & 121 \\
\hline $\mathrm{H}(70 \mathrm{~B})$ & 1576 & 7213 & 4746 & 121 \\
\hline $\mathrm{H}(70 \mathrm{C})$ & 1535 & 6764 & 5087 & 121 \\
\hline $\mathrm{H}(71 \mathrm{~A})$ & 3130 & 7133 & 4113 & 69 \\
\hline $\mathrm{H}(71 \mathrm{~B})$ & 3508 & 6934 & 3445 & 69 \\
\hline $\mathrm{H}(72 \mathrm{~A})$ & 2651 & 7590 & 3259 & 110 \\
\hline $\mathrm{H}(72 \mathrm{~B})$ & 1430 & 7439 & 3558 & 110 \\
\hline $\mathrm{H}(72 \mathrm{C})$ & 1813 & 7242 & 2891 & 110 \\
\hline H(49A) & 3335 & 1074 & 10468 & 118 \\
\hline $\mathrm{H}(49 \mathrm{~B})$ & 3661 & 1551 & 10331 & 118 \\
\hline $\mathrm{H}(50 \mathrm{~A})$ & 5533 & 1165 & 10560 & 157 \\
\hline $\mathrm{H}(50 \mathrm{~B})$ & 5485 & 1324 & 9822 & 157 \\
\hline $\mathrm{H}(50 \mathrm{C})$ & 5163 & 848 & 9977 & 157 \\
\hline $\mathrm{H}(51 \mathrm{~A})$ & 4266 & 1382 & 8825 & 110 \\
\hline $\mathrm{H}(51 \mathrm{~B})$ & 2909 & 1493 & 8541 & 110 \\
\hline
\end{tabular}




\begin{tabular}{|c|c|c|c|}
\hline $\mathrm{H}(52 \mathrm{~A})$ & 4009 & 2105 & 8790 \\
\hline $\mathrm{H}(52 \mathrm{~B})$ & 4246 & 1958 & 9531 \\
\hline $\mathrm{H}(52 \mathrm{C})$ & 2897 & 2070 & 9240 \\
\hline $\mathrm{H}(53 \mathrm{~A})$ & 1298 & 1193 & 9979 \\
\hline $\mathrm{H}(53 \mathrm{~B})$ & 1597 & 1666 & 9801 \\
\hline $\mathrm{H}(54 \mathrm{~A})$ & -165 & 1431 & 9110 \\
\hline $\mathrm{H}(54 \mathrm{~B})$ & 613 & 1057 & 8839 \\
\hline $\mathrm{H}(54 \mathrm{C})$ & 916 & 1530 & 8659 \\
\hline $\mathrm{H}(55 \mathrm{~A})$ & 2656 & 740 & 8813 \\
\hline $\mathrm{H}(55 \mathrm{~B})$ & 3978 & 718 & 9185 \\
\hline $\mathrm{H}(56 \mathrm{~A})$ & 2771 & 131 & 9494 \\
\hline $\mathrm{H}(56 \mathrm{~B})$ & 1794 & 442 & 9765 \\
\hline $\mathrm{H}(56 \mathrm{C})$ & 3132 & 410 & 10124 \\
\hline $\mathrm{H}(57 \mathrm{~A})$ & 6081 & 3327 & 2559 \\
\hline $\mathrm{H}(57 \mathrm{~B})$ & 6816 & 2923 & 2816 \\
\hline $\mathrm{H}(58 \mathrm{~A})$ & 8145 & 3407 & 2434 \\
\hline $\mathrm{H}(58 \mathrm{~B})$ & 7399 & 3412 & 1736 \\
\hline $\mathrm{H}(58 \mathrm{C})$ & 8128 & 3001 & 1979 \\
\hline $\mathrm{H}(59 \mathrm{~A})$ & 7197 & 2377 & 1969 \\
\hline $\mathrm{H}(60 \mathrm{~A})$ & 6107 & 1771 & 1443 \\
\hline $\mathrm{H}(60 \mathrm{~B})$ & 5196 & 2097 & 1073 \\
\hline $\mathrm{H}(60 \mathrm{C})$ & 4916 & 1921 & 1773 \\
\hline
\end{tabular}




\begin{tabular}{|c|c|c|c|}
\hline $\mathrm{H}(61 \mathrm{~A})$ & 4175 & 2969 & 2514 \\
\hline $\mathrm{H}(61 \mathrm{~B})$ & 3937 & 2558 & 2088 \\
\hline $\mathrm{H}(62 \mathrm{~A})$ & 3962 & 2386 & 3191 \\
\hline $\mathrm{H}(62 \mathrm{~B})$ & 5297 & 2578 & 3327 \\
\hline $\mathrm{H}(62 \mathrm{C})$ & 5098 & 2164 & 2896 \\
\hline $\mathrm{H}(63 \mathrm{~A})$ & 4700 & 2825 & 1107 \\
\hline $\mathrm{H}(63 \mathrm{~B})$ & 6012 & 3036 & 1096 \\
\hline $\mathrm{H}(64 \mathrm{~A})$ & 4472 & 3573 & 985 \\
\hline $\mathrm{H}(64 \mathrm{~B})$ & 5281 & 3646 & 1654 \\
\hline $\mathrm{H}(64 \mathrm{C})$ & 3967 & 3435 & 1659 \\
\hline $\mathrm{H}(73 \mathrm{~A})$ & 9770 & 8294 & 2317 \\
\hline $\mathrm{H}(73 \mathrm{~B})$ & 9909 & 7815 & 2119 \\
\hline $\mathrm{H}(74 \mathrm{~A})$ & 11830 & 8117 & 2339 \\
\hline $\mathrm{H}(74 \mathrm{~B})$ & 11529 & 8461 & 1786 \\
\hline $\mathrm{H}(74 \mathrm{C})$ & 11670 & 7980 & 1590 \\
\hline $\mathrm{H}(75 \mathrm{~A})$ & 10010 & 8748 & 1067 \\
\hline $\mathrm{H}(75 \mathrm{~B})$ & 8697 & 8680 & 692 \\
\hline $\mathrm{H}(76 \mathrm{~A})$ & 8614 & 9220 & 1448 \\
\hline $\mathrm{H}(76 \mathrm{~B})$ & 9091 & 8911 & 2023 \\
\hline $\mathrm{H}(76 \mathrm{C})$ & 7777 & 8841 & 1651 \\
\hline $\mathrm{H}(77 \mathrm{~A})$ & 7998 & 7713 & 1583 \\
\hline $\mathrm{H}(77 \mathrm{~B})$ & 7642 & 8161 & 1857 \\
\hline
\end{tabular}




\begin{tabular}{|c|c|c|c|c|}
\hline $\mathrm{H}(78 \mathrm{~A})$ & 6211 & 7951 & 1013 & 133 \\
\hline $\mathrm{H}(78 \mathrm{~B})$ & 7263 & 7923 & 522 & 133 \\
\hline $\mathrm{H}(78 \mathrm{C})$ & 6886 & 8366 & 805 & 133 \\
\hline $\mathrm{H}(79 \mathrm{~A})$ & 9229 & 7952 & 389 & 98 \\
\hline H(79B) & 10556 & 8036 & 727 & 98 \\
\hline $\mathrm{H}(80 \mathrm{~A})$ & 10246 & 7342 & 525 & 127 \\
\hline $\mathrm{H}(80 \mathrm{~B})$ & 9127 & 7340 & 974 & 127 \\
\hline $\mathrm{H}(80 \mathrm{C})$ & 10478 & 7422 & 1291 & 127 \\
\hline $\mathrm{H}(1 \mathrm{SA})$ & 7091 & 9218 & 3199 & 261 \\
\hline $\mathrm{H}(1 \mathrm{SB})$ & 7675 & 9453 & 3834 & 261 \\
\hline $\mathrm{H}(1 \mathrm{SC})$ & 7023 & 9716 & 3245 & 261 \\
\hline $\mathrm{H}(2 \mathrm{SA})$ & 5101 & 9424 & 3458 & 215 \\
\hline $\mathrm{H}(2 \mathrm{SB})$ & 5740 & 9147 & 4032 & 215 \\
\hline $\mathrm{H}(3 \mathrm{SA})$ & 3737 & 9848 & 4128 & 179 \\
\hline $\mathrm{H}(3 \mathrm{SB})$ & 4101 & 9536 & 4723 & 179 \\
\hline $\mathrm{H}(4 \mathrm{SA})$ & 3519 & 10183 & 5083 & 254 \\
\hline $\mathrm{H}(4 \mathrm{SB})$ & 4616 & 10391 & 4735 & 254 \\
\hline $\mathrm{H}(4 \mathrm{SC})$ & 4896 & 10077 & 5331 & 254 \\
\hline
\end{tabular}


Table S54. Torsion Angles, ${ }^{\circ}$, for $\left[\left(\mathrm{L}^{1}{ }_{\mathrm{re}}\right)(\mathrm{Cl}) \mathrm{Fe}(\mathrm{II})\right]\left[\mathrm{Et}_{4} \mathrm{~N}\right]_{2}(7)$

\begin{tabular}{|c|c|c|c|}
\hline N4-Fe1-N1-C1 & $-110.6(9)$ & N1A-Fe1A-N3A-C13A & $67.5(10)$ \\
\hline $\mathrm{N} 2-\mathrm{Fe} 1-\mathrm{N} 1-\mathrm{C} 1$ & $5.1(9)$ & N2A-Fe1A-N3A-C13A & $91.1(7)$ \\
\hline Cl1-Fe1-N1-C1 & $114.9(8)$ & C11A-Fe1A-N3A-C13A & $-158.7(6)$ \\
\hline N3-Fe1-N1-C1 & $-15.8(13)$ & N1A-Fe1A-N4A-C14A & $-108.6(9)$ \\
\hline N4-Fe1-N1-C19 & $59.6(12)$ & $\mathrm{N} 2 \mathrm{~A}-\mathrm{Fe} 1 \mathrm{~A}-\mathrm{N} 4 \mathrm{~A}-\mathrm{C} 14 \mathrm{~A}$ & $-19.2(10)$ \\
\hline N2-Fe1-N1-C19 & $175.3(12)$ & C11A-Fe1A-N4A-C14A & $123.2(8)$ \\
\hline C11-Fe1-N1-C19 & $-74.9(11)$ & N3A-Fe1A-N4A-C14A & $36.7(8)$ \\
\hline N3-Fe1-N1-C19 & $154.5(10)$ & N1A-Fe1A-N4A-C39A & $67.4(11)$ \\
\hline N4-Fe1-N2-C7 & $-86.8(10)$ & N2A-Fe1A-N4A-C39A & $156.7(10)$ \\
\hline N1-Fe1-N2-C7 & $160.6(9)$ & C11A-Fe1A-N4A-C39A & $-60.9(11)$ \\
\hline $\mathrm{C} 11-\mathrm{Fe} 1-\mathrm{N} 2-\mathrm{C} 7$ & $56.6(9)$ & N3A-Fe1A-N4A-C39A & $-147.4(10)$ \\
\hline N3-Fe1-N2-C7 & $-31.0(8)$ & C19A-N1A-C1A-C2A & $176.9(12)$ \\
\hline N4-Fe1-N2-C2 & $110.8(8)$ & Fe1A-N1A-C1A-C2A & $8.7(16)$ \\
\hline N1-Fe1-N2-C2 & $-1.8(8)$ & C19A-N1A-C1A-C6A & $-9(2)$ \\
\hline $\mathrm{C} 11-\mathrm{Fe} 1-\mathrm{N} 2-\mathrm{C} 2$ & $-105.8(8)$ & Fe1A-N1A-C1A-C6A & $-177.4(11)$ \\
\hline N3-Fe1-N2-C2 & $166.5(9)$ & C6A-C1A-C2A-C3A & $4(2)$ \\
\hline N4-Fe1-N3-C29 & $-79.7(9)$ & $\mathrm{N} 1 \mathrm{~A}-\mathrm{C} 1 \mathrm{~A}-\mathrm{C} 2 \mathrm{~A}-\mathrm{C} 3 \mathrm{~A}$ & $178.4(12)$ \\
\hline N2-Fe1-N3-C29 & $151.0(9)$ & C6A-C1A-C2A-N2A & $177.4(11)$ \\
\hline N1-Fe1-N3-C29 & $173.1(8)$ & N1A-C1A-C2A-N2A & $-8.1(18)$ \\
\hline C11-Fe1-N3-C29 & $40.3(8)$ & C7A-N2A-C2A-C3A & $-22(2)$ \\
\hline N4-Fe1-N3-C8 & $155.2(7)$ & Fe1A-N2A-C2A-C3A & $176.3(11)$ \\
\hline
\end{tabular}




\begin{tabular}{|c|c|c|c|}
\hline N2-Fe1-N3-C8 & $25.8(7)$ & C7A-N2A-C2A-C1A & $165.4(12)$ \\
\hline N1-Fe1-N3-C8 & $48.0(10)$ & Fe1A-N2A-C2A-C1A & $3.7(15)$ \\
\hline C11-Fe1-N3-C8 & $-84.9(7)$ & $\mathrm{C} 1 \mathrm{~A}-\mathrm{C} 2 \mathrm{~A}-\mathrm{C} 3 \mathrm{~A}-\mathrm{C} 4 \mathrm{~A}$ & $-1(2)$ \\
\hline N4-Fe1-N3-C13 & $39.1(6)$ & $\mathrm{N} 2 \mathrm{~A}-\mathrm{C} 2 \mathrm{~A}-\mathrm{C} 3 \mathrm{~A}-\mathrm{C} 4 \mathrm{~A}$ & $-173.6(13)$ \\
\hline N2-Fe1-N3-C13 & $-90.2(7)$ & $\mathrm{C} 2 \mathrm{~A}-\mathrm{C} 3 \mathrm{~A}-\mathrm{C} 4 \mathrm{~A}-\mathrm{C} 5 \mathrm{~A}$ & $-1(2)$ \\
\hline N1-Fe1-N3-C13 & $-68.0(9)$ & C3A-C4A-C5A-C6A & $1(2)$ \\
\hline Cl1-Fe1-N3-C13 & $159.1(6)$ & C4A-C5A-C6A-C1A & $2(2)$ \\
\hline N2-Fe1-N4-C39 & $-152.9(9)$ & C2A-C1A-C6A-C5A & $-5(2)$ \\
\hline N1-Fe1-N4-C39 & $-60.5(10)$ & N1A-C1A-C6A-C5A & $-178.6(13)$ \\
\hline Cl1-Fe1-N4-C39 & $67.1(10)$ & C2A-N2A-C7A-C12A & $-19.4(19)$ \\
\hline N3-Fe1-N4-C39 & $153.0(10)$ & Fe1A-N2A-C7A-C12A & $141.7(10)$ \\
\hline N2-Fe1-N4-C14 & $16.0(9)$ & C2A-N2A-C7A-C8A & $165.7(11)$ \\
\hline N1-Fe1-N4-C14 & $108.4(8)$ & Fe1A-N2A-C7A-C8A & $-33.2(15)$ \\
\hline Cl1-Fe1-N4-C14 & $-124.0(7)$ & C12A-C7A-C8A-C9A & $5.7(18)$ \\
\hline N3-Fe1-N4-C14 & $-38.1(8)$ & N2A-C7A-C8A-C9A & $-178.8(12)$ \\
\hline C19-N1-C1-C6 & $8(2)$ & C12A-C7A-C8A-N3A & $-172.6(11)$ \\
\hline Fe1-N1-C1-C6 & $178.2(11)$ & $\mathrm{N} 2 \mathrm{~A}-\mathrm{C} 7 \mathrm{~A}-\mathrm{C} 8 \mathrm{~A}-\mathrm{N} 3 \mathrm{~A}$ & $2.9(17)$ \\
\hline C19-N1-C1-C2 & $-177.9(12)$ & C29A-N3A-C8A-C9A & $-35.7(17)$ \\
\hline Fe1-N1-C1-C2 & $-7.3(14)$ & C13A-N3A-C8A-C9A & $99.5(14)$ \\
\hline $\mathrm{C} 7-\mathrm{N} 2-\mathrm{C} 2-\mathrm{C} 3$ & $23.5(19)$ & Fe1A-N3A-C8A-C9A & $-159.7(10)$ \\
\hline Fe1-N2-C2-C3 & $-175.4(10)$ & C29A-N3A-C8A-C7A & $142.6(12)$ \\
\hline $\mathrm{C} 7-\mathrm{N} 2-\mathrm{C} 2-\mathrm{C} 1$ & $-162.4(11)$ & C13A-N3A-C8A-C7A & $-82.2(15)$ \\
\hline
\end{tabular}




\begin{tabular}{|c|c|c|c|}
\hline $\mathrm{Fe} 1-\mathrm{N} 2-\mathrm{C} 2-\mathrm{C} 1$ & $-1.2(13)$ & Fe1A-N3A-C8A-C7A & $18.7(12)$ \\
\hline $\mathrm{N} 1-\mathrm{C} 1-\mathrm{C} 2-\mathrm{N} 2$ & $5.9(17)$ & C7A-C8A-C9A-C10A & $-4(2)$ \\
\hline $\mathrm{C} 6-\mathrm{C} 1-\mathrm{C} 2-\mathrm{N} 2$ & $-178.9(11)$ & N3A-C8A-C9A-C10A & $174.3(12)$ \\
\hline $\mathrm{N} 1-\mathrm{C} 1-\mathrm{C} 2-\mathrm{C} 3$ & $-179.9(11)$ & C8A-C9A-C10A-C11A & $1(2)$ \\
\hline $\mathrm{C} 6-\mathrm{C} 1-\mathrm{C} 2-\mathrm{C} 3$ & $-4.7(18)$ & C9A-C10A-C11A-C12A & $0(2)$ \\
\hline $\mathrm{N} 2-\mathrm{C} 2-\mathrm{C} 3-\mathrm{C} 4$ & $179.2(12)$ & C10A-C11A-C12A-C7A & $2(2)$ \\
\hline $\mathrm{C} 1-\mathrm{C} 2-\mathrm{C} 3-\mathrm{C} 4$ & $5(2)$ & $\mathrm{N} 2 \mathrm{~A}-\mathrm{C} 7 \mathrm{~A}-\mathrm{C} 12 \mathrm{~A}-\mathrm{C} 11 \mathrm{~A}$ & $-179.8(13)$ \\
\hline $\mathrm{C} 2-\mathrm{C} 3-\mathrm{C} 4-\mathrm{C} 5$ & $-4(2)$ & C8A-C7A-C12A-C11A & $-4.8(19)$ \\
\hline $\mathrm{C} 3-\mathrm{C} 4-\mathrm{C} 5-\mathrm{C} 6$ & $3(2)$ & C8A-N3A-C13A-C18A & $-50.5(16)$ \\
\hline $\mathrm{C} 4-\mathrm{C} 5-\mathrm{C} 6-\mathrm{C} 1$ & $-3(2)$ & C29A-N3A-C13A-C18A & $87.4(14)$ \\
\hline $\mathrm{N} 1-\mathrm{C} 1-\mathrm{C} 6-\mathrm{C} 5$ & $178.1(12)$ & Fe1A-N3A-C13A-C18A & $-153.5(10)$ \\
\hline $\mathrm{C} 2-\mathrm{C} 1-\mathrm{C} 6-\mathrm{C} 5$ & $3.5(18)$ & C8A-N3A-C13A-C14A & $137.2(12)$ \\
\hline $\mathrm{C} 2-\mathrm{N} 2-\mathrm{C} 7-\mathrm{C} 8$ & $-166.9(12)$ & C29A-N3A-C13A-C14A & $-84.9(14)$ \\
\hline $\mathrm{Fe} 1-\mathrm{N} 2-\mathrm{C} 7-\mathrm{C} 8$ & $33.4(15)$ & Fe1A-N3A-C13A-C14A & $34.3(10)$ \\
\hline C2-N2-C7-C12 & $16.9(18)$ & C18A-C13A-C14A-N4A & $177.1(11)$ \\
\hline Fe1-N2-C7-C12 & $-142.7(10)$ & $\mathrm{N} 3 \mathrm{~A}-\mathrm{C} 13 \mathrm{~A}-\mathrm{C} 14 \mathrm{~A}-\mathrm{N} 4 \mathrm{~A}$ & $-10.6(16)$ \\
\hline $\mathrm{N} 2-\mathrm{C} 7-\mathrm{C} 8-\mathrm{C} 9$ & $177.8(12)$ & C18A-C13A-C14A-C15A & $0.8(17)$ \\
\hline $\mathrm{C} 12-\mathrm{C} 7-\mathrm{C} 8-\mathrm{C} 9$ & $-5.8(19)$ & N3A-C13A-C14A-C15A & 173.2(9) \\
\hline N2-C7-C8-N3 & $-2.3(18)$ & C39A-N4A-C14A-C13A & $151.0(12)$ \\
\hline $\mathrm{C} 12-\mathrm{C} 7-\mathrm{C} 8-\mathrm{N} 3$ & $174.1(10)$ & Fe1A-N4A-C14A-C13A & $-32.7(14)$ \\
\hline C29-N3-C8-C9 & $35.0(17)$ & C39A-N4A-C14A-C15A & $-32.8(16)$ \\
\hline C13-N3-C8-C9 & $-99.5(14)$ & Fe1A-N4A-C14A-C15A & $143.5(9)$ \\
\hline
\end{tabular}




\begin{tabular}{|c|c|c|c|}
\hline Fe1-N3-C8-C9 & $160.3(11)$ & C13A-C14A-C15A-C16A & $-2.8(16)$ \\
\hline C29-N3-C8-C7 & $-145.0(11)$ & N4A-C14A-C15A-C16A & $-179.1(10)$ \\
\hline C13-N3-C8-C7 & $80.6(14)$ & C14A-C15A-C16A-C17A & $5.0(17)$ \\
\hline Fe1-N3-C8-C7 & $-19.7(12)$ & C15A-C16A-C17A-C18A & $-4.8(18)$ \\
\hline $\mathrm{C} 7-\mathrm{C} 8-\mathrm{C} 9-\mathrm{C} 10$ & $4(2)$ & C16A-C17A-C18A-C13A & 2.6(19) \\
\hline N3-C8-C9-C10 & $-176.3(12)$ & C14A-C13A-C18A-C17A & $-1(2)$ \\
\hline C8-C9-C10-C11 & $-1(2)$ & N3A-C13A-C18A-C17A & $-172.5(11)$ \\
\hline C9-C10-C11-C12 & $0(2)$ & C1A-N1A-C19A-C24A & $-44(2)$ \\
\hline C10-C11-C12-C7 & $-2(2)$ & Fe1A-N1A-C19A-C24A & $123.2(14)$ \\
\hline N2-C7-C12-C11 & $-178.6(12)$ & C1A-N1A-C19A-C20A & $138.7(13)$ \\
\hline C8-C7-C12-C11 & $5.1(17)$ & Fe1A-N1A-C19A-C20A & $-54.2(17)$ \\
\hline C29-N3-C13-C18 & $-86.4(14)$ & N1A-C19A-C20A-C21A & $177.8(13)$ \\
\hline C8-N3-C13-C18 & $48.8(15)$ & C24A-C19A-C20A-C21A & $0(2)$ \\
\hline Fe1-N3-C13-C18 & $153.4(10)$ & C19A-C20A-C21A-C22A & $-2(2)$ \\
\hline C29-N3-C13-C14 & $84.6(13)$ & C20A-C21A-C22A-C23A & $0(2)$ \\
\hline C8-N3-C13-C14 & $-140.2(11)$ & C20A-C21A-C22A-C25A & $-171.2(15)$ \\
\hline Fe1-N3-C13-C14 & $-35.6(10)$ & C21A-C22A-C23A-C24A & $4(2)$ \\
\hline C39-N4-C14-C15 & $27.1(16)$ & $\mathrm{C} 25 \mathrm{~A}-\mathrm{C} 22 \mathrm{~A}-\mathrm{C} 23 \mathrm{~A}-\mathrm{C} 24 \mathrm{~A}$ & $175.6(14)$ \\
\hline Fe1-N4-C14-C15 & $-143.2(10)$ & C22A-C23A-C24A-C19A & $-6(2)$ \\
\hline C39-N4-C14-C13 & $-157.2(10)$ & N1A-C19A-C24A-C23A & $-173.3(14)$ \\
\hline Fe1-N4-C14-C13 & $32.5(12)$ & C20A-C19A-C24A-C23A & $4(2)$ \\
\hline C18-C13-C14-N4 & $-176.1(10)$ & $\mathrm{C} 21 \mathrm{~A}-\mathrm{C} 22 \mathrm{~A}-\mathrm{C} 25 \mathrm{~A}-\mathrm{C} 27 \mathrm{~A}$ & $-7(3)$ \\
\hline
\end{tabular}




\begin{tabular}{|c|c|c|c|}
\hline N3-C13-C14-N4 & $12.6(15)$ & $\mathrm{C} 23 \mathrm{~A}-\mathrm{C} 22 \mathrm{~A}-\mathrm{C} 25 \mathrm{~A}-\mathrm{C} 27 \mathrm{~A}$ & $-177.7(18)$ \\
\hline C18-C13-C14-C15 & $0.1(15)$ & $\mathrm{C} 21 \mathrm{~A}-\mathrm{C} 22 \mathrm{~A}-\mathrm{C} 25 \mathrm{~A}-\mathrm{C} 26 \mathrm{~A}$ & $157.0(19)$ \\
\hline N3-C13-C14-C15 & $-171.2(10)$ & $\mathrm{C} 23 \mathrm{~A}-\mathrm{C} 22 \mathrm{~A}-\mathrm{C} 25 \mathrm{~A}-\mathrm{C} 26 \mathrm{~A}$ & $-14(3)$ \\
\hline N4-C14-C15-C16 & $172.4(11)$ & $\mathrm{C} 21 \mathrm{~A}-\mathrm{C} 22 \mathrm{~A}-\mathrm{C} 25 \mathrm{~A}-\mathrm{C} 28 \mathrm{~A}$ & $-105(2)$ \\
\hline C13-C14-C15-C16 & $-3.4(17)$ & C23A-C22A-C25A-C28A & $84(2)$ \\
\hline $\mathrm{C} 14-\mathrm{C} 15-\mathrm{C} 16-\mathrm{C} 17$ & $5.9(18)$ & C8A-N3A-C29A-C34A & $137.1(13)$ \\
\hline C15-C16-C17-C18 & $-5.1(18)$ & C13A-N3A-C29A-C34A & $0.8(17)$ \\
\hline $\mathrm{C} 16-\mathrm{C} 17-\mathrm{C} 18-\mathrm{C} 13$ & $1.9(17)$ & Fe1A-N3A-C29A-C34A & $-106.8(12)$ \\
\hline N3-C13-C18-C17 & $171.4(10)$ & C8A-N3A-C29A-C30A & $-41.9(16)$ \\
\hline C14-C13-C18-C17 & $0.5(16)$ & C13A-N3A-C29A-C30A & $-178.2(11)$ \\
\hline C1-N1-C19-C20 & $-140.5(14)$ & Fe1A-N3A-C29A-C30A & $74.2(12)$ \\
\hline Fe1-N1-C19-C20 & $50.0(18)$ & C34A-C29A-C30A-C31A & $-6.3(18)$ \\
\hline C1-N1-C19-C24 & $44(2)$ & N3A-C29A-C30A-C31A & $172.8(11)$ \\
\hline Fe1-N1-C19-C24 & $-125.3(13)$ & C29A-C30A-C31A-C32A & $2.9(19)$ \\
\hline N1-C19-C20-C21 & $-176.0(13)$ & C30A-C31A-C32A-C33A & $5(2)$ \\
\hline C24-C19-C20-C21 & $-1(2)$ & C30A-C31A-C32A-C35A & $-180.0(12)$ \\
\hline C19-C20-C21-C22 & $-3(2)$ & C31A-C32A-C33A-C34A & $-9.9(19)$ \\
\hline $\mathrm{C} 20-\mathrm{C} 21-\mathrm{C} 22-\mathrm{C} 23$ & $1(2)$ & C35A-C32A-C33A-C34A & $175.8(12)$ \\
\hline $\mathrm{C} 20-\mathrm{C} 21-\mathrm{C} 22-\mathrm{C} 25$ & $180.0(15)$ & C30A-C29A-C34A-C33A & $1.7(19)$ \\
\hline $\mathrm{C} 21-\mathrm{C} 22-\mathrm{C} 23-\mathrm{C} 24$ & $4(2)$ & N3A-C29A-C34A-C33A & $-177.3(11)$ \\
\hline $\mathrm{C} 25-\mathrm{C} 22-\mathrm{C} 23-\mathrm{C} 24$ & $-175.1(15)$ & C32A-C33A-C34A-C29A & $7(2)$ \\
\hline C22-C23-C24-C19 & $-7(2)$ & C33A-C32A-C35A-C36A & $131.3(15)$ \\
\hline
\end{tabular}




\begin{tabular}{|c|c|c|c|}
\hline C20-C19-C24-C23 & $6(2)$ & C31A-C32A-C35A-C36A & $-42.9(18)$ \\
\hline N1-C19-C24-C23 & $-179.2(13)$ & C33A-C32A-C35A-C37A & $-108.2(16)$ \\
\hline $\mathrm{C} 21-\mathrm{C} 22-\mathrm{C} 25-\mathrm{C} 27$ & $170.5(18)$ & C31A-C32A-C35A-C37A & $77.6(16)$ \\
\hline $\mathrm{C} 23-\mathrm{C} 22-\mathrm{C} 25-\mathrm{C} 27$ & $-11(3)$ & C33A-C32A-C35A-C38A & $13.5(18)$ \\
\hline $\mathrm{C} 21-\mathrm{C} 22-\mathrm{C} 25-\mathrm{C} 28$ & $53(2)$ & C31A-C32A-C35A-C38A & $-160.8(12)$ \\
\hline $\mathrm{C} 23-\mathrm{C} 22-\mathrm{C} 25-\mathrm{C} 28$ & $-128.7(17)$ & C14A-N4A-C39A-C44A & $-41.9(19)$ \\
\hline $\mathrm{C} 21-\mathrm{C} 22-\mathrm{C} 25-\mathrm{C} 26$ & $-70(2)$ & Fe1A-N4A-C39A-C44A & $142.3(12)$ \\
\hline $\mathrm{C} 23-\mathrm{C} 22-\mathrm{C} 25-\mathrm{C} 26$ & $108.2(18)$ & C14A-N4A-C39A-C40A & $145.4(13)$ \\
\hline C8-N3-C29-C30 & $44.6(17)$ & Fe1A-N4A-C39A-C40A & $-30.4(18)$ \\
\hline C13-N3-C29-C30 & $179.5(12)$ & C44A-C39A-C40A-C41A & $1(2)$ \\
\hline Fe1-N3-C29-C30 & $-73.0(14)$ & N4A-C39A-C40A-C41A & $174.5(12)$ \\
\hline C8-N3-C29-C34 & $-138.9(12)$ & C39A-C40A-C41A-C42A & $2(2)$ \\
\hline C13-N3-C29-C34 & $-4.1(17)$ & C40A-C41A-C42A-C43A & $-3(2)$ \\
\hline Fe1-N3-C29-C34 & $103.5(11)$ & $\mathrm{C} 40 \mathrm{~A}-\mathrm{C} 41 \mathrm{~A}-\mathrm{C} 42 \mathrm{~A}-\mathrm{C} 45 \mathrm{~A}$ & $176.6(15)$ \\
\hline C34-C29-C30-C31 & $7(2)$ & C41A-C42A-C43A-C44A & $0(2)$ \\
\hline N3-C29-C30-C31 & $-176.6(12)$ & $\mathrm{C} 45 \mathrm{~A}-\mathrm{C} 42 \mathrm{~A}-\mathrm{C} 43 \mathrm{~A}-\mathrm{C} 44 \mathrm{~A}$ & $-179.7(14)$ \\
\hline C29-C30-C31-C32 & $-5(2)$ & C42A-C43A-C44A-C39A & $4(2)$ \\
\hline C30-C31-C32-C33 & $3(2)$ & C40A-C39A-C44A-C43A & $-4(2)$ \\
\hline C30-C31-C32-C35 & $174.8(14)$ & N4A-C39A-C44A-C43A & $-177.4(13)$ \\
\hline C31-C32-C33-C34 & $-3(2)$ & C41A-C42A-C45A-C47A & $-77(3)$ \\
\hline C35-C32-C33-C34 & $-175.5(13)$ & C43A-C42A-C45A-C47A & $103(2)$ \\
\hline C32-C33-C34-C29 & $5(2)$ & C41A-C42A-C45A-C48A & $143.7(19)$ \\
\hline
\end{tabular}




\begin{tabular}{|c|c|c|c|}
\hline C30-C29-C34-C33 & $-7.1(19)$ & C43A-C42A-C45A-C48A & $-37(3)$ \\
\hline N3-C29-C34-C33 & $176.4(12)$ & C41A-C42A-C45A-C46A & $40(3)$ \\
\hline C31-C32-C35-C36 & $45(2)$ & $\mathrm{C} 43 \mathrm{~A}-\mathrm{C} 42 \mathrm{~A}-\mathrm{C} 45 \mathrm{~A}-\mathrm{C} 46 \mathrm{~A}$ & $-140.8(16)$ \\
\hline C33-C32-C35-C36 & $-144.0(14)$ & C71-N7-C65-C66 & $-67.2(15)$ \\
\hline C31-C32-C35-C38 & $168.9(15)$ & C67-N7-C65-C66 & $52.0(16)$ \\
\hline C33-C32-C35-C38 & $-20(2)$ & C69-N7-C65-C66 & $171.4(12)$ \\
\hline C31-C32-C35-C37 & $-74.8(18)$ & C71-N7-C67-C68 & $170.5(12)$ \\
\hline C33-C32-C35-C37 & $96.6(16)$ & C65-N7-C67-C68 & $52.9(16)$ \\
\hline C14-N4-C39-C40 & $-140.2(12)$ & C69-N7-C67-C68 & $-67.2(16)$ \\
\hline Fe1-N4-C39-C40 & $28.7(17)$ & C71-N7-C69-C70 & $-60.9(16)$ \\
\hline C14-N4-C39-C44 & $46.0(16)$ & C67-N7-C69-C70 & $179.1(12)$ \\
\hline Fe1-N4-C39-C44 & $-145.2(10)$ & C65-N7-C69-C70 & $56.4(15)$ \\
\hline C44-C39-C40-C41 & $-4.8(19)$ & C67-N7-C71-C72 & $66.4(15)$ \\
\hline N4-C39-C40-C41 & $-178.7(11)$ & C65-N7-C71-C72 & $-171.7(12)$ \\
\hline C39-C40-C41-C42 & $6(2)$ & C69-N7-C71-C72 & $-52.8(16)$ \\
\hline C40-C41-C42-C43 & $-2(2)$ & C51-N5-C49-C50 & $-54.8(19)$ \\
\hline C40-C41-C42-C45 & $178.2(13)$ & C53-N5-C49-C50 & $179.8(14)$ \\
\hline C41-C42-C43-C44 & $-5(2)$ & C55-N5-C49-C50 & $57.1(19)$ \\
\hline C45-C42-C43-C44 & $175.5(13)$ & C49-N5-C51-C52 & $-54.0(19)$ \\
\hline C42-C43-C44-C39 & $6(2)$ & C53-N5-C51-C52 & $67.8(18)$ \\
\hline C40-C39-C44-C43 & $-1(2)$ & C55-N5-C51-C52 & $-167.7(15)$ \\
\hline N4-C39-C44-C43 & $172.9(11)$ & C49-N5-C53-C54 & $-176.8(13)$ \\
\hline
\end{tabular}




\begin{tabular}{|c|c|c|c|}
\hline $\mathrm{C} 43-\mathrm{C} 42-\mathrm{C} 45-\mathrm{C} 48$ & $169.7(14)$ & C51-N5-C53-C54 & $56.4(16)$ \\
\hline $\mathrm{C} 41-\mathrm{C} 42-\mathrm{C} 45-\mathrm{C} 48$ & $-10(2)$ & C55-N5-C53-C54 & $-60.5(17)$ \\
\hline $\mathrm{C} 43-\mathrm{C} 42-\mathrm{C} 45-\mathrm{C} 47$ & $-68.8(19)$ & C49-N5-C55-C56 & $59(2)$ \\
\hline $\mathrm{C} 41-\mathrm{C} 42-\mathrm{C} 45-\mathrm{C} 47$ & $111.4(16)$ & C51-N5-C55-C56 & $-179.9(17)$ \\
\hline $\mathrm{C} 43-\mathrm{C} 42-\mathrm{C} 45-\mathrm{C} 46$ & $53.2(19)$ & C53-N5-C55-C56 & $-57(2)$ \\
\hline C41-C42-C45-C46 & $-126.6(14)$ & C63-N6-C57-C58 & $-53.0(15)$ \\
\hline N4A-Fe1A-N1A-C19A & $-58.9(11)$ & C59-N6-C57-C58 & $65.3(15)$ \\
\hline N2A-Fe1A-N1A-C19A & $-172.9(11)$ & C61-N6-C57-C58 & $-176.4(12)$ \\
\hline C11A-Fe1A-N1A-C19A & $77.5(10)$ & C63-N6-C59-C60 & $-68.2(14)$ \\
\hline N3A-Fe1A-N1A-C19A & $-150.2(9)$ & C57-N6-C59-C60 & $170.5(11)$ \\
\hline N4A-Fe1A-N1A-C1A & 109.1(9) & C61-N6-C59-C60 & $52.6(15)$ \\
\hline N2A-Fe1A-N1A-C1A & $-4.9(9)$ & C63-N6-C61-C62 & $170.4(13)$ \\
\hline Cl1A-Fe1A-N1A-C1A & $-114.5(8)$ & C57-N6-C61-C62 & $-65.6(16)$ \\
\hline N3A-Fe1A-N1A-C1A & $17.8(13)$ & C59-N6-C61-C62 & $51.9(16)$ \\
\hline N4A-Fe1A-N2A-C7A & $86.2(10)$ & C57-N6-C63-C64 & $-55.7(14)$ \\
\hline N1A-Fe1A-N2A-C7A & $-161.6(10)$ & C59-N6-C63-C64 & $-174.3(11)$ \\
\hline C11A-Fe1A-N2A-C7A & $-60.0(9)$ & C61-N6-C63-C64 & $66.2(15)$ \\
\hline N3A-Fe1A-N2A-C7A & $31.0(9)$ & C77-N8-C73-C74 & $168.9(13)$ \\
\hline N4A-Fe1A-N2A-C2A & $-111.5(9)$ & C79-N8-C73-C74 & $51.5(17)$ \\
\hline N1A-Fe1A-N2A-C2A & $0.8(8)$ & C75-N8-C73-C74 & $-66.3(17)$ \\
\hline Cl1A-Fe1A-N2A-C2A & $102.4(8)$ & C77-N8-C75-C76 & $55.5(18)$ \\
\hline N3A-Fe1A-N2A-C2A & $-166.6(9)$ & C73-N8-C75-C76 & $-65.4(17)$ \\
\hline
\end{tabular}




$\begin{array}{lrlr}\text { N4A-Fe1A-N3A-C8A } & -153.6(7) & \text { C79-N8-C75-C76 } & 178.7(14) \\ \text { N1A-Fe1A-N3A-C8A } & -48.7(11) & \text { C73-N8-C77-C78 } & 179.6(13) \\ \text { N2A-Fe1A-N3A-C8A } & -25.1(7) & \text { C79-N8-C77-C78 } & -66.6(16) \\ \text { C11A-Fe1A-N3A-C8A } & 85.1(7) & \text { C75-N8-C77-C78 } & 55.3(18) \\ \text { N4A-Fe1A-N3A-C29A } & 79.8(8) & \text { C77-N8-C79-C80 } & -57.9(17) \\ \text { N1A-Fe1A-N3A-C29A } & -175.2(8) & \text { C73-N8-C79-C80 } & 56.7(17) \\ \text { N2A-Fe1A-N3A-C29A } & -151.7(9) & \text { C75-N8-C79-C80 } & 177.3(14) \\ \text { C11A-Fe1A-N3A-C29A } & -41.4(8) & \text { C3S-O1S-C2S-C1S } & 167.9(18) \\ \text { N4A-Fe1A-N3A-C13A } & -37.4(6) & \text { C2S-O1S-C3S-C4S } & -179.4(18)\end{array}$


Table S55. Atomic coordinates $\left(\times 10^{4}\right)$ and equivalent isotropic displacement parameters $\left(\AA^{2} \times 10^{3}\right)$ for $\left[\left(\mathrm{L}_{\mathrm{re}}^{1}\right) \mathrm{Fe}(\mathrm{III}) \mathrm{Cl}\right]\left(\mathrm{NEt}_{4}\right)(\mathbf{8})$. $\mathrm{U}(\mathrm{eq})$ is defined as one third of the trace of the orthogonalized $\mathrm{U}^{\mathrm{ij}}$ tensor

\begin{tabular}{|c|c|c|c|c|}
\hline & $\mathrm{x}$ & $\mathrm{y}$ & $\mathrm{z}$ & $\mathrm{U}(\mathrm{eq})$ \\
\hline $\mathrm{Fe}(1)$ & $2477(1)$ & $5217(1)$ & $2463(1)$ & $24(1)$ \\
\hline $\mathrm{Cl}(1)$ & $4400(2)$ & $5845(1)$ & $2425(1)$ & $31(1)$ \\
\hline $\mathrm{N}(1)$ & $1026(5)$ & 5889(3) & $2322(2)$ & $21(1)$ \\
\hline $\mathrm{N}(2)$ & $2289(5)$ & $5210(3)$ & 1621(1) & $20(1)$ \\
\hline $\mathrm{N}(3)$ & $2262(5)$ & $4152(3)$ & $2298(2)$ & $23(1)$ \\
\hline $\mathrm{N}(4)$ & $2562(5)$ & $4766(3)$ & 3081(1) & $23(1)$ \\
\hline $\mathrm{N}(5)$ & $2698(5)$ & $8578(2)$ & $2419(2)$ & $25(1)$ \\
\hline $\mathrm{C}(1)$ & $857(6)$ & $6188(4)$ & 1893(2) & $23(2)$ \\
\hline $\mathrm{C}(2)$ & $1519(6)$ & $5887(4)$ & $1529(2)$ & $20(2)$ \\
\hline$C(3)$ & $1424(6)$ & 6199(3) & $1104(2)$ & $26(2)$ \\
\hline $\mathrm{C}(4)$ & $641(7)$ & 6812(3) & $1016(2)$ & $28(2)$ \\
\hline$C(5)$ & $-41(6)$ & $7108(3)$ & $1358(2)$ & $28(2)$ \\
\hline$C(6)$ & $74(6)$ & 6815(3) & $1799(2)$ & $21(2)$ \\
\hline$C(7)$ & $1523(7)$ & $4519(4)$ & $1570(2)$ & $23(2)$ \\
\hline $\mathrm{C}(8)$ & $1573(6)$ & $3969(3)$ & 1907(2) & $20(2)$ \\
\hline $\mathrm{C}(9)$ & $847(6)$ & $3294(3)$ & $1843(2)$ & $23(2)$ \\
\hline$C(10)$ & $150(6)$ & $3187(4)$ & $1449(2)$ & $25(2)$ \\
\hline $\mathrm{C}(11)$ & $145(6)$ & $3732(4)$ & $1116(2)$ & $28(2)$ \\
\hline
\end{tabular}




\begin{tabular}{|c|c|c|c|c|}
\hline$C(12)$ & $835(7)$ & $4386(4)$ & $1176(2)$ & $27(2)$ \\
\hline$C(13)$ & $2595(7)$ & $3636(3)$ & $2630(2)$ & $19(1)$ \\
\hline$C(14)$ & $2752(6)$ & $3971(3)$ & $3065(2)$ & $23(2)$ \\
\hline$C(15)$ & $3141(6)$ & $3511(3)$ & $3429(2)$ & $25(2)$ \\
\hline$C(16)$ & $3374(6)$ & $2735(4)$ & $3366(2)$ & $27(2)$ \\
\hline$C(17)$ & $3249(6)$ & $2422(3)$ & $2947(2)$ & $29(2)$ \\
\hline$C(18)$ & $2870(5)$ & $2857(3)$ & $2581(2)$ & $27(2)$ \\
\hline C(19) & 109(7) & $6063(3)$ & $2667(2)$ & $22(2)$ \\
\hline$C(20)$ & $-1220(7)$ & $6013(3)$ & $2599(2)$ & $27(2)$ \\
\hline$C(21)$ & $-2039(6)$ & $6121(3)$ & $2959(2)$ & $24(2)$ \\
\hline$C(22)$ & $-1624(7)$ & $6244(3)$ & $3401(2)$ & $22(2)$ \\
\hline$C(23)$ & $-293(7)$ & $6291(3)$ & $3457(2)$ & $26(2)$ \\
\hline$C(24)$ & $524(6)$ & $6219(3)$ & $3092(2)$ & $24(2)$ \\
\hline$C(25)$ & $-2531(8)$ & $6324(3)$ & $3794(2)$ & $28(2)$ \\
\hline$C(26)$ & $-3456(6)$ & $7000(3)$ & $3701(2)$ & $39(2)$ \\
\hline$C(27)$ & $-1851(7)$ & $6483(4)$ & $4243(2)$ & $47(2)$ \\
\hline$C(28)$ & $-3288(7)$ & $5584(3)$ & $3849(2)$ & $39(2)$ \\
\hline C(29) & $3485(6)$ & $5176(4)$ & $1385(2)$ & $25(2)$ \\
\hline$C(30)$ & $4181(7)$ & $5829(4)$ & $1305(2)$ & $27(2)$ \\
\hline$C(31)$ & $5281(7)$ & $5805(4)$ & $1046(2)$ & $31(2)$ \\
\hline$C(32)$ & $5791(6)$ & $5128(4)$ & $870(2)$ & $28(2)$ \\
\hline$C(33)$ & $5147(7)$ & $4471(4)$ & $992(2)$ & $31(2)$ \\
\hline
\end{tabular}




\begin{tabular}{|c|c|c|c|c|}
\hline$C(34)$ & 3991(7) & $4505(4)$ & $1244(2)$ & $26(2)$ \\
\hline$C(35)$ & $6934(6)$ & $5147(4)$ & $557(2)$ & $32(2)$ \\
\hline$C(36)$ & $8043(6)$ & $5568(4)$ & $780(2)$ & $41(2)$ \\
\hline$C(37)$ & $6573(7)$ & $5569(4)$ & $127(2)$ & $52(2)$ \\
\hline$C(38)$ & $7335(8)$ & $4358(4)$ & $420(2)$ & $45(2)$ \\
\hline C(39) & $2601(7)$ & $5124(3)$ & $3507(2)$ & $21(2)$ \\
\hline$C(40)$ & $1839(6)$ & $4908(3)$ & $3867(2)$ & $27(2)$ \\
\hline$C(41)$ & $1820(6)$ & $5329(4)$ & $4262(2)$ & $28(2)$ \\
\hline$C(42)$ & $2532(8)$ & $5978(3)$ & $4323(2)$ & $29(2)$ \\
\hline$C(43)$ & $3292(6)$ & $6175(3)$ & $3961(2)$ & $27(2)$ \\
\hline$C(44)$ & $3337(6)$ & $5765(3)$ & $3556(2)$ & $24(2)$ \\
\hline$C(45)$ & $2498(10)$ & $6461(4)$ & $4744(2)$ & $44(2)$ \\
\hline$C(46)$ & $3817(7)$ & $6601(5)$ & $4914(2)$ & $78(3)$ \\
\hline$C(47)$ & $1778(10)$ & $6125(6)$ & $5112(3)$ & $123(5)$ \\
\hline$C(48)$ & $1867(9)$ & $7238(5)$ & $4638(3)$ & $101(4)$ \\
\hline C(49) & $1303(6)$ & $8646(4)$ & $2255(2)$ & $31(2)$ \\
\hline$C(50)$ & $1063(6)$ & $9151(3)$ & $1865(2)$ & $33(2)$ \\
\hline$C(51)$ & $3570(7)$ & $8441(3)$ & $2020(2)$ & $31(2)$ \\
\hline$C(52)$ & $3299(7)$ & $7715(3)$ & $1766(2)$ & $33(2)$ \\
\hline$C(53)$ & $2741(7)$ & $7931(3)$ & $2758(2)$ & $30(2)$ \\
\hline$C(54)$ & $4000(7)$ & $7804(4)$ & $2983(2)$ & $47(2)$ \\
\hline$C(55)$ & $3116(6)$ & $9316(3)$ & $2636(2)$ & $32(2)$ \\
\hline
\end{tabular}




\begin{tabular}{lrrrr}
$\mathrm{C}(56)$ & $2479(8)$ & $9530(3)$ & $3073(2)$ & $41(2)$ \\
$\mathrm{O}(1 \mathrm{~S})$ & $4359(6)$ & $8097(4)$ & $128(2)$ & $86(2)$ \\
$\mathrm{C}(1 \mathrm{~S})$ & $5365(11)$ & $9248(5)$ & $374(3)$ & $113(4)$ \\
$\mathrm{C}(2 \mathrm{~S})$ & $4674(11)$ & $8538(6)$ & $516(3)$ & $98(4)$ \\
$\mathrm{C}(3 \mathrm{~S})$ & $3643(10)$ & $7416(6)$ & $212(3)$ & $99(4)$ \\
$\mathrm{C}(4 \mathrm{~S})$ & $3354(13)$ & $6997(6)$ & $-223(4)$ & $169(7)$ \\
\hline
\end{tabular}


Table S56. Bond Distances, $\AA$, for $\left[\left(\mathrm{L}^{1}{ }_{\mathrm{re}}\right) \mathrm{Fe}(\mathrm{III}) \mathrm{Cl}\right]\left(\mathrm{NEt}_{4}\right)(\mathbf{8})$

\begin{tabular}{|c|c|c|c|}
\hline $\mathrm{Fe}(1)-\mathrm{N}(3)$ & $1.949(5)$ & $C(32)-C(35)$ & $1.515(8)$ \\
\hline $\mathrm{Fe}(1)-\mathrm{N}(1)$ & $1.971(5)$ & $C(33)-C(34)$ & $1.423(9)$ \\
\hline $\mathrm{Fe}(1)-\mathrm{N}(4)$ & $1.996(4)$ & $\mathrm{C}(33)-\mathrm{H}(33)$ & 0.9500 \\
\hline $\mathrm{Fe}(1)-\mathrm{Cl}(1)$ & $2.301(2)$ & $\mathrm{C}(34)-\mathrm{H}(34)$ & 0.9500 \\
\hline $\mathrm{Fe}(1)-\mathrm{N}(2)$ & $2.501(4)$ & $\mathrm{C}(35)-\mathrm{C}(38)$ & $1.507(8)$ \\
\hline $\mathrm{N}(1)-\mathrm{C}(1)$ & $1.385(7)$ & $C(35)-C(37)$ & $1.522(8)$ \\
\hline $\mathrm{N}(1)-\mathrm{C}(19)$ & $1.434(7)$ & $C(35)-C(36)$ & $1.528(8)$ \\
\hline $\mathrm{N}(2)-\mathrm{C}(29)$ & $1.437(7)$ & $\mathrm{C}(36)-\mathrm{H}(36 \mathrm{~A})$ & 0.9800 \\
\hline $\mathrm{N}(2)-\mathrm{C}(2)$ & $1.463(8)$ & $\mathrm{C}(36)-\mathrm{H}(36 \mathrm{~B})$ & 0.9800 \\
\hline $\mathrm{N}(2)-\mathrm{C}(7)$ & $1.466(8)$ & $\mathrm{C}(36)-\mathrm{H}(36 \mathrm{C})$ & 0.9800 \\
\hline $\mathrm{N}(3)-\mathrm{C}(13)$ & $1.383(6)$ & $\mathrm{C}(37)-\mathrm{H}(37 \mathrm{~A})$ & 0.9800 \\
\hline $\mathrm{N}(3)-\mathrm{C}(8)$ & $1.402(7)$ & $\mathrm{C}(37)-\mathrm{H}(37 \mathrm{~B})$ & 0.9800 \\
\hline $\mathrm{N}(4)-\mathrm{C}(39)$ & $1.407(6)$ & $\mathrm{C}(37)-\mathrm{H}(37 \mathrm{C})$ & 0.9800 \\
\hline $\mathrm{N}(4)-\mathrm{C}(14)$ & $1.413(6)$ & $\mathrm{C}(38)-\mathrm{H}(38 \mathrm{~A})$ & 0.9800 \\
\hline $\mathrm{N}(5)-\mathrm{C}(51)$ & $1.512(7)$ & $\mathrm{C}(38)-\mathrm{H}(38 \mathrm{~B})$ & 0.9800 \\
\hline $\mathrm{N}(5)-\mathrm{C}(55)$ & $1.513(6)$ & $\mathrm{C}(38)-\mathrm{H}(38 \mathrm{C})$ & 0.9800 \\
\hline $\mathrm{N}(5)-\mathrm{C}(53)$ & $1.519(6)$ & $\mathrm{C}(39)-\mathrm{C}(44)$ & $1.373(8)$ \\
\hline $\mathrm{N}(5)-\mathrm{C}(49)$ & $1.545(8)$ & $\mathrm{C}(39)-\mathrm{C}(40)$ & $1.386(8)$ \\
\hline $\mathrm{C}(1)-\mathrm{C}(2)$ & $1.387(8)$ & $\mathrm{C}(40)-\mathrm{C}(41)$ & $1.385(7)$ \\
\hline$C(1)-C(6)$ & $1.403(8)$ & $\mathrm{C}(40)-\mathrm{H}(40)$ & 0.9500 \\
\hline $\mathrm{C}(2)-\mathrm{C}(3)$ & $1.377(8)$ & $\mathrm{C}(41)-\mathrm{C}(42)$ & $1.375(8)$ \\
\hline
\end{tabular}




\begin{tabular}{|c|c|c|c|}
\hline$C(3)-C(4)$ & $1.380(8)$ & $\mathrm{C}(41)-\mathrm{H}(41)$ & 0.9500 \\
\hline $\mathrm{C}(3)-\mathrm{H}(3)$ & 0.9500 & $\mathrm{C}(42)-\mathrm{C}(43)$ & $1.378(8)$ \\
\hline$C(4)-C(5)$ & $1.346(8)$ & $C(42)-C(45)$ & $1.509(8)$ \\
\hline $\mathrm{C}(4)-\mathrm{H}(4)$ & 0.9500 & $C(43)-C(44)$ & $1.401(7)$ \\
\hline$C(5)-C(6)$ & $1.406(8)$ & $\mathrm{C}(43)-\mathrm{H}(43)$ & 0.9500 \\
\hline $\mathrm{C}(5)-\mathrm{H}(5)$ & 0.9500 & $\mathrm{C}(44)-\mathrm{H}(44)$ & 0.9500 \\
\hline $\mathrm{C}(6)-\mathrm{H}(6)$ & 0.9500 & $C(45)-C(47)$ & $1.450(10)$ \\
\hline$C(7)-C(8)$ & $1.391(7)$ & $C(45)-C(46)$ & $1.492(11)$ \\
\hline$C(7)-C(12)$ & $1.390(8)$ & $\mathrm{C}(45)-\mathrm{C}(48)$ & $1.550(10)$ \\
\hline$C(8)-C(9)$ & $1.423(7)$ & $\mathrm{C}(46)-\mathrm{H}(46 \mathrm{~A})$ & 0.9800 \\
\hline C(9)-C(10) & $1.388(8)$ & $\mathrm{C}(46)-\mathrm{H}(46 \mathrm{~B})$ & 0.9800 \\
\hline $\mathrm{C}(9)-\mathrm{H}(9)$ & 0.9500 & $\mathrm{C}(46)-\mathrm{H}(46 \mathrm{C})$ & 0.9800 \\
\hline $\mathrm{C}(10)-\mathrm{C}(11)$ & $1.374(8)$ & $\mathrm{C}(47)-\mathrm{H}(47 \mathrm{~A})$ & 0.9800 \\
\hline $\mathrm{C}(10)-\mathrm{H}(10)$ & 0.9500 & $\mathrm{C}(47)-\mathrm{H}(47 \mathrm{~B})$ & 0.9800 \\
\hline $\mathrm{C}(11)-\mathrm{C}(12)$ & $1.371(8)$ & $\mathrm{C}(47)-\mathrm{H}(47 \mathrm{C})$ & 0.9800 \\
\hline $\mathrm{C}(11)-\mathrm{H}(11)$ & 0.9500 & $\mathrm{C}(48)-\mathrm{H}(48 \mathrm{~A})$ & 0.9800 \\
\hline $\mathrm{C}(12)-\mathrm{H}(12)$ & 0.9500 & $\mathrm{C}(48)-\mathrm{H}(48 \mathrm{~B})$ & 0.9800 \\
\hline $\mathrm{C}(13)-\mathrm{C}(18)$ & $1.407(7)$ & $\mathrm{C}(48)-\mathrm{H}(48 \mathrm{C})$ & 0.9800 \\
\hline$C(13)-C(14)$ & $1.426(7)$ & $C(49)-C(50)$ & $1.480(7)$ \\
\hline$C(14)-C(15)$ & $1.408(7)$ & $\mathrm{C}(49)-\mathrm{H}(49 \mathrm{~A})$ & 0.9900 \\
\hline$C(15)-C(16)$ & $1.399(7)$ & $\mathrm{C}(49)-\mathrm{H}(49 \mathrm{~B})$ & 0.9900 \\
\hline $\mathrm{C}(15)-\mathrm{H}(15)$ & 0.9500 & $\mathrm{C}(50)-\mathrm{H}(50 \mathrm{~A})$ & 0.9800 \\
\hline
\end{tabular}




\begin{tabular}{|c|c|c|c|}
\hline$C(16)-C(17)$ & $1.362(8)$ & $\mathrm{C}(50)-\mathrm{H}(50 \mathrm{~B})$ & 0.9800 \\
\hline $\mathrm{C}(16)-\mathrm{H}(16)$ & 0.9500 & $\mathrm{C}(50)-\mathrm{H}(50 \mathrm{C})$ & 0.9800 \\
\hline$C(17)-C(18)$ & $1.385(8)$ & $\mathrm{C}(51)-\mathrm{C}(52)$ & $1.509(7)$ \\
\hline $\mathrm{C}(17)-\mathrm{H}(17)$ & 0.9500 & $\mathrm{C}(51)-\mathrm{H}(51 \mathrm{~A})$ & 0.9900 \\
\hline $\mathrm{C}(18)-\mathrm{H}(18)$ & 0.9500 & $\mathrm{C}(51)-\mathrm{H}(51 \mathrm{~B})$ & 0.9900 \\
\hline$C(19)-C(24)$ & $1.360(8)$ & $\mathrm{C}(52)-\mathrm{H}(52 \mathrm{~A})$ & 0.9800 \\
\hline$C(19)-C(20)$ & $1.410(8)$ & $\mathrm{C}(52)-\mathrm{H}(52 \mathrm{~B})$ & 0.9800 \\
\hline $\mathrm{C}(20)-\mathrm{C}(21)$ & $1.382(8)$ & $\mathrm{C}(52)-\mathrm{H}(52 \mathrm{C})$ & 0.9800 \\
\hline $\mathrm{C}(20)-\mathrm{H}(20)$ & 0.9500 & $\mathrm{C}(53)-\mathrm{C}(54)$ & $1.495(8)$ \\
\hline$C(21)-C(22)$ & $1.395(8)$ & $\mathrm{C}(53)-\mathrm{H}(53 \mathrm{~A})$ & 0.9900 \\
\hline $\mathrm{C}(21)-\mathrm{H}(21)$ & 0.9500 & $\mathrm{C}(53)-\mathrm{H}(53 \mathrm{~B})$ & 0.9900 \\
\hline$C(22)-C(23)$ & $1.408(9)$ & $\mathrm{C}(54)-\mathrm{H}(54 \mathrm{~A})$ & 0.9800 \\
\hline$C(22)-C(25)$ & $1.509(8)$ & $\mathrm{C}(54)-\mathrm{H}(54 \mathrm{~B})$ & 0.9800 \\
\hline$C(23)-C(24)$ & $1.386(8)$ & $\mathrm{C}(54)-\mathrm{H}(54 \mathrm{C})$ & 0.9800 \\
\hline $\mathrm{C}(23)-\mathrm{H}(23)$ & 0.9500 & $C(55)-C(56)$ & $1.505(7)$ \\
\hline $\mathrm{C}(24)-\mathrm{H}(24)$ & 0.9500 & $\mathrm{C}(55)-\mathrm{H}(55 \mathrm{~A})$ & 0.9900 \\
\hline$C(25)-C(28)$ & $1.533(8)$ & $\mathrm{C}(55)-\mathrm{H}(55 \mathrm{~B})$ & 0.9900 \\
\hline$C(25)-C(27)$ & $1.533(8)$ & $\mathrm{C}(56)-\mathrm{H}(56 \mathrm{~A})$ & 0.9800 \\
\hline$C(25)-C(26)$ & $1.559(9)$ & $\mathrm{C}(56)-\mathrm{H}(56 \mathrm{~B})$ & 0.9800 \\
\hline $\mathrm{C}(26)-\mathrm{H}(26 \mathrm{~A})$ & 0.9800 & $\mathrm{C}(56)-\mathrm{H}(56 \mathrm{C})$ & 0.9800 \\
\hline $\mathrm{C}(26)-\mathrm{H}(26 \mathrm{~B})$ & 0.9800 & $\mathrm{O}(1 \mathrm{~S})-\mathrm{C}(2 \mathrm{~S})$ & $1.423(9)$ \\
\hline $\mathrm{C}(26)-\mathrm{H}(26 \mathrm{C})$ & 0.9800 & $\mathrm{O}(1 \mathrm{~S})-\mathrm{C}(3 \mathrm{~S})$ & $1.434(10)$ \\
\hline
\end{tabular}




$\begin{array}{lclr}\mathrm{C}(27)-\mathrm{H}(27 \mathrm{~A}) & 0.9800 & \mathrm{C}(1 \mathrm{~S})-\mathrm{C}(2 \mathrm{~S}) & 1.503(12) \\ \mathrm{C}(27)-\mathrm{H}(27 \mathrm{~B}) & 0.9800 & \mathrm{C}(1 \mathrm{~S})-\mathrm{H}(1 \mathrm{~S} 1) & 0.9800 \\ \mathrm{C}(27)-\mathrm{H}(27 \mathrm{C}) & 0.9800 & \mathrm{C}(1 \mathrm{~S})-\mathrm{H}(1 \mathrm{~S} 2) & 0.9800 \\ \mathrm{C}(28)-\mathrm{H}(28 \mathrm{~A}) & 0.9800 & \mathrm{C}(1 \mathrm{~S})-\mathrm{H}(1 \mathrm{~S} 3) & 0.9800 \\ \mathrm{C}(28)-\mathrm{H}(28 \mathrm{~B}) & \mathrm{C}(2 \mathrm{~S})-\mathrm{H}(2 \mathrm{~S} 1) & 0.9900 \\ \mathrm{C}(28)-\mathrm{H}(28 \mathrm{C}) & \mathrm{C}(2 \mathrm{~S})-\mathrm{H}(2 \mathrm{~S} 2) & 0.9900 \\ \mathrm{C}(29)-\mathrm{C}(34) & \mathrm{C}(3 \mathrm{~S})-\mathrm{C}(4 \mathrm{~S}) & 0.9900 \\ \mathrm{C}(29)-\mathrm{C}(30) & 0.9800 & \mathrm{C}(3 \mathrm{~S})-\mathrm{H}(3 \mathrm{~S} 1) & 0.9900 \\ \mathrm{C}(30)-\mathrm{C}(31) & 1.360(8) & \mathrm{C}(3 \mathrm{~S})-\mathrm{H}(3 \mathrm{~S} 2) & 0.9800 \\ \mathrm{C}(30)-\mathrm{H}(30) & 1.381(9) & \mathrm{C}(4 \mathrm{~S})-\mathrm{H}(4 \mathrm{~S} 1) & 0.9800 \\ \mathrm{C}(31)-\mathrm{C}(32) & 1.385(8) & \mathrm{C}(4 \mathrm{~S})-\mathrm{H}(4 \mathrm{~S} 2) & 0.9800 \\ \mathrm{C}(31)-\mathrm{H}(31) & 0.9500 & \mathrm{C}(4 \mathrm{~S})-\mathrm{H}(4 \mathrm{~S} 3) & \\ \mathrm{C}(32)-\mathrm{C}(33) & 1.406(8) & & \end{array}$


Table S57. Angles, ${ }^{\circ}$, for $\left[\left(\mathrm{L}^{1}\right.\right.$ re $\left.) \mathrm{Fe}(\mathrm{III}) \mathrm{Cl}\right]\left(\mathrm{NEt}_{4}\right)(8)$

\begin{tabular}{|c|c|c|c|}
\hline $\mathrm{N}(3)-\mathrm{Fe}(1)-\mathrm{N}(1)$ & $115.7(2)$ & $\mathrm{C}(29)-\mathrm{C}(34)-\mathrm{C}(33)$ & $121.9(6)$ \\
\hline $\mathrm{N}(3)-\mathrm{Fe}(1)-\mathrm{N}(4)$ & $81.59(18)$ & $\mathrm{C}(29)-\mathrm{C}(34)-\mathrm{H}(34)$ & 119.0 \\
\hline $\mathrm{N}(1)-\mathrm{Fe}(1)-\mathrm{N}(4)$ & $117.9(2)$ & $\mathrm{C}(33)-\mathrm{C}(34)-\mathrm{H}(34)$ & 119.0 \\
\hline $\mathrm{N}(3)-\mathrm{Fe}(1)-\mathrm{Cl}(1)$ & $123.38(18)$ & $\mathrm{C}(38)-\mathrm{C}(35)-\mathrm{C}(32)$ & $111.4(6)$ \\
\hline N(1)-Fe(1)-Cl(1) & $112.20(16)$ & $\mathrm{C}(38)-\mathrm{C}(35)-\mathrm{C}(37)$ & $107.0(5)$ \\
\hline N(4)-Fe(1)-Cl(1) & $101.30(17)$ & $\mathrm{C}(32)-\mathrm{C}(35)-\mathrm{C}(37)$ & 109.5 \\
\hline $\mathrm{N}(3)-\mathrm{Fe}(1)-\mathrm{N}(2)$ & $74.71(17)$ & $\mathrm{C}(38)-\mathrm{C}(35)-\mathrm{C}(36)$ & 109.5 \\
\hline $\mathrm{N}(1)-\mathrm{Fe}(1)-\mathrm{N}(2)$ & $74.36(19)$ & $\mathrm{C}(32)-\mathrm{C}(35)-\mathrm{C}(36)$ & 109.5 \\
\hline $\mathrm{N}(4)-\mathrm{Fe}(1)-\mathrm{N}(2)$ & $156.29(18)$ & $\mathrm{C}(37)-\mathrm{C}(35)-\mathrm{C}(36)$ & 109.5 \\
\hline $\mathrm{Cl}(1)-\mathrm{Fe}(1)-\mathrm{N}(2)$ & $91.29(14)$ & $\mathrm{C}(35)-\mathrm{C}(36)-\mathrm{H}(36 \mathrm{~A})$ & 109.5 \\
\hline $\mathrm{C}(1)-\mathrm{N}(1)-\mathrm{C}(19)$ & $119.0(5)$ & $\mathrm{C}(35)-\mathrm{C}(36)-\mathrm{H}(36 \mathrm{~B})$ & 109.5 \\
\hline $\mathrm{C}(1)-\mathrm{N}(1)-\mathrm{Fe}(1)$ & $121.4(4)$ & $\mathrm{H}(36 \mathrm{~A})-\mathrm{C}(36)-\mathrm{H}(36 \mathrm{~B})$ & 109.5 \\
\hline $\mathrm{C}(19)-\mathrm{N}(1)-\mathrm{Fe}(1)$ & $119.6(4)$ & $\mathrm{C}(35)-\mathrm{C}(36)-\mathrm{H}(36 \mathrm{C})$ & 109.5 \\
\hline $\mathrm{C}(29)-\mathrm{N}(2)-\mathrm{C}(2)$ & $115.1(5)$ & $\mathrm{H}(36 \mathrm{~A})-\mathrm{C}(36)-\mathrm{H}(36 \mathrm{C})$ & 109.5 \\
\hline $\mathrm{C}(29)-\mathrm{N}(2)-\mathrm{C}(7)$ & $113.1(5)$ & $\mathrm{H}(36 \mathrm{~B})-\mathrm{C}(36)-\mathrm{H}(36 \mathrm{C})$ & 109.5 \\
\hline $\mathrm{C}(2)-\mathrm{N}(2)-\mathrm{C}(7)$ & $110.7(5)$ & $\mathrm{C}(35)-\mathrm{C}(37)-\mathrm{H}(37 \mathrm{~A})$ & 109.5 \\
\hline $\mathrm{C}(29)-\mathrm{N}(2)-\mathrm{Fe}(1)$ & $114.7(4)$ & $\mathrm{C}(35)-\mathrm{C}(37)-\mathrm{H}(37 \mathrm{~B})$ & 109.5 \\
\hline $\mathrm{C}(2)-\mathrm{N}(2)-\mathrm{Fe}(1)$ & $103.0(3)$ & $\mathrm{H}(37 \mathrm{~A})-\mathrm{C}(37)-\mathrm{H}(37 \mathrm{~B})$ & 109.5 \\
\hline $\mathrm{C}(7)-\mathrm{N}(2)-\mathrm{Fe}(1)$ & $98.7(3)$ & $\mathrm{C}(35)-\mathrm{C}(37)-\mathrm{H}(37 \mathrm{C})$ & 109.5 \\
\hline $\mathrm{C}(13)-\mathrm{N}(3)-\mathrm{C}(8)$ & $124.6(5)$ & $\mathrm{H}(37 \mathrm{~A})-\mathrm{C}(37)-\mathrm{H}(37 \mathrm{C})$ & 109.5 \\
\hline $\mathrm{C}(13)-\mathrm{N}(3)-\mathrm{Fe}(1)$ & $115.0(3)$ & $\mathrm{H}(37 \mathrm{~B})-\mathrm{C}(37)-\mathrm{H}(37 \mathrm{C})$ & 109.5 \\
\hline
\end{tabular}




\begin{tabular}{|c|c|c|c|}
\hline $\mathrm{C}(8)-\mathrm{N}(3)-\mathrm{Fe}(1)$ & $119.2(4)$ & $\mathrm{C}(35)-\mathrm{C}(38)-\mathrm{H}(38 \mathrm{~A})$ & 109.5 \\
\hline $\mathrm{C}(39)-\mathrm{N}(4)-\mathrm{C}(14)$ & $117.9(4)$ & $\mathrm{C}(35)-\mathrm{C}(38)-\mathrm{H}(38 \mathrm{~B})$ & $117.8(6)$ \\
\hline $\mathrm{C}(39)-\mathrm{N}(4)-\mathrm{Fe}(1)$ & $130.1(4)$ & $\mathrm{H}(38 \mathrm{~A})-\mathrm{C}(38)-\mathrm{H}(38 \mathrm{~B})$ & $118.6(6)$ \\
\hline $\mathrm{C}(14)-\mathrm{N}(4)-\mathrm{Fe}(1)$ & $111.6(3)$ & $\mathrm{C}(35)-\mathrm{C}(38)-\mathrm{H}(38 \mathrm{C})$ & $123.3(6)$ \\
\hline $\mathrm{C}(51)-\mathrm{N}(5)-\mathrm{C}(55)$ & $107.0(5)$ & $\mathrm{H}(38 \mathrm{~A})-\mathrm{C}(38)-\mathrm{H}(38 \mathrm{C})$ & $120.8(6)$ \\
\hline $\mathrm{C}(51)-\mathrm{N}(5)-\mathrm{C}(53)$ & $112.3(5)$ & $\mathrm{H}(38 \mathrm{~B})-\mathrm{C}(38)-\mathrm{H}(38 \mathrm{C})$ & 119.6 \\
\hline $\mathrm{C}(55)-\mathrm{N}(5)-\mathrm{C}(53)$ & $110.7(4)$ & $\mathrm{C}(44)-\mathrm{C}(39)-\mathrm{C}(40)$ & 119.6 \\
\hline $\mathrm{C}(51)-\mathrm{N}(5)-\mathrm{C}(49)$ & $109.9(5)$ & $\mathrm{C}(44)-\mathrm{C}(39)-\mathrm{N}(4)$ & $123.1(6)$ \\
\hline $\mathrm{C}(55)-\mathrm{N}(5)-\mathrm{C}(49)$ & $109.9(5)$ & $\mathrm{C}(40)-\mathrm{C}(39)-\mathrm{N}(4)$ & 118.4 \\
\hline $\mathrm{C}(53)-\mathrm{N}(5)-\mathrm{C}(49)$ & $107.0(5)$ & $\mathrm{C}(39)-\mathrm{C}(40)-\mathrm{C}(41)$ & 118.4 \\
\hline $\mathrm{N}(1)-\mathrm{C}(1)-\mathrm{C}(2)$ & $120.3(6)$ & $\mathrm{C}(39)-\mathrm{C}(40)-\mathrm{H}(40)$ & $114.9(5)$ \\
\hline $\mathrm{N}(1)-\mathrm{C}(1)-\mathrm{C}(6)$ & $123.8(6)$ & $\mathrm{C}(41)-\mathrm{C}(40)-\mathrm{H}(40)$ & $124.2(7)$ \\
\hline$C(2)-C(1)-C(6)$ & $115.9(6)$ & $\mathrm{C}(42)-\mathrm{C}(41)-\mathrm{C}(40)$ & $120.9(7)$ \\
\hline $\mathrm{C}(3)-\mathrm{C}(2)-\mathrm{C}(1)$ & $121.5(6)$ & $\mathrm{C}(42)-\mathrm{C}(41)-\mathrm{H}(41)$ & $123.7(6)$ \\
\hline $\mathrm{C}(3)-\mathrm{C}(2)-\mathrm{N}(2)$ & $122.3(6)$ & $\mathrm{C}(40)-\mathrm{C}(41)-\mathrm{H}(41)$ & 118.1 \\
\hline $\mathrm{C}(1)-\mathrm{C}(2)-\mathrm{N}(2)$ & $116.2(6)$ & $\mathrm{C}(41)-\mathrm{C}(42)-\mathrm{C}(43)$ & 118.1 \\
\hline$C(2)-C(3)-C(4)$ & $121.8(6)$ & $\mathrm{C}(41)-\mathrm{C}(42)-\mathrm{C}(45)$ & $109.0(6)$ \\
\hline $\mathrm{C}(2)-\mathrm{C}(3)-\mathrm{H}(3)$ & 119.1 & $C(43)-C(42)-C(45)$ & $110.5(6)$ \\
\hline $\mathrm{C}(4)-\mathrm{C}(3)-\mathrm{H}(3)$ & 119.1 & $\mathrm{C}(42)-\mathrm{C}(43)-\mathrm{C}(44)$ & $110.5(5)$ \\
\hline$C(5)-C(4)-C(3)$ & $118.4(6)$ & $\mathrm{C}(42)-\mathrm{C}(43)-\mathrm{H}(43)$ & $108.3(6)$ \\
\hline $\mathrm{C}(5)-\mathrm{C}(4)-\mathrm{H}(4)$ & 120.8 & $\mathrm{C}(44)-\mathrm{C}(43)-\mathrm{H}(43)$ & 109.5 \\
\hline $\mathrm{C}(3)-\mathrm{C}(4)-\mathrm{H}(4)$ & 120.8 & $\mathrm{C}(39)-\mathrm{C}(44)-\mathrm{C}(43)$ & $119.7(6)$ \\
\hline
\end{tabular}




\begin{tabular}{|c|c|c|c|}
\hline$C(4)-C(5)-C(6)$ & $120.7(6)$ & $\mathrm{C}(39)-\mathrm{C}(44)-\mathrm{H}(44)$ & 120.2 \\
\hline $\mathrm{C}(4)-\mathrm{C}(5)-\mathrm{H}(5)$ & 119.6 & $\mathrm{C}(43)-\mathrm{C}(44)-\mathrm{H}(44)$ & 120.2 \\
\hline $\mathrm{C}(6)-\mathrm{C}(5)-\mathrm{H}(5)$ & 119.6 & $C(47)-C(45)-C(46)$ & $107.3(7)$ \\
\hline $\mathrm{C}(1)-\mathrm{C}(6)-\mathrm{C}(5)$ & $121.6(6)$ & $\mathrm{C}(47)-\mathrm{C}(45)-\mathrm{C}(42)$ & $113.8(7)$ \\
\hline $\mathrm{C}(1)-\mathrm{C}(6)-\mathrm{H}(6)$ & 119.2 & $\mathrm{C}(46)-\mathrm{C}(45)-\mathrm{C}(42)$ & $110.5(7)$ \\
\hline $\mathrm{C}(5)-\mathrm{C}(6)-\mathrm{H}(6)$ & 119.2 & $\mathrm{C}(47)-\mathrm{C}(45)-\mathrm{C}(48)$ & $106.8(8)$ \\
\hline $\mathrm{C}(8)-\mathrm{C}(7)-\mathrm{C}(12)$ & $120.3(6)$ & $\mathrm{C}(46)-\mathrm{C}(45)-\mathrm{C}(48)$ & $108.5(7)$ \\
\hline $\mathrm{C}(8)-\mathrm{C}(7)-\mathrm{N}(2)$ & $118.8(6)$ & $\mathrm{C}(42)-\mathrm{C}(45)-\mathrm{C}(48)$ & $109.8(6)$ \\
\hline $\mathrm{C}(12)-\mathrm{C}(7)-\mathrm{N}(2)$ & $120.7(6)$ & $\mathrm{C}(45)-\mathrm{C}(46)-\mathrm{H}(46 \mathrm{~A})$ & 109.5 \\
\hline$C(7)-C(8)-N(3)$ & $116.9(5)$ & $\mathrm{C}(45)-\mathrm{C}(46)-\mathrm{H}(46 \mathrm{~B})$ & 109.5 \\
\hline$C(7)-C(8)-C(9)$ & $117.6(6)$ & $\mathrm{H}(46 \mathrm{~A})-\mathrm{C}(46)-\mathrm{H}(46 \mathrm{~B})$ & 109.5 \\
\hline $\mathrm{N}(3)-\mathrm{C}(8)-\mathrm{C}(9)$ & $125.3(5)$ & $\mathrm{C}(45)-\mathrm{C}(46)-\mathrm{H}(46 \mathrm{C})$ & 109.5 \\
\hline $\mathrm{C}(10)-\mathrm{C}(9)-\mathrm{C}(8)$ & $120.4(6)$ & $\mathrm{H}(46 \mathrm{~A})-\mathrm{C}(46)-\mathrm{H}(46 \mathrm{C})$ & 109.5 \\
\hline $\mathrm{C}(10)-\mathrm{C}(9)-\mathrm{H}(9)$ & 119.8 & $\mathrm{H}(46 \mathrm{~B})-\mathrm{C}(46)-\mathrm{H}(46 \mathrm{C})$ & 109.5 \\
\hline $\mathrm{C}(8)-\mathrm{C}(9)-\mathrm{H}(9)$ & 119.8 & $\mathrm{C}(45)-\mathrm{C}(47)-\mathrm{H}(47 \mathrm{~A})$ & 109.5 \\
\hline $\mathrm{C}(11)-\mathrm{C}(10)-\mathrm{C}(9)$ & $120.7(6)$ & $\mathrm{C}(45)-\mathrm{C}(47)-\mathrm{H}(47 \mathrm{~B})$ & 109.5 \\
\hline $\mathrm{C}(11)-\mathrm{C}(10)-\mathrm{H}(10)$ & 119.7 & $\mathrm{H}(47 \mathrm{~A})-\mathrm{C}(47)-\mathrm{H}(47 \mathrm{~B})$ & 109.5 \\
\hline $\mathrm{C}(9)-\mathrm{C}(10)-\mathrm{H}(10)$ & 119.7 & $\mathrm{C}(45)-\mathrm{C}(47)-\mathrm{H}(47 \mathrm{C})$ & 109.5 \\
\hline $\mathrm{C}(12)-\mathrm{C}(11)-\mathrm{C}(10)$ & $119.4(6)$ & $\mathrm{H}(47 \mathrm{~A})-\mathrm{C}(47)-\mathrm{H}(47 \mathrm{C})$ & 109.5 \\
\hline $\mathrm{C}(12)-\mathrm{C}(11)-\mathrm{H}(11)$ & 120.3 & $\mathrm{H}(47 \mathrm{~B})-\mathrm{C}(47)-\mathrm{H}(47 \mathrm{C})$ & 109.5 \\
\hline $\mathrm{C}(10)-\mathrm{C}(11)-\mathrm{H}(11)$ & 120.3 & $\mathrm{C}(45)-\mathrm{C}(48)-\mathrm{H}(48 \mathrm{~A})$ & 109.5 \\
\hline$C(11)-C(12)-C(7)$ & $121.5(6)$ & $\mathrm{C}(45)-\mathrm{C}(48)-\mathrm{H}(48 \mathrm{~B})$ & 109.5 \\
\hline
\end{tabular}




\begin{tabular}{|c|c|c|c|}
\hline $\mathrm{C}(11)-\mathrm{C}(12)-\mathrm{H}(12)$ & 119.2 & $\mathrm{H}(48 \mathrm{~A})-\mathrm{C}(48)-\mathrm{H}(48 \mathrm{~B})$ & 109.5 \\
\hline $\mathrm{C}(7)-\mathrm{C}(12)-\mathrm{H}(12)$ & 119.2 & $\mathrm{C}(45)-\mathrm{C}(48)-\mathrm{H}(48 \mathrm{C})$ & 109.5 \\
\hline $\mathrm{N}(3)-\mathrm{C}(13)-\mathrm{C}(18)$ & $128.1(5)$ & $\mathrm{H}(48 \mathrm{~A})-\mathrm{C}(48)-\mathrm{H}(48 \mathrm{C})$ & 109.5 \\
\hline $\mathrm{N}(3)-\mathrm{C}(13)-\mathrm{C}(14)$ & $113.6(4)$ & $\mathrm{H}(48 \mathrm{~B})-\mathrm{C}(48)-\mathrm{H}(48 \mathrm{C})$ & 109.5 \\
\hline $\mathrm{C}(18)-\mathrm{C}(13)-\mathrm{C}(14)$ & $118.2(5)$ & $\mathrm{C}(50)-\mathrm{C}(49)-\mathrm{N}(5)$ & $116.8(5)$ \\
\hline$C(15)-C(14)-N(4)$ & $125.7(5)$ & $\mathrm{C}(50)-\mathrm{C}(49)-\mathrm{H}(49 \mathrm{~A})$ & 108.1 \\
\hline$C(15)-C(14)-C(13)$ & $119.1(5)$ & $\mathrm{N}(5)-\mathrm{C}(49)-\mathrm{H}(49 \mathrm{~A})$ & 108.1 \\
\hline $\mathrm{N}(4)-\mathrm{C}(14)-\mathrm{C}(13)$ & $115.1(5)$ & $\mathrm{C}(50)-\mathrm{C}(49)-\mathrm{H}(49 \mathrm{~B})$ & 108.1 \\
\hline $\mathrm{C}(16)-\mathrm{C}(15)-\mathrm{C}(14)$ & $120.6(6)$ & $\mathrm{N}(5)-\mathrm{C}(49)-\mathrm{H}(49 \mathrm{~B})$ & 108.1 \\
\hline $\mathrm{C}(16)-\mathrm{C}(15)-\mathrm{H}(15)$ & 119.7 & $\mathrm{H}(49 \mathrm{~A})-\mathrm{C}(49)-\mathrm{H}(49 \mathrm{~B})$ & 107.3 \\
\hline $\mathrm{C}(14)-\mathrm{C}(15)-\mathrm{H}(15)$ & 119.7 & $\mathrm{C}(49)-\mathrm{C}(50)-\mathrm{H}(50 \mathrm{~A})$ & 109.5 \\
\hline $\mathrm{C}(17)-\mathrm{C}(16)-\mathrm{C}(15)$ & $120.0(6)$ & $\mathrm{C}(49)-\mathrm{C}(50)-\mathrm{H}(50 \mathrm{~B})$ & 109.5 \\
\hline $\mathrm{C}(17)-\mathrm{C}(16)-\mathrm{H}(16)$ & 120.0 & $\mathrm{H}(50 \mathrm{~A})-\mathrm{C}(50)-\mathrm{H}(50 \mathrm{~B})$ & 109.5 \\
\hline $\mathrm{C}(15)-\mathrm{C}(16)-\mathrm{H}(16)$ & 120.0 & $\mathrm{C}(49)-\mathrm{C}(50)-\mathrm{H}(50 \mathrm{C})$ & 109.5 \\
\hline $\mathrm{C}(16)-\mathrm{C}(17)-\mathrm{C}(18)$ & $121.1(6)$ & $\mathrm{H}(50 \mathrm{~A})-\mathrm{C}(50)-\mathrm{H}(50 \mathrm{C})$ & 109.5 \\
\hline $\mathrm{C}(16)-\mathrm{C}(17)-\mathrm{H}(17)$ & 119.5 & $\mathrm{H}(50 \mathrm{~B})-\mathrm{C}(50)-\mathrm{H}(50 \mathrm{C})$ & 109.5 \\
\hline $\mathrm{C}(18)-\mathrm{C}(17)-\mathrm{H}(17)$ & 119.5 & $\mathrm{~N}(5)-\mathrm{C}(51)-\mathrm{C}(52)$ & $114.2(5)$ \\
\hline $\mathrm{C}(17)-\mathrm{C}(18)-\mathrm{C}(13)$ & $121.1(6)$ & $\mathrm{N}(5)-\mathrm{C}(51)-\mathrm{H}(51 \mathrm{~A})$ & 108.7 \\
\hline $\mathrm{C}(17)-\mathrm{C}(18)-\mathrm{H}(18)$ & 119.5 & $\mathrm{C}(52)-\mathrm{C}(51)-\mathrm{H}(51 \mathrm{~A})$ & 108.7 \\
\hline $\mathrm{C}(13)-\mathrm{C}(18)-\mathrm{H}(18)$ & 119.5 & $\mathrm{~N}(5)-\mathrm{C}(51)-\mathrm{H}(51 \mathrm{~B})$ & 108.7 \\
\hline $\mathrm{C}(24)-\mathrm{C}(19)-\mathrm{C}(20)$ & $117.4(6)$ & $\mathrm{C}(52)-\mathrm{C}(51)-\mathrm{H}(51 \mathrm{~B})$ & 108.7 \\
\hline $\mathrm{C}(24)-\mathrm{C}(19)-\mathrm{N}(1)$ & $119.1(6)$ & $\mathrm{H}(51 \mathrm{~A})-\mathrm{C}(51)-\mathrm{H}(51 \mathrm{~B})$ & 107.6 \\
\hline
\end{tabular}




\begin{tabular}{|c|c|c|c|}
\hline $\mathrm{C}(20)-\mathrm{C}(19)-\mathrm{N}(1)$ & $123.2(6)$ & $\mathrm{C}(51)-\mathrm{C}(52)-\mathrm{H}(52 \mathrm{~A})$ & 109.5 \\
\hline$C(21)-C(20)-C(19)$ & $119.7(6)$ & $\mathrm{C}(51)-\mathrm{C}(52)-\mathrm{H}(52 \mathrm{~B})$ & 109.5 \\
\hline $\mathrm{C}(21)-\mathrm{C}(20)-\mathrm{H}(20)$ & 120.1 & $\mathrm{H}(52 \mathrm{~A})-\mathrm{C}(52)-\mathrm{H}(52 \mathrm{~B})$ & 109.5 \\
\hline $\mathrm{C}(19)-\mathrm{C}(20)-\mathrm{H}(20)$ & 120.1 & $\mathrm{C}(51)-\mathrm{C}(52)-\mathrm{H}(52 \mathrm{C})$ & 109.5 \\
\hline $\mathrm{C}(20)-\mathrm{C}(21)-\mathrm{C}(22)$ & $123.4(6)$ & $\mathrm{H}(52 \mathrm{~A})-\mathrm{C}(52)-\mathrm{H}(52 \mathrm{C})$ & 109.5 \\
\hline $\mathrm{C}(20)-\mathrm{C}(21)-\mathrm{H}(21)$ & 118.3 & $\mathrm{H}(52 \mathrm{~B})-\mathrm{C}(52)-\mathrm{H}(52 \mathrm{C})$ & 109.5 \\
\hline $\mathrm{C}(22)-\mathrm{C}(21)-\mathrm{H}(21)$ & 118.3 & $\mathrm{C}(54)-\mathrm{C}(53)-\mathrm{N}(5)$ & $115.7(6)$ \\
\hline $\mathrm{C}(21)-\mathrm{C}(22)-\mathrm{C}(23)$ & $115.5(6)$ & $\mathrm{C}(54)-\mathrm{C}(53)-\mathrm{H}(53 \mathrm{~A})$ & 108.4 \\
\hline $\mathrm{C}(21)-\mathrm{C}(22)-\mathrm{C}(25)$ & $122.7(6)$ & $\mathrm{N}(5)-\mathrm{C}(53)-\mathrm{H}(53 \mathrm{~A})$ & 108.4 \\
\hline $\mathrm{C}(23)-\mathrm{C}(22)-\mathrm{C}(25)$ & $121.8(6)$ & $\mathrm{C}(54)-\mathrm{C}(53)-\mathrm{H}(53 \mathrm{~B})$ & 108.4 \\
\hline $\mathrm{C}(24)-\mathrm{C}(23)-\mathrm{C}(22)$ & $120.9(6)$ & $\mathrm{N}(5)-\mathrm{C}(53)-\mathrm{H}(53 \mathrm{~B})$ & 108.4 \\
\hline $\mathrm{C}(24)-\mathrm{C}(23)-\mathrm{H}(23)$ & 119.5 & $\mathrm{H}(53 \mathrm{~A})-\mathrm{C}(53)-\mathrm{H}(53 \mathrm{~B})$ & 107.4 \\
\hline $\mathrm{C}(22)-\mathrm{C}(23)-\mathrm{H}(23)$ & 119.5 & $\mathrm{C}(53)-\mathrm{C}(54)-\mathrm{H}(54 \mathrm{~A})$ & 109.5 \\
\hline $\mathrm{C}(19)-\mathrm{C}(24)-\mathrm{C}(23)$ & $122.9(7)$ & $\mathrm{C}(53)-\mathrm{C}(54)-\mathrm{H}(54 \mathrm{~B})$ & 109.5 \\
\hline $\mathrm{C}(19)-\mathrm{C}(24)-\mathrm{H}(24)$ & 118.5 & $\mathrm{H}(54 \mathrm{~A})-\mathrm{C}(54)-\mathrm{H}(54 \mathrm{~B})$ & 109.5 \\
\hline $\mathrm{C}(23)-\mathrm{C}(24)-\mathrm{H}(24)$ & 118.5 & $\mathrm{C}(53)-\mathrm{C}(54)-\mathrm{H}(54 \mathrm{C})$ & 109.5 \\
\hline $\mathrm{C}(22)-\mathrm{C}(25)-\mathrm{C}(28)$ & $109.2(5)$ & $\mathrm{H}(54 \mathrm{~A})-\mathrm{C}(54)-\mathrm{H}(54 \mathrm{C})$ & 109.5 \\
\hline $\mathrm{C}(22)-\mathrm{C}(25)-\mathrm{C}(27)$ & $113.1(7)$ & $\mathrm{H}(54 \mathrm{~B})-\mathrm{C}(54)-\mathrm{H}(54 \mathrm{C})$ & 109.5 \\
\hline $\mathrm{C}(28)-\mathrm{C}(25)-\mathrm{C}(27)$ & $107.6(5)$ & $\mathrm{C}(56)-\mathrm{C}(55)-\mathrm{N}(5)$ & $116.8(5)$ \\
\hline $\mathrm{C}(22)-\mathrm{C}(25)-\mathrm{C}(26)$ & $109.1(5)$ & $\mathrm{C}(56)-\mathrm{C}(55)-\mathrm{H}(55 \mathrm{~A})$ & 108.1 \\
\hline$C(28)-C(25)-C(26)$ & $110.1(6)$ & $\mathrm{N}(5)-\mathrm{C}(55)-\mathrm{H}(55 \mathrm{~A})$ & 108.1 \\
\hline $\mathrm{C}(27)-\mathrm{C}(25)-\mathrm{C}(26)$ & $107.7(5)$ & $\mathrm{C}(56)-\mathrm{C}(55)-\mathrm{H}(55 \mathrm{~B})$ & 108.1 \\
\hline
\end{tabular}




\begin{tabular}{|c|c|c|c|}
\hline$C(25)-C(26)-H(26 A)$ & 109.5 & $\mathrm{~N}(5)-\mathrm{C}(55)-\mathrm{H}(55 \mathrm{~B})$ & 108.1 \\
\hline $\mathrm{C}(25)-\mathrm{C}(26)-\mathrm{H}(26 \mathrm{~B})$ & 109.5 & $\mathrm{H}(55 \mathrm{~A})-\mathrm{C}(55)-\mathrm{H}(55 \mathrm{~B})$ & 107.3 \\
\hline $\mathrm{H}(26 \mathrm{~A})-\mathrm{C}(26)-\mathrm{H}(26 \mathrm{~B})$ & 109.5 & $\mathrm{C}(55)-\mathrm{C}(56)-\mathrm{H}(56 \mathrm{~A})$ & 109.5 \\
\hline $\mathrm{C}(25)-\mathrm{C}(26)-\mathrm{H}(26 \mathrm{C})$ & 109.5 & $\mathrm{C}(55)-\mathrm{C}(56)-\mathrm{H}(56 \mathrm{~B})$ & 109.5 \\
\hline $\mathrm{H}(26 \mathrm{~A})-\mathrm{C}(26)-\mathrm{H}(26 \mathrm{C})$ & 109.5 & $\mathrm{H}(56 \mathrm{~A})-\mathrm{C}(56)-\mathrm{H}(56 \mathrm{~B})$ & 109.5 \\
\hline $\mathrm{H}(26 \mathrm{~B})-\mathrm{C}(26)-\mathrm{H}(26 \mathrm{C})$ & 109.5 & $\mathrm{C}(55)-\mathrm{C}(56)-\mathrm{H}(56 \mathrm{C})$ & 109.5 \\
\hline $\mathrm{C}(25)-\mathrm{C}(27)-\mathrm{H}(27 \mathrm{~A})$ & 109.5 & $\mathrm{H}(56 \mathrm{~A})-\mathrm{C}(56)-\mathrm{H}(56 \mathrm{C})$ & 109.5 \\
\hline $\mathrm{C}(25)-\mathrm{C}(27)-\mathrm{H}(27 \mathrm{~B})$ & 109.5 & $\mathrm{H}(56 \mathrm{~B})-\mathrm{C}(56)-\mathrm{H}(56 \mathrm{C})$ & 109.5 \\
\hline $\mathrm{H}(27 \mathrm{~A})-\mathrm{C}(27)-\mathrm{H}(27 \mathrm{~B})$ & 109.5 & $\mathrm{C}(2 \mathrm{~S})-\mathrm{O}(1 \mathrm{~S})-\mathrm{C}(3 \mathrm{~S})$ & $115.8(8)$ \\
\hline $\mathrm{C}(25)-\mathrm{C}(27)-\mathrm{H}(27 \mathrm{C})$ & 109.5 & $\mathrm{C}(2 \mathrm{~S})-\mathrm{C}(1 \mathrm{~S})-\mathrm{H}(1 \mathrm{~S} 1)$ & 109.5 \\
\hline $\mathrm{H}(27 \mathrm{~A})-\mathrm{C}(27)-\mathrm{H}(27 \mathrm{C})$ & 109.5 & $\mathrm{C}(2 \mathrm{~S})-\mathrm{C}(1 \mathrm{~S})-\mathrm{H}(1 \mathrm{~S} 2)$ & 109.5 \\
\hline $\mathrm{H}(27 \mathrm{~B})-\mathrm{C}(27)-\mathrm{H}(27 \mathrm{C})$ & 109.5 & $\mathrm{H}(1 \mathrm{~S} 1)-\mathrm{C}(1 \mathrm{~S})-\mathrm{H}(1 \mathrm{~S} 2)$ & 109.5 \\
\hline $\mathrm{C}(25)-\mathrm{C}(28)-\mathrm{H}(28 \mathrm{~A})$ & 109.5 & $\mathrm{C}(2 \mathrm{~S})-\mathrm{C}(1 \mathrm{~S})-\mathrm{H}(1 \mathrm{~S} 3)$ & 109.5 \\
\hline $\mathrm{C}(25)-\mathrm{C}(28)-\mathrm{H}(28 \mathrm{~B})$ & 109.5 & $\mathrm{H}(1 \mathrm{~S} 1)-\mathrm{C}(1 \mathrm{~S})-\mathrm{H}(1 \mathrm{~S} 3)$ & 109.5 \\
\hline $\mathrm{H}(28 \mathrm{~A})-\mathrm{C}(28)-\mathrm{H}(28 \mathrm{~B})$ & 109.5 & $\mathrm{H}(1 \mathrm{~S} 2)-\mathrm{C}(1 \mathrm{~S})-\mathrm{H}(1 \mathrm{~S} 3)$ & 109.5 \\
\hline $\mathrm{C}(25)-\mathrm{C}(28)-\mathrm{H}(28 \mathrm{C})$ & 109.5 & $\mathrm{O}(1 \mathrm{~S})-\mathrm{C}(2 \mathrm{~S})-\mathrm{C}(1 \mathrm{~S})$ & $109.9(8)$ \\
\hline $\mathrm{H}(28 \mathrm{~A})-\mathrm{C}(28)-\mathrm{H}(28 \mathrm{C})$ & 109.5 & $\mathrm{O}(1 \mathrm{~S})-\mathrm{C}(2 \mathrm{~S})-\mathrm{H}(2 \mathrm{~S} 1)$ & 109.7 \\
\hline $\mathrm{H}(28 \mathrm{~B})-\mathrm{C}(28)-\mathrm{H}(28 \mathrm{C})$ & 109.5 & $\mathrm{C}(1 \mathrm{~S})-\mathrm{C}(2 \mathrm{~S})-\mathrm{H}(2 \mathrm{~S} 1)$ & 109.7 \\
\hline $\mathrm{C}(34)-\mathrm{C}(29)-\mathrm{C}(30)$ & $117.6(6)$ & $\mathrm{O}(1 \mathrm{~S})-\mathrm{C}(2 \mathrm{~S})-\mathrm{H}(2 \mathrm{~S} 2)$ & 109.7 \\
\hline $\mathrm{C}(34)-\mathrm{C}(29)-\mathrm{N}(2)$ & $121.7(7)$ & $\mathrm{C}(1 \mathrm{~S})-\mathrm{C}(2 \mathrm{~S})-\mathrm{H}(2 \mathrm{~S} 2)$ & 109.7 \\
\hline $\mathrm{C}(30)-\mathrm{C}(29)-\mathrm{N}(2)$ & $120.6(6)$ & $\mathrm{H}(2 \mathrm{~S} 1)-\mathrm{C}(2 \mathrm{~S})-\mathrm{H}(2 \mathrm{~S} 2)$ & 108.2 \\
\hline$C(29)-C(30)-C(31)$ & $120.6(6)$ & $\mathrm{O}(1 \mathrm{~S})-\mathrm{C}(3 \mathrm{~S})-\mathrm{C}(4 \mathrm{~S})$ & $111.3(8)$ \\
\hline
\end{tabular}




$\begin{array}{lrlr}\mathrm{C}(29)-\mathrm{C}(30)-\mathrm{H}(30) & 119.7 & \mathrm{O}(1 \mathrm{~S})-\mathrm{C}(3 \mathrm{~S})-\mathrm{H}(3 \mathrm{~S} 1) & 109.4 \\ \mathrm{C}(31)-\mathrm{C}(30)-\mathrm{H}(30) & 119.7 & \mathrm{C}(4 \mathrm{~S})-\mathrm{C}(3 \mathrm{~S})-\mathrm{H}(3 \mathrm{~S} 1) & 109.4 \\ \mathrm{C}(30)-\mathrm{C}(31)-\mathrm{C}(32) & 123.2(6) & \mathrm{O}(1 \mathrm{~S})-\mathrm{C}(3 \mathrm{~S})-\mathrm{H}(3 \mathrm{~S} 2) & 109.4 \\ \mathrm{C}(30)-\mathrm{C}(31)-\mathrm{H}(31) & 118.4 & \mathrm{C}(4 \mathrm{~S})-\mathrm{C}(3 \mathrm{~S})-\mathrm{H}(3 \mathrm{~S} 2) & 109.4 \\ \mathrm{C}(32)-\mathrm{C}(31)-\mathrm{H}(31) & 118.4 & \mathrm{H}(3 \mathrm{~S} 1)-\mathrm{C}(3 \mathrm{~S})-\mathrm{H}(3 \mathrm{~S} 2) & 108.0 \\ \mathrm{C}(33)-\mathrm{C}(32)-\mathrm{C}(31) & 115.1(6) & \mathrm{C}(3 \mathrm{~S})-\mathrm{C}(4 \mathrm{~S})-\mathrm{H}(4 \mathrm{~S} 1) & 109.5 \\ \mathrm{C}(33)-\mathrm{C}(32)-\mathrm{C}(35) & 124.3(6) & \mathrm{C}(3 \mathrm{~S})-\mathrm{C}(4 \mathrm{~S})-\mathrm{H}(4 \mathrm{~S} 2) & 109.5 \\ \mathrm{C}(31)-\mathrm{C}(32)-\mathrm{C}(35) & 120.5(6) & \mathrm{H}(4 \mathrm{~S} 1)-\mathrm{C}(4 \mathrm{~S})-\mathrm{H}(4 \mathrm{~S} 2) & 109.5 \\ \mathrm{C}(32)-\mathrm{C}(33)-\mathrm{C}(34) & 121.1(6) & \mathrm{C}(3 \mathrm{~S})-\mathrm{C}(4 \mathrm{~S})-\mathrm{H}(4 \mathrm{~S} 3) & 109.5 \\ \mathrm{C}(32)-\mathrm{C}(33)-\mathrm{H}(33) & 119.5 & \mathrm{H}(4 \mathrm{~S} 1)-\mathrm{C}(4 \mathrm{~S})-\mathrm{H}(4 \mathrm{~S} 3) & 109.5 \\ \mathrm{C}(34)-\mathrm{C}(33)-\mathrm{H}(33) & 119.5 & \mathrm{H}(4 \mathrm{~S} 2)-\mathrm{C}(4 \mathrm{~S})-\mathrm{H}(4 \mathrm{~S} 3) & \end{array}$


Table S58. Anisotropic displacement parameters $\left(\AA^{2} \times 10^{3}\right)$ for $\left[\left(\mathrm{L}^{1}{ }_{\mathrm{re}}\right) \mathrm{Fe}(\mathrm{III}) \mathrm{Cl}\right]\left(\mathrm{NEt}_{4}\right)(\mathbf{8})$. The anisotropic displacement factor exponent takes the form: $-2 \pi^{2}\left[\mathrm{~h}^{2} \mathrm{a}^{2} \mathrm{U}^{11}+\ldots+2 \mathrm{hk}\right.$

\begin{tabular}{|c|c|c|c|c|c|c|}
\hline & $\mathrm{U}^{11}$ & $\mathrm{U}^{22}$ & $\mathrm{U}^{33}$ & $\mathrm{U}^{23}$ & $\mathrm{U}^{13}$ & $\mathrm{U}^{12}$ \\
\hline $\mathrm{Fe}(1)$ & $22(1)$ & $22(1)$ & $26(1)$ & $-2(1)$ & $2(1)$ & $-1(1)$ \\
\hline $\mathrm{Cl}(1)$ & $30(1)$ & $35(1)$ & $29(1)$ & $-1(1)$ & $1(1)$ & $-7(1)$ \\
\hline $\mathrm{N}(1)$ & $20(3)$ & $26(3)$ & $18(3)$ & $-5(3)$ & $6(3)$ & $4(3)$ \\
\hline $\mathrm{N}(2)$ & $21(4)$ & $15(3)$ & $25(3)$ & $1(3)$ & $7(3)$ & $3(3)$ \\
\hline $\mathrm{N}(3)$ & $26(4)$ & $24(3)$ & $18(3)$ & $0(2)$ & $-2(3)$ & $0(3)$ \\
\hline $\mathrm{N}(4)$ & $19(3)$ & $26(3)$ & $23(3)$ & $-1(3)$ & $-8(3)$ & 1(4) \\
\hline $\mathrm{N}(5)$ & $19(4)$ & $22(3)$ & $34(3)$ & $4(3)$ & $3(3)$ & $-5(3)$ \\
\hline $\mathrm{C}(1)$ & $9(4)$ & $23(4)$ & $36(4)$ & $-2(4)$ & $-3(3)$ & $-5(3)$ \\
\hline$C(2)$ & $19(4)$ & $19(4)$ & $23(4)$ & $-4(3)$ & $3(3)$ & $-3(4)$ \\
\hline$C(3)$ & $23(5)$ & $22(4)$ & $33(5)$ & $0(3)$ & $3(4)$ & $-2(4)$ \\
\hline$C(4)$ & $27(5)$ & $31(4)$ & $24(4)$ & $5(3)$ & $10(4)$ & $-3(4)$ \\
\hline$C(5)$ & $21(4)$ & $16(4)$ & $47(5)$ & $5(4)$ & $1(4)$ & $-4(4)$ \\
\hline$C(6)$ & $13(4)$ & $23(4)$ & $26(4)$ & $-9(3)$ & $4(3)$ & $-5(3)$ \\
\hline$C(7)$ & $20(4)$ & $23(4)$ & $27(4)$ & $-5(3)$ & $1(4)$ & $3(3)$ \\
\hline$C(8)$ & $21(4)$ & $12(4)$ & $27(4)$ & $-3(3)$ & $1(3)$ & $3(3)$ \\
\hline$C(9)$ & $26(5)$ & $17(4)$ & $25(4)$ & $7(3)$ & $7(3)$ & $4(3)$ \\
\hline$C(10)$ & $5(4)$ & $32(4)$ & $39(5)$ & $-18(4)$ & $-9(3)$ & $5(3)$ \\
\hline $\mathrm{C}(11)$ & $30(5)$ & $34(4)$ & $19(4)$ & $5(4)$ & $2(3)$ & $-3(4)$ \\
\hline
\end{tabular}




\begin{tabular}{|c|c|c|c|c|c|c|}
\hline$C(12)$ & $28(5)$ & $23(4)$ & $28(4)$ & $-9(3)$ & $-1(4)$ & $-10(4)$ \\
\hline$C(13)$ & $17(4)$ & $12(3)$ & $28(4)$ & $-2(3)$ & $5(4)$ & $0(4)$ \\
\hline$C(14)$ & $23(5)$ & $23(4)$ & $23(4)$ & $4(3)$ & $3(3)$ & $-1(3)$ \\
\hline$C(15)$ & $25(5)$ & $28(4)$ & $23(4)$ & $-4(3)$ & $2(3)$ & $0(3)$ \\
\hline$C(16)$ & $20(4)$ & $27(4)$ & $34(5)$ & $6(4)$ & $-6(3)$ & $-1(3)$ \\
\hline$C(17)$ & $16(4)$ & $21(4)$ & $51(5)$ & $1(4)$ & $-3(4)$ & $9(3)$ \\
\hline $\mathrm{C}(18)$ & $17(4)$ & $25(3)$ & $38(4)$ & $-9(4)$ & $-7(3)$ & $-7(3)$ \\
\hline$C(19)$ & $30(5)$ & $17(4)$ & $20(4)$ & $0(3)$ & $4(3)$ & $2(3)$ \\
\hline$C(20)$ & $40(5)$ & $20(4)$ & $21(4)$ & $3(3)$ & $-1(4)$ & $-4(3)$ \\
\hline $\mathrm{C}(21)$ & $21(4)$ & $27(4)$ & $25(4)$ & $4(3)$ & $0(3)$ & $6(3)$ \\
\hline$C(22)$ & $25(5)$ & $15(4)$ & $25(4)$ & $0(3)$ & $7(4)$ & $5(3)$ \\
\hline $\mathrm{C}(23)$ & $37(5)$ & $27(4)$ & $13(4)$ & $1(3)$ & $3(3)$ & $-4(4)$ \\
\hline $\mathrm{C}(24)$ & $15(4)$ & $16(4)$ & $40(4)$ & $4(3)$ & $-4(4)$ & $4(3)$ \\
\hline$C(25)$ & $26(4)$ & $25(3)$ & $33(4)$ & $3(3)$ & $7(4)$ & $-3(4)$ \\
\hline$C(26)$ & $24(5)$ & $42(5)$ & $52(5)$ & $1(4)$ & $10(4)$ & $6(4)$ \\
\hline$C(27)$ & $56(6)$ & $53(5)$ & $33(5)$ & $1(4)$ & $12(4)$ & $4(4)$ \\
\hline $\mathrm{C}(28)$ & $51(6)$ & $28(4)$ & $39(5)$ & $7(4)$ & $13(4)$ & $-1(4)$ \\
\hline$C(29)$ & $17(4)$ & $30(4)$ & $28(4)$ & $-7(4)$ & $2(3)$ & $6(4)$ \\
\hline$C(30)$ & $25(5)$ & $23(4)$ & $35(4)$ & $-2(3)$ & $15(4)$ & $0(4)$ \\
\hline $\mathrm{C}(31)$ & $27(5)$ & $34(4)$ & $31(4)$ & $4(4)$ & $0(4)$ & $-8(4)$ \\
\hline $\mathrm{C}(32)$ & $26(4)$ & $36(4)$ & $21(4)$ & $-4(4)$ & $1(3)$ & $-7(4)$ \\
\hline $\mathrm{C}(33)$ & $32(5)$ & $31(4)$ & $29(4)$ & $-13(3)$ & $-2(4)$ & $8(4)$ \\
\hline
\end{tabular}




\begin{tabular}{|c|c|c|c|c|c|c|}
\hline$C(34)$ & $40(5)$ & $18(4)$ & $21(4)$ & $2(3)$ & $-4(4)$ & $-4(4)$ \\
\hline$C(35)$ & $21(4)$ & $40(5)$ & $35(4)$ & $-2(4)$ & $2(3)$ & $1(4)$ \\
\hline$C(36)$ & $21(5)$ & $61(5)$ & $42(5)$ & $-7(4)$ & $7(4)$ & $-5(4)$ \\
\hline$C(37)$ & $36(5)$ & $85(6)$ & $35(5)$ & $15(5)$ & $10(4)$ & $-12(5)$ \\
\hline$C(38)$ & $25(5)$ & $69(5)$ & $40(4)$ & $-13(4)$ & $15(4)$ & $3(5)$ \\
\hline C(39) & $9(4)$ & $30(4)$ & $24(3)$ & $1(3)$ & $6(4)$ & $-2(4)$ \\
\hline$C(40)$ & $23(4)$ & $22(4)$ & $36(4)$ & $-7(4)$ & $-4(3)$ & $2(3)$ \\
\hline$C(41)$ & $21(4)$ & $44(5)$ & $19(4)$ & $5(4)$ & $4(3)$ & $9(4)$ \\
\hline$C(42)$ & $32(4)$ & $34(4)$ & $22(4)$ & $-11(3)$ & $2(4)$ & $-7(5)$ \\
\hline$C(43)$ & $19(4)$ & $25(4)$ & $36(4)$ & $-10(4)$ & $0(4)$ & $-1(3)$ \\
\hline$C(44)$ & $16(4)$ & $21(4)$ & $35(4)$ & $-2(3)$ & $5(3)$ & $-11(4)$ \\
\hline$C(45)$ & $45(5)$ & $58(5)$ & $30(4)$ & $-5(4)$ & $-4(5)$ & $1(6)$ \\
\hline$C(46)$ & $38(6)$ & $136(10)$ & $61(6)$ & $-65(6)$ & $-12(5)$ & $9(6)$ \\
\hline$C(47)$ & $157(12)$ & $155(11)$ & $57(7)$ & $-63(7)$ & $57(7)$ & $-92(9)$ \\
\hline$C(48)$ & $126(11)$ & $108(8)$ & $69(7)$ & $-55(6)$ & $-37(7)$ & $40(8)$ \\
\hline$C(49)$ & $19(5)$ & $41(5)$ & $33(5)$ & $3(4)$ & $7(4)$ & $10(4)$ \\
\hline$C(50)$ & $35(5)$ & $28(4)$ & $34(4)$ & $0(4)$ & $-10(4)$ & $6(4)$ \\
\hline$C(51)$ & $28(5)$ & $33(4)$ & $32(4)$ & $0(4)$ & $9(4)$ & $-3(4)$ \\
\hline$C(52)$ & $29(5)$ & $39(4)$ & $32(5)$ & $-3(4)$ & $1(4)$ & $-2(4)$ \\
\hline$C(53)$ & $29(5)$ & $22(4)$ & $38(4)$ & $9(3)$ & $2(4)$ & $7(4)$ \\
\hline$C(54)$ & $41(6)$ & $49(5)$ & $51(5)$ & $11(4)$ & $8(4)$ & $13(4)$ \\
\hline$C(55)$ & $40(5)$ & $15(4)$ & $42(5)$ & $-3(3)$ & $-4(4)$ & $1(3)$ \\
\hline
\end{tabular}




\begin{tabular}{lcccccc}
$\mathrm{C}(56)$ & $49(5)$ & $37(4)$ & $36(4)$ & $-1(3)$ & $1(5)$ & $8(5)$ \\
$\mathrm{O}(1 \mathrm{~S})$ & $92(6)$ & $103(5)$ & $62(4)$ & $-2(4)$ & $-6(4)$ & $-34(5)$ \\
$\mathrm{C}(1 \mathrm{~S})$ & $115(11)$ & $111(9)$ & $114(9)$ & $-14(8)$ & $-46(8)$ & $28(9)$ \\
$\mathrm{C}(2 \mathrm{~S})$ & $110(11)$ & $121(10)$ & $63(7)$ & $5(7)$ & $-35(7)$ & $-10(8)$ \\
$\mathrm{C}(3 \mathrm{~S})$ & $72(9)$ & $94(8)$ & $131(10)$ & $75(8)$ & $23(7)$ & $-14(7)$ \\
$\mathrm{C}(4 \mathrm{~S})$ & $278(19)$ & $90(9)$ & $140(11)$ & $4(9)$ & $-90(12)$ & $-60(11)$ \\
\hline
\end{tabular}


Table S59. Hydrogen coordinates $\left(\times 10^{4}\right)$ and isotropic displacement parameters $\left(\AA^{2} \times 10^{3}\right)$ for $\left[\left(\mathrm{L}^{1}{ }_{\mathrm{re}}\right) \mathrm{Fe}(\mathrm{III}) \mathrm{Cl}\right]\left(\mathrm{NEt}_{4}\right)(\mathbf{8})$

\begin{tabular}{|c|c|c|c|c|}
\hline & $\mathrm{X}$ & $\mathrm{y}$ & $\mathrm{z}$ & $\mathrm{U}(\mathrm{eq})$ \\
\hline $\mathrm{H}(3)$ & 1911 & 5986 & 864 & 31 \\
\hline $\mathrm{H}(4)$ & 585 & 7020 & 720 & 33 \\
\hline $\mathrm{H}(5)$ & -607 & 7519 & 1302 & 34 \\
\hline $\mathrm{H}(6)$ & -388 & 7048 & 2037 & 25 \\
\hline $\mathrm{H}(9)$ & 839 & 2914 & 2071 & 27 \\
\hline $\mathrm{H}(10)$ & -327 & 2733 & 1409 & 30 \\
\hline $\mathrm{H}(11)$ & -332 & 3656 & 848 & 33 \\
\hline $\mathrm{H}(12)$ & 843 & 4758 & 943 & 32 \\
\hline $\mathrm{H}(15)$ & 3247 & 3729 & 3720 & 30 \\
\hline $\mathrm{H}(16)$ & 3620 & 2427 & 3614 & 32 \\
\hline $\mathrm{H}(17)$ & 3425 & 1897 & 2906 & 35 \\
\hline $\mathrm{H}(18)$ & 2794 & 2626 & 2293 & 32 \\
\hline $\mathrm{H}(20)$ & -1551 & 5906 & 2307 & 32 \\
\hline $\mathrm{H}(21)$ & -2931 & 6110 & 2903 & 29 \\
\hline $\mathrm{H}(23)$ & 50 & 6374 & 3750 & 31 \\
\hline $\mathrm{H}(24)$ & 1414 & 6282 & 3140 & 28 \\
\hline $\mathrm{H}(26 \mathrm{~A})$ & -2961 & 7465 & 3652 & 59 \\
\hline $\mathrm{H}(26 \mathrm{~B})$ & -4024 & 7069 & 3961 & 59 \\
\hline $\mathrm{H}(26 \mathrm{C})$ & -3965 & 6890 & 3431 & 59 \\
\hline
\end{tabular}




\begin{tabular}{|c|c|c|c|}
\hline $\mathrm{H}(27 \mathrm{~A})$ & -1279 & 6059 & 4314 \\
\hline $\mathrm{H}(27 \mathrm{~B})$ & -2486 & 6538 & 4484 \\
\hline $\mathrm{H}(27 \mathrm{C})$ & -1355 & 6953 & 4217 \\
\hline $\mathrm{H}(28 \mathrm{~A})$ & -3706 & 5457 & 3563 \\
\hline $\mathrm{H}(28 \mathrm{~B})$ & -3934 & 5650 & 4085 \\
\hline $\mathrm{H}(28 \mathrm{C})$ & -2706 & 5172 & 3935 \\
\hline $\mathrm{H}(30)$ & 3902 & 6299 & 1429 \\
\hline $\mathrm{H}(31)$ & 5713 & 6269 & 985 \\
\hline $\mathrm{H}(33)$ & 5483 & 3990 & 907 \\
\hline $\mathrm{H}(34)$ & 3561 & 4045 & 1316 \\
\hline $\mathrm{H}(36 \mathrm{~A})$ & 8324 & 5287 & 1048 \\
\hline $\mathrm{H}(36 \mathrm{~B})$ & 7765 & 6078 & 869 \\
\hline $\mathrm{H}(36 \mathrm{C})$ & 8752 & 5609 & 565 \\
\hline $\mathrm{H}(37 \mathrm{~A})$ & 7301 & 5572 & -80 \\
\hline $\mathrm{H}(37 \mathrm{~B})$ & 6336 & 6094 & 202 \\
\hline $\mathrm{H}(37 \mathrm{C})$ & 5848 & 5314 & -17 \\
\hline $\mathrm{H}(38 \mathrm{~A})$ & 7548 & 4062 & 690 \\
\hline $\mathrm{H}(38 \mathrm{~B})$ & 8085 & 4389 & 223 \\
\hline $\mathrm{H}(38 \mathrm{C})$ & 6635 & 4110 & 258 \\
\hline $\mathrm{H}(40)$ & 1322 & 4467 & 3842 \\
\hline $\mathrm{H}(41)$ & 1291 & 5162 & 4503 \\
\hline $\mathrm{H}(43)$ & 3815 & 6614 & 3989 \\
\hline
\end{tabular}




\begin{tabular}{|c|c|c|c|}
\hline $\mathrm{H}(44)$ & 3874 & 5929 & 3317 \\
\hline $\mathrm{H}(46 \mathrm{~A})$ & 3787 & 6963 & 5165 \\
\hline $\mathrm{H}(46 \mathrm{~B})$ & 4340 & 6812 & 4669 \\
\hline $\mathrm{H}(46 \mathrm{C})$ & 4191 & 6121 & 5017 \\
\hline $\mathrm{H}(47 \mathrm{~A})$ & 2105 & 5614 & 5174 \\
\hline $\mathrm{H}(47 \mathrm{~B})$ & 876 & 6094 & 5027 \\
\hline $\mathrm{H}(47 \mathrm{C})$ & 1868 & 6441 & 5382 \\
\hline $\mathrm{H}(48 \mathrm{~A})$ & 973 & 7158 & 4554 \\
\hline $\mathrm{H}(48 \mathrm{~B})$ & 2320 & 7482 & 4387 \\
\hline $\mathrm{H}(48 \mathrm{C})$ & 1911 & 7565 & 4905 \\
\hline $\mathrm{H}(49 \mathrm{~A})$ & 997 & 8130 & 2176 \\
\hline H(49B) & 779 & 8828 & 2511 \\
\hline $\mathrm{H}(50 \mathrm{~A})$ & 1386 & 9661 & 1931 \\
\hline $\mathrm{H}(50 \mathrm{~B})$ & 144 & 9177 & 1806 \\
\hline $\mathrm{H}(50 \mathrm{C})$ & 1500 & 8949 & 1598 \\
\hline $\mathrm{H}(51 \mathrm{~A})$ & 4463 & 8428 & 2129 \\
\hline $\mathrm{H}(51 \mathrm{~B})$ & 3492 & 8875 & 1809 \\
\hline $\mathrm{H}(52 \mathrm{~A})$ & 3435 & 7279 & 1966 \\
\hline $\mathrm{H}(52 \mathrm{~B})$ & 3874 & 7678 & 1506 \\
\hline $\mathrm{H}(52 \mathrm{C})$ & 2413 & 7718 & 1661 \\
\hline $\mathrm{H}(53 \mathrm{~A})$ & 2095 & 8031 & 2995 \\
\hline $\mathrm{H}(53 \mathrm{~B})$ & 2491 & 7456 & 2602 \\
\hline
\end{tabular}




\begin{tabular}{|c|c|c|c|c|}
\hline $\mathrm{H}(54 \mathrm{~A})$ & 4623 & 7631 & 2759 & 70 \\
\hline $\mathrm{H}(54 \mathrm{~B})$ & 3906 & 7417 & 3219 & 70 \\
\hline $\mathrm{H}(54 \mathrm{C})$ & 4294 & 8281 & 3119 & 70 \\
\hline $\mathrm{H}(55 \mathrm{~A})$ & 4047 & 9288 & 2690 & \\
\hline $\mathrm{H}(55 \mathrm{~B})$ & 2968 & 9732 & 2416 & 39 \\
\hline $\mathrm{H}(56 \mathrm{~A})$ & 1559 & 9586 & 3024 & 61 \\
\hline $\mathrm{H}(56 \mathrm{~B})$ & 2832 & 10011 & 3183 & 61 \\
\hline $\mathrm{H}(56 \mathrm{C})$ & 2630 & 9130 & 3298 & 61 \\
\hline $\mathrm{H}(1 \mathrm{~S} 1)$ & 6081 & 9112 & 177 & 170 \\
\hline $\mathrm{H}(1 \mathrm{~S} 2)$ & 5687 & 9511 & 642 & 170 \\
\hline $\mathrm{H}(1 \mathrm{~S} 3)$ & 4777 & 9582 & 211 & 170 \\
\hline $\mathrm{H}(2 \mathrm{~S} 1)$ & 5221 & 8236 & 721 & 118 \\
\hline $\mathrm{H}(2 \mathrm{~S} 2)$ & 3885 & 8675 & 680 & 118 \\
\hline $\mathrm{H}(3 \mathrm{~S} 1)$ & 2833 & 7549 & 365 & 119 \\
\hline $\mathrm{H}(3 \mathrm{~S} 2)$ & 4136 & 7080 & 416 & 119 \\
\hline $\mathrm{H}(4 \mathrm{~S} 1)$ & 3093 & 7363 & -455 & 254 \\
\hline $\mathrm{H}(4 \mathrm{~S} 2)$ & 2662 & 6633 & -171 & 254 \\
\hline $\mathrm{H}(4 \mathrm{~S} 3)$ & 4118 & 6725 & -324 & 254 \\
\hline
\end{tabular}


Table S60. Torsion Angles, ${ }^{\circ}$, for $\left[\left(\mathrm{L}^{1}{ }_{\mathrm{re}}\right) \mathrm{Fe}(\mathrm{III}) \mathrm{Cl}\right]\left(\mathrm{NEt}_{4}\right)(\mathbf{8})$

\begin{tabular}{|c|c|c|c|}
\hline $\mathrm{N}(3)-\mathrm{Fe}(1)-\mathrm{N}(1)-\mathrm{C}(1)$ & $-82.1(5)$ & $\mathrm{C}(18)-\mathrm{C}(13)-\mathrm{C}(14)-\mathrm{C}(15)$ & $-1.6(10)$ \\
\hline $\mathrm{N}(4)-\mathrm{Fe}(1)-\mathrm{N}(1)-\mathrm{C}(1)$ & $-176.1(4)$ & $\mathrm{N}(3)-\mathrm{C}(13)-\mathrm{C}(14)-\mathrm{N}(4)$ & $-1.0(9)$ \\
\hline $\mathrm{Cl}(1)-\mathrm{Fe}(1)-\mathrm{N}(1)-\mathrm{C}(1)$ & $66.8(5)$ & $\mathrm{C}(18)-\mathrm{C}(13)-\mathrm{C}(14)-\mathrm{N}(4)$ & $174.9(6)$ \\
\hline $\mathrm{N}(2)-\mathrm{Fe}(1)-\mathrm{N}(1)-\mathrm{C}(1)$ & $-18.1(5)$ & $\mathrm{N}(4)-\mathrm{C}(14)-\mathrm{C}(15)-\mathrm{C}(16)$ & $-176.0(6)$ \\
\hline N(3)-Fe(1)-N(1)-C(19) & $96.7(5)$ & $\mathrm{C}(13)-\mathrm{C}(14)-\mathrm{C}(15)-\mathrm{C}(16)$ & $0.1(10)$ \\
\hline N(4)-Fe(1)-N(1)-C(19) & $2.6(5)$ & $\mathrm{C}(14)-\mathrm{C}(15)-\mathrm{C}(16)-\mathrm{C}(17)$ & $1.3(10)$ \\
\hline $\mathrm{Cl}(1)-\mathrm{Fe}(1)-\mathrm{N}(1)-\mathrm{C}(19)$ & $-114.4(4)$ & $\mathrm{C}(15)-\mathrm{C}(16)-\mathrm{C}(17)-\mathrm{C}(18)$ & $-1.2(10)$ \\
\hline $\mathrm{N}(2)-\mathrm{Fe}(1)-\mathrm{N}(1)-\mathrm{C}(19)$ & $160.7(5)$ & $\mathrm{C}(16)-\mathrm{C}(17)-\mathrm{C}(18)-\mathrm{C}(13)$ & $-0.3(10)$ \\
\hline N(3)-Fe(1)-N(2)-C(29) & $-93.2(5)$ & $\mathrm{N}(3)-\mathrm{C}(13)-\mathrm{C}(18)-\mathrm{C}(17)$ & $176.9(7)$ \\
\hline N(1)-Fe(1)-N(2)-C(29) & $143.9(5)$ & $\mathrm{C}(14)-\mathrm{C}(13)-\mathrm{C}(18)-\mathrm{C}(17)$ & $1.7(10)$ \\
\hline N(4)-Fe(1)-N(2)-C(29) & $-91.4(7)$ & $\mathrm{C}(1)-\mathrm{N}(1)-\mathrm{C}(19)-\mathrm{C}(24)$ & $-139.0(6)$ \\
\hline $\mathrm{Cl}(1)-\mathrm{Fe}(1)-\mathrm{N}(2)-\mathrm{C}(29)$ & $31.2(5)$ & $\mathrm{Fe}(1)-\mathrm{N}(1)-\mathrm{C}(19)-\mathrm{C}(24)$ & $42.2(7)$ \\
\hline $\mathrm{N}(3)-\mathrm{Fe}(1)-\mathrm{N}(2)-\mathrm{C}(2)$ & $141.0(4)$ & $\mathrm{C}(1)-\mathrm{N}(1)-\mathrm{C}(19)-\mathrm{C}(20)$ & $47.0(9)$ \\
\hline $\mathrm{N}(1)-\mathrm{Fe}(1)-\mathrm{N}(2)-\mathrm{C}(2)$ & $18.0(4)$ & $\mathrm{Fe}(1)-\mathrm{N}(1)-\mathrm{C}(19)-\mathrm{C}(20)$ & $-131.8(5)$ \\
\hline $\mathrm{N}(4)-\mathrm{Fe}(1)-\mathrm{N}(2)-\mathrm{C}(2)$ & $142.7(5)$ & $\mathrm{C}(24)-\mathrm{C}(19)-\mathrm{C}(20)-\mathrm{C}(21)$ & $-0.1(9)$ \\
\hline $\mathrm{Cl}(1)-\mathrm{Fe}(1)-\mathrm{N}(2)-\mathrm{C}(2)$ & $-94.7(4)$ & $\mathrm{N}(1)-\mathrm{C}(19)-\mathrm{C}(20)-\mathrm{C}(21)$ & $173.9(5)$ \\
\hline $\mathrm{N}(3)-\mathrm{Fe}(1)-\mathrm{N}(2)-\mathrm{C}(7)$ & $27.3(4)$ & $C(19)-C(20)-C(21)-C(22)$ & $-3.2(9)$ \\
\hline $\mathrm{N}(1)-\mathrm{Fe}(1)-\mathrm{N}(2)-\mathrm{C}(7)$ & $-95.7(4)$ & $\mathrm{C}(20)-\mathrm{C}(21)-\mathrm{C}(22)-\mathrm{C}(23)$ & $3.1(9)$ \\
\hline $\mathrm{N}(4)-\mathrm{Fe}(1)-\mathrm{N}(2)-\mathrm{C}(7)$ & $29.0(7)$ & $\mathrm{C}(20)-\mathrm{C}(21)-\mathrm{C}(22)-\mathrm{C}(25)$ & $-177.1(5)$ \\
\hline $\mathrm{Cl}(1)-\mathrm{Fe}(1)-\mathrm{N}(2)-\mathrm{C}(7)$ & $151.6(4)$ & $\mathrm{C}(21)-\mathrm{C}(22)-\mathrm{C}(23)-\mathrm{C}(24)$ & $0.0(9)$ \\
\hline $\mathrm{N}(1)-\mathrm{Fe}(1)-\mathrm{N}(3)-\mathrm{C}(13)$ & $-132.9(5)$ & $\mathrm{C}(25)-\mathrm{C}(22)-\mathrm{C}(23)-\mathrm{C}(24)$ & $-179.7(5)$ \\
\hline
\end{tabular}




\begin{tabular}{|c|c|c|c|}
\hline $\mathrm{N}(4)-\mathrm{Fe}(1)-\mathrm{N}(3)-\mathrm{C}(13)$ & $-16.0(5)$ & $C(20)-C(19)-C(24)-C(23)$ & $3.3(9)$ \\
\hline $\mathrm{Cl}(1)-\mathrm{Fe}(1)-\mathrm{N}(3)-\mathrm{C}(13)$ & $82.1(5)$ & $\mathrm{N}(1)-\mathrm{C}(19)-\mathrm{C}(24)-\mathrm{C}(23)$ & $-171.0(6)$ \\
\hline $\mathrm{N}(2)-\mathrm{Fe}(1)-\mathrm{N}(3)-\mathrm{C}(13)$ & $163.3(5)$ & $C(22)-C(23)-C(24)-C(19)$ & $-3.3(10)$ \\
\hline $\mathrm{N}(1)-\mathrm{Fe}(1)-\mathrm{N}(3)-\mathrm{C}(8)$ & $34.7(5)$ & $\mathrm{C}(21)-\mathrm{C}(22)-\mathrm{C}(25)-\mathrm{C}(28)$ & $62.2(8)$ \\
\hline $\mathrm{N}(4)-\mathrm{Fe}(1)-\mathrm{N}(3)-\mathrm{C}(8)$ & $151.7(5)$ & $\mathrm{C}(23)-\mathrm{C}(22)-\mathrm{C}(25)-\mathrm{C}(28)$ & $-118.0(7)$ \\
\hline $\mathrm{Cl}(1)-\mathrm{Fe}(1)-\mathrm{N}(3)-\mathrm{C}(8)$ & $-110.3(4)$ & $\mathrm{C}(21)-\mathrm{C}(22)-\mathrm{C}(25)-\mathrm{C}(27)$ & $-178.0(5)$ \\
\hline $\mathrm{N}(2)-\mathrm{Fe}(1)-\mathrm{N}(3)-\mathrm{C}(8)$ & $-29.1(4)$ & $\mathrm{C}(23)-\mathrm{C}(22)-\mathrm{C}(25)-\mathrm{C}(27)$ & $1.8(8)$ \\
\hline N(3)-Fe(1)-N(4)-C(39) & $-172.9(6)$ & $\mathrm{C}(21)-\mathrm{C}(22)-\mathrm{C}(25)-\mathrm{C}(26)$ & $-58.2(8)$ \\
\hline N(1)-Fe(1)-N(4)-C(39) & $-58.2(7)$ & $\mathrm{C}(23)-\mathrm{C}(22)-\mathrm{C}(25)-\mathrm{C}(26)$ & $121.5(7)$ \\
\hline $\mathrm{Cl}(1)-\mathrm{Fe}(1)-\mathrm{N}(4)-\mathrm{C}(39)$ & $64.6(6)$ & $\mathrm{C}(2)-\mathrm{N}(2)-\mathrm{C}(29)-\mathrm{C}(34)$ & $-146.3(6)$ \\
\hline N(2)-Fe(1)-N(4)-C(39) & $-174.7(5)$ & $\mathrm{C}(7)-\mathrm{N}(2)-\mathrm{C}(29)-\mathrm{C}(34)$ & $-17.7(9)$ \\
\hline N(3)-Fe(1)-N(4)-C(14) & $14.8(5)$ & $\mathrm{Fe}(1)-\mathrm{N}(2)-\mathrm{C}(29)-\mathrm{C}(34)$ & $94.4(6)$ \\
\hline $\mathrm{N}(1)-\mathrm{Fe}(1)-\mathrm{N}(4)-\mathrm{C}(14)$ & $129.5(4)$ & $\mathrm{C}(2)-\mathrm{N}(2)-\mathrm{C}(29)-\mathrm{C}(30)$ & $35.7(8)$ \\
\hline $\mathrm{Cl}(1)-\mathrm{Fe}(1)-\mathrm{N}(4)-\mathrm{C}(14)$ & $-107.7(4)$ & $\mathrm{C}(7)-\mathrm{N}(2)-\mathrm{C}(29)-\mathrm{C}(30)$ & $164.4(6)$ \\
\hline $\mathrm{N}(2)-\mathrm{Fe}(1)-\mathrm{N}(4)-\mathrm{C}(14)$ & $13.1(8)$ & $\mathrm{Fe}(1)-\mathrm{N}(2)-\mathrm{C}(29)-\mathrm{C}(30)$ & $-83.6(7)$ \\
\hline $\mathrm{C}(19)-\mathrm{N}(1)-\mathrm{C}(1)-\mathrm{C}(2)$ & $-163.6(6)$ & $\mathrm{C}(34)-\mathrm{C}(29)-\mathrm{C}(30)-\mathrm{C}(31)$ & 7.1(10) \\
\hline $\mathrm{Fe}(1)-\mathrm{N}(1)-\mathrm{C}(1)-\mathrm{C}(2)$ & $15.1(8)$ & $\mathrm{N}(2)-\mathrm{C}(29)-\mathrm{C}(30)-\mathrm{C}(31)$ & $-174.9(6)$ \\
\hline$C(19)-N(1)-C(1)-C(6)$ & $19.0(9)$ & $C(29)-C(30)-C(31)-C(32)$ & $-3.2(11)$ \\
\hline $\mathrm{Fe}(1)-\mathrm{N}(1)-\mathrm{C}(1)-\mathrm{C}(6)$ & $-162.2(5)$ & $\mathrm{C}(30)-\mathrm{C}(31)-\mathrm{C}(32)-\mathrm{C}(33)$ & $-3.1(10)$ \\
\hline $\mathrm{N}(1)-\mathrm{C}(1)-\mathrm{C}(2)-\mathrm{C}(3)$ & $-176.4(6)$ & $\mathrm{C}(30)-\mathrm{C}(31)-\mathrm{C}(32)-\mathrm{C}(35)$ & $174.8(6)$ \\
\hline$C(6)-C(1)-C(2)-C(3)$ & $1.2(9)$ & $\mathrm{C}(31)-\mathrm{C}(32)-\mathrm{C}(33)-\mathrm{C}(34)$ & $5.4(9)$ \\
\hline $\mathrm{N}(1)-\mathrm{C}(1)-\mathrm{C}(2)-\mathrm{N}(2)$ & $5.3(9)$ & $C(35)-C(32)-C(33)-C(34)$ & $-172.4(6)$ \\
\hline
\end{tabular}




\begin{tabular}{|c|c|c|c|}
\hline$C(6)-C(1)-C(2)-N(2)$ & $-177.2(5)$ & $\mathrm{C}(30)-\mathrm{C}(29)-\mathrm{C}(34)-\mathrm{C}(33)$ & $-4.8(10)$ \\
\hline$C(29)-N(2)-C(2)-C(3)$ & $39.3(8)$ & $\mathrm{N}(2)-\mathrm{C}(29)-\mathrm{C}(34)-\mathrm{C}(33)$ & $177.2(6)$ \\
\hline $\mathrm{C}(7)-\mathrm{N}(2)-\mathrm{C}(2)-\mathrm{C}(3)$ & $-90.5(7)$ & $\mathrm{C}(32)-\mathrm{C}(33)-\mathrm{C}(34)-\mathrm{C}(29)$ & $-1.6(11)$ \\
\hline $\mathrm{Fe}(1)-\mathrm{N}(2)-\mathrm{C}(2)-\mathrm{C}(3)$ & $164.8(5)$ & $\mathrm{C}(33)-\mathrm{C}(32)-\mathrm{C}(35)-\mathrm{C}(38)$ & $-3.7(10)$ \\
\hline$C(29)-N(2)-C(2)-C(1)$ & $-142.4(6)$ & $\mathrm{C}(31)-\mathrm{C}(32)-\mathrm{C}(35)-\mathrm{C}(38)$ & $178.5(6)$ \\
\hline $\mathrm{C}(7)-\mathrm{N}(2)-\mathrm{C}(2)-\mathrm{C}(1)$ & $87.8(6)$ & $\mathrm{C}(33)-\mathrm{C}(32)-\mathrm{C}(35)-\mathrm{C}(37)$ & $114.2(8)$ \\
\hline $\mathrm{Fe}(1)-\mathrm{N}(2)-\mathrm{C}(2)-\mathrm{C}(1)$ & $-16.8(6)$ & $\mathrm{C}(31)-\mathrm{C}(32)-\mathrm{C}(35)-\mathrm{C}(37)$ & $-63.6(8)$ \\
\hline$C(1)-C(2)-C(3)-C(4)$ & $-1.8(10)$ & $\mathrm{C}(33)-\mathrm{C}(32)-\mathrm{C}(35)-\mathrm{C}(36)$ & $-126.9(7)$ \\
\hline $\mathrm{N}(2)-\mathrm{C}(2)-\mathrm{C}(3)-\mathrm{C}(4)$ & $176.5(6)$ & $\mathrm{C}(31)-\mathrm{C}(32)-\mathrm{C}(35)-\mathrm{C}(36)$ & $55.3(8)$ \\
\hline$C(2)-C(3)-C(4)-C(5)$ & $0.1(10)$ & $\mathrm{C}(14)-\mathrm{N}(4)-\mathrm{C}(39)-\mathrm{C}(44)$ & $131.3(6)$ \\
\hline$C(3)-C(4)-C(5)-C(6)$ & $2.1(10)$ & $\mathrm{Fe}(1)-\mathrm{N}(4)-\mathrm{C}(39)-\mathrm{C}(44)$ & $-40.5(9)$ \\
\hline $\mathrm{N}(1)-\mathrm{C}(1)-\mathrm{C}(6)-\mathrm{C}(5)$ & $178.5(6)$ & $\mathrm{C}(14)-\mathrm{N}(4)-\mathrm{C}(39)-\mathrm{C}(40)$ & $-55.3(9)$ \\
\hline$C(2)-C(1)-C(6)-C(5)$ & $1.0(9)$ & $\mathrm{Fe}(1)-\mathrm{N}(4)-\mathrm{C}(39)-\mathrm{C}(40)$ & $132.8(5)$ \\
\hline$C(4)-C(5)-C(6)-C(1)$ & $-2.7(9)$ & $\mathrm{C}(44)-\mathrm{C}(39)-\mathrm{C}(40)-\mathrm{C}(41)$ & $0.2(10)$ \\
\hline $\mathrm{C}(29)-\mathrm{N}(2)-\mathrm{C}(7)-\mathrm{C}(8)$ & $97.1(7)$ & $\mathrm{N}(4)-\mathrm{C}(39)-\mathrm{C}(40)-\mathrm{C}(41)$ & $-173.2(6)$ \\
\hline $\mathrm{C}(2)-\mathrm{N}(2)-\mathrm{C}(7)-\mathrm{C}(8)$ & $-132.0(6)$ & $C(39)-C(40)-C(41)-C(42)$ & $0.5(10)$ \\
\hline $\mathrm{Fe}(1)-\mathrm{N}(2)-\mathrm{C}(7)-\mathrm{C}(8)$ & $-24.5(6)$ & $\mathrm{C}(40)-\mathrm{C}(41)-\mathrm{C}(42)-\mathrm{C}(43)$ & $-1.2(10)$ \\
\hline $\mathrm{C}(29)-\mathrm{N}(2)-\mathrm{C}(7)-\mathrm{C}(12)$ & $-78.1(8)$ & $\mathrm{C}(40)-\mathrm{C}(41)-\mathrm{C}(42)-\mathrm{C}(45)$ & $178.0(7)$ \\
\hline $\mathrm{C}(2)-\mathrm{N}(2)-\mathrm{C}(7)-\mathrm{C}(12)$ & $52.8(8)$ & $\mathrm{C}(41)-\mathrm{C}(42)-\mathrm{C}(43)-\mathrm{C}(44)$ & $1.2(10)$ \\
\hline $\mathrm{Fe}(1)-\mathrm{N}(2)-\mathrm{C}(7)-\mathrm{C}(12)$ & $160.3(6)$ & $C(45)-C(42)-C(43)-C(44)$ & $-178.0(7)$ \\
\hline $\mathrm{C}(12)-\mathrm{C}(7)-\mathrm{C}(8)-\mathrm{N}(3)$ & $-178.6(6)$ & $\mathrm{C}(40)-\mathrm{C}(39)-\mathrm{C}(44)-\mathrm{C}(43)$ & $-0.2(10)$ \\
\hline $\mathrm{N}(2)-\mathrm{C}(7)-\mathrm{C}(8)-\mathrm{N}(3)$ & $6.2(9)$ & $\mathrm{N}(4)-\mathrm{C}(39)-\mathrm{C}(44)-\mathrm{C}(43)$ & $173.5(6)$ \\
\hline
\end{tabular}




\begin{tabular}{|c|c|c|c|}
\hline$C(12)-C(7)-C(8)-C(9)$ & $-3.1(10)$ & $\mathrm{C}(42)-\mathrm{C}(43)-\mathrm{C}(44)-\mathrm{C}(39)$ & $-0.5(10)$ \\
\hline $\mathrm{N}(2)-\mathrm{C}(7)-\mathrm{C}(8)-\mathrm{C}(9)$ & $-178.4(5)$ & $C(41)-C(42)-C(45)-C(47)$ & $8.3(12)$ \\
\hline $\mathrm{C}(13)-\mathrm{N}(3)-\mathrm{C}(8)-\mathrm{C}(7)$ & $-168.3(6)$ & $C(43)-C(42)-C(45)-C(47)$ & $-172.6(8)$ \\
\hline $\mathrm{Fe}(1)-\mathrm{N}(3)-\mathrm{C}(8)-\mathrm{C}(7)$ & $25.3(7)$ & $C(41)-C(42)-C(45)-C(46)$ & $129.0(8)$ \\
\hline $\mathrm{C}(13)-\mathrm{N}(3)-\mathrm{C}(8)-\mathrm{C}(9)$ & $16.6(10)$ & $\mathrm{C}(43)-\mathrm{C}(42)-\mathrm{C}(45)-\mathrm{C}(46)$ & $-51.9(9)$ \\
\hline $\mathrm{Fe}(1)-\mathrm{N}(3)-\mathrm{C}(8)-\mathrm{C}(9)$ & $-149.8(5)$ & $\mathrm{C}(41)-\mathrm{C}(42)-\mathrm{C}(45)-\mathrm{C}(48)$ & $-111.4(8)$ \\
\hline $\mathrm{C}(7)-\mathrm{C}(8)-\mathrm{C}(9)-\mathrm{C}(10)$ & $1.9(9)$ & $\mathrm{C}(43)-\mathrm{C}(42)-\mathrm{C}(45)-\mathrm{C}(48)$ & $67.7(11)$ \\
\hline $\mathrm{N}(3)-\mathrm{C}(8)-\mathrm{C}(9)-\mathrm{C}(10)$ & $176.9(6)$ & $\mathrm{C}(51)-\mathrm{N}(5)-\mathrm{C}(49)-\mathrm{C}(50)$ & $-47.6(7)$ \\
\hline $\mathrm{C}(8)-\mathrm{C}(9)-\mathrm{C}(10)-\mathrm{C}(11)$ & $-0.3(10)$ & $\mathrm{C}(55)-\mathrm{N}(5)-\mathrm{C}(49)-\mathrm{C}(50)$ & $69.9(6)$ \\
\hline$C(9)-C(10)-C(11)-C(12)$ & $-0.1(10)$ & $\mathrm{C}(53)-\mathrm{N}(5)-\mathrm{C}(49)-\mathrm{C}(50)$ & $-169.8(5)$ \\
\hline $\mathrm{C}(10)-\mathrm{C}(11)-\mathrm{C}(12)-\mathrm{C}(7)$ & $-1.2(10)$ & $\mathrm{C}(55)-\mathrm{N}(5)-\mathrm{C}(51)-\mathrm{C}(52)$ & $179.2(5)$ \\
\hline $\mathrm{C}(8)-\mathrm{C}(7)-\mathrm{C}(12)-\mathrm{C}(11)$ & $2.9(11)$ & $\mathrm{C}(53)-\mathrm{N}(5)-\mathrm{C}(51)-\mathrm{C}(52)$ & $57.5(7)$ \\
\hline $\mathrm{N}(2)-\mathrm{C}(7)-\mathrm{C}(12)-\mathrm{C}(11)$ & $178.0(6)$ & $\mathrm{C}(49)-\mathrm{N}(5)-\mathrm{C}(51)-\mathrm{C}(52)$ & $-61.5(6)$ \\
\hline $\mathrm{C}(8)-\mathrm{N}(3)-\mathrm{C}(13)-\mathrm{C}(18)$ & $31.5(11)$ & $\mathrm{C}(51)-\mathrm{N}(5)-\mathrm{C}(53)-\mathrm{C}(54)$ & $63.3(7)$ \\
\hline $\mathrm{Fe}(1)-\mathrm{N}(3)-\mathrm{C}(13)-\mathrm{C}(18)$ & $-161.6(6)$ & $\mathrm{C}(55)-\mathrm{N}(5)-\mathrm{C}(53)-\mathrm{C}(54)$ & $-56.2(7)$ \\
\hline $\mathrm{C}(8)-\mathrm{N}(3)-\mathrm{C}(13)-\mathrm{C}(14)$ & $-153.1(6)$ & $\mathrm{C}(49)-\mathrm{N}(5)-\mathrm{C}(53)-\mathrm{C}(54)$ & $-176.0(5)$ \\
\hline $\mathrm{Fe}(1)-\mathrm{N}(3)-\mathrm{C}(13)-\mathrm{C}(14)$ & $13.8(8)$ & $\mathrm{C}(51)-\mathrm{N}(5)-\mathrm{C}(55)-\mathrm{C}(56)$ & $-173.4(5)$ \\
\hline $\mathrm{C}(39)-\mathrm{N}(4)-\mathrm{C}(14)-\mathrm{C}(15)$ & $-8.7(10)$ & $\mathrm{C}(53)-\mathrm{N}(5)-\mathrm{C}(55)-\mathrm{C}(56)$ & $-50.7(7)$ \\
\hline $\mathrm{Fe}(1)-\mathrm{N}(4)-\mathrm{C}(14)-\mathrm{C}(15)$ & $164.6(5)$ & $\mathrm{C}(49)-\mathrm{N}(5)-\mathrm{C}(55)-\mathrm{C}(56)$ & $67.3(7)$ \\
\hline $\mathrm{C}(39)-\mathrm{N}(4)-\mathrm{C}(14)-\mathrm{C}(13)$ & $175.0(6)$ & $\mathrm{C}(3 \mathrm{~S})-\mathrm{O}(1 \mathrm{~S})-\mathrm{C}(2 \mathrm{~S})-\mathrm{C}(1 \mathrm{~S})$ & $-177.3(8)$ \\
\hline $\mathrm{Fe}(1)-\mathrm{N}(4)-\mathrm{C}(14)-\mathrm{C}(13)$ & $-11.7(7)$ & $\mathrm{C}(2 \mathrm{~S})-\mathrm{O}(1 \mathrm{~S})-\mathrm{C}(3 \mathrm{~S})-\mathrm{C}(4 \mathrm{~S})$ & $179.5(10)$ \\
\hline $\mathrm{N}(3)-\mathrm{C}(13)-\mathrm{C}(14)-\mathrm{C}(15)$ & $-177.5(6)$ & & \\
\hline
\end{tabular}


Table S61. Atomic coordinates $\left(\times 10^{4}\right)$ and equivalent isotropic displacement parameters $\left(\AA^{2} \times 10^{3}\right)$ for $\left[\left(\mathrm{L}_{\text {re-2 }}^{1} \mathrm{H}\right)(\mathrm{Cl}) \mathrm{Fe}(\mathrm{II})(\mu \leftarrow \mathrm{Cl})_{2} \mathrm{Fe}(\mathrm{II})(\mathrm{Cl})\left(\mathrm{L}_{\mathrm{re}-2}^{1} \mathrm{H}\right)\right](9) . \mathrm{U}(\mathrm{eq})$ is defined as one third of the trace of the orthogonalized $\mathrm{U}^{\mathrm{ij}}$ tensor

\begin{tabular}{|c|c|c|c|c|}
\hline & $\mathrm{x}$ & $\mathrm{y}$ & $\mathrm{z}$ & $\mathrm{U}(\mathrm{eq})$ \\
\hline $\mathrm{Fe}(1)$ & 4342(2) & $379(1)$ & $9116(1)$ & $43(1)$ \\
\hline $\mathrm{Cl}(1)$ & 3653(3) & $-1030(2)$ & $9709(2)$ & $51(1)$ \\
\hline $\mathrm{Cl}(2)$ & $5785(3)$ & 717(3) & $8180(2)$ & $70(1)$ \\
\hline $\mathrm{N}(1)$ & 3444(9) & 1296(6) & $9654(5)$ & $36(2)$ \\
\hline $\mathrm{N}(2)$ & 2462(9) & $-155(6)$ & $8367(5)$ & $37(2)$ \\
\hline $\mathrm{N}(3)$ & 3533(9) & 2048(6) & $7633(5)$ & $50(3)$ \\
\hline $\mathrm{N}(4)$ & 4743(10) & 3093(6) & $9259(5)$ & $38(2)$ \\
\hline $\mathrm{C}(1)$ & 2082(12) & $963(7)$ & $9436(7)$ & $37(3)$ \\
\hline$C(2)$ & $1540(12)$ & $165(8)$ & $8686(7)$ & 41(3) \\
\hline$C(3)$ & 2141(11) & $-830(8)$ & $7569(7)$ & $44(3)$ \\
\hline $\mathrm{C}(4)$ & $1502(11)$ & $-676(8)$ & $6853(7)$ & $51(3)$ \\
\hline $\mathrm{C}(5)$ & $1255(12)$ & $-1316(9)$ & $6073(7)$ & $60(3)$ \\
\hline $\mathrm{C}(6)$ & $1659(13)$ & $-2110(10)$ & $6000(8)$ & $60(4)$ \\
\hline$C(7)$ & 2291(13) & $-2244(9)$ & $6713(8)$ & $62(4)$ \\
\hline$C(8)$ & 2522(11) & $-1625(8)$ & $7469(7)$ & $45(3)$ \\
\hline $\mathrm{C}(9)$ & $1270(18)$ & $-2818(12)$ & $5138(9)$ & $89(5)$ \\
\hline $\mathrm{C}(10)$ & $92(13)$ & $-172(8)$ & $8387(7)$ & $51(3)$ \\
\hline $\mathrm{C}(11)$ & $-723(11)$ & $255(8)$ & $8782(7)$ & $45(3)$ \\
\hline
\end{tabular}




\begin{tabular}{|c|c|c|c|c|}
\hline$C(12)$ & 1133(12) & 1356(7) & $9828(6)$ & $38(3)$ \\
\hline$C(13)$ & $3428(12)$ & 1834(9) & 6753(7) & $53(3)$ \\
\hline$C(14)$ & $2826(15)$ & $2261(10)$ & $6222(8)$ & $84(4)$ \\
\hline$C(16)$ & $3923(12)$ & 1141(9) & $6336(7)$ & $59(4)$ \\
\hline$C(17)$ & $3839(12)$ & 921(9) & $5489(8)$ & $58(3)$ \\
\hline$C(18)$ & 2801(13) & 2496(8) & $8117(7)$ & 41(3) \\
\hline$C(19)$ & $1440(12)$ & 2434(8) & $7825(7)$ & $55(3)$ \\
\hline$C(20)$ & $755(13)$ & 2882(9) & $8315(8)$ & $55(3)$ \\
\hline $\mathrm{C}(21)$ & $1366(12)$ & 3443(8) & $9098(8)$ & $52(3)$ \\
\hline$C(22)$ & $2682(11)$ & $3518(7)$ & $9417(7)$ & $42(3)$ \\
\hline$C(23)$ & $3382(11)$ & 3031(7) & $8942(7)$ & $36(3)$ \\
\hline$C(24)$ & $5868(12)$ & $3758(8)$ & $8978(6)$ & $39(3)$ \\
\hline$C(25)$ & $5705(11)$ & 4442(8) & $8551(7)$ & 44(3) \\
\hline$C(26)$ & $6808(14)$ & $5097(8)$ & $8282(6)$ & 49(3) \\
\hline$C(27)$ & $8162(13)$ & 5099(8) & $8410(7)$ & $44(3)$ \\
\hline$C(28)$ & $8266(12)$ & 4390(8) & $8831(6)$ & $43(3)$ \\
\hline$C(29)$ & $7194(12)$ & $3726(8)$ & $9111(6)$ & $43(3)$ \\
\hline $\mathrm{C}(30)$ & $9338(13)$ & 5814(9) & $8103(7)$ & $51(3)$ \\
\hline $\mathrm{C}(31)$ & $9895(15)$ & $5266(10)$ & $7483(9)$ & $118(6)$ \\
\hline$C(32)$ & 8984(14) & $6571(11)$ & $7736(10)$ & $118(6)$ \\
\hline$C(33)$ & $2750(14)$ & $2032(10)$ & $5352(8)$ & $83(4)$ \\
\hline$C(34)$ & $3224(13)$ & 1349(9) & 4948(8) & 61(4) \\
\hline
\end{tabular}




\begin{tabular}{|c|c|c|c|c|}
\hline$C(35)$ & $3083(14)$ & $1072(11)$ & $3987(7)$ & $65(4)$ \\
\hline$C(36)$ & 1978(19) & $69(10)$ & $3708(8)$ & $135(7)$ \\
\hline$C(37)$ & $2672(15)$ & $1783(10)$ & $3556(7)$ & $98(5)$ \\
\hline$C(38)$ & $4497(17)$ & $1099(15)$ & $3736(8)$ & $169(9)$ \\
\hline C(39) & $10462(15)$ & $6373(11)$ & $8846(10)$ & $117(6)$ \\
\hline$C(40)$ & $4902(10)$ & $3016(8)$ & $10123(6)$ & $29(3)$ \\
\hline$C(41)$ & $5767(11)$ & $3789(8)$ & $10745(7)$ & $43(3)$ \\
\hline$C(42)$ & $2130(30)$ & $-3320(20)$ & $5035(13)$ & $347(19)$ \\
\hline$C(43)$ & $1140(30)$ & $-2383(13)$ & $4434(10)$ & $298(17)$ \\
\hline$C(45)$ & $4134(11)$ & $2124(8)$ & $10324(6)$ & $32(3)$ \\
\hline$C(46)$ & $4178(12)$ & $2053(8)$ & $11159(7)$ & $41(3)$ \\
\hline$C(47)$ & $5010(11)$ & $2806(9)$ & $11768(7)$ & $46(3)$ \\
\hline$C(48)$ & $-202(11)$ & $1023(8)$ & $9506(7)$ & $45(3)$ \\
\hline C(49) & $5812(12)$ & $3672(9)$ & $11573(7)$ & $51(3)$ \\
\hline$C(50)$ & $-90(20)$ & $-3547(14)$ & $5050(13)$ & $233(13)$ \\
\hline$C(1 S)$ & $6240(30)$ & $5020(30)$ & $4026(19)$ & $156(14)$ \\
\hline$C(2 S)$ & $4890(30)$ & $4940(20)$ & $3900(10)$ & $126(11)$ \\
\hline$C(3 S)$ & $4385(15)$ & $4930(20)$ & $4712(9)$ & $102(10)$ \\
\hline
\end{tabular}


Table S62. Bond Distances, $\AA$, for $\left[\left(\mathrm{L}^{1}{ }_{\mathrm{re}-2 \mathrm{H}} \mathrm{H}(\mathrm{Cl}) \mathrm{Fe}(\mathrm{II})(\mu-\mathrm{Cl})_{2} \mathrm{Fe}(\mathrm{II})(\mathrm{Cl})\left(\mathrm{L}_{\mathrm{re}-2}{ }_{\mathrm{H}}\right)\right](9)\right.$

\begin{tabular}{|c|c|c|c|}
\hline $\mathrm{Fe}(1)-\mathrm{N}(1)$ & $2.019(8)$ & $\mathrm{C}(14)-\mathrm{C}(33)$ & $1.389(14)$ \\
\hline $\mathrm{Fe}(1)-\mathrm{N}(2)$ & $2.048(9)$ & $C(16)-C(17)$ & $1.351(13)$ \\
\hline $\mathrm{Fe}(1)-\mathrm{Cl}(2)$ & $2.230(3)$ & $\mathrm{C}(17)-\mathrm{C}(34)$ & $1.405(14)$ \\
\hline $\mathrm{Fe}(1)-\mathrm{Cl}(1)$ & $2.367(3)$ & $\mathrm{C}(18)-\mathrm{C}(23)$ & $1.407(13)$ \\
\hline $\mathrm{Fe}(1)-\mathrm{Cl}(1) \# 1$ & $2.515(3)$ & $\mathrm{C}(18)-\mathrm{C}(19)$ & $1.429(14)$ \\
\hline $\mathrm{Cl}(1)-\mathrm{Fe}(1) \# 1$ & $2.515(3)$ & $C(19)-C(20)$ & $1.350(14)$ \\
\hline $\mathrm{N}(1)-\mathrm{C}(1)$ & $1.332(12)$ & $C(20)-C(21)$ & $1.367(14)$ \\
\hline $\mathrm{N}(1)-\mathrm{C}(45)$ & $1.423(12)$ & $\mathrm{C}(21)-\mathrm{C}(22)$ & $1.391(13)$ \\
\hline $\mathrm{N}(2)-\mathrm{C}(2)$ & $1.315(11)$ & $C(22)-C(23)$ & $1.382(13)$ \\
\hline $\mathrm{N}(2)-\mathrm{C}(3)$ & $1.435(12)$ & $C(24)-C(25)$ & $1.371(12)$ \\
\hline $\mathrm{N}(3)-\mathrm{C}(18)$ & $1.381(12)$ & $\mathrm{C}(24)-\mathrm{C}(29)$ & $1.402(13)$ \\
\hline $\mathrm{N}(3)-\mathrm{C}(13)$ & $1.402(12)$ & $C(25)-C(26)$ & $1.390(13)$ \\
\hline $\mathrm{N}(4)-\mathrm{C}(24)$ & $1.421(12)$ & $\mathrm{C}(26)-\mathrm{C}(27)$ & $1.411(14)$ \\
\hline $\mathrm{N}(4)-\mathrm{C}(40)$ & $1.436(11)$ & $\mathrm{C}(27)-\mathrm{C}(28)$ & $1.379(13)$ \\
\hline $\mathrm{N}(4)-\mathrm{C}(23)$ & $1.439(12)$ & $\mathrm{C}(27)-\mathrm{C}(30)$ & $1.509(14)$ \\
\hline$C(1)-C(12)$ & $1.448(13)$ & $\mathrm{C}(28)-\mathrm{C}(29)$ & $1.386(13)$ \\
\hline $\mathrm{C}(1)-\mathrm{C}(2)$ & $1.464(14)$ & $\mathrm{C}(30)-\mathrm{C}(31)$ & $1.467(14)$ \\
\hline$C(2)-C(10)$ & $1.434(14)$ & $C(30)-C(32)$ & $1.493(14)$ \\
\hline$C(3)-C(8)$ & $1.354(12)$ & $\mathrm{C}(30)-\mathrm{C}(39)$ & $1.515(16)$ \\
\hline$C(3)-C(4)$ & $1.391(13)$ & $\mathrm{C}(33)-\mathrm{C}(34)$ & $1.365(14)$ \\
\hline$C(4)-C(5)$ & $1.395(14)$ & $C(34)-C(35)$ & $1.532(15)$ \\
\hline
\end{tabular}




$\begin{array}{lcll}\mathrm{C}(5)-\mathrm{C}(6) & 1.368(14) & \mathrm{C}(35)-\mathrm{C}(36) & 1.504(18) \\ \mathrm{C}(6)-\mathrm{C}(7) & 1.370(14) & \mathrm{C}(35)-\mathrm{C}(37) & 1.518(15) \\ \mathrm{C}(6)-\mathrm{C}(9) & 1.532(17) & \mathrm{C}(35)-\mathrm{C}(38) & 1.553(16) \\ \mathrm{C}(7)-\mathrm{C}(8) & 1.351(14) & \mathrm{C}(40)-\mathrm{C}(41) & 1.379(13) \\ \mathrm{C}(9)-\mathrm{C}(42) & 1.35(2) & \mathrm{C}(40)-\mathrm{C}(45) & 1.402(13) \\ \mathrm{C}(9)-\mathrm{C}(43) & 1.430(19) & \mathrm{C}(41)-\mathrm{C}(49) & 1.391(13) \\ \mathrm{C}(9)-\mathrm{C}(50) & 1.44(2) & \mathrm{C}(45)-\mathrm{C}(46) & 1.382(12) \\ \mathrm{C}(10)-\mathrm{C}(11) & 1.361(13) & \mathrm{C}(46)-\mathrm{C}(47) & 1.344(14) \\ \mathrm{C}(11)-\mathrm{C}(48) & 1.412(14) & \mathrm{C}(47)-\mathrm{C}(49) & 1.366(17) \\ \mathrm{C}(12)-\mathrm{C}(48) & 1.336(13) & \mathrm{C}(1 \mathrm{~S})-\mathrm{C}(2 \mathrm{~S}) & 1.467(17) \\ \mathrm{C}(13)-\mathrm{C}(14) & 1.385(13) & \mathrm{C}(2 \mathrm{~S})-\mathrm{C}(3 \mathrm{~S}) & 1.460(18) \\ \mathrm{C}(13)-\mathrm{C}(16) & 1.398(13) & \mathrm{C}(3 \mathrm{~S})-\mathrm{C}(3 \mathrm{~S}) \# 2 & \end{array}$

Symmetry transformations used to generate equivalent atoms:

$\# 1-x+1,-y,-z+2 \# 2-x+1,-y+1,-z+1$ 
Table S63. Angles, ${ }^{o}$, for $\left[\left(\mathrm{L}^{1}{ }_{\text {re- } 2} \mathrm{H}\right)(\mathrm{Cl}) \mathrm{Fe}(\mathrm{II})(\mu-\mathrm{Cl})_{2} \mathrm{Fe}(\mathrm{II})(\mathrm{Cl})\left(\mathrm{L}_{\text {re-2 } 2}^{1} \mathrm{H}\right)\right](9)$

\begin{tabular}{|c|c|c|c|}
\hline $\mathrm{N}(1)-\mathrm{Fe}(1)-\mathrm{N}(2)$ & $78.8(3)$ & $\mathrm{C}(16)-\mathrm{C}(17)-\mathrm{C}(34)$ & $123.2(10)$ \\
\hline $\mathrm{N}(1)-\mathrm{Fe}(1)-\mathrm{Cl}(2)$ & $124.4(2)$ & $\mathrm{N}(3)-\mathrm{C}(18)-\mathrm{C}(23)$ & $120.0(11)$ \\
\hline $\mathrm{N}(2)-\mathrm{Fe}(1)-\mathrm{Cl}(2)$ & $102.0(2)$ & $\mathrm{N}(3)-\mathrm{C}(18)-\mathrm{C}(19)$ & $123.0(11)$ \\
\hline $\mathrm{N}(1)-\mathrm{Fe}(1)-\mathrm{Cl}(1)$ & $107.4(2)$ & $\mathrm{C}(23)-\mathrm{C}(18)-\mathrm{C}(19)$ & $117.0(11)$ \\
\hline $\mathrm{N}(2)-\mathrm{Fe}(1)-\mathrm{Cl}(1)$ & $89.2(2)$ & $\mathrm{C}(20)-\mathrm{C}(19)-\mathrm{C}(18)$ & $121.4(11)$ \\
\hline $\mathrm{Cl}(2)-\mathrm{Fe}(1)-\mathrm{Cl}(1)$ & $128.08(13)$ & $\mathrm{C}(19)-\mathrm{C}(20)-\mathrm{C}(21)$ & $120.8(12)$ \\
\hline $\mathrm{N}(1)-\mathrm{Fe}(1)-\mathrm{Cl}(1) \# 1$ & $92.6(3)$ & $\mathrm{C}(20)-\mathrm{C}(21)-\mathrm{C}(22)$ & $120.1(11)$ \\
\hline $\mathrm{N}(2)-\mathrm{Fe}(1)-\mathrm{Cl}(1) \# 1$ & $167.6(3)$ & $\mathrm{C}(23)-\mathrm{C}(22)-\mathrm{C}(21)$ & $120.1(11)$ \\
\hline $\mathrm{Cl}(2)-\mathrm{Fe}(1)-\mathrm{Cl}(1) \# 1$ & $90.25(13)$ & $\mathrm{C}(22)-\mathrm{C}(23)-\mathrm{C}(18)$ & $120.4(11)$ \\
\hline $\mathrm{Cl}(1)-\mathrm{Fe}(1)-\mathrm{Cl}(1) \# 1$ & $84.80(11)$ & $\mathrm{C}(22)-\mathrm{C}(23)-\mathrm{N}(4)$ & $121.2(10)$ \\
\hline $\mathrm{Fe}(1)-\mathrm{Cl}(1)-\mathrm{Fe}(1) \# 1$ & $95.20(11)$ & $\mathrm{C}(18)-\mathrm{C}(23)-\mathrm{N}(4)$ & $118.3(10)$ \\
\hline $\mathrm{C}(1)-\mathrm{N}(1)-\mathrm{C}(45)$ & $121.5(9)$ & $\mathrm{C}(25)-\mathrm{C}(24)-\mathrm{C}(29)$ & $117.2(11)$ \\
\hline $\mathrm{C}(1)-\mathrm{N}(1)-\mathrm{Fe}(1)$ & $113.7(7)$ & $\mathrm{C}(25)-\mathrm{C}(24)-\mathrm{N}(4)$ & $121.8(10)$ \\
\hline $\mathrm{C}(45)-\mathrm{N}(1)-\mathrm{Fe}(1)$ & $123.7(7)$ & $\mathrm{C}(29)-\mathrm{C}(24)-\mathrm{N}(4)$ & $120.9(10)$ \\
\hline$C(2)-N(2)-C(3)$ & $121.7(10)$ & $\mathrm{C}(24)-\mathrm{C}(25)-\mathrm{C}(26)$ & $121.8(10)$ \\
\hline $\mathrm{C}(2)-\mathrm{N}(2)-\mathrm{Fe}(1)$ & $114.0(7)$ & $\mathrm{C}(25)-\mathrm{C}(26)-\mathrm{C}(27)$ & $123.0(10)$ \\
\hline $\mathrm{C}(3)-\mathrm{N}(2)-\mathrm{Fe}(1)$ & $124.2(7)$ & $\mathrm{C}(28)-\mathrm{C}(27)-\mathrm{C}(26)$ & $112.8(11)$ \\
\hline $\mathrm{C}(18)-\mathrm{N}(3)-\mathrm{C}(13)$ & $127.9(9)$ & $\mathrm{C}(28)-\mathrm{C}(27)-\mathrm{C}(30)$ & $125.4(11)$ \\
\hline $\mathrm{C}(24)-\mathrm{N}(4)-\mathrm{C}(40)$ & $117.8(9)$ & $C(26)-C(27)-C(30)$ & $121.8(11)$ \\
\hline $\mathrm{C}(24)-\mathrm{N}(4)-\mathrm{C}(23)$ & $117.1(8)$ & $\mathrm{C}(27)-\mathrm{C}(28)-\mathrm{C}(29)$ & $125.9(11)$ \\
\hline $\mathrm{C}(40)-\mathrm{N}(4)-\mathrm{C}(23)$ & $113.6(8)$ & $\mathrm{C}(28)-\mathrm{C}(29)-\mathrm{C}(24)$ & $119.2(10)$ \\
\hline
\end{tabular}




\begin{tabular}{|c|c|c|c|}
\hline $\mathrm{N}(1)-\mathrm{C}(1)-\mathrm{C}(12)$ & $126.4(10)$ & $\mathrm{C}(31)-\mathrm{C}(30)-\mathrm{C}(32)$ & $109.5(11)$ \\
\hline $\mathrm{N}(1)-\mathrm{C}(1)-\mathrm{C}(2)$ & $115.0(10)$ & $\mathrm{C}(31)-\mathrm{C}(30)-\mathrm{C}(27)$ & $109.2(10)$ \\
\hline$C(12)-C(1)-C(2)$ & $118.4(11)$ & $C(32)-C(30)-C(27)$ & $115.0(11)$ \\
\hline $\mathrm{N}(2)-\mathrm{C}(2)-\mathrm{C}(10)$ & $127.8(11)$ & $\mathrm{C}(31)-\mathrm{C}(30)-\mathrm{C}(39)$ & $108.4(12)$ \\
\hline $\mathrm{N}(2)-\mathrm{C}(2)-\mathrm{C}(1)$ & $114.9(10)$ & $\mathrm{C}(32)-\mathrm{C}(30)-\mathrm{C}(39)$ & $106.3(12)$ \\
\hline $\mathrm{C}(10)-\mathrm{C}(2)-\mathrm{C}(1)$ & $117.3(10)$ & $\mathrm{C}(27)-\mathrm{C}(30)-\mathrm{C}(39)$ & $108.3(10)$ \\
\hline $\mathrm{C}(8)-\mathrm{C}(3)-\mathrm{C}(4)$ & $116.7(11)$ & $\mathrm{C}(34)-\mathrm{C}(33)-\mathrm{C}(14)$ & $123.0(11)$ \\
\hline $\mathrm{C}(8)-\mathrm{C}(3)-\mathrm{N}(2)$ & $121.0(10)$ & $\mathrm{C}(33)-\mathrm{C}(34)-\mathrm{C}(17)$ & $114.2(11)$ \\
\hline $\mathrm{C}(4)-\mathrm{C}(3)-\mathrm{N}(2)$ & $122.2(10)$ & $C(33)-C(34)-C(35)$ & $122.6(12)$ \\
\hline$C(3)-C(4)-C(5)$ & $121.4(11)$ & $\mathrm{C}(17)-\mathrm{C}(34)-\mathrm{C}(35)$ & $123.2(11)$ \\
\hline$C(6)-C(5)-C(4)$ & $120.0(11)$ & $\mathrm{C}(36)-\mathrm{C}(35)-\mathrm{C}(37)$ & $108.7(12)$ \\
\hline$C(5)-C(6)-C(7)$ & $117.5(11)$ & $\mathrm{C}(36)-\mathrm{C}(35)-\mathrm{C}(34)$ & $106.9(11)$ \\
\hline $\mathrm{C}(5)-\mathrm{C}(6)-\mathrm{C}(9)$ & $117.1(13)$ & $\mathrm{C}(37)-\mathrm{C}(35)-\mathrm{C}(34)$ & $112.7(10)$ \\
\hline$C(7)-C(6)-C(9)$ & $125.2(13)$ & $\mathrm{C}(36)-\mathrm{C}(35)-\mathrm{C}(38)$ & $112.7(13)$ \\
\hline$C(8)-C(7)-C(6)$ & $122.3(12)$ & $\mathrm{C}(37)-\mathrm{C}(35)-\mathrm{C}(38)$ & $106.7(12)$ \\
\hline $\mathrm{C}(7)-\mathrm{C}(8)-\mathrm{C}(3)$ & $122.1(11)$ & $\mathrm{C}(34)-\mathrm{C}(35)-\mathrm{C}(38)$ & $109.1(11)$ \\
\hline$C(42)-C(9)-C(43)$ & $110.7(18)$ & $\mathrm{C}(41)-\mathrm{C}(40)-\mathrm{C}(45)$ & $120.4(9)$ \\
\hline$C(42)-C(9)-C(50)$ & $106.3(19)$ & $\mathrm{C}(41)-\mathrm{C}(40)-\mathrm{N}(4)$ & $121.8(10)$ \\
\hline$C(43)-C(9)-C(50)$ & $101.5(18)$ & $\mathrm{C}(45)-\mathrm{C}(40)-\mathrm{N}(4)$ & $117.8(10)$ \\
\hline$C(42)-C(9)-C(6)$ & $111.2(15)$ & $\mathrm{C}(40)-\mathrm{C}(41)-\mathrm{C}(49)$ & $118.3(11)$ \\
\hline$C(43)-C(9)-C(6)$ & $115.1(14)$ & $C(46)-C(45)-C(40)$ & $119.2(10)$ \\
\hline $\mathrm{C}(50)-\mathrm{C}(9)-\mathrm{C}(6)$ & $111.3(13)$ & $\mathrm{C}(46)-\mathrm{C}(45)-\mathrm{N}(1)$ & $122.1(10)$ \\
\hline
\end{tabular}




$\begin{array}{lclr}\mathrm{C}(11)-\mathrm{C}(10)-\mathrm{C}(2) & 120.5(11) & \mathrm{C}(40)-\mathrm{C}(45)-\mathrm{N}(1) & 118.5(9) \\ \mathrm{C}(10)-\mathrm{C}(11)-\mathrm{C}(48) & 122.2(11) & \mathrm{C}(47)-\mathrm{C}(46)-\mathrm{C}(45) & 120.6(11) \\ \mathrm{C}(48)-\mathrm{C}(12)-\mathrm{C}(1) & 121.3(11) & \mathrm{C}(46)-\mathrm{C}(47)-\mathrm{C}(49) & 120.6(11) \\ \mathrm{C}(14)-\mathrm{C}(13)-\mathrm{C}(16) & 114.2(11) & \mathrm{C}(12)-\mathrm{C}(48)-\mathrm{C}(11) & 120.2(11) \\ \mathrm{C}(14)-\mathrm{C}(13)-\mathrm{N}(3) & 124.4(10) & \mathrm{C}(47)-\mathrm{C}(49)-\mathrm{C}(41) & 120.8(11) \\ \mathrm{C}(16)-\mathrm{C}(13)-\mathrm{N}(3) & 121.4(10) & \mathrm{C}(1 \mathrm{~S})-\mathrm{C}(2 \mathrm{~S})-\mathrm{C}(3 \mathrm{~S}) & 107.7(15) \\ \mathrm{C}(13)-\mathrm{C}(14)-\mathrm{C}(33) & 122.6(11) & \mathrm{C}(3 \mathrm{~S}) \# 2-\mathrm{C}(3 \mathrm{~S})-\mathrm{C}(2 \mathrm{~S}) & 102.5(14) \\ \mathrm{C}(17)-\mathrm{C}(16)-\mathrm{C}(13) & 122.7(11) & & \end{array}$

Symmetry transformations used to generate equivalent atoms:

$\# 1-\mathrm{x}+1,-\mathrm{y},-\mathrm{z}+2 \# 2-\mathrm{x}+1,-\mathrm{y}+1,-\mathrm{z}+1$ 
Table S64. Anisotropic displacement parameters $\left(\AA^{2} \times 10^{3}\right)$ for $\left[\left(\mathrm{L}^{1}{ }_{\mathrm{re}-2} \mathrm{H}\right)(\mathrm{Cl}) \mathrm{Fe}(\mathrm{II})(\mu-\right.$ $\left.\mathrm{Cl})_{2} \mathrm{Fe}(\mathrm{II})(\mathrm{Cl})\left(\mathrm{L}^{1}{ }_{\text {re-2 }} \mathrm{H}\right)\right](9)$. The anisotropic displacement factor exponent takes the form: $-2 \pi^{2}\left[h^{2} a^{* 2} U^{11}+\ldots+2 h k a^{*} b^{*} U^{12}\right]$

\begin{tabular}{|c|c|c|c|c|c|c|}
\hline & $\mathrm{U}^{11}$ & $\mathrm{U}^{22}$ & $\mathrm{U}^{33}$ & $\mathrm{U}^{23}$ & $\mathrm{U}^{13}$ & $\mathrm{U}^{12}$ \\
\hline $\mathrm{Fe}(1)$ & $44(1)$ & $40(1)$ & $47(1)$ & $7(1)$ & $5(1)$ & $20(1)$ \\
\hline $\mathrm{Cl}(1)$ & $45(2)$ & 44(2) & $58(2)$ & $10(2)$ & $-2(2)$ & 12(2) \\
\hline $\mathrm{Cl}(2)$ & $58(2)$ & $107(3)$ & $58(2)$ & $30(2)$ & $22(2)$ & $36(2)$ \\
\hline $\mathrm{N}(1)$ & $33(6)$ & 19(5) & $46(6)$ & $9(4)$ & $-4(5)$ & $0(5)$ \\
\hline $\mathrm{N}(2)$ & $38(6)$ & $30(5)$ & $42(6)$ & $5(5)$ & $7(5)$ & $14(5)$ \\
\hline $\mathrm{N}(3)$ & $69(7)$ & 61(7) & $32(6)$ & $13(5)$ & $0(6)$ & $40(6)$ \\
\hline $\mathrm{N}(4)$ & $42(7)$ & $45(6)$ & $30(6)$ & $11(5)$ & $3(5)$ & $19(5)$ \\
\hline $\mathrm{C}(1)$ & $48(8)$ & $24(7)$ & $38(7)$ & $12(6)$ & $14(6)$ & $7(6)$ \\
\hline $\mathrm{C}(2)$ & $28(7)$ & 49(8) & $52(8)$ & $19(6)$ & $0(6)$ & $18(6)$ \\
\hline$C(3)$ & $43(8)$ & $40(8)$ & $45(8)$ & $7(6)$ & $9(6)$ & $12(6)$ \\
\hline $\mathrm{C}(4)$ & $51(8)$ & $55(8)$ & $46(8)$ & $9(7)$ & $3(7)$ & 19(7) \\
\hline$C(5)$ & $55(9)$ & 68(9) & $37(8)$ & $-2(7)$ & $-9(7)$ & 7(7) \\
\hline $\mathrm{C}(6)$ & $50(8)$ & 59(9) & $54(9)$ & $-7(7)$ & $23(7)$ & $6(7)$ \\
\hline$C(7)$ & $79(10)$ & $50(8)$ & $52(9)$ & $-1(7)$ & $7(8)$ & $24(7)$ \\
\hline$C(8)$ & $64(8)$ & $39(7)$ & $40(7)$ & $3(6)$ & $5(6)$ & $29(7)$ \\
\hline $\mathrm{C}(9)$ & $113(13)$ & $84(11)$ & $74(11)$ & $-17(9)$ & $21(10)$ & $54(10)$ \\
\hline$C(10)$ & 66(9) & $36(7)$ & $48(8)$ & $7(6)$ & $0(7)$ & $17(7)$ \\
\hline $\mathrm{C}(11)$ & $35(7)$ & $40(7)$ & $62(8)$ & $14(6)$ & $8(7)$ & $16(6)$ \\
\hline
\end{tabular}




\begin{tabular}{|c|c|c|c|c|c|c|}
\hline$C(12)$ & $48(8)$ & $37(7)$ & $35(7)$ & $12(5)$ & $6(6)$ & $21(6)$ \\
\hline$C(13)$ & $61(9)$ & $60(9)$ & $43(8)$ & $14(7)$ & $-5(7)$ & $28(7)$ \\
\hline$C(14)$ & $132(12)$ & $103(11)$ & $38(8)$ & $-3(8)$ & $-9(9)$ & $85(10)$ \\
\hline$C(16)$ & $67(9)$ & $76(9)$ & $50(8)$ & $6(7)$ & $11(7)$ & $48(8)$ \\
\hline$C(17)$ & $58(9)$ & $73(9)$ & $53(9)$ & $0(7)$ & $14(7)$ & $41(7)$ \\
\hline$C(18)$ & $59(9)$ & $33(7)$ & $35(7)$ & $18(6)$ & $9(7)$ & $14(7)$ \\
\hline C(19) & $44(8)$ & $66(9)$ & $48(8)$ & $8(7)$ & $-17(7)$ & $19(7)$ \\
\hline$C(20)$ & $42(8)$ & $66(9)$ & $59(9)$ & $17(7)$ & $-11(7)$ & $25(7)$ \\
\hline $\mathrm{C}(21)$ & $39(8)$ & $53(8)$ & $65(9)$ & $18(7)$ & $6(7)$ & $18(7)$ \\
\hline$C(22)$ & $41(8)$ & $24(7)$ & $51(8)$ & $5(6)$ & $-8(7)$ & $3(6)$ \\
\hline $\mathrm{C}(23)$ & $39(8)$ & $33(7)$ & $31(7)$ & $6(6)$ & $-3(6)$ & $10(6)$ \\
\hline$C(24)$ & $36(7)$ & $45(8)$ & $34(7)$ & $16(6)$ & $1(6)$ & $11(6)$ \\
\hline$C(25)$ & $26(7)$ & $46(8)$ & $60(8)$ & $27(6)$ & $-1(6)$ & $6(6)$ \\
\hline$C(26)$ & $84(10)$ & $33(7)$ & $37(7)$ & $17(6)$ & $11(7)$ & $26(7)$ \\
\hline $\mathrm{C}(27)$ & $43(8)$ & $44(8)$ & $43(7)$ & $6(6)$ & $-1(6)$ & $18(7)$ \\
\hline$C(28)$ & $38(7)$ & $42(8)$ & $46(7)$ & $8(6)$ & $-1(6)$ & $14(6)$ \\
\hline$C(29)$ & $42(8)$ & $51(8)$ & $45(7)$ & $25(6)$ & $-3(6)$ & $22(7)$ \\
\hline$C(30)$ & $48(9)$ & $53(9)$ & $43(8)$ & $11(7)$ & $18(7)$ & $3(7)$ \\
\hline$C(31)$ & $150(16)$ & $74(11)$ & $110(13)$ & $8(10)$ & $89(12)$ & $6(10)$ \\
\hline$C(32)$ & $66(11)$ & $105(13)$ & $213(18)$ & $110(13)$ & $45(12)$ & $26(10)$ \\
\hline$C(33)$ & $119(12)$ & 101(11) & $45(9)$ & $12(8)$ & $-17(9)$ & $68(10)$ \\
\hline$C(34)$ & $63(9)$ & $73(9)$ & $53(8)$ & $-3(7)$ & $9(7)$ & $39(7)$ \\
\hline
\end{tabular}




\begin{tabular}{|c|c|c|c|c|c|c|}
\hline$C(35)$ & $65(10)$ & $89(11)$ & $32(8)$ & $-10(8)$ & $1(7)$ & $30(8)$ \\
\hline$C(36)$ & $260(20)$ & $60(11)$ & $43(10)$ & $6(8)$ & $-30(12)$ & $15(13)$ \\
\hline$C(37)$ & $127(14)$ & $96(13)$ & $46(9)$ & $22(9)$ & $4(9)$ & $9(11)$ \\
\hline$C(38)$ & $110(15)$ & $340(30)$ & $56(11)$ & $-29(14)$ & $24(11)$ & $103(18)$ \\
\hline C(39) & $77(12)$ & $130(15)$ & $118(14)$ & $43(12)$ & $22(11)$ & $-4(11)$ \\
\hline$C(40)$ & $26(6)$ & $33(7)$ & $28(6)$ & $0(5)$ & $0(5)$ & $15(6)$ \\
\hline$C(41)$ & $52(8)$ & $33(7)$ & $43(8)$ & $1(6)$ & $-1(7)$ & $18(6)$ \\
\hline$C(42)$ & $260(30)$ & $540(40)$ & $180(20)$ & $-280(30)$ & $-120(20)$ & $290(30)$ \\
\hline$C(43)$ & $700(50)$ & $75(15)$ & $53(13)$ & $15(10)$ & $90(20)$ & $60(20)$ \\
\hline$C(45)$ & $37(7)$ & $25(7)$ & $32(7)$ & $-3(6)$ & $-1(6)$ & $17(6)$ \\
\hline$C(46)$ & $50(8)$ & $36(7)$ & $37(7)$ & $10(6)$ & $7(6)$ & $15(6)$ \\
\hline$C(47)$ & $42(8)$ & $50(8)$ & $43(8)$ & $12(7)$ & $-8(6)$ & $14(7)$ \\
\hline$C(48)$ & $27(7)$ & $56(8)$ & $51(8)$ & $17(7)$ & $6(6)$ & $12(6)$ \\
\hline C(49) & $50(8)$ & $45(8)$ & $48(8)$ & $-10(6)$ & $-18(7)$ & $19(7)$ \\
\hline$C(50)$ & $190(20)$ & $148(19)$ & $190(20)$ & $-104(17)$ & 39(19) & $-86(17)$ \\
\hline
\end{tabular}


Table S65. Hydrogen coordinates $\left(\times 10^{4}\right)$ and isotropic displacement parameters $\left(\AA^{2} \times 10^{3}\right)$ for $\left[\left(\mathrm{L}^{1}{ }_{\mathrm{re}-2} \mathrm{H}\right)(\mathrm{Cl}) \mathrm{Fe}(\mathrm{II})(\mu-\mathrm{Cl})_{2} \mathrm{Fe}(\mathrm{II})(\mathrm{Cl})\left(\mathrm{L}^{1}{ }_{\text {re-2 }} \mathrm{H}\right)\right](9)$

\begin{tabular}{|c|c|c|c|c|}
\hline & $\mathrm{x}$ & $\mathrm{y}$ & $\mathrm{z}$ & $\mathrm{U}(\mathrm{eq})$ \\
\hline H3A & 0.4126 & 0.1881 & 0.7904 & 0.060 \\
\hline $\mathrm{H} 4 \mathrm{~A}$ & 0.1233 & -0.0134 & 0.6895 & 0.061 \\
\hline H5A & 0.0815 & -0.1204 & 0.5604 & 0.072 \\
\hline H7A & 0.2572 & -0.2781 & 0.6677 & 0.074 \\
\hline H8A & 0.2955 & -0.1749 & 0.7934 & 0.054 \\
\hline H10A & -0.0293 & -0.0687 & 0.7920 & 0.061 \\
\hline H11A & -0.1655 & 0.0034 & 0.8568 & 0.053 \\
\hline $\mathrm{H} 12 \mathrm{~A}$ & 0.1458 & 0.1851 & 1.0313 & 0.046 \\
\hline H14A & 0.2459 & 0.2719 & 0.6457 & 0.100 \\
\hline H16A & 0.4326 & 0.0818 & 0.6655 & 0.071 \\
\hline H17A & 0.4207 & 0.0464 & 0.5253 & 0.070 \\
\hline H19A & 0.1018 & 0.2078 & 0.7285 & 0.066 \\
\hline $\mathrm{H} 20 \mathrm{~A}$ & -0.0143 & 0.2808 & 0.8118 & 0.066 \\
\hline $\mathrm{H} 21 \mathrm{~A}$ & 0.0901 & 0.3775 & 0.9418 & 0.062 \\
\hline $\mathrm{H} 22 \mathrm{~A}$ & 0.3093 & 0.3896 & 0.9952 & 0.051 \\
\hline $\mathrm{H} 25 \mathrm{~A}$ & 0.4832 & 0.4468 & 0.8440 & 0.053 \\
\hline $\mathrm{H} 26 \mathrm{~A}$ & 0.6646 & 0.5554 & 0.8004 & 0.058 \\
\hline $\mathrm{H} 28 \mathrm{~A}$ & 0.9134 & 0.4354 & 0.8935 & 0.051 \\
\hline H29A & 0.7352 & 0.3265 & 0.9385 & 0.052 \\
\hline \multicolumn{5}{|c|}{ S260 } \\
\hline
\end{tabular}




\begin{tabular}{|c|c|c|c|c|}
\hline H31A & 0.9199 & 0.4917 & 0.7002 & 0.177 \\
\hline H31B & 1.0174 & 0.4808 & 0.7730 & 0.177 \\
\hline $\mathrm{H} 31 \mathrm{C}$ & 1.0675 & 0.5716 & 0.7309 & 0.177 \\
\hline $\mathrm{H} 32 \mathrm{~A}$ & 0.8275 & 0.6251 & 0.7258 & 0.177 \\
\hline H32B & 0.9788 & 0.6995 & 0.7562 & 0.177 \\
\hline $\mathrm{H} 32 \mathrm{C}$ & 0.8663 & 0.6951 & 0.8151 & 0.177 \\
\hline H33A & 0.2358 & 0.2358 & 0.5031 & 0.099 \\
\hline H36A & 0.1129 & 0.0091 & 0.3880 & 0.202 \\
\hline H36B & 0.2242 & -0.0393 & 0.3960 & 0.202 \\
\hline H36C & 0.1856 & -0.0131 & 0.3106 & 0.202 \\
\hline H37A & 0.1787 & 0.1775 & 0.3683 & 0.147 \\
\hline H37B & 0.2625 & 0.1589 & 0.2958 & 0.147 \\
\hline H37C & 0.3343 & 0.2434 & 0.3755 & 0.147 \\
\hline H38A & 0.4791 & 0.0650 & 0.3990 & 0.254 \\
\hline H38B & 0.5165 & 0.1752 & 0.3928 & 0.254 \\
\hline H38C & 0.4410 & 0.0911 & 0.3134 & 0.254 \\
\hline H39A & 1.0721 & 0.5916 & 0.9109 & 0.175 \\
\hline H39B & 1.0128 & 0.6762 & 0.9247 & 0.175 \\
\hline H39C & 1.1248 & 0.6795 & 0.8655 & 0.175 \\
\hline H41A & 0.6307 & 0.4373 & 1.0613 & 0.052 \\
\hline $\mathrm{H} 42 \mathrm{~A}$ & 0.1870 & -0.3750 & 0.4490 & 0.520 \\
\hline H42B & 0.2089 & -0.3691 & 0.5463 & 0.520 \\
\hline
\end{tabular}




\begin{tabular}{|c|c|c|c|c|}
\hline $\mathrm{H} 42 \mathrm{C}$ & 0.3058 & -0.2859 & 0.5079 & 0.520 \\
\hline $\mathrm{H} 43 \mathrm{~A}$ & 0.0903 & -0.2875 & 0.3923 & 0.447 \\
\hline H43B & 0.2002 & -0.1874 & 0.4424 & 0.447 \\
\hline $\mathrm{H} 43 \mathrm{C}$ & 0.0435 & -0.2107 & 0.4483 & 0.447 \\
\hline $\mathrm{H} 46 \mathrm{~A}$ & 0.3626 & 0.1479 & 1.1300 & 0.049 \\
\hline H47A & 0.5045 & 0.2743 & 1.2326 & 0.055 \\
\hline $\mathrm{H} 48 \mathrm{~A}$ & -0.0789 & 0.1297 & 0.9760 & 0.053 \\
\hline H49A & 0.6391 & 0.4184 & 1.2001 & 0.061 \\
\hline H50A & -0.0297 & -0.3988 & 0.4510 & 0.349 \\
\hline H50B & -0.0751 & -0.3229 & 0.5093 & 0.349 \\
\hline $\mathrm{H} 50 \mathrm{C}$ & -0.0129 & -0.3909 & 0.5487 & 0.349 \\
\hline H1S1 & 0.6560 & 0.5165 & 0.4620 & 0.234 \\
\hline H1S2 & 0.6789 & 0.5529 & 0.3778 & 0.234 \\
\hline H1S3 & 0.6309 & 0.4403 & 0.3771 & 0.234 \\
\hline $\mathrm{H} 2 \mathrm{~S} 1$ & 0.4351 & 0.4347 & 0.3491 & 0.151 \\
\hline $\mathrm{H} 2 \mathrm{~S} 2$ & 0.4826 & 0.5500 & 0.3687 & 0.151 \\
\hline H3S1 & 0.4035 & 0.5453 & 0.4865 & 0.122 \\
\hline H3S2 & 0.3664 & 0.4303 & 0.4700 & 0.122 \\
\hline
\end{tabular}


Table S66. Torsion Angles, ${ }^{\circ}$, for $\left[\left(\mathrm{L}_{\mathrm{re}-2}^{1} \mathrm{H}\right)(\mathrm{Cl}) \mathrm{Fe}(\mathrm{II})(\mu-\mathrm{Cl})_{2} \mathrm{Fe}(\mathrm{II})(\mathrm{Cl})\left(\mathrm{L}_{\mathrm{re}-2} \mathrm{H}\right)\right](9)$

\begin{tabular}{lrlr} 
N1-Fe1-C11-Fe1 & \multicolumn{1}{c}{$-91.1(3)$} & C23-C18-C19-C20 & $0.9(15)$ \\
N2-Fe1-C11-Fe1 & $-169.1(3)$ & C18-C19-C20-C21 & $2.6(17)$ \\
C12-Fe1-C11-Fe1 & $86.23(17)$ & C19-C20-C21-C22 & $-3.3(17)$ \\
C11-Fe1-C11-Fe1 & 0.0 & C20-C21-C22-C23 & $0.5(15)$ \\
N2-Fe1-N1-C1 & $16.9(7)$ & C21-C22-C23-C18 & $3.1(15)$ \\
Cl2-Fe1-N1-C1 & $113.9(7)$ & C21-C22-C23-N4 & $-179.7(9)$ \\
C11-Fe1-N1-C1 & $-68.7(7)$ & N3-C18-C23-C22 & $177.1(9)$ \\
C11-Fe1-N1-C1 & $-154.0(7)$ & C19-C18-C23-C22 & $-3.7(14)$ \\
N2-Fe1-N1-C45 & $-174.6(8)$ & N3-C18-C23-N4 & $-0.2(14)$ \\
C12-Fe1-N1-C45 & $-77.7(8)$ & C19-C18-C23-N4 & $179.0(9)$ \\
C11-Fe1-N1-C45 & $99.8(8)$ & C24-N4-C23-C22 & $-99.9(11)$ \\
C11-Fe1-N1-C45 & $14.4(8)$ & C40-N4-C23-C22 & $42.7(13)$ \\
N1-Fe1-N2-C2 & $-14.6(7)$ & C24-N4-C23-C18 & $77.3(12)$ \\
C12-Fe1-N2-C2 & $-137.8(7)$ & C40-N4-C23-C18 & $-140.0(9)$ \\
C11-Fe1-N2-C2 & $93.4(7)$ & C40-N4-C24-C25 & $-129.3(10)$ \\
C11-Fe1-N2-C2 & $32.5(15)$ & C23-N4-C24-C25 & $11.7(14)$ \\
N1-Fe1-N2-C3 & $166.3(8)$ & C40-N4-C24-C29 & $52.0(13)$ \\
C12-Fe1-N2-C3 & $43.1(8)$ & C23-N4-C24-C29 & $-166.9(10)$ \\
C11-Fe1-N2-C3 & $-85.8(7)$ & C29-C24-C25-C26 & $-1.8(16)$ \\
C11-Fe1-N2-C3 & $-146.7(9)$ & N4-C24-C25-C26 & $179.6(10)$ \\
C45-N1-C1-C12 & $-0.8(16)$ & C24-C25-C26-C27 & $1.1(17)$ \\
\hline
\end{tabular}




\begin{tabular}{|c|c|c|c|}
\hline Fe1-N1-C1-C12 & $167.9(8)$ & $\mathrm{C} 25-\mathrm{C} 26-\mathrm{C} 27-\mathrm{C} 28$ & $-0.2(16)$ \\
\hline C45-N1-C1-C2 & $174.5(9)$ & C25-C26-C27-C30 & $179.5(10)$ \\
\hline Fe1-N1-C1-C2 & $-16.8(11)$ & C26-C27-C28-C29 & $0.1(15)$ \\
\hline $\mathrm{C} 3-\mathrm{N} 2-\mathrm{C} 2-\mathrm{C} 10$ & $9.1(16)$ & C30-C27-C28-C29 & $-179.6(11)$ \\
\hline Fe1-N2-C2-C10 & $-170.1(8)$ & C27-C28-C29-C24 & $-0.8(16)$ \\
\hline $\mathrm{C} 3-\mathrm{N} 2-\mathrm{C} 2-\mathrm{C} 1$ & $-170.8(9)$ & C25-C24-C29-C28 & $1.6(15)$ \\
\hline $\mathrm{Fe} 1-\mathrm{N} 2-\mathrm{C} 2-\mathrm{C} 1$ & $10.0(11)$ & N4-C24-C29-C28 & $-179.8(9)$ \\
\hline $\mathrm{N} 1-\mathrm{C} 1-\mathrm{C} 2-\mathrm{N} 2$ & $4.4(13)$ & C28-C27-C30-C31 & $61.9(15)$ \\
\hline $\mathrm{C} 12-\mathrm{C} 1-\mathrm{C} 2-\mathrm{N} 2$ & $-179.9(8)$ & C26-C27-C30-C31 & $-117.7(13)$ \\
\hline $\mathrm{N} 1-\mathrm{C} 1-\mathrm{C} 2-\mathrm{C} 10$ & $-175.5(8)$ & C28-C27-C30-C32 & $-174.6(12)$ \\
\hline $\mathrm{C} 12-\mathrm{C} 1-\mathrm{C} 2-\mathrm{C} 10$ & $0.2(14)$ & C26-C27-C30-C32 & $5.8(16)$ \\
\hline $\mathrm{C} 2-\mathrm{N} 2-\mathrm{C} 3-\mathrm{C} 8$ & $-134.1(11)$ & C28-C27-C30-C39 & $-55.9(15)$ \\
\hline $\mathrm{Fe} 1-\mathrm{N} 2-\mathrm{C} 3-\mathrm{C} 8$ & $45.0(13)$ & C26-C27-C30-C39 & $124.4(12)$ \\
\hline $\mathrm{C} 2-\mathrm{N} 2-\mathrm{C} 3-\mathrm{C} 4$ & $49.6(15)$ & C13-C14-C33-C34 & $2(2)$ \\
\hline Fe1-N2-C3-C4 & $-131.3(9)$ & C14-C33-C34-C17 & $-2(2)$ \\
\hline $\mathrm{C} 8-\mathrm{C} 3-\mathrm{C} 4-\mathrm{C} 5$ & $0.6(17)$ & C14-C33-C34-C35 & $177.5(14)$ \\
\hline $\mathrm{N} 2-\mathrm{C} 3-\mathrm{C} 4-\mathrm{C} 5$ & $177.0(10)$ & C16-C17-C34-C33 & $2(2)$ \\
\hline C3-C4-C5-C6 & $-0.8(18)$ & C16-C17-C34-C35 & $-177.7(12)$ \\
\hline $\mathrm{C} 4-\mathrm{C} 5-\mathrm{C} 6-\mathrm{C} 7$ & $0.6(18)$ & C33-C34-C35-C36 & $-106.5(15)$ \\
\hline C4-C5-C6-C9 & $176.1(12)$ & C17-C34-C35-C36 & $73.0(17)$ \\
\hline C5-C6-C7-C8 & $-0.2(19)$ & C33-C34-C35-C37 & $12.9(19)$ \\
\hline C9-C6-C7-C8 & $-175.3(13)$ & C17-C34-C35-C37 & $-167.6(12)$ \\
\hline
\end{tabular}




\begin{tabular}{|c|c|c|c|}
\hline $\mathrm{C} 6-\mathrm{C} 7-\mathrm{C} 8-\mathrm{C} 3$ & $0.0(19)$ & C33-C34-C35-C38 & $131.3(15)$ \\
\hline $\mathrm{C} 4-\mathrm{C} 3-\mathrm{C} 8-\mathrm{C} 7$ & $-0.2(17)$ & C17-C34-C35-C38 & $-49.2(19)$ \\
\hline $\mathrm{N} 2-\mathrm{C} 3-\mathrm{C} 8-\mathrm{C} 7$ & $-176.7(10)$ & C24-N4-C40-C41 & $27.9(13)$ \\
\hline C5-C6-C9-C42 & $156(2)$ & C23-N4-C40-C41 & $-114.5(11)$ \\
\hline C7-C6-C9-C42 & $-29(3)$ & C24-N4-C40-C45 & $-152.8(9)$ \\
\hline C5-C6-C9-C43 & $29(2)$ & C23-N4-C40-C45 & $64.9(11)$ \\
\hline C7-C6-C9-C43 & $-156.1(18)$ & C45-C40-C41-C49 & $-2.5(14)$ \\
\hline C5-C6-C9-C50 & $-86.0(19)$ & N4-C40-C41-C49 & $176.8(9)$ \\
\hline C7-C6-C9-C50 & $89(2)$ & $\mathrm{C} 41-\mathrm{C} 40-\mathrm{C} 45-\mathrm{C} 46$ & $4.7(14)$ \\
\hline $\mathrm{N} 2-\mathrm{C} 2-\mathrm{C} 10-\mathrm{C} 11$ & $-178.6(10)$ & N4-C40-C45-C46 & $-174.7(8)$ \\
\hline $\mathrm{C} 1-\mathrm{C} 2-\mathrm{C} 10-\mathrm{C} 11$ & $1.3(15)$ & $\mathrm{C} 41-\mathrm{C} 40-\mathrm{C} 45-\mathrm{N} 1$ & $-169.2(9)$ \\
\hline C2-C10-C11-C48 & $-1.5(16)$ & N4-C40-C45-N1 & $11.4(13)$ \\
\hline $\mathrm{N} 1-\mathrm{C} 1-\mathrm{C} 12-\mathrm{C} 48$ & $173.5(10)$ & C1-N1-C45-C46 & $79.7(13)$ \\
\hline $\mathrm{C} 2-\mathrm{C} 1-\mathrm{C} 12-\mathrm{C} 48$ & $-1.7(14)$ & Fe1-N1-C45-C46 & $-87.8(11)$ \\
\hline C18-N3-C13-C14 & $18(2)$ & C1-N1-C45-C40 & $-106.6(11)$ \\
\hline C18-N3-C13-C16 & $-161.3(11)$ & Fe1-N1-C45-C40 & $85.9(10)$ \\
\hline C16-C13-C14-C33 & $-1(2)$ & $\mathrm{C} 40-\mathrm{C} 45-\mathrm{C} 46-\mathrm{C} 47$ & $-4.1(15)$ \\
\hline N3-C13-C14-C33 & $179.6(13)$ & N1-C45-C46-C47 & $169.6(9)$ \\
\hline C14-C13-C16-C17 & $1.0(19)$ & C45-C46-C47-C49 & $1.5(16)$ \\
\hline N3-C13-C16-C17 & $-179.8(12)$ & C1-C12-C48-C11 & $1.6(15)$ \\
\hline C13-C16-C17-C34 & $-1(2)$ & C10-C11-C48-C12 & $0.1(16)$ \\
\hline C13-N3-C18-C23 & $-152.5(11)$ & C46-C47-C49-C41 & $0.7(16)$ \\
\hline
\end{tabular}


C13-N3-C18-C19

N3-C18-C19-C20
28.4(17)

$-179.9(10)$
C40-C41-C49-C47

C1S-C2S-C3S-C3S
$-0.2(15)$

4(4) 